Supporting information for:

\title{
Enantio- and Regioselective Hydrogenation of Di- and Trisubstituted Cycloalkenes
}

Byron K. Peters, Jianguo Liu, Cristiana Margarita, Wangchuk Rabten, Sutthichat Kerdphon, Alexander Orebom, Thomas Morsch and Pher G. Andersson*

Department of Organic Chemistry, Stockholm University, S-10691 Stockholm, Sweden

\section{Table of contents:}

General methods....................................................................................................................... 2

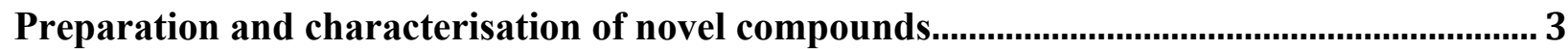

Preparation of aromatic substrates....................................................................................................3

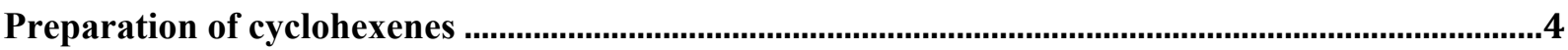

General Procedure for the Birch reduction .............................................................................................

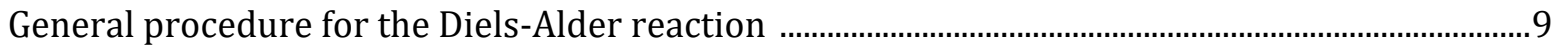

General Procedure for asymmetric hydrogenations....................................................................11

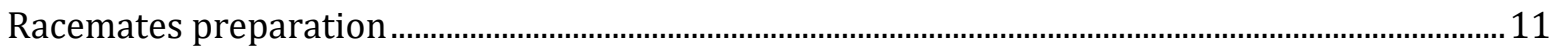

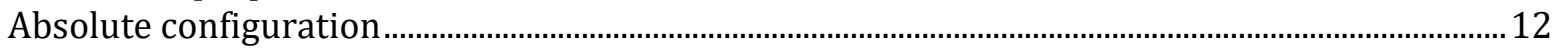

Catalysts.......................................................................................................................................20

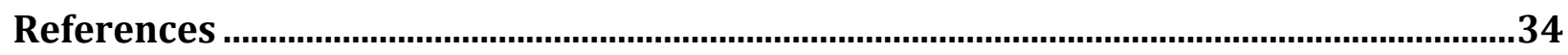

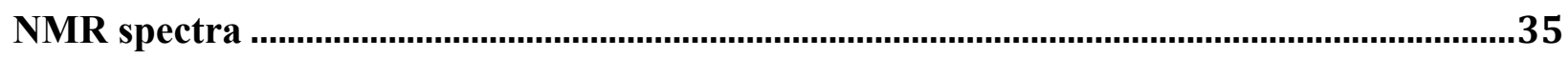

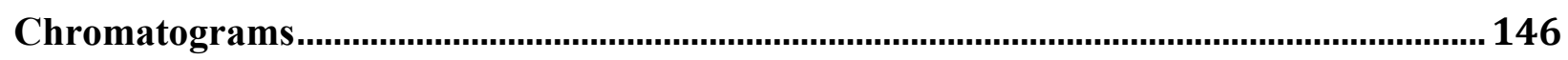




\section{General methods}

All reactions were conducted under dry nitrogen or argon atmosphere using magnetic stirring. $\mathrm{CH}_{2} \mathrm{Cl}_{2}, i-$ PrOH and $t$-BuOH were freshly distilled from $\mathrm{CaH}_{2}$ under nitrogen, THF was freshly distilled from Na and benzophenone under Ar prior to use. All reagents were purchased from commercial suppliers and used without further purification. Li (99.9\%) was purchased from Sigma-Aldrich as a wire with $0.01 \%$ $\mathrm{Na}$.

Chromatographic separations were performed on Kiesel gel $60 \mathrm{H}$ silica gel (particle) size: 0.063-0.100 $\mathrm{mm}$ ). Thin layer chromatography (TLC) was performed on aluminium plates coated with Kieselgel 60 $(0.20 \mathrm{~mm}$, UV254) and visualized under ultraviolet light $(\square=254 \mathrm{~nm})$, or by staining with ethanolic phosphomolybdic acid or Hanessian's stain and heating.

${ }^{1} \mathrm{H}$ NMR spectra were recorded at 500 or $400 \mathrm{MHz}$ in $\mathrm{CDCl}_{3}$ at $25^{\circ} \mathrm{C}$ and referenced internally to the residual $\mathrm{CHCl}_{3}$ peak $(7.26 \mathrm{ppm})$. Prior to use, $\mathrm{CDCl}_{3}$ was neutralised by passage through a short plug of basic alumina. ${ }^{13} \mathrm{C}$ NMR spectra were recorded at 125 or $100 \mathrm{MHz}$ in $\mathrm{CDCl}_{3}$ at $25^{\circ} \mathrm{C}$ and referenced to the central peak of $\mathrm{CDCl}_{3}(77.16 \mathrm{ppm}) .{ }^{31} \mathrm{P}$ NMR spectra were recorded at $121 \mathrm{MHz}$ in $\mathrm{CDCl}_{3}$ or $\mathrm{C}_{6} \mathrm{D}_{6}$. Chemical shifts are reported in ppm ( $\delta$ scale $)$.

Optical rotations were recorded on a thermostated polarimeter using a sodium lamp (589 nm) and a 1.0 $\mathrm{dm}$ cell. IR spectra were measured using an FT-IR apparatus. Enantiomeric excesses were determined using GC (30 m columns, Helium gas carrier at $1 \mathrm{~mL} / \mathrm{min}$, constant pressure) with a MS detector or SFC (250mm Chiralcel columns, $\mathrm{CO}_{2}: \mathrm{MeOH}$ ) with $\mathrm{UV}$ and $\mathrm{MS}$ detectors (see data for individual compounds for details). Racemic compounds were used for comparison.

HRMS was performed on a Bruker micrOTOF with an ESI source and a Waters GCT Premier with a CI source. 


\section{Preparation and characterisation of novel compounds}

\section{Preparation of aromatic substrates}

The following compounds were prepared according to reported procedures:<smiles>Oc1ccc2[nH]ccc2c1</smiles><smiles>CC(C)C(O)c1ccc2ccccc2c1</smiles><smiles>CCCCc1ccc2[nH]ccc2c1</smiles>

5-butyl-1H-indole. The $t$-BuLi solution ( $16 \mathrm{mmol}, 3.2$ equiv., $1.7 \mathrm{M}$ in pentane) was added drop-wise under nitrogen over a period of $0.5 \mathrm{~h}$ to a stirred suspension of substrate $(5 \mathrm{mmol}$, 1 equiv.) in dry THF at $-78^{\circ} \mathrm{C}$ and the resulting mixture was warmed to rt. and stirred for $0.5 \mathrm{~h}$. Then the resulting mixture was cooled to $-78^{\circ} \mathrm{C}$. After adding $n-\mathrm{BuBr}(5.5 \mathrm{mmol}, 1.1$ equiv.) dropwise, the resulting mixture was stirred and let warm from $-78^{\circ} \mathrm{C}$ to room temperature overnight. The reaction was quenched with $\mathrm{NH}_{4} \mathrm{Cl}$, and extracted with $\mathrm{Et}_{2} \mathrm{O}(3 \times 50 \mathrm{~mL})$ three times. The combined organic layers were washed with brine and dried over $\mathrm{MgSO}_{4}$. After concentration under vacuum, the residue was purified by flash chromatography on silica gel using pentane and ethyl acetate as eluent to afford yellow oil, 54\% yield. $\mathrm{R}_{f}=0.64$ (Pentane/EtOAc=7/1). ${ }^{1} \mathbf{H}$ NMR $\left(\mathrm{CDCl}_{3}, 400 \mathrm{MHz}\right): \delta$ 7,99 (bs, 1H), 7,48 (s, 1H), 7,32 - 7,30 (m, 1H), $7,17-7,16(\mathrm{~m}, 1 \mathrm{H}), 7,09-7,07(\mathrm{~m}, 1 \mathrm{H}), 6.53-6.51(\mathrm{~m}, 1 \mathrm{H}), 2.75(\mathrm{t}, J=7.8 \mathrm{~Hz}, 2 \mathrm{H}), 1.72-1.66(\mathrm{~m}$, $2 \mathrm{H}), 1.46-1.39(\mathrm{~m}, 2 \mathrm{H}), 0.98(\mathrm{t}, J=7.4 \mathrm{~Hz}, 3 \mathrm{H}) .{ }^{13} \mathbf{C} \mathbf{N M R}\left(\mathrm{CDCl}_{3}, 100 \mathrm{MHz}\right): \delta 134.36,128.06$, 124.25, 123.13, 119.82, 110.71, 102.24, 35.78, 34.51, 22.44, 14.07. IR $\left(\mathrm{CHCl}_{3}\right.$, neat, $\left.\mathrm{cm}^{-1}\right): v=3411$, 3016, 2928, 1864, 1707, 1578, 1474, 1415, 1334, 1264, 1091, 893, 799, 724. HRMS m/z: $[\mathrm{M}+\mathrm{H}]^{+}$Calc. for $\mathrm{C}_{12} \mathrm{H}_{15} \mathrm{~N}_{1}$ 173.1205; Found 173.1208.<smiles>COc1cc(OCCc2cccc(C)c2)cc(OC)c1OC</smiles>

1,2,3-trimethoxy-5-(3-methylphenethoxy)benzene was prepared from 3,4,5-trimethoxyphenol and 3methylphenethyl alcohol adapting a reported Mitsunobu procedure. ${ }^{3}$ White solid $\left(\mathrm{mp}=64-68{ }^{\circ} \mathrm{C}\right), 72 \%$ yield. $\mathrm{R}_{f}=0.77$ (Pentane:AcOEt, 5:1). ${ }^{1} \mathbf{H}$ NMR $\left(\mathrm{CDCl}_{3}, 400 \mathrm{MHz}\right): \delta 7.23(\mathrm{t}, J=7.5 \mathrm{~Hz}, 1 \mathrm{H}), 7.14-$ $7.04(\mathrm{~m}, 3 \mathrm{H}), 6.16(\mathrm{~s}, 2 \mathrm{H}), 4.14(\mathrm{t}, J=7.3 \mathrm{~Hz}, 2 \mathrm{H}), 3.83(\mathrm{~s}, 6 \mathrm{H}), 3.80(\mathrm{~s}, 3 \mathrm{H}), 3.07$ (t, $J=7.3 \mathrm{~Hz}, 2 \mathrm{H})$, 2.37 (s, 3H). ${ }^{13} \mathbf{C ~ N M R}\left(\mathrm{CDCl}_{3}, 100 \mathrm{MHz}\right): \delta$ 155.58, 153.77, 138.22, 138.04, 132.39, 129.88, 128.53, $127.40,126.09,92.40,69.32,61.10,56.15,35.92,21.49$. IR $\left(\mathrm{NaCl}\right.$, neat, $\left.\mathrm{cm}^{-1}\right): v=2998,2938,2839$, 1594, 1506 1464, 1422, 1389, 1346, 1227, 1195, 1153, 1129, 1053, 1010, 811, 781, 702, 629. HRMSESI m/z: $[\mathrm{M}+\mathrm{Na}]^{+}$Calc. for $\mathrm{C}_{18} \mathrm{H}_{22} \mathrm{O}_{4} \mathrm{Na} 325.1410$; Found 325.1424 .<smiles>CCCCCc1ccc2c(c1)[nH]c1cc(CCCC)ccc12</smiles>

3,6-dibutyl-4a,9a-dihydro-9H-carbazole. A solution of 2,7-dibromocarbazole in dry THF was cooled down to $-78^{\circ} \mathrm{C}$ and $t$-BuLi (5 equiv) was added slowly. The resulting mixture was stirred for $10 \mathrm{~min}$, then warmed to room temperature and stirred for another $20 \mathrm{~min}$. It was then cooled down to $-78^{\circ} \mathrm{C}$ and $n$ - 
$\mathrm{BuBr}$ (2.2 equiv) was added slowly, the mixture then stirred at room temperature overnight. Reaction was quenched with sat. aq. $\mathrm{NH}_{4} \mathrm{Cl}$ and extracted $(3 \times 30 \mathrm{ml})$ with $\mathrm{Et}_{2} \mathrm{O}$. The organic layers were combined and dried over $\mathrm{Na}_{2} \mathrm{SO}_{4}$. After concenttration under vacuum, the residue was purifed by flash chromatography on silica gel using 5\% EtOAc in pentane to give the product as a white solid $\left(\mathrm{mp}=210-214{ }^{\circ} \mathrm{C}\right), 40 \%$ yield. $\mathrm{R}_{\mathrm{f}}=0.53(\mathrm{EtOAc} /$ Pentane $=1 / 9) .{ }^{1} \mathbf{H} \mathbf{~ N M R}\left(\mathrm{CDCl}_{3}, 400 \mathrm{MHz}\right): \delta 7.91(\mathrm{~d}, J=7.9 \mathrm{~Hz}, 2 \mathrm{H}), 7.82(\mathrm{~s}$, $1 \mathrm{H}), 7.19(\mathrm{~s}, 2 \mathrm{H}), 7.05(\mathrm{dd}, J=8.0,1.2 \mathrm{~Hz}, 2 \mathrm{H}), 2.77(\mathrm{~m}, 4 \mathrm{H}), 1.75-1.63(\mathrm{~m}, 4 \mathrm{H}), 1.47-1.34(\mathrm{~m}, 4 \mathrm{H})$, $0.95(\mathrm{t}, J=7.4 \mathrm{~Hz}, 6 \mathrm{H}) .{ }^{13} \mathbf{C}$ NMR $\left(\mathrm{CDCl}_{3}, 100 \mathrm{MHz}\right): \delta 140.74,140.10,121.52,120.40,119.79,110.15$, $36.35,34.29,22.58,14.17$. IR $\left(\mathrm{KBr}\right.$, neat $\left.\mathrm{cm}^{-1}\right): v=3397,2956,2955,2855,1886,1612,1462,1435$, 1322, 1244, 1146, 864, 813. HRMS-EI m/z: [M+Na] ${ }^{+}$Calc. for $\mathrm{C}_{20} \mathrm{H}_{25} \mathrm{NNa}$ 302.1879; Found 302.1882.

\section{Preparation of cyclohexenes}

The following compounds were prepared according to reported procedures:<smiles>CC1=CCC(C)=C(C)C1</smiles>

$18 \mathrm{a}$<smiles>CC1=CCC2=C(CC=CC2)C1</smiles>

2a<smiles>CC1=CCc2[nH]ccc2C1</smiles>

$35 a$<smiles>COC1=CCc2[nH]ccc2C1</smiles>

38a<smiles>COC1=CCc2cc[nH]c2C1</smiles>

$39 a$<smiles>COC1=CCc2c([nH]c3ccccc23)C1</smiles>

9

\section{General Procedure for the Birch reduction}

The reactions were conducted in a 3-necked round-bottomed flask with a dry ice condenser, an $\mathrm{NH}_{3}(\mathrm{~g})$ inlet, and a stopper for $\mathrm{Li}$ or $\mathrm{Na}$ addition. Ammonia was condensed from a commercial $\mathrm{NH}_{3}$ tube into a mixture of: aromatic substrate, alcohol and cosolvent (when used), while cooling the flask in a dry ice/acetone bath. Alcohols used were $t$ - $\mathrm{BuOH}, \mathrm{EtOH}, i-\mathrm{PrOH}, t$-amylOH or $\mathrm{MeOH}$. In some cases a cosolvent was needed $\left(\mathrm{Et}_{2} \mathrm{O}\right.$ or THF) to keep the reaction stirrable in the beginning of the ammonia condensation. Addition of the metal was done at reflux temperature of $\mathrm{NH}_{3}$, with such a speed so as to prevent vigorous reaction/foaming. On discoloration of the reaction mixture, dry ice was removed from the condenser and ammonia was evaporated. If unreacted $\mathrm{Li}$ or $\mathrm{Na}$ was present, it was quenched with $95 \% \mathrm{EtOH}$, or solid $\mathrm{NH}_{4} \mathrm{Cl}$. The reaction was carefully diluted with $\mathrm{H}_{2} \mathrm{O}$, extracted with $\mathrm{Et}_{2} \mathrm{O}$ or pentane three times, the combined organic extracts were washed with brine, and dried $\left(\mathrm{Na}_{2} \mathrm{SO}_{4}\right)$. The solvent was removed and products were purified either by distillation under reduced pressure or by chromatography on dry silica gel (deactivated with $\mathrm{Et}_{3} \mathrm{~N}$ in the case of enol ethers). Products were stable for several months under argon or nitrogen at $-20^{\circ} \mathrm{C}$. At $\mathrm{rt}$ the methoxy enolate dienes disproportionated to a noticeable extent to aromatic starting material and reduced products after weeks.<smiles>[Mg]C1=CCc2[nH]ccc2C1</smiles>

5-hexyl-4,7-dihydro- $1 \mathrm{H}$-indole. According to the general procedure, the following amounts were used: 5-hexyl-1H-indole (107 mg, $0.53 \mathrm{mmol}, 1$ equiv). Ammonia was condensed to a total volume of $10 \mathrm{~mL}$. Li $\left(20 \mathrm{mg}, 3 \mathrm{mmol}, 6\right.$ equiv) was added over $10 \mathrm{~min}$. After stirring at $-30^{\circ} \mathrm{C}$ for 1 hour, the reaction was quenched with $\mathrm{MeOH}$. After general workup and chromatography, the product was obtained as a yellow oil, 31\% yield. $\mathrm{R}_{f}=0.39$ (Pentane/EtOAc=20/1). ${ }^{1} \mathbf{H}$ NMR $\left(\mathrm{CDCl}_{3}, 400 \mathrm{MHz}\right): \delta 7.78(\mathrm{bs}, 1 \mathrm{H}), 6.70(\mathrm{~s}$, 
$1 \mathrm{H}), 6.03(\mathrm{~s}, 1 \mathrm{H}), 5.57-5.55(\mathrm{~m}, 1 \mathrm{H}), 3.27-3.25(\mathrm{~m}, 2 \mathrm{H}), 3.17-3.14(\mathrm{~m}, 2 \mathrm{H}), 2.13(\mathrm{t}, J=7.8 \mathrm{~Hz}, 2 \mathrm{H})$, $1.50-1.44(\mathrm{~m}, 2 \mathrm{H}), 1.35-1.27(\mathrm{~m}, 9 \mathrm{H}), 0.90(\mathrm{t}, J=7.4 \mathrm{~Hz}, 3 \mathrm{H}) .{ }^{13} \mathbf{C} \mathbf{~ N M R}\left(\mathrm{CDCl}_{3}, 100 \mathrm{MHz}\right): \delta$ 137.13, 124.36, 116.81, 116.38, 114.56, 106.57, 37.74, 31.84, 29.05, 27.97, 27.81, 24.31, 22.68, 14.15. IR $\left(\mathrm{CHCl}_{3}\right.$, neat, $\left.\mathrm{cm}^{-1}\right): v=3375,2926,2275,1596,1464,1377,1218,1076,944,829,712 . \mathbf{H R M S ~ m} / \mathrm{z}$ : $[\mathrm{M}+\mathrm{H}]^{+}$Calc. for $\mathrm{C}_{14} \mathrm{H}_{21} \mathrm{~N}_{1}$ 203.1674; Found 203.1685.

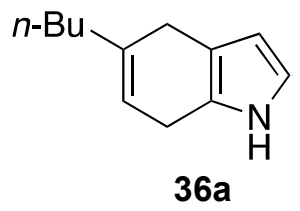

5-butyl-4,7-dihydro-1H-indole. According to the general procedure, the following amounts were used: 5-butyl-1H-indole (540 mg, $3.12 \mathrm{mmol}, 1$ equiv), Ammonia was condensed to a total volume of $50 \mathrm{ml}$. Li ( $86 \mathrm{mg}, 12.5 \mathrm{mmol}, 4$ equiv) was added over $30 \mathrm{~min}$. After general workup and chromatography, the product was obtained as a colorless oil, $68 \%$ yield. $\mathrm{R}_{f}=0.39$ (Pentane/EtOAc $\left.=15 / 1\right) .{ }^{1} \mathbf{H}$ NMR $\left(\mathrm{CDCl}_{3}\right.$, $400 \mathrm{MHz}): \delta 7.76(\mathrm{~s}, 1 \mathrm{H}), 6.71(\mathrm{~s}, 1 \mathrm{H}), 6.04(\mathrm{~s}, 1 \mathrm{H}), 5.58-5.55(\mathrm{~m}, 1 \mathrm{H}), 3.37-3.21(\mathrm{~m}, 2 \mathrm{H}), 3.21-$ $3.10(\mathrm{~m}, 2 \mathrm{H}), 2.15-2.10(\mathrm{~m}, 2 \mathrm{H}), 1.49-1.45(\mathrm{~m}, 2 \mathrm{H}), 1.37-1.33(\mathrm{~m}, 2 \mathrm{H}), 0.99-0.82(\mathrm{~m}, 3 \mathrm{H}) .{ }^{13} \mathrm{C}$ NMR $\left(\mathrm{CDCl}_{3}, 100 \mathrm{MHz}\right): \delta 137.11,124.36,116.83,116.40,114.56,106.58,37.42,30.19,27.83,24.31$, 22.42, 14.03. IR $\left(\mathrm{CHCl}_{3}\right.$, neat, $\left.\mathrm{cm}^{-1}\right): v=3372,2927,1667,1466,1218,1095,947,830,713 . \mathbf{H R M S ~ m} / \mathrm{z}$ : $[\mathrm{M}+\mathrm{H}]^{+}$Calc. for $\mathrm{C}_{12} \mathrm{H}_{17} \mathrm{~N}_{1}$ 176.1434; Found 176.1401.<smiles>COC1=CCC2=C(CC=CC2)C1</smiles>

24a

2-Methoxy-1,4,5,8-tetrahydronaphthalene. ${ }^{10}$ According to the general procedure, the following amounts were used: 2-methoxynaphthalene (1.00 g, $6.32 \mathrm{mmol}, 1$ equiv), THF (5 mL) and EtOH (4 mL, 10 equiv). Ammonia was condensed to a total volume of $50 \mathrm{~mL}$. Li (250 mg, $36.03 \mathrm{mmol}, 5.7$ equiv) was added over $10 \mathrm{~min}$. After general workup and chromatography, the product was obtained as a colorless oil. $88 \%$ yield. $\mathrm{R}_{f}=0.56$ (Pentane). ${ }^{1} \mathbf{H}$ NMR $\left(\mathrm{CDCl}_{3}, 400 \mathrm{MHz}\right): \delta 5.73(\mathrm{~s}, 2 \mathrm{H}), 4.64(\mathrm{t}, J=3.5 \mathrm{~Hz}, 1 \mathrm{H})$, $3.56(\mathrm{~s}, 3 \mathrm{H}), 2.72-2.63(\mathrm{~m}, 2 \mathrm{H}), 2.62-2.52(\mathrm{~m}, 6 \mathrm{H}) .{ }^{13} \mathbf{C} \mathbf{~ N M R}\left(100 \mathrm{MHz}, \mathrm{CDCl}_{3}\right) \delta 153.0,124.6$, 124.2, 123.7, 122.6, 90.6, 53.9, 33.2, 31.4, 30.9, 30.4. IR $\left(\mathrm{NaCl}\right.$, neat, $\left.\mathrm{cm}^{-1}\right): v=3028,2936,2879,2814$, 1715, 1677, 1660, 1434, 1393, 1371, 1218, 1181, 1160, 1124, 1106, 1016, 971, 780, 658. HRMS-EI = $\mathrm{m} / \mathrm{z}$ : $[\mathrm{M}+\mathrm{H}]^{+}$Calc. for $\mathrm{C}_{11} \mathrm{H}_{15} \mathrm{O}$ 163.1123; Found 163.1119.

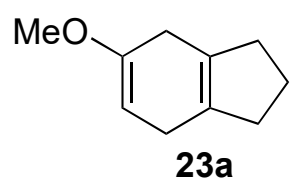

5-methoxy-2,3,4,7-tetrahydro- $1 \mathrm{H}$-indene. ${ }^{11}$ According to the general procedure, the following amounts were used: 5-methoxy-indane $(0.5 \mathrm{~g}, 3.37 \mathrm{mmol}, 1$ equiv) and $i$-PrOH $(5 \mathrm{~mL})$. Ammonia was condensed to a total volume of $15 \mathrm{~mL}$. Li (124 mg, $17.86 \mathrm{mmol}, 5.3$ equiv) was added over $10 \mathrm{~min}$. After general workup and chromatography, the product was obtained as a colorless oil. $76 \%$ yield. $\mathrm{R}_{f}=0.46$ (Pentane). ${ }^{1} \mathrm{H}$ NMR $\left(\mathrm{CDCl}_{3}, 400 \mathrm{MHz}\right): \delta 4.68(\mathrm{t}, J=3.1 \mathrm{~Hz}, 1 \mathrm{H}), 3.57(\mathrm{~s}, 3 \mathrm{H}), 2.75-2.66(\mathrm{~m}, 4 \mathrm{H}), 2.30-2.26$ $(\mathrm{m}, 4 \mathrm{H}), 1.94-1.86(\mathrm{~m}, 2 \mathrm{H}) .{ }^{13} \mathbf{C}$ NMR $\left(\mathrm{CDCl}_{3}, 100 \mathrm{MHz}\right): \delta 154.16,132.43,131.72,91.23,54.32$, 35.37, 35.05, 30.17, 26.97, 22.44. IR (NaCl, neat, $\left.\mathrm{cm}^{-1}\right): v=3055,2994,2948,2888,2841,2824,1704$, 1661, 1466, 1445, 1398, 1368, 1318, 1300, 1219, 1181, 1151, 1134, 1081, 1032, 1002, 909, 783, 713. HRMS-ESI m/z: [M+Na] $]^{+}$Calc. for $\mathrm{C}_{10} \mathrm{H}_{14} \mathrm{ONa}$ 173.0937; Found 173.0935.<smiles>CCCOCCC1=CCC=C(C)C1</smiles>

\section{$27 a$}

tert-butyldimethyl(2-(5-methylcyclohexa-1,4-dien-1-yl)ethoxy)silane was prepared from 2-(5methylcyclohexa-1,4-dien-1-yl)ethan-1-ol adapting a reported procedure. ${ }^{12}$ Colorless oil, $82 \%$ yield. $\mathrm{R}_{f}=$ 0.69 (Pentane:AcOEt; 99:1). ${ }^{1} \mathbf{H}$ NMR $\left(\mathrm{CDCl}_{3}, 400 \mathrm{MHz}\right): \delta 5.48-5.46(\mathrm{~m}, 1 \mathrm{H}), 5.43-5.40(\mathrm{~m}, 1 \mathrm{H})$, $3.73(\mathrm{t}, J=7.1 \mathrm{~Hz}, 2 \mathrm{H}), 2.74-2.64(\mathrm{~m}, 2 \mathrm{H}), 2.54(\mathrm{t}, J=7.7 \mathrm{~Hz}, 2 \mathrm{H}), 2.23(\mathrm{t}, J=7.1 \mathrm{~Hz}, 2 \mathrm{H}), 1.70(\mathrm{~s}$, 
3H), 0.92 (s, 9H), 0.07 (s, 6H). ${ }^{13} \mathbf{C}$ NMR $\left(\mathrm{CDCl}_{3}, 100 \mathrm{MHz}\right): \delta 132.34,131.34,120.12,118.50,62.44$, $40.78,34.60,27.87,26.10,23.35,18.50,-5.13$. IR $\left(\mathrm{NaCl}\right.$, neat, $\left.\mathrm{cm}^{-1}\right): v=2956,2929,2884,2857,2820$, 1472, 1463, 1447, 1388, 1361, 1255, 1099, 1058, 1006, 964, 940, 836, 775, 662. HRMS-ESI m/z: $[\mathrm{M}+\mathrm{Na}]^{+}$Calc. for $\mathrm{C}_{15} \mathrm{H}_{28} \mathrm{ONaSi} 275.1807$; Found 275.1819.<smiles>CC1=CCC=C(CCO)C1</smiles>

2-(5-methylcyclohexa-1,4-dien-1-yl)ethan-1-ol. According to the general procedure, the following amounts were used: 3-methylphenethyl alcohol $(500 \mathrm{mg}, 3.67 \mathrm{mmol}, 1$ equiv) and $i$-PrOH $(7.3 \mathrm{~mL})$. Ammonia was condensed to a total volume of $70 \mathrm{~mL}$. Li (166 mg, $23.86 \mathrm{mmol}, 6.5$ equiv) was added over $15 \mathrm{~min}$. After general workup and chromatography, the product was obtained as a colorless oil. 74\% yield. $\mathrm{R}_{f}=0.47$ (Pentane:AcOEt; 5:1). ${ }^{1} \mathbf{H}$ NMR $\left(\mathrm{CDCl}_{3}, 400 \mathrm{MHz}\right): \delta 5.56-5.52(\mathrm{~m}, 1 \mathrm{H}), 5.42-5.38$ $(\mathrm{m}, 1 \mathrm{H}), 3.70(\mathrm{t}, J=6.3 \mathrm{~Hz}, 2 \mathrm{H}), 2.74-2.65(\mathrm{~m}, 2 \mathrm{H}), 2.50(\mathrm{t}, J=8.0 \mathrm{~Hz}, 2 \mathrm{H}), 2.26(\mathrm{t}, J=6.3 \mathrm{~Hz}, 2 \mathrm{H})$, $1.68(\mathrm{~s}, 3 \mathrm{H}) .{ }^{13} \mathrm{C}$ NMR $\left(\mathrm{CDCl}_{3}, 100 \mathrm{MHz}\right): \delta 131.41,131.08,121.39,118.48,60.12,40.39,33.77,27.81$, 23.25.IR $\left(\mathrm{NaCl}\right.$, neat $\left.\mathrm{cm}^{-1}\right): v=3337,2962,2927,2878,2818,1699,1665,1446,1427,1379,1173$, 1123, 1046, 965, 934 767. HRMS-CI m/z: [M] $]^{+}$Calc. for $\mathrm{C}_{9} \mathrm{H}_{14} \mathrm{O}$ 138.1045; Found 138.1051.<smiles>CCCC[OH+]CCCC1=CCC=C(C)C1</smiles>

tert-butyldimethyl(3-(5-methylcyclohexa-1,4-dien-1-yl)propoxy)silane was prepared from 26a adapting a reported procedure. ${ }^{12}$ Colorless oil, 92\% yield. $\mathrm{R}_{f}=0.70$ (Pentane:AcOEt; 95:5). ${ }^{1} \mathbf{H}$ NMR $\left(\mathrm{CDCl}_{3}, 400 \mathrm{MHz}\right): \delta 5.44-5.39(\mathrm{~m}, 2 \mathrm{H}), 3.61(\mathrm{t}, J=6.5 \mathrm{~Hz}, 2 \mathrm{H}), 2.71-2.64(\mathrm{~m}, 2 \mathrm{H}), 2.48(\mathrm{t}, J=8.0$ $\mathrm{Hz}, 2 \mathrm{H}), 2.02(\mathrm{t}, J=7.6 \mathrm{~Hz}, 2 \mathrm{H}), 1.70-1.61(\mathrm{~m}, 5 \mathrm{H}), 0.90(\mathrm{~s}, 9 \mathrm{H}), 0.05(\mathrm{~s}, 6 \mathrm{H}) .{ }^{13} \mathbf{C} \mathbf{~ N M R}\left(\mathrm{CDCl}_{3}, 100\right.$ MHz): $\delta 134.74,131.43,118.63,118.26,63.09,34.17,33.54,30.73,27.86,26.13,23.39,18.51,-5.10$. IR $\left(\mathrm{NaCl}\right.$, neat, $\left.\mathrm{cm}^{-1}\right): v=2954,2929,2884,2857,2820,1472,1463,1447,1387,1361,1255,1103,1007$, 966, 937, 837, 814, 775, 662. HRMS-ESI m/z: $[\mathrm{M}+\mathrm{Na}]^{+}$Calc. for $\mathrm{C}_{16} \mathrm{H}_{30} \mathrm{ONaSi}$ 289.1958; Found 289.1958 .<smiles>CC1=CCC=C(CCCO)C1</smiles>

26a

3-(5-methylcyclohexa-1,4-dien-1-yl)propan-1-ol. According to the general procedure, the following amounts were used: 3-(3-methylphenyl)propan-1-ol (490 mg, $3.26 \mathrm{mmol}, 1$ equiv) and $i$-PrOH (6.5 mL). Ammonia was condensed to a total volume of $65 \mathrm{~mL}$. Li (147 mg, $21.19 \mathrm{mmol}, 6.5$ equiv) was added over $10 \mathrm{~min}$. After general workup and chromatography, the product was obtained as a colorless oil. 71\% yield. $\mathrm{R}_{f}=0.38$ (Pentane:AcOEt; 8:1). ${ }^{1} \mathbf{H}$ NMR $\left(\mathrm{CDCl}_{3}, 400 \mathrm{MHz}\right): \delta 5.48-5.44(\mathrm{~m}, 1 \mathrm{H}), 5.42-5.38$ $(\mathrm{m}, 1 \mathrm{H}), 3.66(\mathrm{t}, J=6.5 \mathrm{~Hz}, 2 \mathrm{H}), 2.71-2.63(\mathrm{~m}, 2 \mathrm{H}), 2.49(\mathrm{t}, J=8.8 \mathrm{~Hz}, 2 \mathrm{H}), 2.07(\mathrm{t}, J=7.4 \mathrm{~Hz}, 2 \mathrm{H})$, $1.76-1.66(\mathrm{~m}, 5 \mathrm{H}) .{ }^{13} \mathbf{C} \mathbf{~ N M R}\left(\mathrm{CDCl}_{3}, 100 \mathrm{MHz}\right): \delta 134.59,131.31,118.74,118.60,62.99,34.02$, $33.63,30.39,27.84,23.35$. IR $\left(\mathrm{NaCl}\right.$, neat $\left.\mathrm{cm}^{-1}\right): v=3324,2930,2870,2848,2816,1700,1665,1446$, 1381, 1164, 1058, 1013, 965, 935, 771. HRMS-ESI m/z: $[\mathrm{M}+\mathrm{Na}]^{+}$Calc. for $\mathrm{C}_{10} \mathrm{H}_{16} \mathrm{ONa}$ 175.1093; Found 175.1074.<smiles>COc1cc(OC)cc(OCCC2=CCC=C(C)C2)c1</smiles>

$30 a$

1,3-dimethoxy-5-(2-(5-methylcyclohexa-1,4-dien-1-yl)ethoxy)benzene. According to the general procedure, the following amounts were used: 1,2,3-trimethoxy-5-(3-methylphenethoxy)benzene (100 mg, $0.33 \mathrm{mmol}, 1$ equiv), THF $(4 \mathrm{~mL})$ and $t$ - $\mathrm{BuOH}(0.8 \mathrm{~mL})$. Ammonia was condensed to a total volume of $10 \mathrm{~mL}$. Li (57 mg, $8.25 \mathrm{mmol}, 25$ equiv) was added over $10 \mathrm{~min}$. After general workup and 
chromatography, the product was obtained as a colorless oil. $30 \%$ yield. $\mathrm{R}_{f}=0.59$ (Pentane:AcOEt; 8:1). ${ }^{1} \mathbf{H}$ NMR $\left(\mathrm{CDCl}_{3}, 400 \mathrm{MHz}\right): \delta 6.10-6.06(\mathrm{~m}, 3 \mathrm{H}), 5.56-5.52(\mathrm{~m}, 1 \mathrm{H}), 5.43-5.39(\mathrm{~m}, 1 \mathrm{H}), 4.02(\mathrm{t}, J=$ $7.0 \mathrm{~Hz}, 2 \mathrm{H}), 3.76(\mathrm{~s}, 6 \mathrm{H}), 2.74-2.65(\mathrm{~m}, 2 \mathrm{H}), 2.55(\mathrm{t}, J=8.2 \mathrm{~Hz}, 2 \mathrm{H}), 2.46(\mathrm{t}, J=6.7 \mathrm{~Hz}, 2 \mathrm{H}), 1.68(\mathrm{~s}$, $3 \mathrm{H}) .{ }^{13} \mathrm{C}$ NMR $\left(\mathrm{CDCl}_{3}, 100 \mathrm{MHz}\right): \delta 161.43,160.80,131.34,131.16,120.50,118.43,93.22,92.85$, 66.42, 55.45, 36.66, 34.26, 27.78, 23.42. IR $\left(\mathrm{NaCl}\right.$, neat, $\left.\mathrm{cm}^{-1}\right): v=3447,2998,2959,2927,2877,2849$, 2818, 1719, 1663, 1602, 1509, 1471, 1447, 1429, 1385, 1205, 1194, 1155, 1067, 965, 934, 820, 770, 737, 682. HRMS-ESI m/z: [M+Na] ${ }^{+}$Calc. for $\mathrm{C}_{17} \mathrm{H}_{22} \mathrm{O}_{3} \mathrm{Na}$ 297.1461; Found 297.1461.<smiles>CC1=CCC=C(C)C1C(=O)O</smiles>

$32 a$

2,6-dimethylcyclohexa-2,5-diene-1-carboxylic acid. According to the general procedure, the following amounts were used: 2,6-dimethylbenzoic acid (1 equiv), THF $(2.5 \mathrm{~mL} / \mathrm{mmol}$ of substrate) and 2-methyl2-butanol (1.0 equiv). Ammonia was condensed $(7.5 \mathrm{~mL} / \mathrm{mmol}$ of substrate). Li (3.5 equiv) was added over $10 \mathrm{~min}$. After general workup and chromatography, the product was obtained as a white solid $(\mathrm{mp}=$ $\left.106-108^{\circ} \mathrm{C}\right), 90 \%$ yield. $\mathrm{R}_{f}=0.52(\mathrm{MeOH} / \mathrm{DCM}=0.5 / 9.5) .{ }^{1} \mathbf{H} \mathbf{~ N M R}\left(\mathrm{CDCl}_{3}, 400 \mathrm{MHz}\right): \delta 5.82-5.55$ $(\mathrm{m}, 2 \mathrm{H}), 3.70-3.25(\mathrm{~m}, 1 \mathrm{H}), 3.08-2.54(\mathrm{~m}, 2 \mathrm{H}), 1.86-1.59(\mathrm{~m}, 6 \mathrm{H}) .{ }^{13} \mathbf{C} \mathbf{~ N M R}\left(\mathrm{CDCl}_{3}, 100 \mathrm{MHz}\right): \delta$ 178.49, 128.51, 122.51, 52.23, 27.64, 21.93. IR (NaCl, neat, $\mathrm{cm}-1): v=2977,2850,1440,1239,910$, 776. HRMS (EI) m/z: [M+Na]+ Calcd for $\mathrm{C}_{9} \mathrm{H}_{11} \mathrm{Na}_{2} \mathrm{O}_{2}$ 197.0554; Found 197.0543.<smiles>CCCCC1=CCc2c([nH]c3cc(C(C)C)ccc23)C1</smiles>

3,6-dibutyl-4,9-dihydro-1H-carbazole. According to the general procedure, the following amounts were used: 2,7-dibutyl-9H-carbazole (100 mg, $0.36 \mathrm{mmol}, 1$ equiv), and EtOH (0.2 mL, $2.86 \mathrm{mmol})$. Ammonia was condensed to a total volume of $15 \mathrm{~mL}$. Li (17 mg, $2.50 \mathrm{mmol}, 7$ equiv) was added over $10 \mathrm{~min}$. After general workup and chromatography, the product was obtained as a white solid $(\mathrm{mp}=210.4-212.8), 90 \%$ yield. $\mathrm{R}_{\mathrm{f}}=0.56($ EtOAc/Pentane $=1 / 9) .{ }^{1} \mathbf{H} \mathbf{~ N M R}\left(\mathrm{CDCl}_{3}, 400 \mathrm{MHz}\right): \delta 7.59(\mathrm{~s}, 1 \mathrm{H}), 7.37(\mathrm{~d}, J=8.0 \mathrm{~Hz}$, $1 \mathrm{H}), 7.11(\mathrm{~s}, 1 \mathrm{H}), 6.94(\mathrm{dd}, J=8.0,1.4 \mathrm{~Hz}, 1 \mathrm{H}), 5.77-5.72(\mathrm{~m}, 1 \mathrm{H}), 3.43-3.34(\mathrm{~m}, 2 \mathrm{H}), 3.33-3.25$ (m, 2H), $2.74-2.67(\mathrm{~m}, 2 \mathrm{H}), 2.17(\mathrm{t}, J=7.5 \mathrm{~Hz}, 2 \mathrm{H}), 1.70-1.59(\mathrm{~m}, 2 \mathrm{H}), 1.53-1.46(\mathrm{~m}, 2 \mathrm{H}), 1.44-$ $1.30(\mathrm{~m}, 4 \mathrm{H}), 0.99-0.88(\mathrm{~m}, 6 \mathrm{H}) .{ }^{13} \mathbf{C} \mathbf{~ N M R}\left(\mathrm{CDCl}_{3}, 100 \mathrm{MHz}\right): \delta 136.58,136.34,133.93,131.26$, $125.29,120.42,119.72,117.75,110.11,107.46,37.44,36.16,34.51,30.13,27.43,23.74,22.56,22.53$, 14.18. IR $\left(\mathrm{KBr}\right.$, neat, $\left.\mathrm{cm}^{-1}\right): v=3401,2957,2926,2856,1876,1625,1466,1432,1374,1315,1234$, 1162, 1094, 947, 810, 728, 633, 502. HRMS-EI m/z: [M+Na] ${ }^{+}$Calcd for $\mathrm{C}_{20} \mathrm{H}_{27} \mathrm{NNa}$ 304.2036; Found 304.2057.

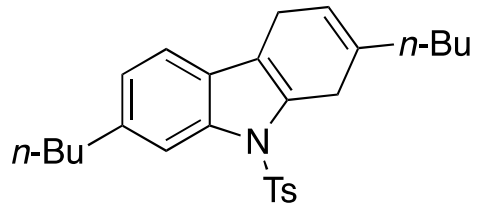

3,6-dibutyl-9-tosyl-4,9-dihydro-1H-carbazole. To a solution of 2,7-dibutyl-4,9-dihydro- $1 H$-carbazole $(86 \mathrm{mg}, 0.31 \mathrm{mmol})$ in dry THF at $-78^{\circ} \mathrm{C}$ was slowly added KHMDS (1M in THF) $(0.4 \mathrm{ml}, 0.37 \mathrm{mmol})$ and the mixture stirred for $10 \mathrm{~min}$. Tosylchloride was added in one portion and the mixture stirred overnight at room temperature. The reaction was quenched with sat. aq. $\mathrm{NaHCO}_{3}$ and extracted $(3 \mathrm{x}$ $35 \mathrm{ml}$ ) with $\mathrm{Et}_{2} \mathrm{O}$. The combined organic layers were dried over $\mathrm{Na}_{2} \mathrm{SO}_{4}$ and concentrated under reduced pressure. The residue was purified by column chromatography on silica gel using EtOAc and Pentane as an eluvent $(1: 49)$ to give the product as a white solid $(\mathrm{mp}=125.4-129.9), 95 \%$ yield. $\mathrm{R}_{\mathrm{f}}=0.40$ $($ EtOAc/Pentane $=1 / 49) .{ }^{1} \mathbf{H}$ NMR $\left(\mathrm{CDCl}_{3}, 400 \mathrm{MHz}\right): \delta 7.97(\mathrm{~s}, 1 \mathrm{H}), 7.64(\mathrm{~d}, J=8.4 \mathrm{~Hz}, 2 \mathrm{H}), 7.23(\mathrm{~d}, J$ $=7.9 \mathrm{~Hz}, 1 \mathrm{H}), 7.17(\mathrm{~d}, J=8.1 \mathrm{~Hz}, 2 \mathrm{H}), 7.06(\mathrm{dd}, J=7.9,1.4 \mathrm{~Hz}, 1 \mathrm{H}), 5.63-5.60(\mathrm{~m}, 1 \mathrm{H}), 3.57(\mathrm{t}, J=7.1$ $\mathrm{Hz}, 2 \mathrm{H}), 3.27-3.22(\mathrm{~m}, 2 \mathrm{H}), 2.74(\mathrm{t}, J=7.6 \mathrm{~Hz}, 2 \mathrm{H}), 2.32(\mathrm{~s}, 3 \mathrm{H}), 2.21-2.10(\mathrm{~m}, 2 \mathrm{H}), 1.68-1.60(\mathrm{~m}, 2 \mathrm{H})$, 1.55-1.47 (m, 2H), 1.40-1.30 (m, 4H), $0.94(\mathrm{t}, J=7.3 \mathrm{~Hz}, 6 \mathrm{H}) .{ }^{13} \mathbf{C ~ N M R}\left(\mathrm{CDCl}_{3}, 100 \mathrm{MHz}\right): \delta 144.52$, 
$139.39,136.90,136.38,135.13,132.44,129.89,127.81,126.51,124.25,117.94,117.26,116.02,114.32$, $37.26,36.29,34.42,30.08,29.41,23.65,22.59,22.41,21.67,14.17$. IR $\left(\mathrm{KBr}\right.$, neat, $\left.\mathrm{cm}^{-1}\right): v=3422,2952$, 2923, 2869, 2855, 1922, 1624, 1597, 1427, 1366, 1301, 1235, 1168, 1120, 987, 943, 813, 760, 685, 664, 590, 546. HRMS-EI m/z: $[\mathrm{M}+\mathrm{Na}]^{+}$Calcd for $\mathrm{C}_{27} \mathrm{H}_{33} \mathrm{NO}_{2} \mathrm{SNa}$ 458.2124; Found 458.2134.<smiles>CC1=CCC=C(CC(=O)O)C1</smiles>

2-(5-methylcyclohexa-1,4-dien-1-yl)acetic acid. According to the general procedure, the following amounts were used: 2 -( $m$-tolyl)acetic acid $(500 \mathrm{mg}, 3.33 \mathrm{mmol}, 1$ equiv) and $t-\mathrm{BuOH}(7 \mathrm{~mL})$. Ammonia was condensed to a total volume of $25 \mathrm{~mL}$. Li $(150 \mathrm{mg}, 21.64 \mathrm{mmol}, 6.5$ equiv) was added over $10 \mathrm{~min}$. After general workup and chromatography, the product was obtained as a white solid $(\mathrm{mp}=113.6-119.7$ $\left.{ }^{\circ} \mathrm{C}\right), 90 \%$ yield. $\mathrm{R}_{\mathrm{f}}=0.26($ EtOAc/Pentane $=1 / 9) .{ }^{1} \mathbf{H}$ NMR $\left(\mathrm{CDCl}_{3}, 400 \mathrm{MHz}\right): \delta 5.67-5.60(\mathrm{~m}, 1 \mathrm{H})$, $5.42-5.37(\mathrm{~m}, 1 \mathrm{H}), 3.04(\mathrm{~s}, 2 \mathrm{H}), 2.78-2.68(\mathrm{~m}, 2 \mathrm{H}), 2.66-2.54(\mathrm{~m}, 2 \mathrm{H}), 1.72-1.65(\mathrm{~m}, 3 \mathrm{H}){ }^{13} \mathrm{C}$ NMR $\left(\mathrm{CDCl}_{3}, 100 \mathrm{MHz}\right): \delta \quad 177.81,130.96,127.82,123.95,118.05,42.71,34.02,27.92,23.22$. IR $\left(\mathrm{KBr}\right.$, neat, $\left.\mathrm{cm}^{-1}\right): v=3414,3092,3033,2966,2735,2659,1880,1694,1405,1341,1247,1031,971$, 942, 909, 898, 778, 719, 682, 582. HRMS-EI m/z: $[\mathrm{M}+\mathrm{Na}]^{+}$Calcd for $\mathrm{C}_{9} \mathrm{H}_{12} \mathrm{O}_{2} \mathrm{Na}$ 175.0730; Found 175.0770 .<smiles>COC(=O)CC1=CCC=C(C)C1</smiles>

29a

methyl 2-(5-methylcyclohexa-1,4-dien-1-yl)acetate. The esterification was carried out as reported in the literature ${ }^{13}$ with little modification. To a solution of 2-(5-methylcyclohexa-1,4-dien-1-yl)acetic acid (54 $\mathrm{mg}, 0.35 \mathrm{mmol})$ in dry $\mathrm{MeOH}(5 \mathrm{ml})$ at $-10^{\circ} \mathrm{C}$ (ice and brine bath) was added $\mathrm{SOCl}_{2}(5 \mu \mathrm{l}, 0.07 \mathrm{mmol})$ slowly. The resulting mixture was stirred at room temperature for $2 \mathrm{~h}$, then it was concentrated in vacuum and sat. aq. $\mathrm{NaHCO}_{3}$ was added. The product was extracted with $\mathrm{CH}_{2} \mathrm{Cl}_{2}(3 \times 5 \mathrm{ml})$. The combine organic layers were dried over $\mathrm{Na}_{2} \mathrm{SO}_{4}$ and concentrated under reduce pressure. The residue was purified using column chromatography on silica gel using EtOAc and pentane as an eluent $(1: 24)$ to give the product as a colorless oil, 95\% yield. $\mathrm{R}_{\mathrm{f}}=0.5($ EtOAc/Pentane $=1 / 24) .{ }^{1} \mathbf{H}$ NMR $\left(\mathrm{CDCl}_{3}, 400 \mathrm{MHz}\right): \delta 5.59-5.53$ $(\mathrm{m}, 1 \mathrm{H}), 5.40-5.33(\mathrm{~m}, 1 \mathrm{H}), 3.67(\mathrm{~s}, 3 \mathrm{H}), 2.99(\mathrm{~s}, 2 \mathrm{H}), 2.76-2.63(\mathrm{~m}, 2 \mathrm{H}), 2.61-2.48(\mathrm{~m}, 2 \mathrm{H}), 1.66(\mathrm{~s}$, $3 \mathrm{H}) .{ }^{13} \mathrm{C}$ NMR $\left(\mathrm{CDCl}_{3}, 100 \mathrm{MHz}\right): \delta 172.16,130.93,128.30,123.16,118.01,51.83,42.85,33.99,27.85$, 23.16. IR $\left(\mathrm{NaCl}\right.$, neat, $\left.\mathrm{cm}^{-1}\right): v=3457,2996,2961,2913,2849,2814,1739,1436,1333,1258,1208$, 1155, 1008, 965, 940. HRMS-EI m/z: [M+Na $]^{+}$Calcd for $\mathrm{C}_{10} \mathrm{H}_{14} \mathrm{O}_{2} \mathrm{Na}$ 189.0886; Found 189.0886.<smiles>CC(C)CC1=CCC2=C(CC=CC2)C1</smiles>

$3 a$

2-isobutyl-1,4,5,8-tetrahydronaphthalene. According to the general procedure, the following amounts were used: 2-methyl-1-(naphthalen-2-yl)propan-1-ol (370 mg, $1.85 \mathrm{mmol}, 1$ equiv), THF (6 mL) and EtOH $(1.4 \mathrm{~mL})$. Ammonia was condensed to a total volume of $20 \mathrm{~mL}$. Na (340 mg, $14.8 \mathrm{mmol}, 8$ equiv) was added over $10 \mathrm{~min}$. After general workup and chromatography, the product was obtained as a colorless oil. $29 \%$ yield, $\mathrm{R}_{f}=0.6$ (Pentane). ${ }^{1} \mathbf{H}$ NMR $\left(\mathrm{CDCl}_{3}, 400 \mathrm{MHz}\right): \delta 0.88(\mathrm{~d}, J=6.47 \mathrm{~Hz}, 6 \mathrm{H})$, $1.78(\mathrm{~m}, 1 \mathrm{H}), 1.83-1.91(\mathrm{~m}, 2 \mathrm{H}), 2.40-2.48(\mathrm{~m}, 2 \mathrm{H}), 2.52-2.62(\mathrm{~m}, 2 \mathrm{H}), 5.42(\mathrm{bs}, 1 \mathrm{H}), 5.74(\mathrm{bs}, 2 \mathrm{H})$. ${ }^{13} \mathrm{C}$ NMR $\left(\mathrm{CDCl}_{3}, 100 \mathrm{MHz}\right): \delta 22.49,25.75,30.59,30.85,31.82,34.02,47.08,77.00,119.48,123.19$, 123.26, 124.39, 124.49, 134.23. IR $\left(\mathrm{NaCl}\right.$, neat, $\left.\mathrm{cm}^{-1}\right): v=2953,2840,2812,1657,1464,1431,971,778$, 659. HRMS-CI m/z: $[\mathrm{M}]^{+}$Calc. for $\mathrm{C}_{14} \mathrm{H}_{20}$ 188.1565; Found 188.1573 .<smiles>CC1=CC(C(=O)O)CC(C)=C1</smiles>

3,5-dimethylcyclohexa-2,5-diene-1-carboxylic acid, ${ }^{14}$ white solid, $85 \%$ yield, $\mathrm{R}_{f}=0.4$ (Pentane:EtOAc, 9:1). ${ }^{1} \mathbf{H}$ NMR $\left(\mathrm{CDCl}_{3}, 400 \mathrm{MHz}\right): \delta 1.75(\mathrm{~s}, 2 \mathrm{H}), 2.40-2.60(\mathrm{~m}, 1 \mathrm{H}), 3.76(\mathrm{~s}, 1 \mathrm{H}), 5.51(\mathrm{~m}, 1 \mathrm{H}) .{ }^{13} \mathrm{C}$ 
NMR $\left(\mathrm{CDCl}_{3}, 100 \mathrm{MHz}\right): \delta 23.16,35.79,44.04,77.16,115.77,134.55,179.68 . \mathbf{I R}\left(\mathrm{NaCl}\right.$, neat, $\left.\mathrm{cm}^{-1}\right): v$ $=2961,1692,1225,927,704$. HRMS-ESI m/z: [M-H] Calc. for $\mathrm{C}_{9} \mathrm{H}_{11} \mathrm{O}_{2}$ 151.0765; Found 151.0764.<smiles>COC1=CCc2c(n(S)c3ccccc23)C1</smiles>

$41 \mathrm{a}$

2-methoxy-9-tosyl-4,9-dihydro-1H-carbazole. The tosylation was carried as in 3,6-dibutyl-9-tosyl-4,9dihydro- $1 H$-carbazole 40a. After general workup and chromatography, the product was obtained as a white solid, $74 \%$ yield, $\mathrm{R}_{f}=0.5$ (Pentane:EtOAc, 5:1). ${ }^{1} \mathbf{H}$ NMR (D6-DMSO, $400 \mathrm{MHz}$ ): $\delta 2.26$ (s, 3H), $3.24-3.32(\mathrm{~m}, 2 \mathrm{H}), 3.59(\mathrm{~s}, 3 \mathrm{H}), 3.65(\mathrm{t}, J=7.24 \mathrm{~Hz}, 2 \mathrm{H}), 4.92(\mathrm{~m}, 1 \mathrm{H}), 7.24(\mathrm{~m}, 1 \mathrm{H}), 7,28-7.35(\mathrm{~m}$, $3 \mathrm{H}), 7.41(\mathrm{~m}, 1 \mathrm{H}), 7.68-7.75(\mathrm{~m}, 2 \mathrm{H}), 8.04(\mathrm{~d}, J=7.86,1 \mathrm{H}) \cdot{ }^{13} \mathbf{C} \mathbf{~ N M R}\left(\mathrm{CDCl}_{3}, 100 \mathrm{MHz}\right): \delta 21.03$, $28.63,54.48,90.13,113.90,115.95,118.84,123.67,124.48,126.26,126.35,128.79,130.17,130.34$, 130.95, 134.73, 135.69, 145.32, 151.73. IR $\left(\mathrm{NaCl}\right.$, neat, $\left.\mathrm{cm}^{-1}\right): v=2916,1677,1452,1364,1168,940$, 746. HRMS-CI m/z: [M] $]^{+}$Calc. for $\mathrm{C}_{14} \mathrm{H}_{24}$ 376.0983; Found 376.0955 .<smiles>CC(=O)c1ccc(C)cc1C(C)=O</smiles>

\section{$34 a$}

dimethyl 4-methylcyclohexa-1,4-diene-1,2-dicarboxylate, ${ }^{15}$ colorless oil, $50 \%$ yield. ${ }^{1} \mathbf{H}$ NMR $\left(\mathrm{CDCl}_{3}\right.$, $400 \mathrm{MHz}): \delta 1.70$ (bs, 3H), $2.84-2.92(\mathrm{~m}, 2 \mathrm{H}), 2.95-3.04(\mathrm{~m}, 2 \mathrm{H}), 3.77(\mathrm{~d}, 2.47 \mathrm{~Hz}, 6 \mathrm{H}), 5.93(\mathrm{~m}, 1 \mathrm{H})$. ${ }^{13} \mathrm{C}$ NMR $\left(\mathrm{CDCl}_{3}, 100 \mathrm{MHz}\right): \delta 22.68,28.71,32.18,52.30,77.16,116.69,128.43,129.80,132.39$, $132.98,168.50,168.74$.<smiles>CC1=CCC2C(=O)CCCC2C1</smiles>

$33 a$

6-methyl-3,4,4a,5,8,8a-hexahydronaphthalen-1(2H)-one, ${ }^{16}$ colorless oil, $98 \%$ yield. $\mathrm{R}_{f}=0.5$ $($ EtOAc/Pentane $=1 / 20) .{ }^{1} \mathbf{H}$ NMR $\left(\mathrm{CDCl}_{3}, 400 \mathrm{MHz}\right): \delta 1.43(\mathrm{~m}, 1 \mathrm{H}), 1.62(\mathrm{~s}, 3 \mathrm{H}), 1.63-1.75(\mathrm{~m}, 2 \mathrm{H})$, $1.84-1.95(\mathrm{~m}, 2 \mathrm{H}), 1.99-2.10(\mathrm{~m}, 2 \mathrm{H}), 2.11-2.16(\mathrm{~m}, 2 \mathrm{H}), 2.28-2.45(\mathrm{~m}, 2 \mathrm{H}), 5.37(\mathrm{~m}, 1 \mathrm{H}) .{ }^{13} \mathbf{C}$ NMR $\left(\mathrm{CDCl}_{3}, 100 \mathrm{MHz}\right): \delta 23.36,24.81,26.24,32.80,38.49,40.61,42.17,50.39,77.16,120.06$, $132.46,212.42$.

\section{General procedure for the Diels-Alder reaction ${ }^{17}$}

In a dry vial, equipped with stirring bar under $\mathrm{Ar}$ atmosphere, $\mathrm{CoBr}_{2}(\mathrm{bpy})(5 \mathrm{~mol} \%), \mathrm{ZnI}_{2}$ (10 mol\%), elemental $\mathrm{Fe}(10 \mathrm{~mol} \%)$ and $\mathrm{Zn}(10 \mathrm{~mol} \%)$ were transferred and dry dichloromethane was added $(0.5$ $\mathrm{ml} / \mathrm{mmol}$ of acetylene). The mixture was heated to boiling point till the reaction colour turned grey-green. The mixture was cooled to room temperature and the acetylene of choice and 2-methyl-1,3-butadiene were added. The reaction was stirred at room temperature for 24 hours. After completion of the reaction, $1.0 \mathrm{~mL}$ of pentane was added and the mixture filtered through silica with dichloromethane. The solvent was removed under vacuum to obtain the crude product. The products were purified by flash chromatography on activated silica (dried at $140^{\circ} \mathrm{C}$ for 17 hours) using pentane as eluent.<smiles>CC1=CCC(C)=C(c2ccccc2)C1</smiles>

1a

3,6-dimethyl-2,5-dihydro-1,1'-biphenyl. According to the general procedure, the following amounts were used: 1-phenyl-1-propyne (371 $\mathrm{mg}, 3.0 \mathrm{mmol}$ ), 2-methyl-1,3-butadiene (409 $\mathrm{mg}, 6.0 \mathrm{mmol}$ ), 
$\mathrm{CoBr}_{2}$ (bpy) (40 mg, $\left.0.11 \mathrm{mmol}\right), \mathrm{ZnI}_{2}$ (71 mg, $\left.0.22 \mathrm{mmol}\right), \mathrm{Fe}$ (14 mg, $\left.0.25 \mathrm{mmol}\right), \mathrm{Zn}(13 \mathrm{mg}, 0.20$ $\mathrm{mmol})$, dichloromethane $(1.5 \mathrm{~mL})$. After column chromatography, the product was obtained as a colorless oil. $61 \%$ yield, $\mathrm{R}_{f}=0.37$ (pentane), ${ }^{1} \mathbf{H}$ NMR $\left(\mathrm{CDCl}_{3}, 400 \mathrm{MHz}\right): \delta 7.33(\mathrm{~m}, 2 \mathrm{H}), 7.23(\mathrm{~m}, 1 \mathrm{H}), 7.18(\mathrm{~m}$, 2H), 5.47(s, 1H), $2.80(\mathrm{~m}, 4 \mathrm{H}), 1.71(\mathrm{~s}, 3 \mathrm{H}), 1.59(\mathrm{~s}, 3 \mathrm{H}) .{ }^{13} \mathbf{C ~ N M R}\left(\mathrm{CDCl}_{3}, 100 \mathrm{MHz}\right): \delta 143.19,131.63$, $129.45,128.13,128.10,127.92,127.89,127.83,126.23,126.20,125.98,125.96,118.85,118.32$, 37.66, 33.81, 22.66, 19.59. IR $\left(\mathrm{NaCl}\right.$, neat, $\left.\mathrm{cm}^{-1}\right): v=: 3075.27,3055.93,2964.48,2924.95,2815.68,2727.39$, 1945.65, 1806.71, 1774.21, 1666.59, 1600.86, 1574.69, 1489.89, 1441.35, 1377.01 1265.49, 1069.92, 918.51, 785.52, 763.00, 700.37. HRMS-CI: m/z: [M] ${ }^{+}$Calc. for $\mathrm{C}_{14} \mathrm{H}_{16}$ 184.1252; Found 184.1258.<smiles>CC1=CCC=C(c2ccc(C(F)(F)F)cc2)C1</smiles>

$10 a$

3-methyl-4'-(trifluoromethyl)-2,5-dihydro-1,1'-biphenyl. According to the general procedure, the following amounts were used: 4-ethynyl- $\alpha, \alpha, \alpha$-trifluorotoluene $(1.022 \mathrm{~g}, 6.0 \mathrm{mmol})$, 2-methyl-1,3butadiene (613 mg, $9.0 \mathrm{mmol}), \mathrm{CoBr}_{2}$ (bpy) $(111 \mathrm{mg}, 0.30 \mathrm{mmol}), \mathrm{ZnI}_{2}(221 \mathrm{mg}, 0.69 \mathrm{mmol}), \mathrm{Fe}(41 \mathrm{mg}$, $0.56 \mathrm{mmol}), \mathrm{Zn}(31 \mathrm{mg}, 0.63 \mathrm{mmol})$, dichloromethane $(3.0 \mathrm{~mL})$. After column chromatography, the product was obtained as a white solid (mp: $41.6-44.2{ }^{0} \mathrm{C}$ ). $65 \%$ yield, $\mathrm{R}_{f}=0.83$ (pentane), ${ }^{1} \mathbf{H}$ NMR $\left(\mathrm{CDCl}_{3}, 400 \mathrm{MHz}\right): \delta 7.55(\mathrm{dd}, 4 \mathrm{H}) 6.23(\mathrm{~m}, 1 \mathrm{H}), 5.50(\mathrm{~m}, 1 \mathrm{H}), 2.94(\mathrm{~m}, 4 \mathrm{H}), 1.79(\mathrm{~s}, 3 \mathrm{H}) .{ }^{13} \mathbf{C} \mathbf{~ N M R}$ $\left(\mathrm{CDCl}_{3}, 100 \mathrm{MHz}\right): \delta 145.06,145.04,133.00,131.20,128.77,125.41,125.37,125.34,125.31,124.05$, 117.96, 32.84, 28.47, 23.46. IR $\left(\mathrm{NaCl}, \mathrm{CCl}_{4}, \mathrm{~cm}^{-1}\right): v=3007.70,3031.62,2969.57,2908.96,2879.51$, 2855.83, 2819.89, 2684.60, 2305.07, 1919.19, 1739.08, 1646.90, 1617.88, 1574.62, 1549.93, 1438.37, 1426.08, 1326.41, 1264.11, 1216.88, 1168.64, 1130.12, 1070.87, 1016.87, 939.01, 853.88. HRMS-CI: $\mathrm{m} / \mathrm{z}:[\mathrm{M}]^{+}$Calc. for $\mathrm{C}_{14} \mathrm{H}_{13} \mathrm{~F}_{3} 238.0969$; Found 238.0983.<smiles>CC1=CCC=C(c2ccc(C)cc2)C1</smiles>

9a

3,4'-dimethyl-2,5-dihydro-1,1'-biphenyl. According to the general procedure, the following amounts were used: 1-ethynyl-4-methylbenzene (696 mg, $6.0 \mathrm{mmol}), 2$-methyl-1,3-butadiene (613 $\mathrm{mg}, 9.0 \mathrm{mmol}$ ), $\mathrm{CoBr}_{2}$ (bpy) (104 mg, $0.28 \mathrm{mmol}$ ), ZnI 2 (214 mg, $0.67 \mathrm{mmol}$ ), Fe (39 mg, $\left.0.70 \mathrm{mmol}\right), \mathrm{Zn} \mathrm{(32} \mathrm{mg,} 0.49$ $\mathrm{mmol})$, dichloromethane $(3.0 \mathrm{~mL})$. After column chromatography, the product was obtained as a colorless oil. $76 \%$ yield. $\mathrm{R}_{\mathrm{f}}=0.32$ (pentane). ${ }^{1} \mathbf{H}$ NMR $\left(\mathrm{CDCl}_{3}, 400 \mathrm{MHz}\right): \delta 7.33(\mathrm{~d}, 2 \mathrm{H}), 7.14(\mathrm{~d}, 2 \mathrm{H}), 6.09(\mathrm{~m}$, 1H), $5.48(\mathrm{~m}, 1 \mathrm{H}), 2.91(\mathrm{~m}, 4 \mathrm{H}), 2.34(\mathrm{~s}, 3 \mathrm{H}), 1.77(\mathrm{~s}, 3 \mathrm{H}) .{ }^{13} \mathbf{C} \mathbf{N M R}\left(\mathrm{CDCl}_{3}, 100 \mathrm{MHz}\right): \delta 138.80$, 136.65, 133.68, 131.51, 129.09, 124.97, 120.91, 118.10, 33.06, 28.42, 23.53, 21.21. IR ( NaCl, $\mathrm{CCl}_{4}, \mathrm{~cm}^{-}$ $\left.{ }^{1}\right): v=3027.67,3050.61,2984.41,2926.56,2819.59,2730.78,2684.96,2305.67,1904.03,1550.06$, 1512.94, 1446.80, 1424.22, 1384.76, 1219.55, 979.81, 895.79. HRMS-CI: m/z: $[\mathrm{M}]^{+}$Calc. for $\mathrm{C}_{14} \mathrm{H}_{16}$ 184.1252; Found 184.1254.<smiles>CC1=CCC=C(c2ccccc2)C1</smiles>

$8 \mathbf{a}$

3-methyl-2,5-dihydro-1,1'-biphenyl. According to the general procedure, the following amounts were used: phenylacetylene $(632 \mathrm{mg}, 6.2 \mathrm{mmol}), 2$-methyl-1,3-butadiene $(545 \mathrm{mg}, 8.0 \mathrm{mmol}), \mathrm{CoBr}_{2}(\mathrm{bpy})$ (117 mg, $0.312 \mathrm{mmol}), \mathrm{ZnI}_{2}(216 \mathrm{mg}, 0.68 \mathrm{mmol}), \mathrm{Fe}(34 \mathrm{mg}, 0.60 \mathrm{mmol}), \mathrm{Zn}(40 \mathrm{mg}, 0.62 \mathrm{mmol})$, dichloromethane $(3.0 \mathrm{~mL})$. After column chromatography, the product was obtained as a colorless oil. $21 \%$ yield. $\mathrm{R}_{f}=0.58$ (pentane), ${ }^{1} \mathbf{H}$ NMR $\left(\mathrm{CDCl}_{3}, 400 \mathrm{MHz}\right): \delta 7.44(\mathrm{~m}, 2 \mathrm{H}), 7.34(\mathrm{~m}, 2 \mathrm{H}), 7.25(\mathrm{~m}, 1 \mathrm{H})$, $6.14(\mathrm{~s}, 1 \mathrm{H}), 5.50(\mathrm{~s}, 1 \mathrm{H}), 2.94(\mathrm{~m}, 4 \mathrm{H}), 1.79(\mathrm{~s}, 3 \mathrm{H}) .{ }^{13} \mathbf{C}$ NMR $\left(\mathrm{CDCl}_{3}, 100 \mathrm{MHz}\right): \delta 141.65,141.50$, $138.46,133.91,131.48,128.82,128.79,128.40,128.13,127.32,127.29,126.99,126.74,125.12,124.41$, $121.77,118.06,34.28,33.03,32.29,29.87,29.09,28.45,23.51,23.06,22.50,21.69,14.22$. IR $(\mathrm{NaCl}$, 
$\left.\mathrm{CCl}_{4}, \mathrm{~cm}^{-1}\right): v=: 3080.66,3057.15,2923.81,2850.91,2728.27,1944.04,1600.80,1494.26,1445.48$, 1384.33, 1267.34, 962.63. HRMS-CI: m/z: [M] Calc. for $\mathrm{C}_{13} \mathrm{H}_{14}$ 170.1096; Found 170.1074.<smiles>CC1=CCC=C(c2ccsc2)C1</smiles>

\section{$42 a$}

3-(5-methylcyclohexa-1,4-dien-1-yl)thiophene. According to the general procedure, the following amounts were used: 3-ethynyl-thiophene $(659 \mathrm{mg}, 6.1 \mathrm{mmol}), 2$-methyl-1,3-butadiene $(613 \mathrm{mg}, 9.0$ $\mathrm{mmol}), \mathrm{CoBr}_{2}$ (bpy) (107 mg, $\left.0.29 \mathrm{mmol}\right), \mathrm{ZnI}_{2}(207 \mathrm{mg}, 0.65 \mathrm{mmol}), \mathrm{Fe}(39 \mathrm{mg}, 0.70 \mathrm{mmol}), \mathrm{Zn}(32 \mathrm{mg}$, $0.49 \mathrm{mmol})$, dichloromethane $(3.0 \mathrm{~mL})$. After column chromatography, the product was obtained as a white solid, (MP: $\left.46.8-51.8{ }^{\circ} \mathrm{C}\right) .18 \%$ yield, $\mathrm{R}_{f}=0.51$ (pentane), ${ }^{1} \mathbf{H} \mathbf{N M R}\left(\mathrm{CDCl}_{3} .400 \mathrm{MHz}\right): \delta 7.30(\mathrm{~m}$, 2H), $7.14(\mathrm{~s}, 1 \mathrm{H}), 6.20(\mathrm{~s}, 1 \mathrm{H}), 5.51(\mathrm{~s}, 1 \mathrm{H}), 2.93(\mathrm{~m}, 4 \mathrm{H}), 1.81(\mathrm{~s}, 3 \mathrm{H}) .{ }^{13} \mathbf{C}$ NMR $\left(\mathrm{CDCl}_{3}, 100 \mathrm{MHz}\right)$ : 143.15, 131.14, 129.25, 125.38, 124.77, 120.75, 118.38, 118.11, 77.48, 77.16, 76.84, 32.97, 27.98, 23.47. IR $\left(\mathrm{NaCl}, \mathrm{CCl}_{4}, \mathrm{~cm}^{-1}\right): \mathrm{v}=: 2324.22,2817.01,2676.24,1751.01,1696.25,1554.18,1445.93,1380.94$, 1406.45, 1334.20, 1249.82, 1150.92, 962.21. HRMS-CI: m/z: [M] ${ }^{+}$Calc. for $\mathrm{C}_{11} \mathrm{H}_{12} \mathrm{~S}$ 179.0660; Found 176.0666 .<smiles>CCC1=C(CC)CC(C)=CC1</smiles>

1,2-diethyl-4-methylcyclohexa-1,4-diene. According to the general procedure, the following amounts were used: 3-hexyne (493 mg, $6.0 \mathrm{mmol})$, 2-methyl-1,3-butadiene (613 mg, $9.0 \mathrm{mmol}$ ), $\mathrm{CoBr}_{2}$ (bpy) (114 $\mathrm{mg}, 0.30 \mathrm{mmol}$ ), ZnI 2 (214mg, $0.67 \mathrm{mmol}$ ), Fe (39 mg, $0.70 \mathrm{mmol}), \mathrm{Zn}(34 \mathrm{mg}, 0.52 \mathrm{mmol}$ ), dichloromethane $(3.0 \mathrm{~mL})$. After column chromatography, the product was obtained as a colorless oil, $60 \%$ yield. $\mathrm{R}_{f}=0.80$ (Pentane). ${ }^{1} \mathbf{H}$ NMR $\left(\mathrm{CDCl}_{3}, 400 \mathrm{MHz}\right): \delta 5.43-5.39(\mathrm{~m}, 1 \mathrm{H}), 2.68-2.50(\mathrm{~m}, 4 \mathrm{H})$, 2.10-2.01 (m, 4H), $1.68(\mathrm{~m}, 3 \mathrm{H}), 1.03-0.93(\mathrm{td}, \mathrm{J}=7.61,7.58,6.78,6 \mathrm{H}) .{ }^{13} \mathbf{C}$ NMR $\left(\mathrm{CDCl}_{3}, 100 \mathrm{MHz}\right)$ : $130.72,127.75,118.04,34.14,30.31,24.46,24.28,22.09,12.33,12.19$. IR $\left(\mathrm{NaCl}\right.$, neat, $\left.\mathrm{cm}^{-1}\right): v=$ 3051.06, 2966.02, 2813.32, 1539.92, 1264.78, 1034.54. HRMS-CI m/z: $[\mathrm{M}+\mathrm{H}]^{+}$Calc. for $\mathrm{C}_{11} \mathrm{H}_{19}$ 151.1487; Found 151.1489.

\section{General Procedure for asymmetric hydrogenations}

A vial was charged with substrate $(0.125 \mathrm{mmol})$ and Ir-complex $(0.5$ to $1 \mathrm{~mol} \%)$. Dry, distilled $\mathrm{CH}_{2} \mathrm{Cl}_{2}$ $(1 \mathrm{~mL})$ was added and the vial was placed in a high-pressure hydrogenation apparatus. The reactor was purged three times with Ar, then filled to the required pressure with $\mathrm{H}_{2}$. The reaction was stirred at room temperature for 16 hours (unless otherwise specified) before the $\mathrm{H}_{2}$ pressure was released and the solvent was removed in vacuo. The crude product was filtered through on a short plug of silica (pentane: $\mathrm{Et}_{2} \mathrm{O}$ 1:1). Conversions were determined by ${ }^{1} \mathrm{H}$ NMR spectroscopy and ee values were determined using chiral GC or SFC.

\section{Racemates preparation}

Racemates were prepared using racemic catalysts ii, $\mathbf{v}$ or Crabtree's catalyst $\left(\left[\operatorname{Ir}(\mathrm{COD})\left(\mathrm{PCy}_{3}\right)(\mathrm{py})\right]^{+} \mathrm{PF}_{6}{ }^{-}\right.$; $4 \mathrm{~mol} \%$ ) according to the general procedure for asymmetric hydrogenation. 


\section{Absolute configuration}

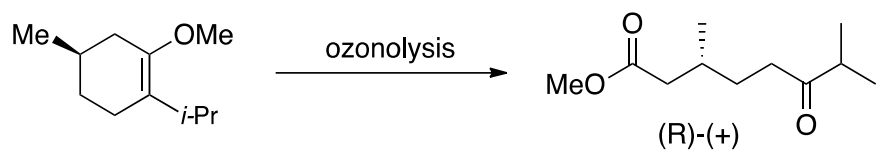

The absolute configuration of compound $\mathbf{2 1 b}$ was obtained by comparing the literature value of optical rotation after oxidative cleavage by ozonolysis $\left(\mathrm{O}_{3}, \mathrm{DCM} / \mathrm{MeOH},-78^{\circ} \mathrm{C}\right.$, then $\mathrm{Me}_{2} \mathrm{~S}$, warm up to r.t.). In this case, the hydrogenation was carried out with catalyst $(S)-\mathbf{v}$.

Characterization data and separation methods for the following compounds have been previously reported: ${ }^{18}$<smiles>CCCCCC1CCCC(CCC)C1</smiles><smiles>COC1CCCC([Pb])C1</smiles><smiles>COC1=C(C)CCC(C)C1</smiles><smiles>CCCC1=C(OC)CC(C)CC1</smiles><smiles>COC1=C(C)CCC(C(C)C)C1</smiles><smiles>COC1CCC2=C(C1)CC(OC)CC2</smiles><smiles>CC1CCC2=C(CCCC2)C1</smiles>

2b

(-)-2-Methyl-1,2,3,4,5,6,7,8-octahydronaphthalene. ${ }^{19}$ According to the general procedure, the following amounts were used: substrate $9 \mathrm{mg}$, Ir-complex $0.5 \mathrm{~mol} \%, \mathrm{CH}_{2} \mathrm{Cl}_{2} 0.5 \mathrm{~mL}, 50$ bar pressure $\mathrm{H}_{2}$. Colorless oil. $\mathrm{R}_{f}=0.97$ (Pentane). ${ }^{1} \mathbf{H}$ NMR $\left(\mathrm{CDCl}_{3}, 400 \mathrm{MHz}\right): \delta 1.84$ (bs, 5H), $1.69-1.45(\mathrm{~m}, 7 \mathrm{H})$, $1.32-1.14(\mathrm{~m}, 3 \mathrm{H}), 0.94(\mathrm{~d}, J=6.3 \mathrm{~Hz}, 3 \mathrm{H}) .{ }^{13} \mathbf{C} \mathbf{N M R}\left(\mathrm{CDCl}_{3}, 100 \mathrm{MHz}\right): \delta 127.65,39.43,31.69$, $30.87,30.51,30.31,29.32,23.42,23.36,22.19$. IR $\left(\mathrm{NaCl}\right.$, neat, $\left.\mathrm{cm}^{-1}\right): v=2925,2855,2831,1675,1456$, 1440, 1375, 1261, 1216, 1166, 1101, 1021, 805, 761, 669. HRMS m/z: $[\mathrm{M}+\mathrm{Na}]^{+}$Calc. for $\mathrm{C}_{9} \mathrm{H}_{16} \mathrm{O}_{2} \mathrm{Na}$ 189.1250; Found 189.1257. $[\boldsymbol{a}]_{\boldsymbol{D}}^{\mathbf{2 3}}=-111.94,\left(c=0.067, \mathrm{CHCl}_{3}\right)$. GC-MS: column Chiraldex $\beta$-DM, $80^{\circ} \mathrm{C}$ isothermal, $\mathrm{t}_{\mathrm{R}}=36.0 \mathrm{~min}$ (major) $/ 37.1$ (minor), $94 \%$ ee.<smiles>COC1CCC2=C(CCCC2)C1</smiles>

2-Methoxy-1,2,3,4,5,6,7,8-octahydronaphthalene, according to the general procedure, the following amounts were used: substrate $20 \mathrm{mg}$, Ir-complex $0.5 \mathrm{~mol} \%, \mathrm{CH}_{2} \mathrm{Cl}_{2} 1 \mathrm{~mL}, 20$ bar pressure $\mathrm{H}_{2}$.

Colorless oil, 98\% yield. $\mathrm{R}_{f}=0.18$ (Pentane). ${ }^{1} \mathbf{H}$ NMR $\left(\mathrm{CDCl}_{3}, 400 \mathrm{MHz}\right): \delta 3.46-3.40(\mathrm{~m}, 1 \mathrm{H}) ; 3.36$ $(\mathrm{s}, 3 \mathrm{H}) ; 2.20-1.46(\mathrm{~m}, 14 \mathrm{H}) .{ }^{13} \mathbf{C} \mathbf{N M R}\left(100 \mathrm{MHz}, \mathrm{CDCl}_{3}\right) \delta 127.84,125.36,76.43,55.83,36.48$, 30.39, 29.97, 28.82, 27.80, 23.18. IR (NaCl, neat, $\left.\mathrm{cm}^{-1}\right): v=2925,2832,1716,1678,1444,1195,1100$. HRMS $=\mathrm{m} / \mathrm{z}:[\mathrm{M}+\mathrm{Na}]^{+}$Calc. for $\mathrm{C}_{11} \mathrm{H}_{18} \mathrm{ONa} 189.1250 ;$ Found 189.1250. $[\boldsymbol{\alpha}]_{\boldsymbol{D}}^{\mathbf{2 3}}=-66.00,(c=0.30$, $\mathrm{CHCl}_{3}$ ). GC-MS: column Hydrodex $\beta-3 \mathrm{P}, 90^{\circ} \mathrm{C}$ isothermal, $\mathrm{t}_{\mathrm{R}}=60.4 \mathrm{~min}$ (major) $/ 63.0$ (minor), $99 \%$ ee.

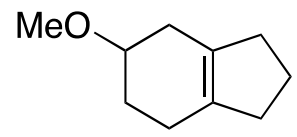

5-methoxy-2,3,4,5,6,7-hexahydro-1H-indene, according to the general procedure, the following amounts were used: substrate $19 \mathrm{mg}$, Ir-complex $0.5 \mathrm{~mol} \%, \mathrm{CH}_{2} \mathrm{Cl}_{2} 1 \mathrm{~mL}, 20$ bar pressure $\mathrm{H}_{2}$. 
Colorless oil, $82 \%$ yield. $\mathrm{R}_{f}=0.15$ (Pentane). ${ }^{1} \mathbf{H}$ NMR $\left(\mathrm{CDCl}_{3}, 400 \mathrm{MHz}\right): \delta 3.61-3.46(\mathrm{~m}, 1 \mathrm{H}), 3.38$ (s, 3H), $2.36-1.61(\mathrm{~m}, 12 \mathrm{H}) .{ }^{13} \mathbf{C}$ NMR $\left(\mathrm{CDCl}_{3}, 100 \mathrm{MHz}\right): \delta 134.09,131.89,76.82,55.94,35.88$, 35.54, 31.89, 27.74, 23.54, 22.19. IR (NaCl, neat, $\left.\mathrm{cm}^{-1}\right): v=3468,2922,2840,1720,1446,1370,1349$, 1194, 1105, 959, 814. HRMS-ESI m/z: $[\mathrm{M}+\mathrm{Na}]^{+}$Calc. for $\mathrm{C}_{10} \mathrm{H}_{16} \mathrm{ONa}$ 175.1093; Found 175.1093. [ $\left.\boldsymbol{\alpha}\right]_{\boldsymbol{D}}^{23}$ $=+22.50,\left(c=0.20, \mathrm{CHCl}_{3}\right)$. GC-MS: column Hydrodex $\beta-3 \mathrm{P}, 90^{\circ} \mathrm{C}$ isothermal, $\mathrm{t}_{\mathrm{R}}=21.3 \mathrm{~min}$ (minor) $/ 22.2 \mathrm{~min}$ (major), $98 \%$ ee.<smiles>CCCOCCC1CCCC(C)C1</smiles>

\section{7b}

tert-butyldimethyl(2-(3-methylcyclohexyl)ethoxy)silane, according to the general procedure, the following amounts were used: substrate $16 \mathrm{mg}$, Ir-complex $0.5 \mathrm{~mol} \%, \mathrm{CH}_{2} \mathrm{Cl}_{2} 0.5 \mathrm{~mL}, 50$ bar pressure $\mathrm{H}_{2}$. Colorless oil, $86 \%$ yield. $\mathrm{R}_{f}=0.21$ (Pentane). Reported as a mixture of isomers (cis:trans, 1:2). ${ }^{1} \mathbf{H}$ NMR $\left(\mathrm{CDCl}_{3}, 400 \mathrm{MHz}\right): \delta 3.76-3.60(\mathrm{~m}, 3 \mathrm{H}), 1.78-1.45(\mathrm{~m}, 10 \mathrm{H}), 1.34-1.07(\mathrm{~m}, 4 \mathrm{H}), 0.95(\mathrm{dd}, J=7.8$, $6.2 \mathrm{~Hz}, 2 \mathrm{H}), 0.92-0.86(\mathrm{~m}, 10 \mathrm{H}), 0.07-0.02(\mathrm{~m}, 6 \mathrm{H}) .{ }^{13} \mathbf{C} \mathbf{N M R}\left(\mathrm{CDCl}_{3}, 100 \mathrm{MHz}\right): \delta 122.37,121.10$, $62.30,61.04,41.53,39.70,37.48,36.96,30.80,30.76,29.00,28.77,25.94,25.92,25.52,25.35,23.75$, 21.91, 18.27, -5.35, -5.37. IR $\left(\mathrm{NaCl}\right.$, neat, $\left.\mathrm{cm}^{-1}\right): v=2953,2927,2857,1255,1101,836,774$. HRMS-CI $\mathrm{m} / \mathrm{z}$ : $[\mathrm{M}-\mathrm{H}]^{-}$Calc. for $\mathrm{C}_{15} \mathrm{H}_{31} \mathrm{OSi}$ 255.2150; Found 255.2159. $[\boldsymbol{\alpha}]_{\boldsymbol{D}}^{23}=-0.50,\left(c=0.170, \mathrm{CHCl}_{3}\right)$. GC-MS: column Hydrodex $\beta-3 \mathrm{P}, 100^{\circ} \mathrm{C}$ isothermal, $\mathrm{t}_{\mathrm{R}}=$ cis $37.1 \mathrm{~min} / 38.8 \mathrm{~min}$, trans $44.5 \mathrm{~min}$ (major) $/ 45.8 \mathrm{~min}$ (minor), $99 \%$ ee.<smiles>COc1cc(OC)cc(OCCC2CCCC(C)C2)c1</smiles>

$30 \mathrm{~b}$

1,3-dimethoxy-5-(2-(3-methylcyclohexyl)ethoxy)benzene, according to the general procedure, the following amounts were used: substrate $9 \mathrm{mg}$, Ir-complex $1 \mathrm{~mol} \%, \mathrm{CH}_{2} \mathrm{Cl}_{2} 0.25 \mathrm{~mL}, 50$ bar pressure $\mathrm{H}_{2}$. Colorless oil, 79\% yield. $\mathrm{R}_{f}=0.63$ (Pentane:AcOEt; 95:5). Reported as a mixture of isomers (cis:trans, 1:3). ${ }^{1} \mathbf{H}$ NMR $\left(\mathrm{CDCl}_{3}, 400 \mathrm{MHz}\right): \delta 6.08(\mathrm{~m}, 3 \mathrm{H}), 3.95(\mathrm{~m}, 2 \mathrm{H}), 3.77(\mathrm{~s}, 6 \mathrm{H}), 1.78-1.62(\mathrm{~m}, 4 \mathrm{H}), 1.54-$ $1.21(\mathrm{~m}, 7 \mathrm{H}), 0.91(\mathrm{~d}, J=6.6 \mathrm{~Hz}, 3 \mathrm{H}$, cis $), 0.88(\mathrm{~d}, J=6.8 \mathrm{~Hz}, 3 \mathrm{H}$, trans $) .{ }^{13} \mathbf{C} \mathbf{N M R}\left(\mathrm{CDCl}_{3}, 100 \mathrm{MHz}\right)$ : $\delta 161.63,161.21,93.54,92.97,66.12,55.47,42.34,39.29,36.90,35.36,34.72,33.99,33.05,32.77$, $31.62,30.48,29.85,29.50,27.36,26.37,23.07,20.98 . \mathbf{I R}\left(\mathrm{NaCl}\right.$, neat, $\left.\mathrm{cm}^{-1}\right): v=2998,2923,2852$, 1602, 1472, 1459, 1391, 1205, 1194, 1153, 1067, 1031, 943, 930, 818, 682. HRMS-ESI m/z: [M+Na $]^{+}$ Calc. for $\mathrm{C}_{17} \mathrm{H}_{26} \mathrm{O}_{3} \mathrm{Na} 301.1780$; Found 301.1771. $[\boldsymbol{\alpha}]_{\boldsymbol{D}}^{23}=-41.57,\left(c=0.170, \mathrm{CHCl}_{3}\right)$.

SFC: Chiralcel ADH column, $95 \% \mathrm{CO}_{2} 5 \% \mathrm{MeOH}, 2 \mathrm{ml} / \mathrm{min}, \mathrm{t}_{R}=$ cis $7.2 \mathrm{~min} / 7.5 \mathrm{~min}$, trans $8.3 \mathrm{~min}$ (major) $/ 9.2 \mathrm{~min}$ (minor), $97 \%$ ee.<smiles>CC1CCCC(CCCO)C1</smiles>

3-(3-methylcyclohexyl)propan-1-ol, according to the general procedure, the following amounts were used: substrate $19 \mathrm{mg}$, Ir-complex $0.5 \mathrm{~mol} \%, \mathrm{CH}_{2} \mathrm{Cl}_{2} 1 \mathrm{~mL}, 50$ bar pressure $\mathrm{H}_{2}$.

Colorless oil, 84\% yield. $\mathrm{R}_{f}=0.16$ (Pentane:AcOEt; 95:5). Reported as a mixture of isomers (cis:trans, 1:7). ${ }^{1} \mathbf{H}$ NMR $\left(\mathrm{CDCl}_{3}, 400 \mathrm{MHz}\right): \delta 3.63(\mathrm{t}, J=6.7 \mathrm{~Hz}, 2 \mathrm{H}), 1.78-1.70(\mathrm{~m}, 1 \mathrm{H}), 1.62-1.48(\mathrm{~m}, 4 \mathrm{H})$, $1.47-1.40(\mathrm{~m}, 2 \mathrm{H}), 1.36-1.07(\mathrm{~m}, 7 \mathrm{H}), 0.89(\mathrm{~d}, J=6.9 \mathrm{~Hz}, 3 \mathrm{H}$, trans $), 0.86(\mathrm{~d}, J=6.6 \mathrm{~Hz}, 3 \mathrm{H}$, cis $) .{ }^{13} \mathbf{C}$ NMR (CDCl $3,100 \mathrm{MHz}): \delta$ 63.55, 42.46, 39.33, 37.67, 35.48, 33.95, 33.66, 33.09, 32.84, 32.40, 31.78, $30.88,30.72,30.29,27.33,26.47,23.10,20.97,20.88 . \mathbf{I R}\left(\mathrm{NaCl}\right.$, neat, $\left.\mathrm{cm}^{-1}\right): v=3327,2922,2866,2848$, 1457, 1377, 1056, 1031. HRMS-ESI m/z: $[\mathrm{M}+\mathrm{K}]^{+}$Calc. for $\mathrm{C}_{10} \mathrm{H}_{20} \mathrm{KO}$ 195.1146; Found 195.1145. [ $\left.\boldsymbol{\alpha}\right]_{\boldsymbol{D}}^{23}$ $=-0.56,\left(c=0.280, \mathrm{CHCl}_{3}\right)$. GC-MS: analysed as 3-(3-methylcyclohexyl)propanal ${ }^{20}$ after PCC oxidation, column Hydrodex $\beta-3 \mathrm{P}, 85^{\circ} \mathrm{C}$ isothermal, $\mathrm{t}_{\mathrm{R}}=$ cis $42.2 \mathrm{~min} / 43.2 \mathrm{~min}$, trans $44.4 \mathrm{~min}$ (major) $/ 48.2 \mathrm{~min}$ (minor), 99\% ee. 
<smiles>CCCOCCCC1CCCC(C)C1</smiles>

tert-butyldimethyl(3-(3-methylcyclohexyl)propoxy)silane, according to the general procedure, the following amounts were used: substrate $33 \mathrm{mg}$, Ir-complex $0.5 \mathrm{~mol} \%, \mathrm{CH}_{2} \mathrm{Cl}_{2} 1 \mathrm{~mL}, 50$ bar pressure $\mathrm{H}_{2}$. Colorless oil, $87 \%$ yield. $\mathrm{R}_{f}=0.75$ (Pentane). Reported as a mixture of isomers (cis:trans, 1:2.5). ${ }^{1} \mathbf{H}$ NMR $\left(\mathrm{CDCl}_{3}, 400 \mathrm{MHz}\right): \delta 3.65-3.61(\mathrm{~m}, 2 \mathrm{H}$, cis $), 3.61-3.56(\mathrm{~m}, 2 \mathrm{H}$, trans $), 1.80-1.64(\mathrm{~m}, 2 \mathrm{H})$, $1.61-1.39(\mathrm{~m}, 8 \mathrm{H}), 1.38-1.04(\mathrm{~m}, 8 \mathrm{H}), 0.94-0.82(\mathrm{~m}, 12 \mathrm{H}), 0.05(\mathrm{~s}, 6 \mathrm{H}) .{ }^{13} \mathbf{C}$ NMR $\left(\mathrm{CDCl}_{3}, 100\right.$ MHz): $\delta 63.88,63.82,63.59,63.57,42.56,42.48,39.41,39.35,37.71,37.69,35.55,35.50,34.02,33.97$, $33.81,33.69,33.16,33.11,32.89,32.86,32.42,32.35,31.86,31.80,30.97,30.91,30.41,30.32,27.37$, $27.35,26.15,26.12,21.02,20.91,18.54,-5.08$. IR $\left(\mathrm{NaCl}\right.$, neat, $\left.\mathrm{cm}^{-1}\right): v=3339,2926,2858,1472,1461$, 1388, 1378, 1361, 1255, 1101, 1060, 975, 951, 934, 836, 813, 775, 713, 663. HRMS-CI m/z: [M] ${ }^{+}$Calc. for $\mathrm{C}_{16} \mathrm{H}_{35} \mathrm{OSi}$ 271.2457; Found 271.2473. $[\alpha]_{D}^{23}=+1.11$, $\left(c=0.720, \mathrm{CHCl}_{3}\right)$. GC-MS: analysed as 3-(3methylcyclohexyl)propana ${ }^{20}$ after PCC oxidation, column Hydrodex $\beta-3 \mathrm{P}, 85^{\circ} \mathrm{C}$ isothermal, $\mathrm{t}_{\mathrm{R}}=c i s 42.2$ $\mathrm{min} / 43.2 \mathrm{~min}$, trans $44.4 \mathrm{~min}$ (major)/48.2 $\mathrm{min}$ (minor), 99\% ee.<smiles>CC1CCCC(C)C1C(=O)O</smiles>

\section{2b}

2,6-dimethylcyclohexane-1-carboxylic acid, according to the general procedure, the following amounts were used: substrate $30 \mathrm{mg}$, Ir-complex $2 \mathrm{~mol} \%, \mathrm{CH}_{2} \mathrm{Cl}_{2} 1.5 \mathrm{~mL}, 100$ bar pressure $\mathrm{H}_{2}, 48 \mathrm{~h}$.

White solid $\left(\mathrm{mp}=76-79{ }^{\circ} \mathrm{C}\right), 98 \%$ yield. $\mathrm{R}_{\mathrm{f}}=0.51(\mathrm{MeOH} / \mathrm{DCM}=0.5 / 9.5) .{ }^{1} \mathbf{H}$ NMR $\left(\mathrm{CDCl}_{3}, 400\right.$ MHz): $\delta 2.31-2.22(\mathrm{~m}, 1 \mathrm{H}), 2.21-2.15(\mathrm{~m}, 1 \mathrm{H}), 1.91-1.39(\mathrm{~m}, 7 \mathrm{H}), 0.98-0.91(\mathrm{~m}, 6 \mathrm{H}) .{ }^{13} \mathbf{C} \mathbf{N M R}$ $\left(\mathrm{CDCl}_{3}, 100 \mathrm{MHz}\right): \delta 181.71,53.92,34.15,32.57,30.79,27.43,21.11,20.02,14.74$. IR (NaCl, neat, $\mathrm{cm}^{-}$ $\left.{ }^{1}\right): v=2985,2929,1702,1416,1385,1224,945$. HRMS (EI) m/z: [M+Na]+ Calcd for $\mathrm{C}_{9} \mathrm{H}_{15} \mathrm{Na}_{2} \mathrm{O}_{2}$ 201.0867; Found 201.0858. $[\boldsymbol{\alpha}]_{\boldsymbol{D}}^{23}=-36.86\left(\mathrm{c}=0.700, \mathrm{CHCl}_{3}\right)$. GC-MS: analysed as the corresponding alcohol after LAH reduction, column Chiraldex $\beta$-DM, $70^{\circ} \mathrm{C}$ isothermal, $\mathrm{t}_{\mathrm{R}}=$ cis $85.8 \mathrm{~min}$ (major) $/ 145.2$ min (minor), trans 178.9 min (major)/207.5 min (minor), 99\% ee.

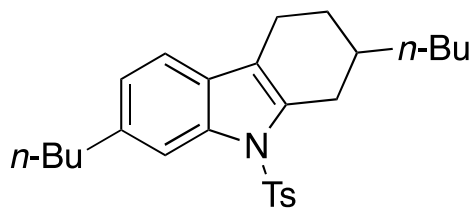

$40 \mathrm{~b}$

3,6-dibutyl-9-tosyl-2,3,4,9-tetrahydro-1H-carbazole, according to the general procedure, the following amounts were used: substrate $20 \mathrm{mg}$, Ir-complex $1 \mathrm{~mol} \%, \mathrm{CH}_{2} \mathrm{Cl}_{2} 0.5 \mathrm{~mL}, 50$ bar pressure $\mathrm{H}_{2}, 24 \mathrm{~h}$.

White solid, $\left(\mathrm{mp}=105-109{ }^{\circ} \mathrm{C}\right), 98 \%$ yield. $\mathrm{R}_{\mathrm{f}}=0.06($ EtOAc/Pentane $=1 / 99) .{ }^{1} \mathbf{H} \mathbf{~ N M R}\left(\mathrm{CDCl}_{3}, 400\right.$ MHz): $\delta 7.96(\mathrm{~d}, J=4.0 \mathrm{~Hz}, 1 \mathrm{H}), 7.63(\mathrm{~d}, J=8.4 \mathrm{~Hz}, 2 \mathrm{H}), 7.22(\mathrm{~d}, J=7.9 \mathrm{~Hz}, 1 \mathrm{H}), 7.17(\mathrm{~d}, J=8.0 \mathrm{~Hz}$, 2H), $7.04(\mathrm{dd}, J=7.9,1.4 \mathrm{~Hz}, 1 \mathrm{H}), 3.22-3.16(\mathrm{~m}, 1 \mathrm{H}), 2.73(\mathrm{t}, J=7.6 \mathrm{~Hz}, 2 \mathrm{H}), 2.66-2.59(\mathrm{~m}, 1 \mathrm{H}), 2.55-$ $2.46(\mathrm{~m}, 2 \mathrm{H}), 2.33(\mathrm{~s}, 3 \mathrm{H}), 1.95-1.89(\mathrm{~m}, 1 \mathrm{H}), 1.82-1.70(\mathrm{~m}, 1 \mathrm{H}), 1.67-1.59(\mathrm{~m}, 2 \mathrm{H}), 1.44-1.29(\mathrm{~m}, 9 \mathrm{H})$, 0.95-0.91 (m, 6H). ${ }^{13} \mathbf{C}$ NMR $\left(\mathrm{CDCl}_{3}, 100 \mathrm{MHz}\right): \delta 144.40,139.14,136.98,136.46,134.88,129.80$, $128.35,126.48,124.14,118.77,117.69,114.46,36.27,35.79,34.89,34.45,31.21,29.50,28.56,23.09$, 22.39, 21.67, 20.73, 14.28, 14.15. IR (KBr, neat, $\left.\mathrm{cm}^{-1}\right): v=3432,2953,2923,2855,1618,1598,1426$, 1362, 1169, 1092, 999, 817, 672, 592, 546. HRMS-EI m/z: [M+Na] ${ }^{+}$Calcd for $\mathrm{C}_{27} \mathrm{H}_{35} \mathrm{NO}_{2} \mathrm{SNa} 460.2281$; Found 460.2290. $[\alpha]_{D}^{23}=-1.96\left(c=0.1015, \mathrm{CHCl}_{3}\right)$. SFC : Chiralcel IC column, $95 \% \mathrm{CO}_{2} 5 \% \mathrm{MeOH}, 2$ $\mathrm{ml} / \mathrm{min}, \mathrm{t}_{R}=18.7 \mathrm{~min}$ (minor)/ $19.6 \mathrm{~min}$ (major), 90\% ee.<smiles>COC(=O)CC1CCCC(C)C1</smiles>

$29 b$

methyl 2-(3-methylcyclohexyl)acetate, according to the general procedure, the following amounts were used: substrate $14.4 \mathrm{mg}$, Ir-complex $0.5 \mathrm{~mol} \%, \mathrm{CH}_{2} \mathrm{Cl}_{2} 0.5 \mathrm{~mL}, 50$ bar pressure $\mathrm{H}_{2}, 24 \mathrm{~h}$. 
Colorless oil, 97\% yield. $\mathrm{R}_{\mathrm{f}}=0.15($ EtOAc/Pentane $=1 / 99) .{ }^{1} \mathbf{H}$ NMR $\left(\mathrm{CDCl}_{3}, 500 \mathrm{MHz}\right): \delta 3.66(\mathrm{~s}, 3 \mathrm{H})$, 2.29-2.25 (m, 1H), $2.17(\mathrm{dd}, J=7.1,2.6 \mathrm{~Hz}, 2 \mathrm{H}), 1.84-1.62(\mathrm{~m}, 5 \mathrm{H}), 1.59-1.08(\mathrm{~m}, 6 \mathrm{H}), 0.91(\mathrm{~d}, J=6.9$ $\mathrm{Hz}, 1 \mathrm{H}), 0.86(\mathrm{~d}, J=6.6 \mathrm{~Hz}, 3 \mathrm{H}) .{ }^{13} \mathbf{C} \mathbf{~ N M R}\left(\mathrm{CDCl}_{3}, 100 \mathrm{MHz}\right): \delta 174.00,173.74,51.51,51.49,42.23$, $41.96,39.60,39.03$, 35.07, 34.96, 33.60, 32.78, 32.61, 31.50, 30.04, 27.32, 26.14, 22.90, 20.82, 20.75. IR $\left(\mathrm{NaCl}\right.$, neat, $\left.\mathrm{cm}^{-1}\right): v=2952,2920,2846,1741,1456,1436,1376,1303,1252,1213,1170,1123,1022$. HRMS-EI $\mathrm{m} / \mathrm{z}$ : $[\mathrm{M}+\mathrm{Na}]^{+}$Calcd for $\mathrm{C}_{10} \mathrm{H}_{18} \mathrm{O}_{2} \mathrm{Na}$ 193.1199; Found 193.1192. $[\boldsymbol{\alpha}]_{\boldsymbol{D}}^{\mathbf{2 3}}=-5.33(c=0.150$, $\mathrm{CHCl}_{3}$ ). GC-MS: column Chiraldex- $\beta$-DM, $80^{\circ} \mathrm{C}$ isothermal, $\mathrm{t}_{\mathrm{R}}=$ cis $33.6 \mathrm{~min} / 34.7 \mathrm{~min}$, trans $39.1 \mathrm{~min}$ (minor)/41.3 min (major), 91\% ee.

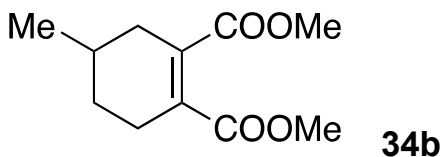

(-)-dimethyl 4-methylcyclohex-1-ene-1,2-dicarboxylate, according to the general procedure, the following amounts were used: substrate $10.5 \mathrm{mg}$, Ir-complex $0.5 \mathrm{~mol} \%, \mathrm{CH}_{2} \mathrm{Cl}_{2} 0.5 \mathrm{~mL}, 50$ bar pressure $\mathrm{H}_{2}$. Colorless oil. $\mathrm{R}_{f}=0.4$ (Pentane:EtOAc, 15:1). ${ }^{1} \mathbf{H}$ NMR $\left(\mathrm{CDCl}_{3}, 400 \mathrm{MHz}\right): \delta 1.01(\mathrm{~d}, J=6.59 \mathrm{~Hz}$, $3 \mathrm{H}), 1.24(\mathrm{~m}, 1 \mathrm{H}), 1.63-1.83(\mathrm{~m}, 2 \mathrm{H}), 1.91(\mathrm{~m}, 1 \mathrm{H}), 2.32(\mathrm{~m}, 1 \mathrm{H}), 2.40-2.52(\mathrm{~m}, 2 \mathrm{H}), 3.76(\mathrm{~s}, 6 \mathrm{H})$. ${ }^{13} \mathrm{C}$ NMR $\left(\mathrm{CDCl}_{3}, 100 \mathrm{MHz}\right): \delta 21.33,26.54,27.63,29.53,34.52,52.27,77.16,135.22,135.24,169.09$, 169.14. IR $\left(\mathrm{NaCl}\right.$, neat, $\left.\mathrm{cm}^{-1}\right): v=2952,2927,1723,1650,1434,1262,1067,1025,742$. HRMS-ESI $\mathrm{m} / \mathrm{z}:[\mathrm{M}+\mathrm{Na}]^{+}$Calc. for $\mathrm{C}_{11} \mathrm{H}_{16} \mathrm{O}_{4}$ 235.0940; Found 235.0941. $[\boldsymbol{a}]_{\boldsymbol{D}}^{23}=-73.13,\left(c=0.160, \mathrm{CHCl}_{3}\right)$. GCMS : column Chiraldex $\beta-\mathrm{DM}, 120^{\circ} \mathrm{C}$ isothermal, $\mathrm{t}_{\mathrm{R}}=55.4 \mathrm{~min}$ (major) $/ 57.1$ (minor), $97 \%$ ee.

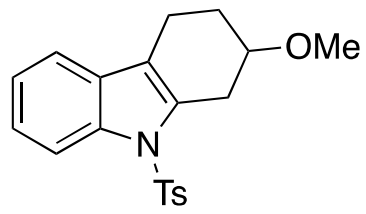

$41 b$

(+)-2-methoxy-9-tosyl-2,3,4,9-tetrahydro- $1 \mathrm{H}$-carbazole, according to the general procedure, the following amounts were used: substrate $10 \mathrm{mg}$, Ir-complex $0.5 \mathrm{~mol} \%, \mathrm{CH}_{2} \mathrm{Cl}_{2} 0.5 \mathrm{ml}, 50$ bar pressure $\mathrm{H}_{2}$. Colorless oil. $\mathrm{R}_{f}=0.4$ (Pentane:EtOAc, 15:1). ${ }^{1} \mathbf{H}$ NMR $\left(\mathrm{CDCl}_{3}, 400 \mathrm{MHz}\right): \delta 1.90(\mathrm{~m}, 1 \mathrm{H}), 2.02(\mathrm{~m}$, $1 \mathrm{H}), 2.32(\mathrm{~s}, 3 \mathrm{H}), 2.58(\mathrm{~m}, 1 \mathrm{H}), 2.72(\mathrm{~m}, 1 \mathrm{H}), 3.00(\mathrm{~m}, 1 \mathrm{H}), 3.35-3,47(\mathrm{~m}, 4 \mathrm{H}), 3.74(\mathrm{~m}, 1 \mathrm{H}), 7.15-$ $7.36(\mathrm{~m}, 5 \mathrm{H}), 7.63-7.68(\mathrm{~m}, 2 \mathrm{H}), 8.14(\mathrm{~m}, 1 \mathrm{H}) .{ }^{13} \mathbf{C} \mathbf{~ N M R}\left(\mathrm{CDCl}_{3}, 100 \mathrm{MHz}\right): \delta 18.20,21.69,27.02$, $30.58,56.46,75.94,77.16,77.36,114.62,118.26,118.28,123.42,124.17,126.52,129.89,129.95$, 132.82, 136.27, 136.78, 144.67. IR ( NaCl, neat, $\left.\mathrm{cm}^{-1}\right): v=2952,2927,1723,1650,1434,1262,1067$, 1025, 742. HRMS-ESI m/z: $[\mathrm{M}+\mathrm{Na}]^{+}$Calc. for $\mathrm{C}_{20} \mathrm{H}_{21} \mathrm{NO}_{3} \mathrm{~S}$ 378.1140; Found 378.1134. [a $]_{\boldsymbol{D}}^{\mathbf{2 3}}=+14.93$, $\left(c=0.100, \mathrm{CHCl}_{3}\right)$. SFC : Chiralcel OJ-H column, $80 \% \mathrm{CO}_{2} 20 \% \mathrm{MeOH}, 1.5 \mathrm{ml} / \mathrm{min}, \mathrm{t}_{R}=8.23 \mathrm{~min}$ (major)/ $8.96 \mathrm{~min}$ (minor), 99\% ee<smiles>CC(C)CC1CCC2=C(CCCC2)C1</smiles>

3b

(+)-2-isobutyl-1,2,3,4,5,6,7,8-octahydronaphthalene, according to the general procedure, the following amounts were used: substrate $9.4 \mathrm{mg}$, Ir-complex $0.5 \mathrm{~mol} \%, \mathrm{CH}_{2} \mathrm{Cl}_{2} 0.5 \mathrm{~mL}, 50$ bar pressure $\mathrm{H}_{2}$.

Colorless oil. $\mathrm{R}_{f}=0.9$ (Pentane). ${ }^{1} \mathbf{H}$ NMR $\left(\mathrm{CDCl}_{3}, 400 \mathrm{MHz}\right): \delta 0.80-0.92(\mathrm{~m}, 6 \mathrm{H}), 1.00-1.19(\mathrm{~m}$, $3 \mathrm{H}), 1.41-2.02(\mathrm{~m}, 15 \mathrm{H}) .{ }^{13} \mathbf{C}$ NMR $\left(\mathrm{CDCl}_{3}, 100 \mathrm{MHz}\right): \delta 22.87,23.28,23.38,23.41,25.03,30.04$, $30.31,30.58,30.86,31.87,37.79,46.61,127.69,128.02$. IR $\left(\mathrm{NaCl}\right.$, neat, $\left.\mathrm{cm}^{-1}\right): v=2952,2921,1365$, 1165, 1102, 822. HRMS-CI m/z: $[\mathrm{M}]^{+}$Calc. for $\mathrm{C}_{14} \mathrm{H}_{24}$ 214.1878; Found 214.1880. $[\boldsymbol{a}]_{\boldsymbol{D}}^{23}=+73.50,(c=$ $0.400, \mathrm{CHCl}_{3}$ ). GC-MS : column Chiraldex $\beta$-DM, $110^{\circ} \mathrm{C}$ isothermal, $\mathrm{t}_{\mathrm{R}}=26.1 \mathrm{~min}$ (major) $/ 27.0$ (minor), $92 \%$ ee. 
<smiles>CC1CC(C)CC(C(=O)O)C1</smiles>

3,5-dimethylcyclohexane-1-carboxylic acid, according to the general procedure, the following amounts were used: substrate $10 \mathrm{mg}$, Ir-complex $1 \mathrm{~mol} \%, \mathrm{CH}_{2} \mathrm{Cl}_{2} 0.5 \mathrm{~mL}, 100$ bar pressure $\mathrm{H}_{2}, 24 \mathrm{~h}$.

White solid, 91\% isolated yield. $\mathrm{R}_{f}=0.5$ (Pentane:EtOAc, 9:1). ${ }^{1} \mathbf{H}$ NMR $\left(\mathrm{CDCl}_{3}, 400 \mathrm{MHz}\right): \delta 2.56$ (tt, $J$ $=12.4,3.6 \mathrm{~Hz}, 1 \mathrm{H}), 2.19-2.01(\mathrm{~m}, 1 \mathrm{H}), 1.99-1.85(\mathrm{~m}, 1 \mathrm{H}), 1.78-1.60(\mathrm{~m}, 2 \mathrm{H}), 1.57(\mathrm{td}, J=12.8,4.7$ $\mathrm{Hz}, 1 \mathrm{H}), 1.50-1.41(\mathrm{~m}, 1 \mathrm{H}), 1.18-0.99(\mathrm{~m}, 2 \mathrm{H}), 0.99(\mathrm{~d}, J=7.2 \mathrm{~Hz}, 3 \mathrm{H}), 0.88(\mathrm{~d}, J=6.5 \mathrm{~Hz}, 3 \mathrm{H}) .{ }^{13} \mathbf{C}$ NMR $\left(\mathrm{CDCl}_{3}, 100 \mathrm{MHz}\right): \delta$ 183.17, 40.08, 37.97, 37.49, 34.04, 27.54, 25.78, 22.74, 18.41. IR ( NaCl, neat, $\left.\mathrm{cm}^{-1}\right): v=3032,2954,2927,1704,1459,1280,1219,940$. HRMS-ESI m/z: $[\mathrm{M}+\mathrm{Na}]^{+} \mathrm{Calc}$. for $\mathrm{C}_{9} \mathrm{H}_{16} \mathrm{O}_{2} \mathrm{Na}$ 179.1048; Found 179.1049. $[\boldsymbol{a}]_{\boldsymbol{D}}^{23}=+3.20,\left(c=0.500, \mathrm{CHCl}_{3}\right)$. GC-MS: analysed as the corresponding methyl ester, column Hydrodex $\beta-6 \mathrm{TBDM}, 90^{\circ} \mathrm{C}$ isothermal, $\mathrm{t}_{\mathrm{R}}=$ trans $17.9 \mathrm{~min}$ (major)/19.5 min (minor), $99 \%$ ee.<smiles>CC1CCC2C(=O)CCCC2C1</smiles>

$33 b-c$

6-Methyloctahydronaphthalen-1(2H)-one, according to the general procedure, the following amounts were used: substrate $5 \mathrm{mg}$, Ir-complex $1 \mathrm{~mol} \%, \mathrm{CH}_{2} \mathrm{Cl}_{2} 0.5 \mathrm{~mL}, 50$ bar pressure $\mathrm{H}_{2}$.

Colorless oil, 98\%yield. $\mathrm{R}_{f}=0.54($ EtOAc/Pentane $=2 / 48) .{ }^{1} \mathbf{H}$ NMR $\left(\mathrm{CDCl}_{3}, 400 \mathrm{MHz}\right): \delta 2.39-2.27(\mathrm{~m}$, 4H), $2.09-1.97(\mathrm{~m}, 3 \mathrm{H}), 1.93-1.85(\mathrm{~m}, 3 \mathrm{H}), 1.79-1.55(\mathrm{~m}, 11 \mathrm{H}), 1.52-1.22(\mathrm{~m}, 9 \mathrm{H}), 0.94(\mathrm{~d}, J=7.2$ $\mathrm{Hz}, 3 \mathrm{H}), 0.88(\mathrm{~d}, J=6.5 \mathrm{~Hz}, 3 \mathrm{H}) .{ }^{13} \mathbf{C}$ NMR $\left(\mathrm{CDCl}_{3}, 100 \mathrm{MHz}\right): \delta 213.03,55.67,54.86,44.85,43.19$, $42.02,41.98,40.17,38.84,34.20,33.33,33.13,32.16,30.78,27.28,26.71,26.68,25.18,22.57,19.33$, 18.00. IR (NaCl, neat, $\left.\mathrm{cm}^{-1}\right): v=3584,2923,2865,1709,1456,1378,1240,1195,1105,1046,947,819$. HRMS-ESI m/z: $[\mathrm{M}+\mathrm{Na}]^{+}$Calc. for $\mathrm{C}_{9} \mathrm{H}_{16} \mathrm{O}_{2} \mathrm{Na}$ 189.1250; Found 189.1257. $[\boldsymbol{a}]_{\boldsymbol{D}}^{\mathbf{2 3}}=-2.48,(c=0.175$, $\mathrm{CHCl}_{3}$ ). GC-MS: mixture of cis-fused $4 R, 8 \mathrm{~S}$ and $4 S, 8 R$ isomers, column Chiraldex $\beta$-DM, $100^{\circ} \mathrm{C}$ isothermal, $\mathrm{t}_{\mathrm{R}}=50.6 \mathrm{~min}$ (major) $/ 53.2 \mathrm{~min}$ (minor), $54.6 \mathrm{~min}$ (major) $/ 60.4 \mathrm{~min}$ (minor), $99 \%$ ee.<smiles>CC1=C(c2ccccc2)CC(C)CC1</smiles>

3,4',6-trimethyl-2,3,4,5-tetrahydro-1,1'-biphenyl, according to the general procedure, the following amounts were used: substrate $10 \mathrm{mg}$, Ir-complex $1 \mathrm{~mol} \%, \mathrm{CH}_{2} \mathrm{Cl}_{2} 1 \mathrm{~mL}, 50$ bar pressure $\mathrm{H}_{2}$,

Colorless oil, $\mathrm{R}_{\mathrm{f}}=0.68$ (pentane), ${ }^{1} \mathbf{H}$ NMR $\left(\mathrm{CDCl}_{3}, 400 \mathrm{MHz}\right): \delta 7.26(\mathrm{~m}, 2 \mathrm{H}), 7.15(\mathrm{~m}, 1 \mathrm{H}), 7.09(\mathrm{~m}$, 2H), 2.33-1.99 (m, 3H), 2.90-1.65 (m, 3H), $1.51(\mathrm{~s}, 3 \mathrm{H}), 1.32-1.19(\mathrm{~m}, 1 \mathrm{H}), 0.94(\mathrm{~d}, 3 \mathrm{H}) . .{ }^{13} \mathbf{C} \mathbf{~ N M R}$ $\left(\mathrm{CDCl}_{3}, 100 \mathrm{MHz}\right): \delta 144.52,132.00,128.80,128.58,128.06,125.94,77.48,77.16,76.84,40.72,31.97$, 31.47, 29.53, 21.89, 20.56. IR $\left(\mathrm{NaCl}\right.$, neat, $\left.\mathrm{cm}^{-1}\right): v=: 3147.85,3076.39,3020.43,2949.67,2911.00$, 2829.59, 2726.16, 1943.13, 1743.51, 1662.77, 1599.50, 1489.89, 1377.06, 1259.59, 1070.36, 1026.92, 803.69. HRMS-CI: $\mathrm{m} / \mathrm{z}:[\mathrm{M}]^{+}$Calc. for $\mathrm{C}_{14} \mathrm{H}_{18} 186.1409$; Found 184.1408. $[\boldsymbol{a}]_{\boldsymbol{D}}^{23}=-73.22,(c=0.183$, $\mathrm{CHCl}_{3}$ ). GC-MS : column Chiraldex $\beta$-DM, $80^{\circ} \mathrm{C}$ isothermal, $\mathrm{t}_{\mathrm{R}}=153.7 \mathrm{~min}$ (major) $/ 156.6 \mathrm{~min}$ (minor), $94 \%$ ee.<smiles>CC1CCCC(c2ccc(C(F)(F)F)cc2)C1</smiles>

$10 \mathrm{~b}$

1-(3-methylcyclohexyl)-4-(trifluoromethyl)benzene, according to the general procedure, the following amounts were used: substrate $10 \mathrm{mg}$, Ir-complex $0.75 \mathrm{~mol} \%, \mathrm{CH}_{2} \mathrm{Cl}_{2} 0.5 \mathrm{~mL}, 50$ bar pressure $\mathrm{H}_{2}$, 
Colorless oil, 94\% yield. $\mathrm{R}_{\mathrm{f}}=0.79$ (pentane). ${ }^{1} \mathbf{H}$ NMR $\left(\mathrm{CDCl}_{3}, 400 \mathrm{MHz}\right): \delta 7.53(\mathrm{~m}, 2 \mathrm{H}), 7.33(\mathrm{~d}, 2 \mathrm{H})$, $2.87(\mathrm{~m}, 1 \mathrm{H}), 2.04(\mathrm{~m}, 1 \mathrm{H}), 1.64(\mathrm{~m}, 8 \mathrm{H}), 1.06(\mathrm{~d}, 3 \mathrm{H}) .{ }^{13} \mathbf{C} \mathbf{~ N M R}\left(\mathrm{CDCl}_{3}, 100 \mathrm{MHz}\right): \delta 151.81,127.50$, $126.78,125.35,125.31,125.28,125.24,77.48,77.36,77.16,76.84,39.82,38.05,35.98,33.75,31.96$, 30.30, 29.04, 27.96, 26.17, 22.08, 21.12, 18.85. IR $\left(\mathrm{NaCl}, \mathrm{CCl}_{4}, \mathrm{~cm}^{-1}\right): v=2958.37,2927.39,2853.94$, 2295.64, 1702.82, 1549.81, 1326.53, 1257.03, 1217.61, 1130.08, 1006.41, 979.13 HRMS-CI: m/z: [M] ${ }^{+}$ Calc. for $\mathrm{C}_{14} \mathrm{H}_{17} \mathrm{~F}_{3} 242.1282$; Found 242.1293. $[\boldsymbol{a}]_{\boldsymbol{D}}^{23}=-7.80,\left(c=0.141, \mathrm{CHCl}_{3}\right)$. GC-MS : column Chiraldex $\beta$-DM, $85^{\circ} \mathrm{C}$ isothermal, $\mathrm{t}_{\mathrm{R}}=231.8 \mathrm{~min}$ (major) $/ 243.2 \mathrm{~min}$ (minor), $99 \%$ ee.<smiles>Cc1ccc(C2CCCC(C)C2)cc1</smiles>

$9 b$

1-methyl-4-(3-methylcyclohexyl)benzene, according to the general procedure, the following amounts were used: substrate $11 \mathrm{mg}$, Ir-complex $0.5 \mathrm{~mol} \%, \mathrm{CH}_{2} \mathrm{Cl}_{2} 0.5 \mathrm{~mL}, 50$ bar pressure $\mathrm{H}_{2}$.

Colorless oil, $81 \%$ yield. $\mathrm{R}_{\mathrm{f}}=0.24$ (pentane), ${ }^{1} \mathbf{H}$ NMR $\left(\mathrm{CDCl}_{3}, 400 \mathrm{MHz}\right): \delta 7.12(\mathrm{~m}, 4 \mathrm{H}), 2.77(\mathrm{~m}, 1 \mathrm{H})$, $2.32(\mathrm{~s}, 3 \mathrm{H}), 2.04(\mathrm{~m}, 1 \mathrm{H}), 1.78-1.69(\mathrm{~m}, 2 \mathrm{H}), 1.64 .1 .39(\mathrm{~m}, 6 \mathrm{H}), 1.05(\mathrm{~d}, 3 \mathrm{H}) .{ }^{13} \mathbf{C} \mathbf{~ N M R}\left(\mathrm{CDCl}_{3}, 100\right.$ MHz): $\delta$ 144.81, 135.19, 129.11, 129.07, 127.04, 40.14, 37.56, 34.13, 32.14, 29.86, 28.09, 21.32, 21.10, 18.92. IR $\left(\mathrm{NaCl}, \mathrm{CCl}_{4}, \mathrm{~cm}^{-1}\right): v=: 2925.01,2852.84,1560.04,1545.88,1459.84,1377.79,1256.76$, 1006.25 HRMS-CI: $\mathrm{m} / \mathrm{z}:[\mathrm{M}]^{+}$Calc. for $\mathrm{C}_{14} \mathrm{H}_{20}$ 188.1565; Found 188.1575. $[\boldsymbol{a}]_{\boldsymbol{D}}^{\mathbf{2 3}}=1.94,(c=0.103$, $\mathrm{CHCl}_{3}$ ). GC-MS : column Chiraldex $\beta$-DM, $80^{\circ} \mathrm{C}$ isothermal, $\mathrm{t}_{\mathrm{R}}=$ cis 208.9 min (major) $/ 215.5$ min (minor), trans 263.7 min (major)/272.5 min (minor), 99\% ee.<smiles>CC1CCCC(c2ccccc2)C1</smiles>

\section{$8 b$}

(3-methylcyclohexyl)benzene, according to the general procedure, the following amounts were used: substrate $10 \mathrm{mg}$, Ir-complex $0.5 \mathrm{~mol} \%, \mathrm{CH}_{2} \mathrm{Cl}_{2} 0.5 \mathrm{~mL}, 50$ bar pressure $\mathrm{H}_{2}$.

Colorless oil, 91\% yield. $\mathrm{R}_{\mathrm{f}}=0.29$ (pentane). ${ }^{1} \mathbf{H}$ NMR $\left(\mathrm{CDCl}_{3}, 400 \mathrm{MHz}\right): \delta 7.36-7.21(\mathrm{~m}, 4 \mathrm{H}), 7.21-$ $7.14(\mathrm{~m}, 1 \mathrm{H}), 2.819 \mathrm{~m}, 1 \mathrm{H}), 2.05(\mathrm{~m}, 1 \mathrm{H}), 1.89-1.71(\mathrm{~m}, 2 \mathrm{H}), 1.67-1.39(\mathrm{~m}, 6 \mathrm{H}), 1.06(\mathrm{~d}, 3 \mathrm{H}) .{ }^{13} \mathbf{C} \mathbf{N M R}$ $\left(\mathrm{CDCl}_{3}, 100 \mathrm{MHz}\right): \delta 147.65,128.68,128.22,127.98,127.17,127.15,127.02,125.63,77.33,77.21$, 77.01, 76.69, 39.89, 37.86, 33.90, 31.96, 27.93, 21.14, 18.74. IR ( NaCl, $\left.\mathrm{CCl}_{4}, \mathrm{~cm}^{-1}\right): v=: 3584.01$, 3063.88, 3028.79, 2958.61, 2945.44, 2851.77, 1601.65, 1583.79, 1493.13, 1459.90, 1378.26, 1260.56, 1157.33, 1072.20, 1025.88. HRMS-CI: $\mathrm{m} / \mathrm{z}$ : $[\mathrm{M}]^{+}$Calc. for $\mathrm{C}_{13} \mathrm{H}_{18}$ 175.1487; Found 175.1499. $[\boldsymbol{a}]_{\boldsymbol{D}}^{\mathbf{2 3}}=-$ 1.22, $\left(c=0.163, \mathrm{CHCl}_{3}\right)$. GC-MS : column Chiraldex $\beta$-DM, $80^{\circ} \mathrm{C}$ isothermal, $\mathrm{t}_{\mathrm{R}}=$ cis $149.8 \mathrm{~min}$ (major)/146.3 min (minor), trans $198.6 \mathrm{~min}$ (major)/191.9 $\mathrm{min}$ (minor), 99\% ee.<smiles>CC1CCCC(c2ccsc2)C1</smiles>

3-(3-methylcyclohexyl)thiophene, Colorless oil. ${ }^{1} \mathbf{H}$ NMR $\left(\mathrm{CDCl}_{3} .400 \mathrm{MHz}\right): \delta 7.24(\mathrm{~m}, 2 \mathrm{H}), 7.00(\mathrm{~m}$, 1H), $6.95(\mathrm{~m}, 1 \mathrm{H}), 2.95(\mathrm{~m}, 1 \mathrm{H}), 2.24(\mathrm{~m}, 1 \mathrm{H}), 2.04-1.28(\mathrm{~m}, 8 \mathrm{H}), 0.99(\mathrm{~d}, 3 \mathrm{H}) \cdot{ }^{13} \mathbf{C} \mathbf{~ N M R}\left(\mathrm{CDCl}_{3}, 400\right.$ MHz): 141.65, 141.50, 138.46, 133.91, 131.48, 128.82, 128.79, 128.40, 128.13, 127.32, 127.29, 126.99, $126.74,125.12,124.41,121.77,118.06,34.28,33.03,32.29,29.87,29.09,28.45,23.51,23.06,22.50$, 21.69, 14.22. IR $\left(\mathrm{NaCl}, \mathrm{CCl}_{4}, \mathrm{~cm}^{-1}\right): \mathrm{v}=: 3049,12,2926.52,2853.10,1551.37,1458.18,1377.51,1264.22$, 1218.63, 1009.09. $[\boldsymbol{a}]_{\boldsymbol{D}}^{23}=-8.176,\left(c=0.159, \mathrm{CHCl}_{3}\right)$. 


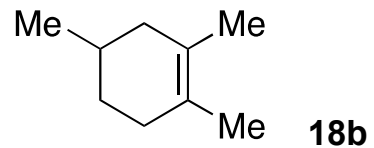

1,2,4-trimethylcyclohex-1-ene, ${ }^{21}$ according to the general procedure, the following amounts were used: substrate $12 \mathrm{mg}$, Ir-complex $0.5 \mathrm{~mol} \%, \mathrm{CH}_{2} \mathrm{Cl}_{2} 1 \mathrm{ml}, 20$ bar pressure $\mathrm{H}_{2}$.

$[\boldsymbol{a}]_{\boldsymbol{D}}^{\mathbf{2 3}}=+2.00\left(c=0.05, \mathrm{CHCl}_{3}\right)$. GC-MS: column Chiraldex- $\beta$-DM, $40{ }^{\circ} \mathrm{C}$ isothermal, $1 \mathrm{ml} / \mathrm{min}(\mathrm{He}), \mathrm{t}_{R}=$ $34.4 \mathrm{~min}$ (minor) $/ 35.3 \mathrm{~min}$ (major), $92 \%$ ee.<smiles>CC1CCc2[nH]ccc2C1</smiles>

5-methyl-4, 5,6,7-tetrahydro- $1 \mathrm{H}$-indole, ${ }^{22,23}$ according to the general procedure, the following amounts were used: substrate $13 \mathrm{mg}$, Ir-complex $0.5 \mathrm{~mol} \%, \mathrm{CH}_{2} \mathrm{Cl}_{2} 1 \mathrm{ml}, 50$ bar pressure $\mathrm{H}_{2}$.

98\% yield. $[\boldsymbol{a}]_{\boldsymbol{D}}^{\mathbf{2 3}}=+99.00\left(c=0.20, \mathrm{CHCl}_{3}\right)$. GC-MS: column Chiraldex- $\beta$-DM, $90^{\circ} \mathrm{C}$ isothermal, 1 $\mathrm{ml} / \mathrm{min}(\mathrm{He}), \mathrm{t}_{R}=31.2 \mathrm{~min}$ (major) $/ 35.9 \mathrm{~min}$ (minor), $98 \%$ ee.<smiles>COC1CCc2[nH]ccc2C1</smiles>

38b

5-methoxy-4,5,6,7-tetrahydro-1 $\mathrm{H}$-indole, ${ }^{23}$ according to the general procedure, the following amounts were used: substrate $15 \mathrm{mg}$, Ir-complex $2 \mathrm{~mol} \%, \mathrm{CH}_{2} \mathrm{Cl}_{2} 1 \mathrm{ml}, 100$ bar pressure $\mathrm{H}_{2}$.

$[\boldsymbol{a}]_{\boldsymbol{D}}^{\mathbf{2 3}}=-24.55\left(c=0.22, \mathrm{CHCl}_{3}\right)$. GC-MS: column Chiraldex- $\beta$-DM, $110^{\circ} \mathrm{C}$ isothermal, $1 \mathrm{ml} / \mathrm{min}(\mathrm{He}), \mathrm{t}_{R}$ $=60.7 \mathrm{~min}$ (major) $/ 62.8 \mathrm{~min}$ (minor), $99 \%$ ee.<smiles>CCCCC1CCc2[nH]ccc2C1</smiles>

$36 b$

5-butyl-4,5,6,7-tetrahydro-1H-indole, according to the general procedure, the following amounts were used: substrate $16 \mathrm{mg}$, Ir-complex $0.5 \mathrm{~mol} \%, \mathrm{CH}_{2} \mathrm{Cl}_{2} 1 \mathrm{ml}, 50$ bar pressure $\mathrm{H}_{2}$.

Colorless oil, 85\% yield. $\mathrm{R}_{f}=0.50$ (Pentane/EtOAc $\left.=15 / 1\right) .{ }^{1} \mathbf{H}$ NMR $\left(\mathrm{CDCl}_{3}, 400 \mathrm{MHz}\right): \delta 7.70(\mathrm{~s}, 1 \mathrm{H})$, $6.62(\mathrm{~s}, 1 \mathrm{H}), 5.97(\mathrm{~s}, 1 \mathrm{H}), 2.75-2.50(\mathrm{~m}, 3 \mathrm{H}), 2.18-2.09(\mathrm{~m}, 1 \mathrm{H}), 1.99-1.84(\mathrm{~m}, 1 \mathrm{H}), 1.74-1.60(\mathrm{~m}$, $1 \mathrm{H}), 1.59-1.20(\mathrm{~m}, 9 \mathrm{H}), 1.02-0.83(\mathrm{~m}, 4 \mathrm{H}) .{ }^{13} \mathbf{C} \mathbf{~ N M R}\left(\mathrm{CDCl}_{3}, 100 \mathrm{MHz}\right): \delta 126.92,116.88,115.80$, 107.37, 36.09, 35.19, 30.01, 29.52, 29.49, 22.98, 22.52, 14.18. IR $\left(\mathrm{CHCl}_{3}\right.$, neat, $\left.\mathrm{cm}^{-1}\right): v=3351,2927$, 1690, 1456, 1216, 1095, 757, 665. HRMS m/z: $[\mathrm{M}+\mathrm{H}]^{+}$Calc. for $\mathrm{C}_{12} \mathrm{H}_{19} \mathrm{~N}_{1}$ 177.1517; Found 177.1512. $[\boldsymbol{a}]_{\boldsymbol{D}}^{\mathbf{2}}=+18.70\left(c=0.20, \mathrm{CHCl}_{3}\right)$. SFC: Chiralcel OJ-H column, $95 \% \mathrm{CO}_{2} 5 \% \mathrm{MeOH}, 2 \mathrm{ml} / \mathrm{min}, \mathrm{t}_{R}=9.29$ min (major)/9.95 min (minor), $90 \%$ ee.<smiles>COC1CCc2cc[nH]c2C1</smiles>

\section{$39 \mathrm{~b}$}

5-butyl-4,5,6,7-tetrahydro-1 $\mathrm{H}$-indole, according to the general procedure, the following amounts were used: substrate $15 \mathrm{mg}$, Ir-complex $2 \mathrm{~mol} \%, \mathrm{CH}_{2} \mathrm{Cl}_{2} 1 \mathrm{ml}, 100$ bar pressure $\mathrm{H}_{2}$.

Colorless oil, 93\% yield. $\mathrm{R}_{f}=0.34$ (Pentane/EtOAc=9/1). ${ }^{1} \mathbf{H}$ NMR $\left(\mathrm{C}_{6} \mathrm{D}_{6}, 400 \mathrm{MHz}\right): \delta 6.50(\mathrm{~s}, 1 \mathrm{H})$, $6.33(\mathrm{~s}, 1 \mathrm{H}), 6.09(\mathrm{~s}, 1 \mathrm{H}), 3.47-3.36(\mathrm{~m}, 1 \mathrm{H}), 3.17(\mathrm{~s}, 3 \mathrm{H}), 2.76-2.34(\mathrm{~m}, 4 \mathrm{H}), 2.01-1.95(\mathrm{~m}, 1 \mathrm{H})$, $1.77-1.65(\mathrm{~m}, 1 \mathrm{H}) .{ }^{13} \mathbf{C}$ NMR $\left(126 \mathrm{MHz}, \mathrm{C}_{6} \mathrm{D} 6\right): \delta 123.91,116.35,116.15,107.06,77.38,55.56,29.51$, 29.31, 20.99. IR $\left(\mathrm{CHCl}_{3}\right.$, neat, $\left.\mathrm{cm}^{-1}\right): v=3368,2922,1593,1463,1088,966,906,826,710 . \mathbf{H R M S} \mathrm{m} / \mathrm{z}$ : $[\mathrm{M}+\mathrm{H}]^{+}$Calc. for $\mathrm{C}_{9} \mathrm{H}_{13} \mathrm{~N}_{1} \mathrm{O}_{1}$ 151.0997; Found 151.1097. $[\boldsymbol{a}]_{\boldsymbol{D}}^{23}=-58.70\left(c=0.15, \mathrm{CHCl}_{3}\right)$. GC-MS: column Chiraldex- $\beta-\mathrm{DM}, 120^{\circ} \mathrm{C}$ isothermal, $1 \mathrm{ml} / \mathrm{min}(\mathrm{He}), \mathrm{t}_{R}=41.4 \mathrm{~min}$ (major) $/ 44.7 \mathrm{~min}$ (minor), $99 \%$ ee. 


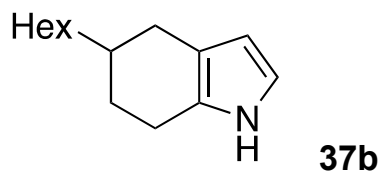

5-hexyl-4,5,6,7-tetrahydro-1H-indole, according to the general procedure, the following amounts were used: substrate $20 \mathrm{mg}$, Ir-complex $0.5 \mathrm{~mol} \%, \mathrm{CH}_{2} \mathrm{Cl}_{2} 1 \mathrm{ml}, 50$ bar pressure $\mathrm{H}_{2}$.

Colorless oil, $83 \%$ yield. $\mathrm{R}_{f}=0.55($ Pentane/EtOAc $=20 / 1) .{ }^{1} \mathbf{H}$ NMR $\left(\mathrm{CDCl}_{3}, 400 \mathrm{MHz}\right): \delta 7.70(\mathrm{~s}, 1 \mathrm{H})$, $6.62(\mathrm{~s}, 1 \mathrm{H}), 5.97(\mathrm{~s}, 1 \mathrm{H}), 2.70-2.54(\mathrm{~m}, 3 \mathrm{H}), 2.15-2.09(\mathrm{~m}, 2 \mathrm{H}), 1.93-1.87(\mathrm{~m}, 1 \mathrm{H}), 1.55-1.08(\mathrm{~m}$, 21H), $0.90-0.87(\mathrm{~m}, 6 \mathrm{H}) .{ }^{13} \mathrm{C}$ NMR $\left(\mathrm{CDCl}_{3}, 100 \mathrm{MHz}\right): \delta 126.92,116.89,115.79,107.37,36.39,35.18$, 31.94, 30.01, 29.59, 29.49, 27.18, 22.70, 22.51, 14.13. IR $\left(\mathrm{CHCl}_{3}\right.$, neat, $\left.\mathrm{cm}^{-1}\right): v=3378,2924,1673$, 1466, 1261, 1216, 1094, 1030, 759, 711, 666. HRMS m/z: $[\mathrm{M}+\mathrm{H}]^{+}$Calc. for $\mathrm{C}_{14} \mathrm{H}_{23} \mathrm{~N}_{1}$ 206.1903; Found 206.1932. $[\boldsymbol{a}]_{\boldsymbol{D}}^{23}=+17.60\left(c=0.25, \mathrm{CHCl}_{3}\right)$. SFC: Chiralcel OJ-H column, $95 \% \mathrm{CO}_{2} 5 \% \mathrm{MeOH}, 2 \mathrm{ml} / \mathrm{min}$, $\mathrm{t}_{R}=8.76 \mathrm{~min}$ (major) $/ 10.88 \mathrm{~min}$ (minor), $92 \%$ ee.<smiles>CCC1=C(CC)CC(C)CC1</smiles>

\section{$19 b$}

1,2-diethyl-4-methylcyclohex-1-ene, according to the general procedure, the following amounts were used: substrate $20 \mathrm{mg}$, Ir-complex $2 \mathrm{~mol} \%, \mathrm{CH}_{2} \mathrm{Cl}_{2} 1 \mathrm{~mL}, 50$ bar pressure $\mathrm{H}_{2}$.

Colorless oil. ${ }^{1} \mathbf{H}$ NMR $\left(\mathrm{CDCl}_{3}, 400 \mathrm{MHz}\right): \delta 2.08-1.88(\mathrm{~m}, 7 \mathrm{H}), 1.70-1.52(\mathrm{~m}, 3 \mathrm{H}), 1.24-1.17(\mathrm{~m}, 1 \mathrm{H})$, 0.96-0.91 (m, 9H). ${ }^{13} \mathbf{C}$ NMR $\left(\mathrm{CDCl}_{3}, 100 \mathrm{MHz}\right): \delta 130.85,130.82,37.75,31.81,29.39,29.22,25.94$, 25.75, 22.18, 13.56, 13.47. IR $\left(\mathrm{NaCl}\right.$, neat, $\left.\mathrm{cm}^{-1}\right): v=3026.21,2964.15,2928.19,2725.21,2052.06$, 1231.75, 1294.15. HRMS-CI m/z: $[\mathrm{M}]^{+}$Calc. for $\mathrm{C}_{11} \mathrm{H}_{22}$ 154.1721; Found 154.1736. $[\boldsymbol{a}]_{\boldsymbol{D}}^{23}=-89.266,(c$ $\left.=0.230, \mathrm{CHCl}_{3}\right)$. GC-MS: column Chiraldex- $\beta-\mathrm{DM}, 55^{\circ} \mathrm{C}$ isothermal, $1 \mathrm{ml} / \mathrm{min}(\mathrm{He}), \mathrm{t}_{R}=39.1 \mathrm{~min}$ (minor) $/ 40.1 \mathrm{~min}$ (major), 99\% ee.<smiles>CC1CCC=C(c2ccsc2)C1</smiles>

\section{$42 c$}

3-(5-methylcyclohex-1-en-1-yl)thiophene, according to the general procedure, the following amounts were used: substrate $25 \mathrm{mg}$, Ir-complex $0.5 \mathrm{~mol} \%, \mathrm{CH}_{2} \mathrm{Cl}_{2} 1 \mathrm{~mL}, 50$ bar pressure $\mathrm{H}_{2}, 5 \mathrm{~h}$.

Colorless oil, $\mathrm{R}_{f}=0.68$ (pentane). ${ }^{1} \mathbf{H}$ NMR $\left(\mathrm{CDCl}_{3} .400 \mathrm{MHz}\right): \delta 7.26-7.23(\mathrm{~m}, 2 \mathrm{H}), 7.09(\mathrm{t}, 1 \mathrm{H}), 6.18-$ $6.13(\mathrm{~m}, 1 \mathrm{H}), 2.54-2.44(\mathrm{~m}, 1 \mathrm{H}), 2.31-2.13(\mathrm{~m}, 2 \mathrm{H}), 2.06-1.94(\mathrm{~m}, 1 \mathrm{H}), 1.91-1.69(\mathrm{~m}, 2 \mathrm{H}), 1.68-1.47(\mathrm{~m}$, $1 \mathrm{H}), 1.08-1.01(\mathrm{~d}, \mathrm{~J}=6.55 \mathrm{~Hz}, 3 \mathrm{H}) \cdot{ }^{13} \mathbf{C} \mathbf{N M R}\left(\mathrm{CDCl}_{3}, 400 \mathrm{MHz}\right): \delta .143 .92,131.59,127.40,125.16$, $124.82,124.77,123.46,117.84,39.63,35.90,34.13,33.79,30.46,28.81,27.70,25.69,22.35,22.01$, 21.17, 14.07. IR $\left(\mathrm{NaCl}, \mathrm{CCl}_{4}, \mathrm{~cm}^{-1}\right): \mathrm{v}=: 3049.17,2950.41,2922.85,2683.38,1456.08,1434.73,1264.15$, 863.92. HRMS: $\mathrm{m} / \mathrm{z}$ : $[\mathrm{M}]^{+}$Calc. 178.0828 Found ; 178.0816. $[\boldsymbol{a}]_{\boldsymbol{D}}^{\mathbf{2 3}}=-26.901,\left(c=0.342, \mathrm{CHCl}_{3}\right)$. SFC: Chiralcel IF column, $95 \% \mathrm{CO}_{2} 5 \% \mathrm{MeOH}, 2 \mathrm{ml} / \mathrm{min}, \mathrm{t}_{R}=8.8 \mathrm{~min}$ (major) $/ 9.2 \mathrm{~min}$ (minor), $99 \%$ ee.<smiles>CC1CCC=C(c2ccc(C(F)(F)F)cc2)C1</smiles>

$10 \mathrm{c}$

3-methyl-4'-(trifluoromethyl)-2,3,4,5-tetrahydro-1,1'-biphenyl, according to the general procedure, the following amounts were used: substrate $25 \mathrm{mg}$, Ir-complex $0.5 \mathrm{~mol} \%, \mathrm{CH}_{2} \mathrm{Cl}_{2} 1 \mathrm{~mL}, 5$ bar pressure $\mathrm{H}_{2}$, 0.5h. Colorless oil, $\mathrm{R}_{f}=0.81$ (pentane). ${ }^{1} \mathbf{H}$ NMR $\left(\mathrm{CDCl}_{3} .400 \mathrm{MHz}\right): \delta$ 7.57-7.51 (m, 2H), 7.49-7.44 (m, $2 \mathrm{H}), 6.21-6.16(\mathrm{~m}, 1 \mathrm{H}), 2.50-2.41(\mathrm{~m}, 1 \mathrm{H}), 2.36-2.19(\mathrm{~m}, 2 \mathrm{H}), 2.09-1.98(\mathrm{~m}, 2 \mathrm{H}), 1.34-1.20(\mathrm{~m}, 1 \mathrm{H})$, $1.07(\mathrm{~d}, \mathrm{~J}=6.6 \mathrm{~Hz}, 3 \mathrm{H}) .{ }^{13} \mathrm{C} \mathbf{~ N M R}\left(\mathrm{CDCl}_{3}, 100 \mathrm{MHz}\right): \delta 146.00,135.33,126.63,125.16,125.12,125.08$, 125.04, 99.98, 35.83, 30.16, 28.90, 26.02, 21.93. IR $\left(\mathrm{NaCl}, \mathrm{CCl}_{4}, \mathrm{~cm}^{-1}\right): \mathrm{v}=: 3004.00,2927.09,2646.30$, 1800.82, 1614.93, 1326.34, 1069.41, 1165.51, 1123.36, 825.70. HRMS: Calc ; 240.1126 for $\mathrm{C}_{14} \mathrm{H}_{15} \mathrm{~F}_{3}$ Found ; 240.1140. $[\boldsymbol{a}]_{\boldsymbol{D}}^{\mathbf{2 3}}=-47.423,\left(c=0.194, \mathrm{CHCl}_{3}\right)$. GC-MS: column Chiraldex- $\beta-\mathrm{DM}, 85^{\circ} \mathrm{C}$ isothermal, $1 \mathrm{ml} / \mathrm{min}\left(\mathrm{He}\right.$ ), $\mathrm{t}_{R}=231.8 \mathrm{~min}$ (major) $/ 243.2 \mathrm{~min}$ (minor), $99 \%$ ee. 


\section{Catalysts}

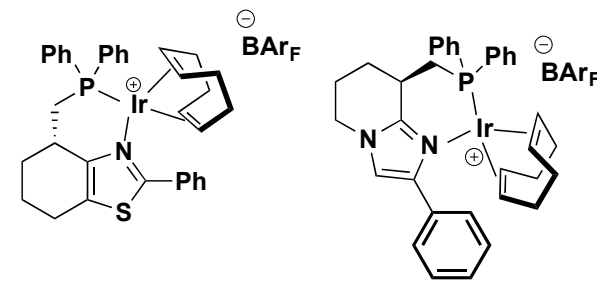

i

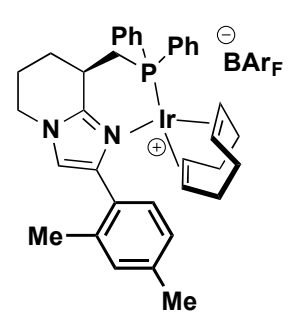

$\mathbf{v}$ ii

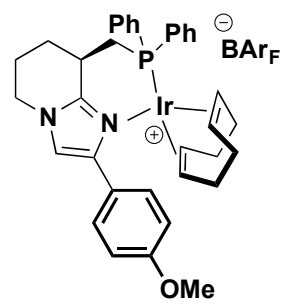

vi

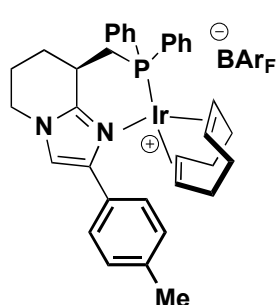

iii

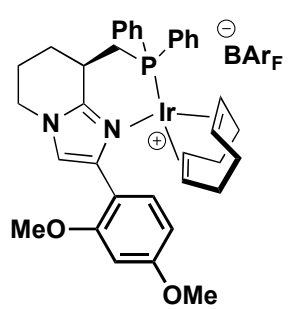

vii

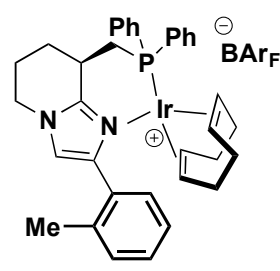

iv

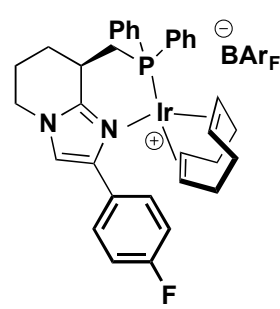

viii

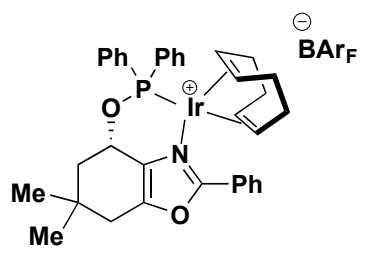

X

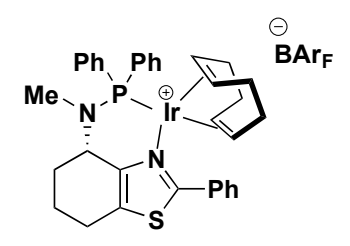

Ix

Novel catalysts iii-viii were prepared according to previously reported procedures. ${ }^{24}$<smiles>CCOC(=O)c1cccn2cc(-c3ccc(C)cc3)nc12</smiles>

ethyl 2-(p-tolyl)imidazo[1,2-a]pyridine-8-carboxylate. Yellow oil, 79\% yield. $\mathrm{R}_{f}=0.44$ (DCM:MeOH= 50:1). ${ }^{1} \mathbf{H}$ NMR $\left(400 \mathrm{MHz}, \mathrm{CDCl}_{3}\right) \delta 8.26(\mathrm{dd}, J=6.7,1.2 \mathrm{~Hz}, 1 \mathrm{H}), 7.92-7.87(\mathrm{~m}, 1 \mathrm{H}), 7.24-7.22(\mathrm{~m}$, $2 \mathrm{H}), 6.80(\mathrm{t}, J=6.9 \mathrm{~Hz}, 1 \mathrm{H}), 4.52(\mathrm{q}, J=7.1 \mathrm{~Hz}, 2 \mathrm{H}), 2.38(\mathrm{~s}, 3 \mathrm{H}), 1.49(\mathrm{t}, J=7.1 \mathrm{~Hz}, 3 \mathrm{H}) .{ }^{13} \mathbf{C} \mathbf{~ N M R}$ $\left(100 \mathrm{MHz}, \mathrm{CDCl}_{3}\right) \delta 164.61,147.04,142.95,138.07,130.56,129.32,129.21,128.77,126.26,119.77$, 111.07, 108.16, 61.59, 21.37, 14.36. IR (neat, $\mathrm{cm}^{-1}$ ): $v=3132,2920,2236,1903,1729,1620,913,825$, 670. HRMS-ESI m/z: $[\mathrm{M}+\mathrm{Na}]^{+}$Calc. for $\mathrm{C}_{17} \mathrm{H}_{16} \mathrm{~N}_{2} \mathrm{Na}_{1} \mathrm{O}_{2}$ 303.1109; Found 303.1118.<smiles>CCOC(=O)c1cccn2cc(-c3ccccc3C)nc12</smiles>

ethyl 2-(o-tolyl)imidazo[1,2-a]pyridine-8-carboxylate. Yellow oil, $40 \%$ yield. $\mathrm{R}_{f}=0.54$ (AcOEt:Pentane, 1:1). ${ }^{1} \mathbf{H}$ NMR $\left(400 \mathrm{MHz}, \mathrm{CDCl}_{3}\right) \delta 8.30$ (dd, $\left.J=6.7,1.3 \mathrm{~Hz}, 1 \mathrm{H}\right), 8.00-7.95(\mathrm{~m}, 1 \mathrm{H})$, $7.92(\mathrm{dd}, J=7.2,1.3 \mathrm{~Hz}, 1 \mathrm{H}), 7.76(\mathrm{~s}, 1 \mathrm{H}), 7.31-7.22(\mathrm{~m}, 3 \mathrm{H}), 6.83-6.77(\mathrm{~m}, 1 \mathrm{H}), 4.51(\mathrm{q}, J=7.1 \mathrm{~Hz}$, 
2H), $2.58(\mathrm{~s}, 3 \mathrm{H}), 1.48(\mathrm{t}, J=7.1 \mathrm{~Hz}, 3 \mathrm{H}) .{ }^{13} \mathrm{C}$ NMR (100 MHz, $\left.\mathrm{CDCl}_{3}\right) \delta 164.71,146.55,141.93$, 136.02, 132.96, 130.86, 129.90, 129.35, 128.77, 127.90, 125.91, 119.90, 111.13, 110.99, 61.51, 21.67, 14.34. IR $\left(\mathrm{NaCl}\right.$, neat, $\left.\mathrm{cm}^{-1}\right): v=3067,2981,2933,1724,1548,1496,14651361,1284,1265,1186$, 1144, 1040, 756. HRMS-ESI m/z: $[\mathrm{M}+\mathrm{Na}]^{+}$Calc. for $\mathrm{C}_{17} \mathrm{H}_{16} \mathrm{~N}_{2} \mathrm{NaO}_{2} 303.1104$; Found 303.1111.

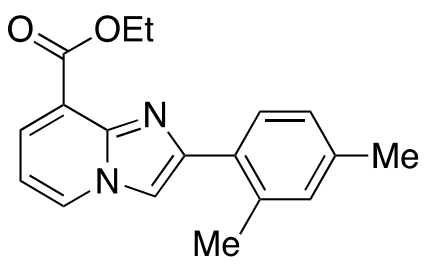

ethyl 2-(2,4-dimethylphenyl)imidazo[1,2-a]pyridine-8-carboxylate. Yellow oil, $66 \%$ yield. $\mathrm{R}_{f}=0.63$ (AcOEt:Pentane, 2:1). ${ }^{1} \mathbf{H}$ NMR $\left(400 \mathrm{MHz}, \mathrm{CDCl}_{3}\right) \delta 8.32(\mathrm{dd}, J=6.7,1.3 \mathrm{~Hz}, 1 \mathrm{H}), 7.93(\mathrm{dd}, J=7.2,1.3$ $\mathrm{Hz}, 1 \mathrm{H}), 7.88(\mathrm{~d}, J=7.7 \mathrm{~Hz}, 1 \mathrm{H}), 7.78(\mathrm{~s}, 1 \mathrm{H}), 7.13-7.07(\mathrm{~m}, 2 \mathrm{H}), 6.87-6.83(\mathrm{~m}, 1 \mathrm{H}), 4.51$ (q, $J=7.1$ $\mathrm{Hz}, 2 \mathrm{H}), 2.58(\mathrm{~s}, 3 \mathrm{H}), 2.36(\mathrm{~s}, 3 \mathrm{H}), 1.48(\mathrm{t}, J=7.1 \mathrm{~Hz}, 3 \mathrm{H}) .{ }^{13} \mathbf{C} \mathbf{N M R}\left(100 \mathrm{MHz}, \mathrm{CDCl}_{3}\right) \delta 164.76$, 146.63, 141.87, 137.56, 135.74, 131.64, 130.07, 129.79, 129.29, 128.64, 126.67, 119.73, 110.84, 61.47, 21.61, 21.12, 14.32. IR $\left(\mathrm{NaCl}\right.$, neat, $\left.\mathrm{cm}^{-1}\right): v=2979,1724,1621,1547,1484,1360,1284,1266,1187$, 1144, 1039, 757. HRMS-ESI m/z: [M+Na] ${ }^{+}$Calc. for $\mathrm{C}_{18} \mathrm{H}_{18} \mathrm{~N}_{2} \mathrm{NaO}_{2} 317.1260$; Found 317.1267.

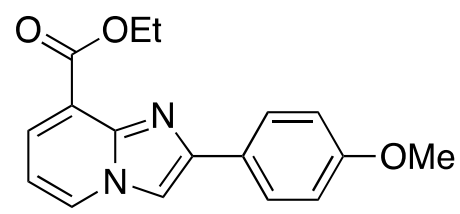

ethyl 2-(4-methoxyphenyl)imidazo[1,2-a]pyridine-8-carboxylate. Yellow oil, $96 \%$ yield. $\mathrm{R}_{f}=0.54$ (AcOEt:Pentane, 2:1). ${ }^{1} \mathbf{H}$ NMR $\left(400 \mathrm{MHz}, \mathrm{CDCl}_{3}\right) \delta 8.26(\mathrm{dd}, J=6.7,1.4 \mathrm{~Hz}, 1 \mathrm{H}), 8.00-7.93(\mathrm{~m}, 2 \mathrm{H})$, $7.91(\mathrm{dd}, J=7.2,1.3 \mathrm{~Hz}, 1 \mathrm{H}), 7.84(\mathrm{~s}, 1 \mathrm{H}), 6.99-6.92(\mathrm{~m}, 2 \mathrm{H}), 6.81(\mathrm{t}, J=6.9 \mathrm{~Hz}, 1 \mathrm{H}), 4.52(\mathrm{q}, J=7.1$ $\mathrm{Hz}, 2 \mathrm{H}), 3.84(\mathrm{~s}, 3 \mathrm{H}), 1.49$ (t, $J=7.2 \mathrm{~Hz}, 3 \mathrm{H}) .{ }^{13} \mathbf{C}$ NMR $\left(100 \mathrm{MHz}, \mathrm{CDCl}_{3}\right) \delta 164.75,159.91,146.96$, 143.07, 129.26, 128.84, 127.78, 126.21, 119.72, 114.14, 111.15, 107.74, 61.71, 55.42, 14.48. IR ( NaCl, neat, $\left.\mathrm{cm}^{-1}\right): v=2981,1725,1614,1545,1489,1365,1248,1186,1144,1033,838,756$. HRMS-ESI m/z: $[\mathrm{M}+\mathrm{Na}]^{+}$Calc. for $\mathrm{C}_{17} \mathrm{H}_{16} \mathrm{~N}_{2} \mathrm{NaO}_{3} 319.1053$; Found 319.1043.<smiles>CCOC(=O)c1cccn2cc(-c3ccc(OC)cc3OC)nc12</smiles>

ethyl 2-(2,4-dimethoxyphenyl)imidazo[1,2-a]pyridine-8-carboxylate, Yellow solid, MP:154.3 $158.3^{0} \mathrm{C}, 78 \%$ yield. $\mathrm{R}_{f}=0,1$ (Pentane: Ethylacetate, $\left.5: 1\right) .{ }^{1} \mathbf{H}$ NMR $\left(\mathrm{CDCl}_{3}, 400 \mathrm{MHz}\right): \delta 8.47(\mathrm{~d}, J=8.5$ $\mathrm{Hz} 1 \mathrm{H}), 8.23$ (dd, $J=6.7,1.4 \mathrm{~Hz}, 1 \mathrm{H}), 8.14(\mathrm{~s}, 1 \mathrm{H}), 7.88(\mathrm{dd}, J=7.2 \mathrm{~Hz}, 1.21 \mathrm{~Hz}, 1 \mathrm{H}), 6.74(\mathrm{t}, 1 \mathrm{H}), 6.64$ $(\mathrm{dd}, J=8.7 \mathrm{~Hz}, 2.4 \mathrm{~Hz}, 1 \mathrm{H}), 6.52(\mathrm{~d}, J=2.4,1 \mathrm{H}), 4.50(\mathrm{q}, 2 \mathrm{H}), 3.93(\mathrm{~s}, 3 \mathrm{H}), 3.83(\mathrm{~s}, 3 \mathrm{H}), 1.48(\mathrm{t}, 3 \mathrm{H})$. ${ }^{13} \mathbf{C}$ NMR $\left(\mathrm{CDCl}_{3}, 400 \mathrm{MHz}\right): 164.87,160.77,158.04,142.57,141.69,130.36,129.30,128.69,119.14$, 115.28, 111.67, 110.56, 104.97, 98.46, 61.51, 55.46, 55.44, 14.43. IR $\left(\mathrm{NaCl}\right.$, neat, $\left.\mathrm{cm}^{-1}\right): v=2982.99$, 2838.95, 2725.06, 1611.26, 1579.45, 1491.19, 1357.55, 1293.64, 1209.83. HRMS m/z: [M+H] ${ }^{+}$Calc. for $\mathrm{C}_{18} \mathrm{H}_{18} \mathrm{~N}_{2} \mathrm{O}_{4} \mathrm{H}$ 327.1339; Found 327.1346.<smiles>CCOC(=O)c1cccn2cc(-c3ccc(F)cc3)nc12</smiles>

ethyl 2-(4-fluorophenyl)imidazo[1,2-a]pyridine-8-carboxylate. $91 \%$ yield. Yellowish oil, $\mathrm{R}_{f}=0.23$ (50\% EtOAc in Pentane). ${ }^{1} \mathrm{H}$ NMR $\left(\mathrm{CDCl}_{3}, 400 \mathrm{MHz}\right): \delta 8.29$ (dd, $\left.J=8.0,4.0 \mathrm{~Hz}, 1 \mathrm{H}\right), 8.01-7.95(\mathrm{~m}$, 
$3 \mathrm{H}), 7.89(\mathrm{~s}, 1 \mathrm{H}), 7.11(\mathrm{t}, J=12.0 \mathrm{~Hz}, 2 \mathrm{H}), 6.86(\mathrm{t}, J=8.0 \mathrm{~Hz}, 1 \mathrm{H}), 4.51(\mathrm{q}, J=8.0 \mathrm{~Hz}, 2 \mathrm{H}), 1.49(\mathrm{t}, J=$ $4.0 \mathrm{~Hz}, 3 \mathrm{H}) .{ }^{13} \mathrm{C} \mathrm{NMR}\left(\mathrm{CDCl}_{3}, 100 \mathrm{MHz}\right.$ ): $\delta 164.57,162.85(\mathrm{~d}, J=246 \mathrm{~Hz}), 145.95,143.04,129.43(\mathrm{~d}$, $J=12 \mathrm{~Hz}), 128.06(\mathrm{~d}, J=8 \mathrm{~Hz}), 119.61,115.78,115.56,111.47,108.32,61.85,14.45$. IR ( NaCl, neat, $\left.\mathrm{cm}^{-1}\right): v=3431,3133,3103,2978,2927,1893,1606,1542,1488,1452,1370,1288,1222,1185,1151$, 1064, 1030, 1007, 934, 839, 803, 770, 744, 674. HRMS (EI) m/z: $[\mathrm{M}+\mathrm{H}]^{+}$Calc. for $\mathrm{C}_{16} \mathrm{H}_{14} \mathrm{FN}_{2} \mathrm{O}_{2}=$ 285.1034 Found 285.1042.<smiles>CCOC(=O)C1CCCn2cc(-c3ccc(C)cc3)nc21</smiles>

ethyl 2-(p-tolyl)-5,6,7,8-tetrahydroimidazo[1,2-a]pyridine-8-carboxylate. Yellow oil, $91 \%$ yield. $\mathrm{R}_{f}=$ 0.60 (AcOEt:Pentane= 2:1). ${ }^{1} \mathbf{H}$ NMR $\left(\mathrm{CDCl}_{3}, 400 \mathrm{MHz}\right): \delta 7.66-7.64(\mathrm{~m}, 2 \mathrm{H}), 7.18-7.16(\mathrm{~m}, 2 \mathrm{H})$, $7.08(\mathrm{~s}, 1 \mathrm{H}), 4.28-4.18(\mathrm{~m}, 3 \mathrm{H}), 4.13-4.07(\mathrm{~m}, 1 \mathrm{H}), 4.02-3.93(\mathrm{~m}, 1 \mathrm{H}), 2.34(\mathrm{~s}, 3 \mathrm{H}), 2.21-2.10(\mathrm{~m}$, $2 \mathrm{H}), 2.04-1.93(\mathrm{~m}, 1 \mathrm{H}), 1.30(\mathrm{t}, J=7.1 \mathrm{~Hz}, 3 \mathrm{H}) .{ }^{13} \mathbf{C} \mathbf{~ N M R}\left(\mathrm{CDCl}_{3}, 100 \mathrm{MHz}\right): \delta 141.48,129.56$, 129.30, 125.07, 113.92, 61.66, 44.86, 40.74, 24.67, 21.24, 20.16, 14.18. IR (neat, $\mathrm{cm}^{-1}$ ): $v=2955,1731$, $1511,1443,1380,1329,1255,1177,1029,950,824,757$.

HRMS (EI) m/z: [M+H] Calc. for $\mathrm{C}_{17} \mathrm{H}_{21} \mathrm{~N}_{2} \mathrm{O}_{2}$ 285.1598; Found 285.1641.<smiles>CCOC(=O)C1CCCn2cc(-c3ccccc3C)nc21</smiles>

ethyl 2-(o-tolyl)-5,6,7,8-tetrahydroimidazo[1,2-a]pyridine-8-carboxylate, Brown oil, $81 \%$ yield. $\mathrm{R}_{f}$ $=0,70(100 \%$ Ethylacetate $) .{ }^{1} \mathbf{H}$ NMR $\left(\mathrm{CDCl}_{3}, 400 \mathrm{MHz}\right): \delta 7.75(\mathrm{~m}, 1 \mathrm{H}), 7.23-7,13(\mathrm{~m}, 3 \mathrm{H}), 6.94(\mathrm{~s}$, $1 \mathrm{H}), 4.29-4.16(\mathrm{~m}, 2 \mathrm{H}), 4.15-4.04(\mathrm{~m}, 2 \mathrm{H}), 4.03-3.94(\mathrm{~m}, 1 \mathrm{H}), 2.46(\mathrm{~s}, 3 \mathrm{H}), 2.37-2.28(\mathrm{~m}, 1 \mathrm{H}), 2.26-2.13$ (m, 2H), 2.06-1.96 (m, 1H), $1.29(\mathrm{t}, 3 \mathrm{H}) .{ }^{13} \mathbf{C ~ N M R}\left(\mathrm{CDCl}_{3}, 400 \mathrm{MHz}\right): 172.10,140.73,140.42,134.89$, $133.73,130.52,128.62,126.58,125.72,116.92,77.48,77.16,76.84,61.32,44.56,41.25,25.08,21.62$, 20.51, 14.17, 14.06. IR $\left(\mathrm{NaCl}\right.$, neat, $\left.\mathrm{cm}^{-1}\right): v=3348.71,3145.11,3059.97,2959.01,2871.63,1921.37$, 1704.55, 1694.57, 1505.63, 1480.48, 1463.44, 1379.52, 1286.03, 1253.45, 1137.78, 1094.93, 1029.26, 948.10. HRMS m/z: [M+H] ${ }^{+}$Calc. for $\mathrm{C}_{17} \mathrm{H}_{21} \mathrm{O}_{2} \mathrm{~N}_{2} \mathrm{H} 285.1595$; Found 285,1607.<smiles>CCOC(=O)C1CCCn2cc(-c3ccc(C)cc3C)nc21</smiles>

ethyl 2-(2,4-dimethylphenyl)-5,6,7,8-tetrahydroimidazo[1,2-a]pyridine-8-carboxylate. Yellow oil, $74 \%$ yield. $\mathrm{R}_{f}=0.59$ (AcOEt:Pentane, $\left.2: 1\right) .{ }^{1} \mathbf{H}$ NMR $\left(400 \mathrm{MHz}, \mathrm{CDCl}_{3}\right) \delta 7.68-7.60(\mathrm{~m}, 1 \mathrm{H}), 7.05-$ $6.98(\mathrm{~m}, 2 \mathrm{H}), 6.89(\mathrm{~s}, 1 \mathrm{H}), 4.21(\mathrm{q}, J=7.1 \mathrm{~Hz}, 2 \mathrm{H}), 4.12-3.99(\mathrm{~m}, 2 \mathrm{H}), 3.99-3.89(\mathrm{~m}, 1 \mathrm{H}), 2.42(\mathrm{~s}$, $3 \mathrm{H}), 2.31(\mathrm{~s}, 4 \mathrm{H}), 2.29-2.10(\mathrm{~m}, 3 \mathrm{H}), 2.03-1.92(\mathrm{~m}, 1 \mathrm{H}), 1.28(\mathrm{t}, J=7.1 \mathrm{~Hz}, 3 \mathrm{H}) .{ }^{13} \mathbf{C}$ NMR $(100$ $\left.\mathrm{MHz}, \mathrm{CDCl}_{3}\right) \delta 172.17,140.66,140.62,136.17,134.76,131.38,130.98,128.65,126.54,116.62,61.36$, 44.61, 41.32, 25.21, 21.59, 21.11, 20.60, 14.26. IR $\left(\mathrm{NaCl}\right.$, neat, $\left.\mathrm{cm}^{-1}\right): v=2954,1735,1508,1443,1377$, 1319, 1254, 1177, 1030, 953, 828, 757. HRMS-ESI m/z: $[\mathrm{M}+\mathrm{H}]^{+}$Calc. for $\mathrm{C}_{18} \mathrm{H}_{23} \mathrm{~N}_{2} \mathrm{O}_{2}$ 299.1754; Found 299.1749 . 


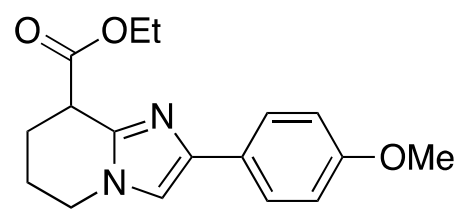

ethyl 2-(4-methoxyphenyl)-5,6,7,8-tetrahydroimidazo[1,2-a]pyridine-8-carboxylate. Pale yellow solid (mp $\left.110-114{ }^{\circ} \mathrm{C}\right), 73 \%$ yield. $\mathrm{R}_{f}=0.44($ AcOEt:Pentane, $2: 1) .{ }^{1} \mathbf{H}$ NMR $\left(400 \mathrm{MHz}, \mathrm{CDCl}_{3}\right) \delta 7.67$ $-7.60(\mathrm{~m}, 2 \mathrm{H}), 7.14(\mathrm{~s}, 1 \mathrm{H}), 6.98-6.92(\mathrm{~m}, 2 \mathrm{H}), 4.63-4.55(\mathrm{~m}, 1 \mathrm{H}), 4.27-4.13(\mathrm{~m}, 3 \mathrm{H}), 4.09-3.99$ $(\mathrm{m}, 1 \mathrm{H}), 3.82(\mathrm{~s}, 3 \mathrm{H}), 2.45-2.36(\mathrm{~m}, 1 \mathrm{H}), 2.21-2.07(\mathrm{~m}, 3 \mathrm{H}), 1.27(\mathrm{t}, J=7.1 \mathrm{~Hz}, 3 \mathrm{H}) .{ }^{13} \mathbf{C} \mathbf{~ N M R}(100$ $\left.\mathrm{MHz}, \mathrm{CDCl}_{3}\right) \delta 169.26,160.46,140.95,135.07,127.15,119.46,114.61,114.41,62.56,55.40,45.67$, 38.66, 23.05, 19.44, 13.87. IR $\left(\mathrm{NaCl}\right.$, neat, $\left.\mathrm{cm}^{-1}\right): v=2955,1735,1560,1509,1441,1380,1245,1175$, 1030, 837, 759. HRMS-ESI m/z: [M+Na] $]^{+}$Calc. for $\mathrm{C}_{17} \mathrm{H}_{20} \mathrm{~N}_{2} \mathrm{NaO}_{3} 323.1366$; Found 323.1366.<smiles>CCOC(=O)C1CCCn2cc(-c3ccc(OC)cc3OC)nc21</smiles>

ethyl-2-(2,4-dimethoxyphenyl)-5,6,7,8-tetrahydroimidazo[1,2-a]pyridine-8-carboxylate,

Yellow/brownish solid, MP: $112.6-119.8^{0} \mathrm{C}, 79 \%$ yield. $\mathrm{R}_{f}=0,44$ (ethylacetate). ${ }^{1} \mathbf{H}$ NMR $\left(\mathrm{CDCl}_{3}, 400\right.$ MHz): $\delta 8.05(\mathrm{~d}, J=8.6 \mathrm{~Hz}, 1 \mathrm{H}), 7.30(\mathrm{~s}, 1 \mathrm{H}), 6.55(\mathrm{dd}, J=8.6 \mathrm{~Hz}, 2.5 \mathrm{~Hz}, 1 \mathrm{H}), 6.50(\mathrm{~d}, J=2.4 \mathrm{~Hz}, 1 \mathrm{H})$, 4.28-4.15 (m, 2H), 4.10-4.00 (m, 2H), 3.98-3.91 (m, 1H), 3.89 (s, 3H), $3.82(\mathrm{~s}, 3 \mathrm{H}), 2.33-2.25(\mathrm{~m}, 1 \mathrm{H})$, 2.24-2.08 (m, 2H), 2.01-1.92 (m, 2H), 1.29 (t, 3H). ${ }^{13} \mathbf{C}$ NMR $\left(\mathrm{CDCl}_{3}, 400 \mathrm{MHz}\right): 171.51,158.66$, $156.25,139.65,135.95,127.70,116.75,115.77,103.91,97.83,60.63,54.76,54.67,43.87,40.67,24.61$, 19.91, 13.60. IR $\left(\mathrm{NaCl}\right.$, neat, $\left.\mathrm{cm}^{-1}\right): v=2940.19,2869.41,2051.02,1716.64,1614.44,1582.97,1464.74$, 1289.79. HRMS m/z: $[\mathrm{M}+\mathrm{H}]^{+}$Calc. for $\mathrm{C}_{18} \mathrm{H}_{22} \mathrm{O}_{4} \mathrm{~N}_{2} \mathrm{H}$ 331.1652; Found 331,1663.

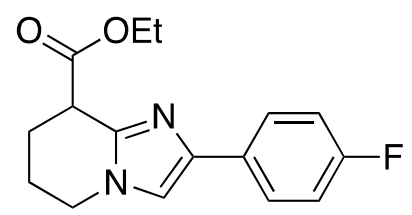

ethyl-2-(4-fluorophenyl)-5,6,7,8-tetrahydroimidazo[1,2-a]pyridine-8-carboxylate. $\quad 98 \%$ yield. Yellowish oil. ${ }^{1} \mathbf{H}$ NMR $\left(\mathrm{CDCl}_{3}, 400 \mathrm{MHz}\right): \delta$ 7.68-7.63 (m, 2H), 7.04-6.96 (m, 3H), 4.25-4.13 (m, 2H), 4.05-3.96 (m, 2H), 3.94-3.84 (m, 1H), 2.31-2.23 (m, 1H), 2.19-2.05 (m, 2H), 2.00-1.88 (m, 1H), $1.27(\mathrm{t}, J$ $=4 \mathrm{~Hz}, 3 \mathrm{H}) .{ }^{13} \mathbf{C}$ NMR $\left(\mathrm{CDCl}_{3}, 100 \mathrm{MHz},\right): \delta 172.11,161.71(\mathrm{~d}, J=243 \mathrm{~Hz}), 141.68,140.20,130.46(\mathrm{~d}$, $J=3 \mathrm{~Hz}), 126.40(\mathrm{~d}, J=8 \mathrm{~Hz}), 115.25(\mathrm{~d}, J=21 \mathrm{~Hz}), 114.17,61.47,44.65,41.18,25.04,20.26,14.20$. IR $\left(\mathrm{NaCl}\right.$, neat, $\left.\mathrm{cm}^{-1}\right): v=2961,2034,1897,1733,1559,1508,1478,1441,1379,1323,1219,1179$, 1093, 1028, 948, 843, 813, 755. HRMS (EI) m/z: $[\mathrm{M}+\mathrm{H}]^{+}$Calc. for $\mathrm{C}_{16} \mathrm{H}_{18} \mathrm{FN}_{2} \mathrm{O}_{2}=289.1347$ Found 289.1344 .<smiles>Cc1ccc(-c2cn3c(n2)C(CO)CCC3)cc1</smiles>

(R)-(2-(p-tolyl)-5,6,7,8-tetrahydroimidazo[1,2-a]pyridin-8-yl)methanol, White foam, $81 \%$ yield. $\mathrm{R}_{f}=$ $0.70(\mathrm{MeOH} / \mathrm{DCM}=0.5 / 9.5) .{ }^{1} \mathbf{H}$ NMR $\left(\mathrm{CDCl}_{3}, 400 \mathrm{MHz}\right): \delta 7.63-7.60(\mathrm{~m}, 2 \mathrm{H}), 7.17-7.15(\mathrm{~m}, 2 \mathrm{H})$, $7.01(\mathrm{~s}, 1 \mathrm{H}), 4.03-3.93(\mathrm{~m}, 1 \mathrm{H}), 3.85-3.79(\mathrm{~m}, 2 \mathrm{H}), 3.74-3.60(\mathrm{~m}, 1 \mathrm{H}), 3.11-3.03(\mathrm{~m}, 1 \mathrm{H}), 2.34(\mathrm{~s}$, $3 \mathrm{H}), 2.12-2.06(\mathrm{~m}, 1 \mathrm{H}), 2.04-1.91(\mathrm{~m}, 2 \mathrm{H}), 1.52-1.42(\mathrm{~m}, 1 \mathrm{H}) .{ }^{13} \mathbf{C}$ NMR $\left(\mathrm{CDCl}_{3}, 100 \mathrm{MHz}\right): \delta$ 147.82, 140.50, 136.36, 131.07, 129.20, 124.64, 113.14, 67.97, 65.83, 44.52, 37.27, 30.92, 25.61, 23.55, 22.22, 21.20. IR (neat, $\left.\mathrm{cm}^{-1}\right): v=3338,2969,1715,1448,1378,1291,1217,1104,951,755$.

$[\propto]_{\mathbf{D}}^{\mathbf{2 3}}=-44\left(c=0.2, \mathrm{CHCl}_{3}\right)$. HRMS (EI) m/z: $[\mathrm{M}+\mathrm{H}]^{+}$Calc. for $\mathrm{C}_{15} \mathrm{H}_{19} \mathrm{~N}_{2} \mathrm{O}_{1}$ 243.1492; Found 243.1489. 


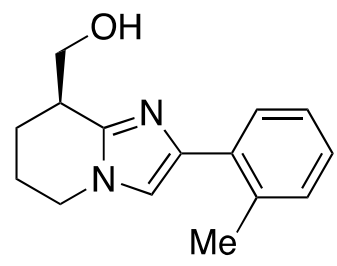

(R)-(2-(o-tolyl)-5,6,7,8-tetrahydroimidazo[1,2-a]pyridin-8-yl)methanol, Brown solid, 51\% yield. $\mathrm{R}_{f}=$ 0.26 (ethylacetate). ${ }^{1} \mathbf{H}$ NMR $\left(\mathrm{CDCl}_{3}, 400 \mathrm{MHz}\right): \delta 7.81(\mathrm{dd}, J=7.6 \mathrm{~Hz}, 1.4 \mathrm{~Hz}, 1 \mathrm{H}), 7.25-7.14(\mathrm{~m}, 3 \mathrm{H})$, $6.96(\mathrm{~s}, 1 \mathrm{H}), 5.59$ (bs, 1H), 4.09 (m, 1H), 3.95-3.86 (td, $J=11.9,4,0 \mathrm{~Hz}, 1 \mathrm{H}), 3.86-3.78$ (dd, J=10.7 Hz, $4.6 \mathrm{~Hz}, 1 \mathrm{H}), 3.7(\mathrm{t}, 1 \mathrm{H}), 3.11(\mathrm{sep}, J=5.2 \mathrm{~Hz}, 1 \mathrm{H}), 2.48(\mathrm{~s}, 3 \mathrm{H}), 2.20-2.11(\mathrm{~m}, 1 \mathrm{H}), 2.08-1.95(\mathrm{~m}, 2 \mathrm{H})$, $1.46(\mathrm{dq}, J=20.0 \mathrm{~Hz}, 2.1 \mathrm{~Hz}, 1 \mathrm{H}) .{ }^{13} \mathrm{C} \mathbf{N M R}\left(\mathrm{CDCl}_{3}, 400 \mathrm{MHz}\right): \delta 172.10,140.73,140.42,134.89$, $133.73,130.52,128.62,126.58,125.72,116.92,77.48,77.16,76.84,61.32,44.56,41.25,25.08,21.62$, 20.51, 14.17, 14.06. IR ( NaCl, neat, $\left.\mathrm{cm}^{-1}\right): v=3359.84,3054.69,2949.85,2865.65,1667.77,1603.76$, 1511.44, 1442.45, 1378.96, 1202.97, 1089.85, 1059.27. HRMS m/z: $[\mathrm{M}+\mathrm{H}]^{+}$Calc. for 243.1492 ; Found 243.1491. $[\propto]_{\mathbf{D}}^{23}=65.421\left(c=0.214, \mathrm{CHCl}_{3}\right)$.<smiles>Cc1ccc(-c2cn3c(n2)[C@@H](CO)CCC3)c(C)c1</smiles>

(R)-(2-(2,4-dimethylphenyl)-5,6,7,8-tetrahydroimidazo[1,2-a]pyridin-8-yl)methanol, White foam, $84 \%$ yield. $\mathrm{R}_{f}=0.70(\mathrm{MeOH} / \mathrm{DCM}=0.5 / 9.5) .{ }^{1} \mathbf{H} \mathbf{~ N M R}\left(\mathrm{CDCl}_{3}, 400 \mathrm{MHz}\right): \delta 7.70-7.65(\mathrm{~m}, 1 \mathrm{H}), 7.07$ $-7.01(\mathrm{~m}, 2 \mathrm{H}), 6.91(\mathrm{~s}, 1 \mathrm{H}), 4.13-4.03(\mathrm{~m}, 1 \mathrm{H}), 3.96-3.86(\mathrm{~m}, 1 \mathrm{H}), 3.83(\mathrm{dd}, J=10.5,4.6 \mathrm{~Hz}, 1 \mathrm{H})$, $3.73(\mathrm{t}, J=10.1 \mathrm{~Hz}, 1 \mathrm{H}), 3.12(\mathrm{tt}, J=10.4,5.2 \mathrm{~Hz}, 1 \mathrm{H}), 2.45(\mathrm{~s}, 3 \mathrm{H}), 2.33(\mathrm{~s}, 3 \mathrm{H}), 2.20-2.10(\mathrm{~m}, 1 \mathrm{H})$, $2.09-1.96(\mathrm{~m}, 1 \mathrm{H}), 1.55-1.42(\mathrm{~m}, 1 \mathrm{H}) .{ }^{13} \mathbf{C ~ N M R}\left(\mathrm{CDCl}_{3}, 100 \mathrm{MHz}\right): \delta 147.2,139.9,136.5,134.8$, $131.7,130.3,128.4,126.8,115.9,66.1,44.6,37.3,23.7,22.4,21.9,21.2 . \mathbf{I R}\left(\mathrm{NaCl}\right.$, neat, $\left.\mathrm{cm}^{-1}\right): v=3354$, 2947, 2864, 1510, 1438, 1376, 1195, 1059, 826. $[\propto]_{\mathbf{D}}^{23}=-66.807\left(c=0.238, \mathrm{CHCl}_{3}\right) . \mathbf{H R M S}(\mathbf{E I}) \mathrm{m} / \mathrm{z}$ : $[\mathrm{M}+\mathrm{H}]^{+}$Calc. for $\mathrm{C}_{16} \mathrm{H}_{21} \mathrm{~N}_{2} \mathrm{O}$ 257.1648; Found 257.1637.<smiles>COc1ccc(-c2cn3c(n2)[C@@H](CO)CCC3)cc1</smiles>

(R)-(2-(4-methoxyphenyl)-5,6,7,8-tetrahydroimidazo[1,2-a]pyridin-8-yl)methanol, White foam, 82\% yield. $\mathrm{R}_{\mathrm{f}}=0.50(\mathrm{MeOH} / \mathrm{DCM}=0.5 / 9.5) .{ }^{1} \mathrm{H}$ NMR $\left(\mathrm{CDCl}_{3}, 400 \mathrm{MHz}\right): \delta 7.64(\mathrm{~d}, J=8.4 \mathrm{~Hz}, 2 \mathrm{H}), 7.00-$ $6.82(\mathrm{~m}, 3 \mathrm{H}), 4.04-3.92(\mathrm{~m}, 1 \mathrm{H}), 3.89-3.68(\mathrm{~m}, 6 \mathrm{H}), 3.13-2.98(\mathrm{~m}, 1 \mathrm{H}), 2.17-1.85(\mathrm{~m}, 3 \mathrm{H}), 1.54-$ $1.39(\mathrm{~m}, 1 \mathrm{H}) .{ }^{13} \mathbf{C}$ NMR $\left(\mathrm{CDCl}_{3}, 100 \mathrm{MHz}\right): \delta 158.7,147.7,140.2,126.8,126.0,114.0,112.7,65.8,55.3$, 44.5, 37.3, 23.6, 22.2. IR $\left(\mathrm{NaCl}\right.$, neat, $\left.\mathrm{cm}^{-1}\right): v=3403,2931,1615,1510,1439,1275,1260,750 .[\propto]_{\mathbf{D}}^{23}=$ -59.387 $\left(c=0.261, \mathrm{CHCl}_{3}\right)$. HRMS (EI) m/z: $[\mathrm{M}+\mathrm{H}]^{+}$Calc. for $\mathrm{C}_{15} \mathrm{H}_{18} \mathrm{~N}_{2} \mathrm{O}_{2}$ 259.1441; Found 259.1444.<smiles>COc1ccc(-c2cn3c(n2)[C@@H](CO)CCC3)c(OC)c1</smiles>

(R)-(2-(2,4-dimethoxyphenyl)-5,6,7,8-tetrahydroimidazo[1,2-a]pyridin-8-yl)methanol, Light yellow solid, MP: $159.8-163.8^{0} \mathrm{C}, 54 \%$ yield. $\mathrm{R}_{f}=0,36$ (Ethylacetate). ${ }^{1} \mathbf{H}$ NMR $\left(\mathrm{CDCl}_{3}, 400 \mathrm{MHz}\right): \delta 8.04$ (d, $J$ $=7.5 \mathrm{~Hz}, 1 \mathrm{H}), 7.28(\mathrm{~s}, 1 \mathrm{H}), 6.57(\mathrm{dd}, J=8.7 \mathrm{~Hz}, 2.5 \mathrm{~Hz}, 1 \mathrm{H}), 6.51(\mathrm{~d}, J=2.3 \mathrm{~Hz}, 1 \mathrm{H}), 5.51(\mathrm{bs}, 1 \mathrm{H})$, 4.09-4.00 (m, 1H), $3.91(\mathrm{~s}, 3 \mathrm{H}), 3.83(\mathrm{~s}, 3 \mathrm{H}), 3.70(\mathrm{t}, 1 \mathrm{H}), 3.15-3.05(\mathrm{~h}, J=14.66 \mathrm{~Hz}, 5.2 \mathrm{~Hz}, 1 \mathrm{H}), 2.16-$ 
$1.92(\mathrm{~m}, 3 \mathrm{H}), 1.51-1.38(\mathrm{~m}, 1 \mathrm{H}), 1.29-1.17(\mathrm{~m}, 2 \mathrm{H}) .{ }^{13} \mathbf{C} \mathbf{N M R}\left(\mathrm{CDCl}_{3}, 400 \mathrm{MHz}\right): 159.56,157.00$, $146.80,135.80,128.18,116.69,115.89,104.74,98.68,66.10,55.55,55.43,44.51,37.22,29.84,23.75$, 22.44. IR $\left(\mathrm{NaCl}\right.$, neat, $\left.\mathrm{cm}^{-1}\right): v=3349.93,2998.13,2941.91,2837.07,1673.86,1614.69,1583.01$, $1557.09,1482.65,1436.76,1376.35,1290.69,1208.37,1035.42$. HRMS $\mathrm{m} / \mathrm{z}:[\mathrm{M}+\mathrm{H}]^{+}$Calc. for $\mathrm{C}_{16} \mathrm{H}_{20} \mathrm{O}_{3} \mathrm{~N}_{2} \mathrm{H}$ 289.1547; Found 289,1560. [ $\left.\propto\right]_{\mathbf{D}}^{23}=53.478\left(c=0.230, \mathrm{CHCl}_{3}\right)$.

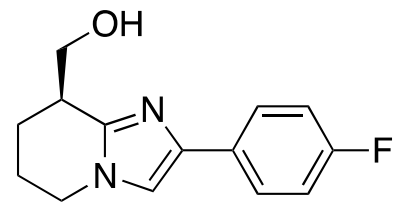

(R)-(2-(4-fluorophenyl)-5,6,7,8-tetrahydroimidazo[1,2-a]pyridin-8-yl)methanol. $78 \%$ yield. White foam, $\mathrm{R}_{f}=0.26(5 \% \mathrm{MeOH}$ in $\mathrm{DCM}) .{ }^{1} \mathbf{H} \mathbf{~ N M R}\left(\mathrm{CDCl}_{3}, 400 \mathrm{MHz}\right): \delta 7.71-7.66(\mathrm{~m}, 2 \mathrm{H}), 7.06-7.00(\mathrm{~m}$, $3 \mathrm{H}), 5.25(\mathrm{bs}, 1 \mathrm{H}), 4.06-4.01(\mathrm{~m}, 1 \mathrm{H}), 3.90-3.80(\mathrm{~m}, 2 \mathrm{H}), 3.74-3.69(\mathrm{~m}, 1 \mathrm{H}), 3.11-3.04(\mathrm{~m}, 1 \mathrm{H}), 2.17-$ $2.09(\mathrm{~m}, 1 \mathrm{H}), 2.06-1.93(\mathrm{~m}, 2 \mathrm{H}), 1.52-1.42(\mathrm{~m}, 1 \mathrm{H}) .{ }^{13} \mathbf{C}$ NMR $\left(\mathrm{CDCl}_{3}, 100 \mathrm{MHz},\right): \delta 161.76(\mathrm{~d}, J=293$ Hz), 148.20, 139.70, 130.22 (d, $J=3 \mathrm{~Hz}), 126.38$ (d, $J=8 \mathrm{~Hz}), 115.59,115.37,113.39,65.95,44.69$, 37.44, 23.63, 22.32. IR $\left(\mathrm{NaCl}\right.$, neat, $\left.\mathrm{cm}^{-1}\right): v=3340,2948,2867,1560,1509,1479,1439,1378,1323$, $1219,1155,1094,1058,958,841,755 .[\propto]_{\mathbf{D}}^{23}=63.478\left(c=0.1153, \mathrm{CHCl}_{3}\right)$. HRMS (EI) m/z: $[\mathrm{M}+\mathrm{H}]^{+}$ Calc. for $\mathrm{C}_{14} \mathrm{H}_{16} \mathrm{FN}_{2} \mathrm{O}=247.1241$ Found 247.1250.<smiles>Cc1ccc(-c2cn3c(n2)C(C[O+])CCC3)cc1</smiles>

(R)-(2-(p-tolyl)-5,6,7,8-tetrahydroimidazo[1,2-a]pyridin-8-yl)methyl 4-methylbenzenesulfonate, White foam, $78 \%$ yield. $\mathrm{R}_{f}=0.60(\mathrm{MeOH} / \mathrm{DCM}=0.5 / 9.5) .{ }^{1} \mathbf{H}$ NMR $\left(\mathrm{CDCl}_{3}, 400 \mathrm{MHz}\right): \delta 7.76-7.74$ (m, 2H), $7.53-7.51(\mathrm{~m}, 2 \mathrm{H}), 7.29-7.27(\mathrm{~m}, 2 \mathrm{H}), 7.15-7.13(\mathrm{~m}, 2 \mathrm{H}), 7.00(\mathrm{~s}, 1 \mathrm{H}), 4.59$ (dd, $J=9.6$, $3.8 \mathrm{~Hz}, 1 \mathrm{H}), 4.27(\mathrm{t}, J=8.7 \mathrm{~Hz}, 1 \mathrm{H}), 3.99-3.90(\mathrm{~m}, 2 \mathrm{H}), 3.33-3.27(\mathrm{~m}, 1 \mathrm{H}), 2.40(\mathrm{~s}, 3 \mathrm{H}), 2.34(\mathrm{~s}, 3 \mathrm{H})$, $2.32(\mathrm{~s}, 3 \mathrm{H}), 2.21-2.10(\mathrm{~m}, 2 \mathrm{H}), 1.95-1.83(\mathrm{~m}, 2 \mathrm{H}) .{ }^{13} \mathbf{C} \mathbf{N M R}\left(\mathrm{CDCl}_{3}, 100 \mathrm{MHz}\right): \delta$ 144.80, 143.01, $140.92,136.18,132.61,131.41,129.88,129.16,127.97,124.65,114.06,71.63,53.51,44.73,34.93$, 30.91, 24.04, 21.60, 21.20, 21.16. IR (neat, $\left.\mathrm{cm}^{-1}\right): v=2954,1598,1360,1175,957,753 .[\propto]_{\mathbf{D}}^{23}=-24(c=$ 0.175, $\mathrm{CHCl}_{3}$ ). HRMS (EI) m/z: $[\mathrm{M}+\mathrm{H}]^{+}$Calc. for $\mathrm{C}_{22} \mathrm{H}_{25} \mathrm{~N}_{2} \mathrm{O}_{3} \mathrm{~S}_{1}$ 397.1580; Found 397.1572.<smiles>Cc1ccccc1-c1cn2c(n1)[C@H](CO[S-])CCC2</smiles>

(R)-(2-(o-tolyl)-5,6,7,8-tetrahydroimidazo[1,2-a]pyridin-8-yl)methyl 4-methylbenzenesulfonate. 88\% yield. White foam, $\mathrm{R}_{f}=0.33(2.5 \% \mathrm{MeOH}$ in $\mathrm{DCM}) .{ }^{1} \mathrm{H} \mathrm{NMR}\left(\mathrm{CDCl}_{3}, 400 \mathrm{MHz}\right): \delta 7.76(\mathrm{~d}, J=8 \mathrm{~Hz}$, $2 \mathrm{H}), 7.67-7.65(\mathrm{~m}, 1 \mathrm{H}), 7.28(\mathrm{~d}, J=8.0 \mathrm{~Hz}, 2 \mathrm{H}), 7.23-7.11(\mathrm{~m}, 3 \mathrm{H}), 6.89(\mathrm{~s}, 1 \mathrm{H}), 4.61(\mathrm{dd}, J=9.6,3.8$ $\mathrm{Hz}, 1 \mathrm{H}), 4.27(\mathrm{t}, J=12 \mathrm{~Hz}, 1 \mathrm{H}), 4.10-3.89(\mathrm{~m}, 2 \mathrm{H}), 3.33-3.26(\mathrm{~m}, 1 \mathrm{H}), 2.41(\mathrm{~s}, 3 \mathrm{H}), 2.40(\mathrm{~s}, 3 \mathrm{H}), 2.28-$ $2.18(\mathrm{~m}, 1 \mathrm{H}), 2.18-2.09(\mathrm{~m}, 1 \mathrm{H}), 2.01-1.80(\mathrm{~m}, 2 \mathrm{H}) .{ }^{13} \mathrm{C} \mathrm{NMR}\left(\mathrm{CDCl}_{3}, 100 \mathrm{MHz},\right): \delta .144 .91,142.41$, $140.28,134.85,133.65,132.77,130.76,129.97,128.46,128.11,126.74,125.92,117.14,71.73,44.83$, 35.06, 24.27, 21.84, 21.73, 21.41. IR $\left(\mathrm{NaCl}\right.$, neat, $\left.\mathrm{cm}^{-1}\right): v=2953,2922,1598,1448,1359,1189,1176$, $1121,1097,1043,953,833,811,742 .[\propto]_{\mathbf{D}}^{23.2}=17.021\left(c=0.4698, \mathrm{CHCl}_{3}\right)$. HRMS (EI) m/z: $[\mathrm{M}+\mathrm{H}]^{+}$ Calc. for $\mathrm{C}_{22} \mathrm{H}_{25} \mathrm{~N}_{2} \mathrm{O}_{3} \mathrm{~S}=397.1580$ Found 397.1581 . 
<smiles>Cc1ccc(-c2cn3c(n2)[C@H](C[O-])CCC3)c(C)c1</smiles>

(R)-(2-(2,4-dimethylphenyl)-5,6,7,8-tetrahydroimidazo[1,2-a]pyridin-8-yl)methyl methylbenzenesulfonate, White foam, $91 \%$ yield. $\mathrm{R}_{f}=0.60(\mathrm{MeOH} / \mathrm{DCM}=0.5 / 9.5) .{ }^{1} \mathbf{H} \mathrm{NMR}\left(\mathrm{CDCl}_{3}\right.$, $400 \mathrm{MHz}): \delta 7.79-7.74(\mathrm{~m}, 2 \mathrm{H}), 7.57-7.52(\mathrm{~m}, 1 \mathrm{H}), 7.31-7.27(\mathrm{~m}, 2 \mathrm{H}), 7.04-6.98(\mathrm{~m}, 2 \mathrm{H}), 6.85(\mathrm{~s}$, $1 \mathrm{H}), 4.61(\mathrm{dd}, J=9.6,3.8 \mathrm{~Hz}, 1 \mathrm{H}), 4.28(\mathrm{t}, J=9.0 \mathrm{~Hz}, 1 \mathrm{H}), 4.06-3.90(\mathrm{~m}, 2 \mathrm{H}), 3.32(\mathrm{q}, J=6.6,5.0 \mathrm{~Hz}$, $1 \mathrm{H}), 2.41(\mathrm{~s}, 3 \mathrm{H}), 2.38(\mathrm{~s}, 3 \mathrm{H}), 2.32(\mathrm{~s}, 3 \mathrm{H}), 2.28-2.09(\mathrm{~m}, 2 \mathrm{H}), 2.04-1.83(\mathrm{~m}, 2 \mathrm{H}) .{ }^{13} \mathbf{C} \mathbf{~ N M R}\left(\mathrm{CDCl}_{3}\right.$, $100 \mathrm{MHz}): \delta 144.92,142.31,136.40,134.69,132.82,131.56,130.00,128.48,128.16,126.71,116.80$, 71.81, 44.86, 35.03, 24.28, 21.76, 21.75, 21.41, 21.18.. IR $\left(\mathrm{NaCl}\right.$, neat, $\left.\mathrm{cm}^{-1}\right): v=2952,1597,1359$, 1176, 957, 749. [ $\propto]_{\mathbf{D}}^{23}=-26.217\left(c=0.365, \mathrm{CHCl}_{3}\right)$. HRMS (EI) $\mathrm{m} / \mathrm{z}:[\mathrm{M}+\mathrm{H}]^{+}$Calc. for $\mathrm{C}_{23} \mathrm{H}_{27} \mathrm{~N}_{2} \mathrm{O}_{3} \mathrm{~S}$ 411.1737; Found 411.1736.

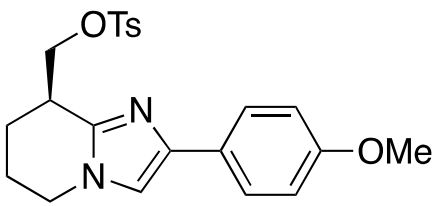

(R)-(2-(4-methoxyphenyl)-5,6,7,8-tetrahydroimidazo[1,2-a]pyridin-8-yl)methyl

4-methyl benzenesulfonate, White foam, $86 \%$ yield. $\mathrm{R}_{\mathrm{f}}=0.61(\mathrm{MeOH} / \mathrm{DCM}=0.5 / 9.5) .{ }^{1} \mathbf{H}$ NMR $\left(\mathrm{CDCl}_{3}, 400\right.$ $\mathrm{MHz}): \delta 7.79-7.72(\mathrm{~m}, 2 \mathrm{H}), 7.58-7.52(\mathrm{~m}, 2 \mathrm{H}), 7.32-7.27(\mathrm{~m}, 2 \mathrm{H}), 6.95(\mathrm{~s}, 1 \mathrm{H}), 6.92-6.86(\mathrm{~m}, 2 \mathrm{H})$, $4.58(\mathrm{dd}, J=9.5,3.8 \mathrm{~Hz}, 1 \mathrm{H}), 4.25(\mathrm{t}, J=9.0 \mathrm{~Hz}, 1 \mathrm{H}), 4.02-3.87(\mathrm{~m}, 2 \mathrm{H}), 3.81(\mathrm{~s}, 3 \mathrm{H}), 3.33-3.26(\mathrm{~m}$, $1 \mathrm{H}), 2.41(\mathrm{~s}, 3 \mathrm{H}), 2.28-2.06(\mathrm{~m}, 2 \mathrm{H}), 2.00-1.82(\mathrm{~m}, 2 \mathrm{H}) .{ }^{13} \mathbf{C} \mathbf{~ N M R}\left(\mathrm{CDCl}_{3}, 100 \mathrm{MHz}\right): \delta 144.9$, 143.1, 132.8, 130.0, 128.2, 126.2, 114.1, 113.6, 71.8, 55.5, 44.9, 35.0, 24.2, 21.8, 21.3. IR (NaCl, neat, $\left.\mathrm{cm}^{-1}\right): v=2958,2836,1560,1510,1440,1358,1245,1175,912,740 .[\propto]_{\mathbf{D}}^{23}=-36.800\left(c=0.375, \mathrm{CHCl}_{3}\right)$. HRMS (EI) m/z: [M+H] $]^{+}$Calc. for $\mathrm{C}_{22} \mathrm{H}_{25} \mathrm{~N}_{2} \mathrm{O}_{4} \mathrm{~S}$ 413.1530; Found 413.1544.<smiles>COC[C@H]1CCCn2cc(-c3ccc(OC)cc3OC)nc21</smiles>

(R)-(2-(2,4-dimethoxyphenyl)-5,6,7,8-tetrahydroimidazo[1,2-a]pyridin-8-yl)methyl

methanesulfonate. White foam, $88 \%$ yield. $\mathrm{R}_{f}=0.06\left(2.5 \%\right.$ EtOAc in DCM). ${ }^{1} \mathbf{H}$ NMR $\left(\mathrm{CDCl}_{3}, 400\right.$ MHz): $\delta 8.00(\mathrm{~d}, J=12.0 \mathrm{~Hz}, 1 \mathrm{H}), 7.29(\mathrm{~s}, 1 \mathrm{H}), 6.56(\mathrm{dd}, J=8.6,2.4,1 \mathrm{H}), 6.51(\mathrm{~d}, J=2.3 \mathrm{~Hz}, 1 \mathrm{H})$, 4.64-4.73 (m, 2H), 4.04-4.00 (m, 1H), 3.98-3.91 (m, 1H), $3.90(\mathrm{~s}, 3 \mathrm{H}), 3.83(\mathrm{~s}, 3 \mathrm{H}), 3.36-3.30(\mathrm{~m}, 1 \mathrm{H})$, $2.99(\mathrm{~s}, 3 \mathrm{H}), 2.23-2.12(\mathrm{~m}, 2 \mathrm{H}), 2.01-1.86(\mathrm{~m}, 3 \mathrm{H}) .{ }^{13} \mathbf{C} \mathbf{~ N M R}\left(\mathrm{CDCl}_{3}, 100 \mathrm{MHz},\right): \delta 159.52,157.01$, 142.18, 136.36, 127.82, 117.68, 116.24, 104.79, 98.68, 71.82, 55.52, 55.41, 44.77, 37.14, 35.45,24.31, 21.77. IR $\left(\mathrm{NaCl}\right.$, neat, $\left.\mathrm{cm}^{-1}\right): v=2926,1613,1580,1552,1511,1481,1458,1438,1413,1348,1288$, $1208,1174,1069,1032,950,901,832 .[\propto]_{\mathbf{D}}^{\mathbf{2 3 . 0}}=75.263\left(c=0.1897, \mathrm{CHCl}_{3}\right) . \mathbf{H R M S}(\mathbf{E I}) \mathrm{m} / \mathrm{z}:[\mathrm{M}+\mathrm{H}]^{+}$ Calc. for $\mathrm{C}_{17} \mathrm{H}_{23} \mathrm{~N}_{2} \mathrm{O}_{5} \mathrm{~S}=367.1322$; Found 367.1328.

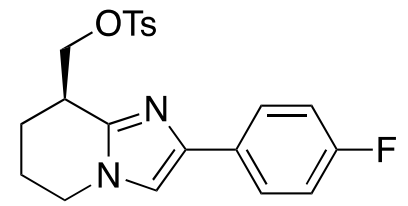

(R)-(2-(4-fluorophenyl)-5,6,7,8-tetrahydroimidazo[1,2-a]pyridin-8-yl)methyl methylbenzenesulfonate. $92 \%$ yield. White foam, $\mathrm{R}_{f}=0.16(2.5 \% \mathrm{MeOH}$ in DCM $) .{ }^{1} \mathbf{H}$ NMR $\left(\mathrm{CDCl}_{3}\right.$, $400 \mathrm{MHz}): \delta$ 7.77-7.74 (m, 2H), 7.61-7.56 (m, 2H), 7.30-7.27 (m, 2H), 7.04-6.99 (m, 3H), $4.58(\mathrm{dd}, J=$ 8.0, $4.0 \mathrm{~Hz} 1 \mathrm{H}), 4.26(\mathrm{dd}, J=12.0,8.0 \mathrm{~Hz} 1 \mathrm{H}), 4.01-3.89(\mathrm{~m}, 2 \mathrm{H}), 3.31-3.25(\mathrm{~m}, 1 \mathrm{H}), 2.40(\mathrm{~s}, 3 \mathrm{H}), 2.23-$ $2.09(\mathrm{~m}, 2 \mathrm{H}), 1.97-1.81(\mathrm{~m}, 2 \mathrm{H}) .{ }^{13} \mathbf{C} \mathbf{N M R}\left(\mathrm{CDCl}_{3}, 100 \mathrm{MHz},\right): \delta 161.99(\mathrm{~d}, J=244 \mathrm{~Hz}), 144.92$, 
143.45, 140.24, 132.77, 129.98, 128.14, 126.43 (d, $J=8 \mathrm{~Hz}), 115.56,115.34,114.22,71.64,44.94$, 35.09, 24.18, 21.76, 21.36. IR $\left(\mathrm{NaCl}\right.$, neat $\left.\mathrm{cm}^{-1}\right): v=2922,2849,1598,1557,1505,1437,1349,1213$, 1172, 1095, 949, 901, 839, 809, 744. [ $\propto]_{\mathbf{D}}^{23}=33.577\left(c=0.1373, \mathrm{CHCl}_{3}\right)$. HRMS (EI) m/z: $[\mathrm{M}+\mathrm{H}]^{+} \mathrm{Calc}$. for $\mathrm{C}_{21} \mathrm{H}_{22} \mathrm{FN}_{2} \mathrm{O}_{3} \mathrm{~S}=401.1330$ Found 401.1343 .

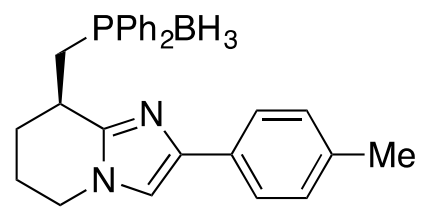

(R)-(8)-((diphenylphosphino)methyl)-2-(p-tolyl)-5,6,7,8-tetrahydroimidazo [1,2-a]pyridine Borane, White foam, $48 \%$ yield. $\mathrm{R}_{\mathrm{f}}=0.20($ EtOAc/Pentane $=1 / 5) .{ }^{1} \mathbf{H}$ NMR $\left(\mathrm{CDCl}_{3}, 400 \mathrm{MHz}\right): \delta 8.00-7.96(\mathrm{~m}$, $2 \mathrm{H}), 7.79-7.75(\mathrm{~m}, 2 \mathrm{H}), 7.67-7.65(\mathrm{~m}, 2 \mathrm{H}), 7.51-7.42(\mathrm{~m}, 7 \mathrm{H}), 7.19-7.17(\mathrm{~m}, 2 \mathrm{H}), 7.00(\mathrm{~s}, 1 \mathrm{H})$, $3.96-3.92(\mathrm{~m}, 1 \mathrm{H}), 3.87-3.81(\mathrm{~m}, 1 \mathrm{H}), 3.71-3.65(\mathrm{~m}, 1 \mathrm{H}), 3.31-3.23(\mathrm{~m}, 1 \mathrm{H}), 2.42-2.29(\mathrm{~m}, 5 \mathrm{H})$, $2.00-1.94(\mathrm{~m}, 1 \mathrm{H}), 1.87-1.80(\mathrm{~m}, 1 \mathrm{H}), 1.53-1.45(\mathrm{~m}, 1 \mathrm{H}) .{ }^{13} \mathbf{C} \mathbf{N M R}\left(\mathrm{CDCl}_{3}, 100 \mathrm{MHz}\right): \delta 147.68$, $147.56,140.75,136.15,132.63,132.56,132.16,132.09,131.79,131.31,131.29,131.19,131.17,130.99$, $130.55,129.40,129.26,129.00,128.92,128.87,128.84,128.79,128.71,124.76,114.03,60.45,44.87$, 32.09, 32.07, 29.76, 29.47, 28.54, 22.15, 21.30, 21.12, 14.28. ${ }^{31} \mathbf{P}$ NMR $\left(\mathrm{CDCl}_{3}, 100 \mathrm{MHz}\right): \delta 13.89$. IR $\left(\mathrm{NaCl}\right.$, neat, $\left.\mathrm{cm}^{-1}\right): v=3057,2919,2374,1508,1436,1380,1265,1187,1107,1062,825,746,691$. $[\propto]_{\mathbf{D}}^{23}=-8.4\left(c=0.26, \mathrm{CHCl}_{3}\right)$. HRMS (EI) $\mathrm{m} / \mathrm{z}$ : $[\mathrm{M}+\mathrm{H}]^{+}$Calc. for $\mathrm{C}_{27} \mathrm{H}_{31} \mathrm{~B}_{1} \mathrm{~N}_{2} \mathrm{P}_{1}$ 425.2317; Found 425.2295 .

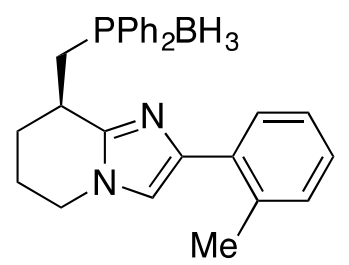

(R)-(8)-((diphenylphosphanyl)methyl)-2-(o-tolyl)-5,6,7,8-tetrahydroimidazo[1,2-a]pyridine borane adduct. $94 \%$ yield. White foam, $\mathrm{R}_{f}=0.23(1 \% \mathrm{MeOH}$ in $\mathrm{DCM}) .{ }^{1} \mathrm{H} \mathrm{NMR}\left(\mathrm{CDCl}_{3}, 400 \mathrm{MHz}\right): \delta 8.03-$ $7.95(\mathrm{~m}, 2 \mathrm{H}), 7.81(\mathrm{dd}, J=7.7,1.4 \mathrm{~Hz}, 1 \mathrm{H}), 7.77-7.72(\mathrm{~m}, 2 \mathrm{H}), 7.52-7.39(\mathrm{~m}, 6 \mathrm{H}), 7.28-7.14(\mathrm{~m}, 3 \mathrm{H})$, $6.89(\mathrm{~s}, 1 \mathrm{H}), 4.003-3.97(\mathrm{~m}, 1 \mathrm{H}), 3.90(\mathrm{td}, J=11.5,4.9 \mathrm{~Hz}, 1 \mathrm{H}), 3.78-3.67(\mathrm{~m}, 1 \mathrm{H}), 3.30-3.20(\mathrm{~m}, 1 \mathrm{H})$, $2.51(\mathrm{~s}, 3 \mathrm{H}), 2.45-2.36(\mathrm{~m}, 1 \mathrm{H}), 2.36-2.27(\mathrm{~m}, 1 \mathrm{H}), 2.06-1.98(\mathrm{~m}, 1 \mathrm{H}), 1.95-1.82(\mathrm{~m}, 1 \mathrm{H}), 1.59-1.47(\mathrm{~m}$, $1 \mathrm{H}) .{ }^{13} \mathrm{C} \mathrm{NMR}\left(\mathrm{CDCl}_{3}, 125 \mathrm{MHz}\right.$ ): $\delta 146.97(\mathrm{~d}, J=15.1 \mathrm{~Hz}), 140.16,135.08,133.91,132.73(\mathrm{~d}, J=9.2$ $\mathrm{Hz}), 132.15(\mathrm{~d}, J=9.2 \mathrm{~Hz}), 131.39(\mathrm{~d}, J=2.5 \mathrm{~Hz}), 131.22$ (d, $J=2.4 \mathrm{~Hz}), 131.11,130.86,130.67$, $129.04(\mathrm{~d}, J=10.0 \mathrm{~Hz}), 128.88(\mathrm{~d}, J=10.0 \mathrm{~Hz}), 128.70,128.57,126.77,126.00,117.08,44.96,32.08(\mathrm{~d}$, $J=3.3 \mathrm{~Hz}), 29.58(\mathrm{~d}, J=36.9 \mathrm{~Hz}), 28.82,22.34,21.91 .{ }^{31} \mathrm{P}$ NMR: $\delta 14.38$. IR $\left(\mathrm{NaCl}\right.$, neat, $\left.\mathrm{cm}^{-1}\right): v=$ 3057, 3007, 2951, 2379, 1603, 1548, 1505, 1480, 1437, 1379, 1319, 1216, 1187, 1107, 1059, 999, 947, 748. $[\propto]_{\mathbf{D}}^{23}=-10.280\left(c=0.1065, \mathrm{CHCl}_{3}\right)$. HRMS (EI) $\mathrm{m} / \mathrm{z}$ : $[\mathrm{M}+\mathrm{H}]^{+}$Calc. for $\mathrm{C}_{27} \mathrm{H}_{31} \mathrm{BN}_{2} \mathrm{P}=425.2317$ Found 425.2333 .<smiles>Cc1ccc(-c2cn3c(n2)[C@H](CP)CCC3)c(C)c1</smiles>

(R)-(8)-((diphenylphosphino)methyl)-2-(p-2',4'-dimethyl-phenyl)-5,6,7,8-tetrahydro imidazo [1,2-a] pyridine Borane, White foam, $73 \%$ yield. $\mathrm{R}_{f}=0.80($ EtOAc/Pentane $=1 / 1) .{ }^{1} \mathbf{H}$ NMR $\left(\mathrm{CDCl}_{3}, 400\right.$ MHz): $\delta 8.05-7.95(\mathrm{~m}, 2 \mathrm{H}), 7.80-7.67(\mathrm{~m}, 3 \mathrm{H}), 7.54-7.37(\mathrm{~m}, 6 \mathrm{H}), 7.06(\mathrm{~d}, J=7.6 \mathrm{~Hz}, 2 \mathrm{H}), 6.86(\mathrm{~s}$, $1 \mathrm{H}), 3.99$ (ddd, $J=12.2,5.8,3.0 \mathrm{~Hz}, 1 \mathrm{H}), 3.89$ (td, $J=11.5,4.9 \mathrm{~Hz}, 1 \mathrm{H}), 3.74$ (td, $J=14.7,2.2 \mathrm{~Hz}, 1 \mathrm{H})$, $3.32-3.17(\mathrm{~m}, 1 \mathrm{H}), 2.48(\mathrm{~s}, 3 \mathrm{H}), 2.46-2.27(\mathrm{~m}, 5 \mathrm{H}), 2.07-1.97(\mathrm{~m}, 1 \mathrm{H}), 1.95-1.81(\mathrm{~m}, 1 \mathrm{H}), 1.60-$ $1.47(\mathrm{~m}, 1 \mathrm{H}) .{ }^{13} \mathbf{C}$ NMR $\left(\mathrm{CDCl}_{3}, 100 \mathrm{MHz}\right): \delta 146.78(\mathrm{~d}, J=15.0 \mathrm{~Hz}), 140.20,136.26,134.84,132.70(\mathrm{~d}$, $J=9.2 \mathrm{~Hz}), 132.12(\mathrm{~d}, J=9.2 \mathrm{~Hz}), 131.60,131.34(\mathrm{~d}, J=2.4 \mathrm{~Hz}), 131.25-130.99(\mathrm{~m}), 130.62,129.31-$ 128.59 (m), 128.48, 126.69, 116.68, 44.88, 32.04 (d, $J=3.0 \mathrm{~Hz}), 29.55$ (d, $J=37.0 \mathrm{~Hz}), 28.83,22.32$, 
21.78, 21.17. ${ }^{31} \mathbf{P}$ NMR $\left(\mathrm{CDCl}_{3}, 100 \mathrm{MHz}\right): \delta 14.2 . \mathbf{I R}\left(\mathrm{NaCl}\right.$, neat, $\left.\mathrm{cm}^{-1}\right): v=3053,2950,2373,1436$, 1377, 1194, 1061, 827, 736, 702. $[\propto]_{\mathbf{D}}^{23}=-8.903\left(c=0.775, \mathrm{CHCl}_{3}\right)$. HRMS (EI) m/z: $[\mathrm{M}+\mathrm{H}]^{+}$Calc. for $\mathrm{C}_{28} \mathrm{H}_{33} \mathrm{BN}_{2} \mathrm{P}$ 439.2474; Found 439.2485.<smiles>COc1ccc(-c2cn3c(n2)C(CP)CCC3)cc1</smiles>

(R)-(8)-((diphenylphosphino)methyl)-2-(p-methoxy-phenyl)-5,6,7,8-tetrahydroimidazo

$[1,2-$ a]pyridine Borane, White foam, $49 \%$ yield. $\mathrm{R}_{\mathrm{f}}=0.70($ EtOAc/Pentane $=3 / 7) .{ }^{1} \mathbf{H}$ NMR $\left(\mathrm{CDCl}_{3}, 400\right.$ MHz): $\delta 8.02-7.93(\mathrm{~m}, 2 \mathrm{H}), 7.80-7.72(\mathrm{~m}, 2 \mathrm{H}), 7.72-7.65(\mathrm{~m}, 2 \mathrm{H}), 7.53-7.39(\mathrm{~m}, 6 \mathrm{H}), 6.96-6.88$ $(\mathrm{m}, 3 \mathrm{H}), 4.00-3.93(\mathrm{~m}, 1 \mathrm{H}), 3.83(\mathrm{~s}, 4 \mathrm{H}), 3.78-3.58(\mathrm{~m}, 1 \mathrm{H}), 3.26(\mathrm{~s}, 1 \mathrm{H}), 2.46-2.26(\mathrm{~m}, 1 \mathrm{H}), 2.08-$ $1.95(\mathrm{~m}, 1 \mathrm{H}), 1.94-1.76(\mathrm{~m}, 1 \mathrm{H}), 1.66-1.39(\mathrm{~m}, 2 \mathrm{H}) .{ }^{13} \mathbf{C} \mathbf{N M R}\left(\mathrm{CDCl}_{3}, 100 \mathrm{MHz}\right): \delta 158.5,147.5$, $132.7(\mathrm{~d}, J=9.2 \mathrm{~Hz}), 132.2(\mathrm{~d}, J=9.2 \mathrm{~Hz}), 129.1,129.0,128.9,128.8,114.1,113.5,55.5,45.0,32.1$, 29.9, 29.5, 28.6, 22.2. ${ }^{31} \mathbf{P}$ NMR $\left(\mathrm{CDCl}_{3}, 100 \mathrm{MHz}\right): \delta 14.0$. IR $\left(\mathrm{NaCl}\right.$, neat, $\left.\mathrm{cm}^{-1}\right): v=3428,2954,2375$, 1616, 1507, 1437, 1380, 1245, 911, 734. $[\propto]_{\mathrm{D}}^{\mathbf{2 3}}=-11.333\left(c=0.300, \mathrm{CHCl}_{3}\right) . \mathbf{H R M S}(\mathbf{E I}) \mathrm{m} / \mathrm{z}:[\mathrm{M}+\mathrm{H}]^{+}$ Calc. for $\mathrm{C}_{27} \mathrm{H}_{31} \mathrm{BN}_{2} \mathrm{OP}$ 441.226; Found 441.2265.<smiles>COc1ccc(-c2cn3c(n2)C(CP)CCC3)c(OC)c1</smiles>

(R)-(2-(2,4-dimethoxyphenyl)-8-((diphenylphosphanyl)methyl)-5,6,7,8-tetrahydroimidazo[1,2a]pyridine borane adduct. White foam, $85 \%$ yield. $\mathrm{R}_{f}=0.06\left(2.5 \%\right.$ EtOAc in DCM). ${ }^{1} \mathbf{H} \mathbf{~ N M R}\left(\mathrm{CDCl}_{3}\right.$, $500 \mathrm{MHz}): \delta 8.12(\mathrm{~d}, J=8.4 \mathrm{~Hz}, 1 \mathrm{H}), 8.13-8.11(\mathrm{~m}, 2 \mathrm{H}), 7.78-7.73(\mathrm{~m}, 2 \mathrm{H}), 7.50-7.47(\mathrm{~m}, 3 \mathrm{H}), 7.47-$ $7.40(\mathrm{~m}, 3 \mathrm{H}), 7.23(\mathrm{~s}, 1 \mathrm{H}), 6.61(\mathrm{dd}, J=8.5,2.5 \mathrm{~Hz}, 1 \mathrm{H}), 6.52(\mathrm{~d}, J=2.5 \mathrm{~Hz}, 1 \mathrm{H}), 3.99-3.93(\mathrm{~m}, 1 \mathrm{H})$, $3.90(\mathrm{~s}, 3 \mathrm{H}), 3.89-3.85(\mathrm{~m}, 1 \mathrm{H}) 3.84(\mathrm{~s}, 3 \mathrm{H}), 3.73-3.66(\mathrm{~m}, 1 \mathrm{H}), 3.30-3.20(\mathrm{~m}, 1 \mathrm{H}), 2.39-2.30(\mathrm{~m}, 2 \mathrm{H})$, 2.02-1.95 (m, 1H), 1.89-1.79 (m, 1H), 1.53-1.44 (m, 1H). ${ }^{13} \mathbf{C}$ NMR $\left(\mathrm{CDCl}_{3}, 125 \mathrm{MHz},\right): \delta 159.42$, $157.02,146.49(\mathrm{~d}, J=15.0 \mathrm{~Hz}), 135.98,132.70(\mathrm{~d}, J=9.2 \mathrm{~Hz}), 132.18(\mathrm{~d}, J=9.2 \mathrm{~Hz}), 131.32(\mathrm{~d}, J=2.5$ $\mathrm{Hz}), 131.19(\mathrm{~d}, J=2.5 \mathrm{~Hz}), 131.15,130.71,129.35,129.01(\mathrm{~d}, J=10.1 \mathrm{~Hz}), 128.87(\mathrm{~d}, J=10.0 \mathrm{~Hz})$, 128.13, 117.51, 116.59, 104.86, 98.59, 55.54, 55.43, 44.84, 32.02 (d, $J=2.5 \mathrm{~Hz}), 29.69$ (d, $J=36.2 \mathrm{~Hz})$, 28.78, 22.29. ${ }^{31} \mathrm{P}$ NMR: $\delta$ 14.09. IR $\left(\mathrm{NaCl}\right.$, neat, $\left.\mathrm{cm}^{-1}\right): v=3159,3056,3005,2953,2376,1967,1890$, 1818, 1771, 1708, 1614, 1582, 1555, 1507, 1465, 1436, 1378, 1289, 1208, 1181, 1158, 1108, 1068, 1033, 952, 828, 752, 702. $[\propto]_{\mathbf{D}}^{23}=-0.971\left(c=0.1030, \mathrm{CHCl}_{3}\right)$. HRMS (EI) $\mathrm{m} / \mathrm{z}:[\mathrm{M}+\mathrm{H}]^{+}$Calc. for $\mathrm{C}_{28} \mathrm{H}_{33} \mathrm{BN}_{2} \mathrm{O}_{2} \mathrm{P}=471.2372$; Found 471.2356.<smiles>Fc1ccc(-c2cn3c(n2)C(CP)CCC3)cc1</smiles>

(R)-(8-((diphenylphosphanyl)methyl)-2-(4-fluorophenyl)-5,6,7,8-tetrahydroimidazo[1,2-a]pyridine borane adduct. White foam, $\mathrm{R}_{f}=0.05(2.5 \%$ EtOAc in DCM $) .{ }^{1} \mathbf{H}$ NMR $\left(\mathrm{CDCl}_{3}, 400 \mathrm{MHz}\right): \delta 7.97-$ $7.92(\mathrm{~m}, 2 \mathrm{H}), 7.77-7.69(\mathrm{~m}, 4 \mathrm{H}), 7.51-7.40(\mathrm{~m}, 6 \mathrm{H}), 7.05(\mathrm{t}, J=8.0 \mathrm{~Hz}, 2 \mathrm{H}), 6.98(\mathrm{~s}, 1 \mathrm{H}), 4.00-3.94(\mathrm{~m}$, $1 \mathrm{H}), 3.89-3.83(\mathrm{~m}, 1 \mathrm{H}), 3.68-3.60(\mathrm{~m}, 1 \mathrm{H}), 3.31-3.21(\mathrm{~m}, 1 \mathrm{H}), 2.41-2.30(\mathrm{~m}, 2 \mathrm{H}), 2.04-1.95(\mathrm{~m}, 1 \mathrm{H})$, $1.91-1.80(\mathrm{~m}, 1 \mathrm{H}), 1.54-1.44(\mathrm{~m}, 1 \mathrm{H}) .{ }^{13} \mathbf{C}$ NMR $\left(\mathrm{CDCl}_{3}, 100 \mathrm{MHz},\right): \delta 161.98(\mathrm{~d}, J=243 \mathrm{~Hz}), 148.05$, 147.90, 139.97, 132.66 (d, $J=9 \mathrm{~Hz}), 132.19$ (d, $J=9 \mathrm{~Hz}), 131.41$ (d, $J=3 \mathrm{~Hz}), 131.28$ (d, $J=3 \mathrm{~Hz})$, $129.06(\mathrm{~d}, J=10 \mathrm{~Hz}), 128.92(\mathrm{~d}, J=10 \mathrm{~Hz}), 126.46(\mathrm{~d}, J=8 \mathrm{~Hz}), 45.02,32.14,29.90,29.53,28.59$, 22.24. IR $\left(\mathrm{NaCl}\right.$, neat, $\left.\mathrm{cm}^{-1}\right): v=3057,3006,2954,2865,2374,1896,1559,1505,1437,1381,1218$, 1189, 1153, 1107, 1060, 841, 753, 701. [ $\propto]_{\mathbf{D}}^{23}=9.167\left(c=0.1195, \mathrm{CHCl}_{3}\right) . \mathbf{H R M S}(\mathbf{E I}) \mathrm{m} / \mathrm{z}:[\mathrm{M}+\mathrm{H}]^{+}$ Calc. for $\mathrm{C}_{26} \mathrm{H}_{28} \mathrm{BFN}_{2} \mathrm{P}=429.2066$ Found 429.2080 . 


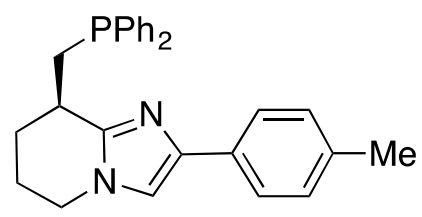

(R)-8-((diphenylphosphanyl)methyl)-2-(p-tolyl)-5,6,7,8-tetrahydroimidazo[1,2-a]pyridine, White foam, 54\% yield. $\mathrm{R}_{f}=0.30($ EtOAc/Pentane $=1 / 5) .{ }^{1} \mathbf{H} \mathbf{~ N M R}\left(\mathrm{CDCl}_{3}, 400 \mathrm{MHz}\right): \delta 7.65-7.59(\mathrm{~m}, 4 \mathrm{H})$, $7.50-7.46(\mathrm{~m}, 2 \mathrm{H}), 7.38-7.29(\mathrm{~m}, 6 \mathrm{H}), 7.17-7.15(\mathrm{~m}, 2 \mathrm{H}), 6.99(\mathrm{~s}, 1 \mathrm{H}), 3.98-3.87(\mathrm{~m}, 2 \mathrm{H}), 3.31$ (dt, $J=14.2,4.1 \mathrm{~Hz}, 1 \mathrm{H}), 3.14-3.05(\mathrm{~m}, 1 \mathrm{H}), 2.35(\mathrm{~s}, 3 \mathrm{H}), 2.30-2.23(\mathrm{~m}, 2 \mathrm{H}), 2.09-2.02(\mathrm{~m}, 1 \mathrm{H}), 1.91-$ $1.80(\mathrm{~m}, 1 \mathrm{H}), 1.76-1.67(\mathrm{~m}, 1 \mathrm{H}) .{ }^{13} \mathbf{C}$ NMR $\left(\mathrm{CDCl}_{3}, 100 \mathrm{MHz}\right): \delta 133.30,133.11,132.86,132.67$, $129.15,128.76,128.60,128.53,128.43,128.38,128.31,124.79,113.57,49.17,44.93,32.99,32.86$, 31.94, 29.72, 29.38, 22.71, 21.85, 21.22, 14.14, 11.95. ${ }^{31} \mathbf{P}$ NMR $\left(\mathrm{CDCl}_{3}, 100 \mathrm{MHz}\right): \delta$-20.6. IR (neat, $\left.\mathrm{cm}^{-1}\right): v=2924,2386,1434,1169,823,749,697 .[\propto]_{\mathbf{D}}^{23}=-5.682\left(c=0.088, \mathrm{CHCl}_{3}\right) . \mathbf{H R M S}(\mathbf{E I}) \mathrm{m} / \mathrm{z}:$ $[\mathrm{M}+\mathrm{H}]^{+}$Calc. for $\mathrm{C}_{27} \mathrm{H}_{28} \mathrm{~N}_{2} \mathrm{P}_{1}$ 411.1985; Found 411.1958.

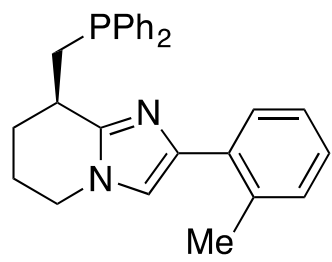

(R)-(8)-((diphenylphosphanyl)methyl)-2-(o-tolyl)-5,6,7,8-tetrahydroimidazo[1,2-a]pyridine. $\quad 72 \%$ yield. White foam, $\mathrm{R}_{f}=0.11\left(2.5 \%\right.$ EtOAc in DCM). ${ }^{1} \mathbf{H}$ NMR $\left(\mathrm{CDCl}_{3}, 500 \mathrm{MHz}\right): \delta 7.82(\mathrm{~d}, J=7.7 \mathrm{~Hz}$, $1 \mathrm{H})$, 7.66-7.59 (m, 2H), 7.50-7.46 (m, 2H), 7.41-7.33 (m, 3H), 7.32-7.29 (m, 3H), 7.25-7.20 (m, 2H), 7.17-7.14 (m, 1H), $6.88(\mathrm{~s}, 1 \mathrm{H}), 4.02-3.91(\mathrm{~m}, 2 \mathrm{H}), 3.32(\mathrm{dt}, J=14.3,4.0 \mathrm{~Hz}, 1 \mathrm{H}), 3.12-3.04(\mathrm{~m}, 1 \mathrm{H})$, $2.48(\mathrm{~s}, 3 \mathrm{H}), 2.36-2.23(\mathrm{~m}, 2 \mathrm{H}), 2.14-2.02(\mathrm{~m}, 1 \mathrm{H}), 1.95-1.83(\mathrm{~m}, 1 \mathrm{H}), 1.76-1.69(\mathrm{~m}, 1 \mathrm{H}) .{ }^{13} \mathbf{C} \mathbf{~ N M R}$ $\left(\mathrm{CDCl}_{3}, 125 \mathrm{MHz}\right.$ ): $\delta 147.88(\mathrm{~d}, J=11.8 \mathrm{~Hz}), 140.06,139.38(\mathrm{~d}, J=11.7 \mathrm{~Hz}), 137.84(\mathrm{~d}, J=12.6 \mathrm{~Hz})$, 134.91, 134.10, 133.31 (d, $J=19.3 \mathrm{~Hz}), 132.82$ (d, $J=19.1 \mathrm{~Hz}), 130.70,128.88,128.67$ (d, $J=6.8 \mathrm{~Hz})$, $128.55(\mathrm{~d}, J=10.0 \mathrm{~Hz}), 128.44(\mathrm{~d}, J=6.7 \mathrm{~Hz}), 126.52,125.93,116.67,44.96,33.69$ (d, $J=17.7 \mathrm{~Hz})$, $32.93(\mathrm{~d}, J=12.9 \mathrm{~Hz}), 28.07(\mathrm{~d}, J=10.9 \mathrm{~Hz}), 22.12,21.91 .{ }^{31} \mathbf{P}$ NMR: $\delta-20.66$. IR $\left(\mathrm{NaCl}\right.$, neat, $\left.\mathrm{cm}^{-1}\right): v$ $=3053,3012,2947,2858,2384,1954,1889,1813,1720,1671,1603,1585,1505,1479,1434,1379$, 1317, 1267, 1186, 1121, 1094, 1068, 1044, 947. [ $\propto]_{\mathbf{D}}^{23}=54.286\left(c=0.1750, \mathrm{CHCl}_{3}\right)$. HRMS (EI) m/z: $[\mathrm{M}+\mathrm{H}]^{+}$Calc. for $\mathrm{C}_{27} \mathrm{H}_{28} \mathrm{~N}_{2} \mathrm{P}=411.1985$ Found 411.1999 .<smiles>Cc1ccc(-c2cn3c(n2)C(Cc2ccccc2)CCC3)c(C)c1</smiles>

(R)-2-(2,4-dimethylphenyl)-8-((diphenylphosphanyl)methyl)-5,6,7,8-tetrahydroimidazo

$[1,2-$ a]pyridine, White foam, 90\% yield. $\mathrm{R}_{f}=0.54$ (EtOAc/Pentane $\left.=3 / 7\right) .{ }^{1} \mathbf{H}$ NMR $\left(\mathrm{CDCl}_{3}, 400 \mathrm{MHz}\right): \delta$ $7.70(\mathrm{dd}, J=7.7,1.4 \mathrm{~Hz}, 1 \mathrm{H}), 7.62(\mathrm{tt}, J=7.6,1.3 \mathrm{~Hz}, 2 \mathrm{H}), 7.52-7.42(\mathrm{~m}, 2 \mathrm{H}), 7.42-7.23(\mathrm{~m}, 6 \mathrm{H})$, $7.07-7.00(\mathrm{~m}, 2 \mathrm{H}), 6.84(\mathrm{~s}, 1 \mathrm{H}), 4.04-3.87(\mathrm{~m}, 2 \mathrm{H}), 3.31(\mathrm{dt}, J=14.2,4.1 \mathrm{~Hz}, 1 \mathrm{H}), 3.15-3.00(\mathrm{~m}$, $1 \mathrm{H}), 2.44(\mathrm{~s}, 3 \mathrm{H}), 2.32(\mathrm{~s}, 3 \mathrm{H}), 2.36-2.20(\mathrm{~m}, 2 \mathrm{H}), 2.12-2.01(\mathrm{~m}, 1 \mathrm{H}), 1.97-1.81(\mathrm{~m}, 1 \mathrm{H}), 1.79-1.64$ $(\mathrm{m}, 1 \mathrm{H}) .{ }^{13} \mathbf{C} \mathbf{N M R}\left(\mathrm{CDCl}_{3}, 100 \mathrm{MHz}\right): \delta 147.8(\mathrm{~d}, J=11.8 \mathrm{~Hz}), 140.1,139.4(\mathrm{~d}, J=11.4 \mathrm{~Hz}), 137.9(\mathrm{~d}, J$ $=12.8 \mathrm{~Hz}), 136.1,134.7,133.1(\mathrm{dd}, J=48.4,19.0 \mathrm{~Hz}), 131.5,131.3,128.9,128.8-128.2(\mathrm{~m}), 126.7$, 116.3, 44.9, $33.7(\mathrm{~d}, J=17.6 \mathrm{~Hz}), 32.9(\mathrm{~d}, J=12.7 \mathrm{~Hz}), 28.1(\mathrm{~d}, J=10.8 \mathrm{~Hz}), 22.1,21.8,21.2 .{ }^{31} \mathbf{P}$ NMR $\left(\mathrm{CDCl}_{3}, 100 \mathrm{MHz}\right): \delta-20.6$. IR $\left(\mathrm{NaCl}\right.$, neat, $\left.\mathrm{cm}^{-1}\right): v=2944,2353,1434,1191,740,697 .[\propto]_{\mathbf{D}}^{23}=-49.833$ $\left(c=0.600, \mathrm{CHCl}_{3}\right)$. HRMS (EI) m/z: $[\mathrm{M}+\mathrm{H}]^{+}$Calc. for $\mathrm{C}_{28} \mathrm{H}_{30} \mathrm{~N}_{2} \mathrm{P} 425.2141$; Found 425.2164 . 


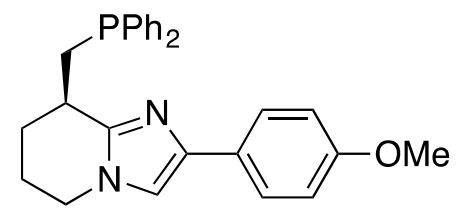

(R)-8-((diphenylphosphanyl)methyl)-2-(4-methoxyphenyl)-5,6,7,8-tetrahydroimidazo

$[1,2-$ a]pyridine, White foam, $78 \%$ yield. $\mathrm{R}_{\mathrm{f}}=0.50($ EtOAc/Pentane $=1 / 1) .{ }^{1} \mathbf{H}$ NMR $\left(\mathrm{CDCl}_{3}, 400 \mathrm{MHz}\right): \delta$ $7.70-7.64(\mathrm{~m}, 2 \mathrm{H}), 7.64-7.58(\mathrm{~m}, 2 \mathrm{H}), 7.48(\mathrm{td}, J=7.5,2.1 \mathrm{~Hz}, 2 \mathrm{H}), 7.43-7.28(\mathrm{~m}, 6 \mathrm{H}), 6.93(\mathrm{~s}, 1 \mathrm{H})$, $6.92-6.87(\mathrm{~m}, 2 \mathrm{H}), 4.02-3.86(\mathrm{~m}, 2 \mathrm{H}), 3.82(\mathrm{~s}, 3 \mathrm{H}), 3.27(\mathrm{dt}, J=14.2,3.9 \mathrm{~Hz}, 1 \mathrm{H}), 3.19-3.00(\mathrm{~m}$, $1 \mathrm{H}), 2.35-2.22(\mathrm{~m}, 2 \mathrm{H}), 2.11-1.97(\mathrm{~m}, 1 \mathrm{H}), 1.91-1.77(\mathrm{~m}, 1 \mathrm{H}), 1.77-1.64(\mathrm{~m}, 1 \mathrm{H}) .{ }^{13} \mathbf{C} \mathbf{~ N M R}$ $\left(\mathrm{CDCl}_{3}, 100 \mathrm{MHz}\right): \delta 158.5,148.7(\mathrm{~d}, J=11.8 \mathrm{~Hz}), 140.7,139.4$ (d, $\left.J=11.6 \mathrm{~Hz}\right), 137.9$ (d, $\left.J=12.7 \mathrm{~Hz}\right)$, $133.1(\mathrm{dd}, J=43.3,19.0 \mathrm{~Hz}), 128.9,128.7(\mathrm{~d}, J=7.0 \mathrm{~Hz}), 128.6-128.3(\mathrm{~m}), 126.2,114.0,113.1,55.4$, 45.0, $33.7(\mathrm{~d}, J=17.9 \mathrm{~Hz}), 33.1(\mathrm{~d}, J=13.0 \mathrm{~Hz}), 26.0(\mathrm{~d}, J=11.3 \mathrm{~Hz}), 21.99 .{ }^{31} \mathbf{P}$ NMR $\left(\mathrm{CDCl}_{3}, 100\right.$ MHz): $\delta$-20.6. IR $\left(\mathrm{NaCl}\right.$, neat, $\left.\mathrm{cm}^{-1}\right): v=2946,2856,1507,1434,1275,1171,764 .[\propto]_{\mathbf{D}}^{23}=-58.857(c=$ 0.175, $\mathrm{CHCl}_{3}$ ). HRMS (EI) m/z: [M+H] ${ }^{+}$Calc. for $\mathrm{C}_{27} \mathrm{H}_{27} \mathrm{~N}_{2} \mathrm{OP}$ 427.1934; Found 427.1939.<smiles>COc1ccc(-c2cn3c(n2)C(Cc2ccccc2)CCC3)c(OC)c1</smiles>

(R)-2-(2,4-dimethoxyphenyl)-8-((diphenylphosphanyl)methyl)-5,6,7,8-tetrahydroimidazo[1,2-

a]pyridine. White foam, $\mathrm{R}_{f}=0.06\left(2.5 \%\right.$ EtOAc in DCM). ${ }^{1} \mathbf{H}$ NMR $\left(\mathrm{CDCl}_{3}, 500 \mathrm{MHz}\right): \delta 8.10(\mathrm{~d}, J=$ $10.0 \mathrm{~Hz}, 1 \mathrm{H}), 7.64-7.59(\mathrm{~m}, 2 \mathrm{H}), 7.50-7.46(\mathrm{~m}, 2 \mathrm{H}), 7.40-7.33(\mathrm{~m}, 3 \mathrm{H}), 7.33-7.28(\mathrm{~m}, 3 \mathrm{H}), 7.24(\mathrm{~s}, 1 \mathrm{H})$, $6.59(\mathrm{dd}, J=8.7,2.4 \mathrm{~Hz}, 1 \mathrm{H}), 6.51(\mathrm{~d}, J=2.6 \mathrm{~Hz}, 1 \mathrm{H}), 4.00-3.94(\mathrm{~m}, 1 \mathrm{H}), 3.94-3.87(\mathrm{~m}, 1 \mathrm{H}), 3.89(\mathrm{~s}$, $3 \mathrm{H}) 3.83(\mathrm{~s}, 3 \mathrm{H}), 3.29(\mathrm{dt}, J=14.2,3.9 \mathrm{~Hz}, 1 \mathrm{H}), 3.13-3.04(\mathrm{~m}, 1 \mathrm{H}), 2.31-2.23(\mathrm{~m}, 2 \mathrm{H}), 2.08-2.01(\mathrm{~m}$, $1 \mathrm{H}), 1.90-1.80(\mathrm{~m}, 1 \mathrm{H}), 1.74-1.65(\mathrm{~m}, 1 \mathrm{H}) .{ }^{13} \mathbf{C}$ NMR $\left(\mathrm{CDCl}_{3}, 125 \mathrm{MHz},\right): \delta 159.28,156.96,147.49(\mathrm{~d}, J$ $=11.2 \mathrm{~Hz}), 139.47,(\mathrm{~d}, J=11.2 \mathrm{~Hz}), 138.03(\mathrm{~d}, 12.5 \mathrm{~Hz}), 136.02,133.30(\mathrm{~d}, J=18.7 \mathrm{~Hz}), 132.88(\mathrm{~d}, J=$ $18.7 \mathrm{~Hz}), 128.83,128.60(\mathrm{~d}, J=6.2 \mathrm{~Hz}), 128.51,128.44$ (d, $J=6.2 \mathrm{~Hz}), 128.26,117.10,116.76,104.78$, 98.56, 55.50, 55.40, 44.88, 33.68 (d, $J=18.7 \mathrm{~Hz}), 33.09$ (d, $J=12.5 \mathrm{~Hz}), 28.08$ (d, $J=11.2 \mathrm{~Hz}), 22.08$. ${ }^{31}$ P NMR: $\delta$ - 20.47. IR $\left(\mathrm{NaCl}\right.$, neat, $\left.\mathrm{cm}^{-1}\right): v=3210,3052,2941,2380,2358,1890,1817,1613,1582$, $1555,1508,1466,1434,1377,1288,1207,1179,1158,1069,1034,952,831,741,698 .[\propto]_{\mathbf{D}}^{23}=-39.583$ $\left(c=0.2880, \mathrm{CHCl}_{3}\right)$. HRMS (EI) m/z: $[\mathrm{M}+\mathrm{H}]^{+}$Calc. for $\mathrm{C}_{28} \mathrm{H}_{30} \mathrm{~N}_{2} \mathrm{O}_{2} \mathrm{P}=457.2039$; Found 457.2044.

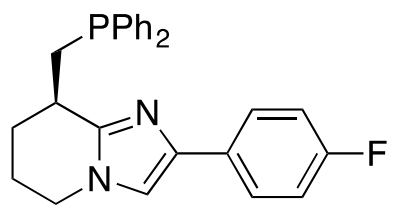

(R)-(8)-((diphenylphosphanyl)methyl)-2-(4-fluorophenyl)-5,6,7,8-tetrahydroimidazo[1,2-a]pyridine. White foam, $\mathrm{R}_{f}=0.04\left(2.5 \%\right.$ EtOAc in DCM). ${ }^{1} \mathbf{H} \mathbf{~ N M R}\left(\mathrm{CDCl}_{3}, 500 \mathrm{MHz}\right): \delta$ 7.72-7.66 (m, 2H), 7.63$7.57(\mathrm{~m}, 2 \mathrm{H}), 7.49-7.45(\mathrm{~m}, 2 \mathrm{H}), 7.40-7.33(\mathrm{~m}, 3 \mathrm{H}), 7.33-7.27(\mathrm{~m}, 3 \mathrm{H}), 7.05-7.01(\mathrm{~m}, 2 \mathrm{H}), 6.97(\mathrm{~s}, 1 \mathrm{H})$, 3.99-3.88 (m, 2H), $3.25(\mathrm{dt}, J=14.1,3.9 \mathrm{~Hz}, 1 \mathrm{H}), 3.12-3.02(\mathrm{~m}, 1 \mathrm{H}), 2.31-2.26(\mathrm{~m}, 2 \mathrm{H}), 2.11-2.02(\mathrm{~m}$, $1 \mathrm{H}), 1.87(\mathrm{~m}, 1 \mathrm{H}), 1.75-1.68(\mathrm{~m}, 1 \mathrm{H}) .{ }^{13} \mathbf{C}$ NMR $\left(\mathrm{CDCl}_{3}, 125 \mathrm{MHz},\right): \delta 161.91(\mathrm{~d}, J=244.7 \mathrm{~Hz}), 148.97$ $(\mathrm{d}, J=11.6 \mathrm{~Hz}), 140.00,139.33(\mathrm{~d}, J=11.7 \mathrm{~Hz}), 137.88(\mathrm{~d}, J=12.6 \mathrm{~Hz}), 133.31(\mathrm{~d}, J=18.9 \mathrm{~Hz}), 132.87$ $(\mathrm{d}, J=18.6 \mathrm{~Hz}), 128.93,128.71(\mathrm{~d}, J=6.9 \mathrm{~Hz}), 128.58,128.48(\mathrm{~d}, J=6.8 \mathrm{~Hz}), 126.49(\mathrm{~d}, J=7.8 \mathrm{~Hz})$, $115.48,115.39(\mathrm{~d}, J=21.3 \mathrm{~Hz}), 113.76,45.07,33.76(\mathrm{~d}, J=17.8 \mathrm{~Hz}), 33.09(\mathrm{~d}, J=12.9 \mathrm{~Hz}), 27.95(\mathrm{~d}, J$ $=11.1 \mathrm{~Hz}), 22.03 .{ }^{31} \mathbf{P}$ NMR: $\delta-20.68 . \mathbf{I R}\left(\mathrm{NaCl}\right.$, neat, $\left.\mathrm{cm}^{-1}\right): v=3392,3055,2949,1713,1598,1559$, 
$1506,1479,1436,1379,1218,1186,1154,1119,1072,1027,999,949 .[\propto]_{\mathbf{D}}^{23}=13.383(c=0.2685$, $\mathrm{CHCl}_{3}$ ). HRMS (EI) m/z: [M+H] $]^{+}$Calc. for $\mathrm{C}_{26} \mathrm{H}_{25} \mathrm{FN}_{2} \mathrm{P}=415.1734$ Found 415.1713 .

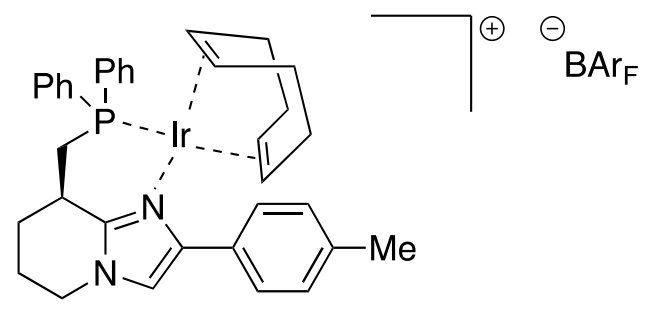

Complex-(R)-iii, Orange solid, $46 \%$ yield. $\mathrm{R}_{f}=0.46(\mathrm{DCM} / \mathrm{Pentane}=1 / 1) .{ }^{1} \mathbf{H}$ NMR $\left(\mathrm{CDCl}_{3}, 500 \mathrm{MHz}\right)$ : $\delta 7.85-7.81(\mathrm{~m}, 2 \mathrm{H}), 7.72-7.70(\mathrm{~m}, 10 \mathrm{H}), 7.56-7.48(\mathrm{~m}, 10 \mathrm{H}), 7.43-7.41(\mathrm{~m}, 3 \mathrm{H}), 7.27(\mathrm{~d}, J=7.9$, $3 \mathrm{H}), 7.16-7.12(\mathrm{~m}, 2 \mathrm{H}), 6.89(\mathrm{~s}, 1 \mathrm{H}), 4.63(\mathrm{~s}, 1 \mathrm{H}), 3.99-3.89(\mathrm{~m}, 3 \mathrm{H}), 3.82-3.79(\mathrm{~m}, 1 \mathrm{H}), 3.76-$ $3.71(\mathrm{~m}, 1 \mathrm{H}), 2.69-2.64(\mathrm{~m}, 1 \mathrm{H}), 2.51-2.40(\mathrm{~m}, 6 \mathrm{H}), 2.35-2.22(\mathrm{~m}, 3 \mathrm{H}), 2.16-2.08(\mathrm{~m}, 2 \mathrm{H}), 2.01-$ $1.95(\mathrm{~m}, 2 \mathrm{H}), 1.90-1.83(\mathrm{~m}, 1 \mathrm{H}), 1.77-1.71(\mathrm{~m}, 1 \mathrm{H}), 1.51-1.43(\mathrm{~m}, 1 \mathrm{H}), 1.33-1.22(\mathrm{~m}, 8 \mathrm{H}), 0.89-$ $0.85(\mathrm{~m}, 3 \mathrm{H}) .{ }^{13} \mathrm{C}$ NMR $\left(\mathrm{CDCl}_{3}, 126 \mathrm{MHz}\right): \delta 162.32,161.92,161.52,161.13,147.05,142.38,139.67$, $134.88,134.83,134.79,132.62,132.43,132.21,131.22,130.80,130.73,129.96,129.60,129.54,129.51$, $129.46,129.09,129.01,128.81,128.02,127.86,127.82,125.66,123.49,121.32,117.71,117.51,100.02$, $97.31,97.23,92.19,92.08,63.97,61.58,45.97,36.65,35.42,35.37,33.02,29.76,28.68,28.40,28.24$, 28.12, 25.89, 21.34, 20.44, 1.08. ${ }^{31} \mathbf{P}$ NMR $\left(\mathrm{CDCl}_{3}, 100 \mathrm{MHz}\right): \delta 14.2$. IR (neat, $\left.\mathrm{cm}^{-1}\right): v=2923,2024$, 1610, 1434, 1360, 1276, 1124, 887, 740, 670. [ $\propto]_{\mathbf{D}}^{23}=-8.16\left(c=0.625, \mathrm{CHCl}_{3}\right) . \mathbf{H R M S}(\mathbf{E I}) \mathrm{m} / \mathrm{z}:[\mathrm{M}-$ $\left.\mathrm{BAr}_{\mathrm{F}}\right]^{+}$Calc. for $\mathrm{C}_{35} \mathrm{H}_{39} \mathrm{IrN}_{2} \mathrm{P}=711.2476$; Found 711.2416.

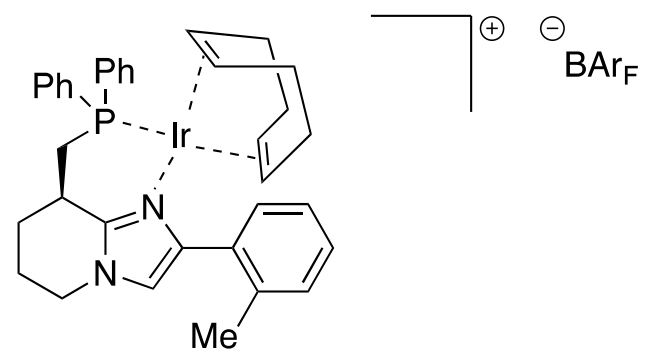

Complex-(R)-iv, $89 \%$ yield. Orange foam, $\mathrm{R}_{f}=0.13\left(\mathrm{CH}_{2} \mathrm{Cl}_{2} /\right.$ Pentane $\left.4: 6\right) .{ }^{1} \mathbf{H}$ NMR $\left(\mathrm{CDCl}_{3}, 500\right.$ MHz): $\delta$ 7.89-7.85 (m, 2H), $7.71(\mathrm{bs}, 8 \mathrm{H}), 7.62-7.56(\mathrm{~m}, 3 \mathrm{H}), 7.52(\mathrm{~s}, 4 \mathrm{H}), 7.43-7.36(\mathrm{~m}, 4 \mathrm{H}), 7.32-7.24$ (m, 4H), 7.13-7.08 (m, 2H), 7.13-7.08 (m, 2H), $6.85(\mathrm{~s}, 1 \mathrm{H}), 4.63(\mathrm{bs}, 1 \mathrm{H}), 4.02-3.90(\mathrm{~m}, 3 \mathrm{H}), 3.55-3.47$ (m, 2H), 2.62 (ddd, $J=14.7,11.4,3.1 \mathrm{~Hz}, 1 \mathrm{H}), 2.47-2.39(\mathrm{~m}, 2 \mathrm{H}), 2.34-2.25(\mathrm{~m}, 6 \mathrm{H}), 2.19-2.10(\mathrm{~m}, 2 \mathrm{H})$, 2.03-1.98 (m, 2H), 1.93-1.87 (m, 1H), 1.75-1.66 (m, 1H), $1.54(\mathrm{~s}, 1 \mathrm{H}), 1.38-1.17(\mathrm{~m}, 5 \mathrm{H}) .{ }^{13} \mathbf{C}$ NMR $\left(\mathrm{CDCl}_{3}, 125 \mathrm{MHz}\right.$ ): $\delta 162.43,162.03,161.63,161.24,147.27(\mathrm{~d}, J=3.7 \mathrm{~Hz}), 140.72,136.53,135.23(\mathrm{~d}$, $J=12.5 \mathrm{~Hz}), 134.94,132.66,132.49,132.07,131.33,130.89(\mathrm{dd}, J=8.7,3.7 \mathrm{~Hz}), 130.69,130.11$, $129.76,129.69,129.65,129.57,129.19,129.15,129.07,128.90,127.93,125.87,125.77,123.60,121.43$, 119.59, 117.64-117.52 (m), $96.95(\mathrm{~d}, J=10.0 \mathrm{~Hz}), 93.95(\mathrm{~d}, J=13.7 \mathrm{~Hz}), 63.47,59.98,46.14,36.41(\mathrm{~d}$, $J=5.0 \mathrm{~Hz}$ ), 35.56 (d, $J=6.2 \mathrm{~Hz}), 32.65,28.73,28.44$ (d, $J=5.0 \mathrm{~Hz}), 28.29,28.22,26.45,20.69,20.37$. ${ }^{31} \mathbf{P}$ NMR $\delta$ 13.36. IR $\left(\mathrm{NaCl}\right.$, neat, $\left.\mathrm{cm}^{-1}\right): v=3064,2959,2927,2888,2060,2023,1784,1610,1487$, $1438,1355,1278,1126,1000,982,931,887,839,742,713,696,682,670 .[\propto]_{\mathrm{D}}^{23}=-12.712(c=0.1177$, $\mathrm{CHCl}_{3}$ ). HRMS (EI) m/z: [M-BAr $]^{+}$Calc. for $\mathrm{C}_{35} \mathrm{H}_{39} \mathrm{IrN}_{2} \mathrm{P}=711.2476$; Found 711.2480. 


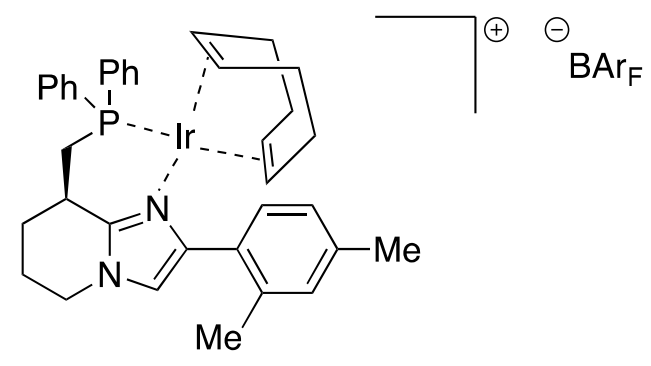

Complex-(R)-v, Orange solid, $51 \%$ yield. $\mathrm{R}_{f}=0.50(\mathrm{DCM} / \mathrm{Pentane}=1 / 1) .{ }^{1} \mathbf{H}$ NMR $\left(\mathrm{CDCl}_{3}, 400 \mathrm{MHz}\right)$ : $\delta 7.97-7.81(\mathrm{~m}, 2 \mathrm{H}), 7.71(\mathrm{p}, J=2.2 \mathrm{~Hz}, 8 \mathrm{H}), 7.58(\mathrm{dp}, J=5.1,2.9 \mathrm{~Hz}, 3 \mathrm{H}), 7.52(\mathrm{~s}, 4 \mathrm{H}), 7.40(\mathrm{dq}, J=$ 5.6, $2.7 \mathrm{~Hz}, 3 \mathrm{H}), 7.19-7.06(\mathrm{~m}, 5 \mathrm{H}), 6.81(\mathrm{~s}, 1 \mathrm{H}), 4.67-4.56(\mathrm{~m}, 1 \mathrm{H}), 4.07-3.87(\mathrm{~m}, 3 \mathrm{H}), 3.66-3.54$ (m, 1H), $3.51-3.45(\mathrm{~m}, 1 \mathrm{H}), 2.62$ (ddd, $J=14.7,11.5,3.1 \mathrm{~Hz}, 1 \mathrm{H}), 2.49-2.36(\mathrm{~m}, 5 \mathrm{H}), 2.36-2.27(\mathrm{~m}$, $2 \mathrm{H}), 2.24(\mathrm{~s}, 3 \mathrm{H}), 2.17-2.09(\mathrm{~m}, 2 \mathrm{H}), 2.06-1.85(\mathrm{~m}, 3 \mathrm{H}), 1.76-1.64(\mathrm{~m}, 1 \mathrm{H}), 1.63-1.54(\mathrm{~m}, 1 \mathrm{H})$, $1.45-1.12(\mathrm{~m}, 3 \mathrm{H}) .{ }^{13} \mathbf{C} \mathbf{N M R}\left(\mathrm{CDCl}_{3}, 100 \mathrm{MHz}\right): \delta 161.8(\mathrm{dd}, J=99.7,49.9 \mathrm{~Hz}), 147.1(\mathrm{~d}, J=3.7 \mathrm{~Hz})$, $140.8,139.9,136.3,135.2(\mathrm{~d}, J=12.3 \mathrm{~Hz}), 134.9,133.2-132.2(\mathrm{~m}), 132.0,131.6,131.3(\mathrm{~d}, J=2.5 \mathrm{~Hz})$, $131.0-130.7(\mathrm{~m}), 130.2,129.9-129.2(\mathrm{~m}), 129.2-128.4(\mathrm{~m}), 127.7,126.5,126.0,123.3,120.6,119.4$, $117.6(\mathrm{~d}, J=5.4 \mathrm{~Hz}), 96.9(\mathrm{~d}, J=10.5 \mathrm{~Hz}), 94.2(\mathrm{~d}, J=13.2 \mathrm{~Hz}), 63.3,59.7,46.1,36.4(\mathrm{~d}, J=4.6 \mathrm{~Hz})$, $35.6(\mathrm{~d}, J=6.2 \mathrm{~Hz}), 32.6,30.4-29.4(\mathrm{~m}), 29.0-28.0(\mathrm{~m}), 26.4,22.9,21.3,20.5(\mathrm{~d}, J=24.0 \mathrm{~Hz}) .{ }^{31} \mathbf{P}$ NMR $\left(\mathrm{CDCl}_{3}, 100 \mathrm{MHz}\right): \delta$-13.4. IR $\left(\mathrm{NaCl}\right.$, neat, $\left.\mathrm{cm}^{-1}\right): v=2944,2858,1505,1434,1376,1191,740$, 697. $[\propto]_{\mathbf{D}}^{23}=-14.222\left(c=0.225, \mathrm{CHCl}_{3}\right)$. HRMS (EI) $\mathrm{m} / \mathrm{z}$ : $\left.[\mathrm{M}-\mathrm{BAr}]_{\mathrm{F}}\right]^{+}$Calc. for $\mathrm{C}_{36} \mathrm{H}_{41} \mathrm{IrN}_{2} \mathrm{P} 725.2633$; Found 725.2627.

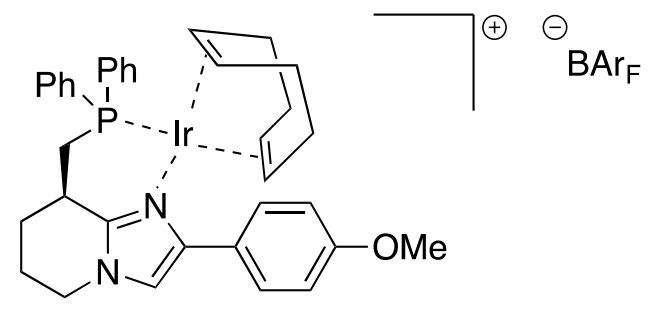

Complex-(R)-vi, Orange solid, 55\% yield. $\mathrm{R}_{f}=0.50(\mathrm{DCM} / \mathrm{Pentane}=1 / 1) .{ }^{\mathbf{1}} \mathbf{H} \mathbf{N M R}\left(\mathrm{CDCl}_{3}, 400 \mathrm{MHz}\right)$ : $\delta 7.87-7.78(\mathrm{~m}, 2 \mathrm{H}), 7.76-7.68(\mathrm{~m}, 8 \mathrm{H}), 7.59-7.47(\mathrm{~m}, 9 \mathrm{H}), 7.46-7.39(\mathrm{~m}, 3 \mathrm{H}), 7.20-7.11(\mathrm{~m}$, 2H), $7.04-6.97(\mathrm{~m}, 2 \mathrm{H}), 6.85(\mathrm{~s}, 1 \mathrm{H}), 4.74-4.59(\mathrm{~m}, 1 \mathrm{H}), 4.05-3.90(\mathrm{~m}, 2 \mathrm{H}), 3.87(\mathrm{~s}, 3 \mathrm{H}), 3.84-3.75$ (m, 2H), $2.72-2.61(\mathrm{~m}, 1 \mathrm{H}), 2.54-2.39(\mathrm{~m}, 3 \mathrm{H}), 2.38-2.22(\mathrm{~m}, 2 \mathrm{H}), 2.20-2.06(\mathrm{~m}, 2 \mathrm{H}), 2.05-1.98$ $(\mathrm{m}, 2 \mathrm{H}), 1.93-1.84(\mathrm{~m}, 1 \mathrm{H}), 1.82-1.69(\mathrm{~m}, 1 \mathrm{H}), 1.60-1.46(\mathrm{~m}, 2 \mathrm{H}), 1.42-1.21(\mathrm{~m}, 2 \mathrm{H}) .{ }^{13} \mathbf{C} \mathbf{~ N M R}$ $\left(\mathrm{CDCl}_{3}, 100 \mathrm{MHz}\right): \delta 161.84(\mathrm{dd}, J=99.7,49.8 \mathrm{~Hz}), 160.71,147.01(\mathrm{~d}, J=3.8 \mathrm{~Hz}), 142.24,134.90(\mathrm{~d}, J$ $=11.9 \mathrm{~Hz}), 132.53(\mathrm{dd}, J=27.1,24.5 \mathrm{~Hz}), 131.31(\mathrm{~d}, J=2.5 \mathrm{~Hz}), 130.88(\mathrm{~d}, J=9.4 \mathrm{~Hz}), 130.09,129.79$ - $129.36(\mathrm{~m}), 129.33-129.06(\mathrm{~m}), 129.06-128.84(\mathrm{~m}), 128.76,128.59,126.05,123.38(\mathrm{~d}, J=8.8 \mathrm{~Hz})$, 120.63, $117.95-117.25(\mathrm{~m}), 114.29,97.38$ (d, $J=10.0 \mathrm{~Hz}), 92.27$ (d, $J=14.2 \mathrm{~Hz}), 64.05,61.65,55.60$, 46.03, $36.77(\mathrm{~d}, J=4.6 \mathrm{~Hz}), 35.48(\mathrm{~d}, J=5.6 \mathrm{~Hz}), 33.09,28.81,28.49-28.12(\mathrm{~m}), 26.10,20.53 .{ }^{31} \mathbf{P}$ NMR (CDCl $3,100 \mathrm{MHz}): \delta-14.2$. IR $\left(\mathrm{NaCl}\right.$, neat, $\left.\mathrm{cm}^{-1}\right): v=3054,2985,1610,1354,1278,1125,740$. $[\propto]_{\mathbf{D}}^{23}=-20.606\left(c=0.165, \mathrm{CHCl}_{3}\right)$. HRMS (EI) m/z: [M-BAr $]^{+}$Calc. for $\mathrm{C}_{35} \mathrm{H}_{39} \mathrm{IrN}_{2} \mathrm{OP} 727.2425$; Found 727.2449.

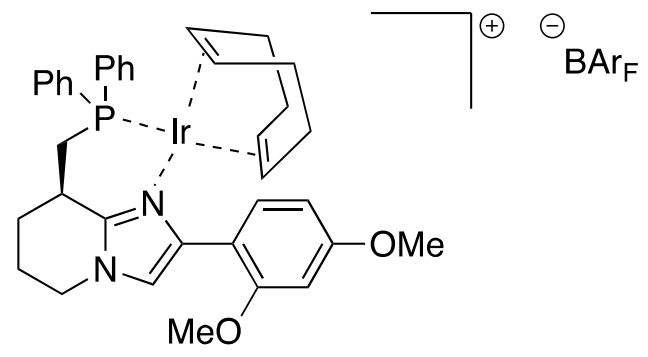

Complex-(R)-vii, Orange foam. $51 \%$ yield. ${ }^{1} \mathbf{H}$ NMR $\left(\mathrm{CDCl}_{3}, 500 \mathrm{MHz}\right): \delta 7.83-7.79(\mathrm{~m}, 2 \mathrm{H}), 7.71(\mathrm{~s}$, $8 \mathrm{H}), 7.61(\mathrm{~d}, J=8.4 \mathrm{~Hz}, 1 \mathrm{H}), 7.62-7.53(\mathrm{~m}, 3 \mathrm{H}), 7.52(\mathrm{~s}, 4 \mathrm{H}), 7.43-7.39(\mathrm{~m}, 3 \mathrm{H}), 7.18-7.10(\mathrm{~m}, 2 \mathrm{H}), 7.06$ (s, $1 \mathrm{H}), 6.62(\mathrm{dd}, J=8.4,2.3 \mathrm{~Hz}, 1 \mathrm{H}), 6.52(\mathrm{~d}, J=2.3 \mathrm{~Hz}, 1 \mathrm{H}), 4.64(\mathrm{~s}, 1 \mathrm{H}), 4.05-4.00(\mathrm{~m}, 1 \mathrm{H}), 3.99-$ 
$3.90(\mathrm{~m}, 2 \mathrm{H}), 3.89(\mathrm{~s}, 3 \mathrm{H}), 3.87-3.80(\mathrm{~m}, 1 \mathrm{H}), 3.78(\mathrm{~s}, 3 \mathrm{H}), 3.72(\mathrm{~s}, 1 \mathrm{H}), 2.64$ (ddd, $J=14.7,11.3,3.3$ $\mathrm{Hz}, 1 \mathrm{H}), 2.51-2.40(\mathrm{~m}, 3 \mathrm{H}), 2.37-2.20(\mathrm{~m}, 2 \mathrm{H}), 2.20-2.07$ (m, 2H), 2.00 (s, 2H), 1.88-1.81 (m, 1H), 1.77$1.71(\mathrm{~m}, 1 \mathrm{H}), 1.56-1.50(\mathrm{~m}, 3 \mathrm{H}) .{ }^{13} \mathbf{C} \mathbf{N M R}\left(\mathrm{CDCl}_{3}, 125 \mathrm{MHz}\right): \delta 159.28,156.96,147.49(\mathrm{~d}, J=11.2$ $\mathrm{Hz}), 139.47,(\mathrm{~d}, J=11.2 \mathrm{~Hz}), 138.03(\mathrm{~d}, 12.5 \mathrm{~Hz}), 136.02,133.30(\mathrm{~d}, J=18.7 \mathrm{~Hz}), 132.88$ (d, $J=18.7$ $\mathrm{Hz}), 128.83,128.60(\mathrm{~d}, J=6.2 \mathrm{~Hz}), 128.51,128.44(\mathrm{~d}, J=6.2 \mathrm{~Hz}), 128.26,117.10,116.76,104.78$, 98.56, 55.50, 55.40, 44.88, $33.68(\mathrm{~d}, J=18.7 \mathrm{~Hz}), 33.09$ (d, $J=12.5 \mathrm{~Hz}), 28.08(\mathrm{~d}, J=11.2 \mathrm{~Hz}), 22.08$. ${ }^{31}$ P NMR: $\delta$ 13.49. IR $\left(\mathrm{NaCl}\right.$, neat, $\left.\mathrm{cm}^{-1}\right): v=2962,2888,2840,1613,1582,1497,1465,1438,1355$, $1277,1212,1160,1124,1032,1000,984,931,887,839,740,713,696,682 .[\propto]_{\mathrm{D}}^{23}=-11.304(c=0.2880$, $\mathrm{CHCl}_{3}$ ). HRMS (EI) m/z: [M-BAr $]^{+}$Calc. for $\mathrm{C}_{36} \mathrm{H}_{41} \mathrm{IrN}_{2} \mathrm{O}_{2} \mathrm{P}=757.2531$; Found 757.2523.

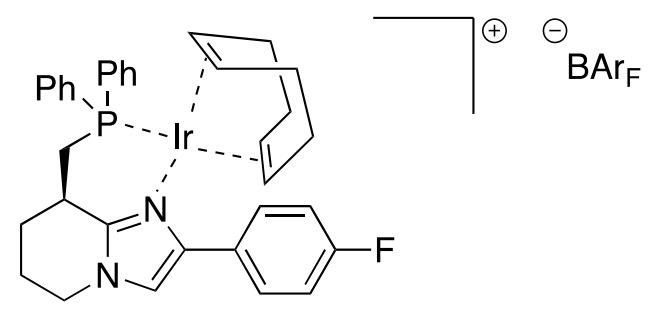

Complex-(R)-viii, $92 \%$ yield. Orange foam, $\mathrm{R}_{f}=0.06(100 \% \mathrm{DCM}) .{ }^{1} \mathbf{H}$ NMR $\left(\mathrm{CDCl}_{3}, 500 \mathrm{MHz}\right): \delta$ 7.83-7.77 (m, 2H), 7.71-7.70 (m, 8H), 7.59-7.53 (m, 5H), 7.51 (s, 4H), 7.45-7.40 (m, 3H), 7.19-7.11 (m, $4 \mathrm{H}), 6.87(\mathrm{~s}, 1 \mathrm{H}), 4.65-4.60(\mathrm{~m}, 1 \mathrm{H}), 3.99-3.86(\mathrm{~m}, 3 \mathrm{H}), 3.84-3.80(\mathrm{~m}, 1 \mathrm{H}), 3.66(\mathrm{p}, J=7.4 \mathrm{~Hz}, 1 \mathrm{H}), 2.66$ (ddd, $J=14.7,11.1,3.5 \mathrm{~Hz}, 1 \mathrm{H}), 2.53-2.08(\mathrm{~m}, 6 \mathrm{H}), 1.99-1.94(\mathrm{~m}, 2 \mathrm{H}), 1.89-1.82(\mathrm{~m}, 1 \mathrm{H}), 1.79-1.71(\mathrm{~m}$, $1 \mathrm{H}), 1.55-1.49(\mathrm{~m}, 2 \mathrm{H}), 1.42-1.32(\mathrm{~m}, 1 \mathrm{H}), 1.31-1.22(\mathrm{~m}, 1 \mathrm{H}) .{ }^{13} \mathbf{C} \mathbf{N M R}\left(\mathrm{CDCl}_{3}, 125 \mathrm{MHz},\right): \delta 164.37$, $162.41,162.02,161.62,161.22,147.53(\mathrm{~d}, J=3.7 \mathrm{~Hz}), 141.35$, 134.93, 134.86, 134.76, 132.64, 132.58, 132.17, 131.42, $130.86(\mathrm{~d}, J=8.7 \mathrm{~Hz}), 129.83(\mathrm{~d}, J=8.7 \mathrm{~Hz}), 129.72(\mathrm{~d}, J=11.2 \mathrm{~Hz}), 129.37,129.18$, $128.90,127.92,127.26(\mathrm{~d}, J=3.7 \mathrm{~Hz}), 125.75,123.59,121.42,118.20,117.61,116.18,116.01,97.43(\mathrm{~d}$, $J=8.7 \mathrm{~Hz}), 91.94(\mathrm{~d}, J=13.7 \mathrm{~Hz}), 64.38,62.00,46.12,36.83(\mathrm{~d}, J=5.0 \mathrm{~Hz}), 35.50(\mathrm{~d}, J=5.0 \mathrm{~Hz})$, 33.07, 28.62, 28.34, $28.22(\mathrm{t}, J=7.5 \mathrm{~Hz}), 26.01,20.47 .{ }^{31} \mathbf{P}$ NMR $\delta 14.07 . \mathbf{I R}\left(\mathrm{NaCl}\right.$, neat, $\left.\mathrm{cm}^{-1}\right): v=$ $3065,2965,2883,2832,1611,1518,1495,1353,1275,1121,888,837,742,710,680 .[\propto]_{\mathbf{D}}^{23}=-9.756(c=$ 0.1225, $\mathrm{CHCl}_{3}$ ). HRMS (EI) m/z: [M-BAr $]^{+}$Calc. for $\mathrm{C}_{34} \mathrm{H}_{36} \mathrm{FIrN}_{2} \mathrm{P}=715.2226$; Found 715.2227. 


\section{References}

${ }^{1}$ Gore, V.; Patel, P.; Chang, C.-T.; Sivendran, S.; Kang, N.; Ouedraogo, Y. P.; Gravel, S.; Powell, W. S.; Rokach, J. J. Med. Chem. 2013, 56, 3725.

${ }^{2}$ S. Kulasegaram, R. J. Kulawiec J. Org. Chem. 1997, 62, 6547-6561.

${ }^{3}$ H. I. Duynstee, M. C. de Koning, G. A. van der Marel, J. H. van Boom, Tetrahedron, 1999, 55, 9881.

${ }^{4}$ B. C. Söederberg, B. Åakermark, S. S. Hall, J. Org. Chem. 1988, 53, 2925-2937.

${ }^{5}$ C. Petrier, K. Suslick, Ultrason. Sonochem. 2000, 7, 53-61.

${ }^{6}$ N. Takenaka, J. Chen, B. Captain, R. S. Sarangthem, A. Chandrakumar, J. Am. Chem. Soc. 2010, 132, 4536-4537.

${ }^{7}$ W. A. Remers, G. J. Gibs, C. Pidacks, M. J. Weiss, J. Am. Chem. Soc. 1967, 89, 5513-5514.

${ }^{8}$ W. A. Remers, G. J. Gibs, C. Pidacks, M. J. Weiss, J. Org. Chem. 1971, 36, 279.

${ }^{9}$ Bailey, A. S.; Vandrevala, M. H., J. Chem. Soc. Perk. T. 1 1980, 1512-1515.

${ }^{10}$ A. J. Birch, A. R. Murray, H. Smith, J. Chem. Soc. 1951, 1945-1950.

${ }^{11}$ D. H. Hua, W. Y. Gung, R. A. Ostrander, F. Takusagawa, J. Org. Chem., 1987, 52, 2509.

${ }^{12}$ A. S. Pilcher, P. DeShong, J. Org. Chem. 1993, 58, 5130-5134.

${ }^{13}$ D. L. Boger, J. Org. Chem. 1985, 50, 5377-5379.

${ }^{14}$ M. G. Donahue, D. J. Hart, Can. J. Chem. 2004, 82, 314-317.

${ }^{15}$ P. A. Grieco, J. J. Nunes, M. D. Gaul, J. Am. Chem. Soc., 1990, 112, 4595-4596.

${ }^{16}$ F. Fringuelli, R. Girotti, F. Pizzo, L. Vaccaro, Org. Lett. 2006, 8, 2487-2489.

${ }^{17}$ G. Hilt, J. Janikowski, W. Hess, Angew. Chem. Int. Ed. 2006, 45, 5204-5206.

${ }_{18}$ A. Paptchikhine, K. Itto, P. G. Andersson, Chem. Commun. 2011, 47, 3989-3991.

${ }^{19}$ R. A. Benkeser, J. A. Laugal, A. Rappa, US4533752A, 1985.

${ }^{20}$ A-D. Fortineau, WO2008053148A1, 2008.

${ }^{21}$ E. Taarning, R. Madsen, Chem. Eur. J. 2008, 14, 5638-5644.

${ }^{22}$ M. S. R. Murthy, N. A. E. Steenaart, R. A. Johnson, G. C. Shore, WO2001055131A2, 2001.

${ }^{23}$ C. K. Lee, I.-S. H. Lee, W. E. Noland, Heterocycles 2007, 71, 419-428.

${ }^{24}$ P. Kaukoranta, M. Engman, C. Hedberg, J. Bergquist, P. G. Andersson, Adv. Synth. Catal. 2008, 350, 1168-1176. 


\section{NMR spectra}

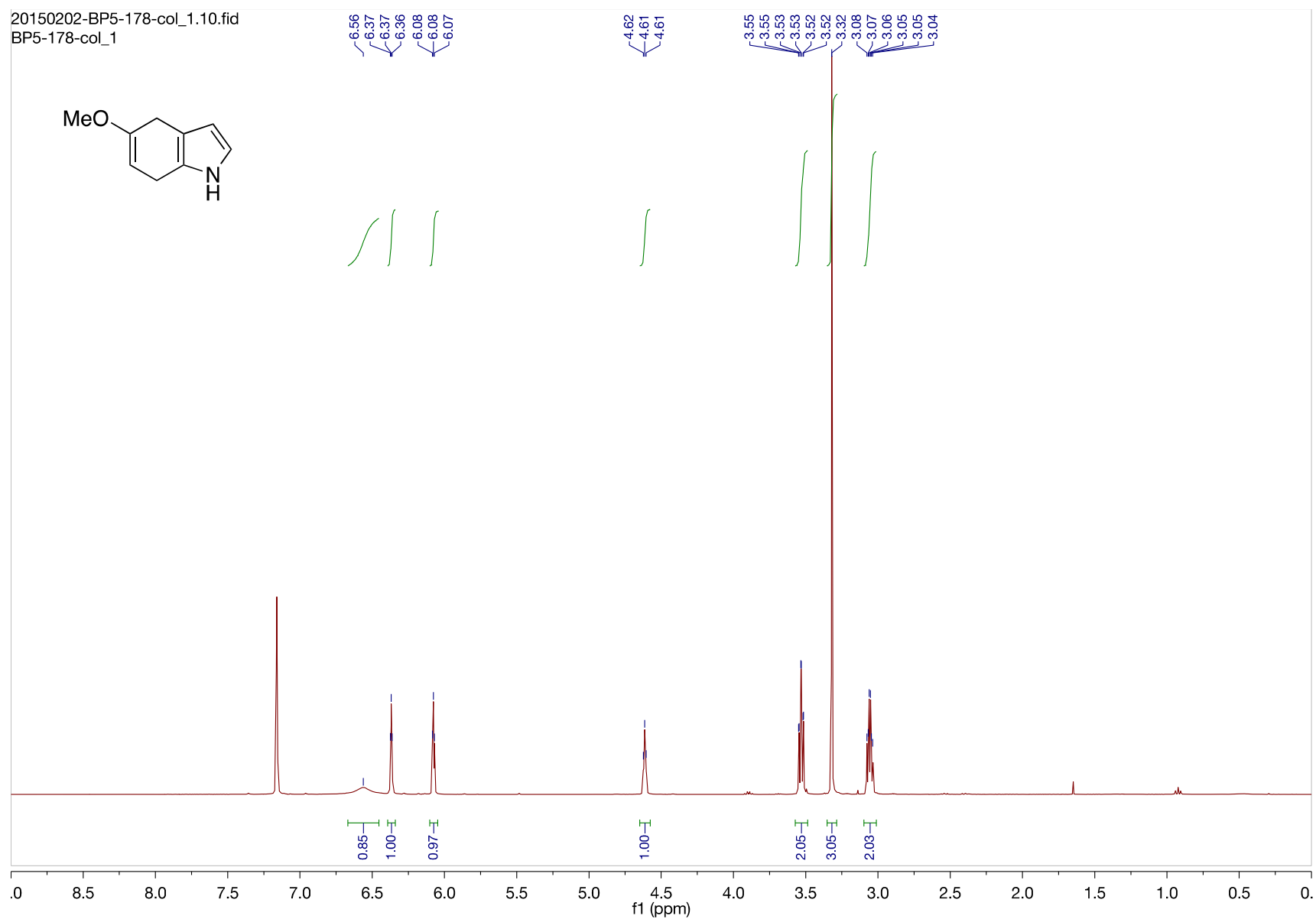

20150203-BP5-178-col_13C.10.fid BP5-178-col_13C

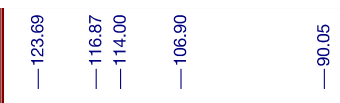

i̊.

$\mathrm{MeO}$<smiles>CC1=CCc2[nH]ccc2C1</smiles>

$\mathrm{H}$ 


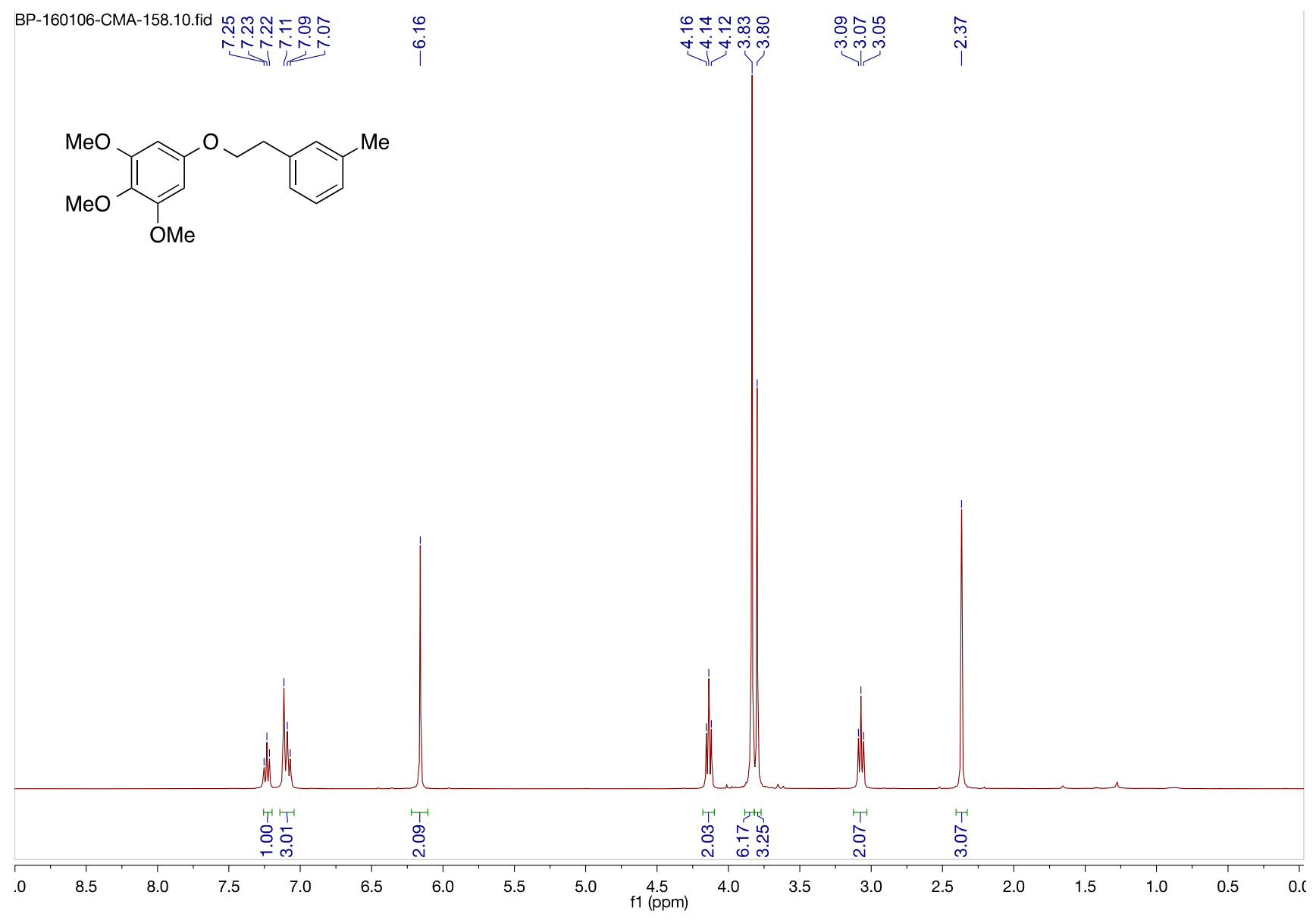<smiles>COc1cc(OCCc2cccc(C)c2)cc(OC)c1OC</smiles>

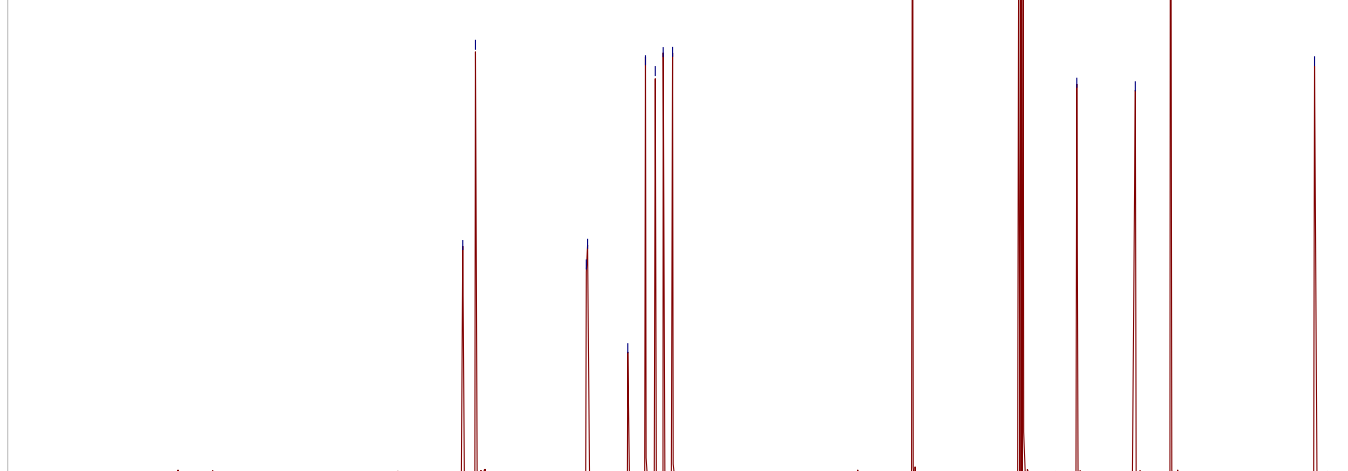

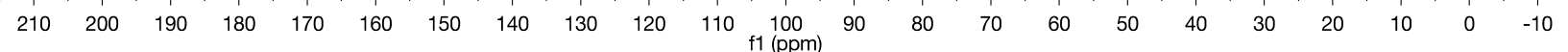




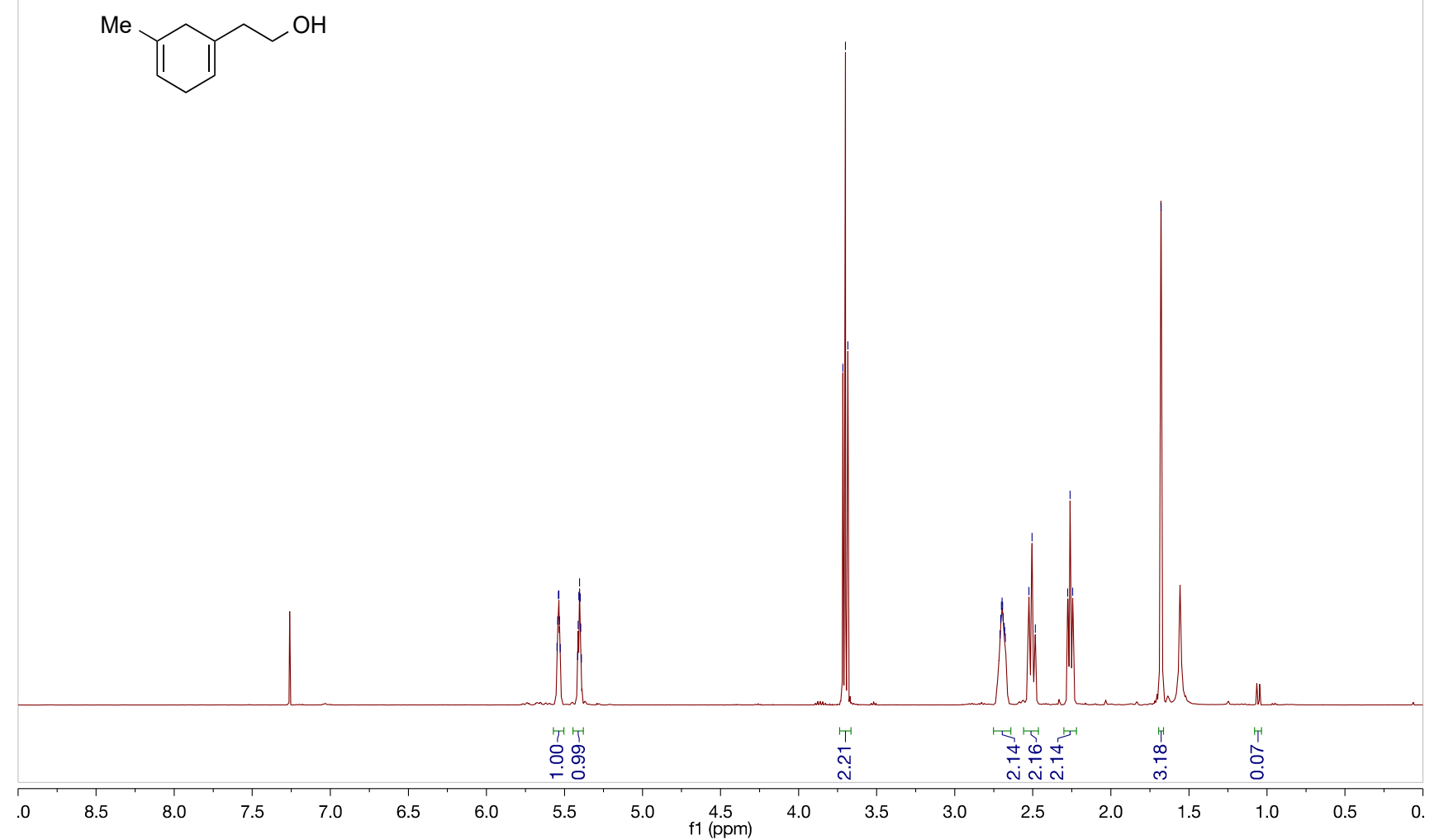

$\mathrm{Me}$ 

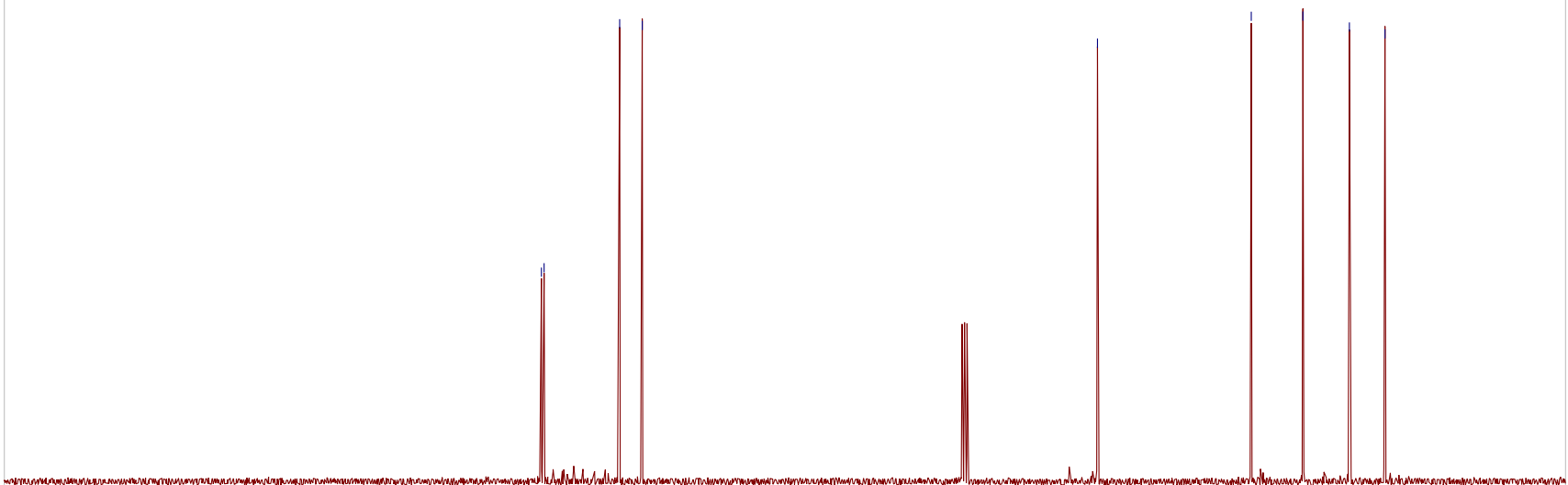


CMA-151211-CMA162_A.10.fid

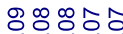

ن

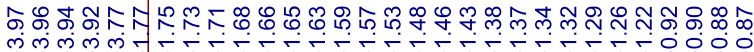

$\mathrm{MeO}$<smiles>COc1cccc(OCCC2CCCC(C)C2)c1</smiles>

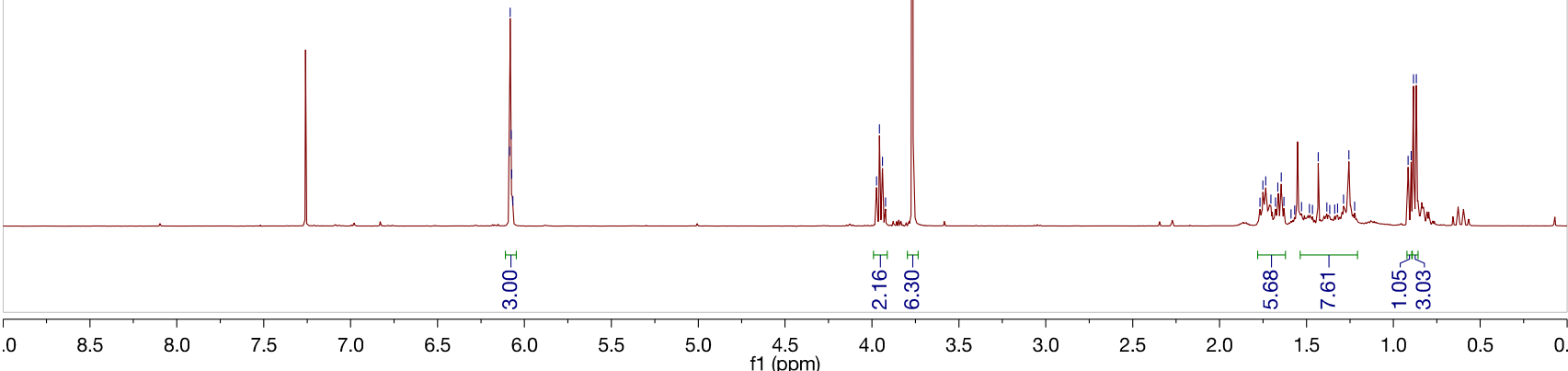

CMA-151211-CMA162_A.11.fid שூ

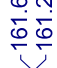

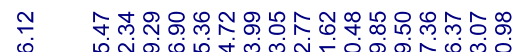

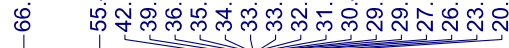

$\mathrm{MeO}$<smiles>COc1cccc(OCCC2CCCC(C)C2)c1</smiles>

$\mathrm{OMe}$
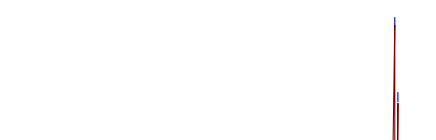
CMA-151215-CMA2-5.10.fid

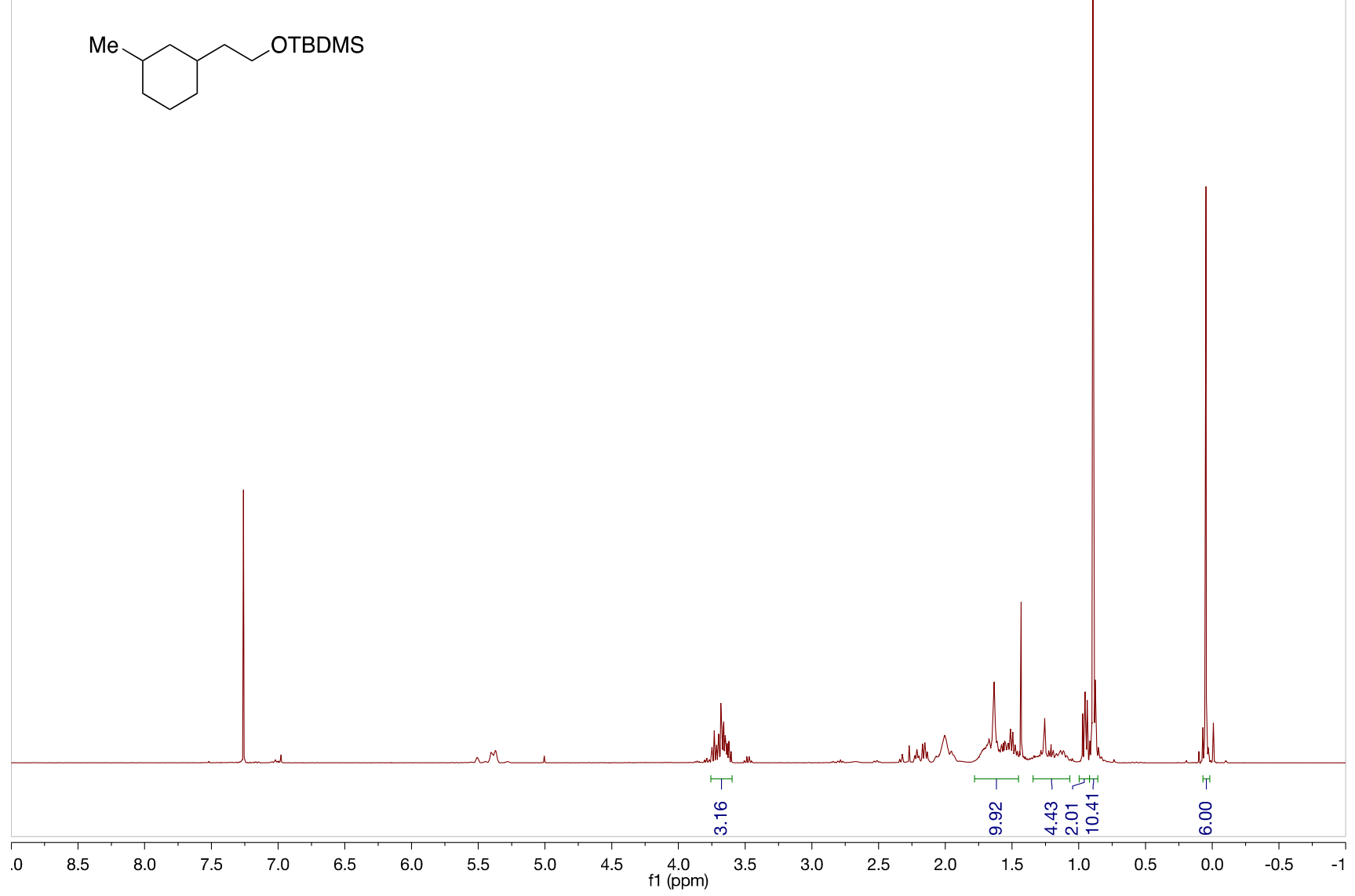

CMA-151218-CMA2-5c.10.fid

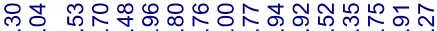

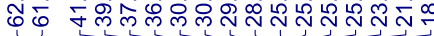

OTBDMS

(1)

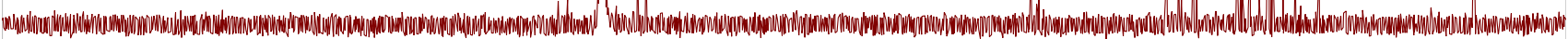

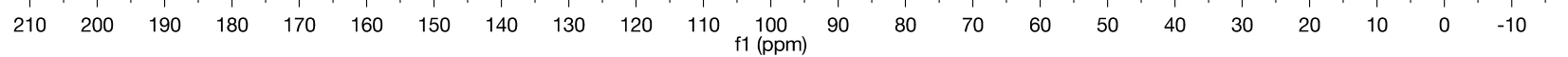


<smiles>C[C@H]1CCC=C(CCCO)C1</smiles>

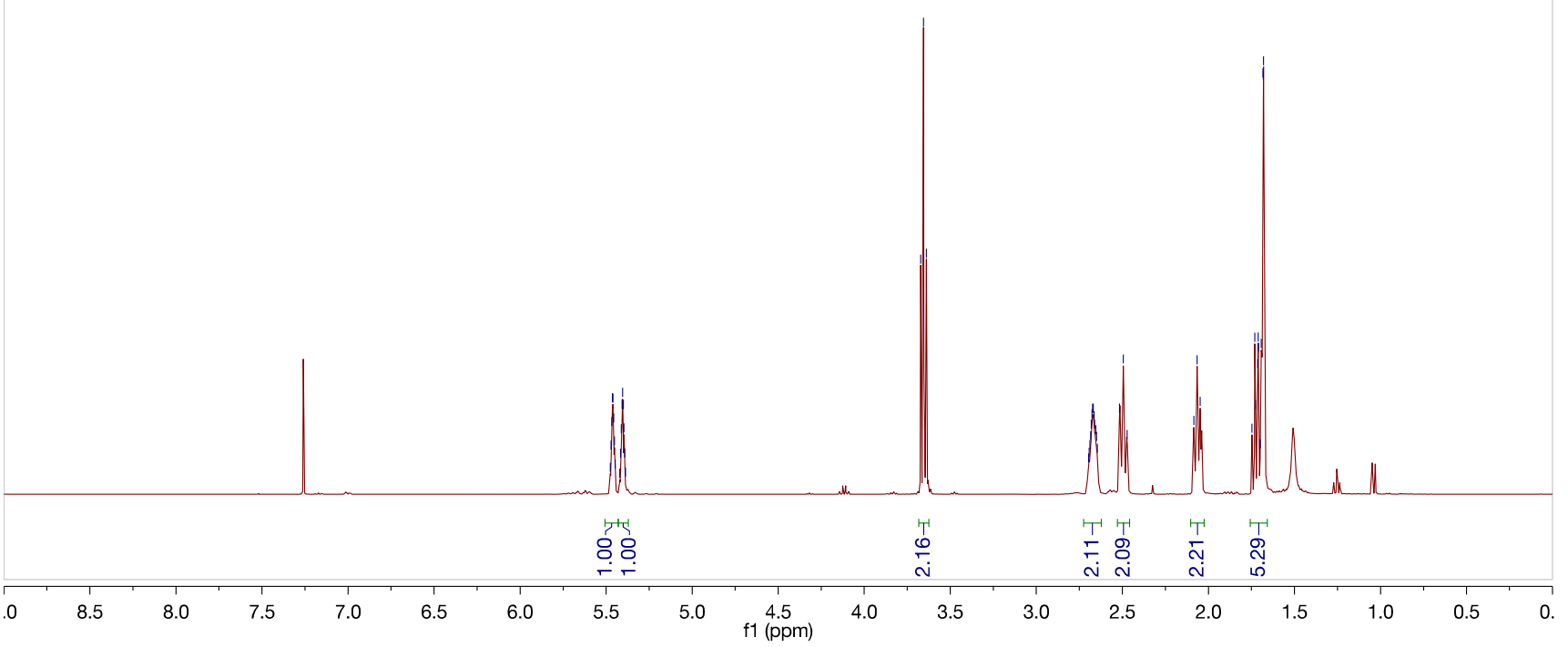

CMA-151201-CMA186-f24-27.11.fid

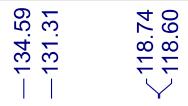

๑

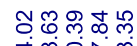

मेल्लंज्ञ

$\mathrm{MPOH}_{\mathrm{OH}}^{\mathrm{Me}}$

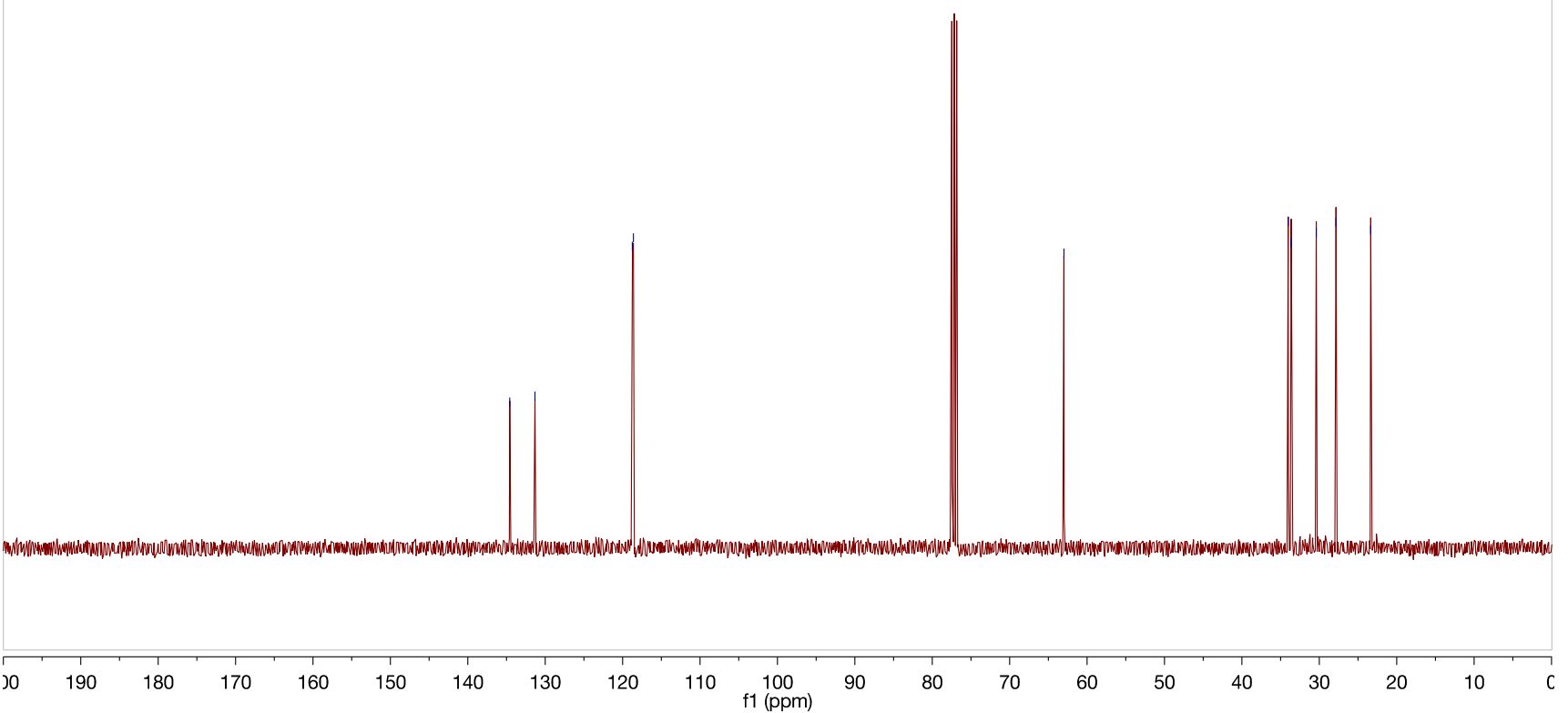


MeTBDMS

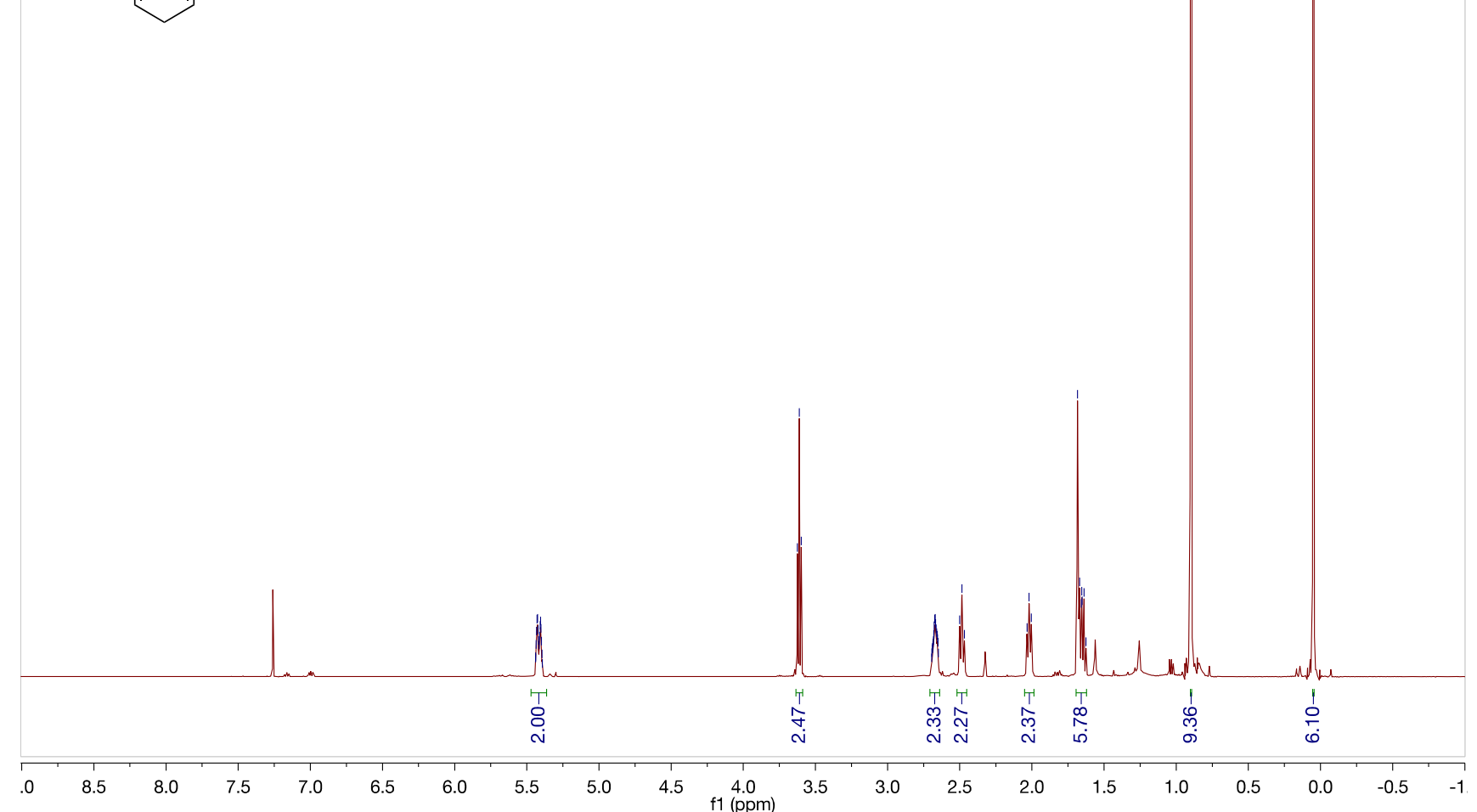

CMA188-f2-3.2.fid

$13 \mathrm{C}$

站辛

울

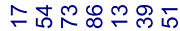

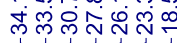

MetBDMS
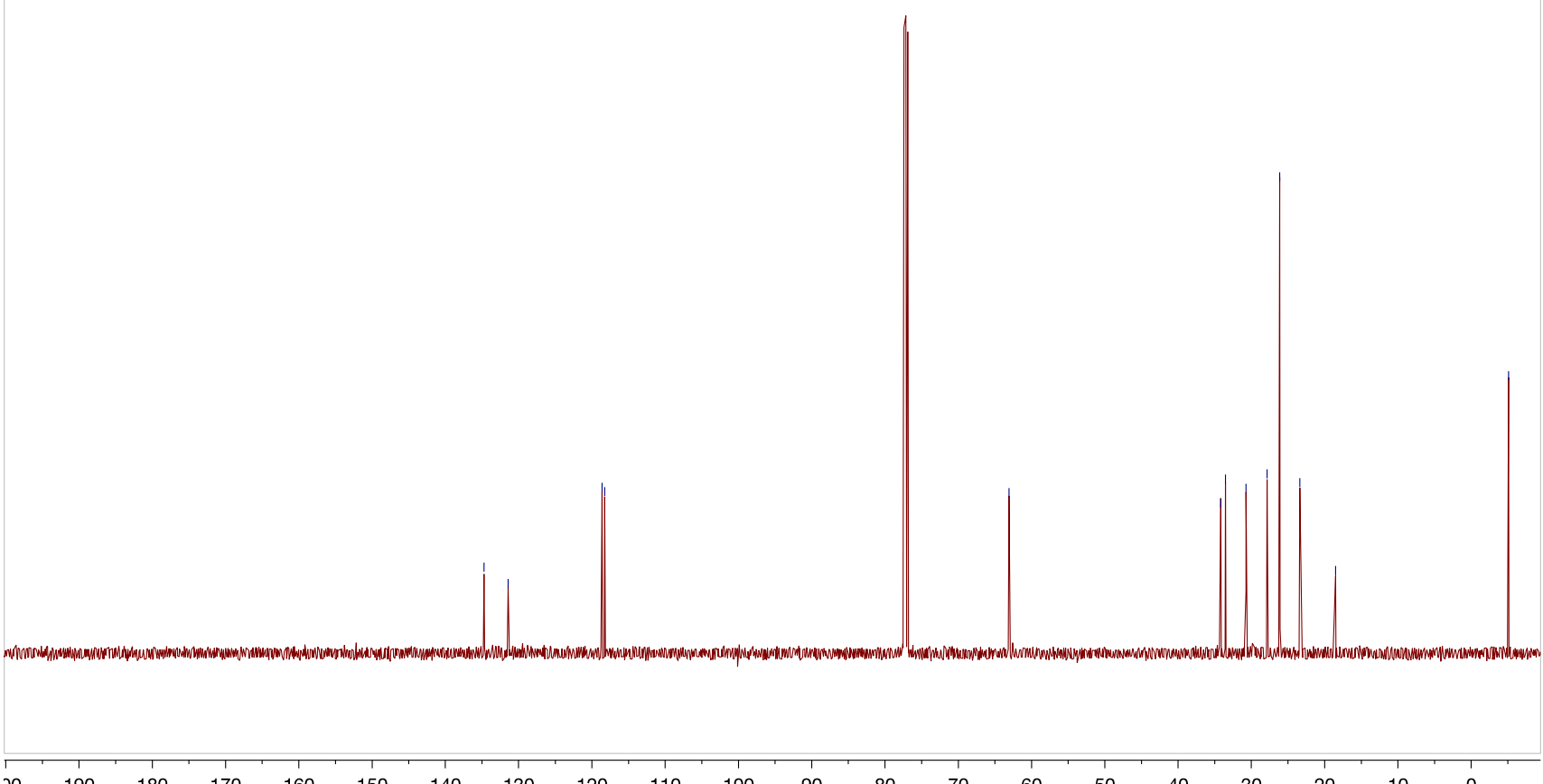

180

160

150

$140 \quad 130$

120

10090

$80 \quad 70$

$60 \quad 50$

$40 \quad 30$

$20 \quad 10$ 


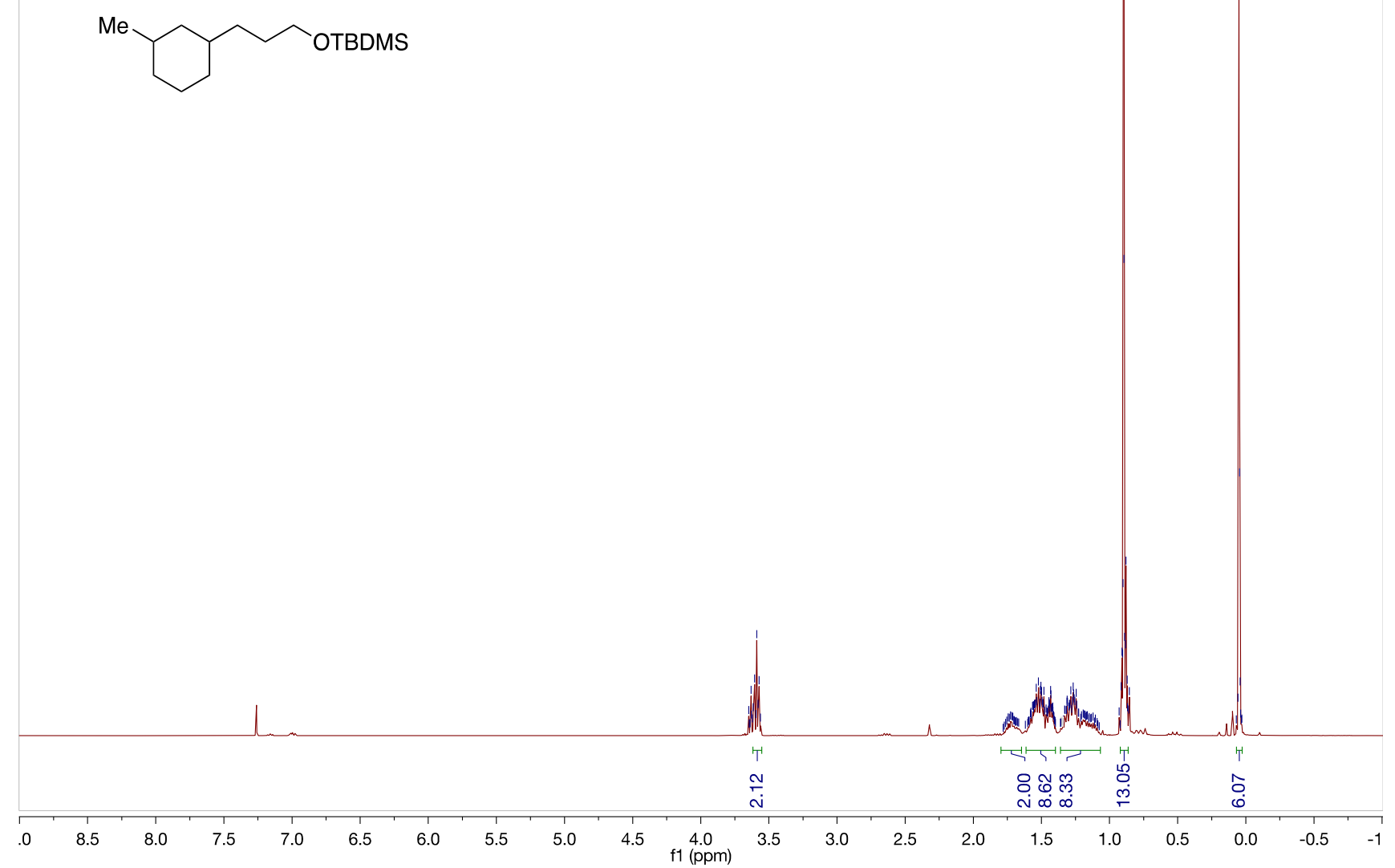<smiles>CCCCOCCCC1CCCC(C)C1</smiles>

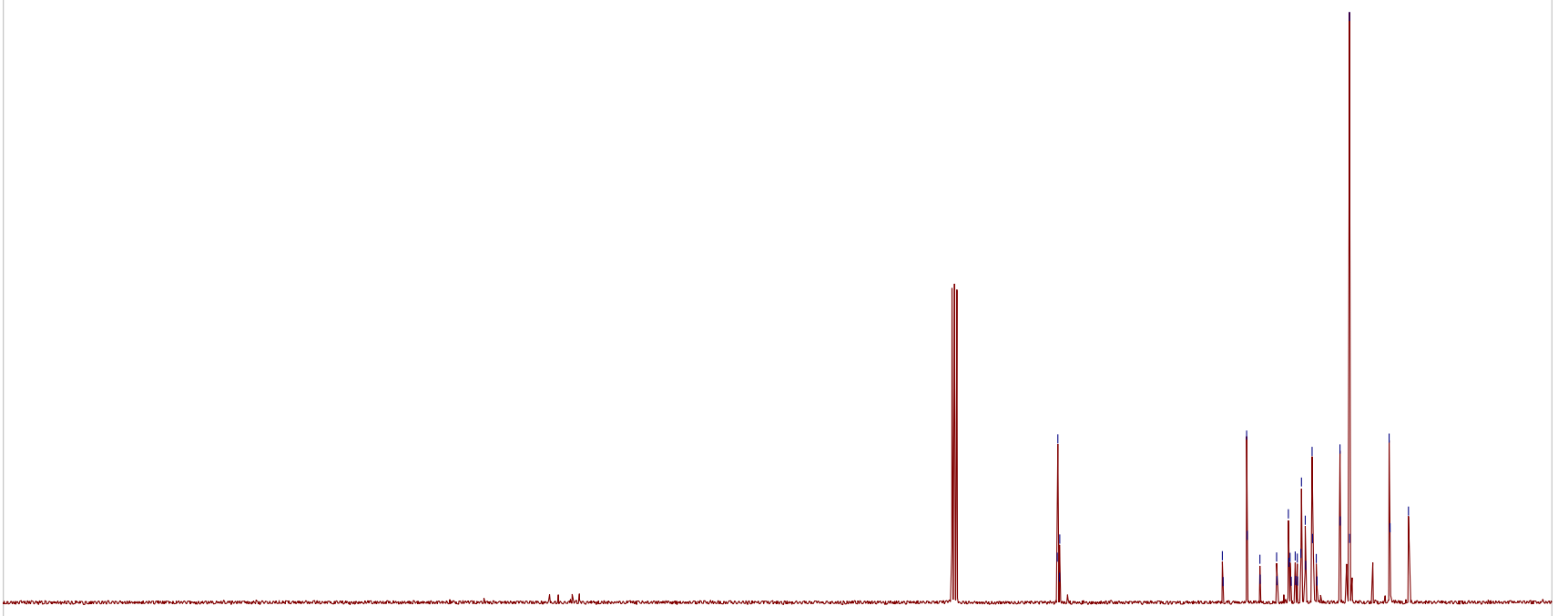


<smiles>CC1CCCC(CCCO)C1</smiles>

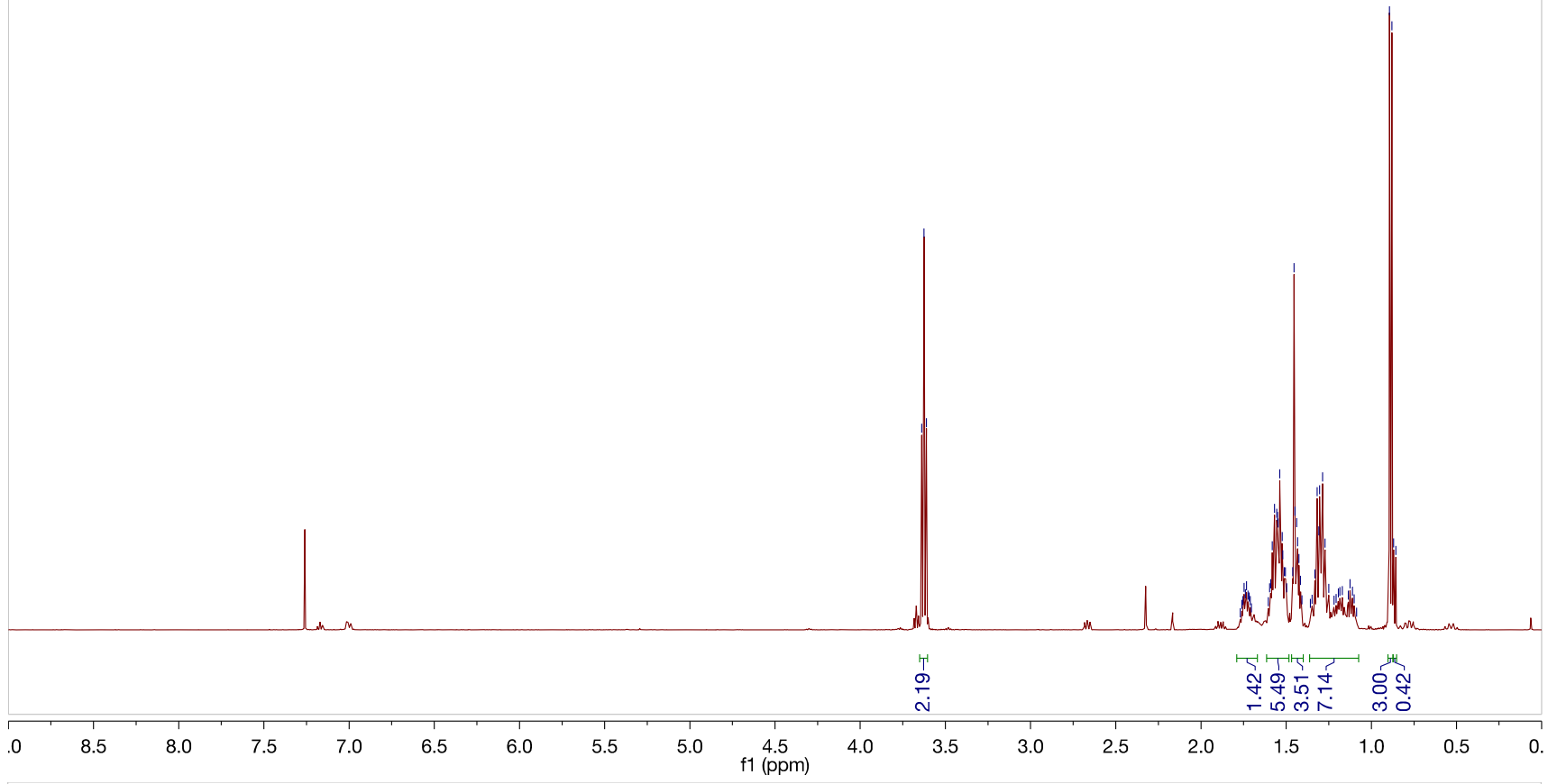

CMA191.2.fid

L<smiles>CC1CCCC(CCCO)C1</smiles>

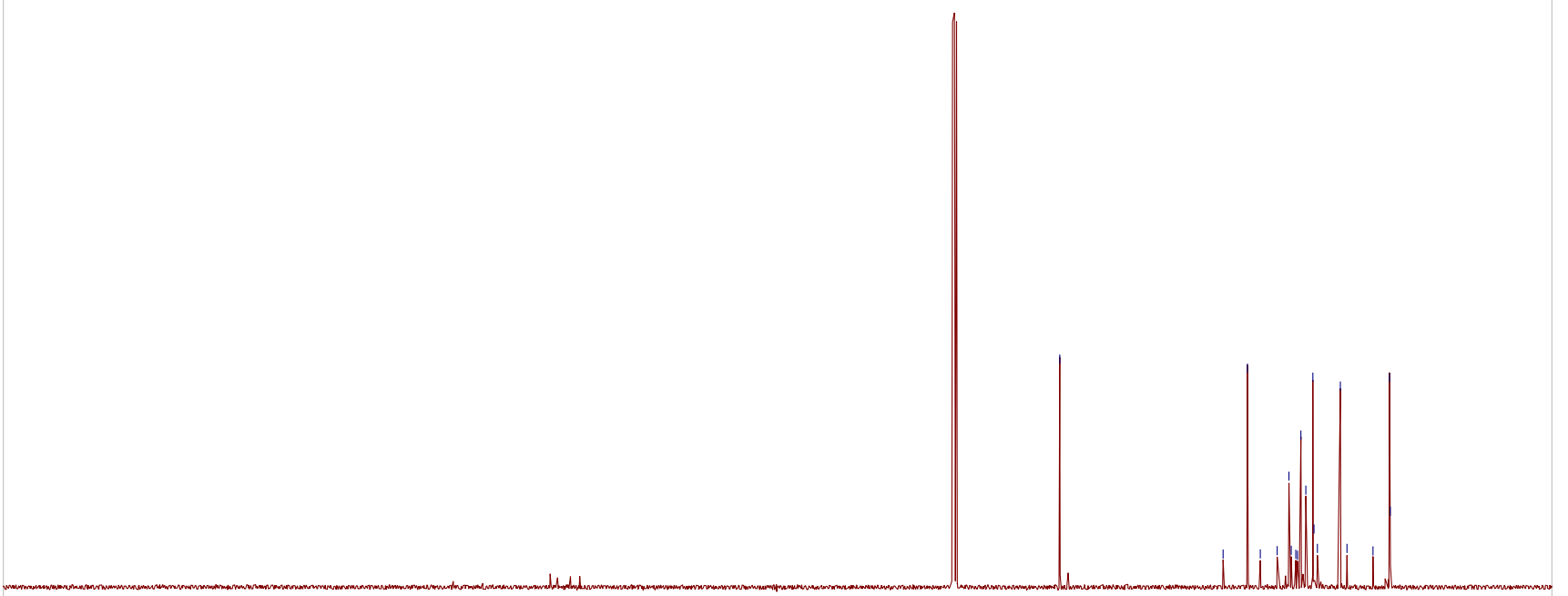


CMA-150113-CMA67_C2bis.10.fid

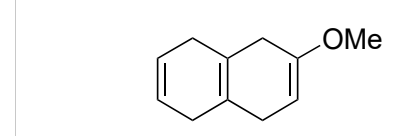

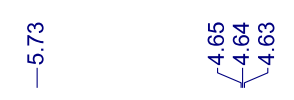

$\stackrel{\circ}{i}$
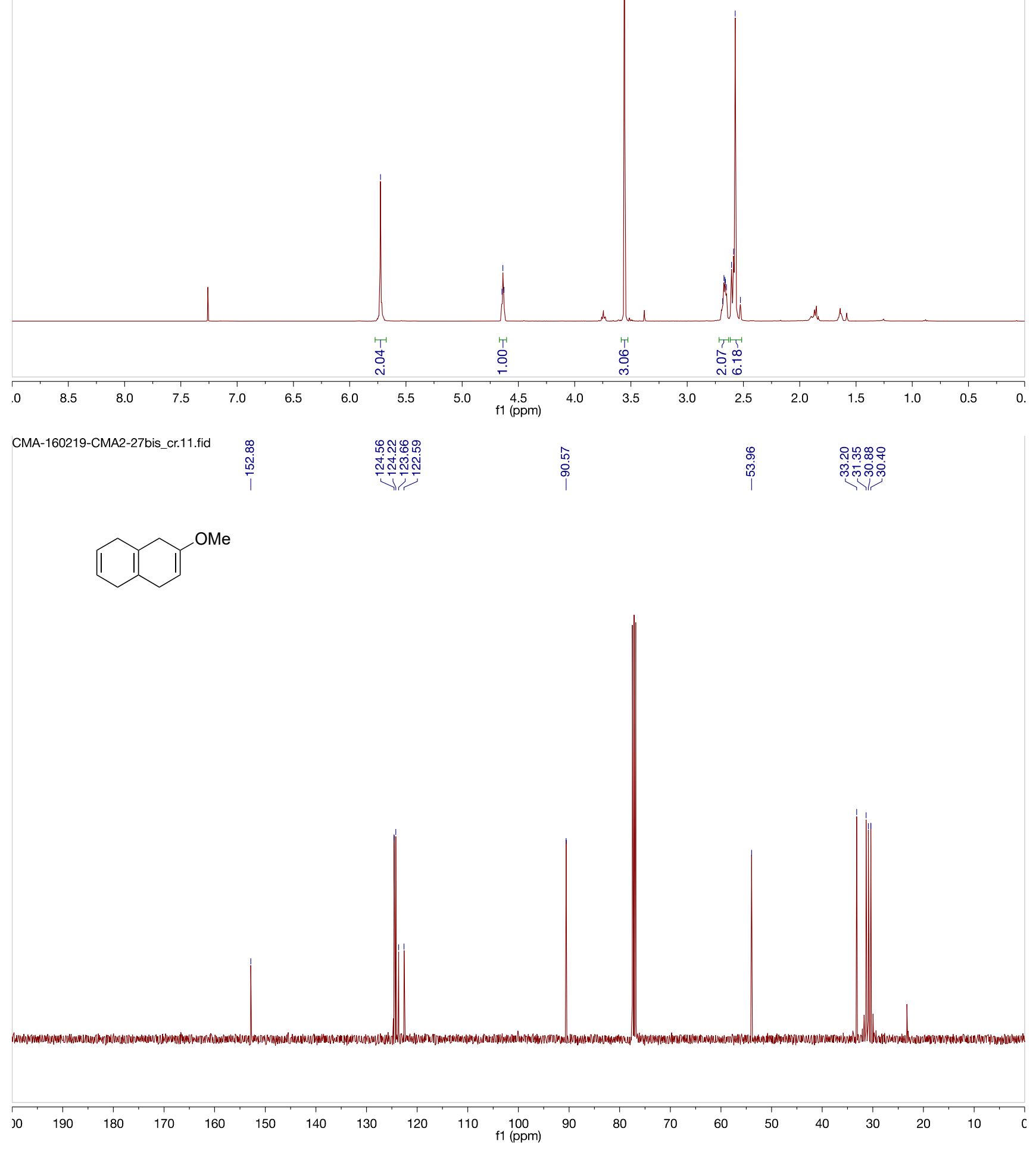

S44 

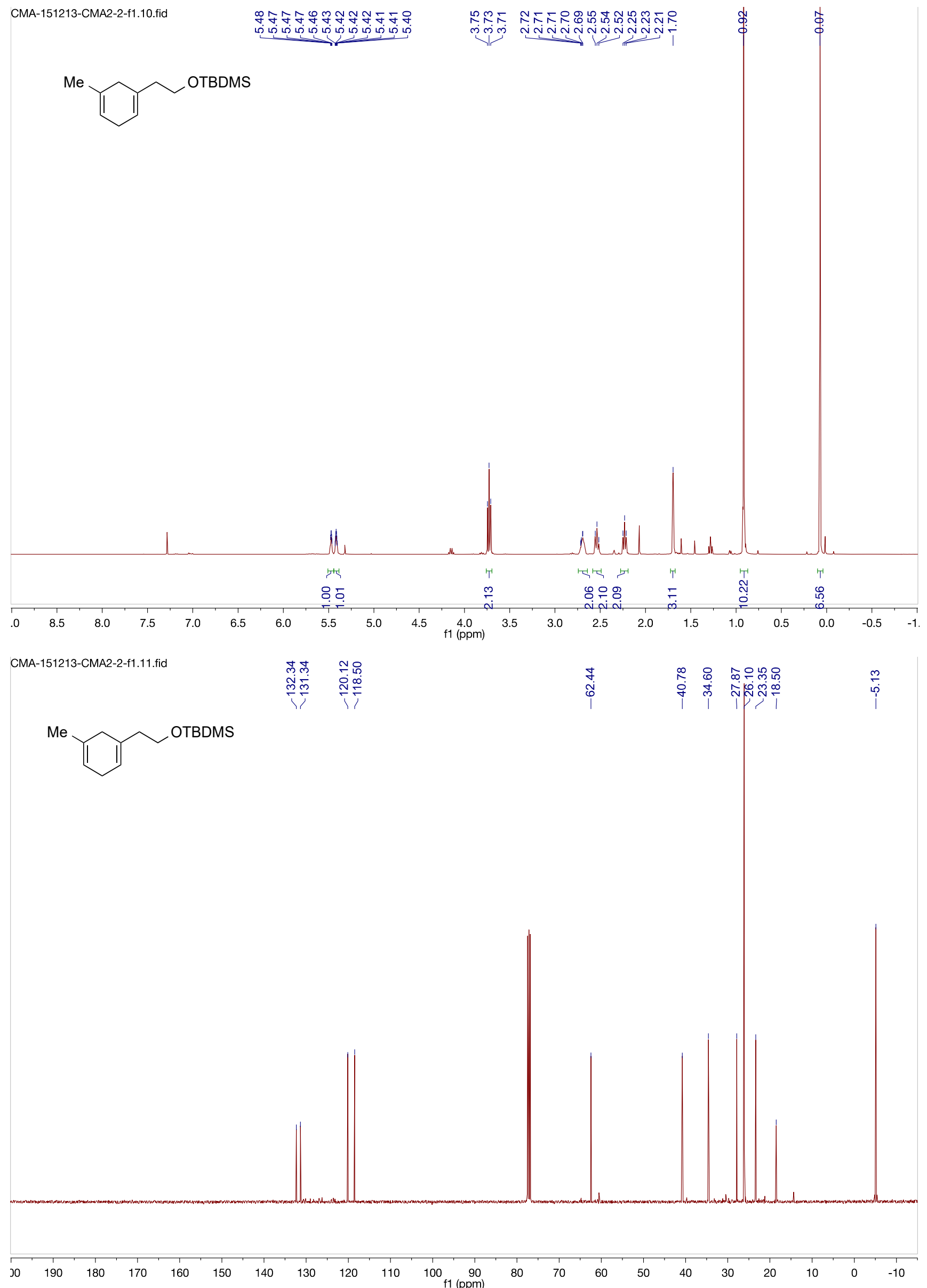
CMA-151216-CMA2-7.10.fid

$\underbrace{8000}$

in

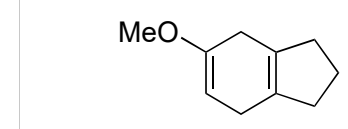


CMA-151217-CMA2-10.10.fid

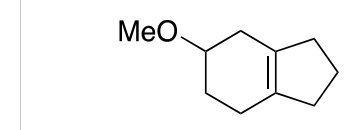

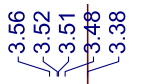

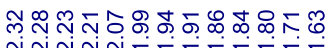

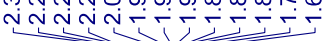
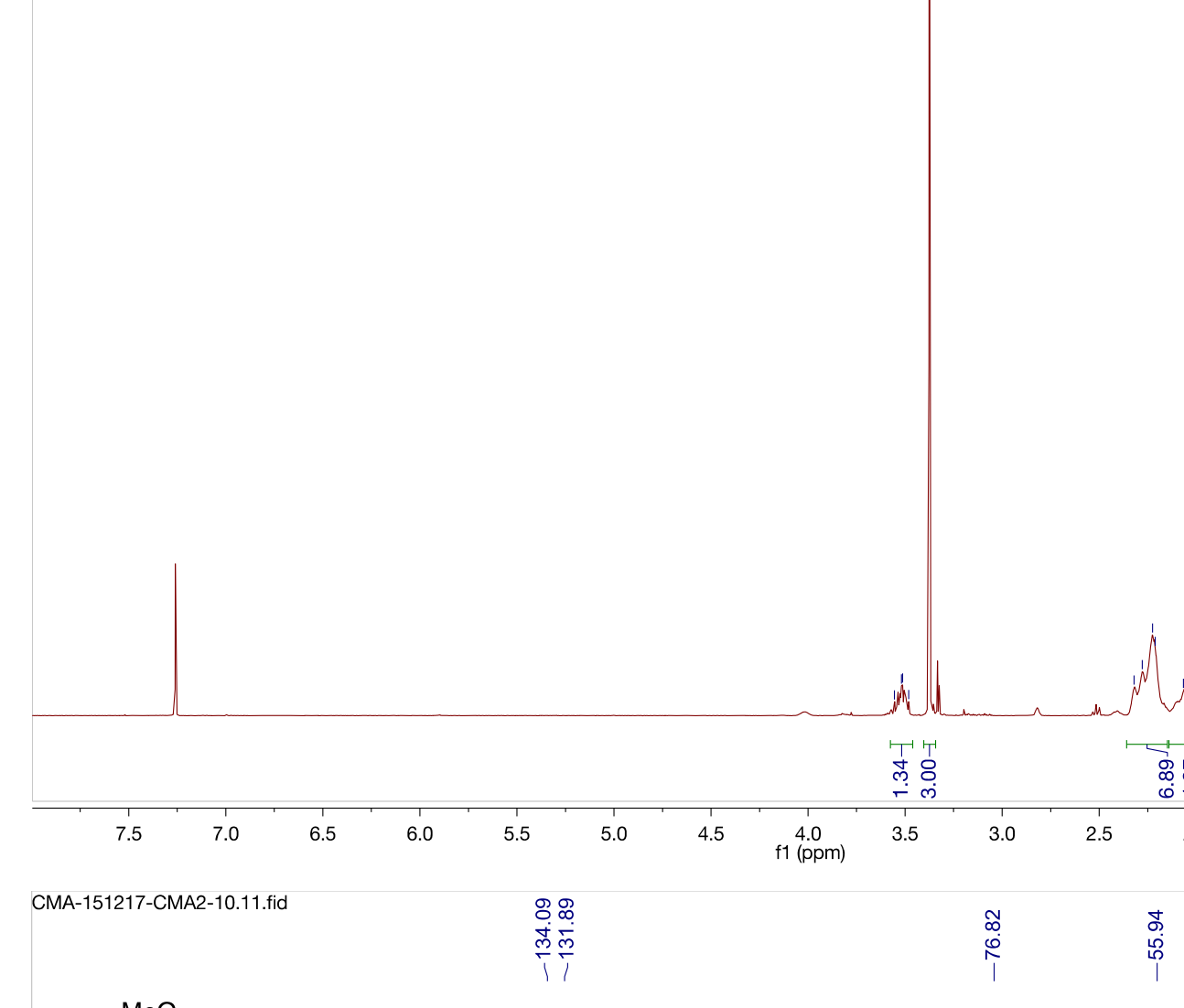

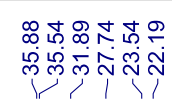
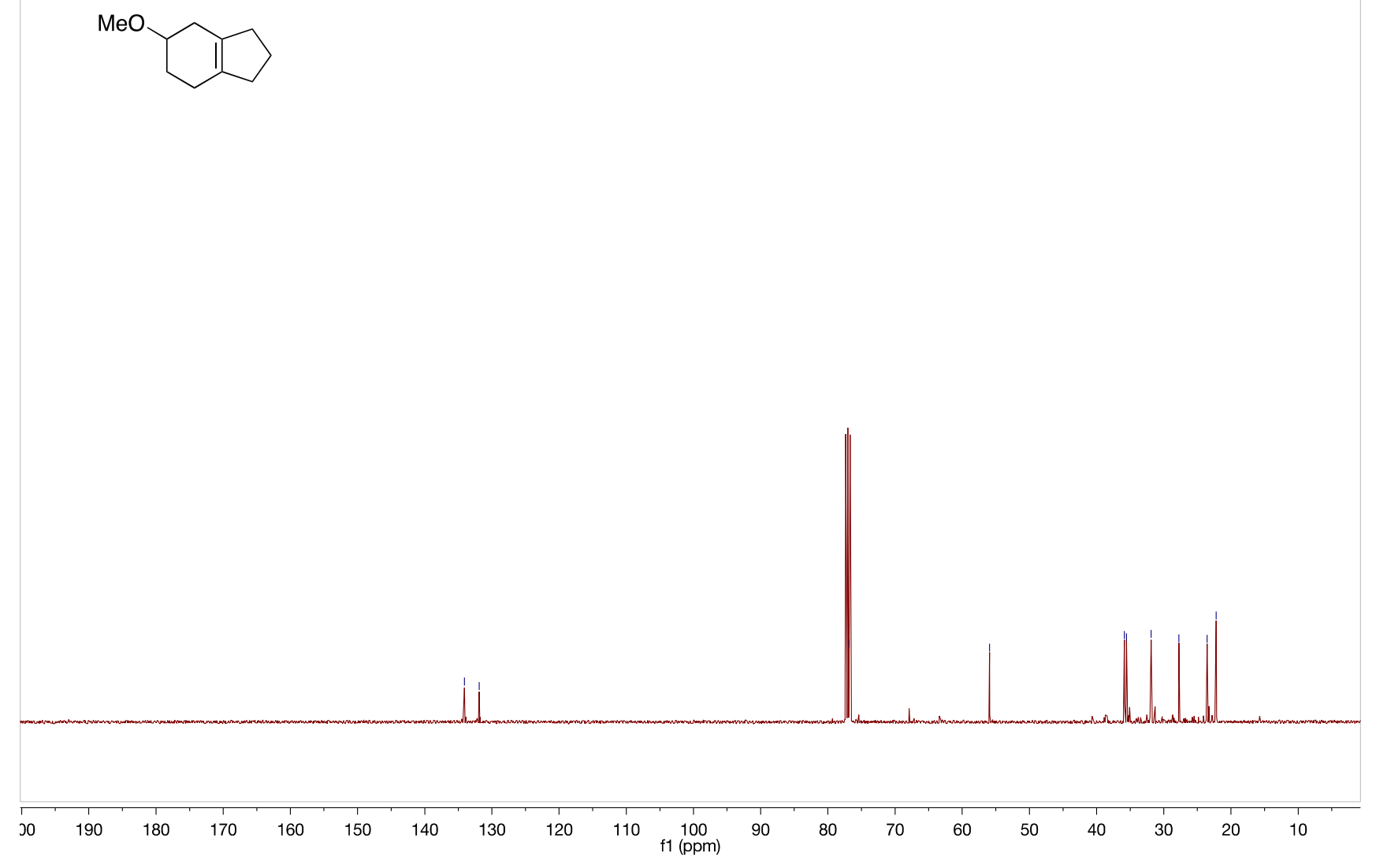

S47 

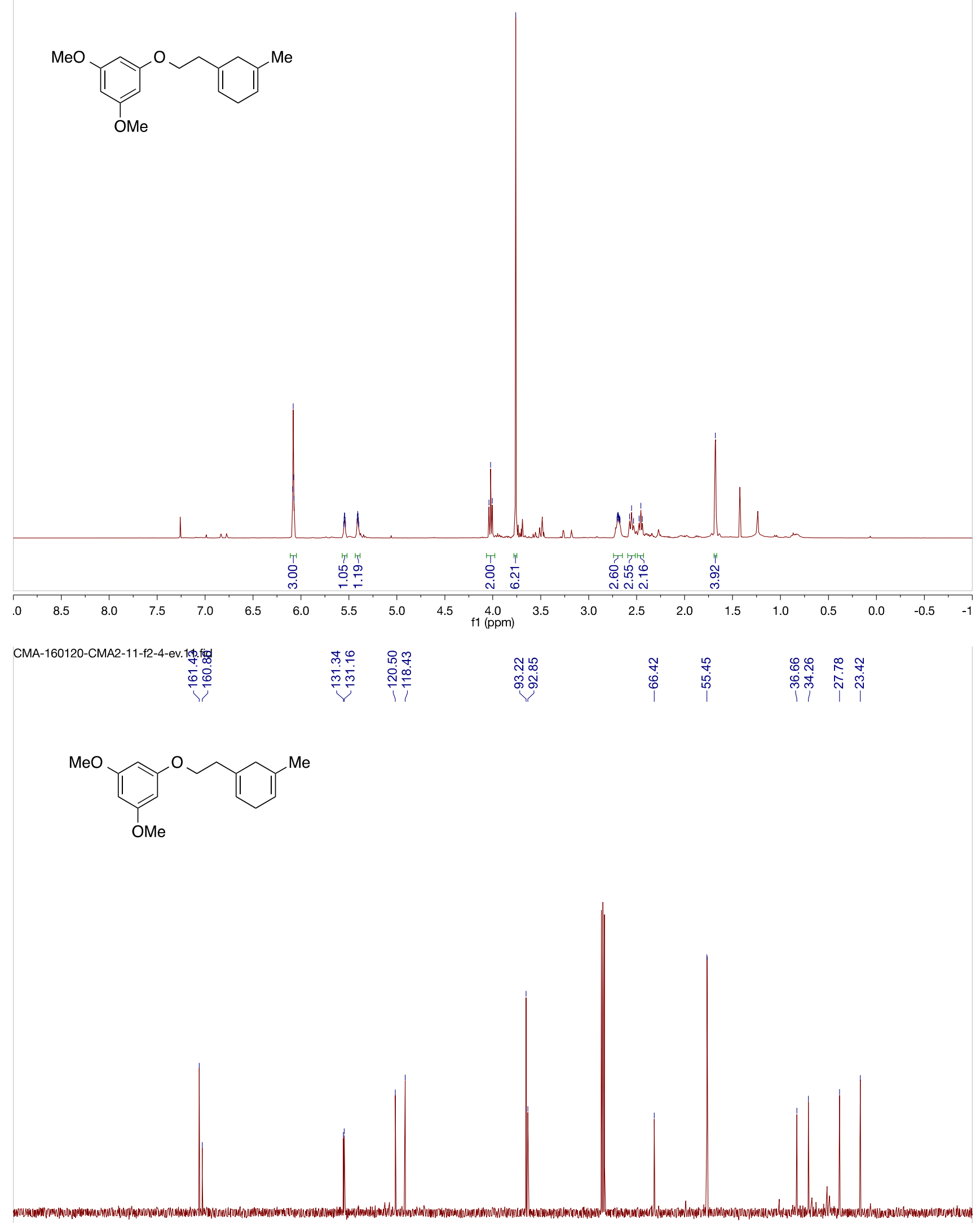


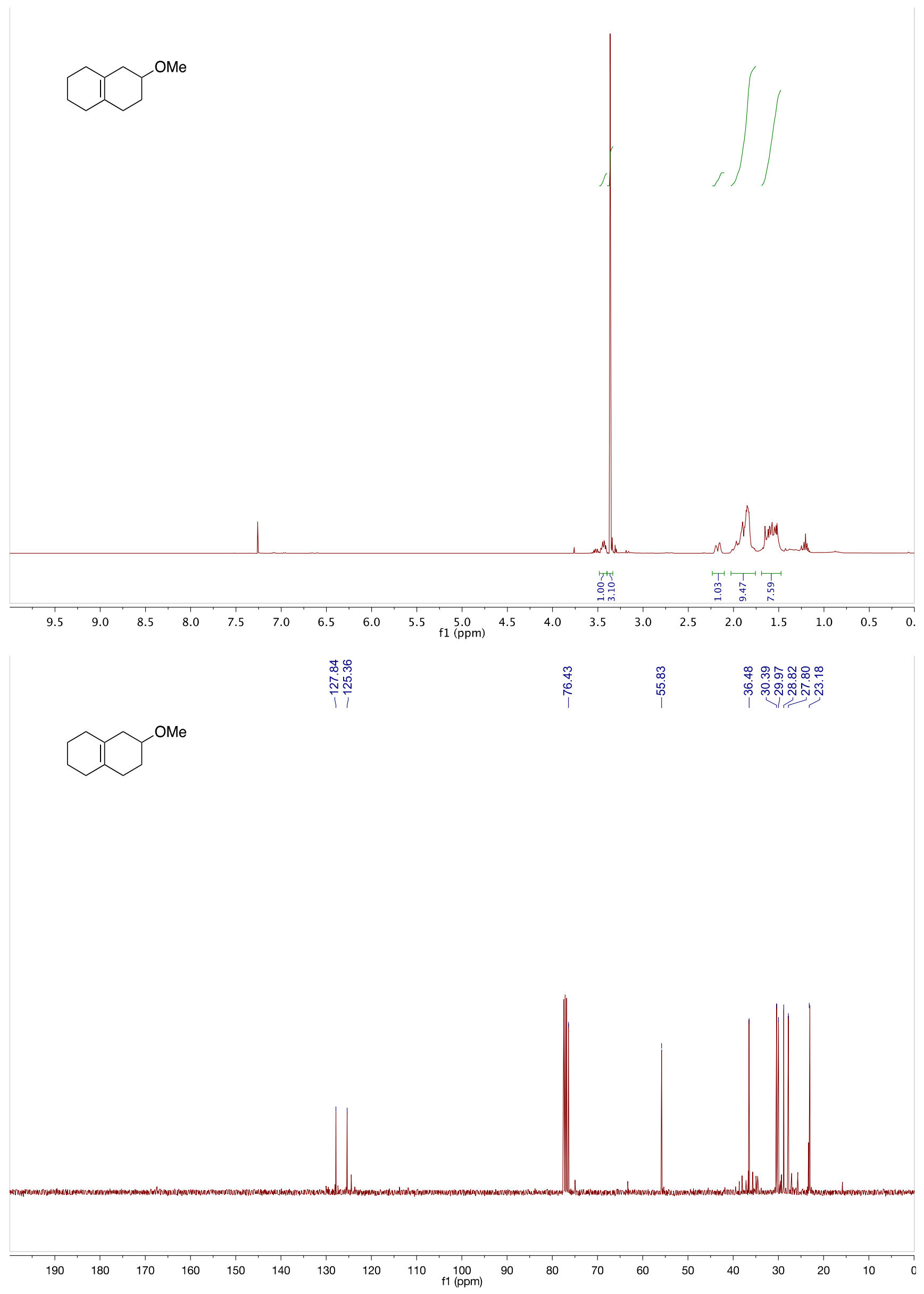

S49 


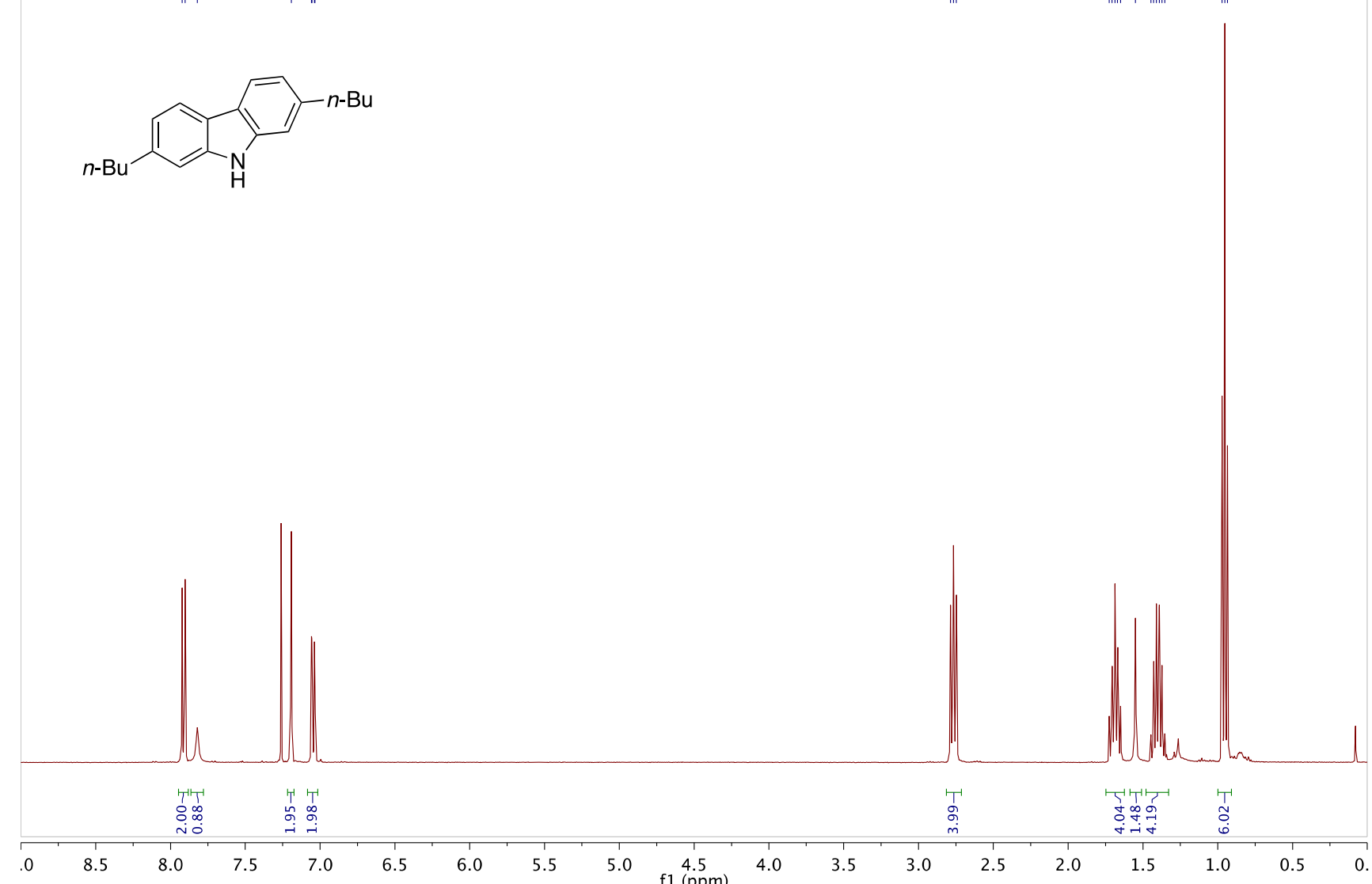

PIV-WR-A-E57-16072015/11 PIV-WR-A-E57pure

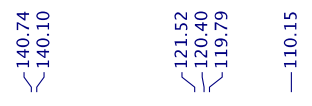<smiles>CCCCCCCCCCCCCCCc1ccc2c(c1)[nH]c1cc(CCCC)ccc12</smiles>

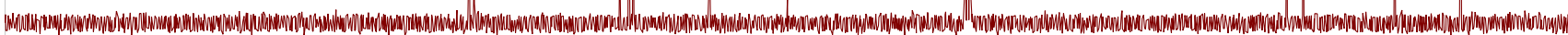


<smiles>CC1=CCC=C(CC(=O)O)C1</smiles>

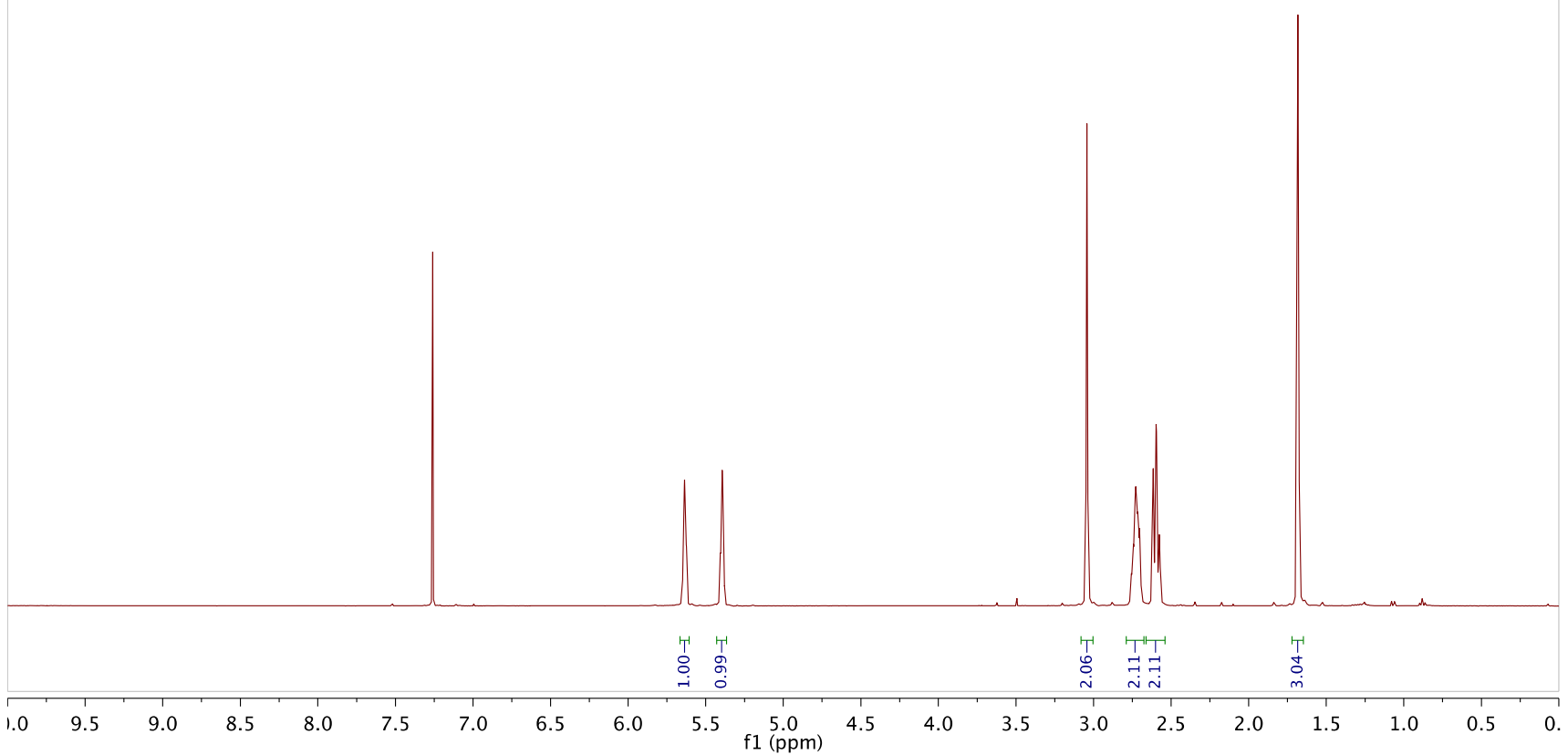

20150505-PIVWR=A-E1/11 PIVWR-A-E1 after ${ }^{\infty}$ *ecrystallization<smiles>CC1=CCC=C(CC(=O)O)C1</smiles>

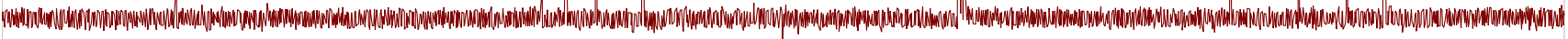

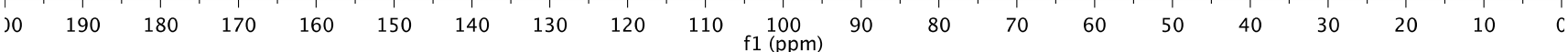


<smiles>CCCCCCCCCCCCCCCCCCCCCCCCC</smiles>

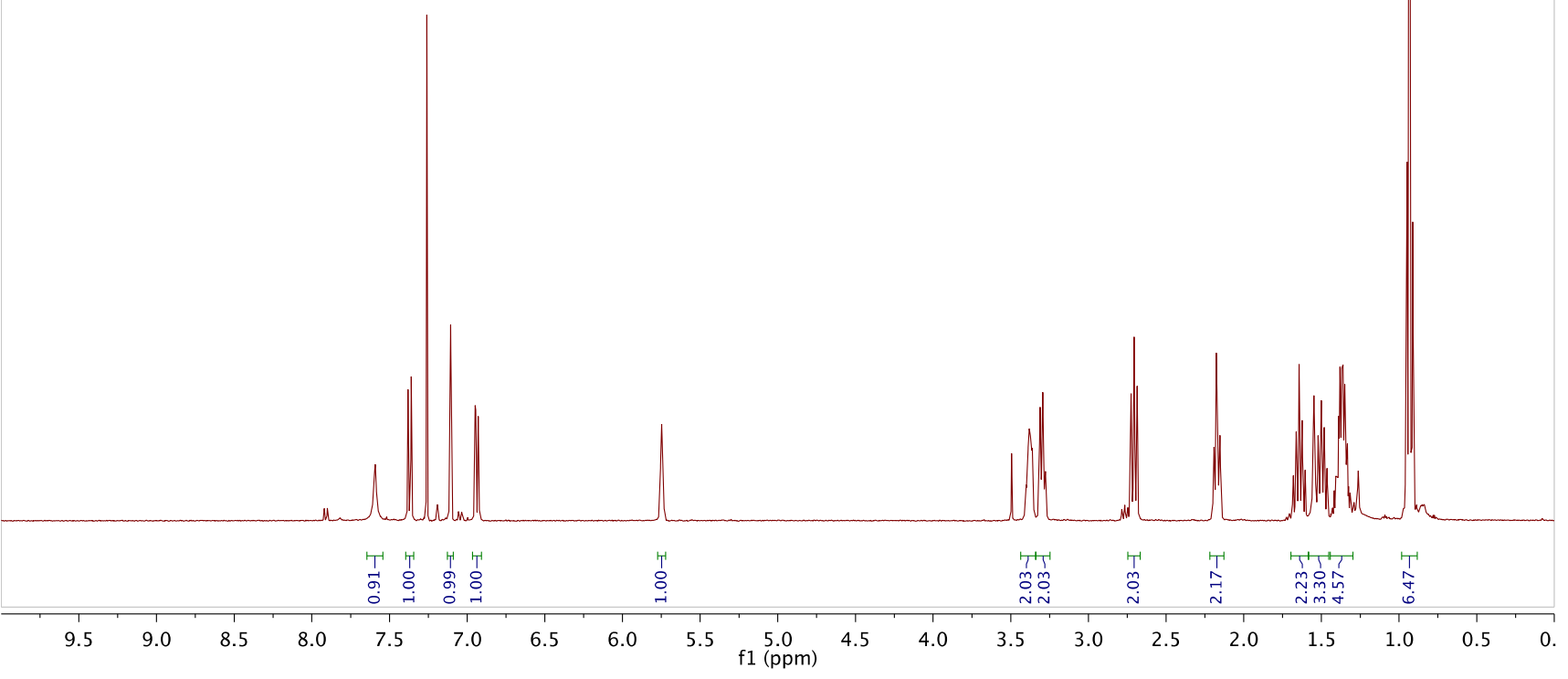

PIV-WR-A-E59-21072015/21 PIV-WR-A-E59 pure

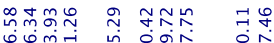

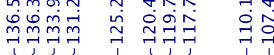<smiles>CCCCCCCCCCCCCCCCCCCCCCCCCCC</smiles>

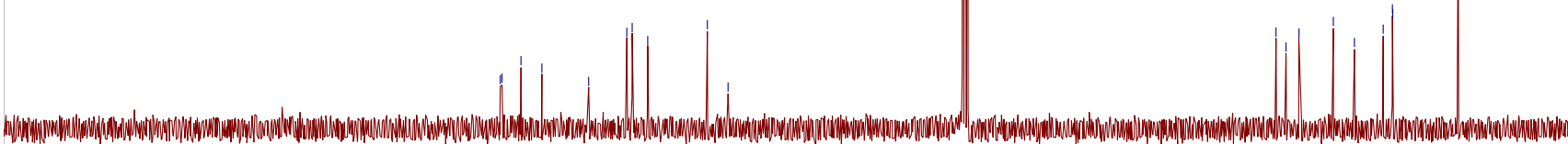

\begin{tabular}{|c|c|c|c|c|c|c|c|c|c|c|c|c|c|c|c|c|c|}
\hline 190 & 180 & 170 & 160 & 150 & 140 & 130 & 120 & 110 & 100 & 90 & 80 & 70 & 60 & 50 & 40 & 30 & 20 \\
\hline
\end{tabular}




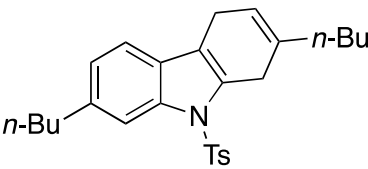

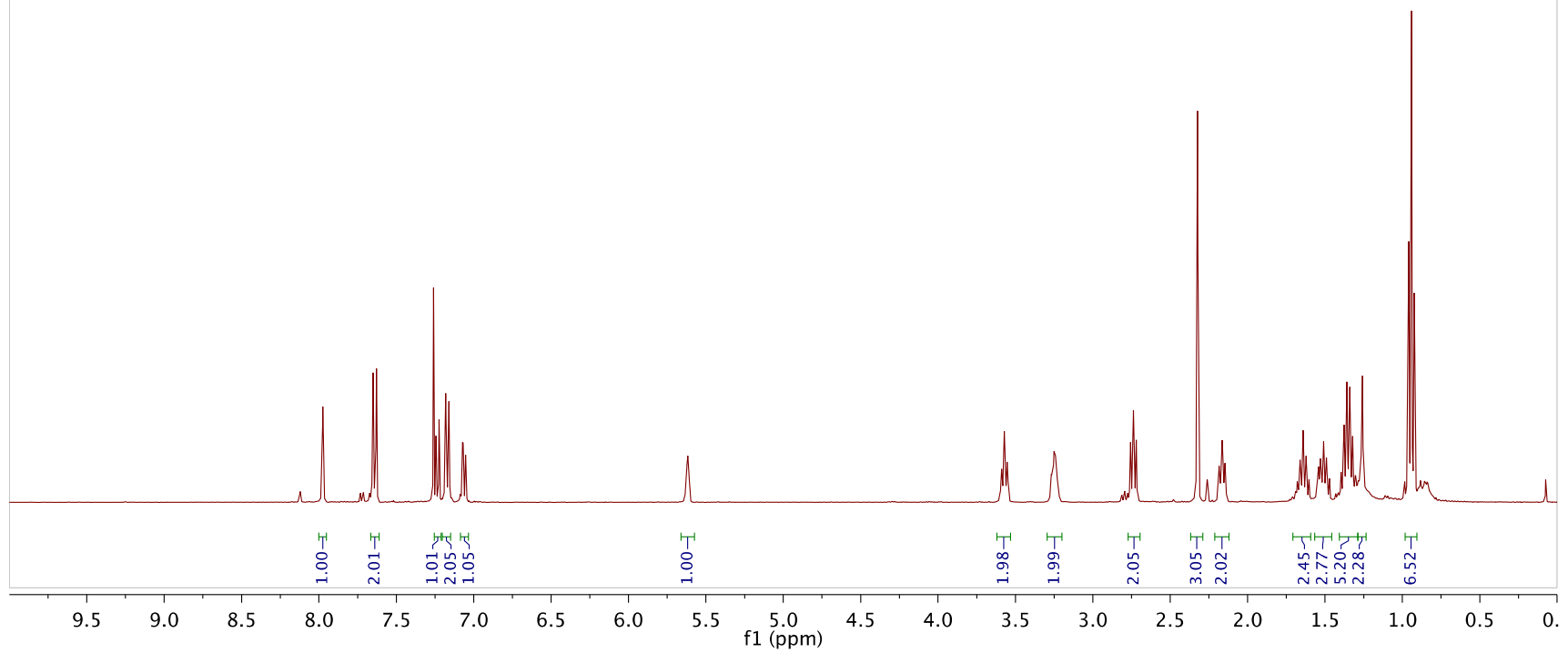

PIV-WR-A-E112-23112015/10 PIV-WR-A-E112-first fraction

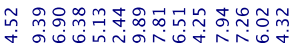

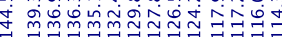

।

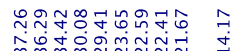
mmm

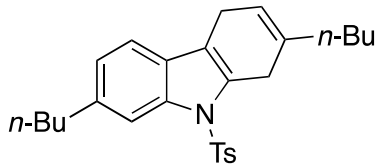

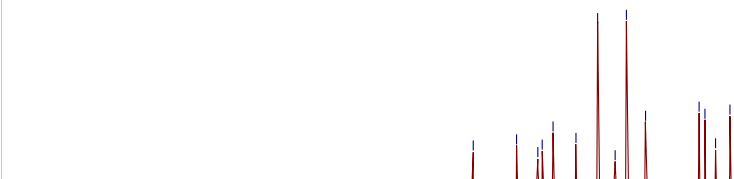


20151124-PIV-WR-A-E114/10

PIV-WR-A-E114 pure

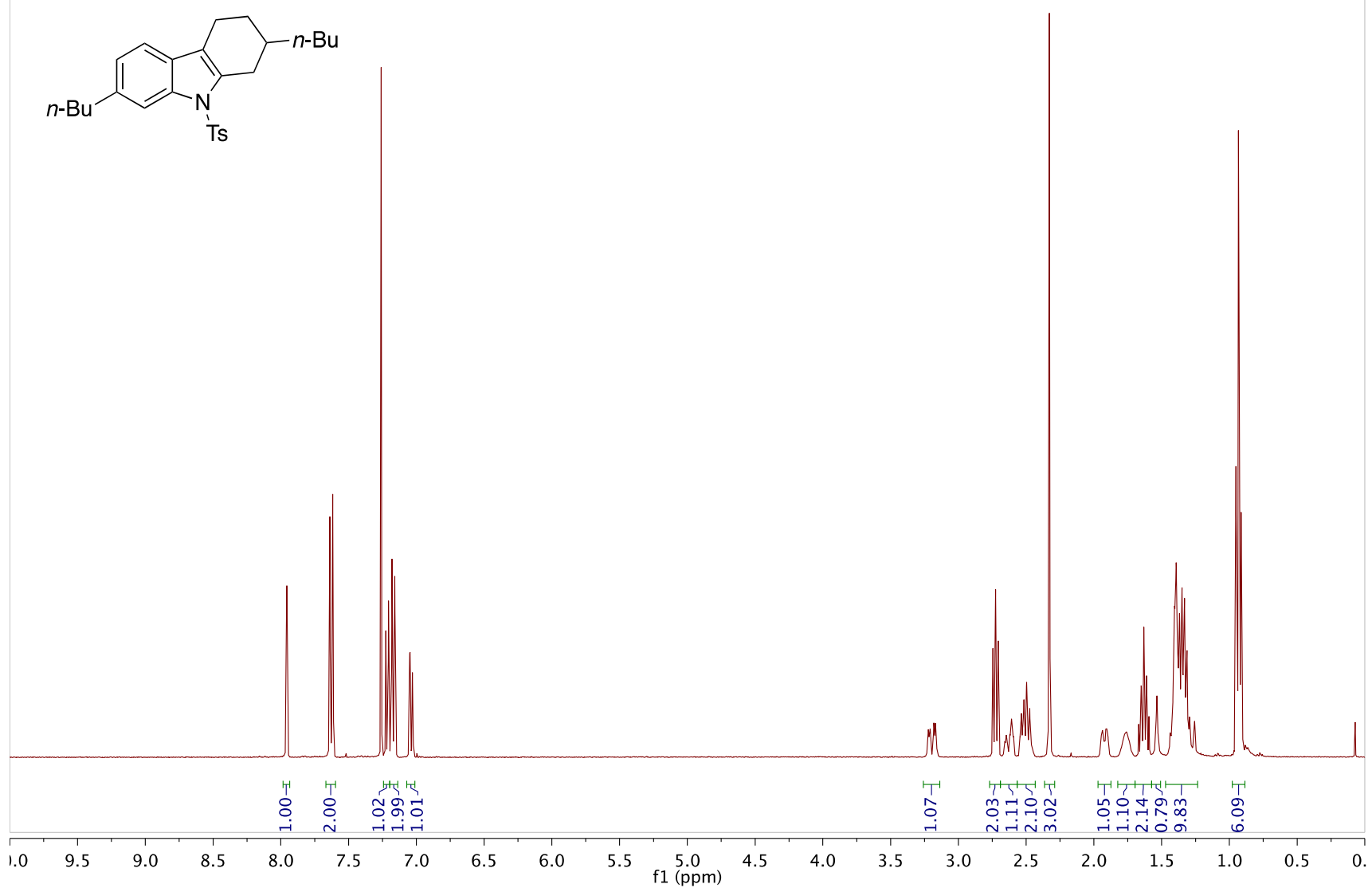

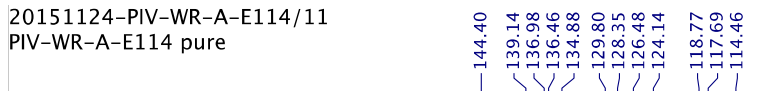<smiles>CCCCC1CCc2c(n([AsH2-])c3cc(C(CCCC)CCCC)ccc23)C1</smiles>

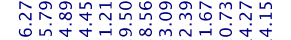

mmmm 서순

mat.

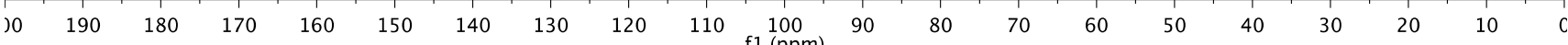


<smiles>COC(=O)CC1=CCC=C(C)C1</smiles>

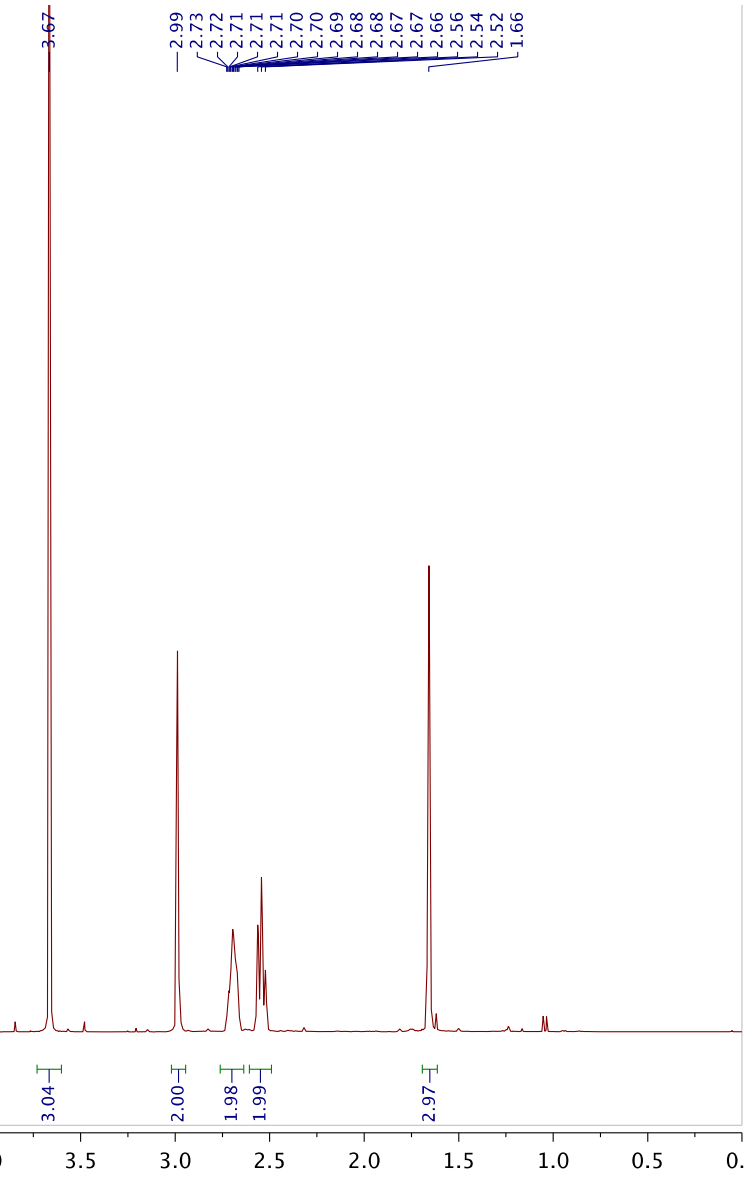

20151201-PIV-WR-A-备120/11 PIV-WR-A-E120<smiles>COC(=O)CC1=CCC=C(C)C1</smiles>

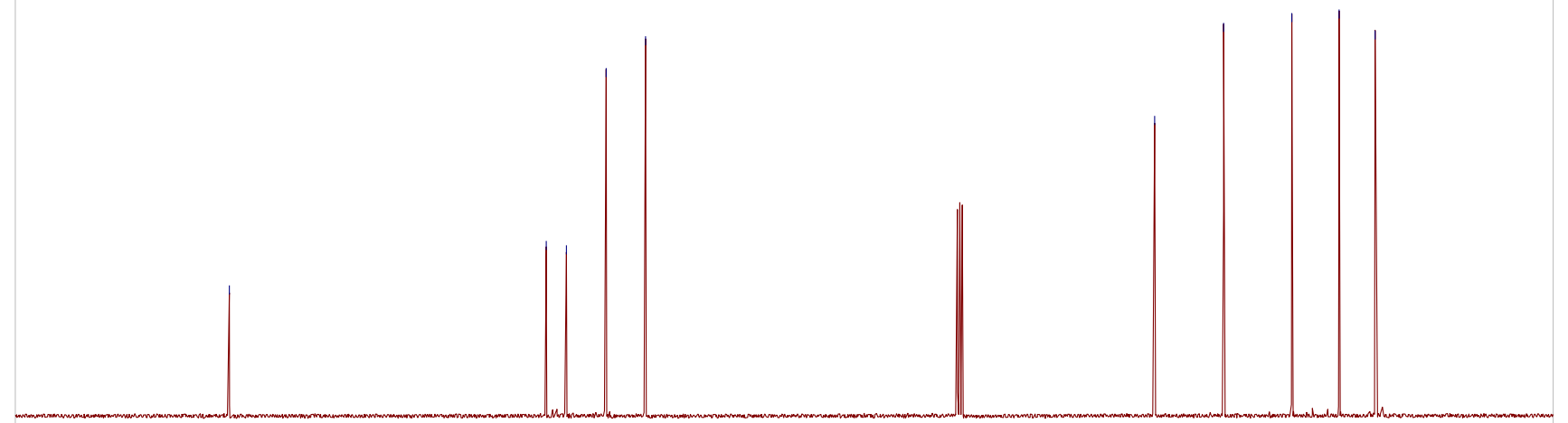


<smiles>COC(=O)CC1CCCC(C)C1</smiles>

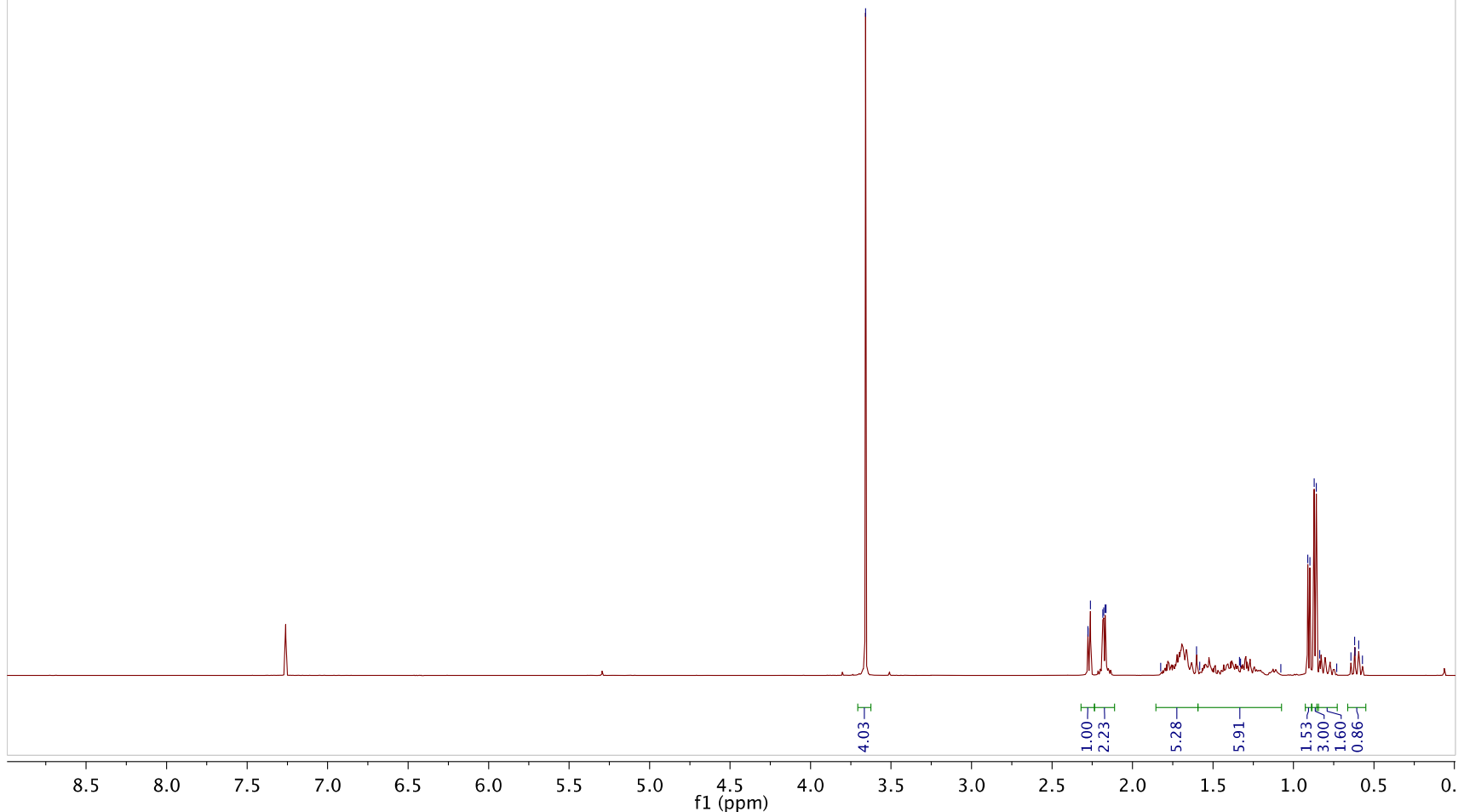

PIV-WR-A-E125-f1/8 PIV-WR-A-E125-f1-ENMR<smiles>COC(=O)CC1CCCC(C)C1</smiles>
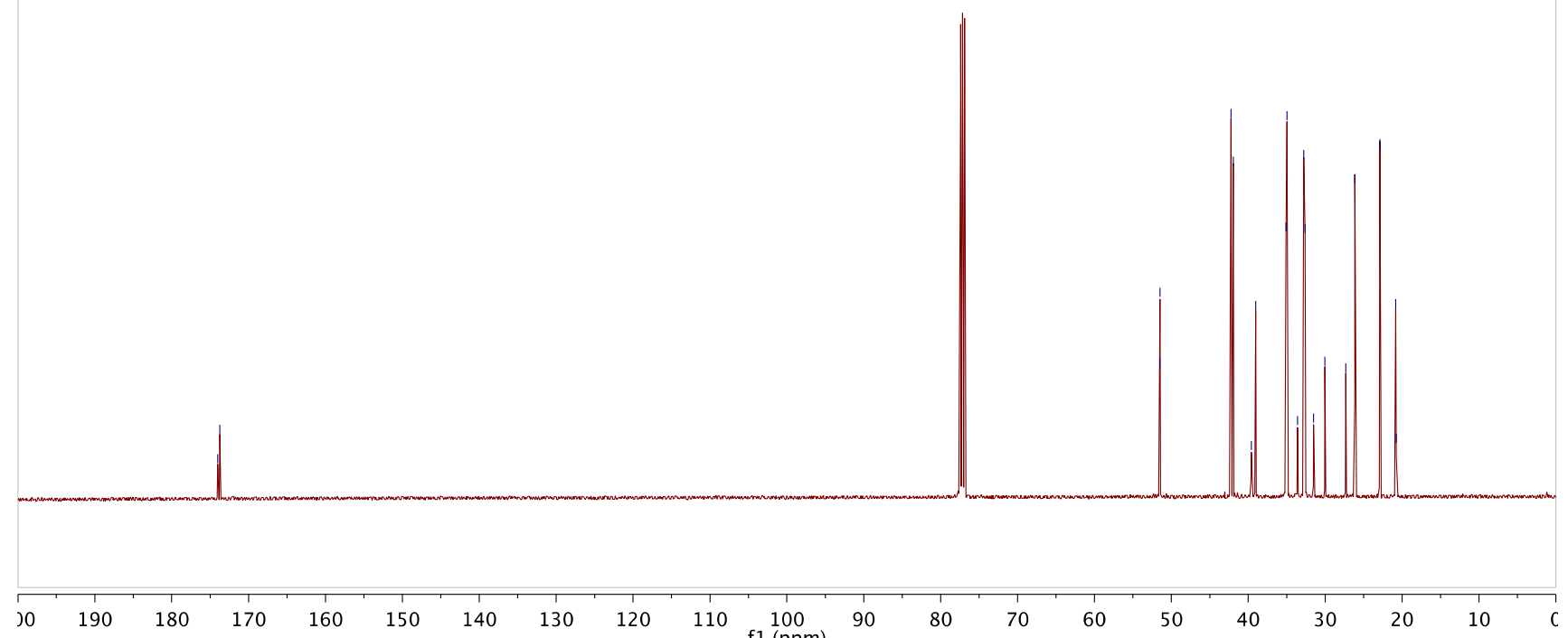

$50 \quad 40$

30

$\begin{array}{lll}1 & 10 & 10\end{array}$




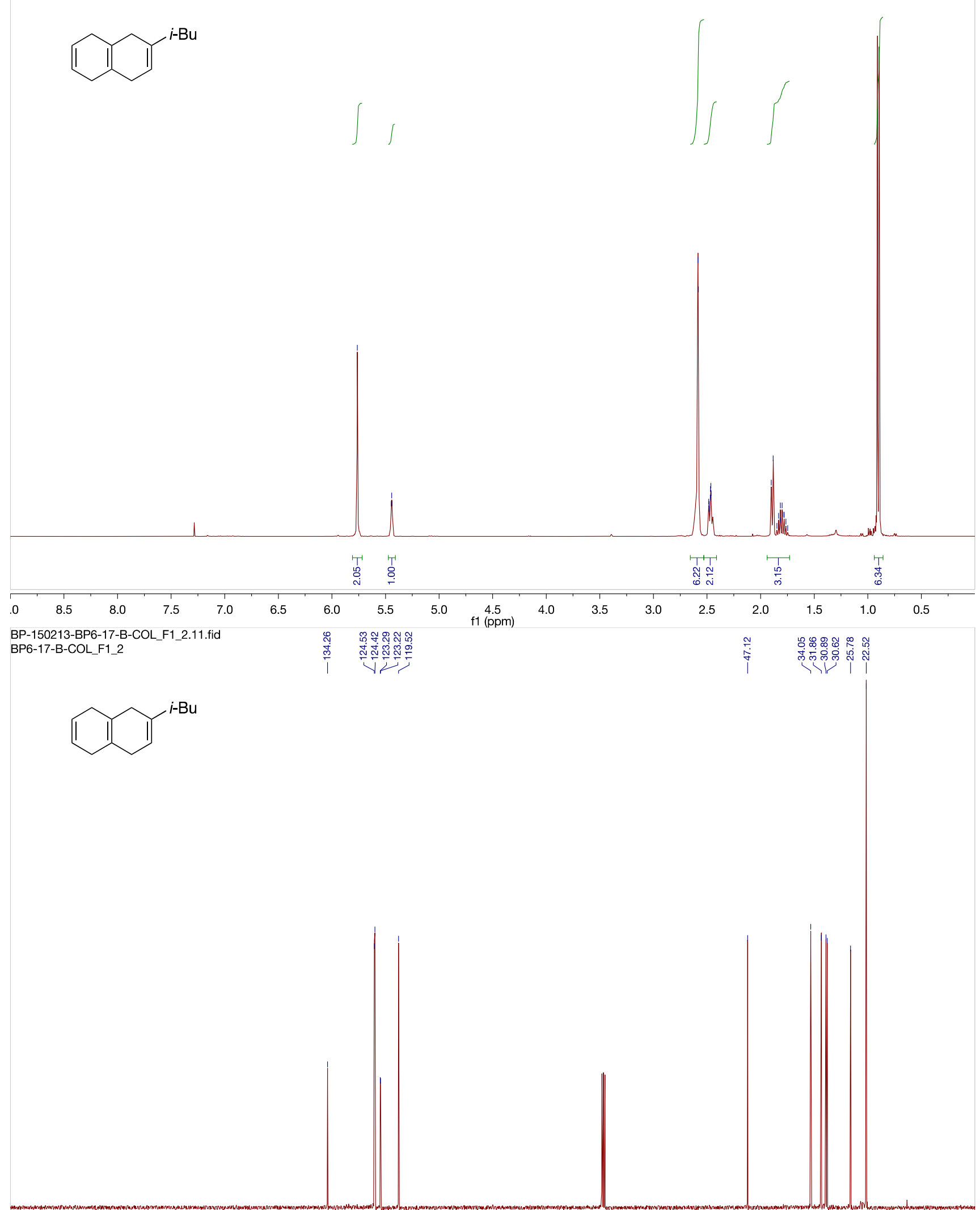

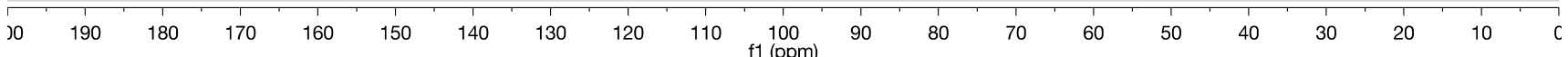


BP-151130-BP7-01B_F24-28.10.fid

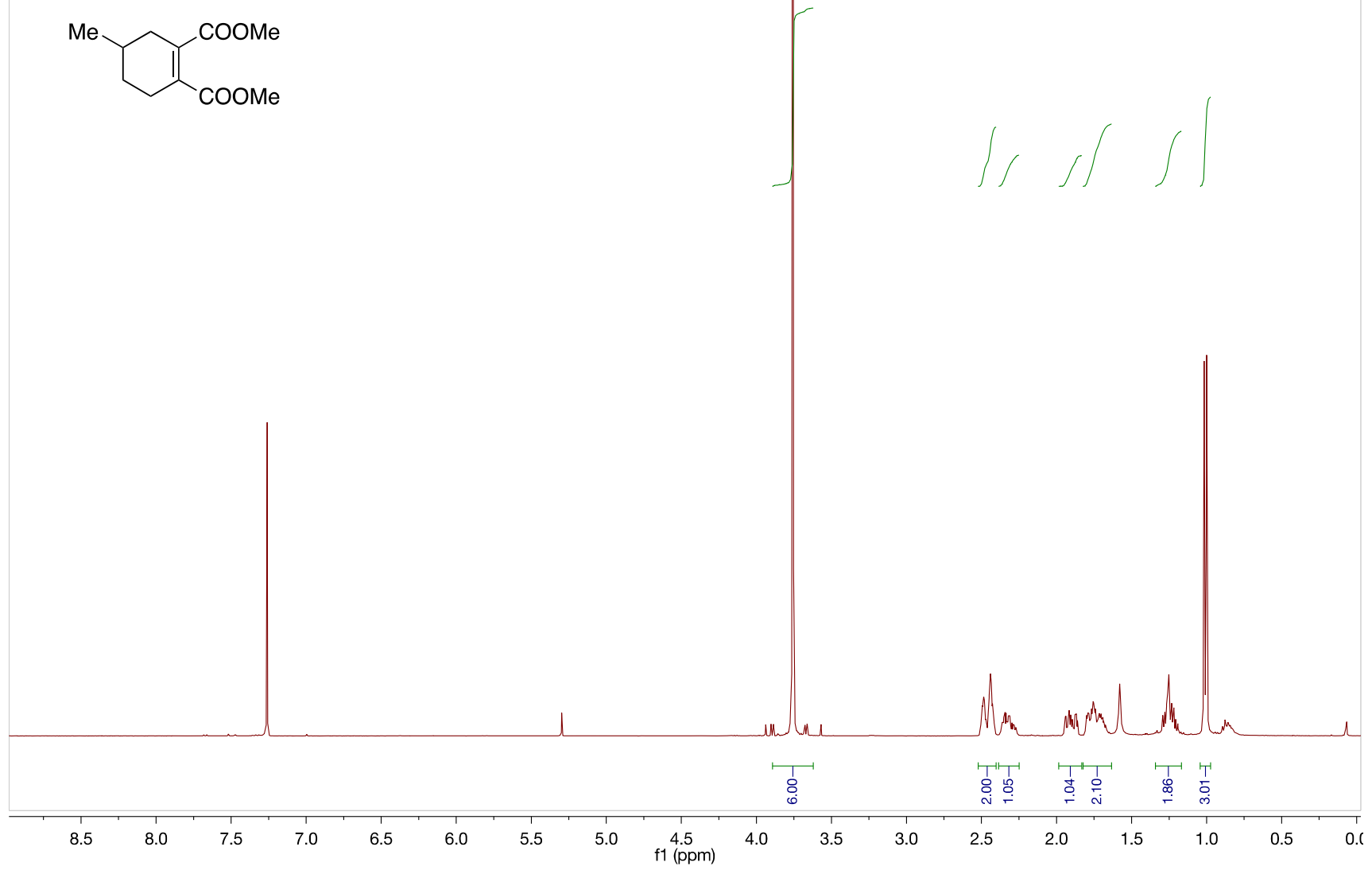

BP-151130-BP7-01B_13C,19. fid

잉

离

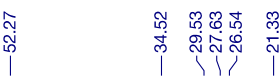<smiles>COC(=O)C1=C(C(=O)OC)CC(C)CC1</smiles>
jo
190
180
$170 \quad 160$
$150 \quad 140$
$130 \quad 120$
$120 \quad 1$
100
$90 \quad 80$
$70 \quad 60$
50
40
$30 \quad 20$
$10 \quad c$ 
BP-151130-BP6-204B-F6-11.10.fid
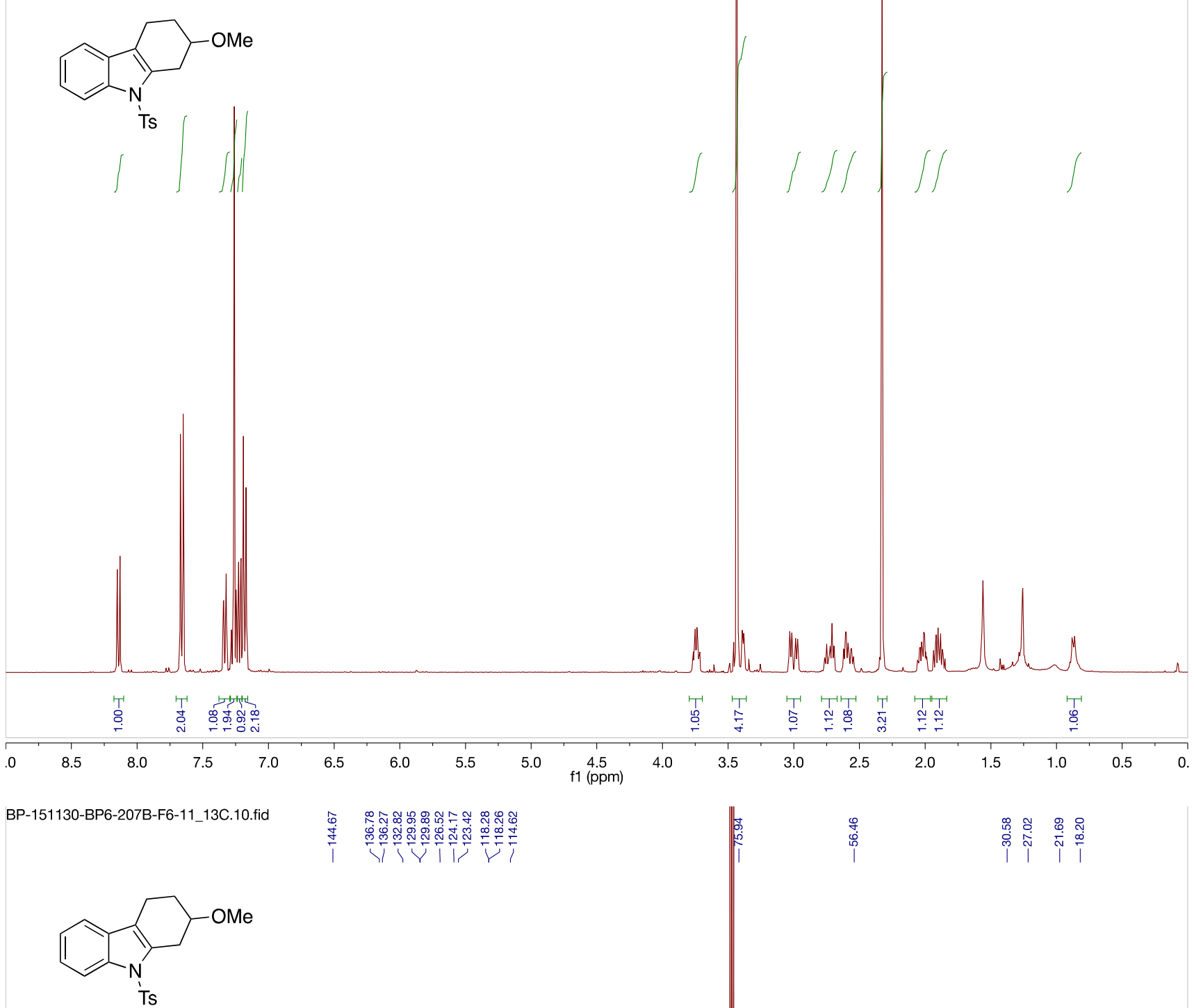

|
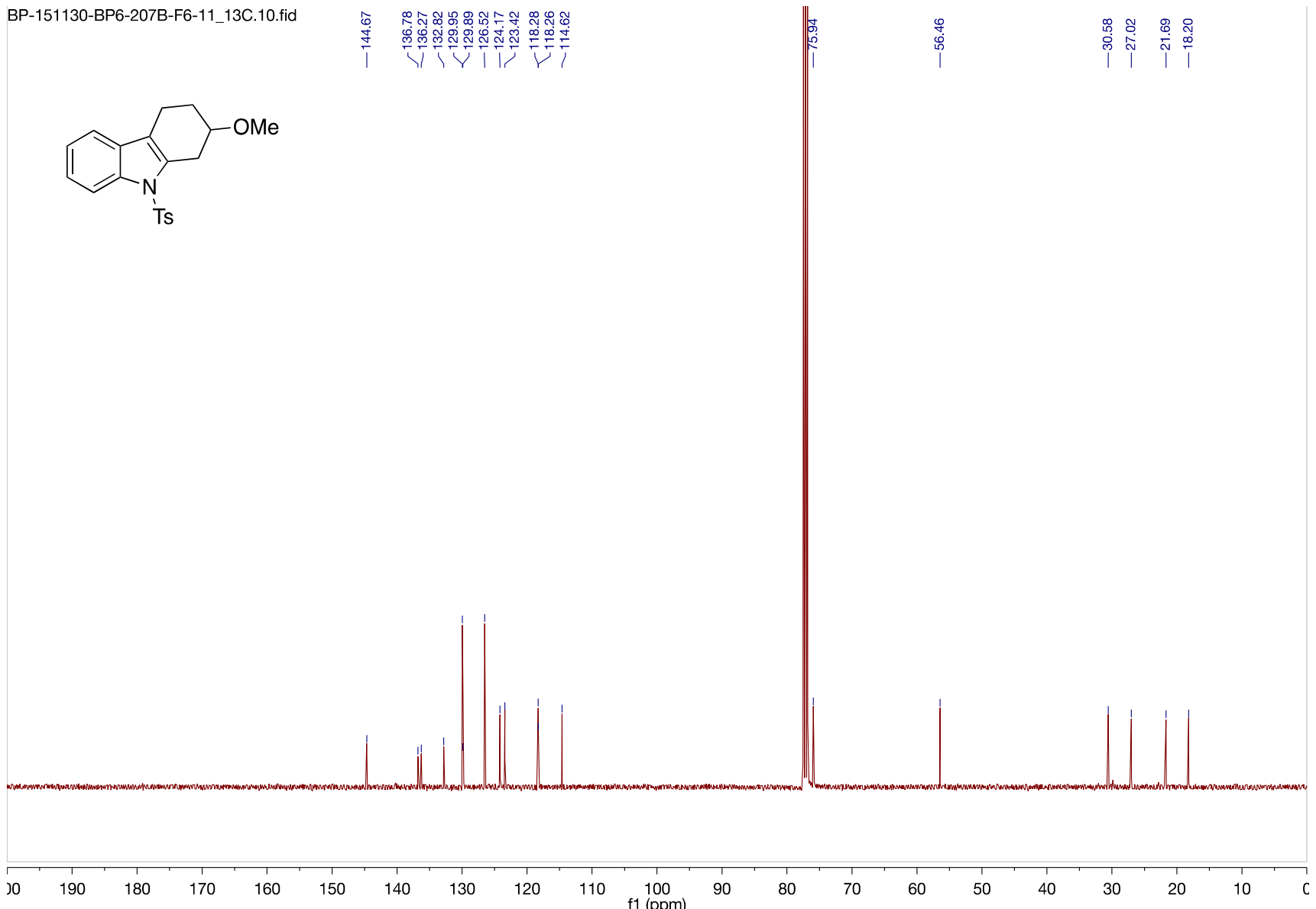

$190 \quad 180$
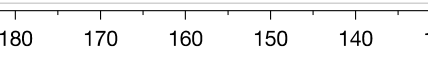

$130 \quad 120$

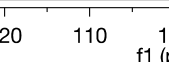

$\begin{array}{llll}100 & 90 & 80 & 70\end{array}$

$\begin{array}{llllll}50 & 40 & 30 & 20 & 10 & \mathrm{C}\end{array}$ 

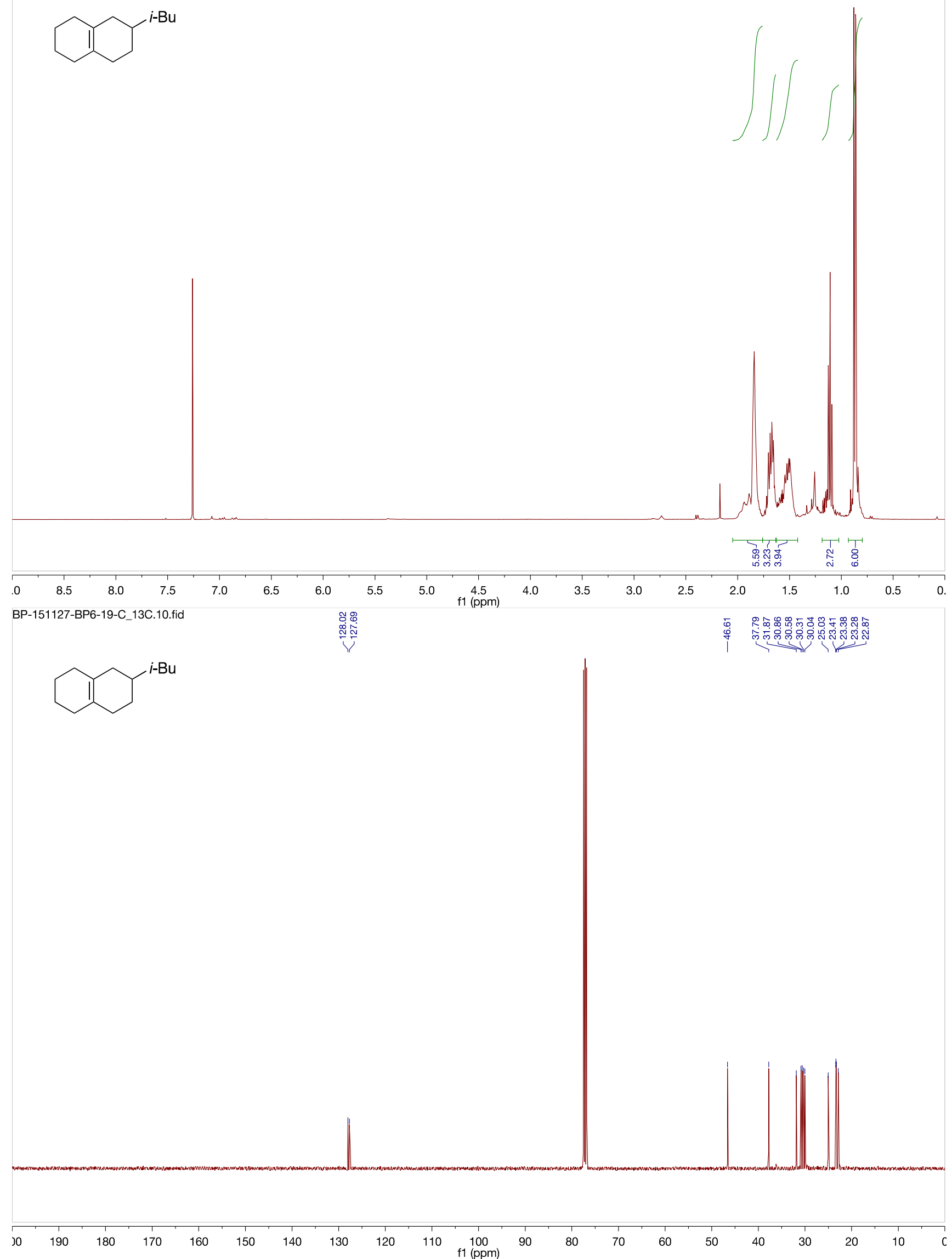
BP-151125-BP6-204_COL.10.fid

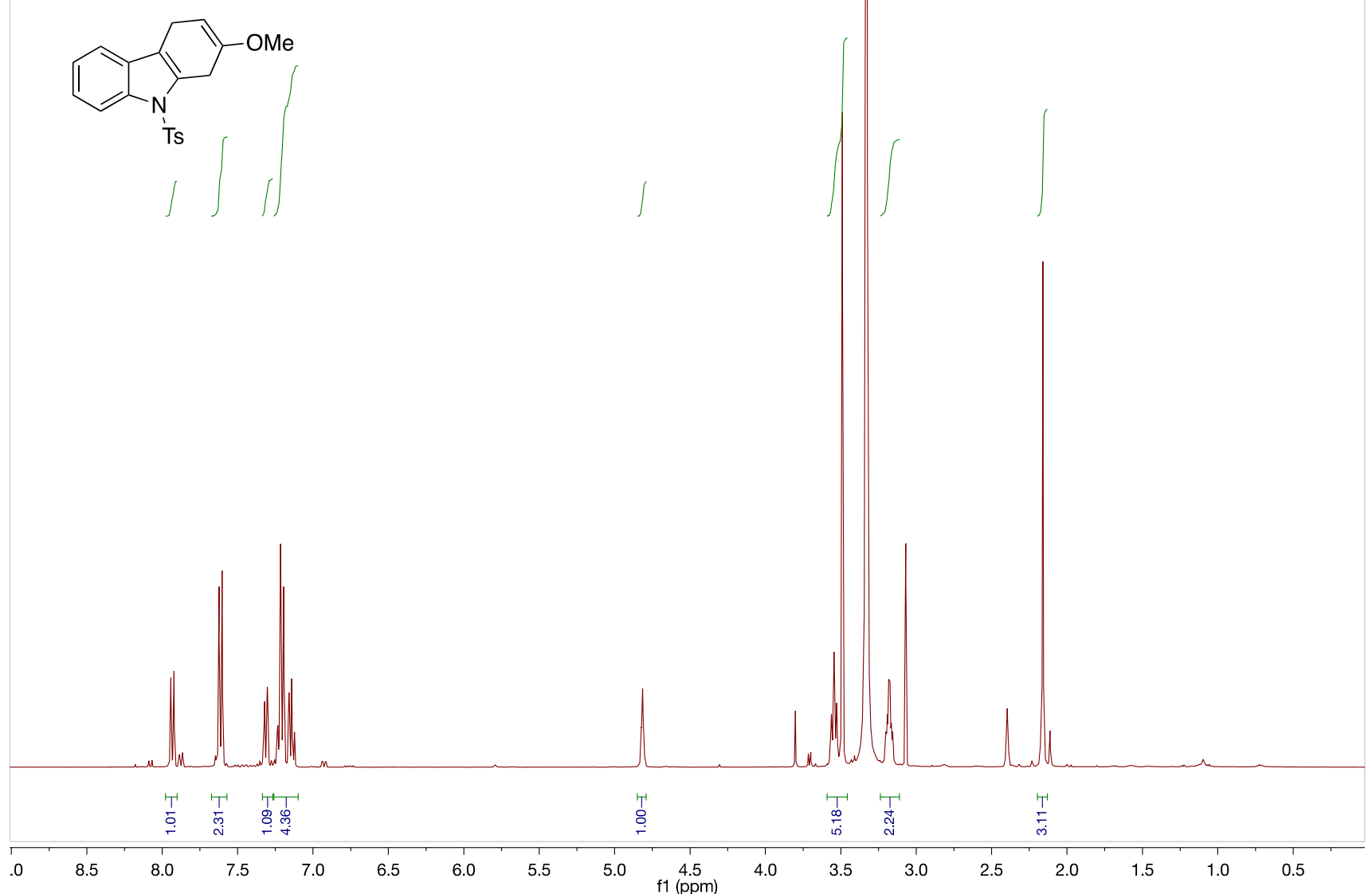
BP-151125-BP6-204_COL.11.fid
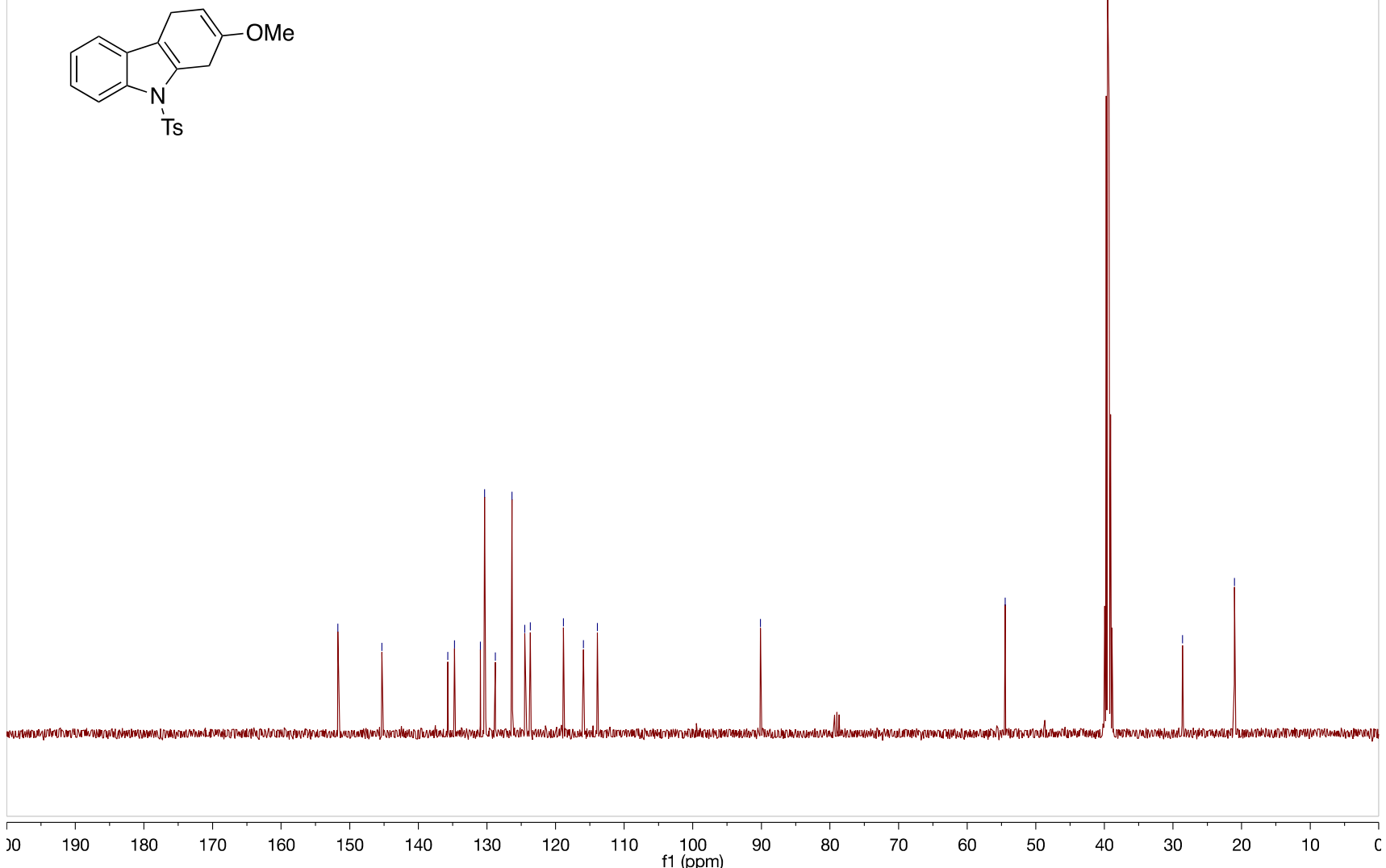
<smiles>CC1CC(C)CC(C(=O)O)C1</smiles>
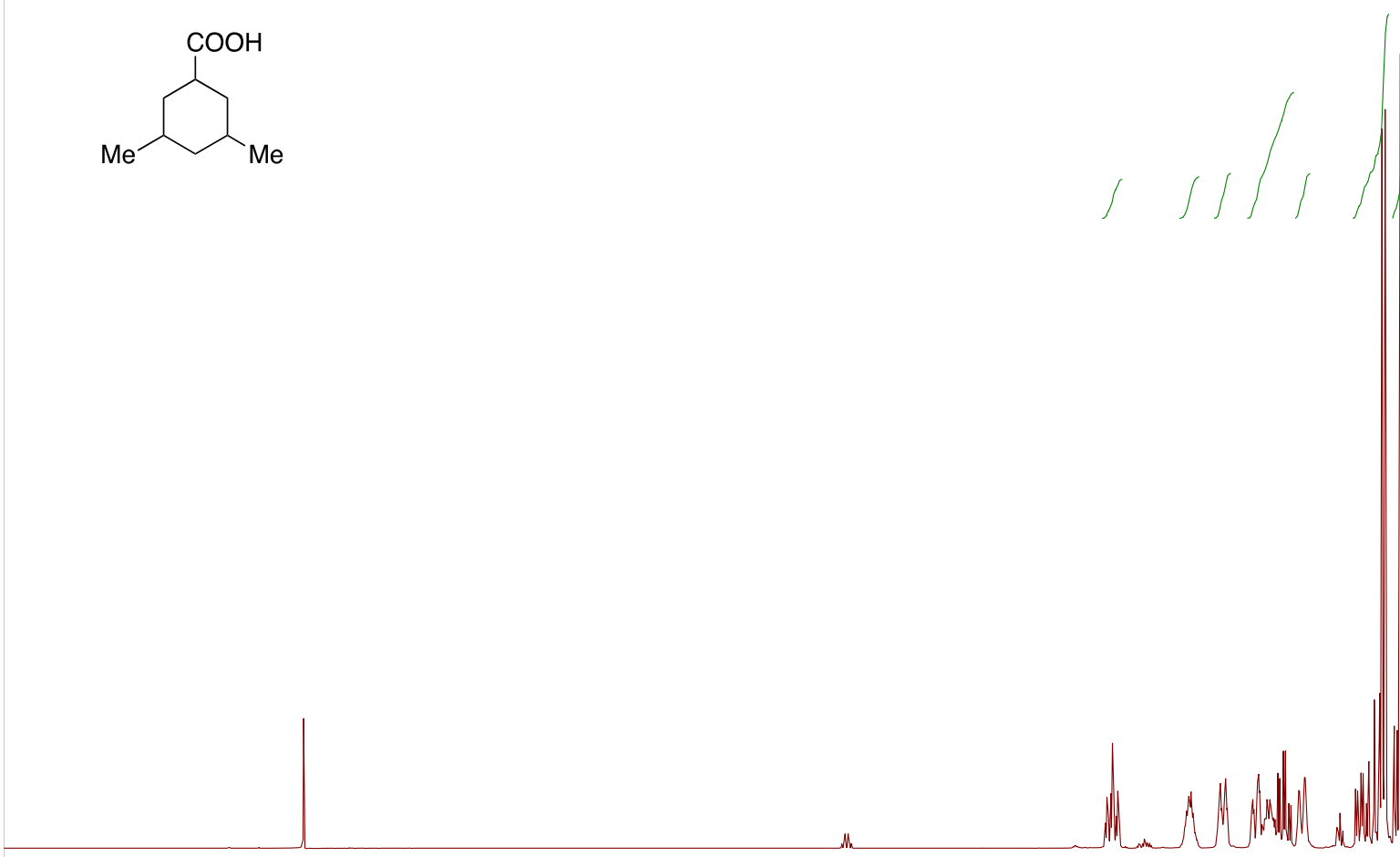

它

8.5

8.0

$\begin{array}{ll}6.5 & 6.0\end{array}$

5.5

5.0 f1 (ppm)

1P13B6-SK-crede-20151025-/11 1P13B6-crude

$$
\text { I }
$$<smiles>CC1CC(C)CC(C(=O)O)C1</smiles>

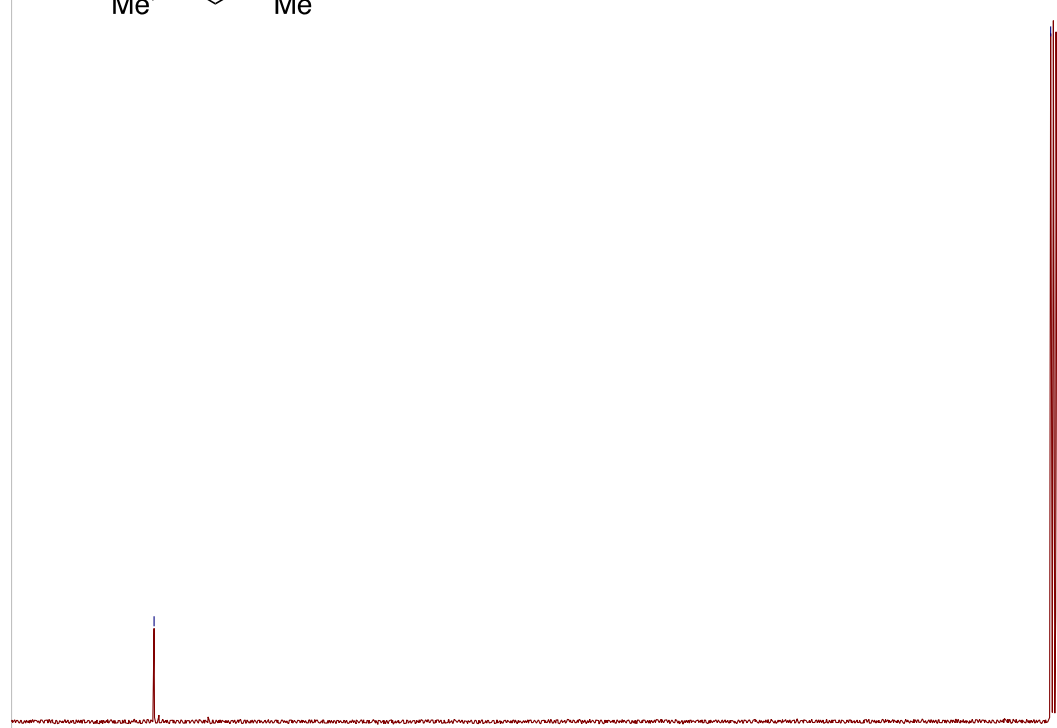

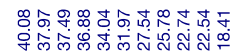

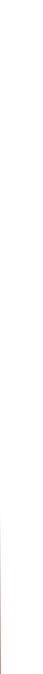

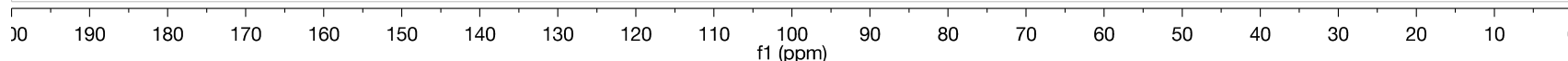



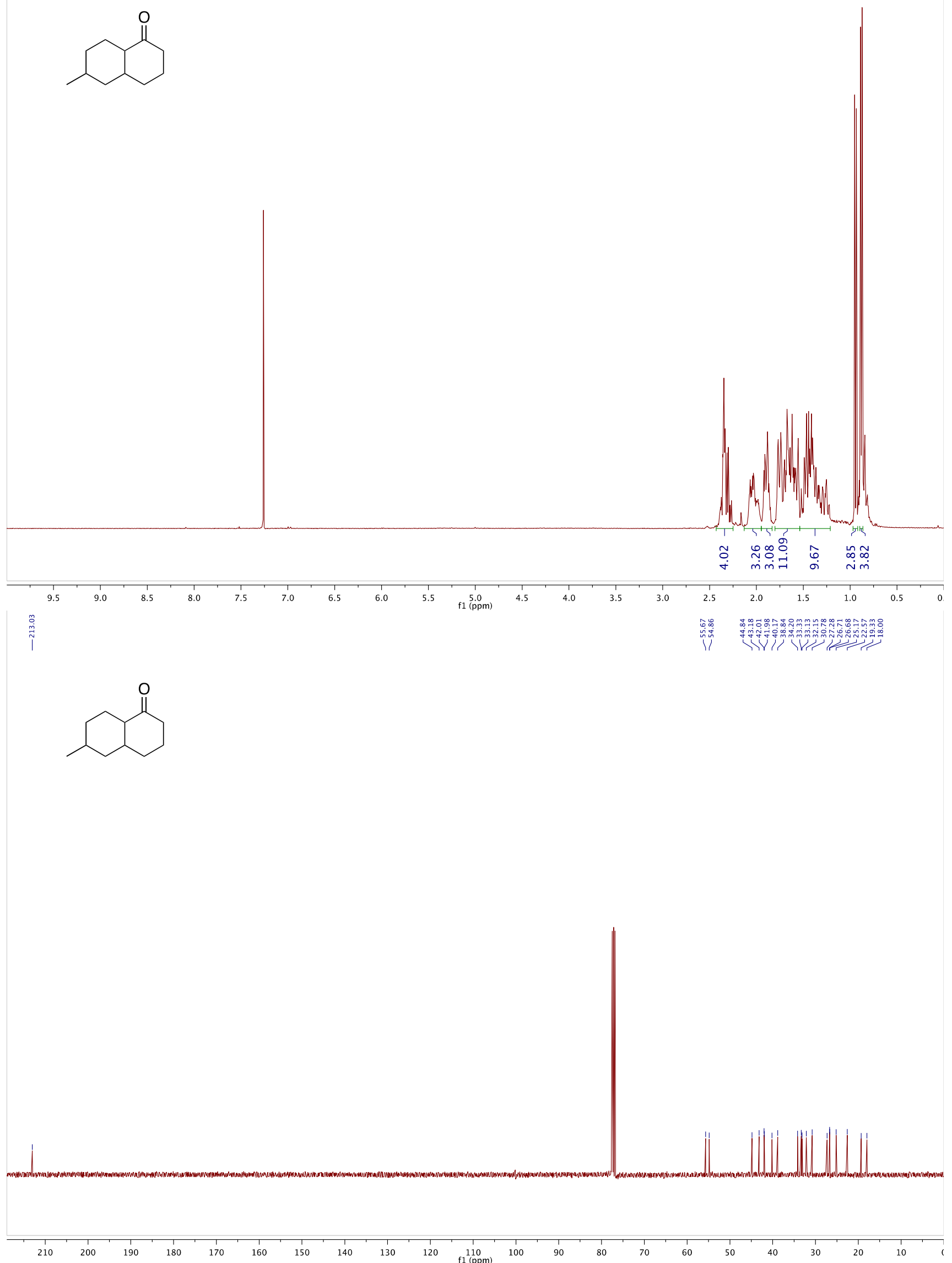

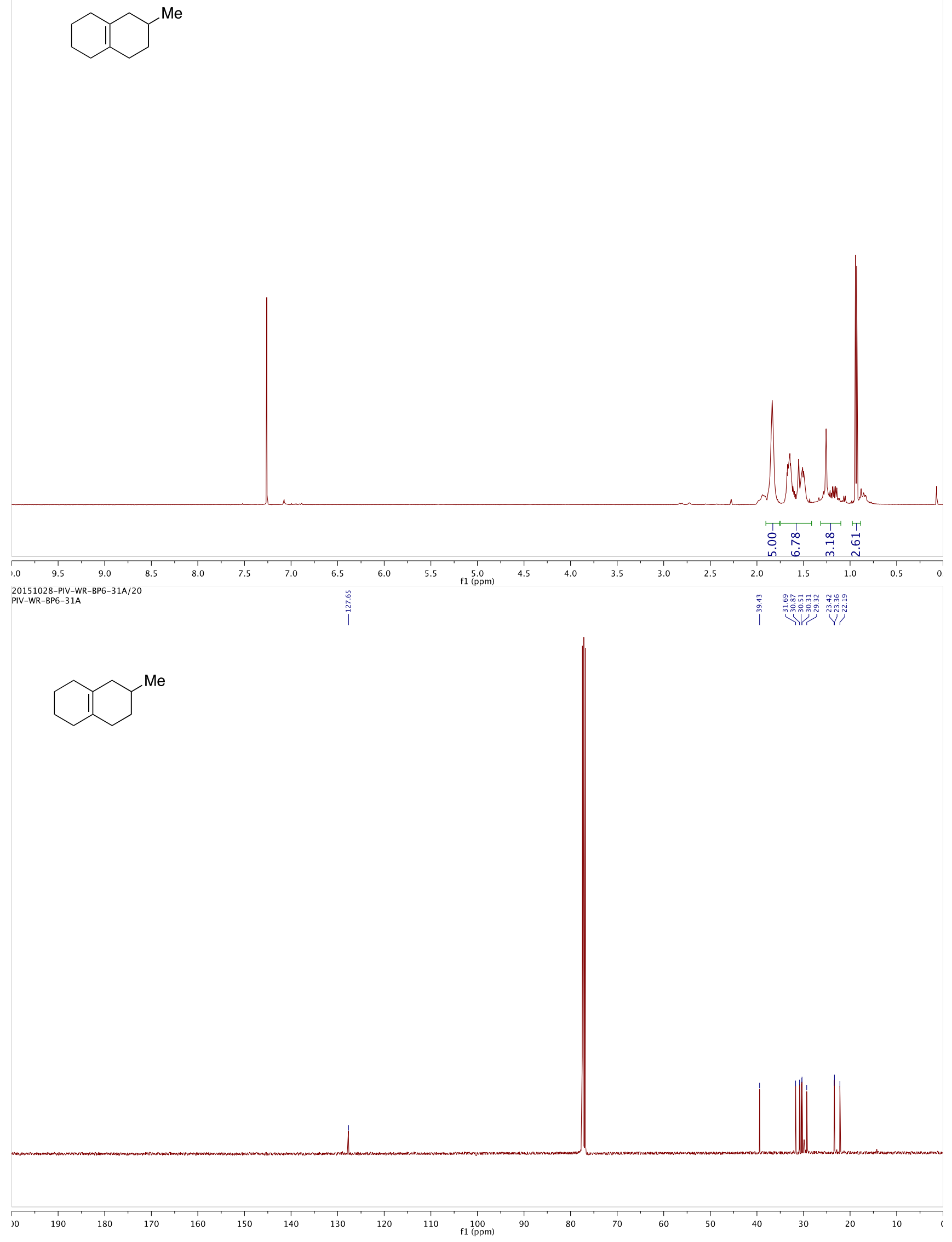

S64 

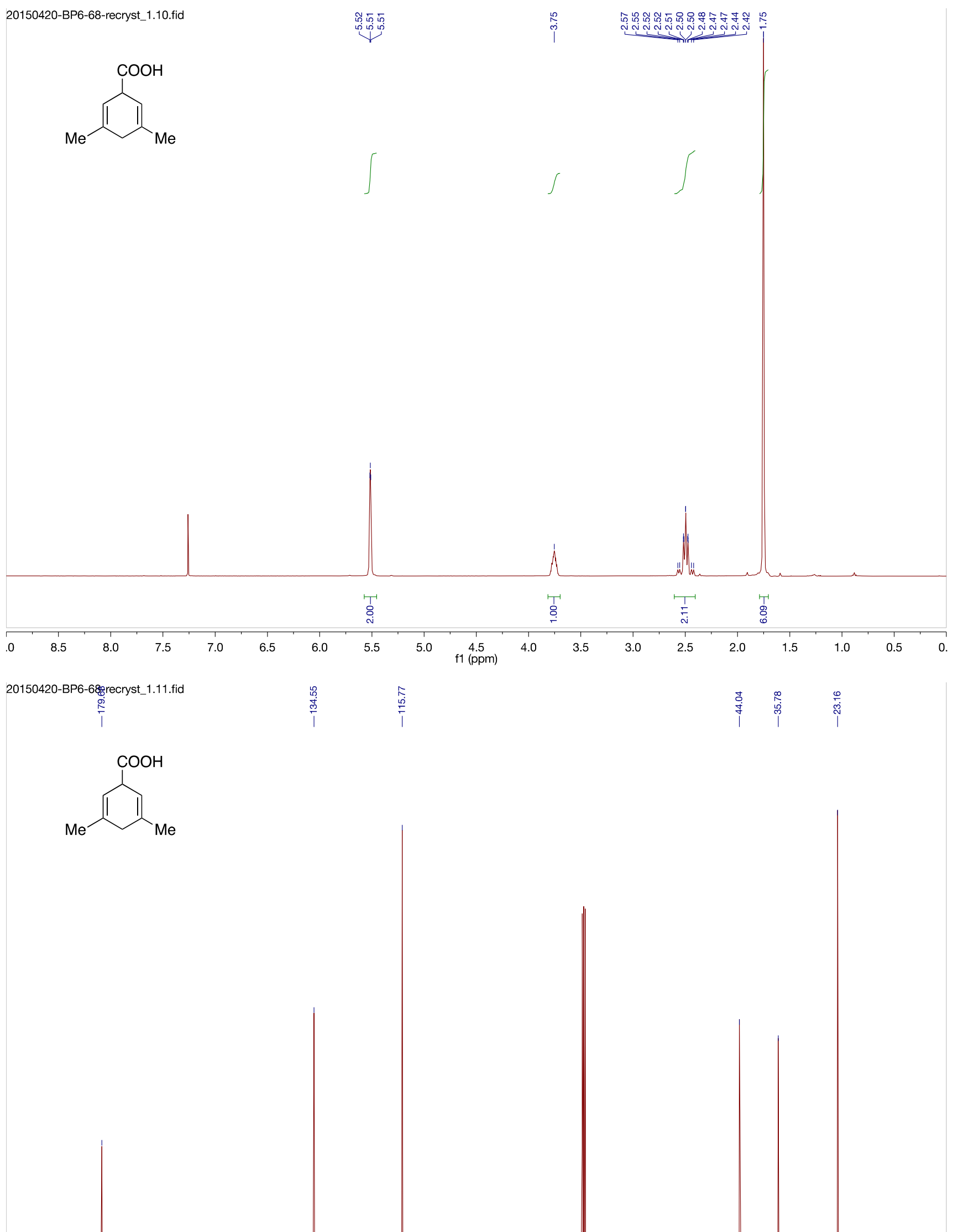

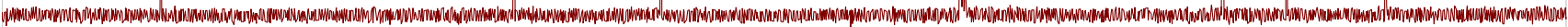

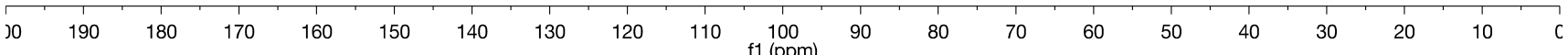




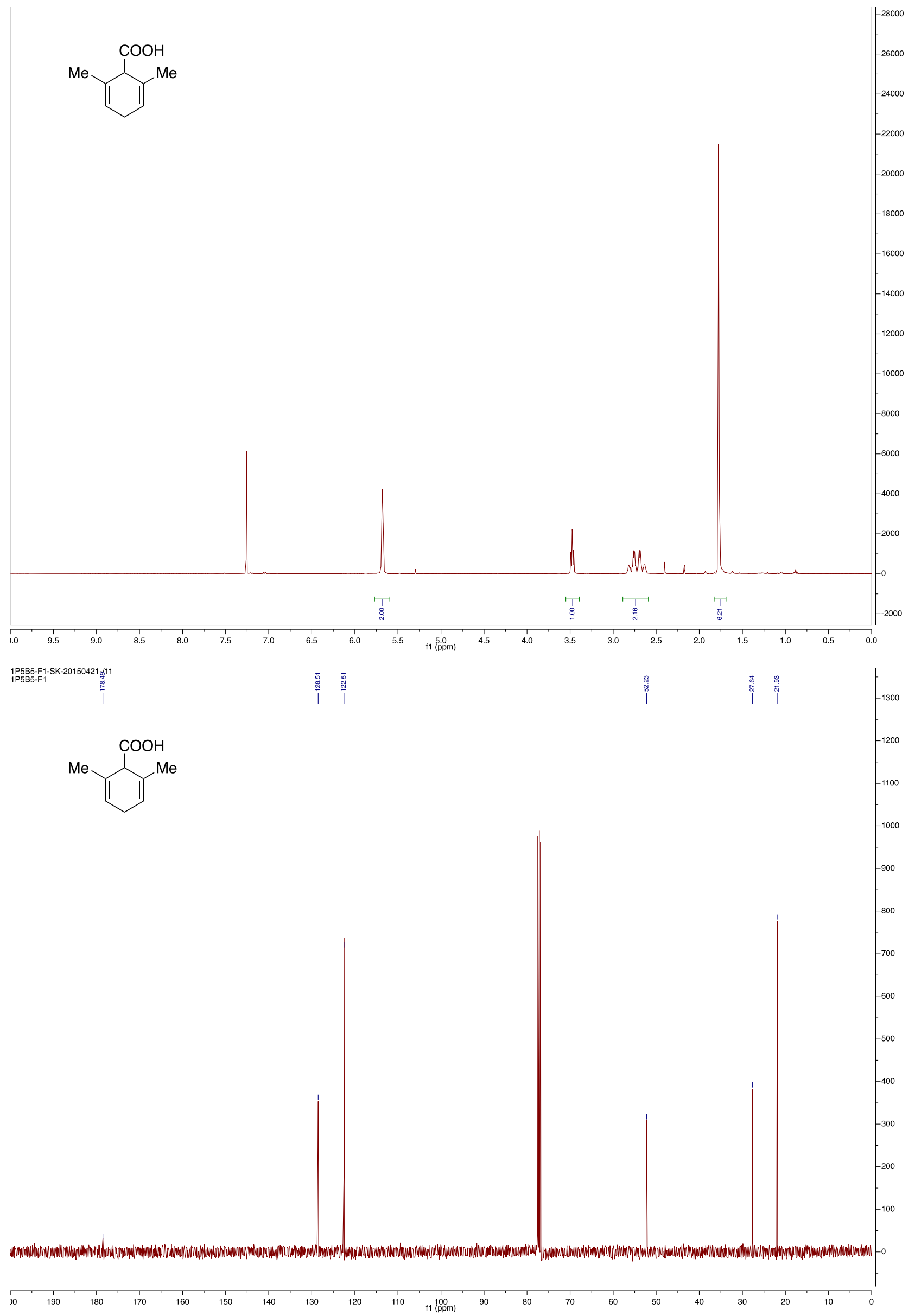

S66 


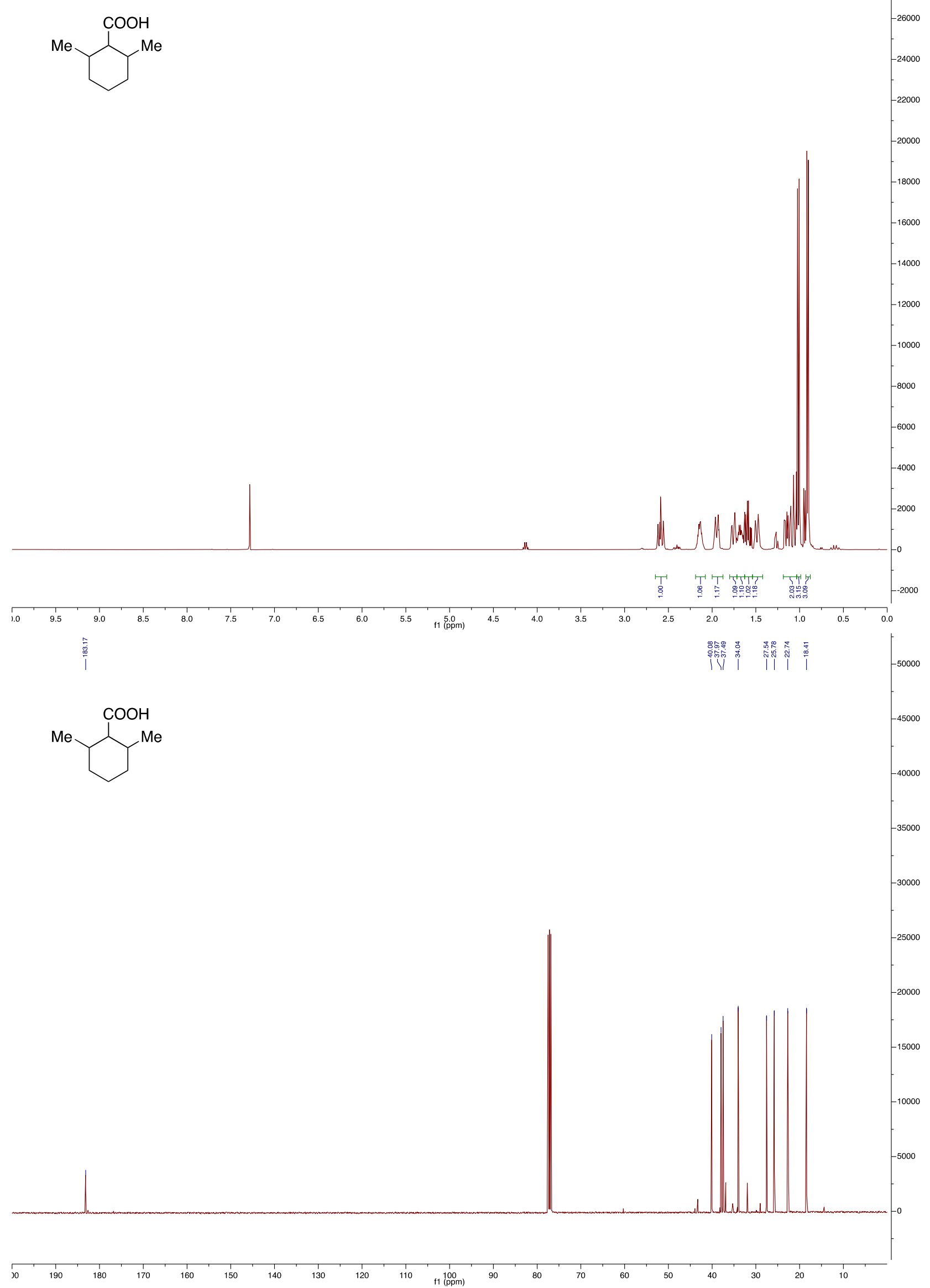


TM-160107-TM59-01/10
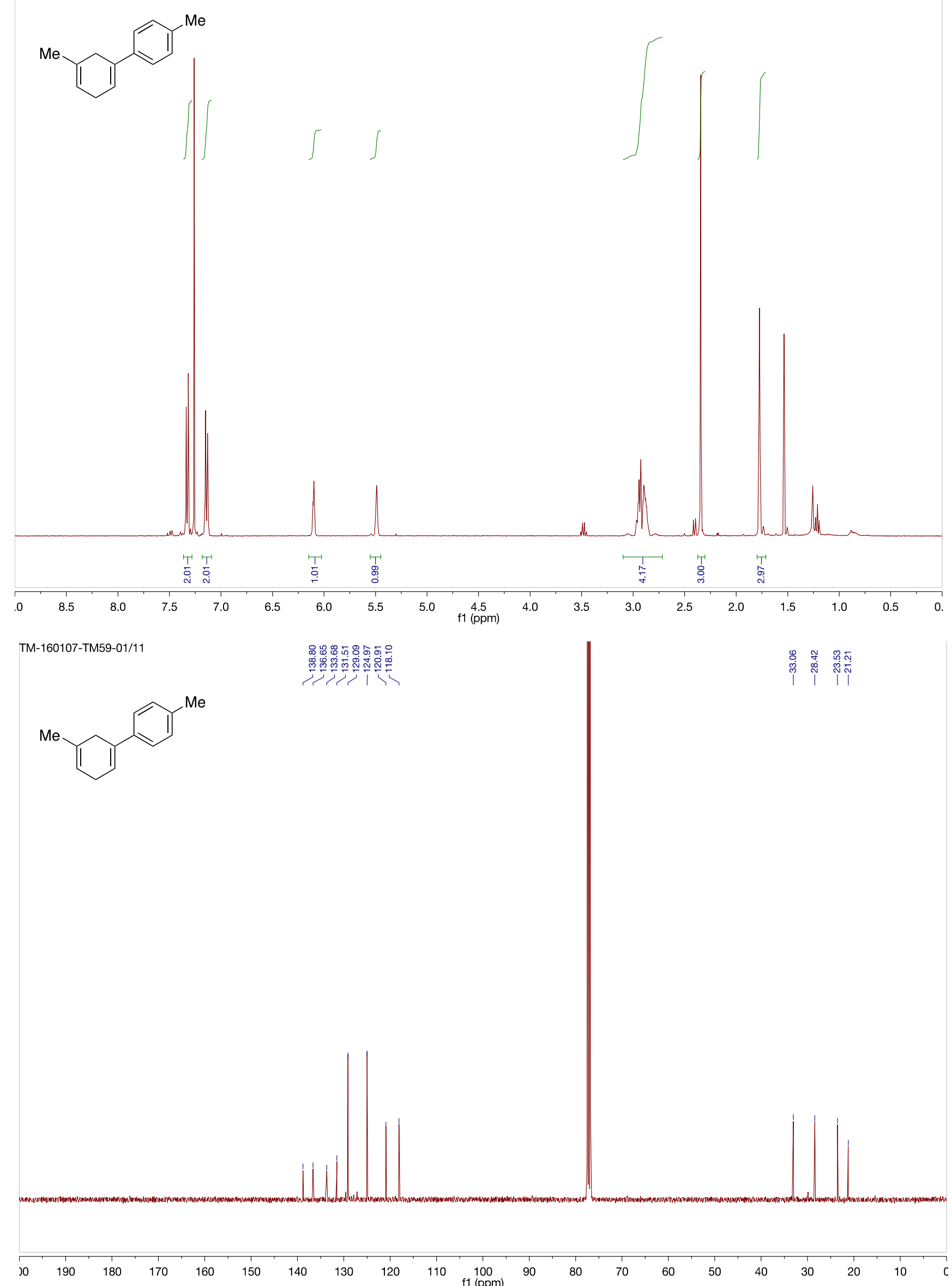


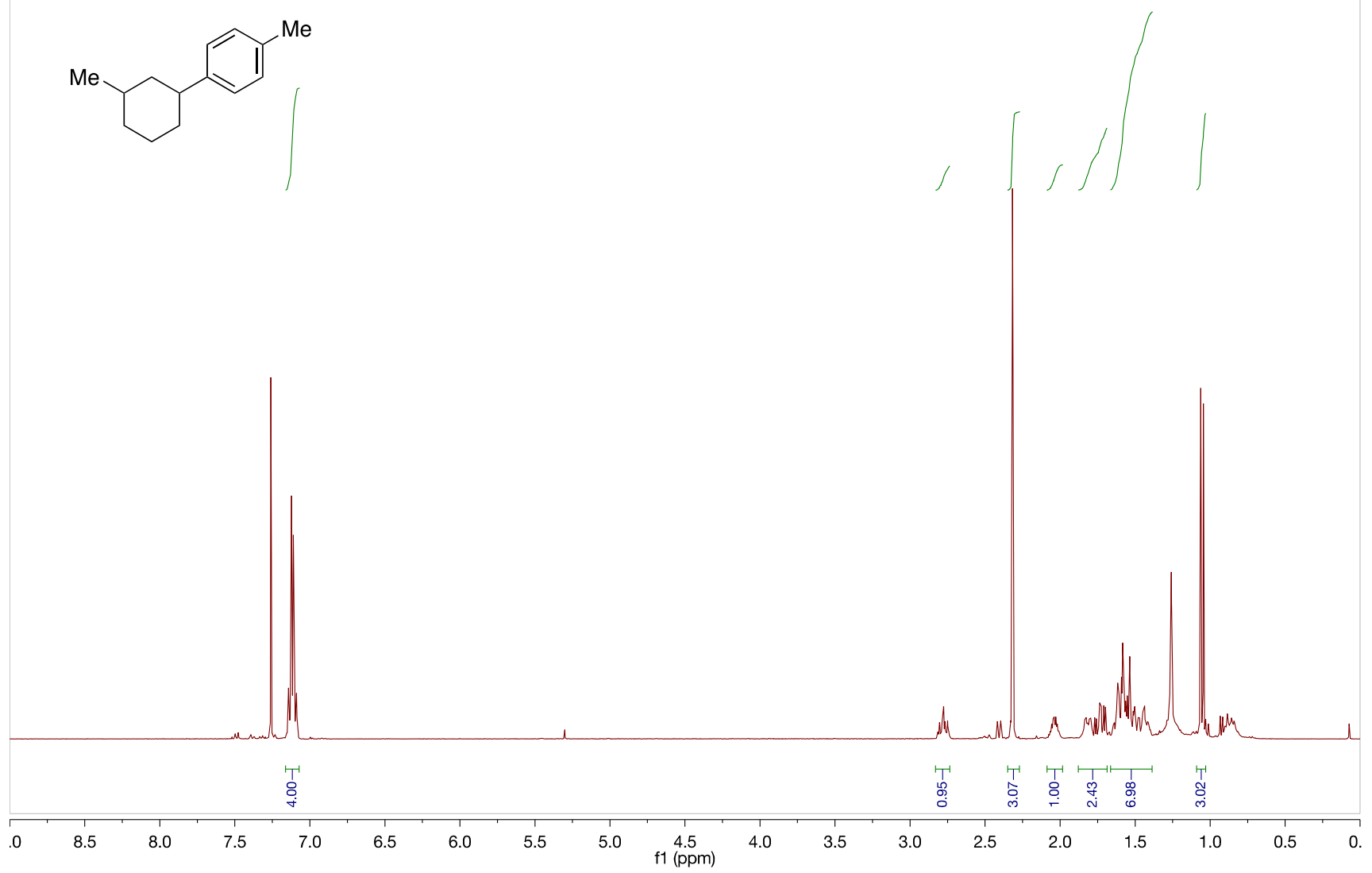

TM-160109-2-TM01-02-A11<smiles>Cc1ccc(C2CCCC(C)C2)cc1</smiles>
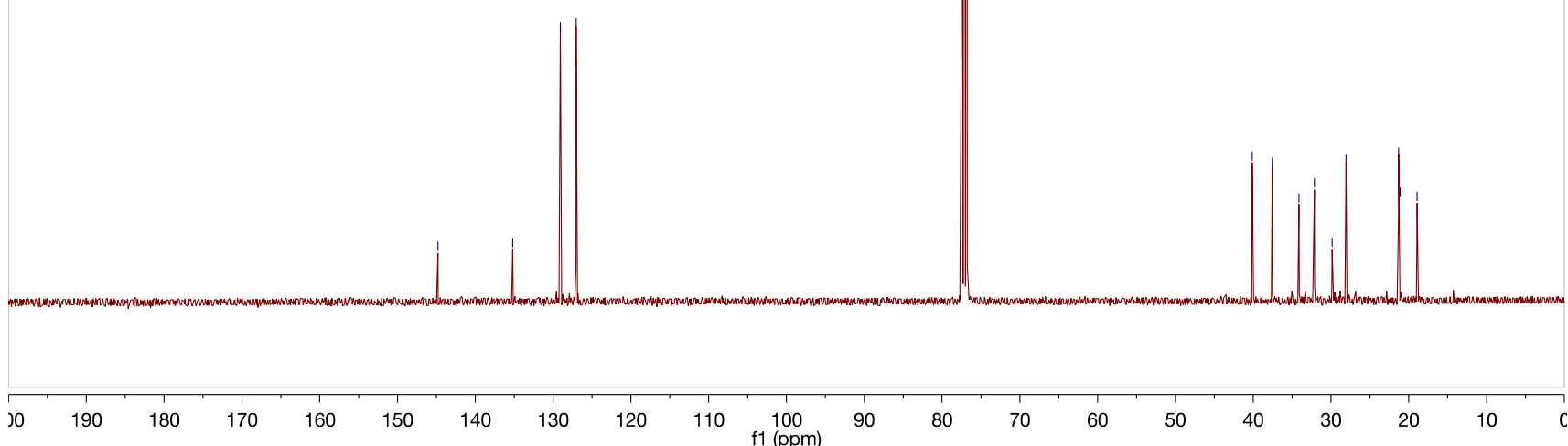


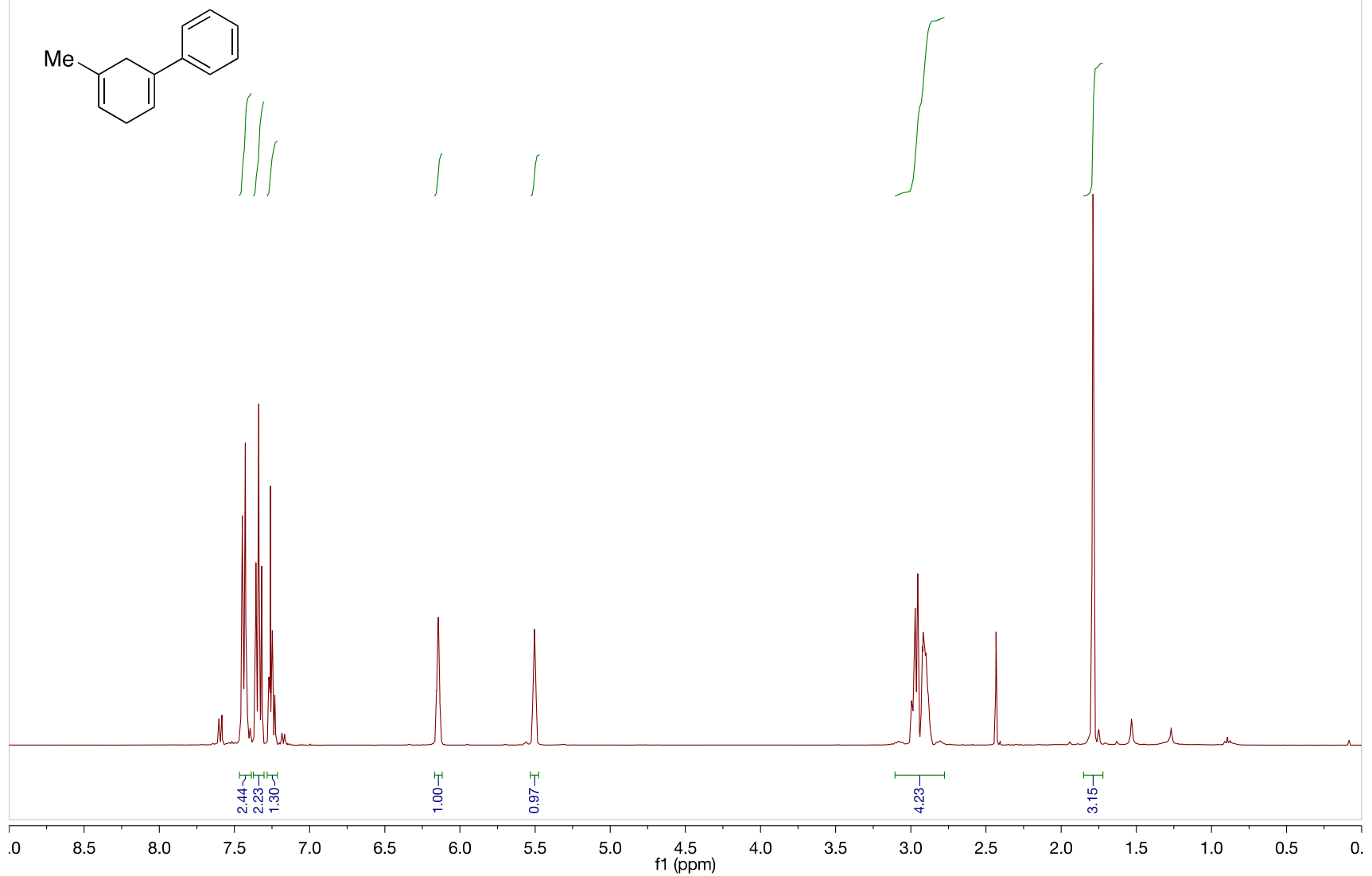

TM-151124-34-01-P/11 竞

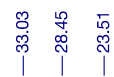<smiles>CC1=CCC=C(c2ccccc2)C1</smiles>

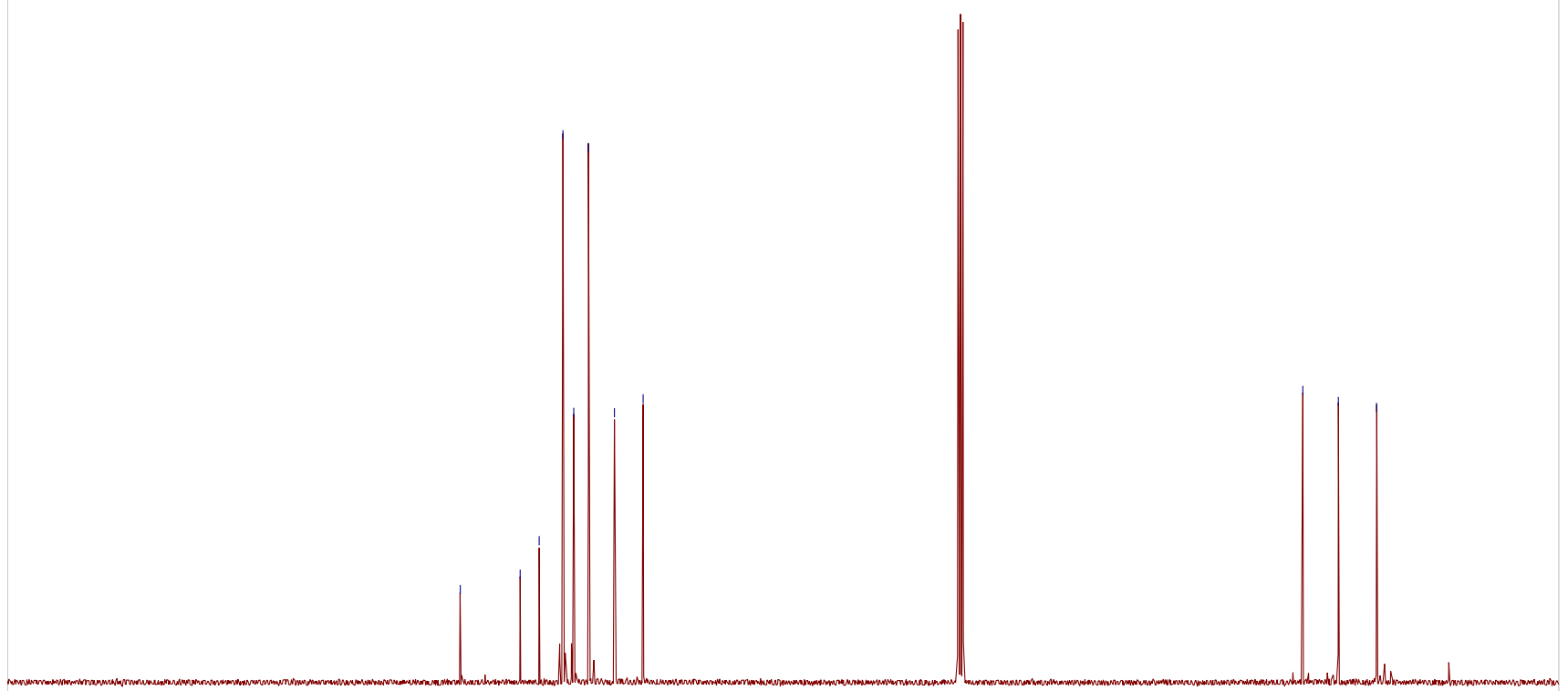

J0 190

$190 \quad 180$

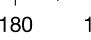

$170 \quad 160$

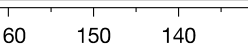

$130 \quad 120$

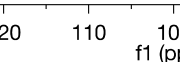

100

$90 \quad 80$

$80+70$

60

$\begin{array}{llllll}1 & 1 & 1 & 1 & 1 \\ 50 & 40 & 30 & 20 & 10 & \mathrm{C}\end{array}$ 


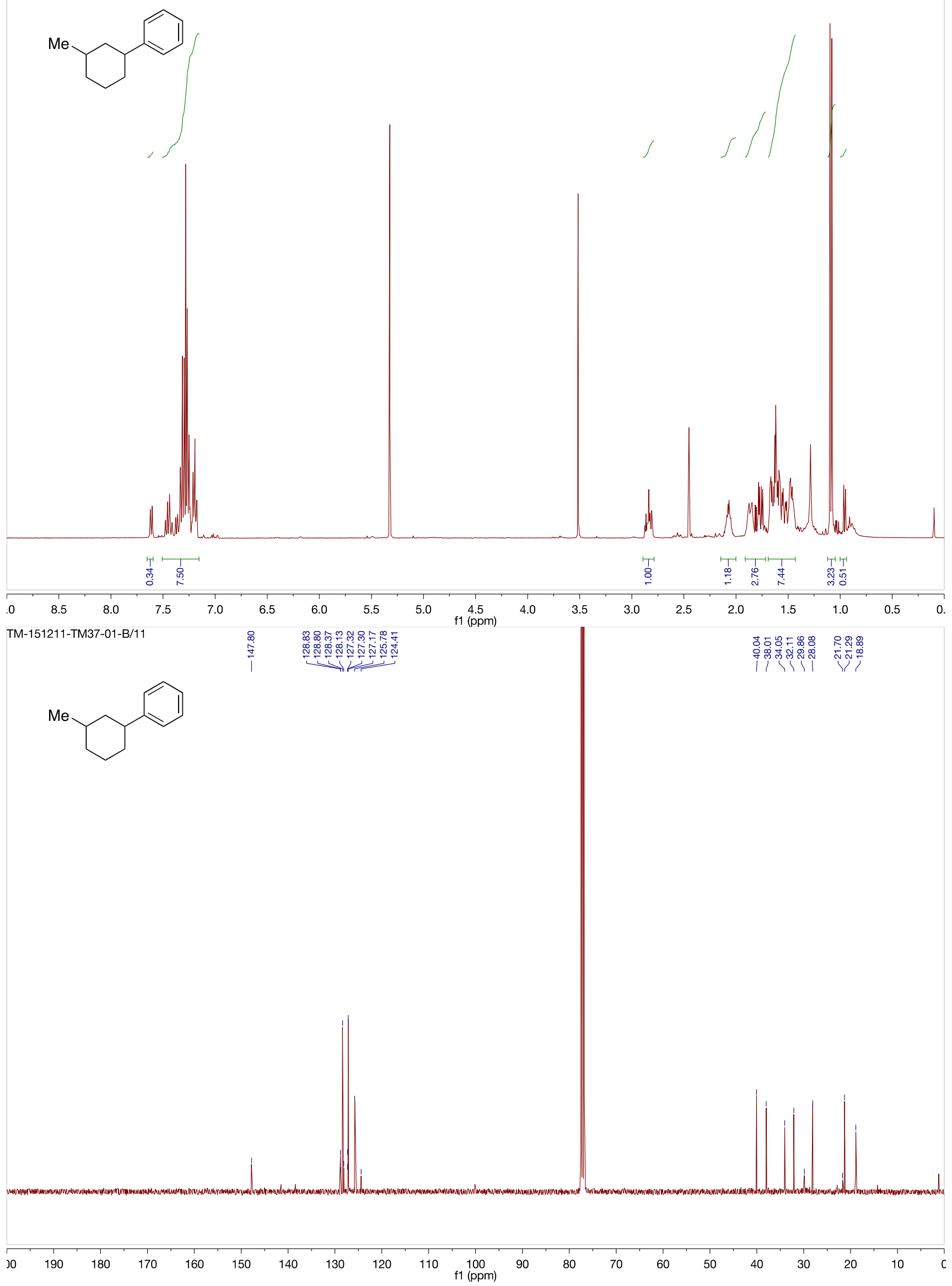




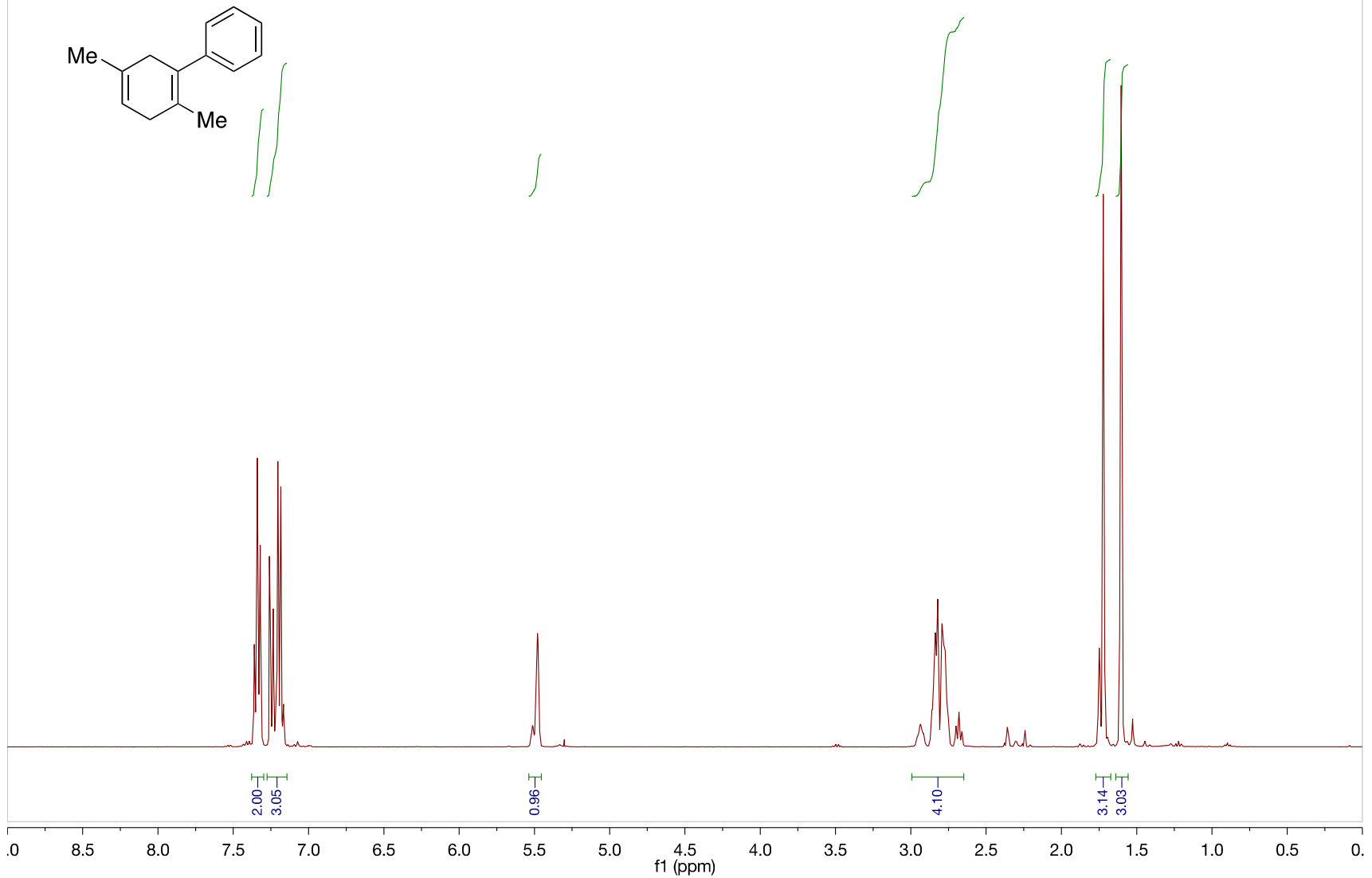

TM-160124-TM50-01-C13/10

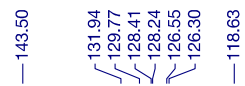<smiles>CC1=CCC(C)=C(c2ccccc2)C1</smiles>

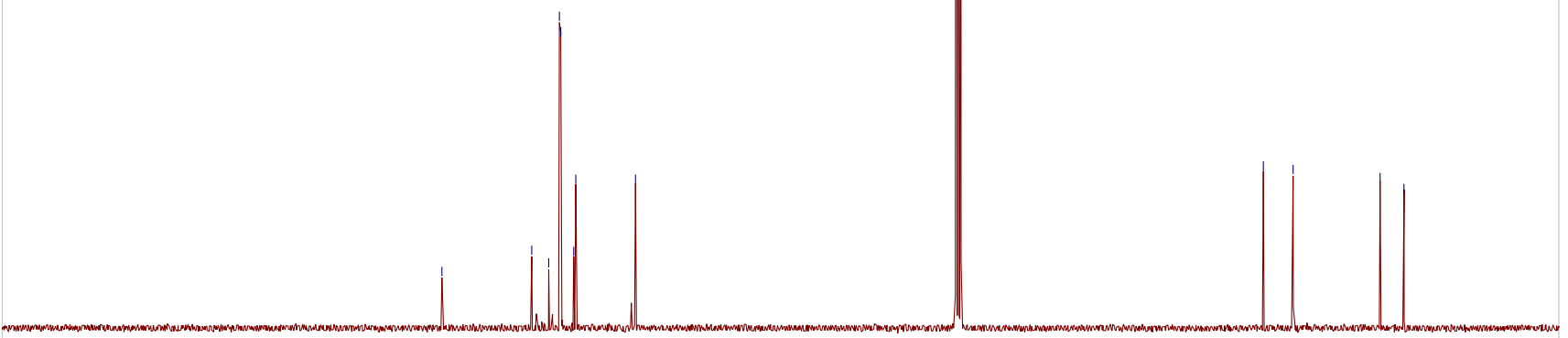

jo 190

$190 \quad 180$

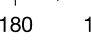

$170 \quad 1$

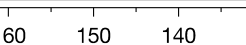

$130 \quad 120$

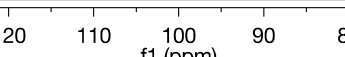

$80 \quad 70$

60

50

$40 \quad 30$

$20+10, c$ 

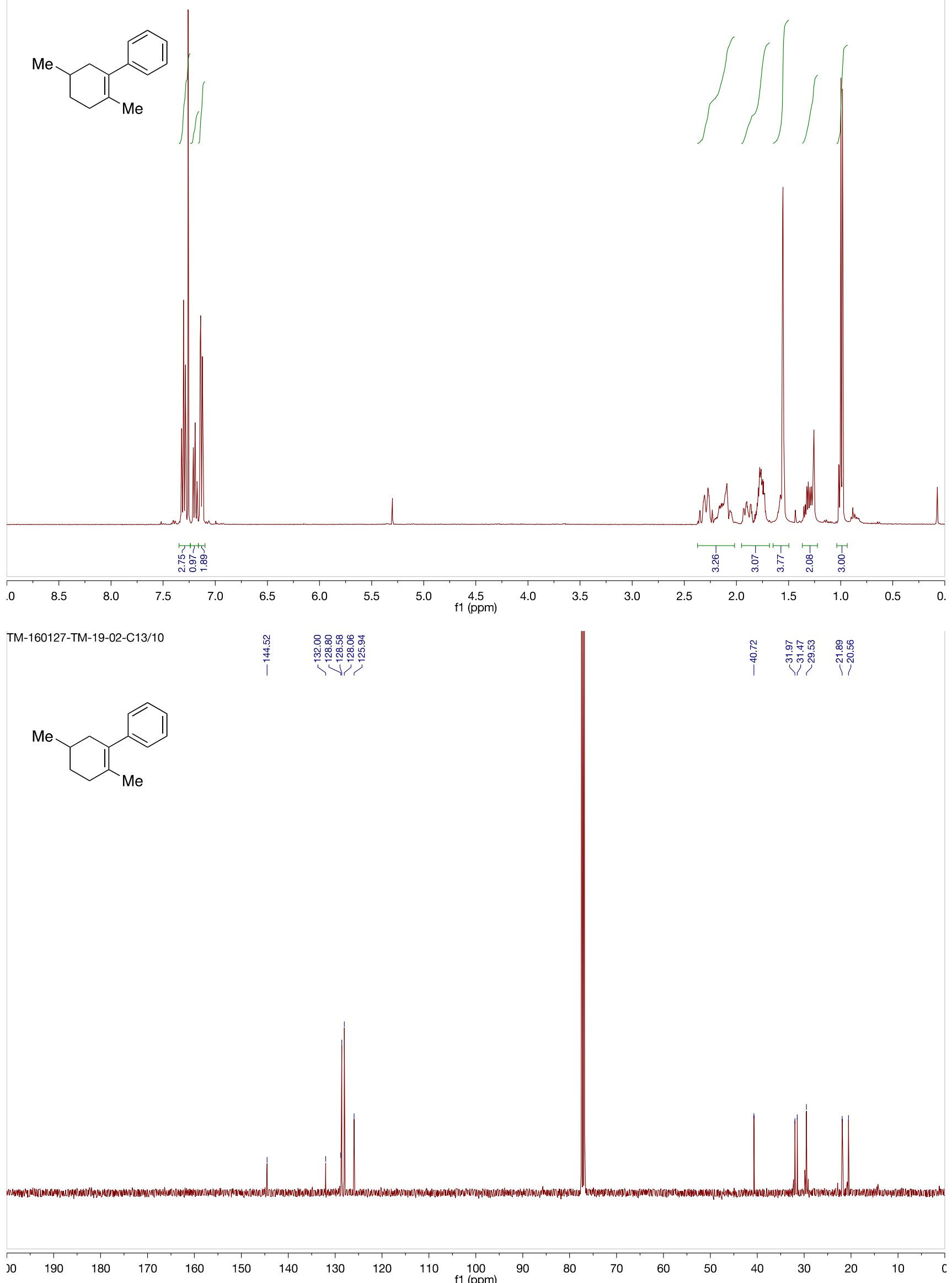

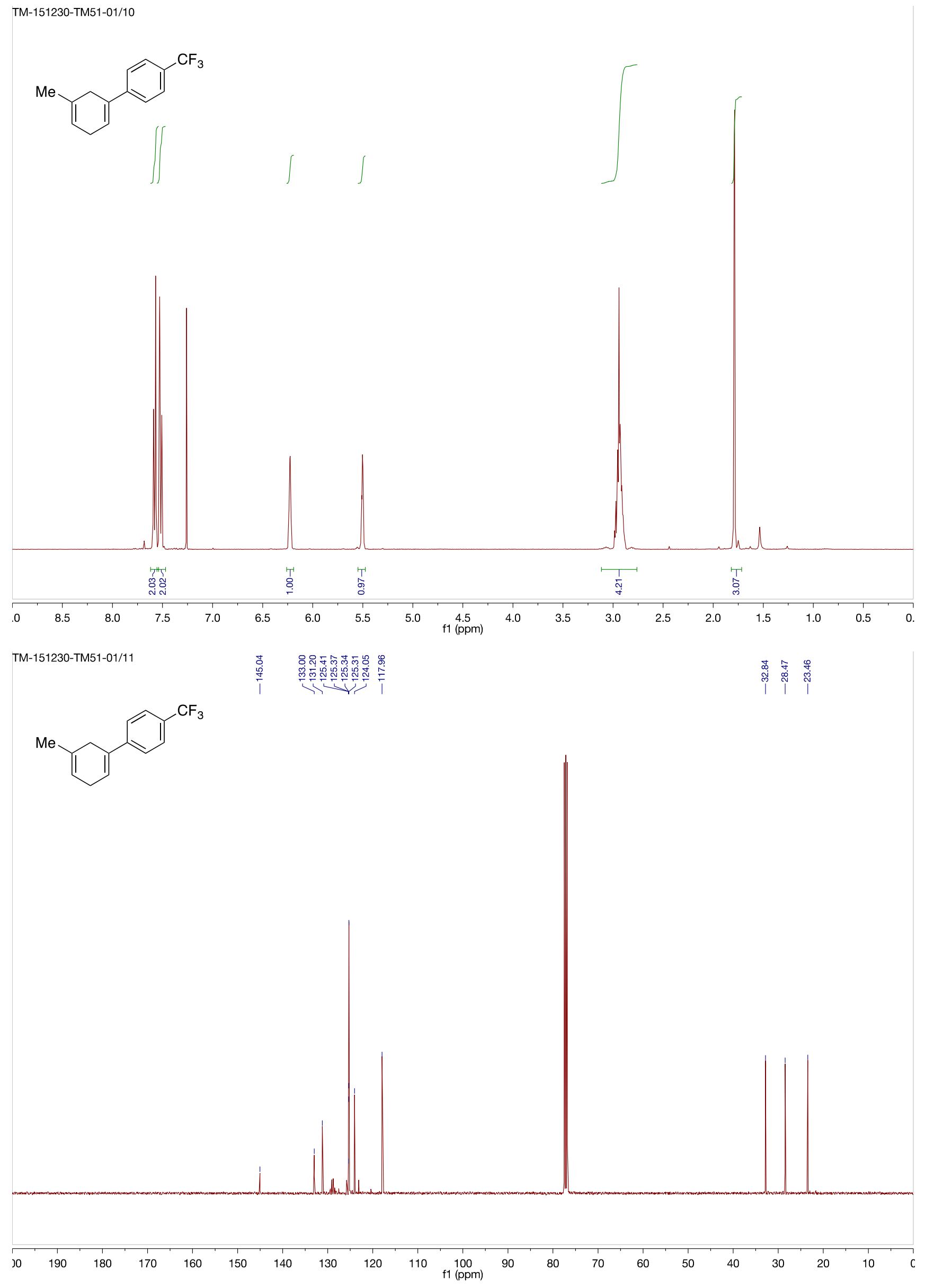

S74 


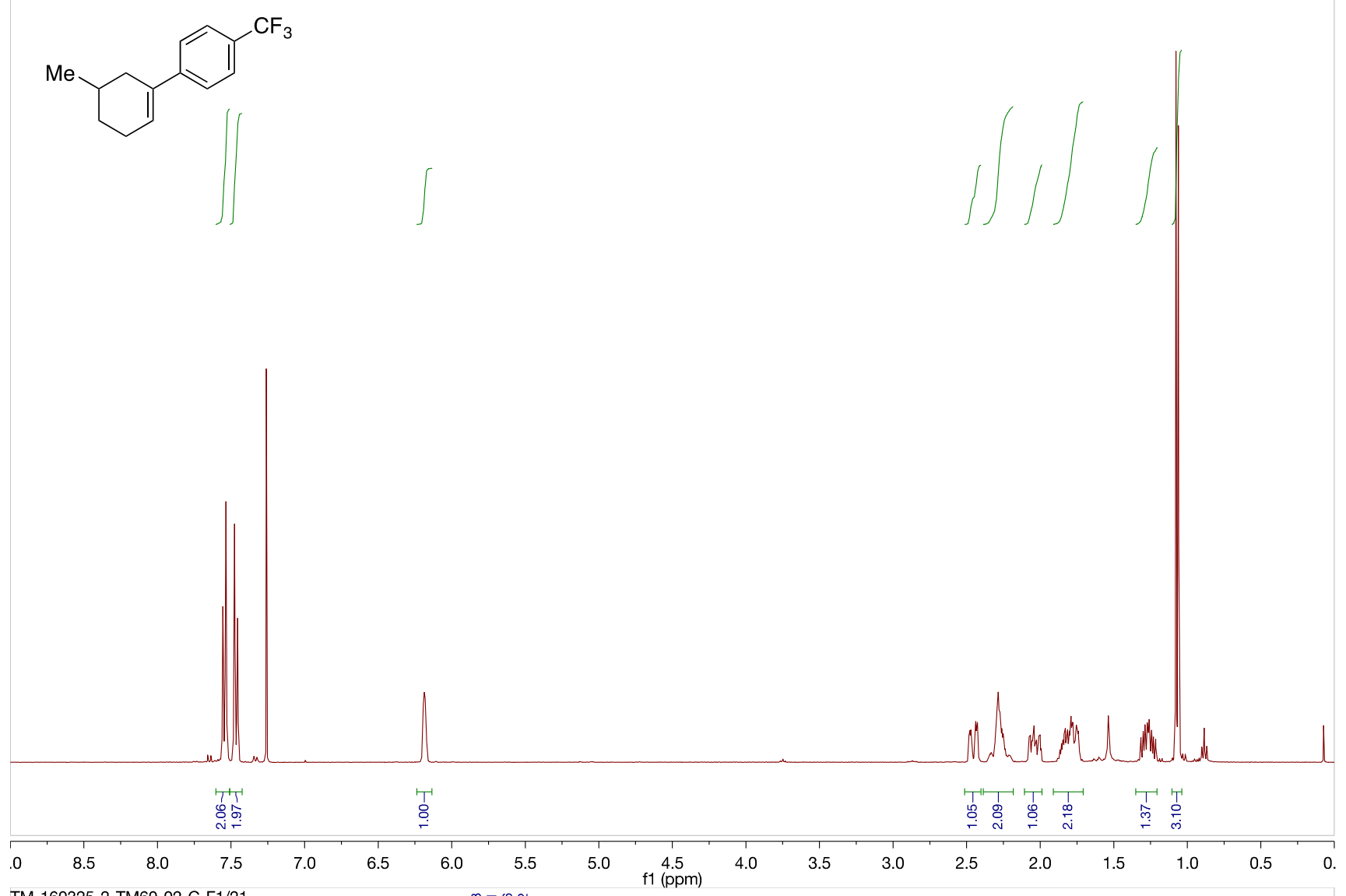

TM-160325-2-TM60-02-C-F1/21<smiles>CC1CCC=C(c2ccc(C(F)(F)F)cc2)C1</smiles>

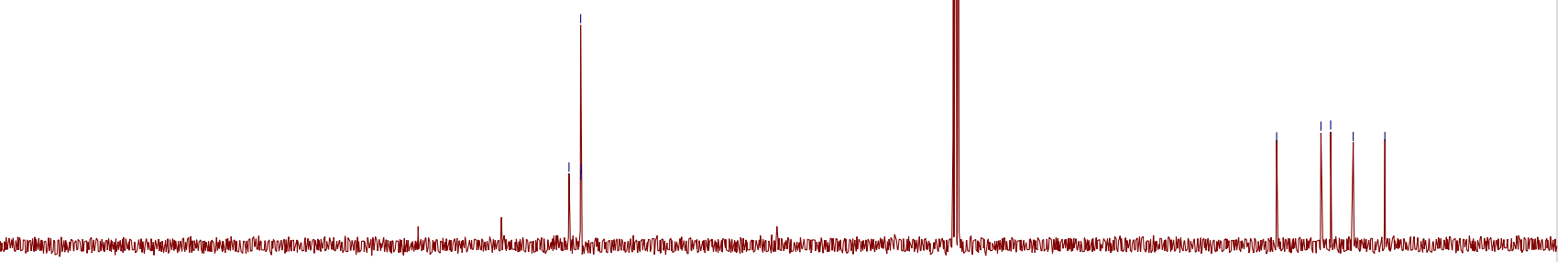

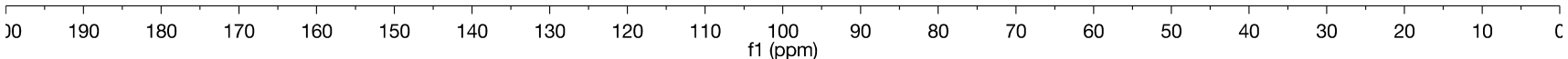



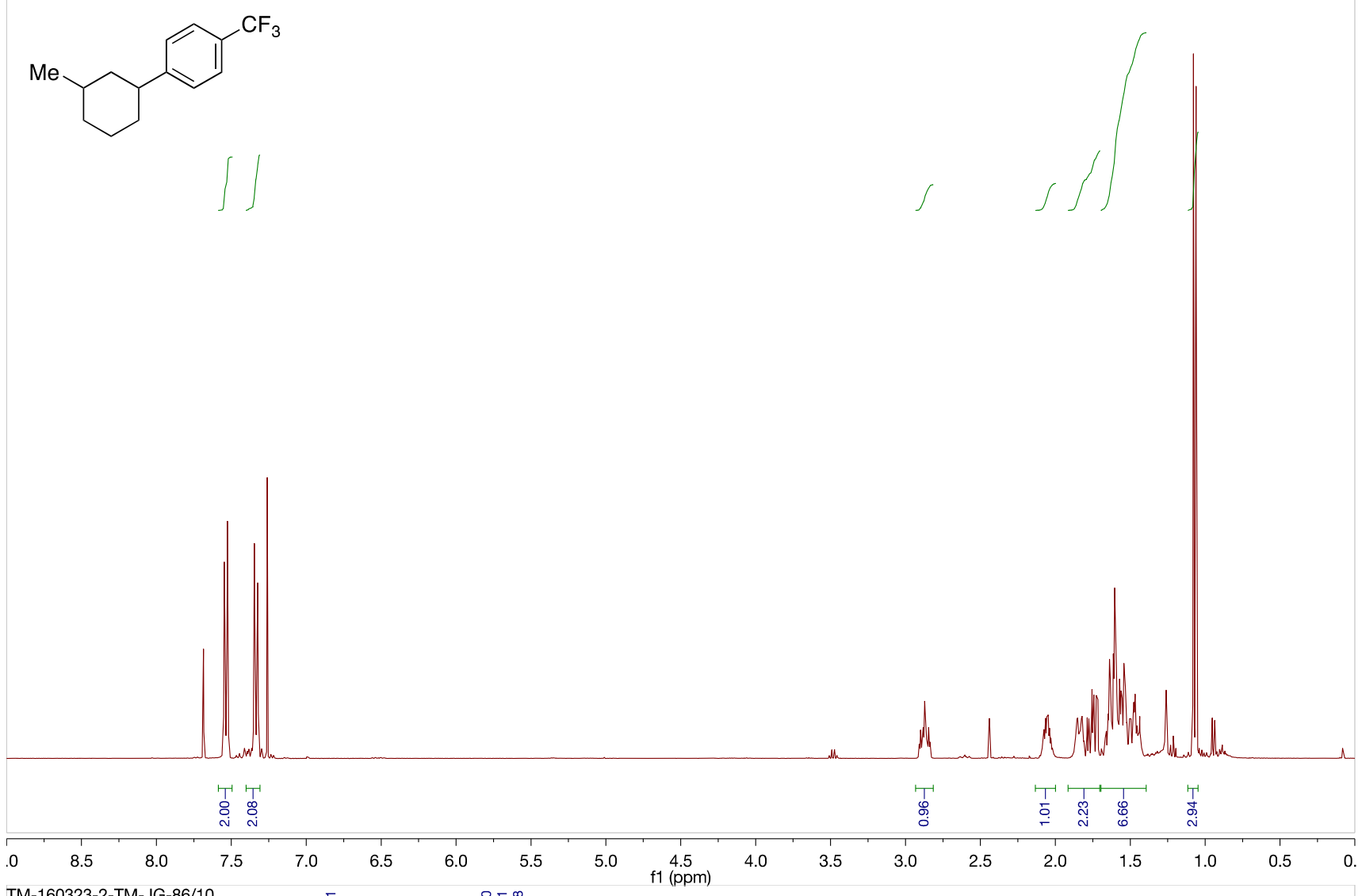
TM-160323-2-TM-JG-86/10 $\frac{\bar{\infty}}{\frac{\infty}{5}}$ \begin{tabular}{l}
5 \\
\hline \\
\hline
\end{tabular}

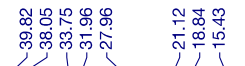<smiles>CC1CCCC(c2ccc(C(F)(F)F)cc2)C1</smiles>

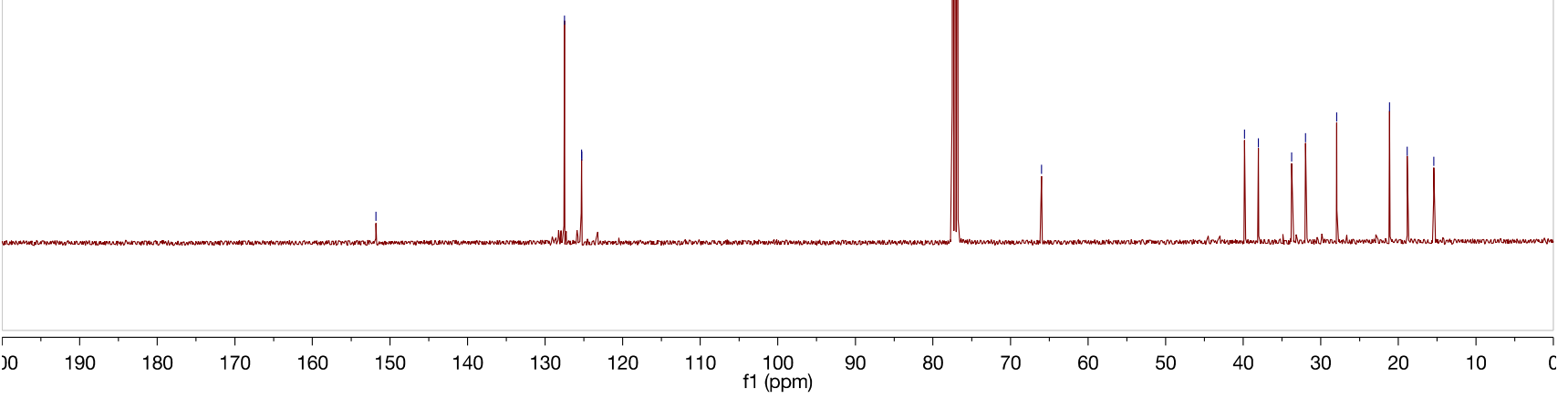


TM-151119-2-TM31-01-P/12

Product
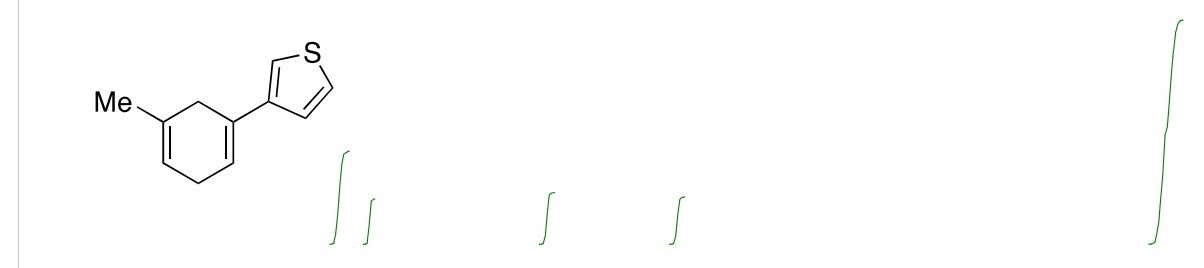

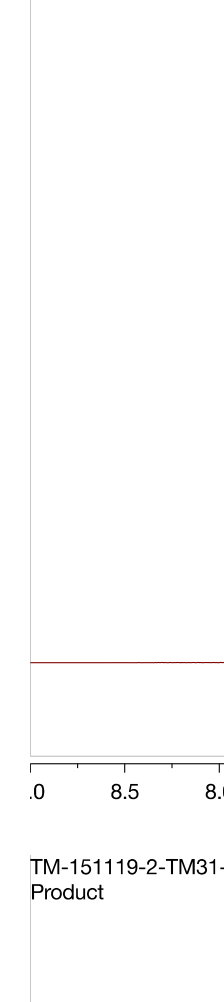

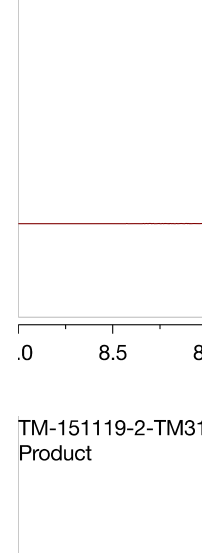

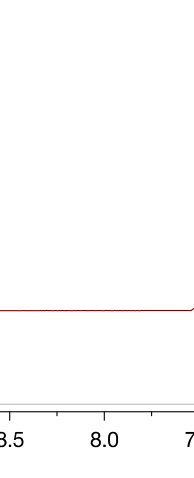

$$
\begin{array}{ll}
\hline \\
\hline \\
\text { TM-1511 } \\
\text { Product } \\
\end{array}
$$


TM-160325-2-TM58-02/10

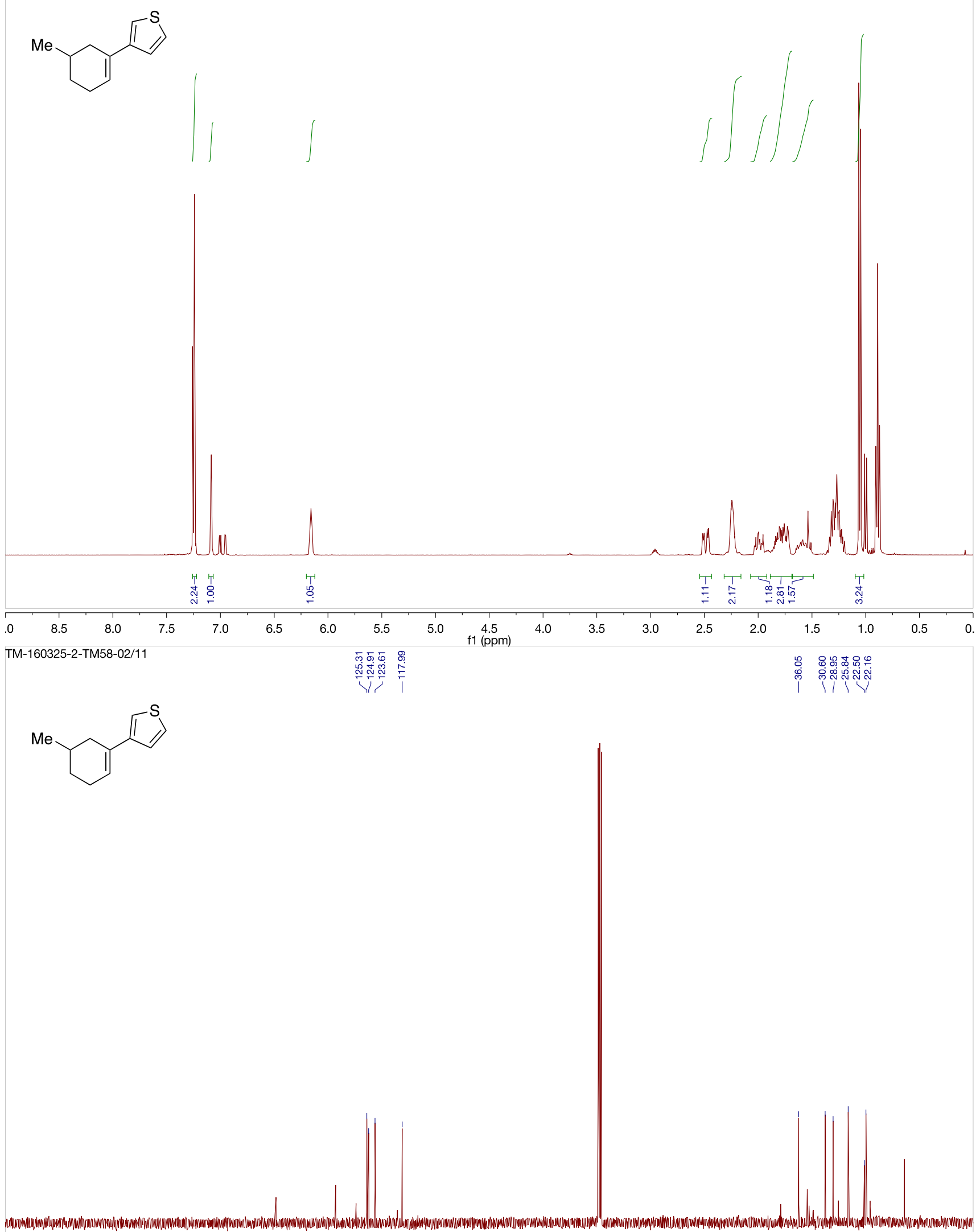

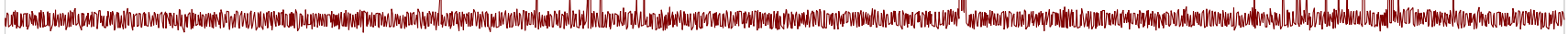

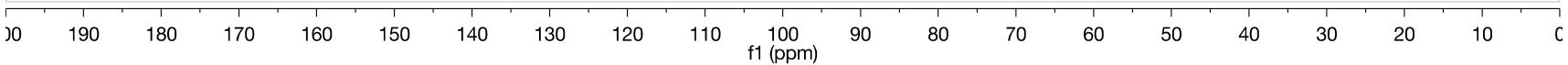


<smiles>CCC1=C(CC)CC(C)CC1</smiles>
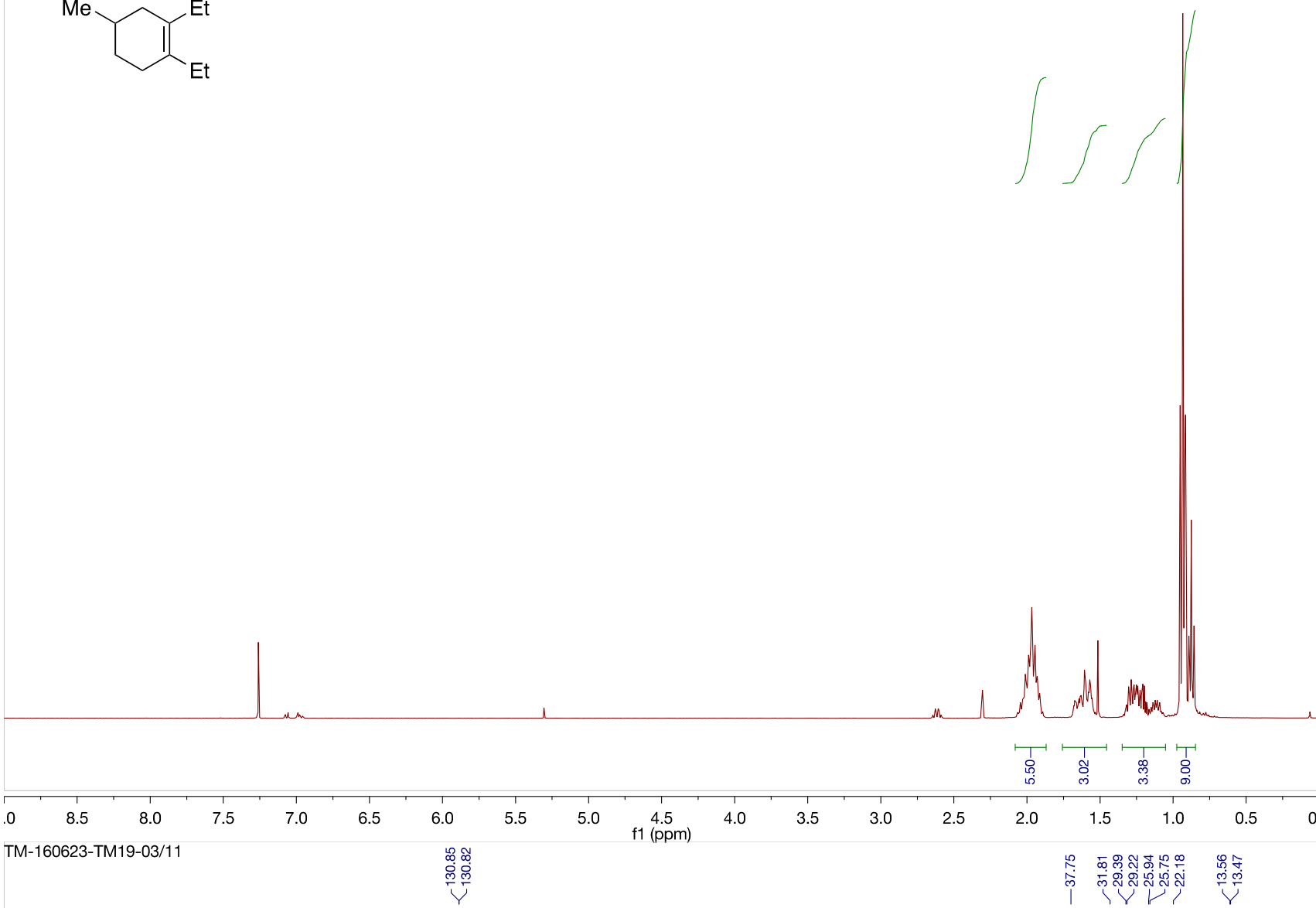<smiles>CCC1=C(CC)CC(C)CC1</smiles>

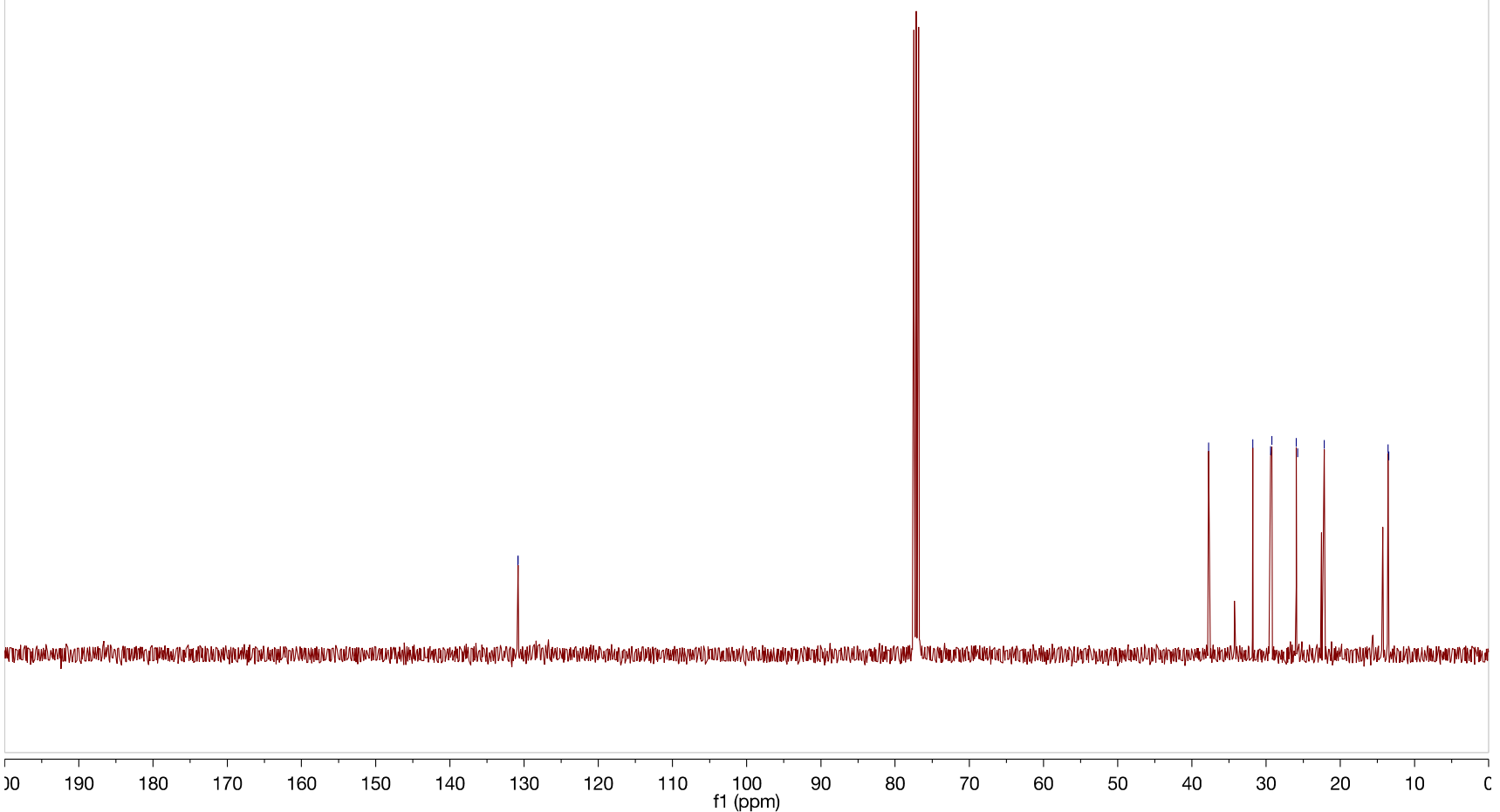


<smiles>CCC1=C(CC)CC(C)=CC1</smiles>
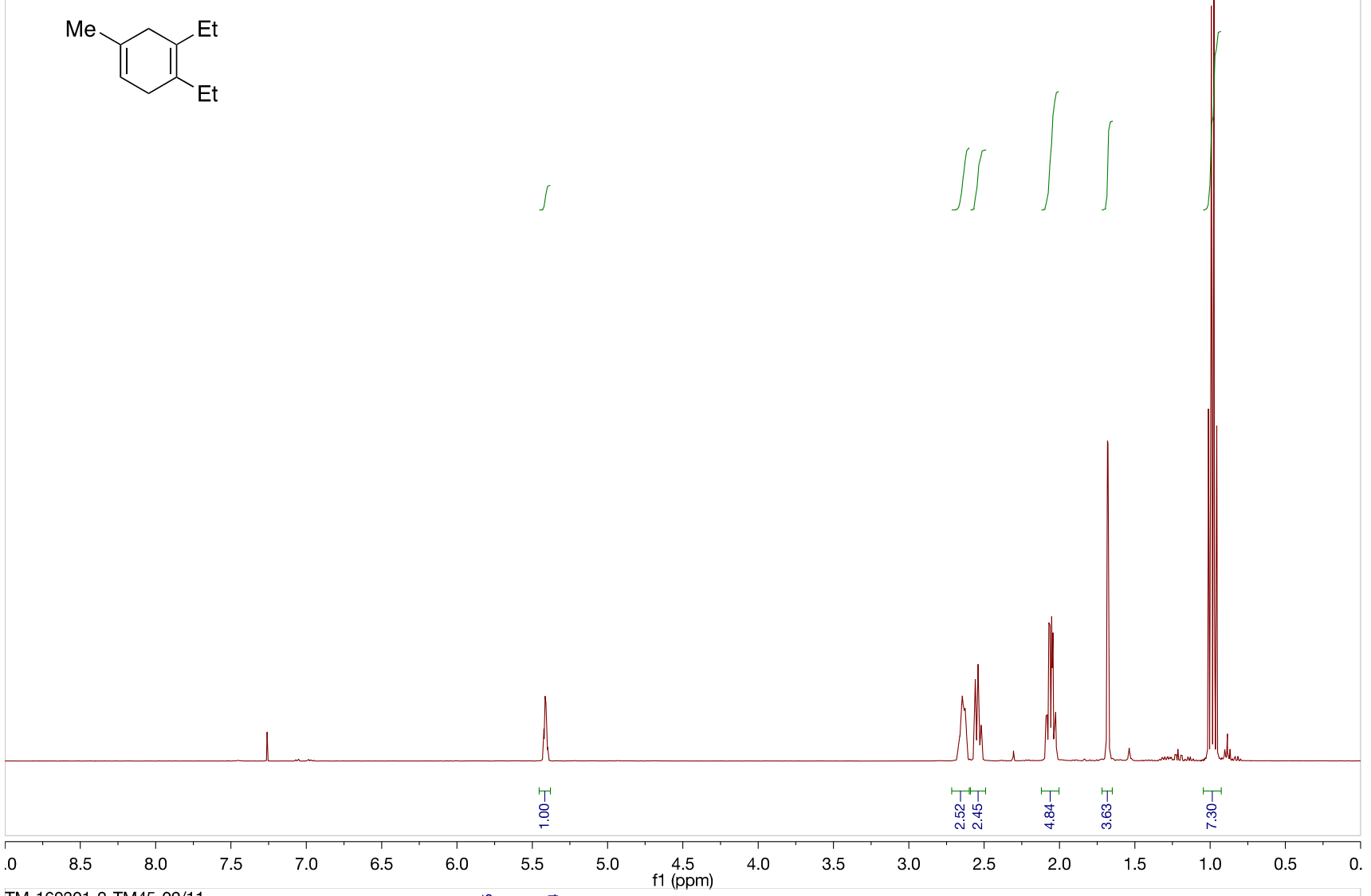
TM-160301-2-TM45-02/11

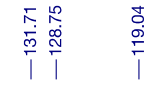
|<smiles>CCC1=C(CC)CC(C)=CC1</smiles>

$$
30
$$




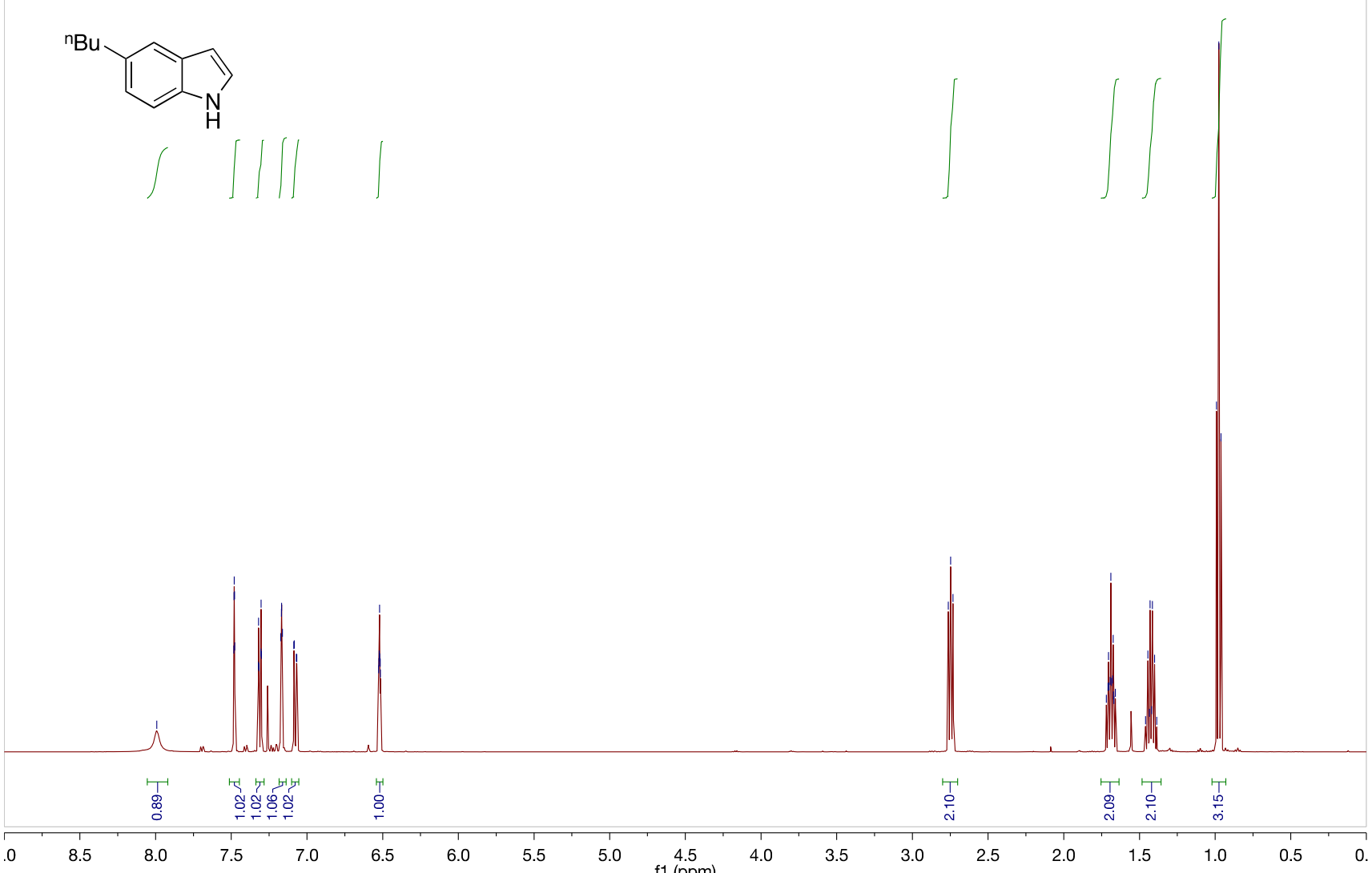
jg5-56-p (c h)/11 jg5-56-p (c h)

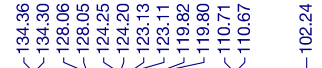

nBu<smiles>Cc1ccc2[nH]ccc2c1</smiles>

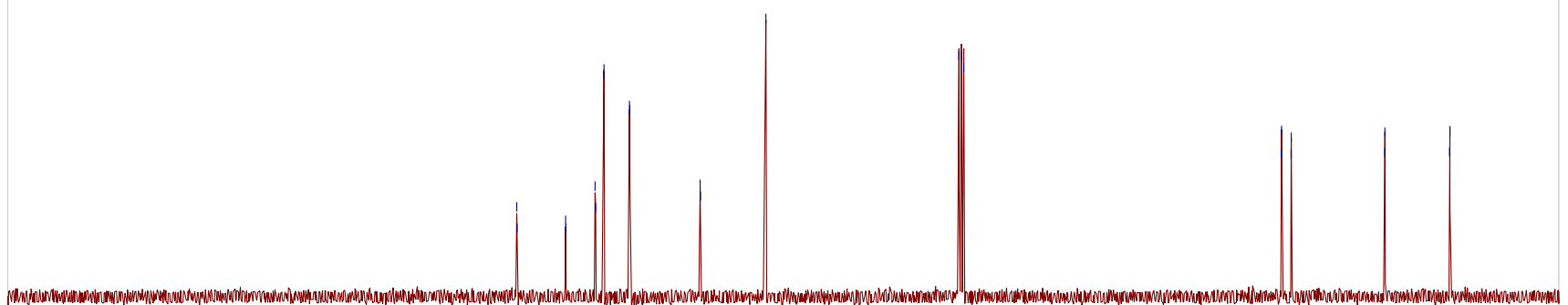

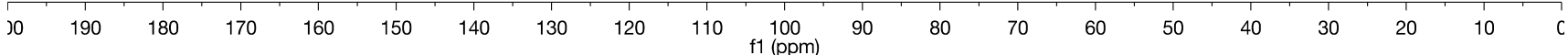


nBu<smiles>CC1=CCc2[nH]ccc2C1</smiles><smiles>C1CCC2CCCC2C1</smiles><smiles>C=CCC</smiles>
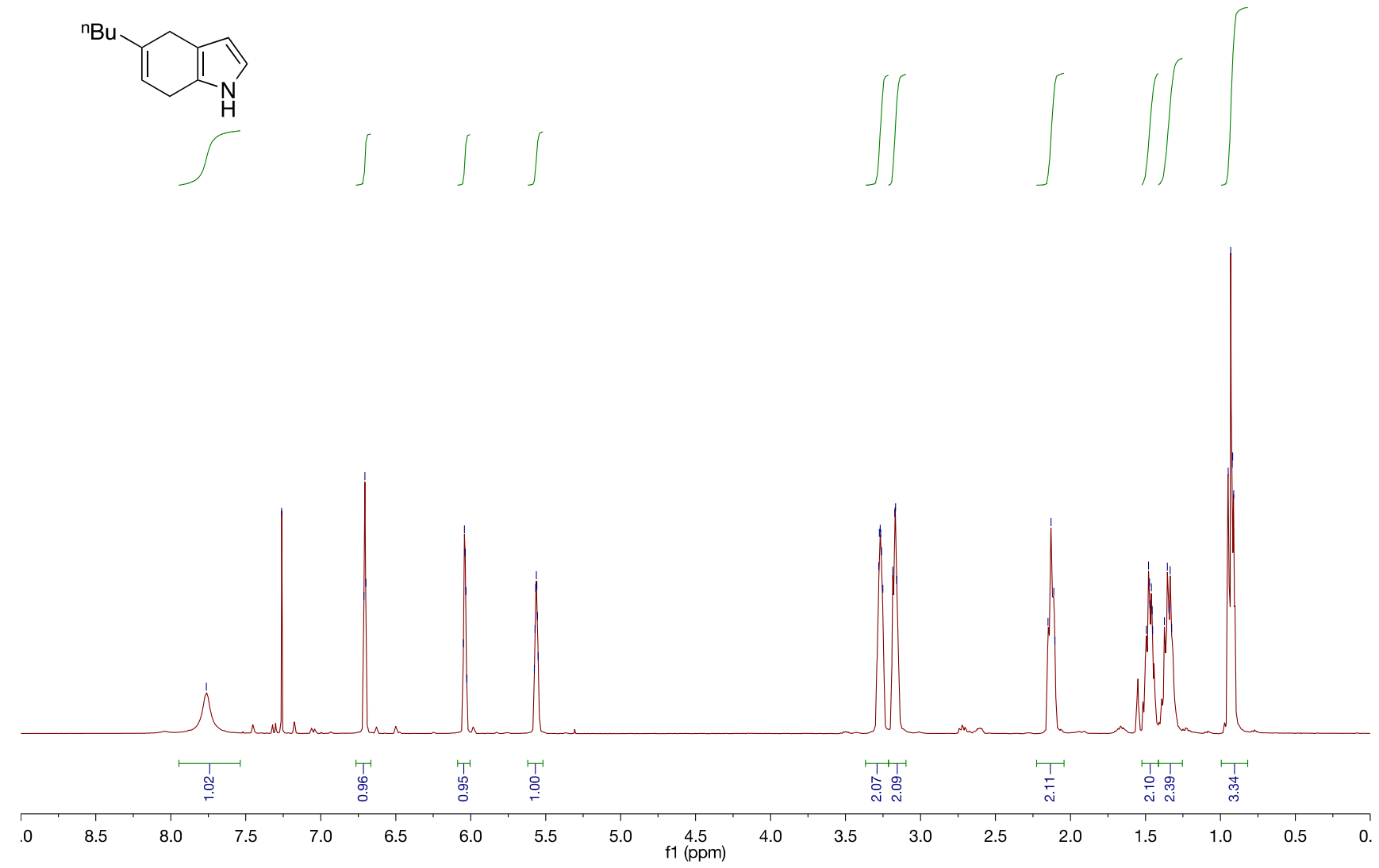

jg5-65-p C H/11 JG5-65-P C H

nBu<smiles>CC1=CCc2[nH]ccc2C1</smiles>

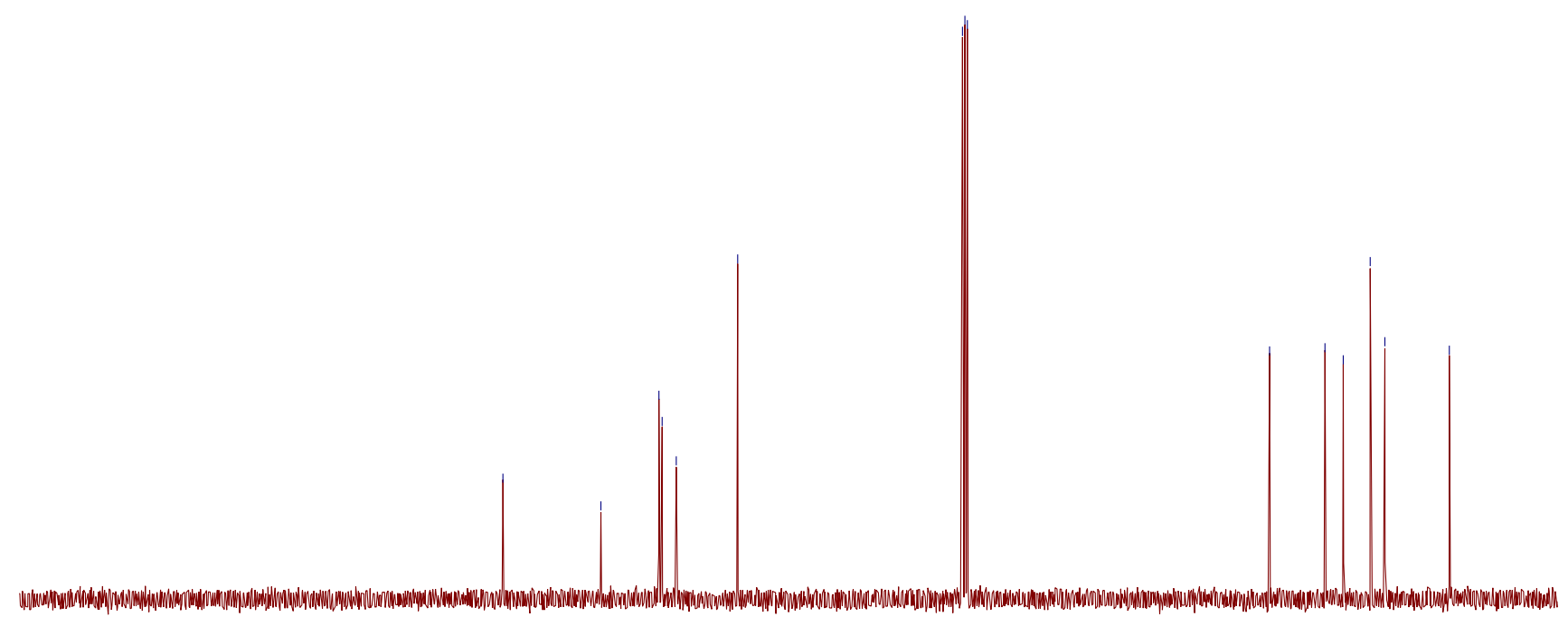

$30 \quad 190$ $180 \quad 170-160$ 150140 $130 \quad 120$ $10 \quad 100$ $90 \quad 80$ $80+70+60$ $60 \quad 50$ 1 


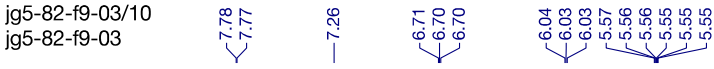<smiles>Oc1ccc2[nH]ccc2c1</smiles>
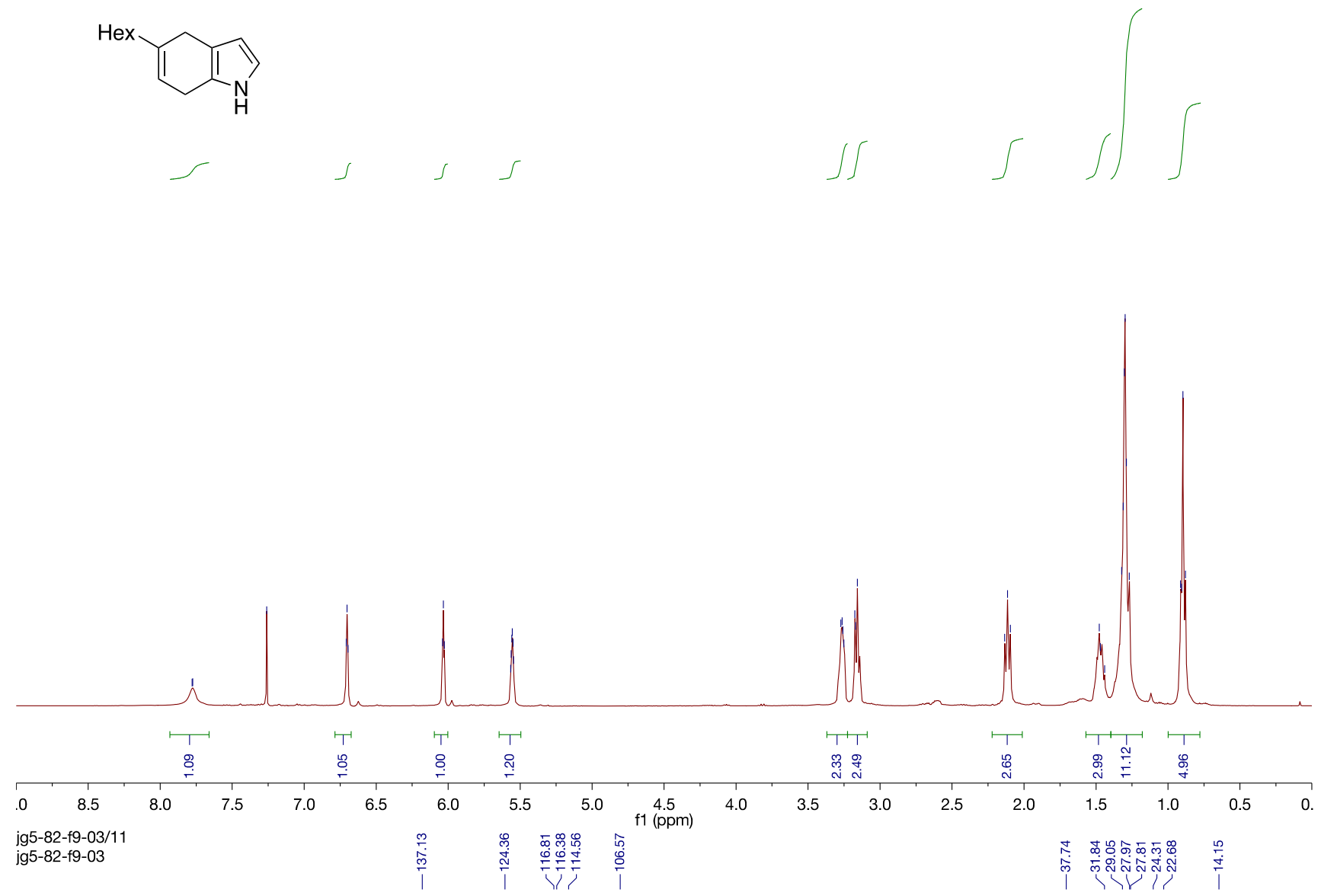

Hex

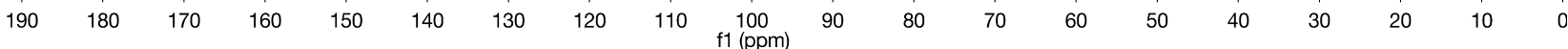




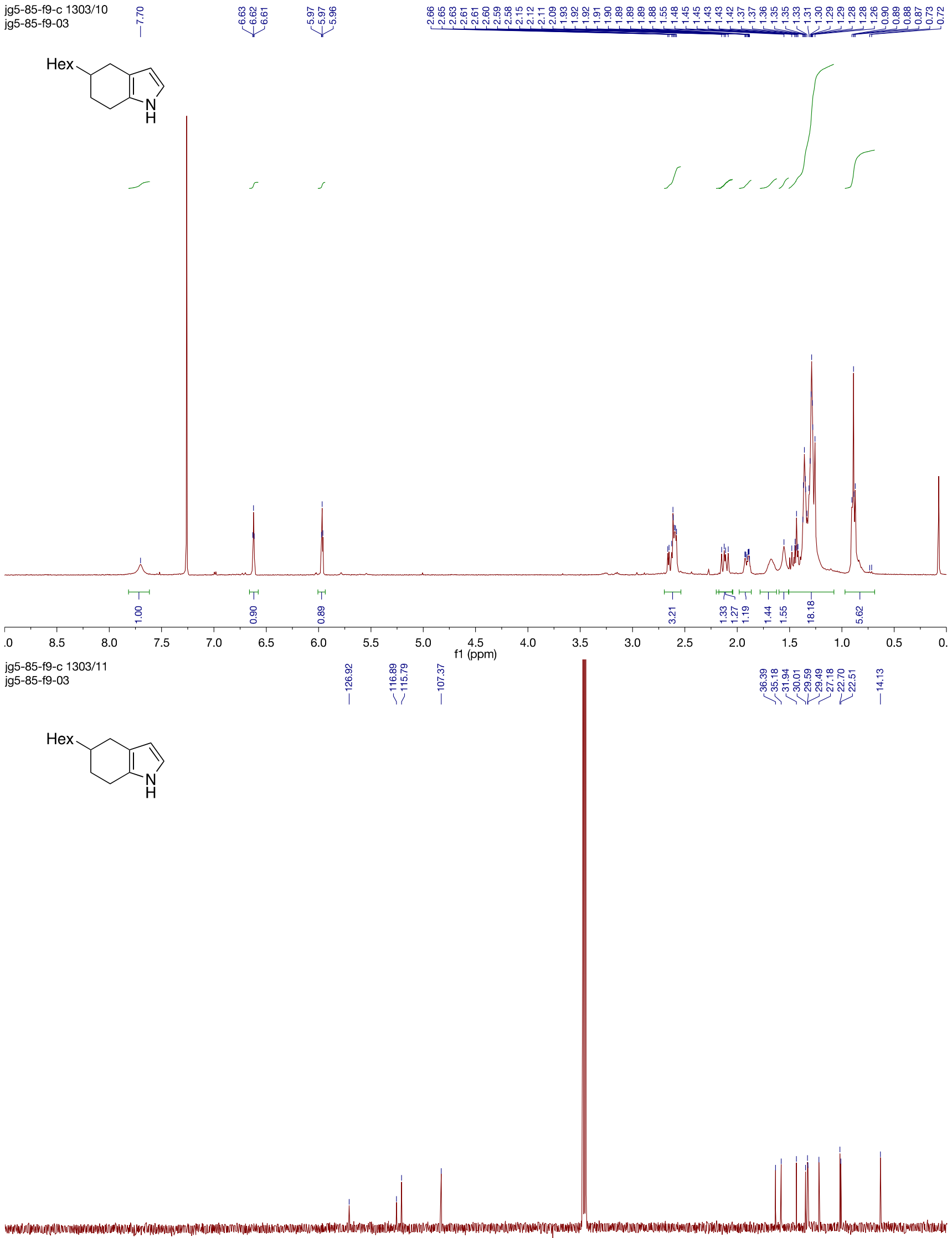

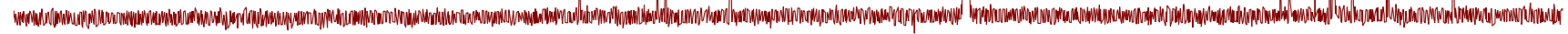

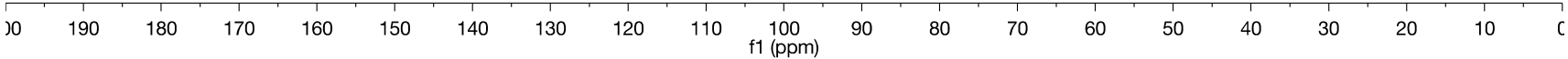




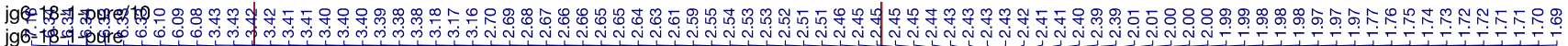
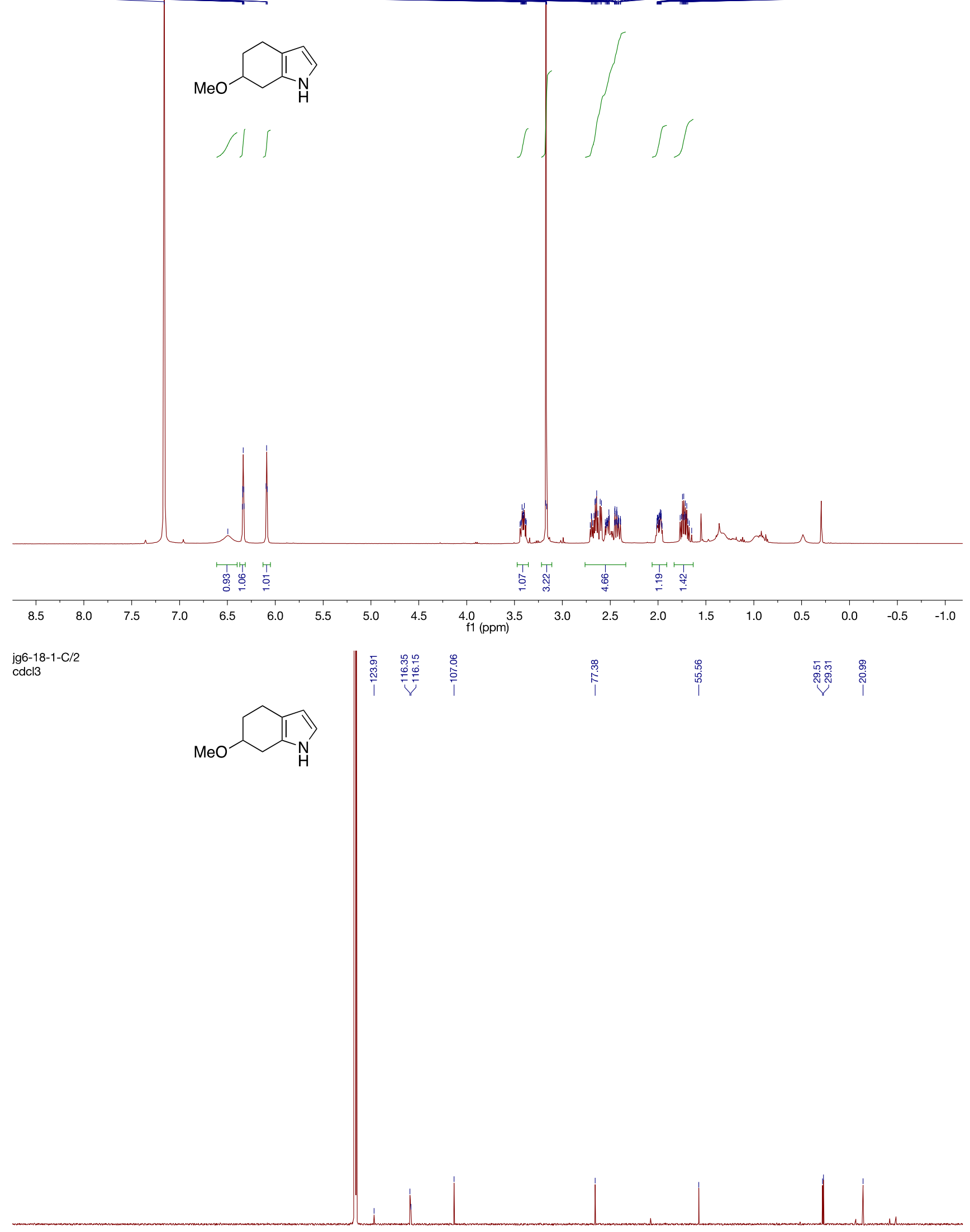

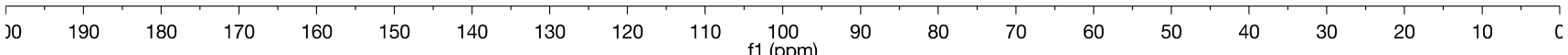




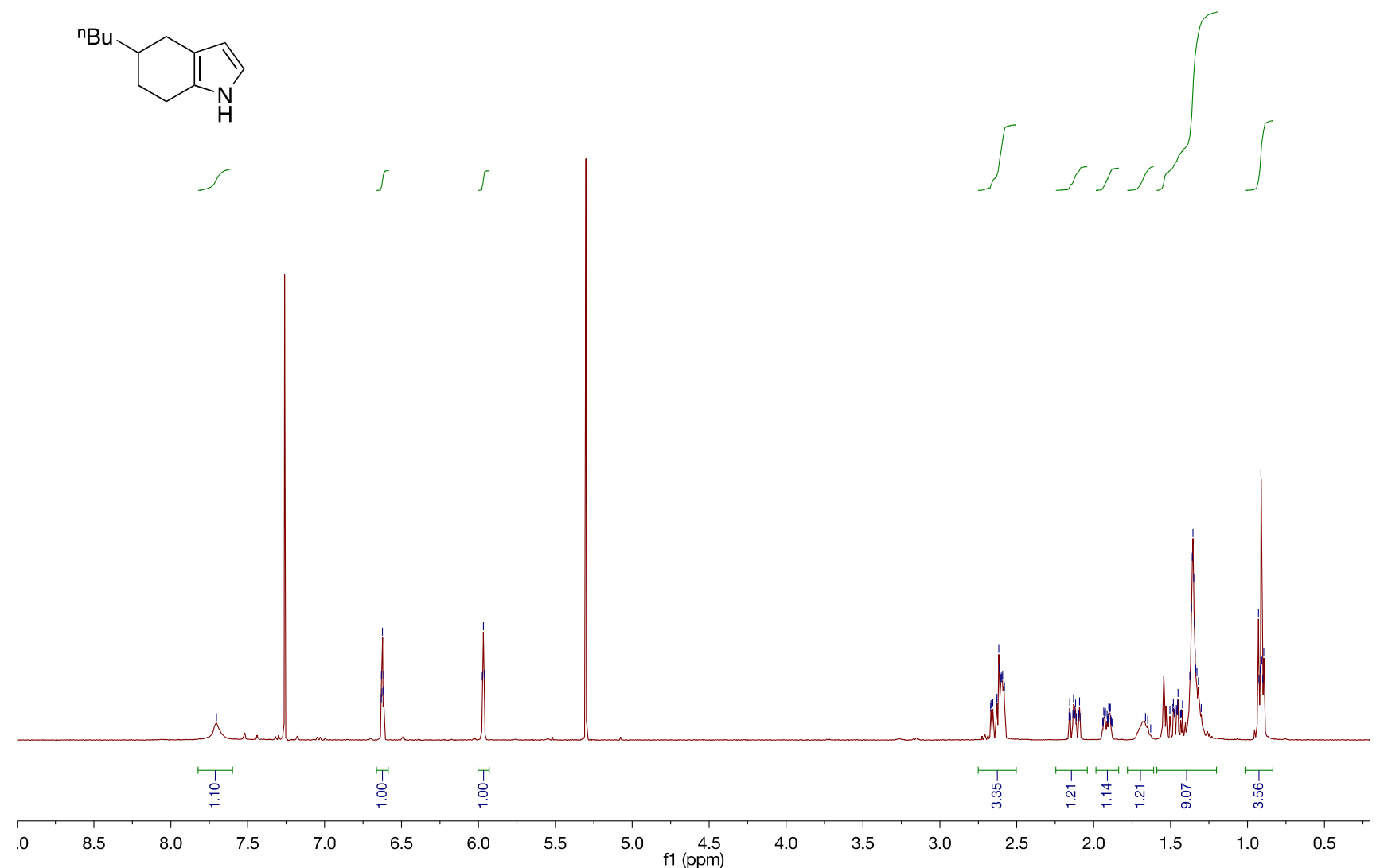

jg5-66-5 $p(\mathrm{CH}) / 11$ jg5-66-5 C h

nBu<smiles>CC1CCc2[nH]ccc2C1</smiles>

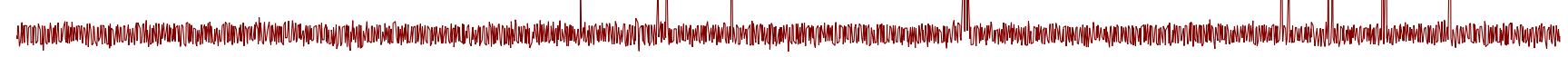

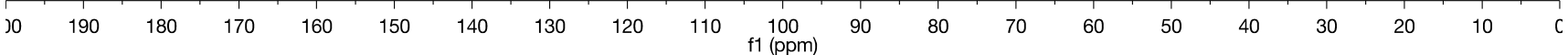




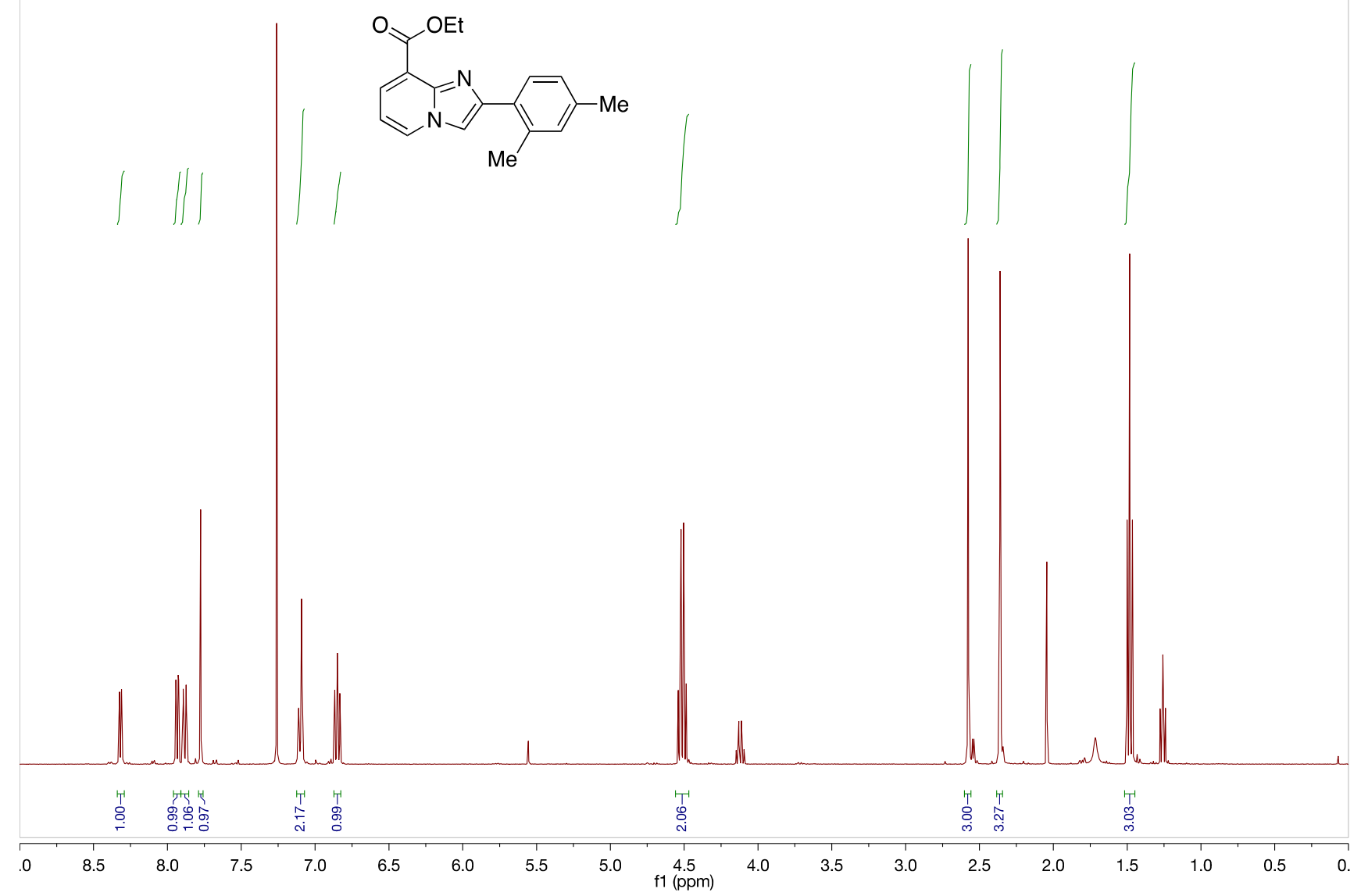

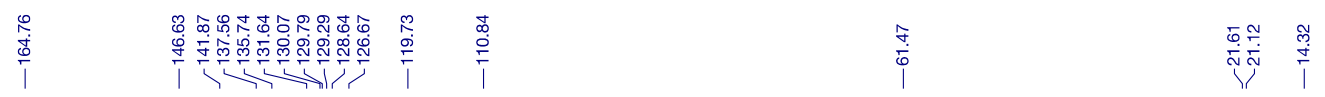
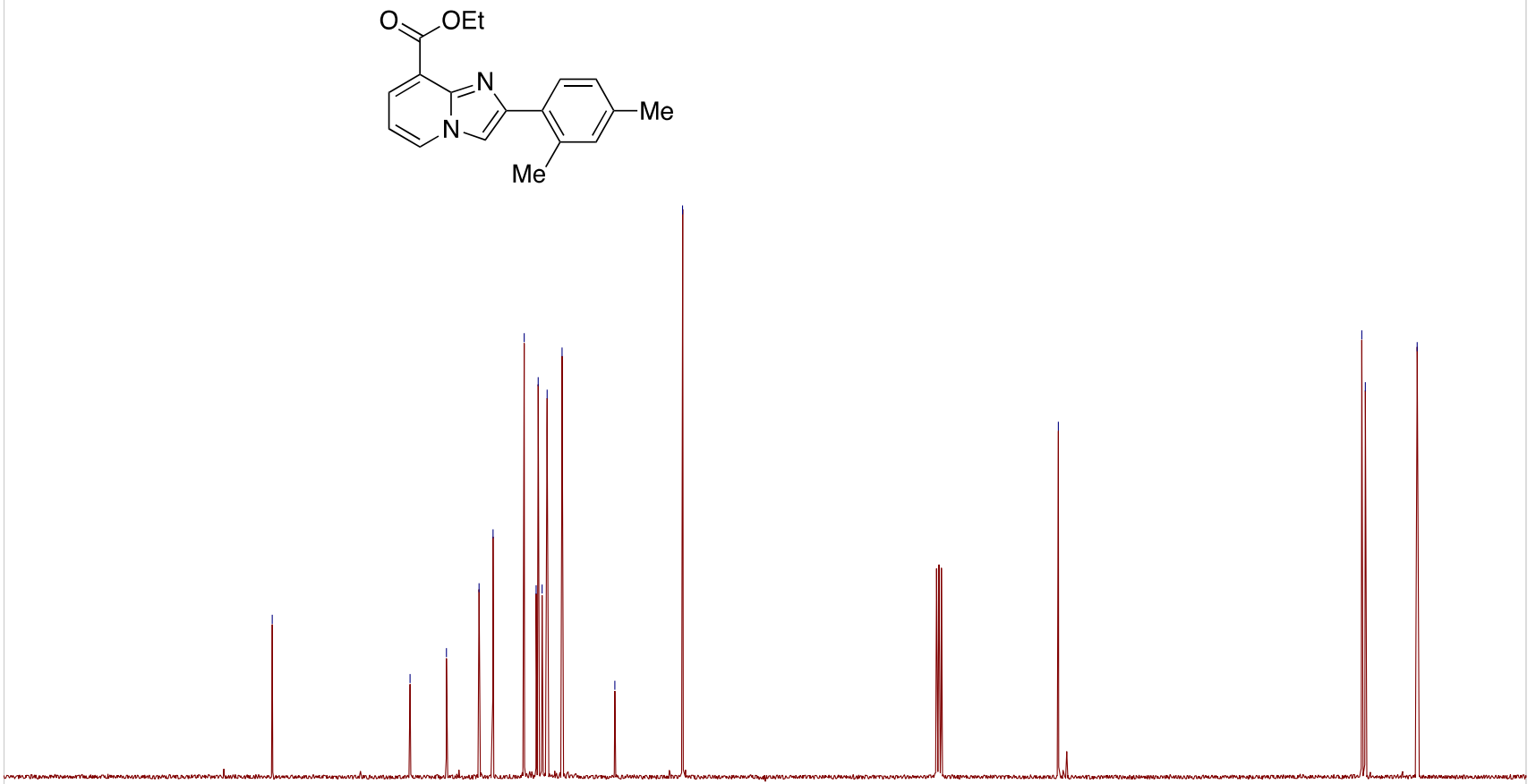

J0 190
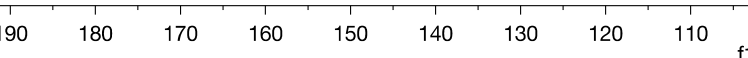

$\begin{array}{ccccc}100 & 90 & 80 & 70 & 60\end{array}$

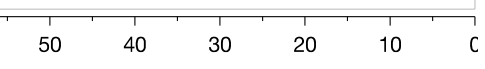



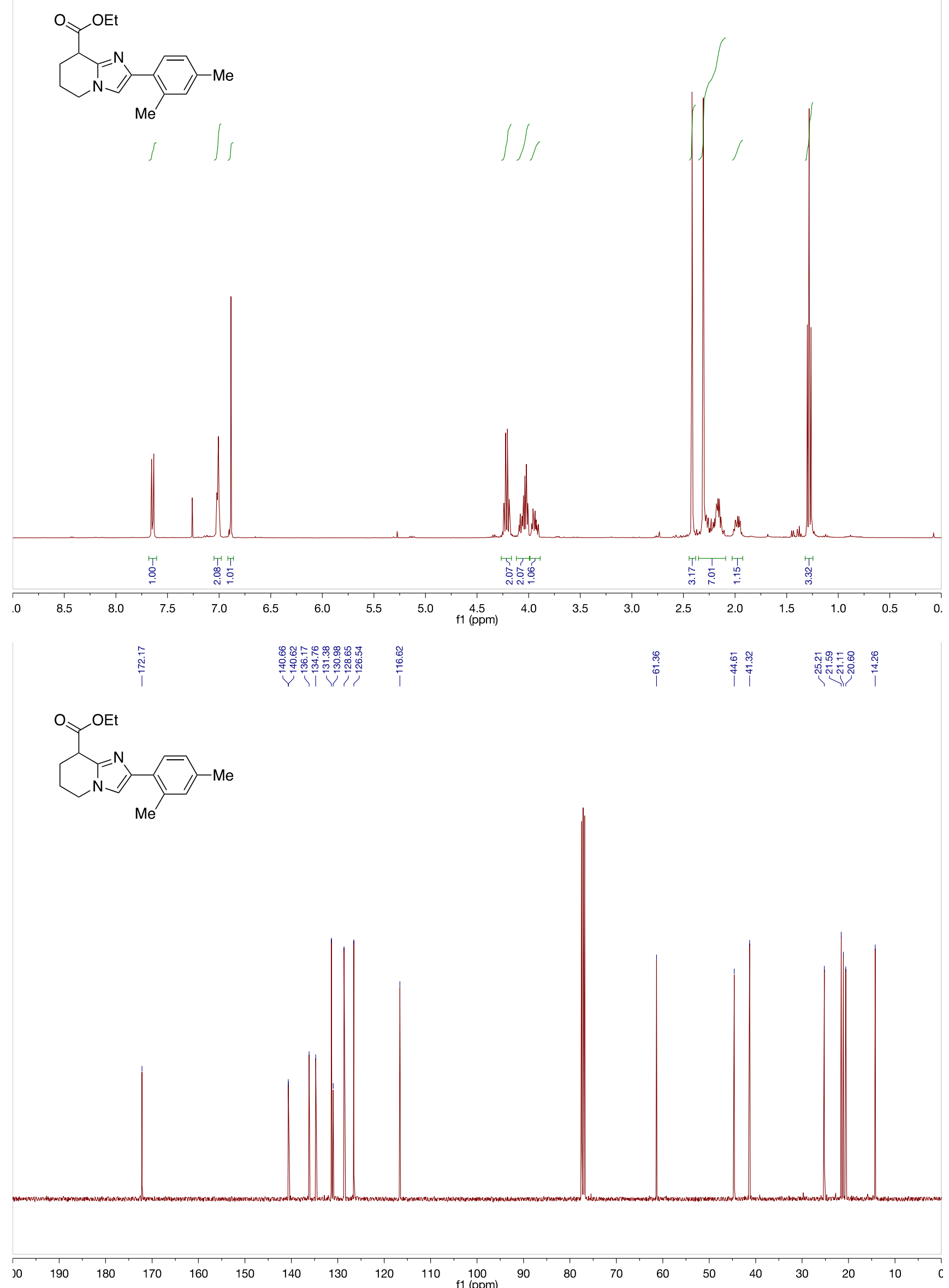


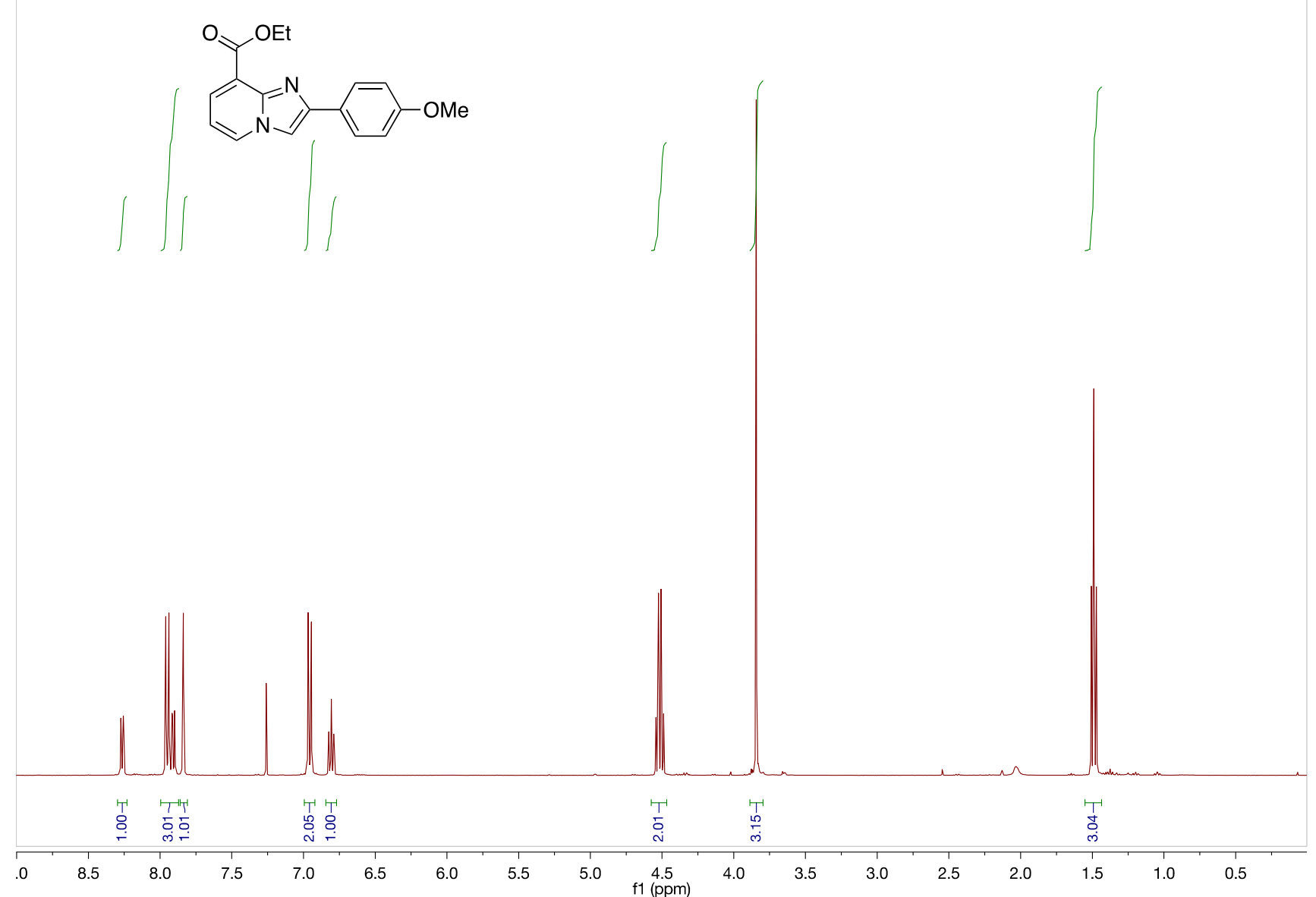

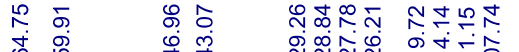
尚

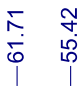
$\stackrel{\substack{i \\ i}}{\stackrel{\infty}{+}}$
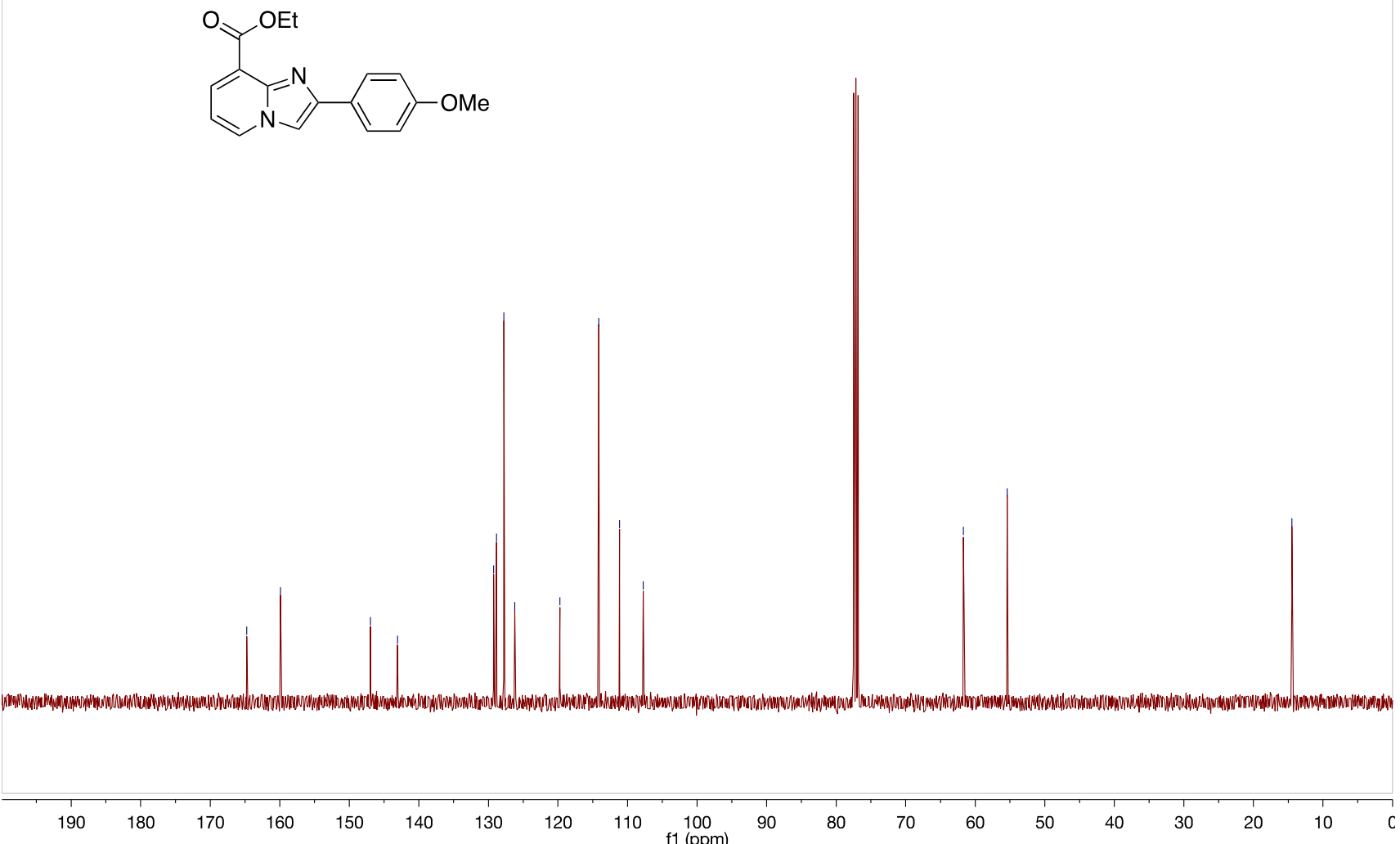

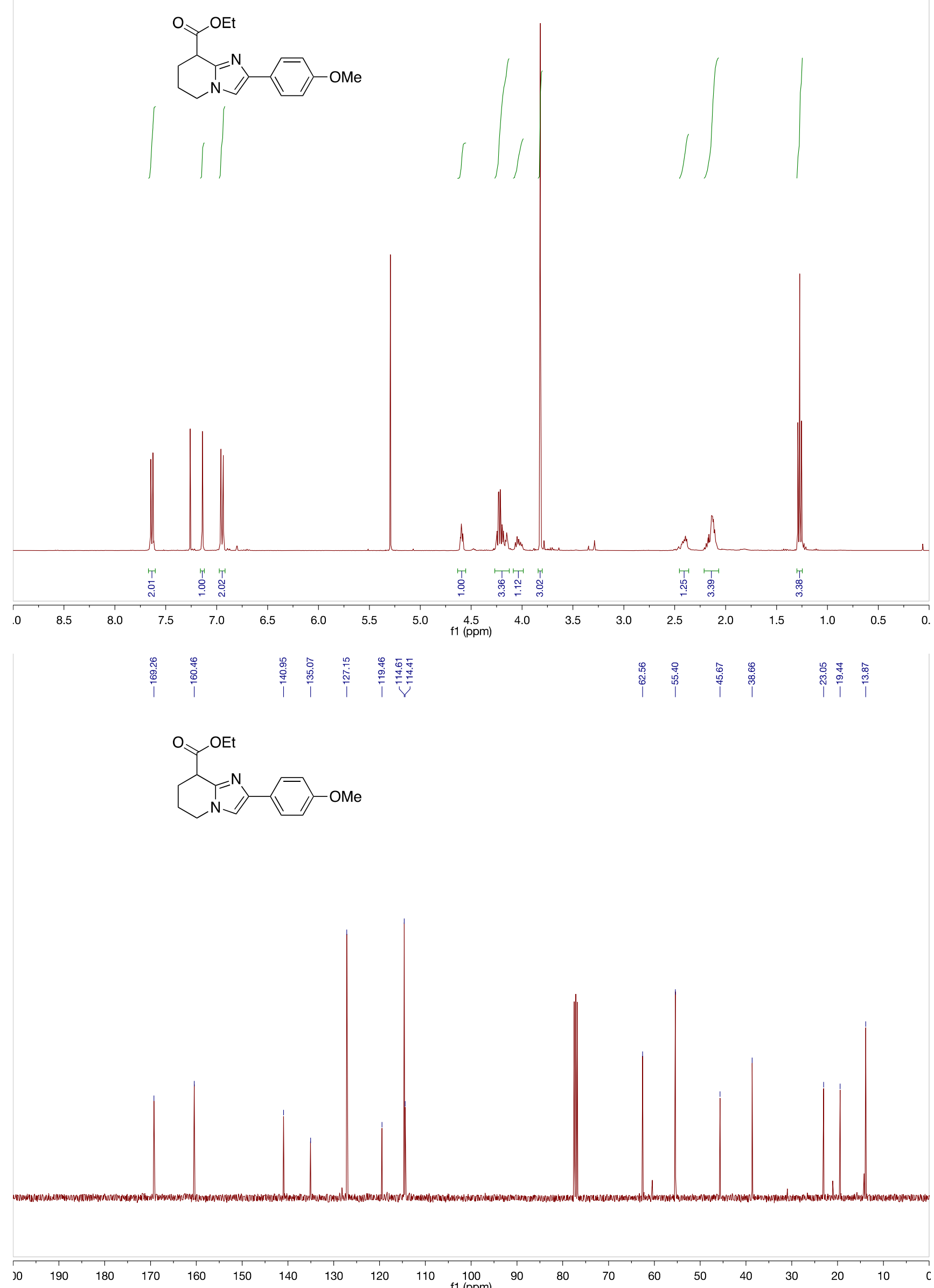

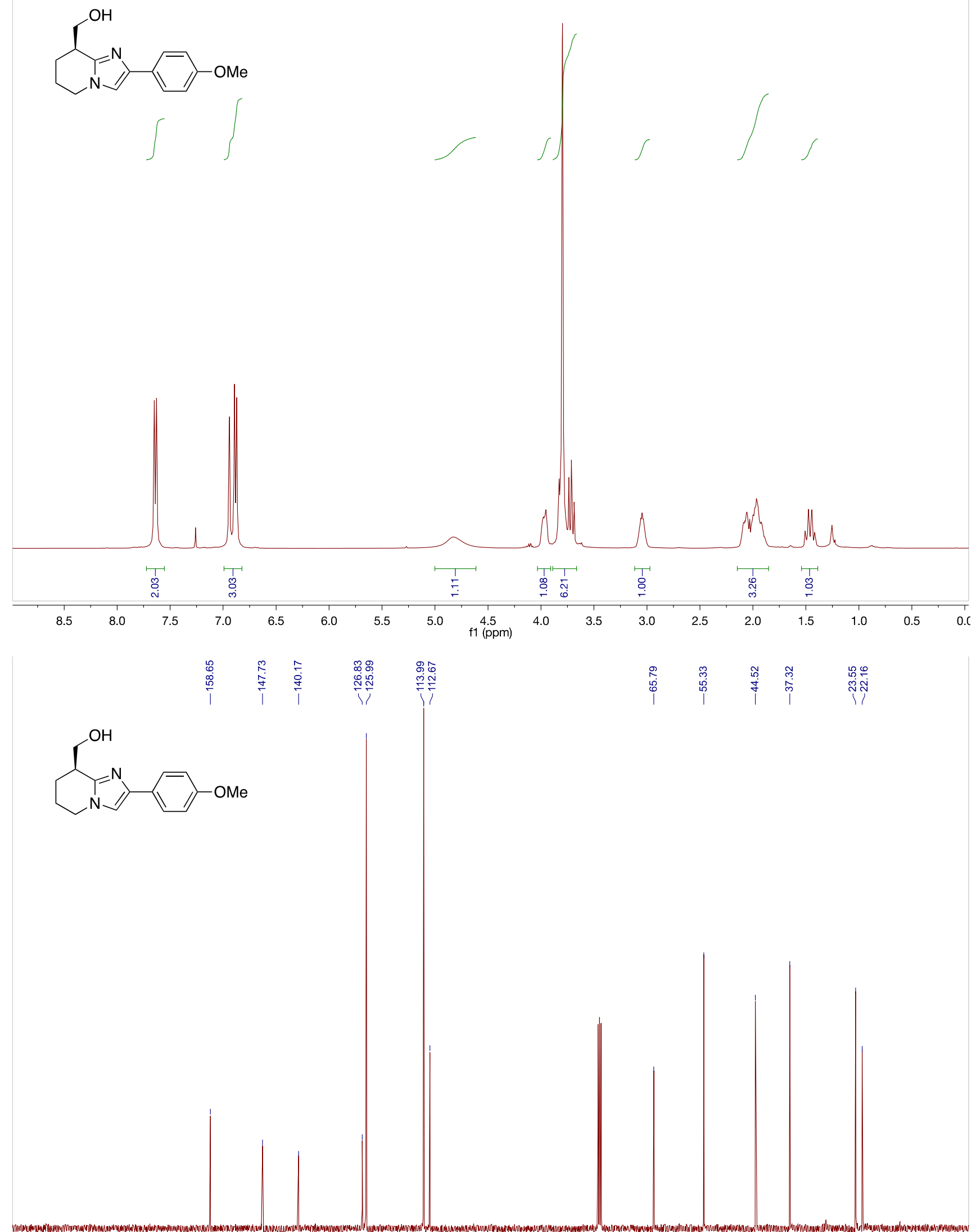

$\begin{array}{lllllllllllllllllllll}30 & 190 & 180 & 170 & 160 & 150 & 140 & 130 & 120 & 110 & \begin{array}{c}100 \\ \mathrm{f} 1(\mathrm{ppm})\end{array} & 90 & 80 & 70 & 60 & 50 & 40 & 30 & 20 & 10 & \mathrm{C}\end{array}$ 

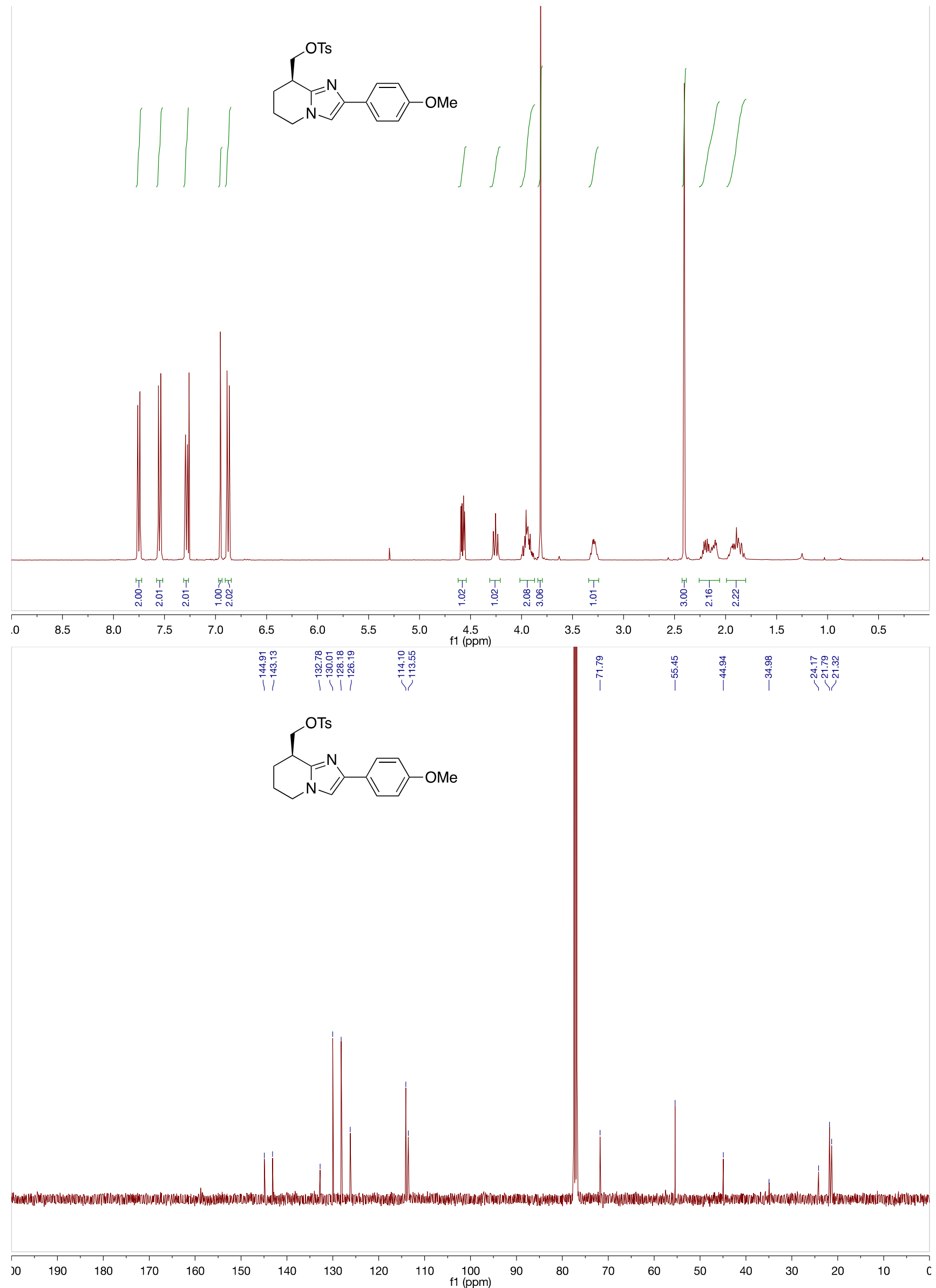


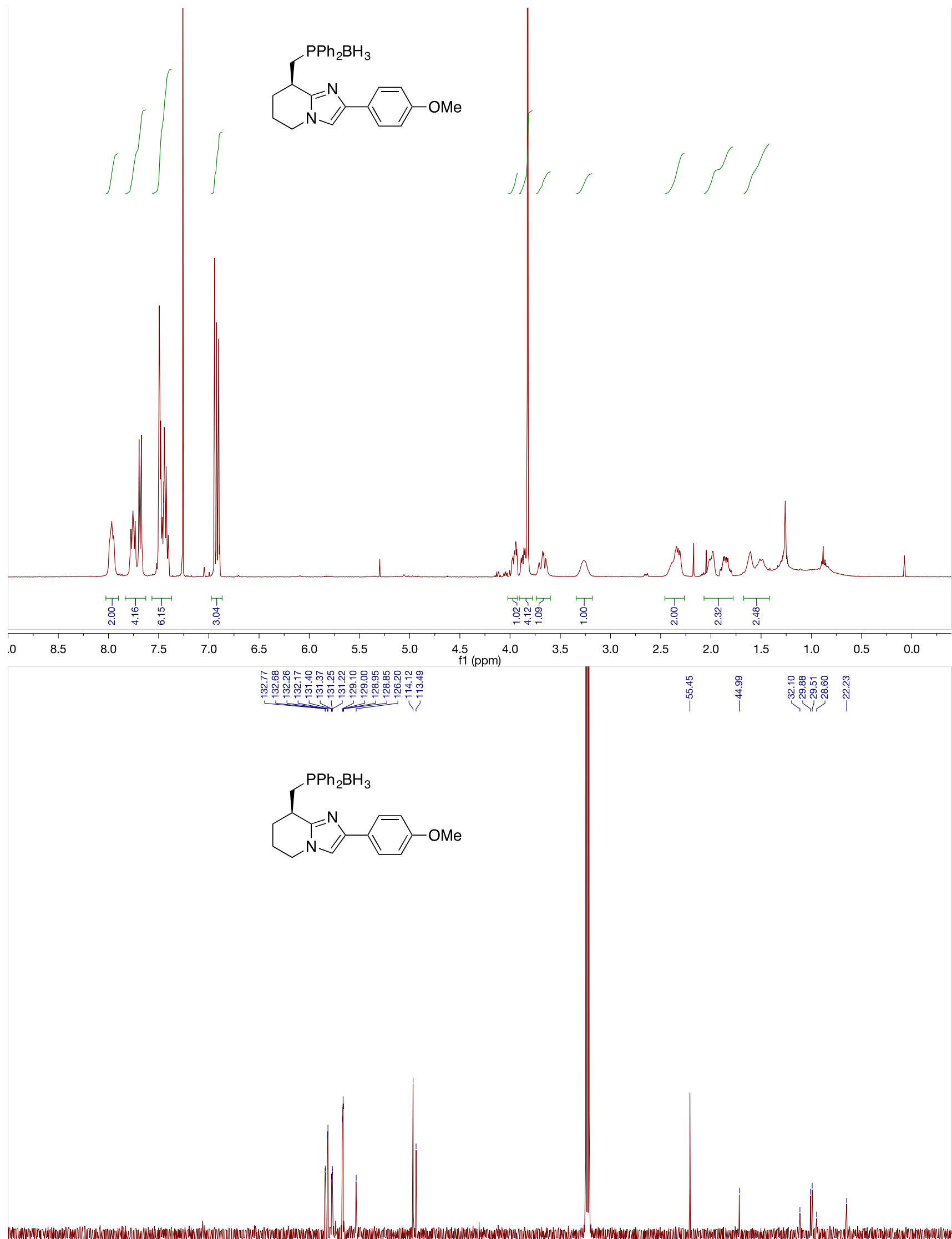

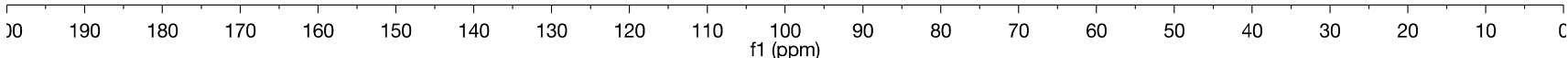


<smiles>COc1ccc(-c2cn3c(n2)C(CP)CCC3)cc1</smiles>

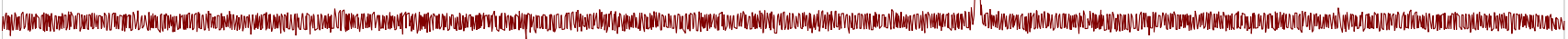
$\begin{array}{lllllllllllllllllllllllllllll}190 & 180 & 170 & 160 & 150 & 140 & 130 & 120 & 110 & 100 & 90 & 80 & 70 & 60 & 50 & 40 & 30 & 20 & 10 & 0 & -10 & -20 & -30 & -40 & -50 & -60 & -70 & -80 & -90\end{array}$ 


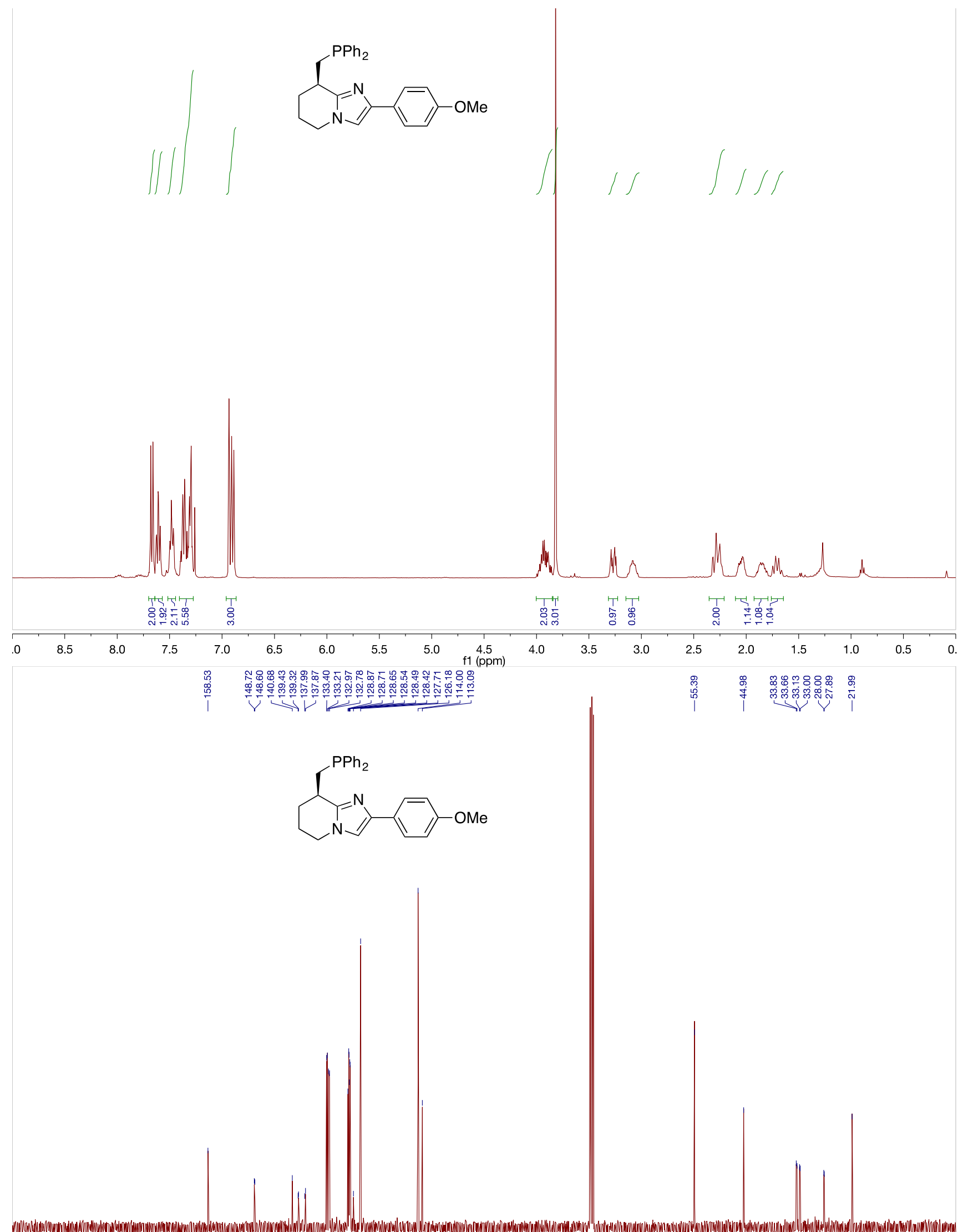

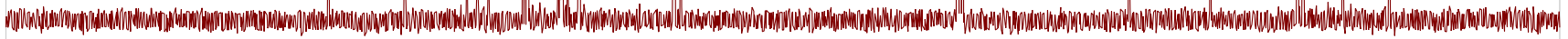

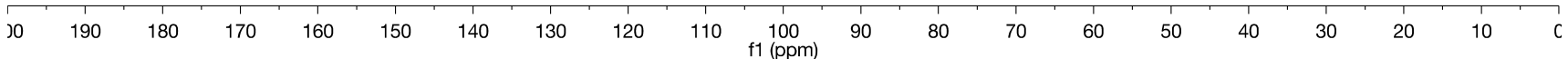




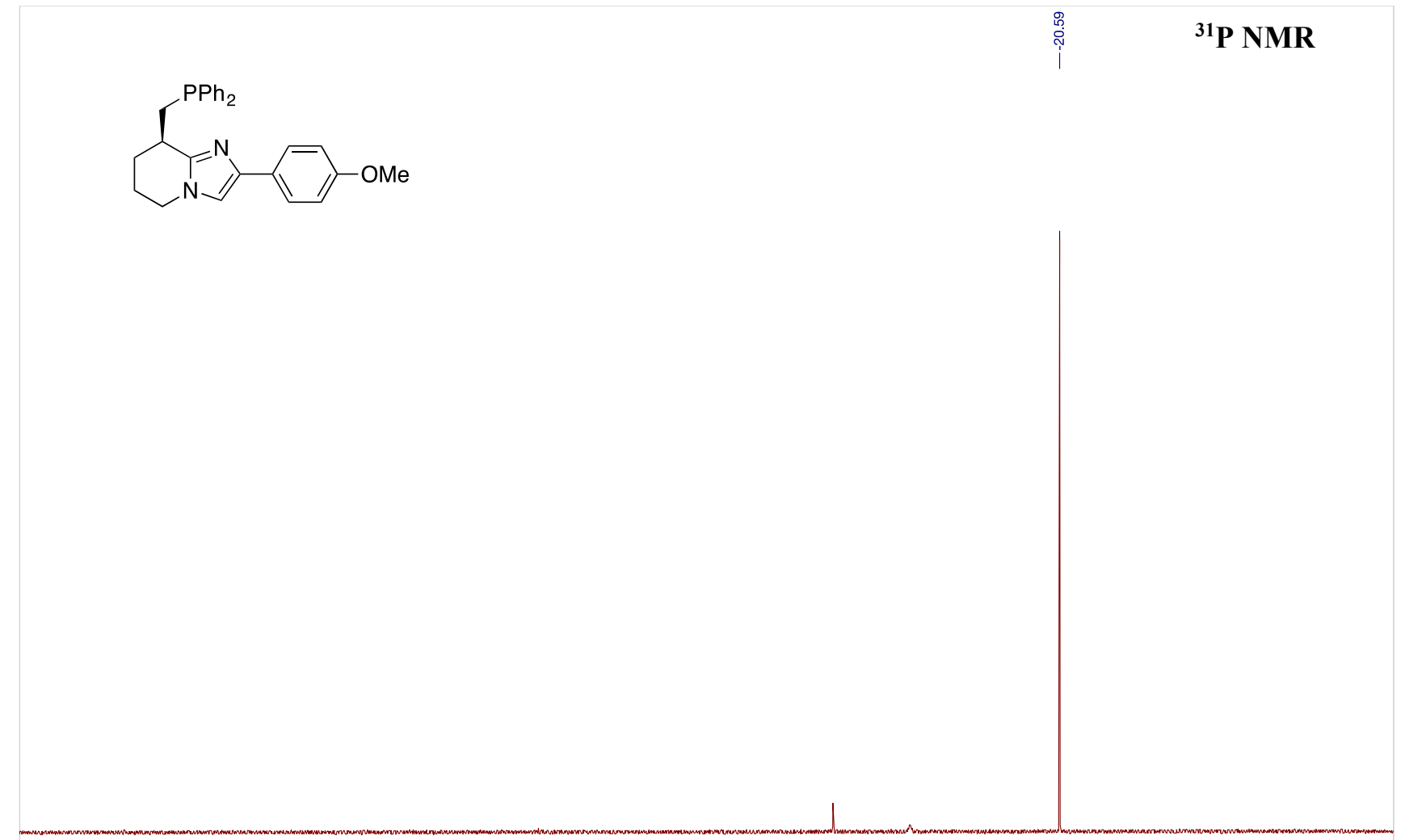

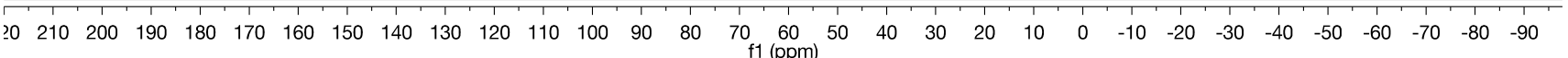



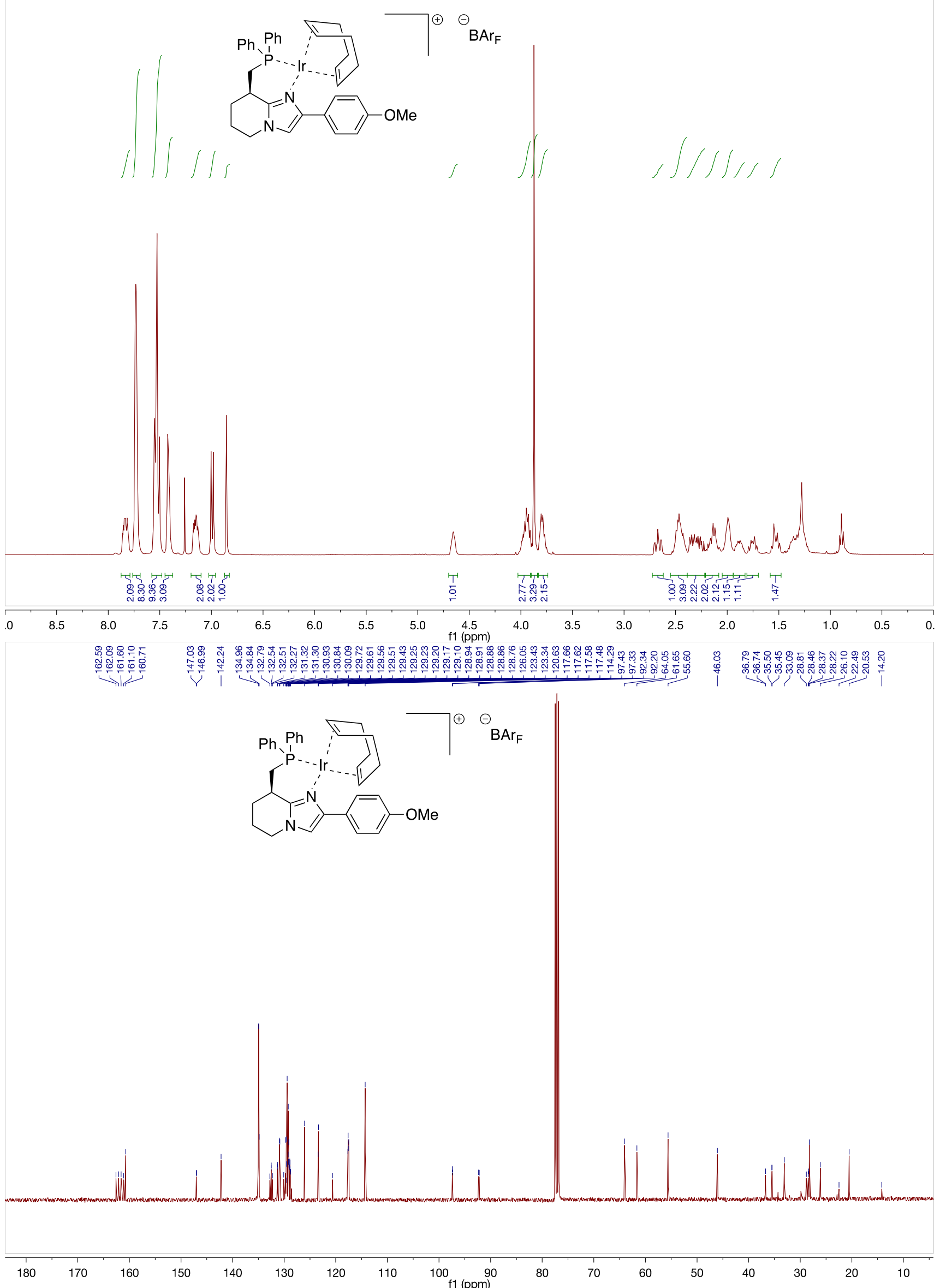


$$
\Theta_{\mathrm{BAr}_{F}}
$$

${ }^{31}$ P NMR

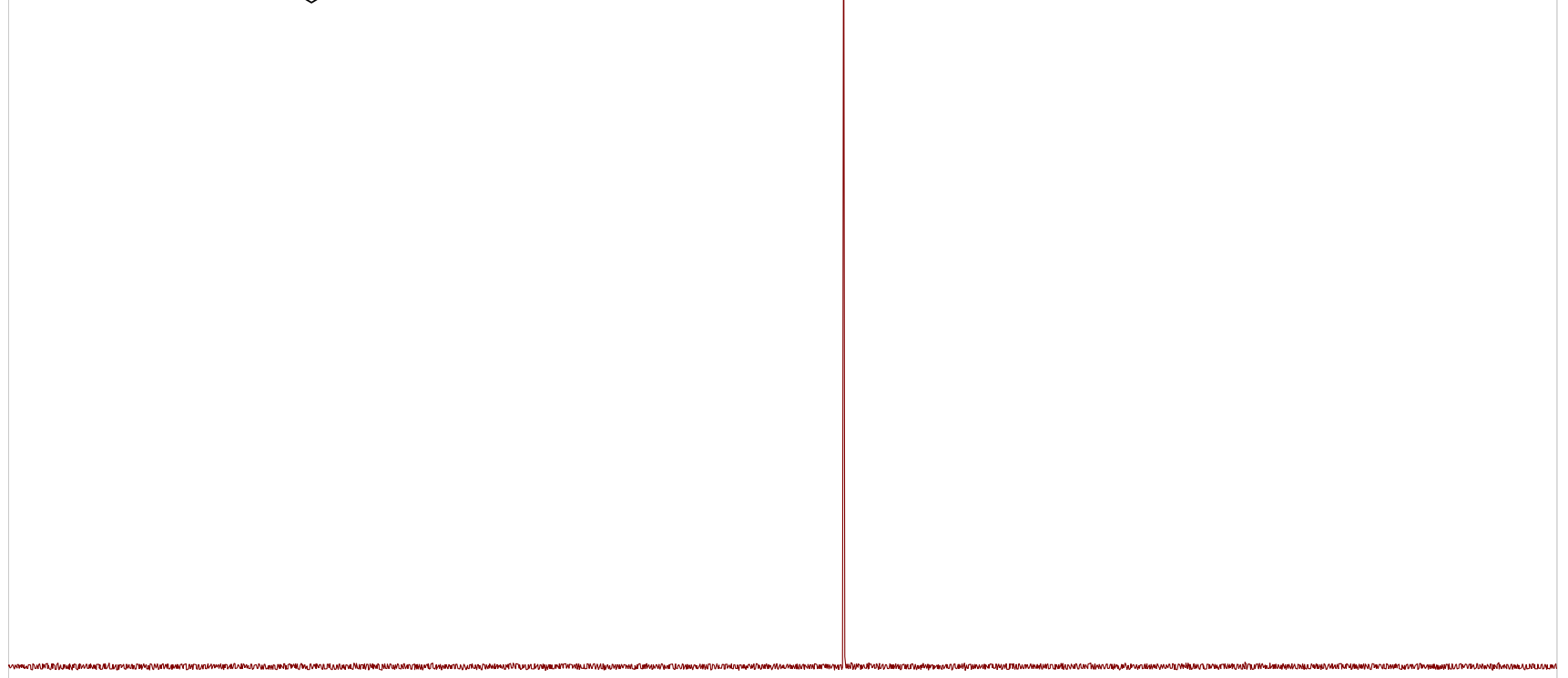

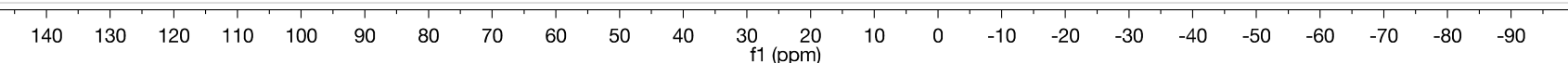



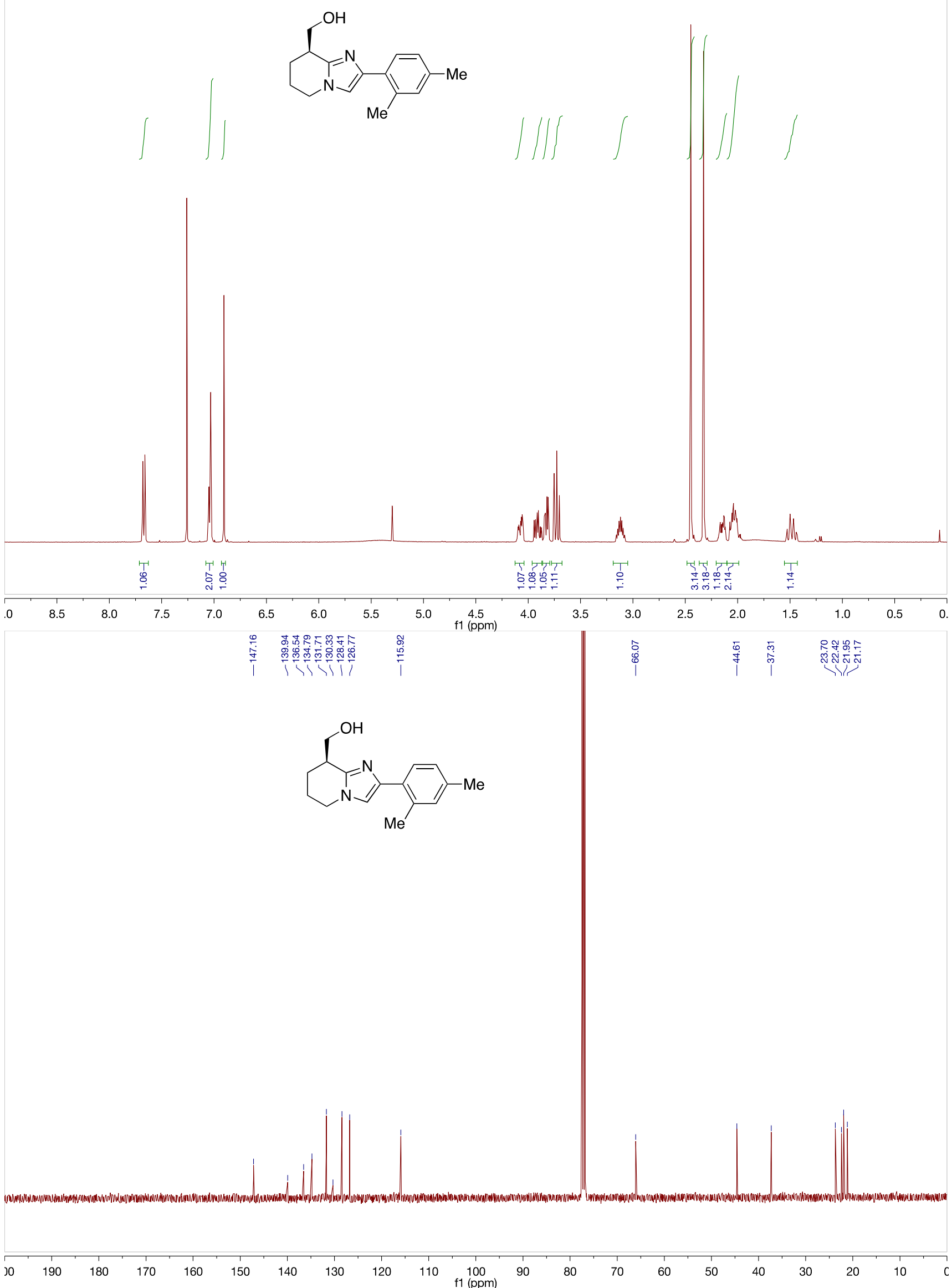

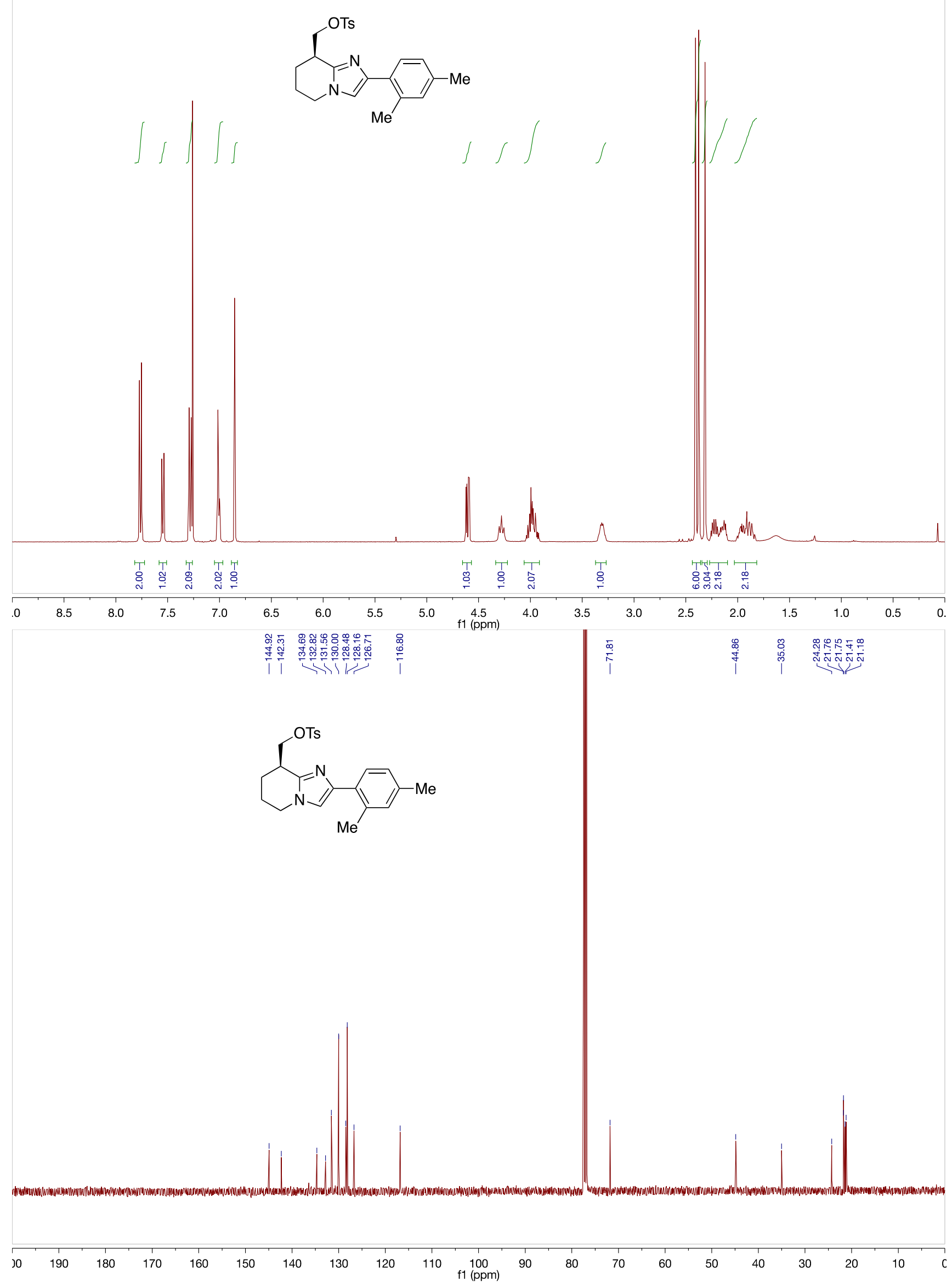


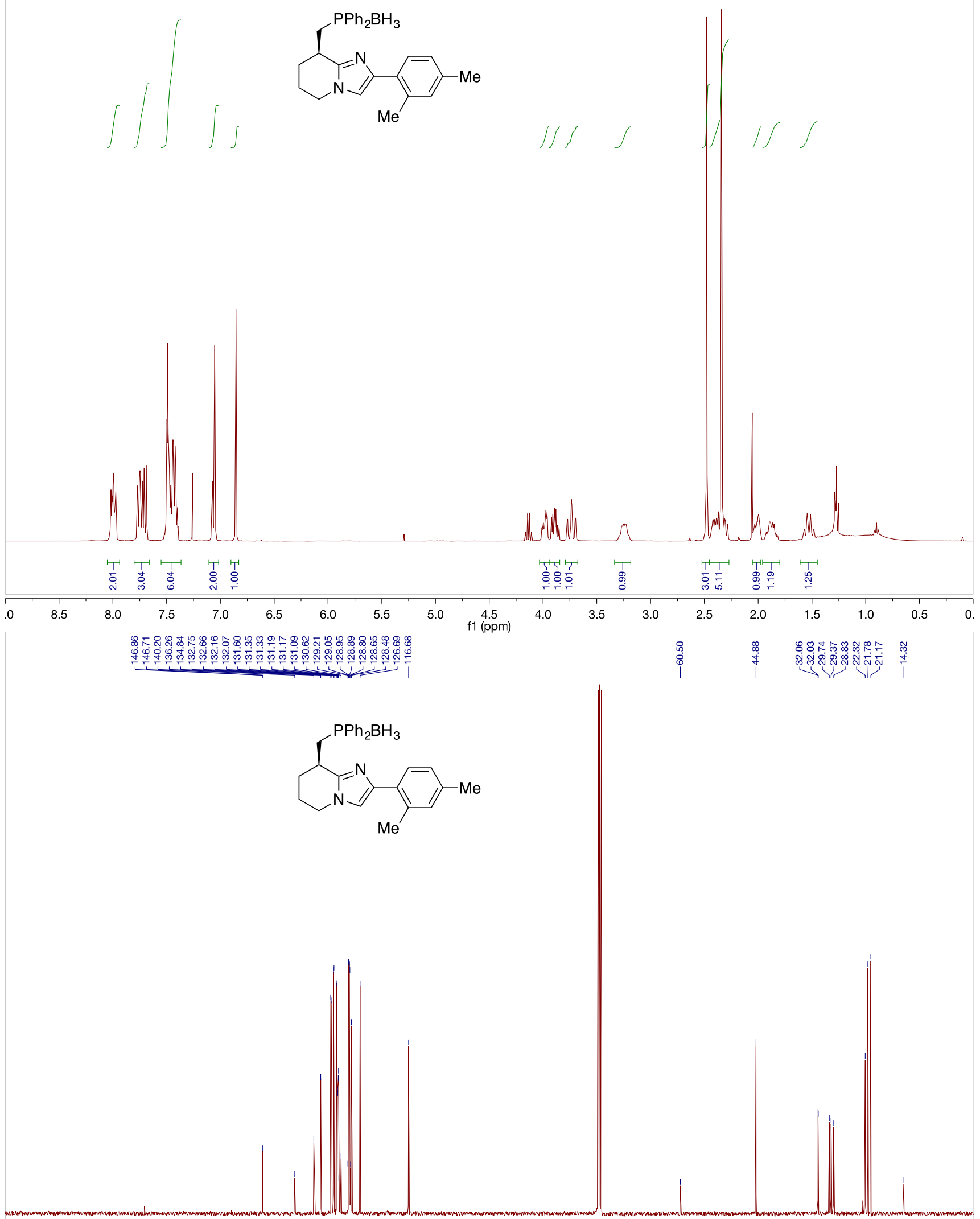

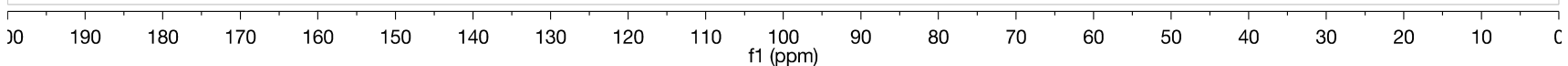



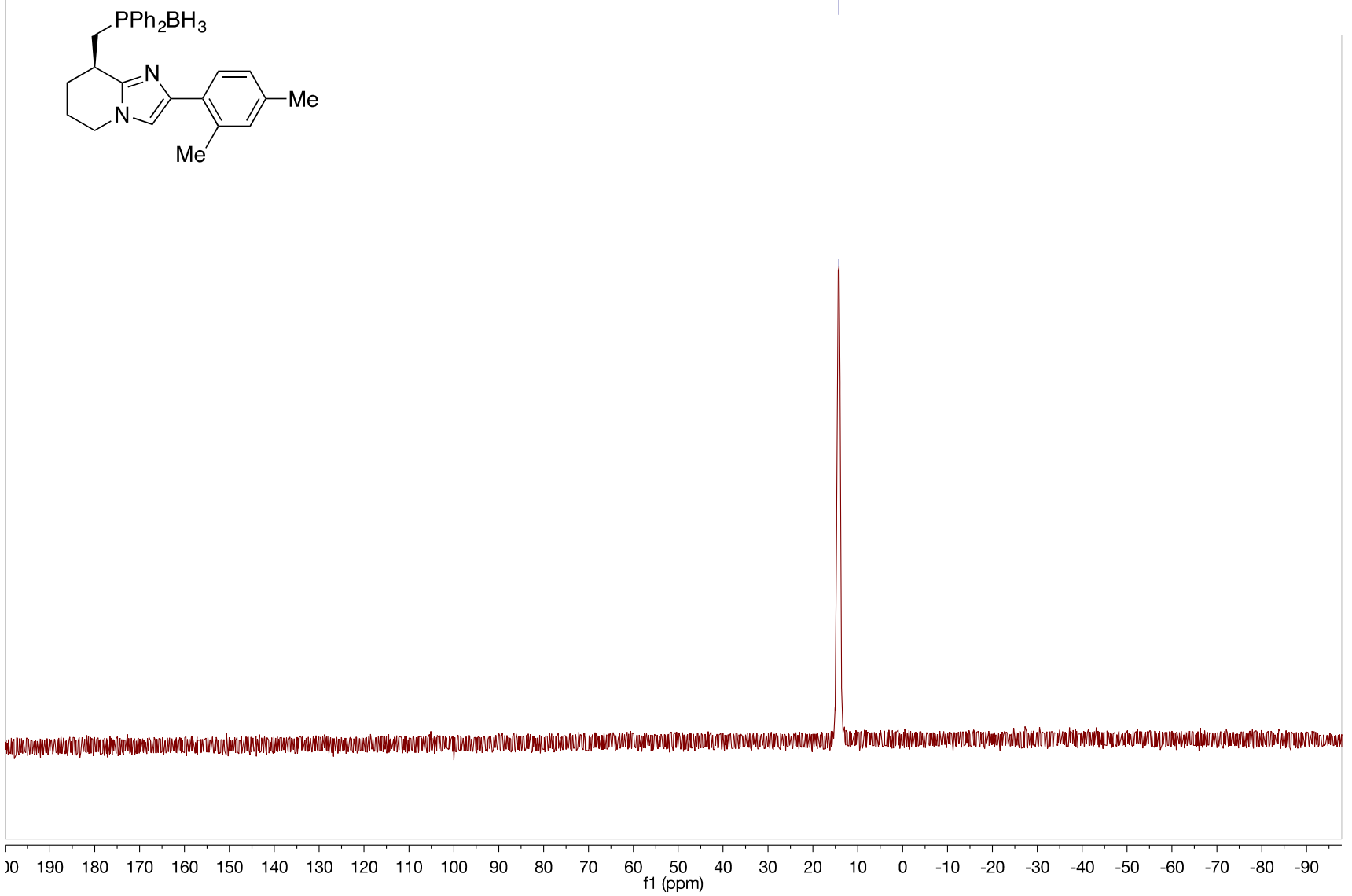


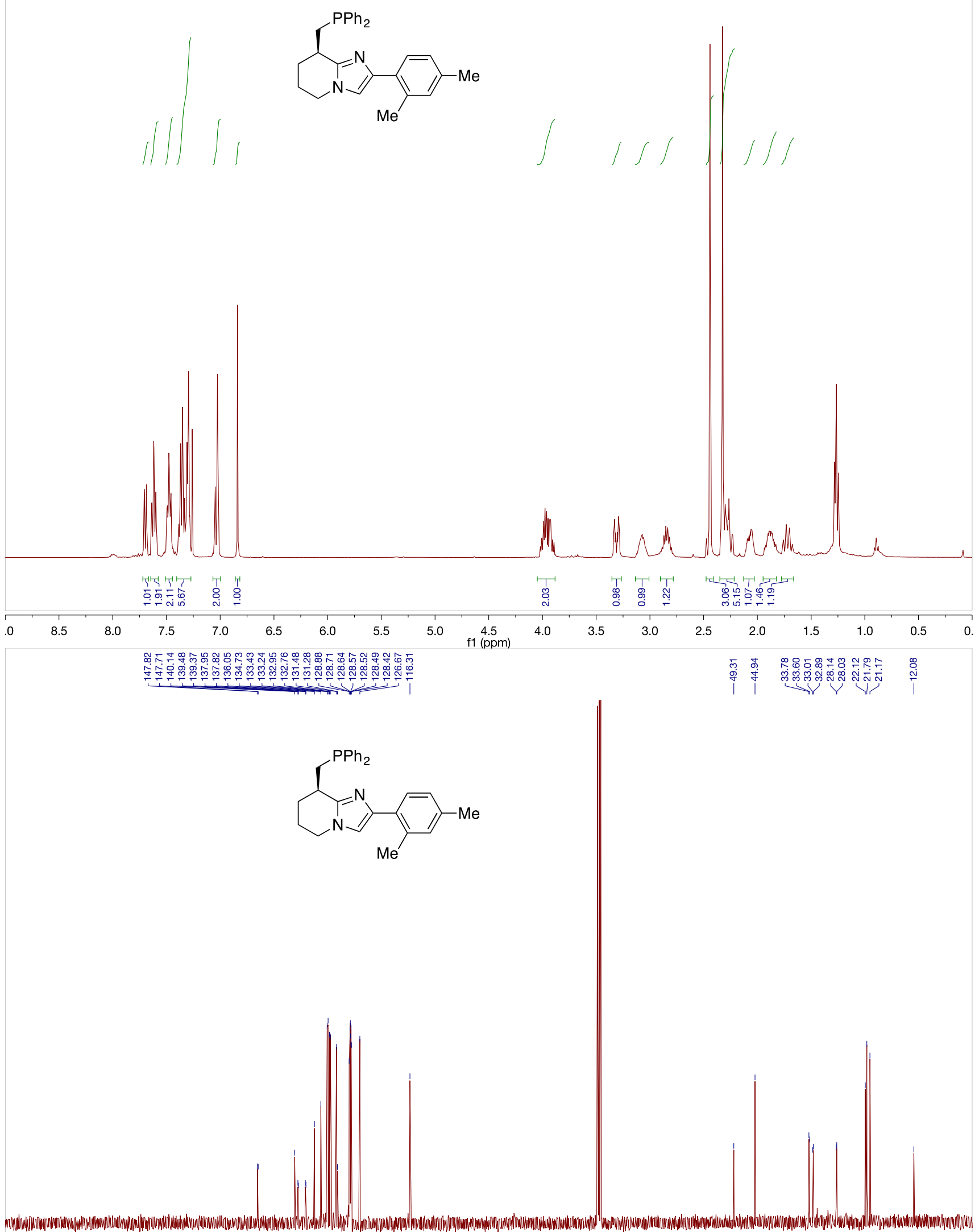

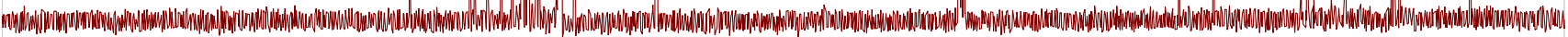

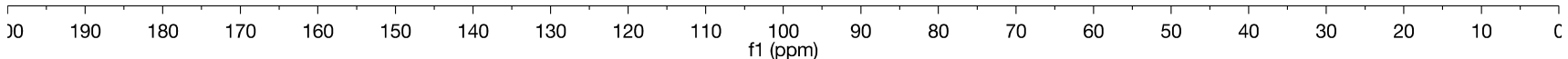




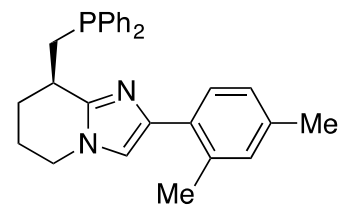

$\begin{array}{rllllllllllllllllllllllllll}140 & 130 & 120 & 110 & 100 & 90 & 80 & 70 & 60 & 50 & 40 & 30 & 20 & 10 & 0 & -10 & -20 & -30 & -40 & -50 & -60 & -70 & -80 & -90 & -11\end{array}$ 

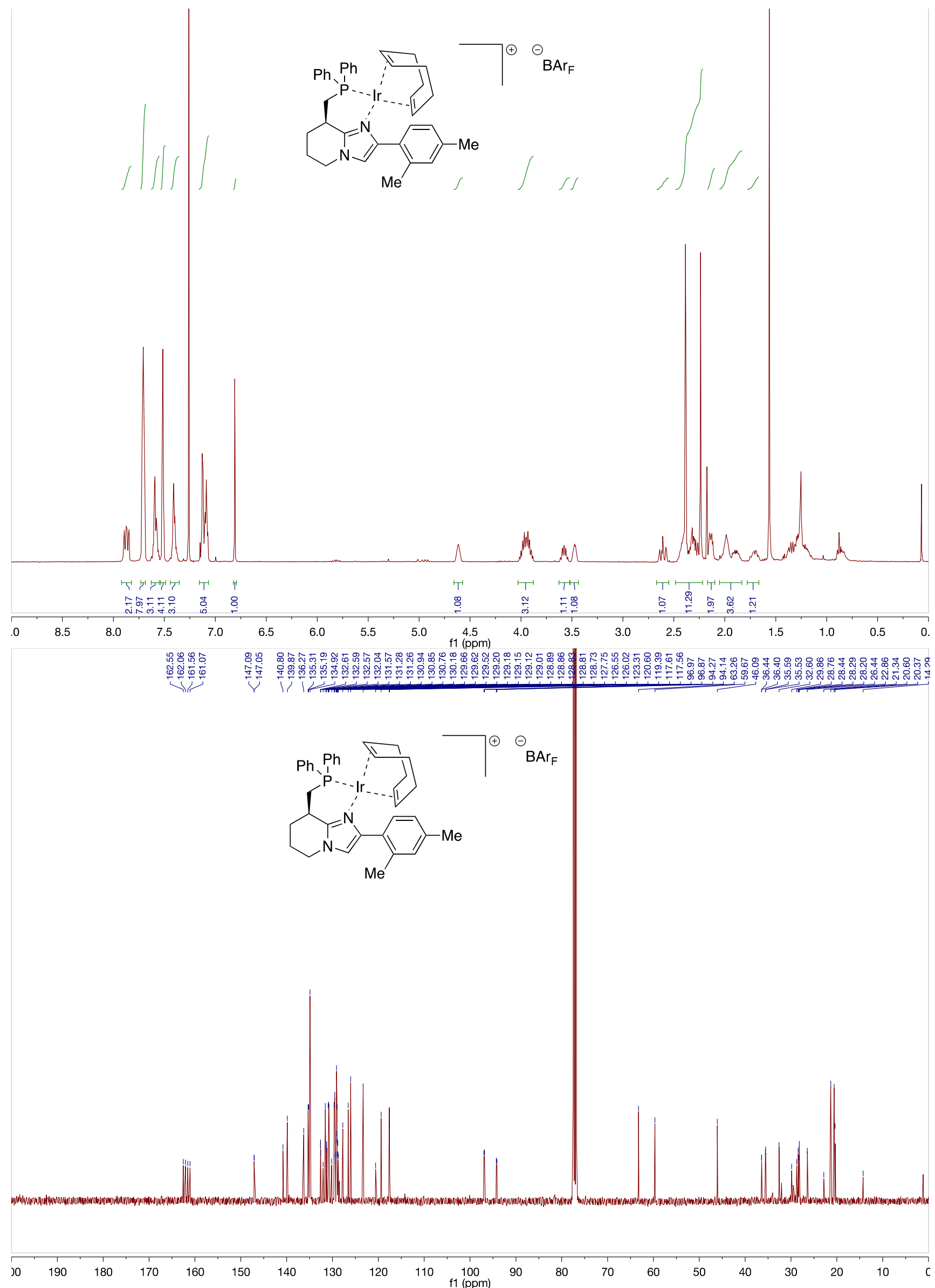


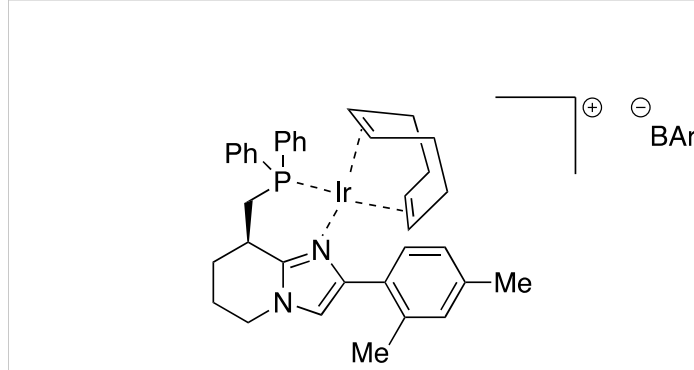

$\mathrm{BAr}_{\mathrm{F}}$

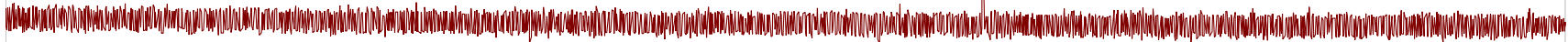

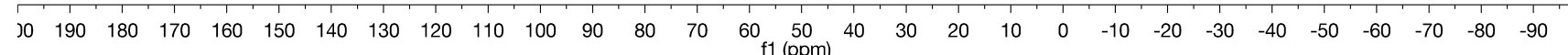




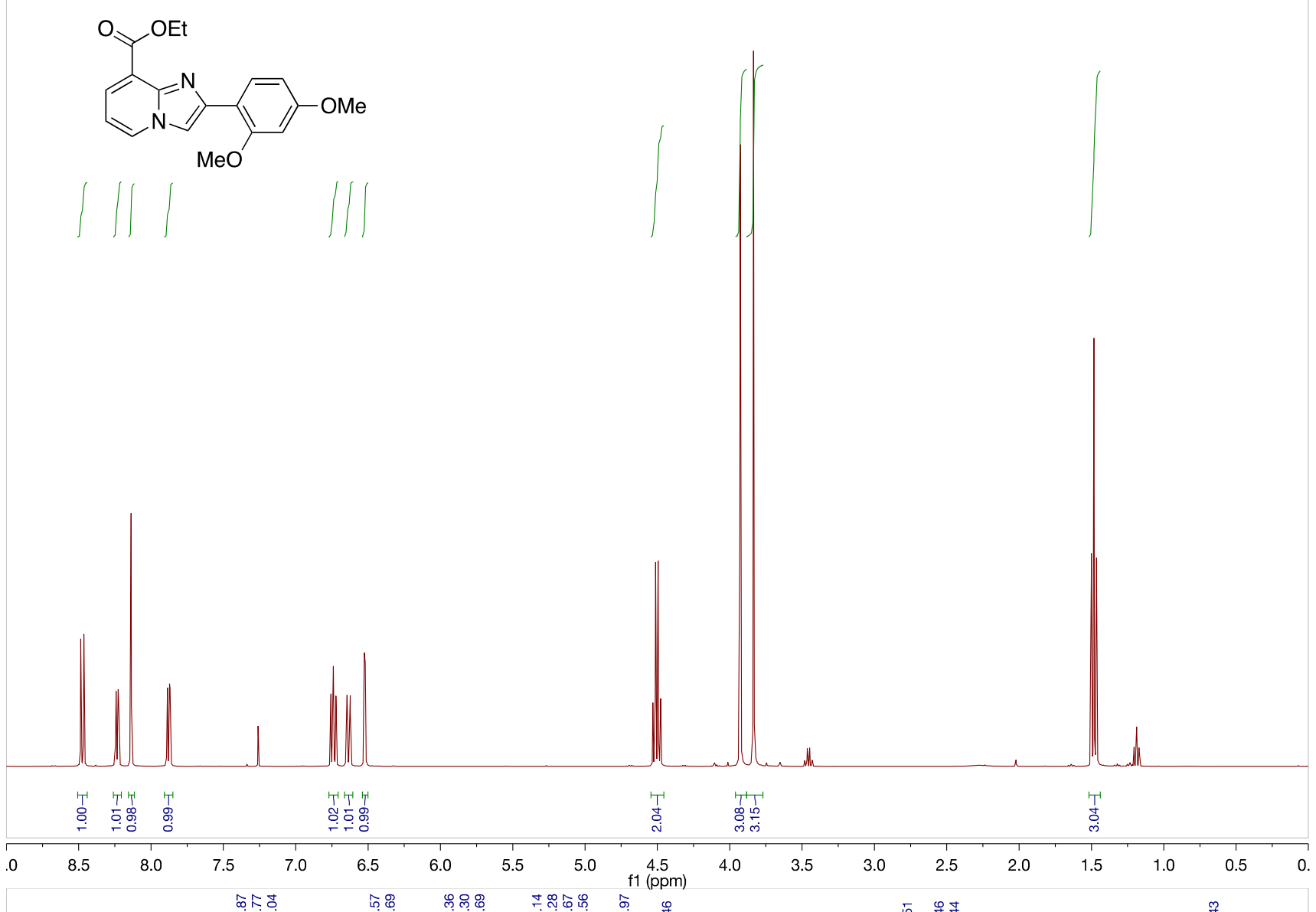

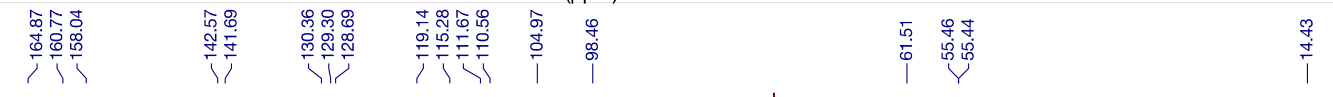<smiles>CCOC(=O)c1cccn2cc(-c3ccc(OC)cc3OC)nc12</smiles>

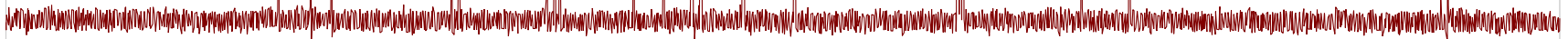

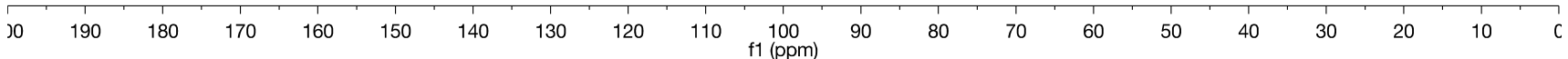




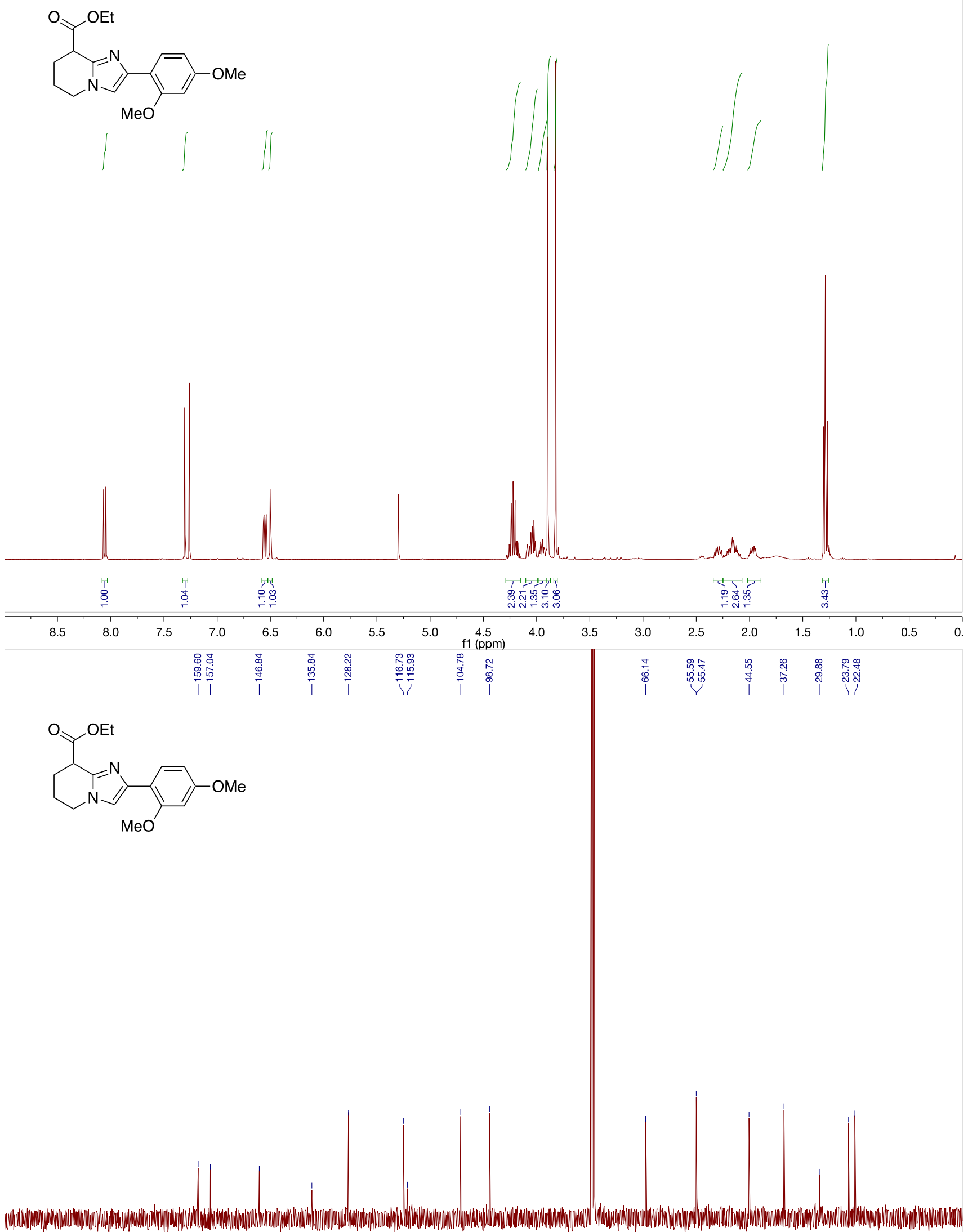

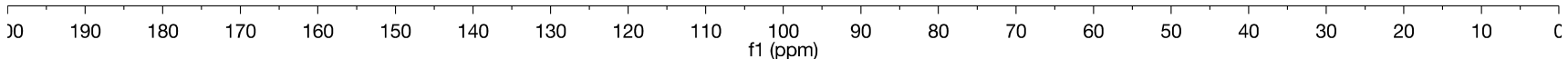




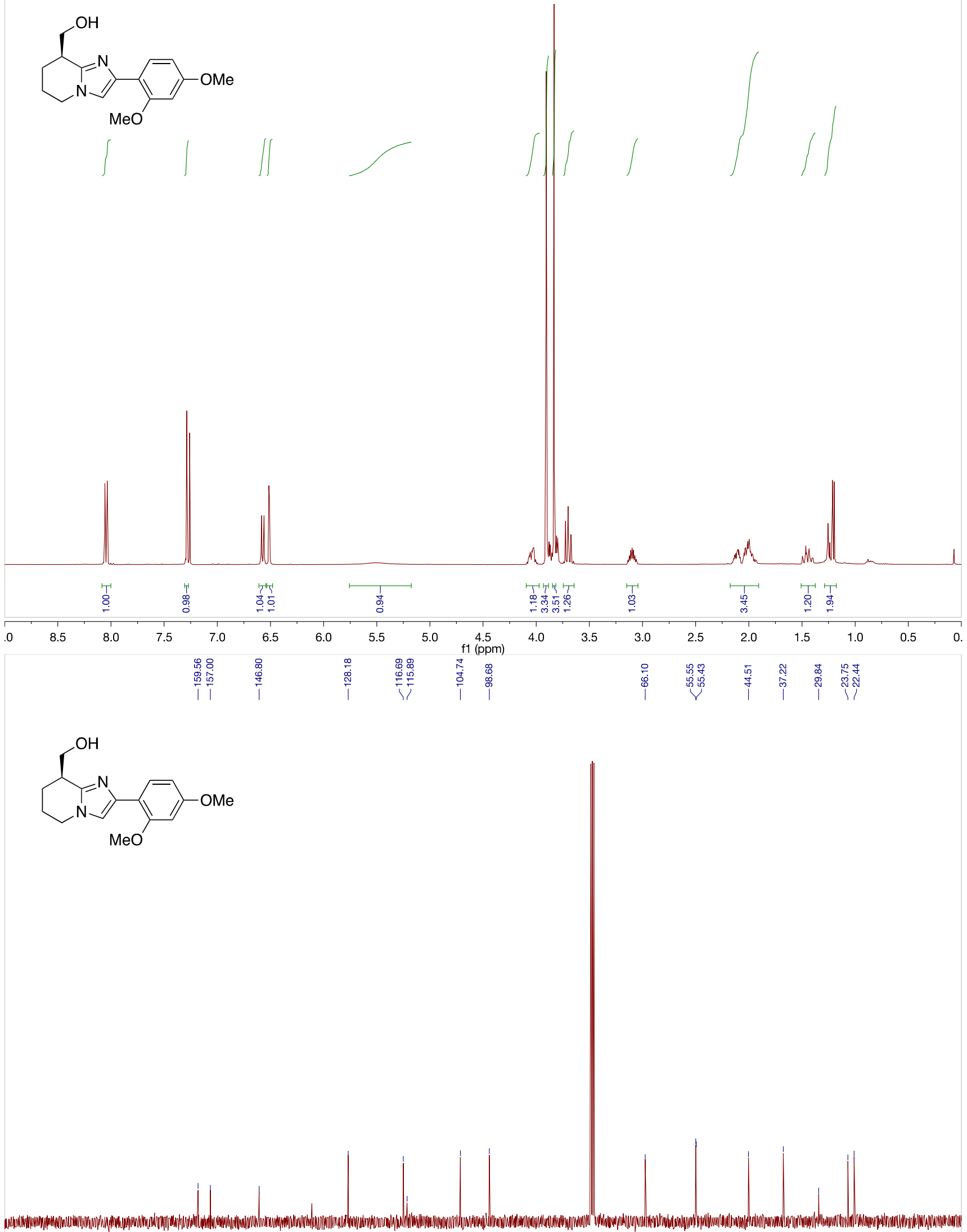

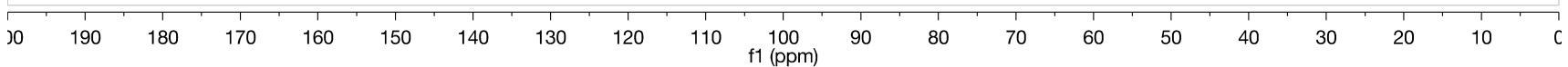




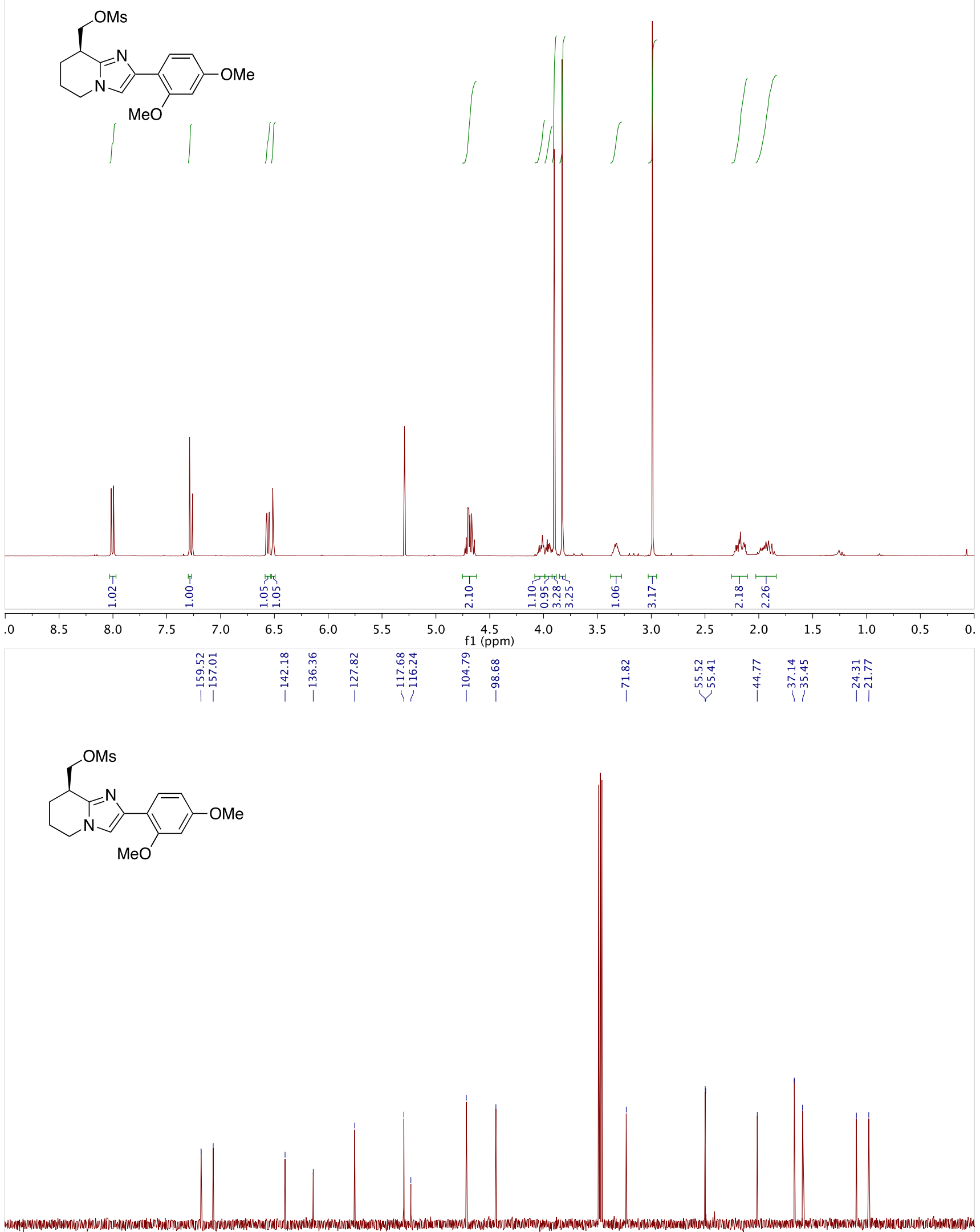

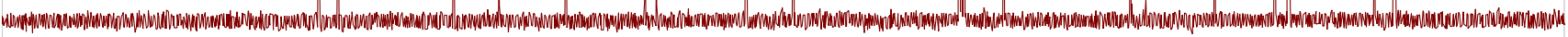

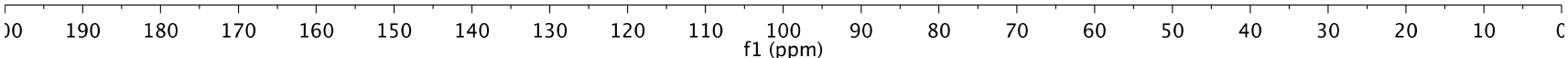




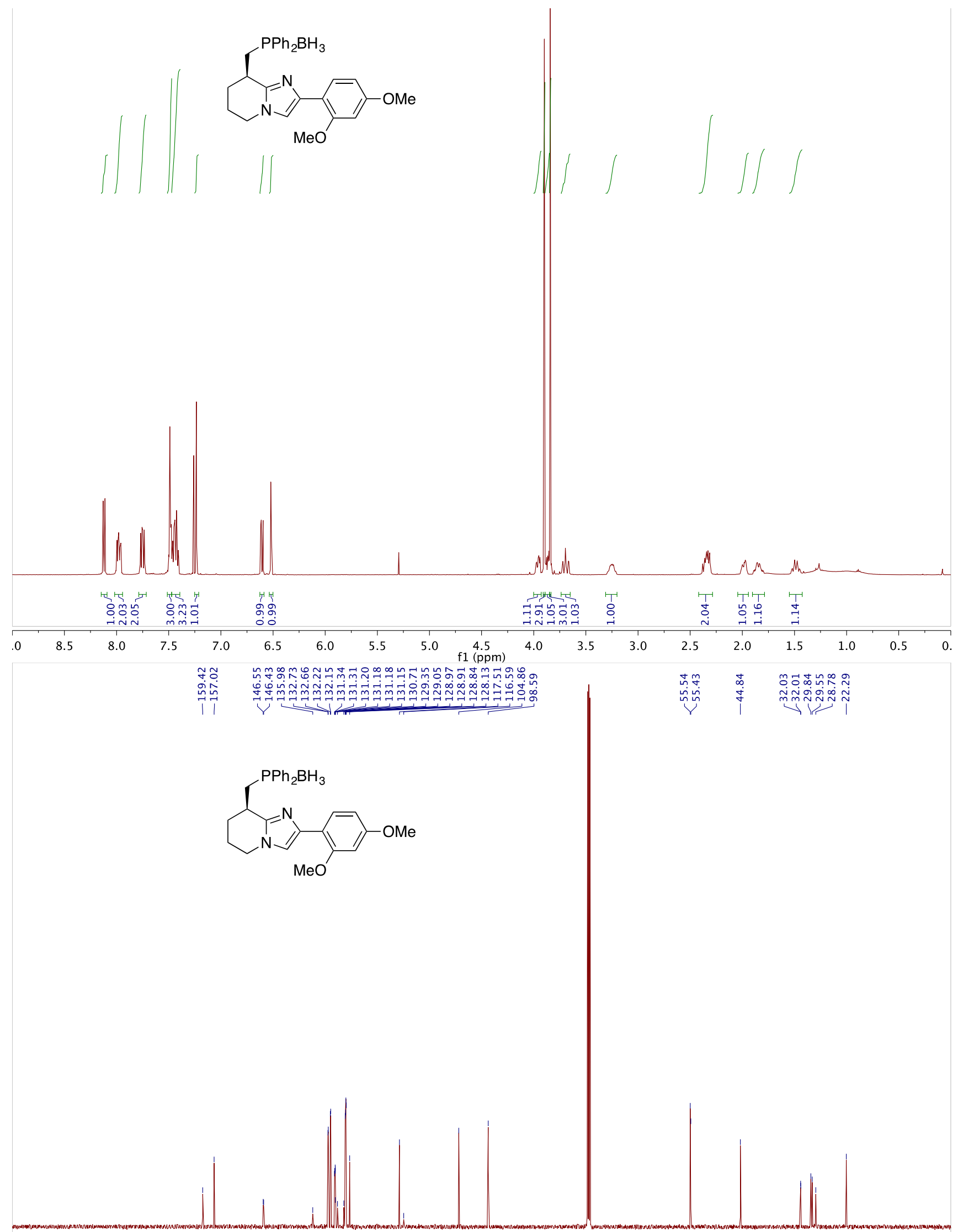

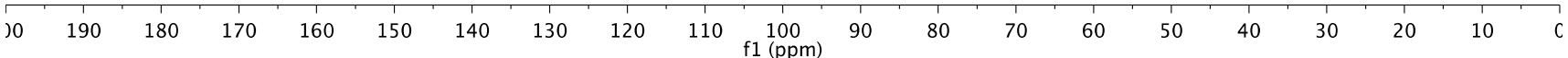


<smiles>COc1ccc(-c2cn3c(n2)CCCC3CP)c(OC)c1</smiles>

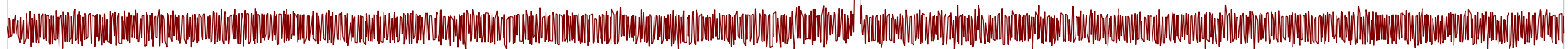

$\begin{array}{lllllllllllllllllllllllllll}50 & 140 & 130 & 120 & 110 & 100 & 90 & 80 & 70 & 60 & 50 & 40 & \begin{array}{l}30 \\ \mathrm{f} 1(\mathrm{ppm})\end{array} & 20 & 10 & 0 & -10 & -20 & -30 & -40 & -50 & -60 & -70 & -80 & -90 & -1\end{array}$ 

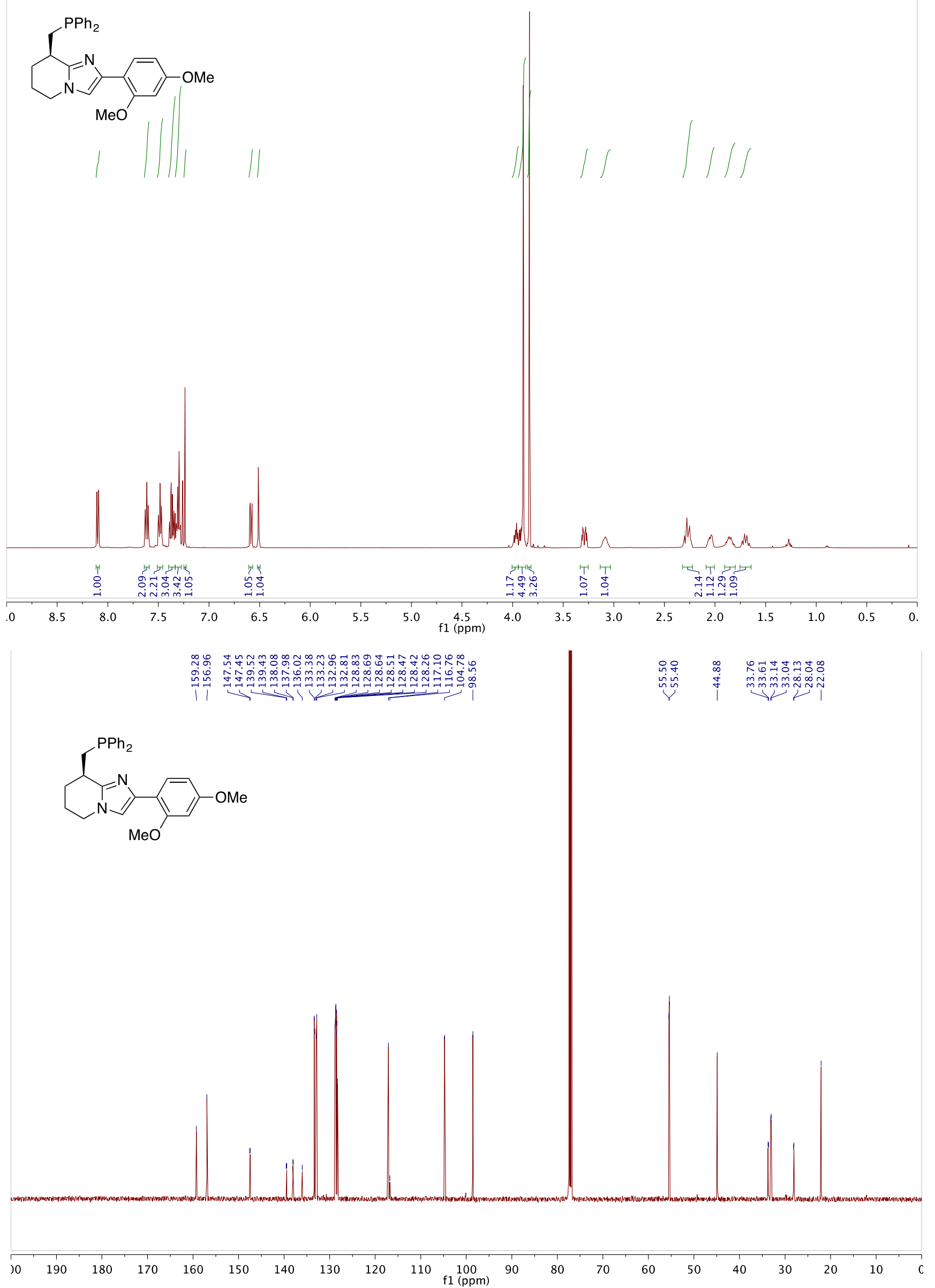
<smiles>COc1ccc(-c2cn3c(n2)[C@@H](Cc2ccccc2)CCC3)c(OC)c1</smiles>

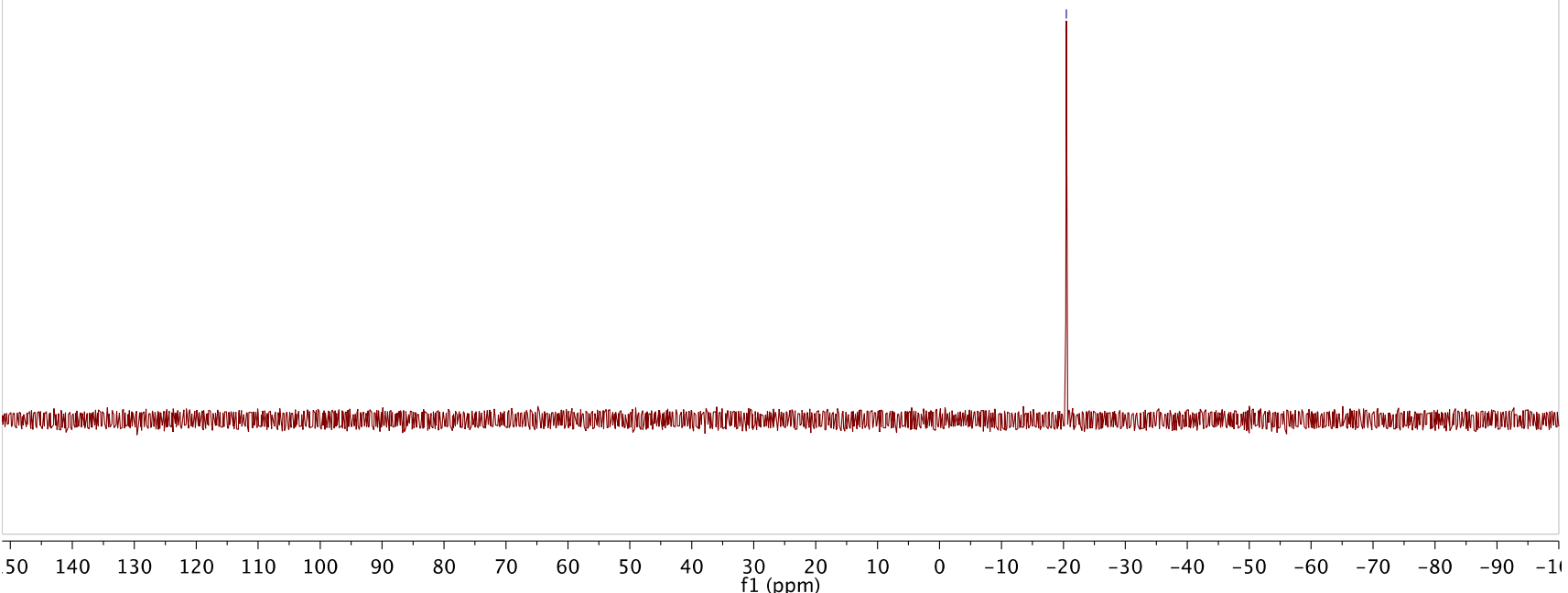




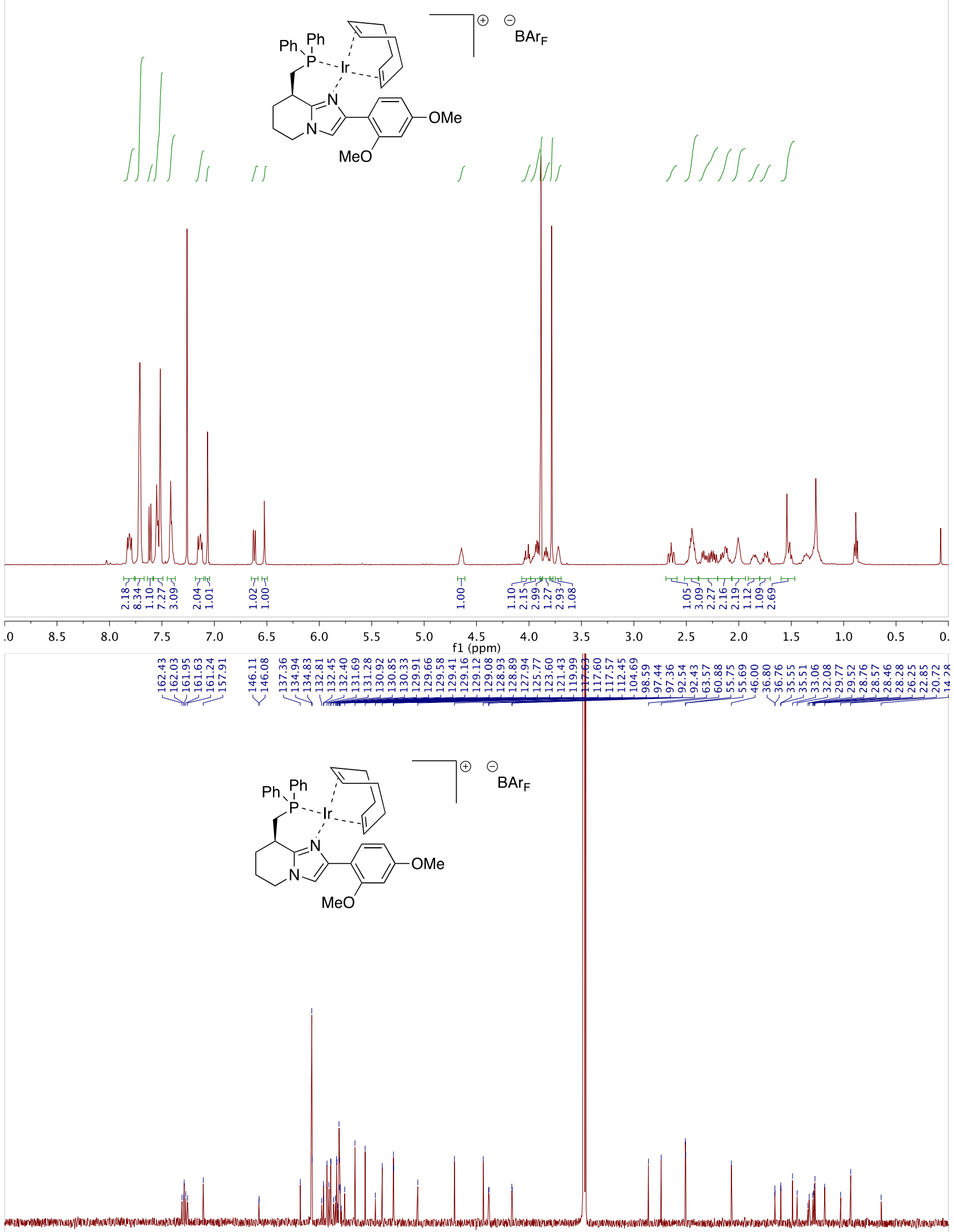

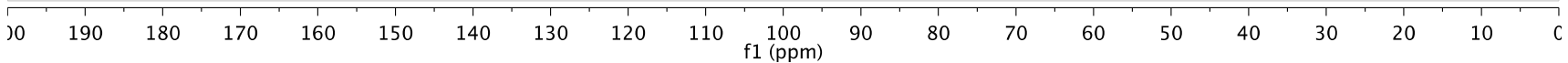




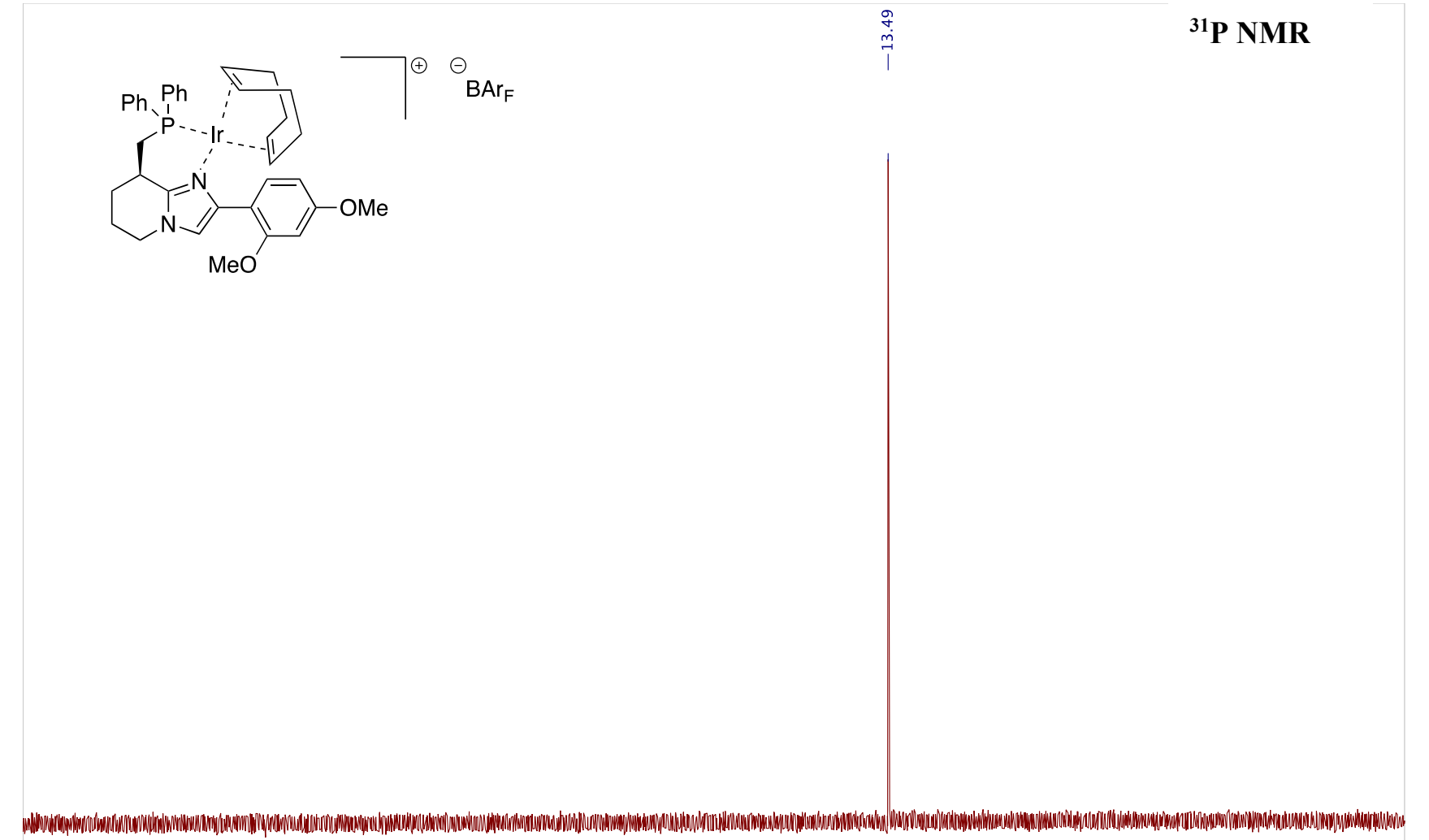

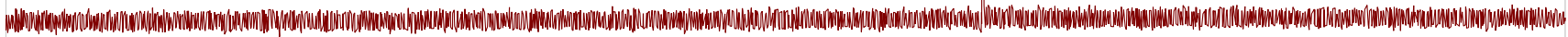

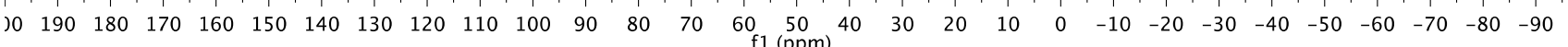




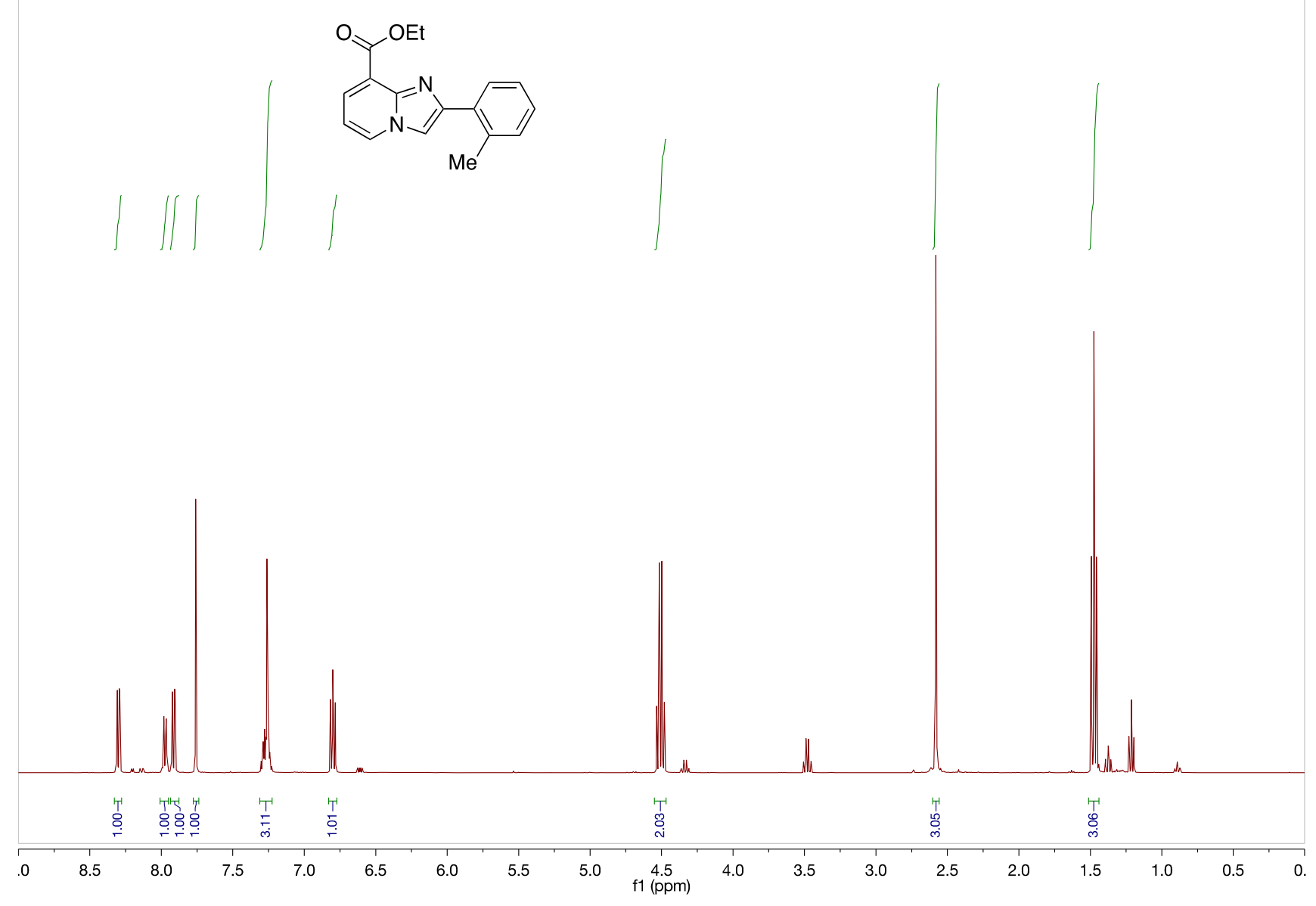

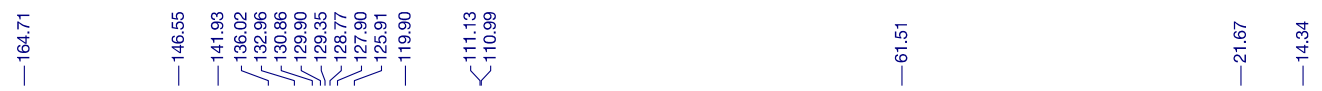
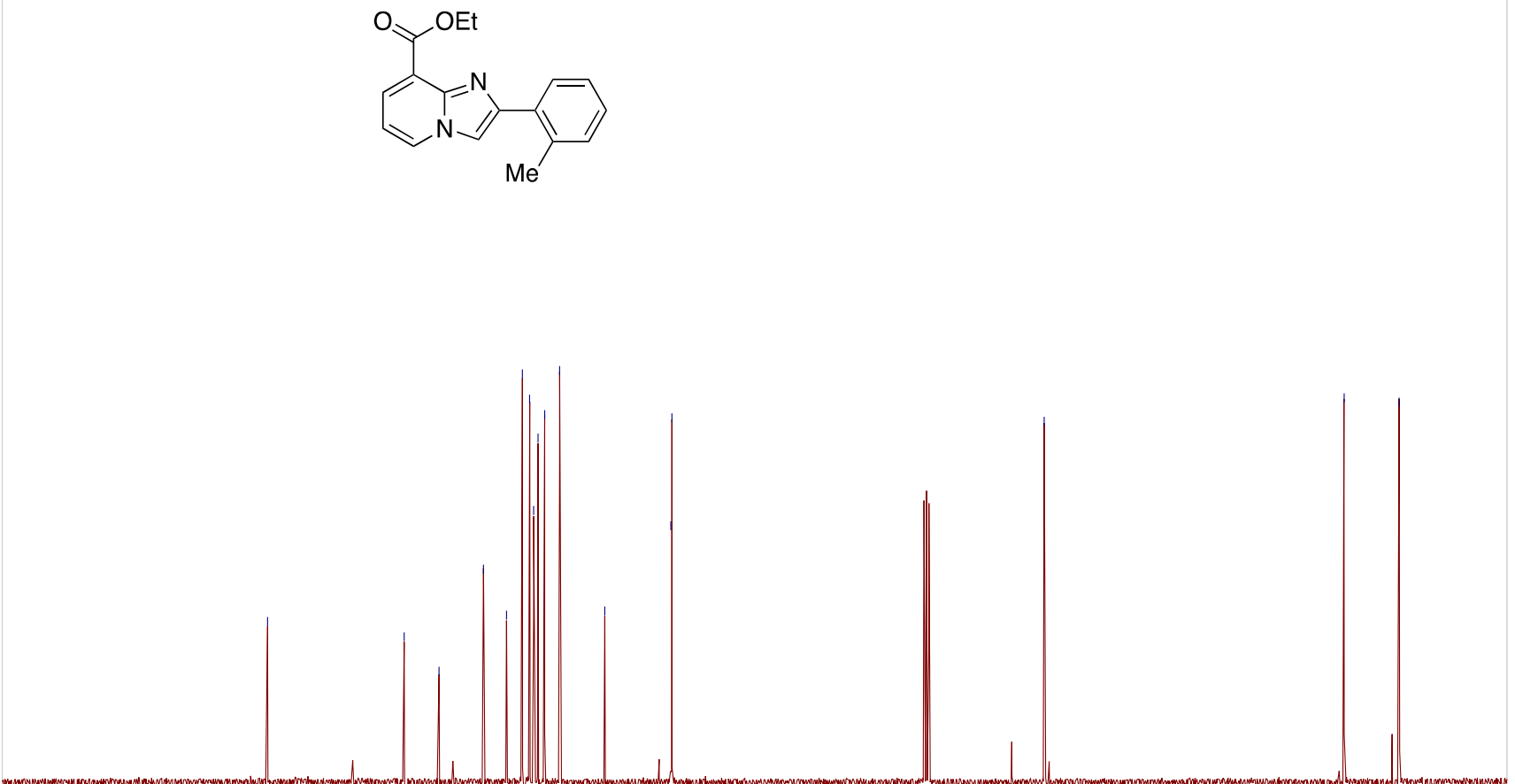

\begin{tabular}{lllllllllllllllllllll}
\hline 0 & 190 & 180 & 170 & 160 & 150 & 140 & 130 & 120 & 110 & 100 & 90 & 80 & 70 & 60 & 50 & 40 & 30 & 20 & 10 & $\mathrm{C}$
\end{tabular} 

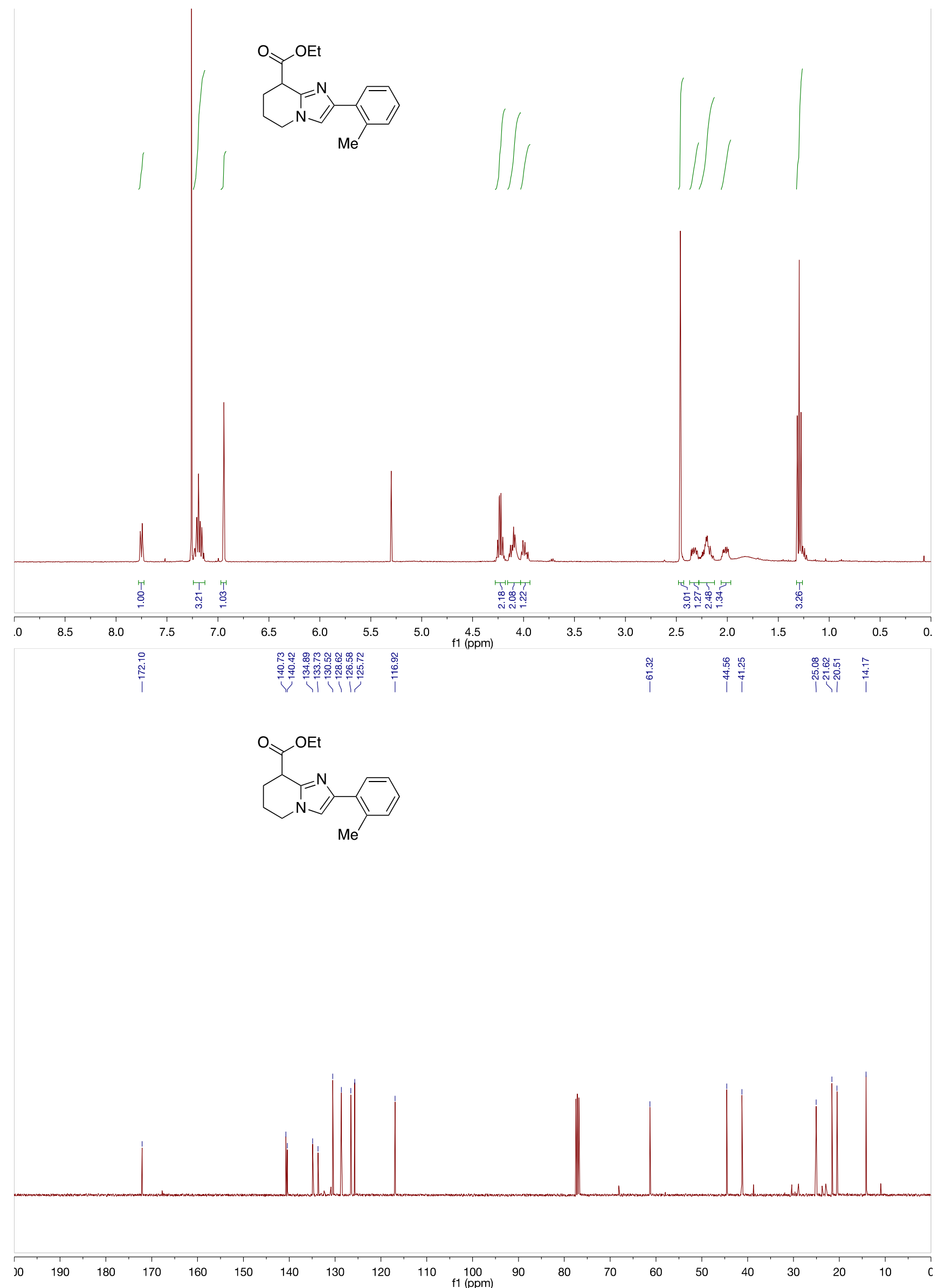

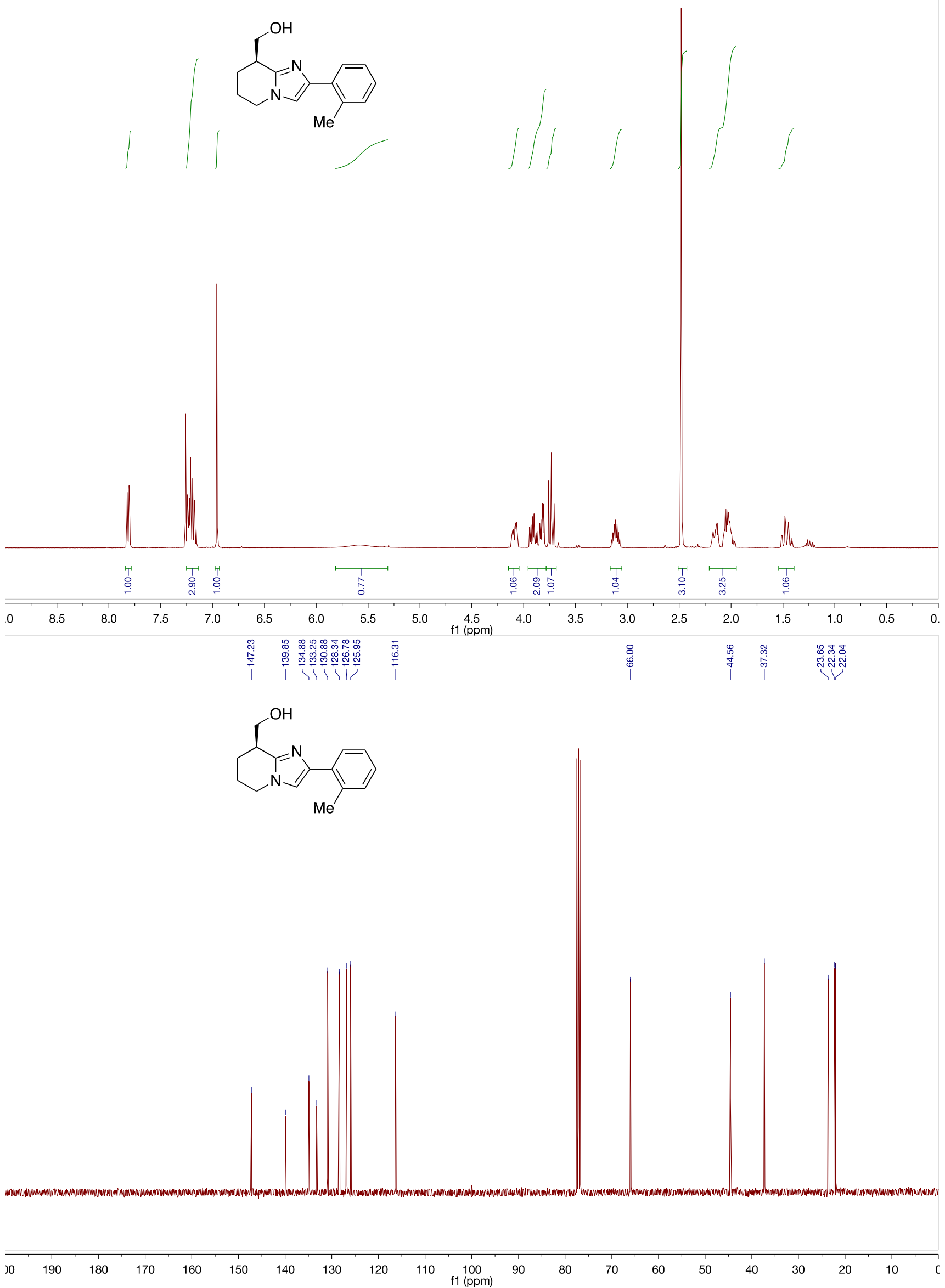


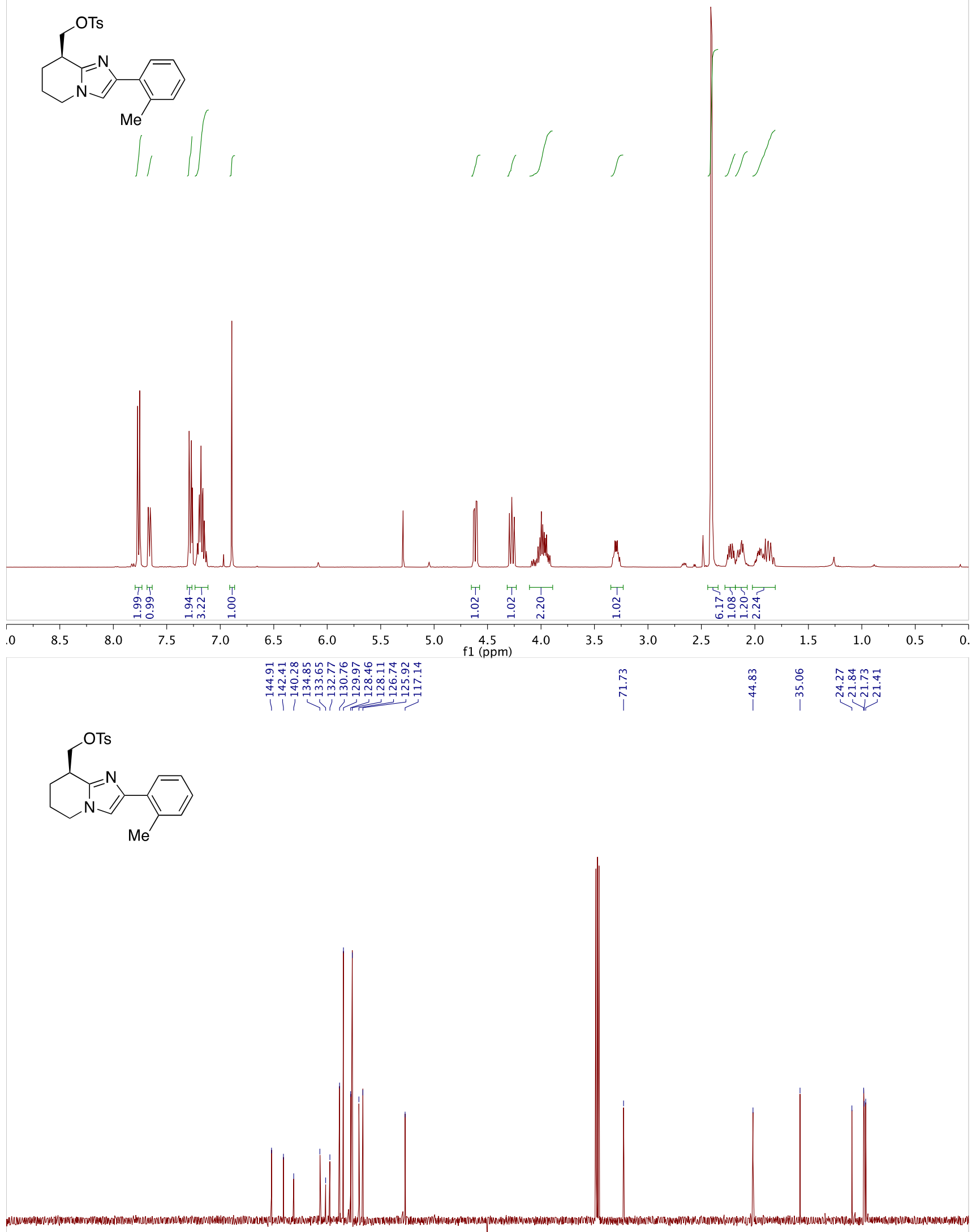

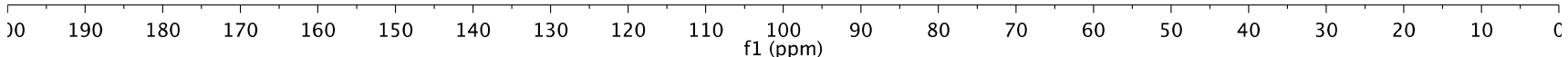




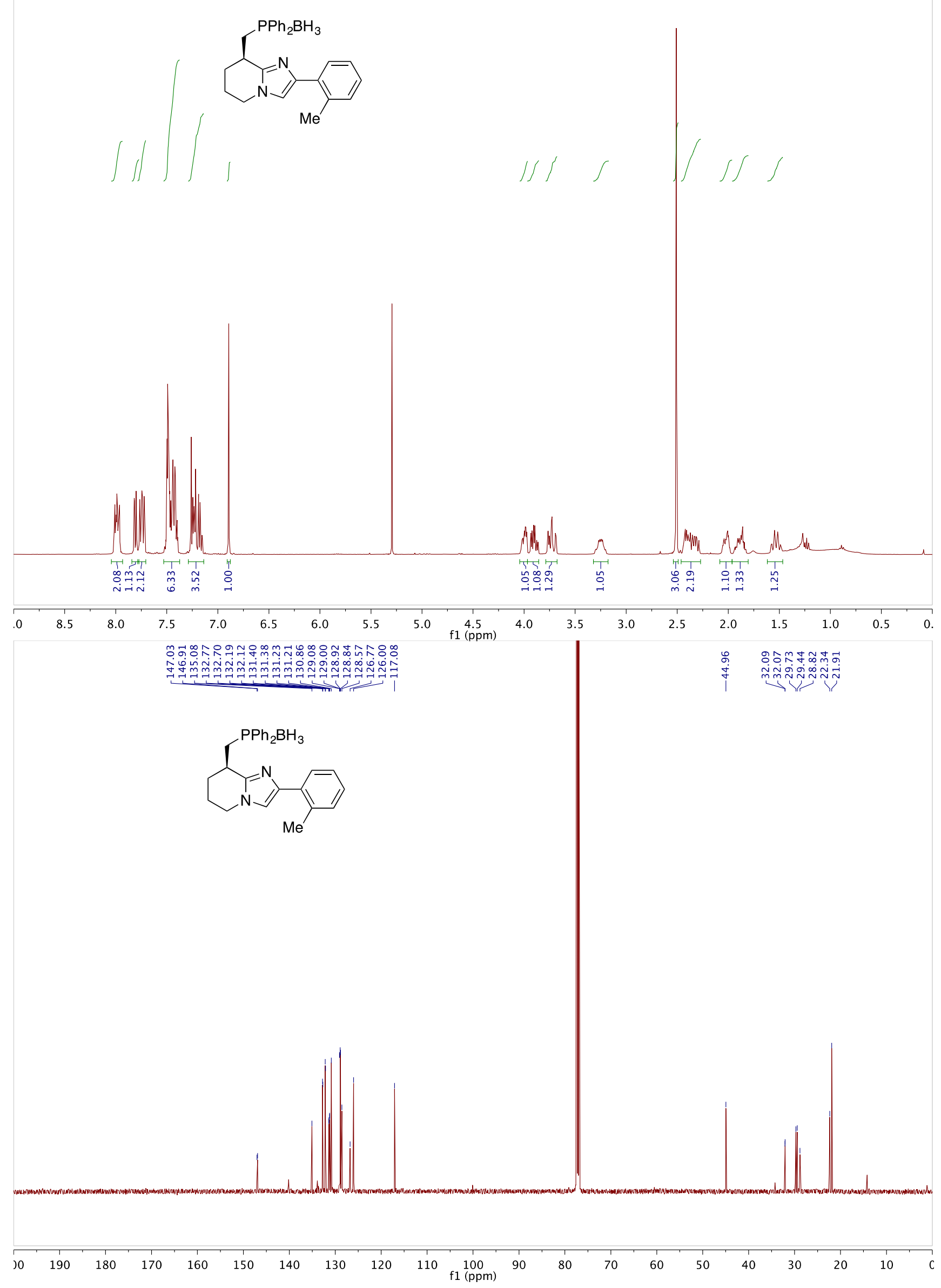



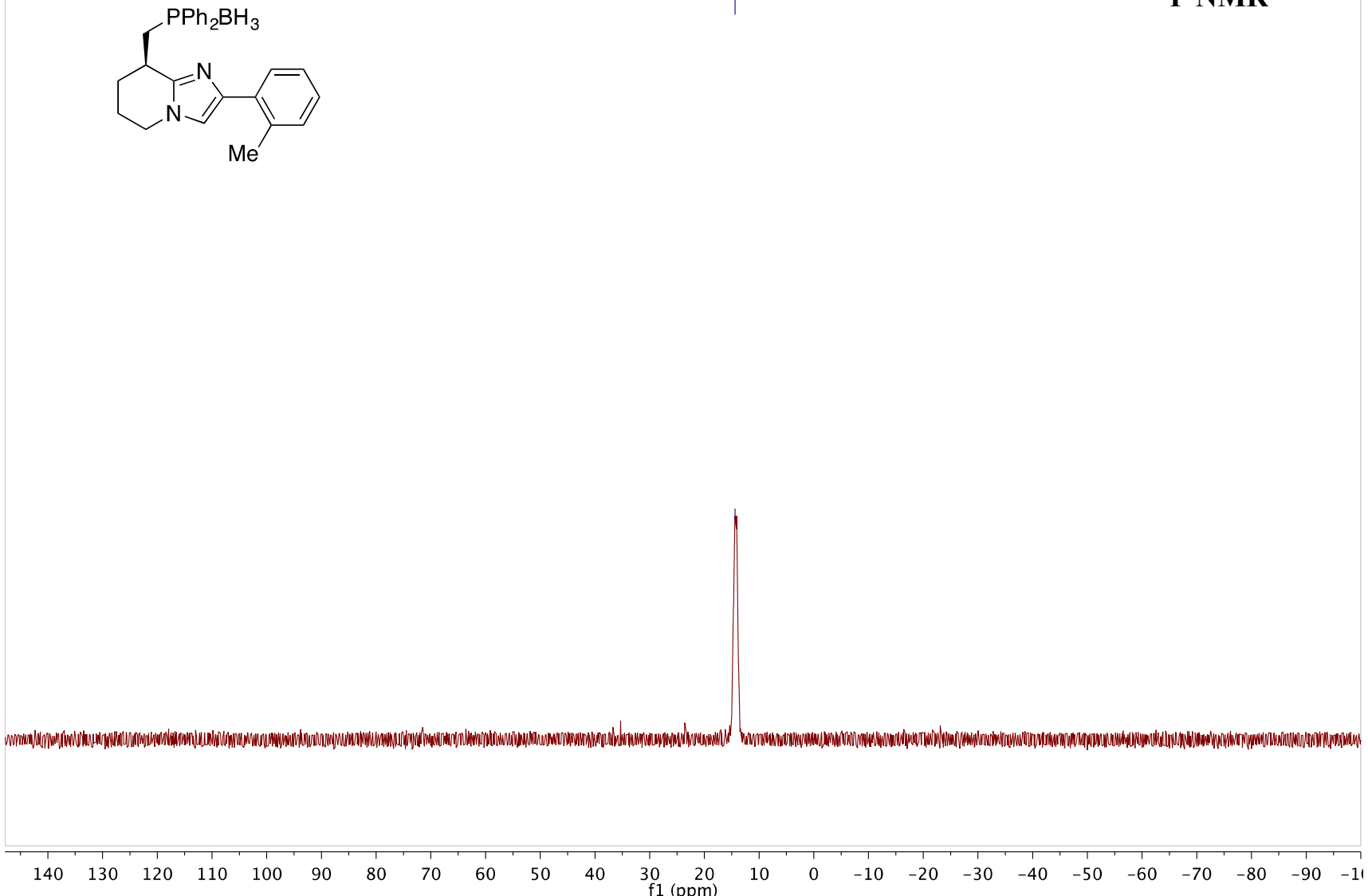


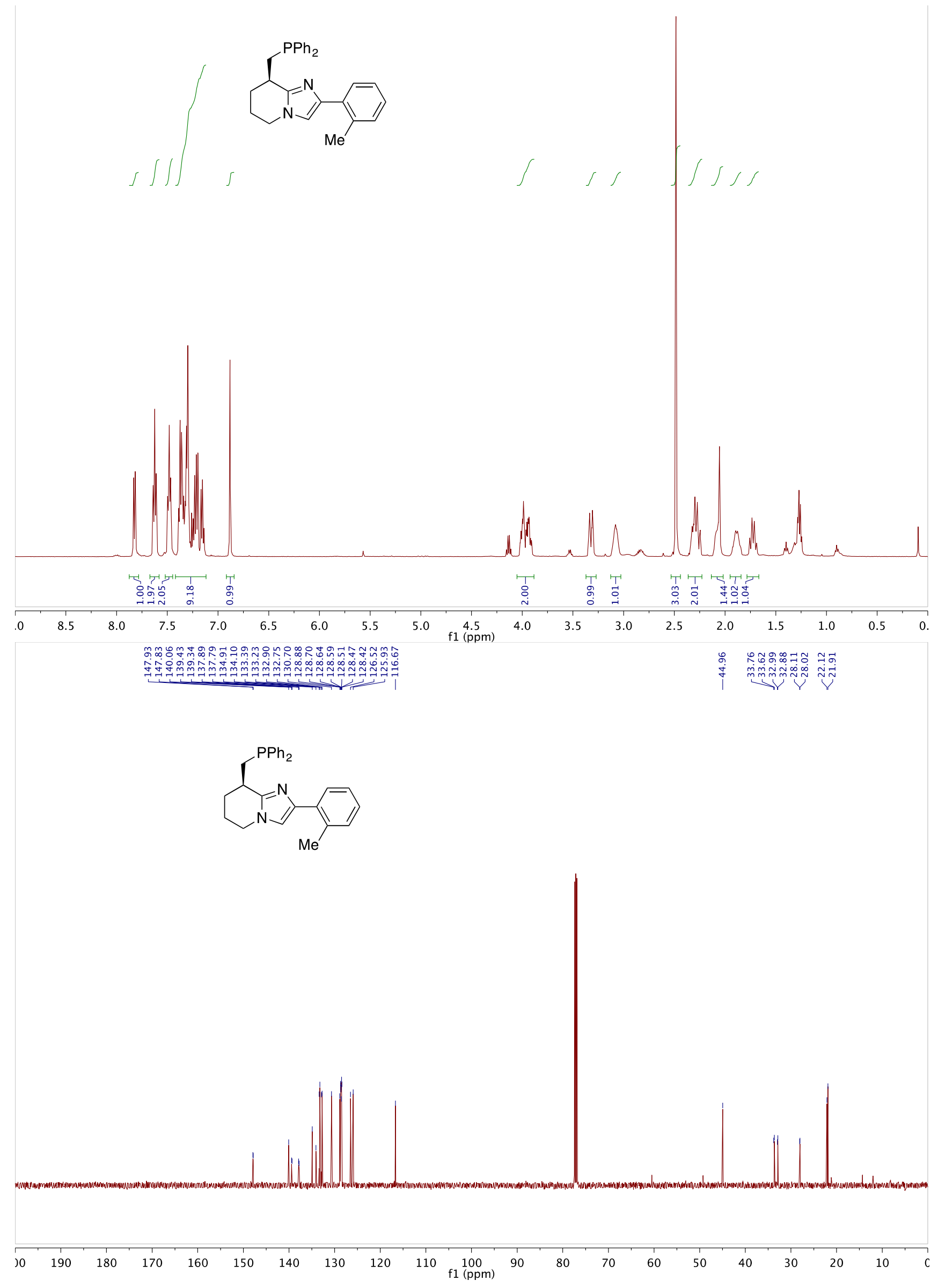


<smiles>Cc1ccccc1-c1cn2c(n1)C(Cc1ccccc1)CCC2</smiles>

${ }^{31}$ P NMR

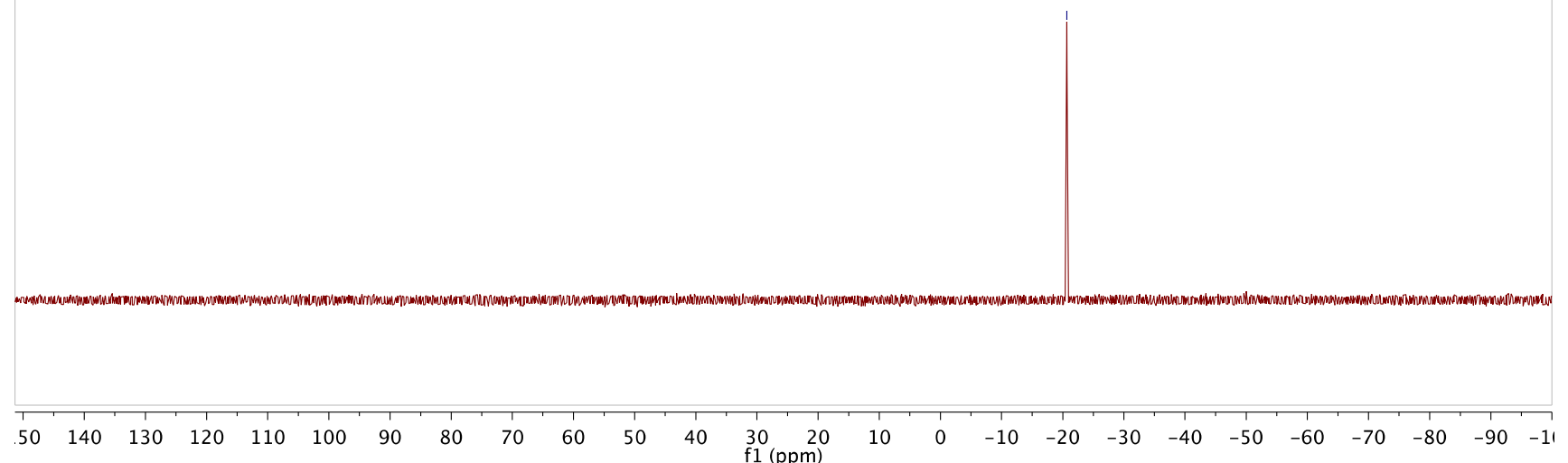




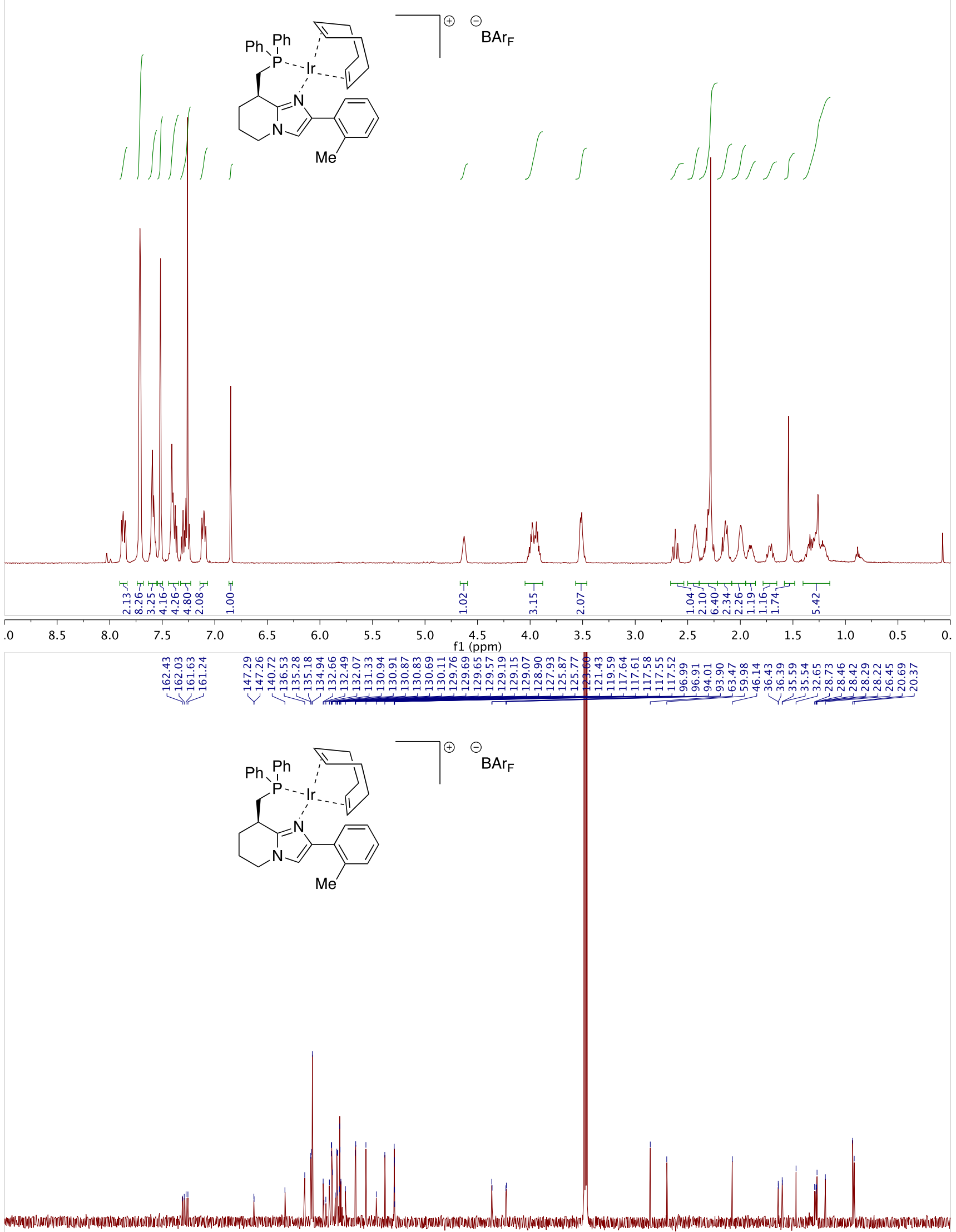

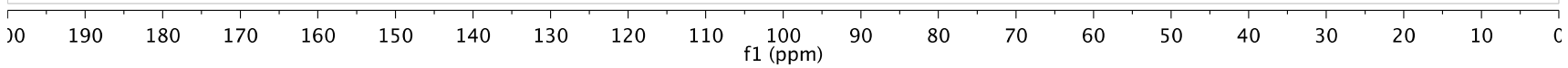




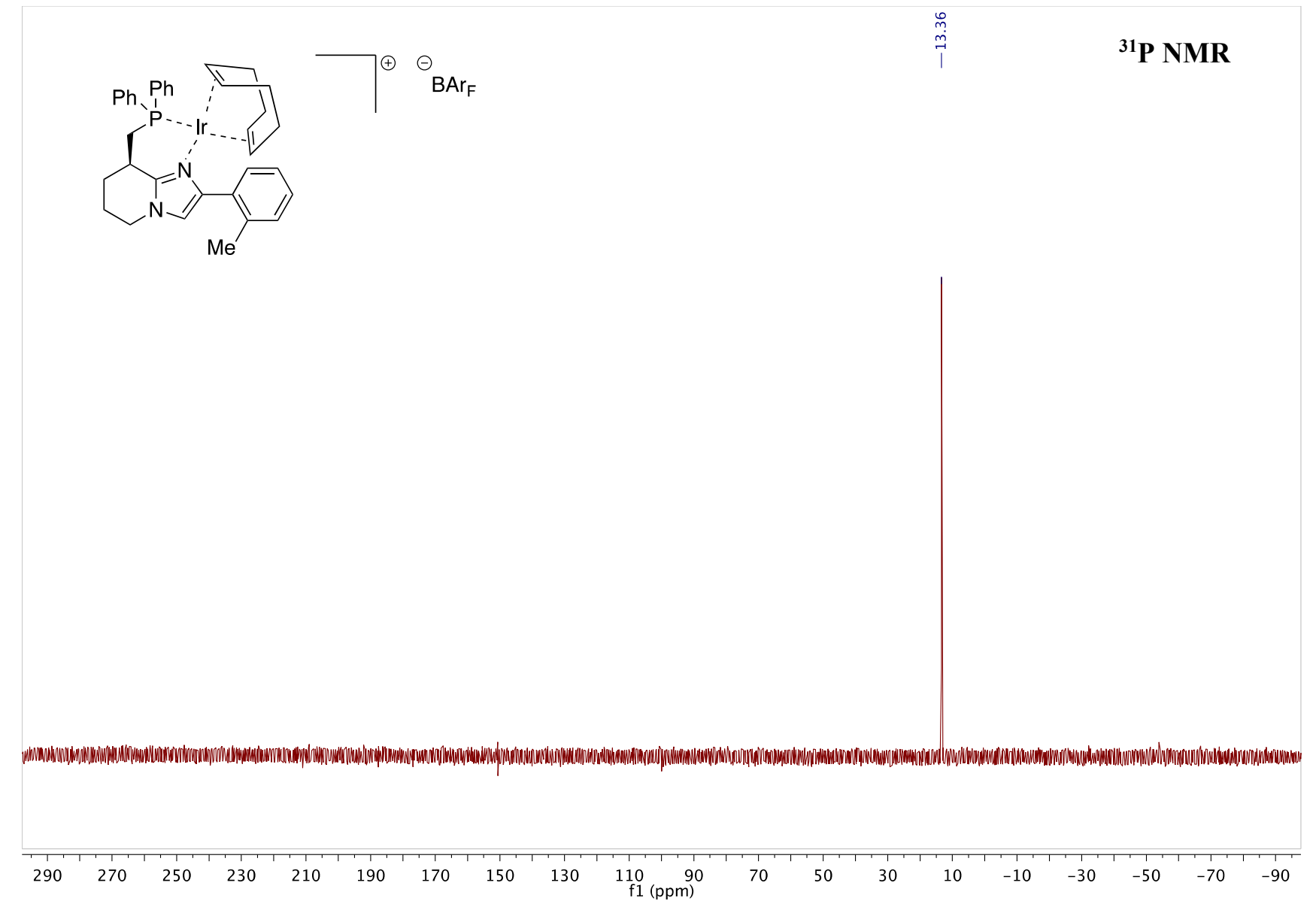




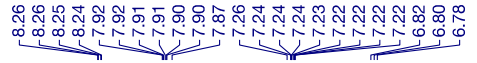
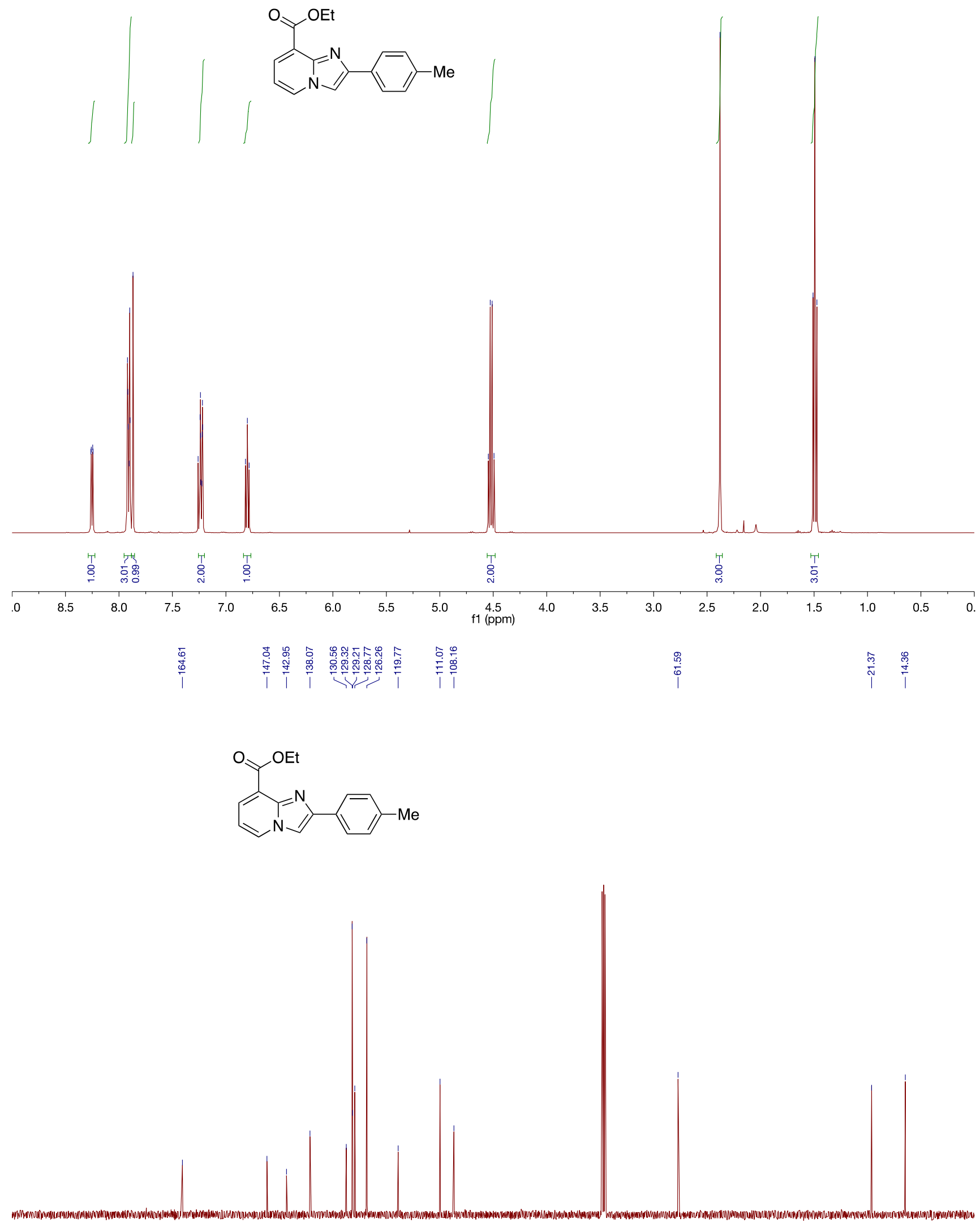

190 $180 \quad 170$ $\begin{array}{lllll}160 & 150 & 140 & 130 & 120\end{array}$ $20 \quad 110 \quad 100 \quad 90 \quad 80$ $80 \quad 70 \quad 60$

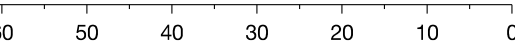



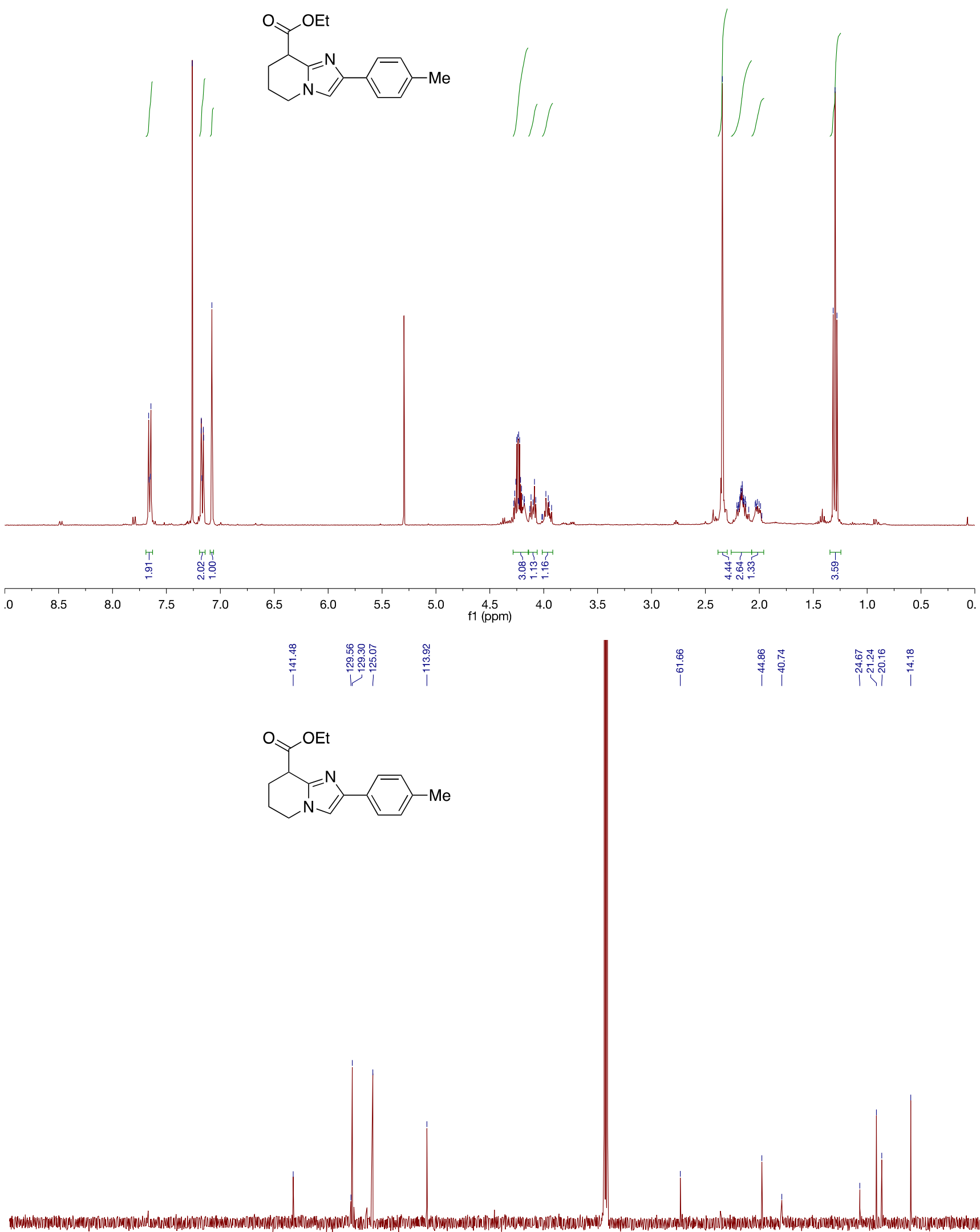

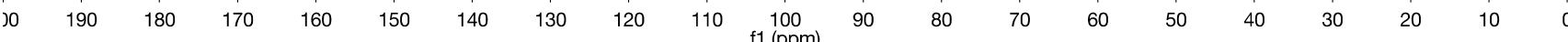




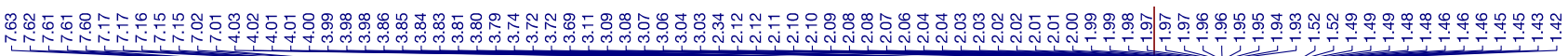
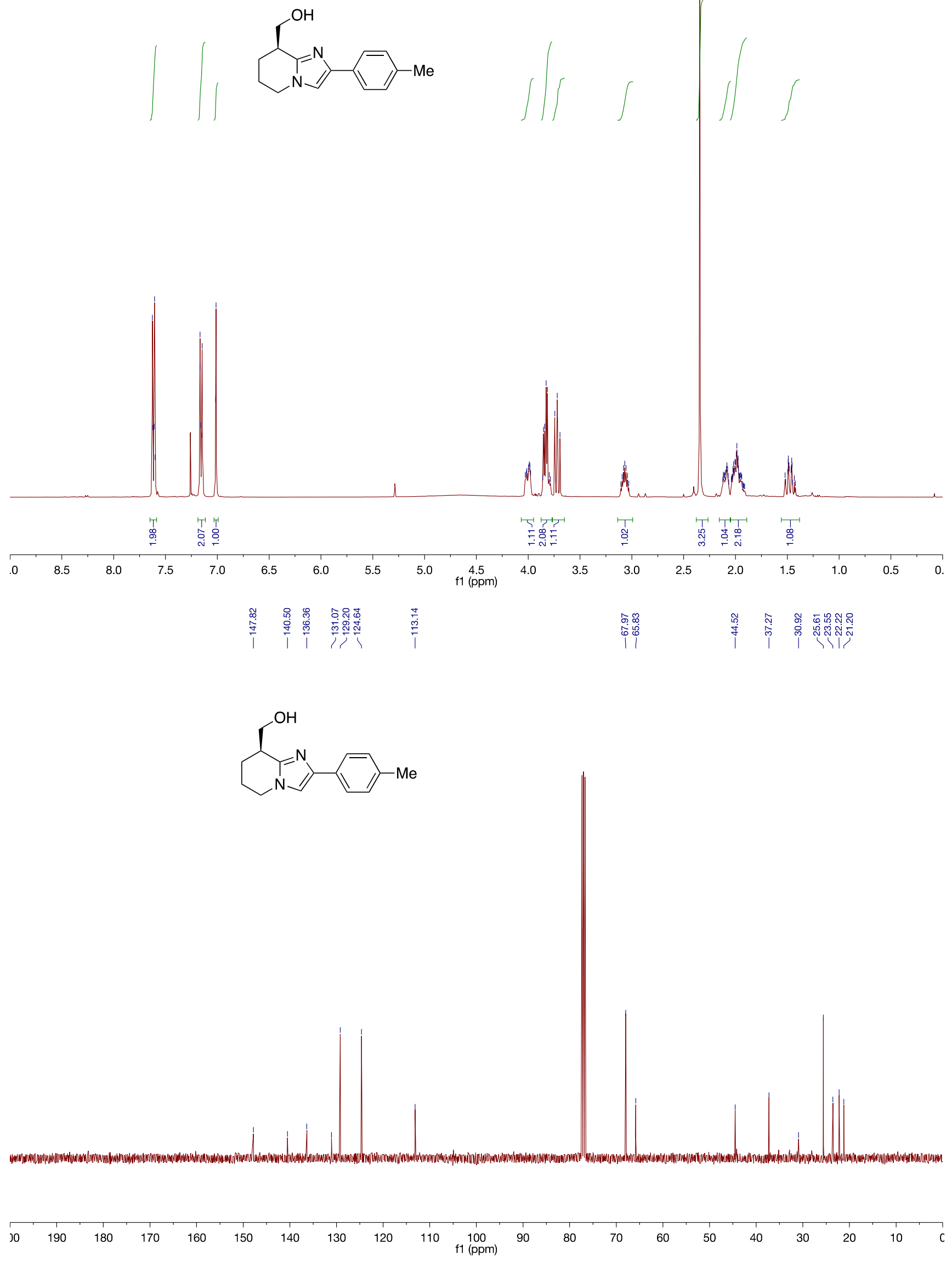

S129 


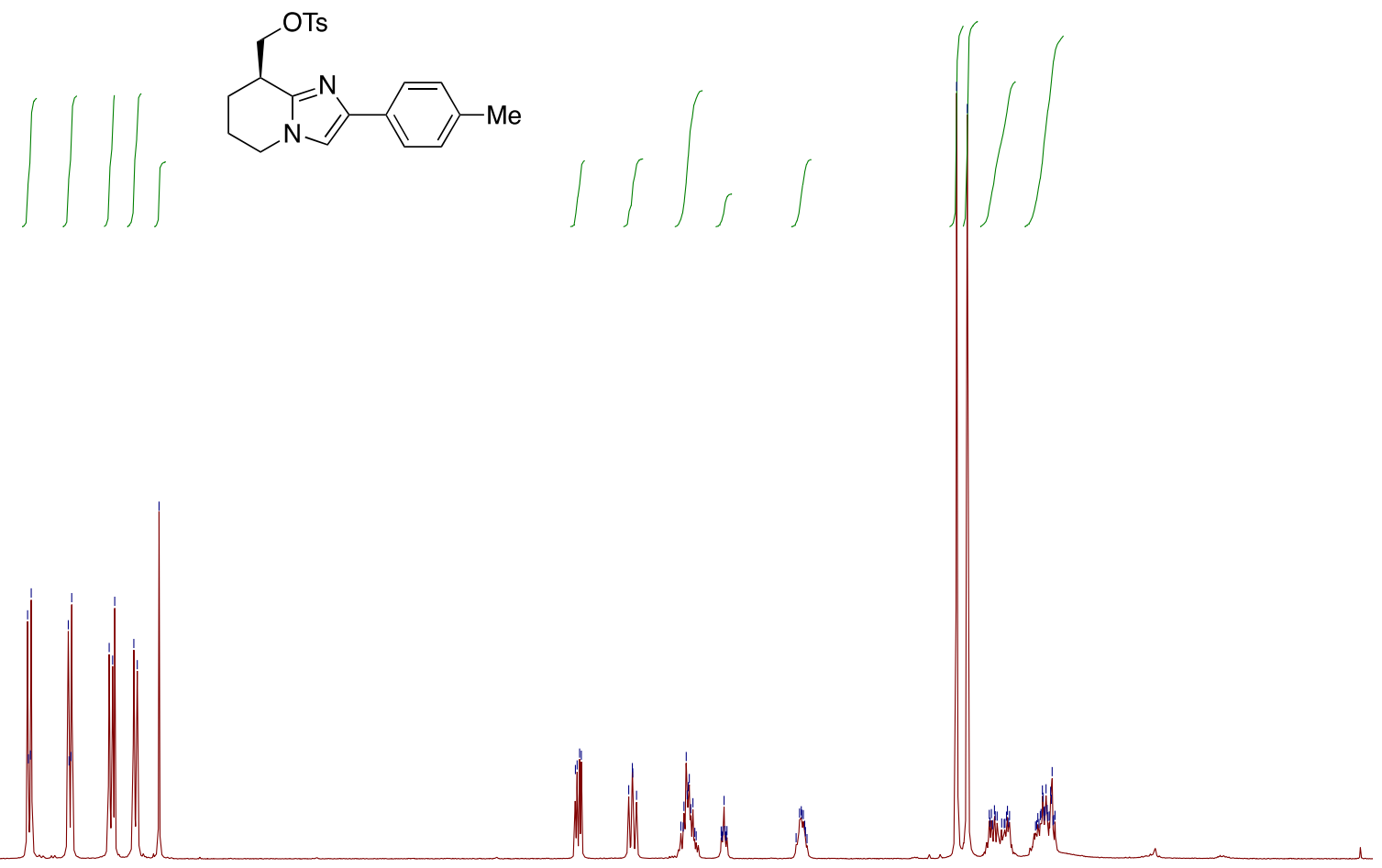

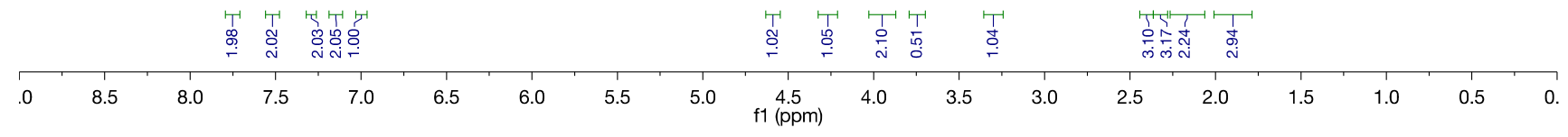

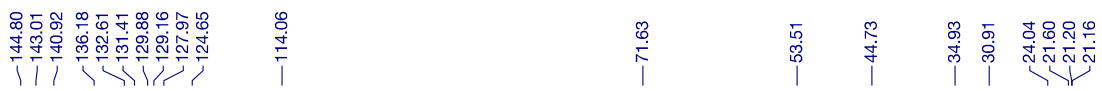

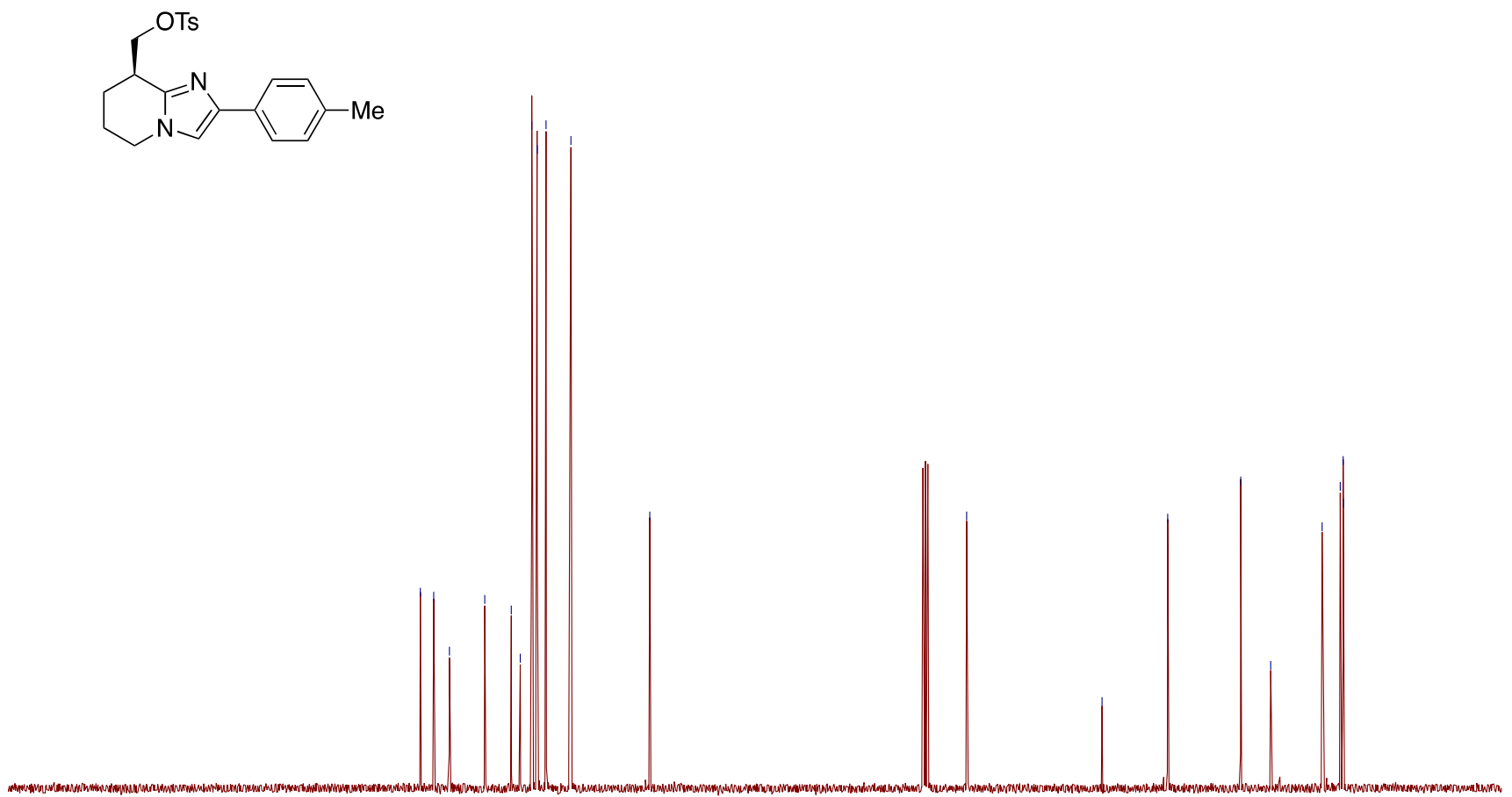

\begin{tabular}{lllllllllllllllllllll}
\hline 0 & 190 & 180 & 170 & 160 & 150 & 140 & 130 & 120 & 110 & 100 & 90 & 80 & 70 & 60 & 50 & 40 & 30 & 20 & 10 & $\mathrm{C}$
\end{tabular} 
<smiles></smiles>

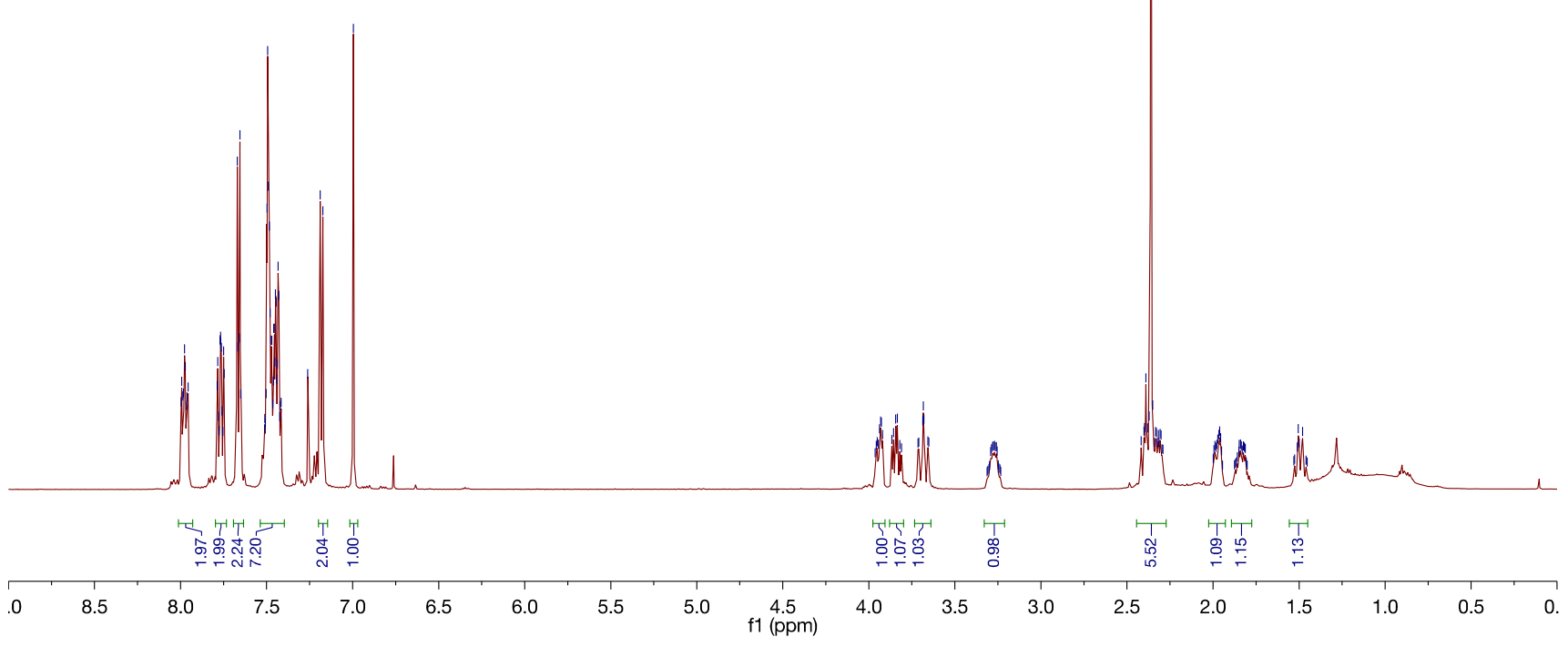

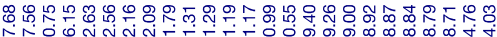
守守宁它<smiles>Cc1ccc(-c2cn3c(n2)C(Cc2ccccc2)CCC3)cc1</smiles>

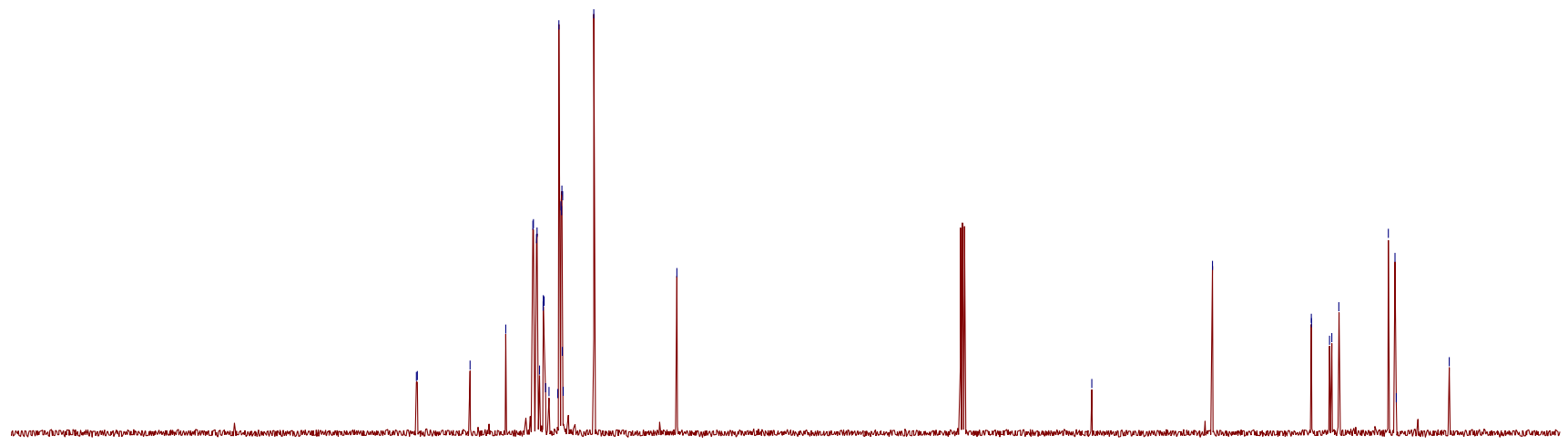

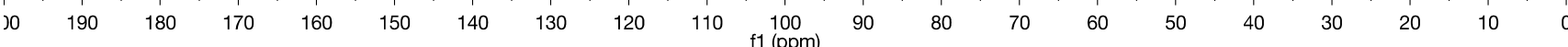



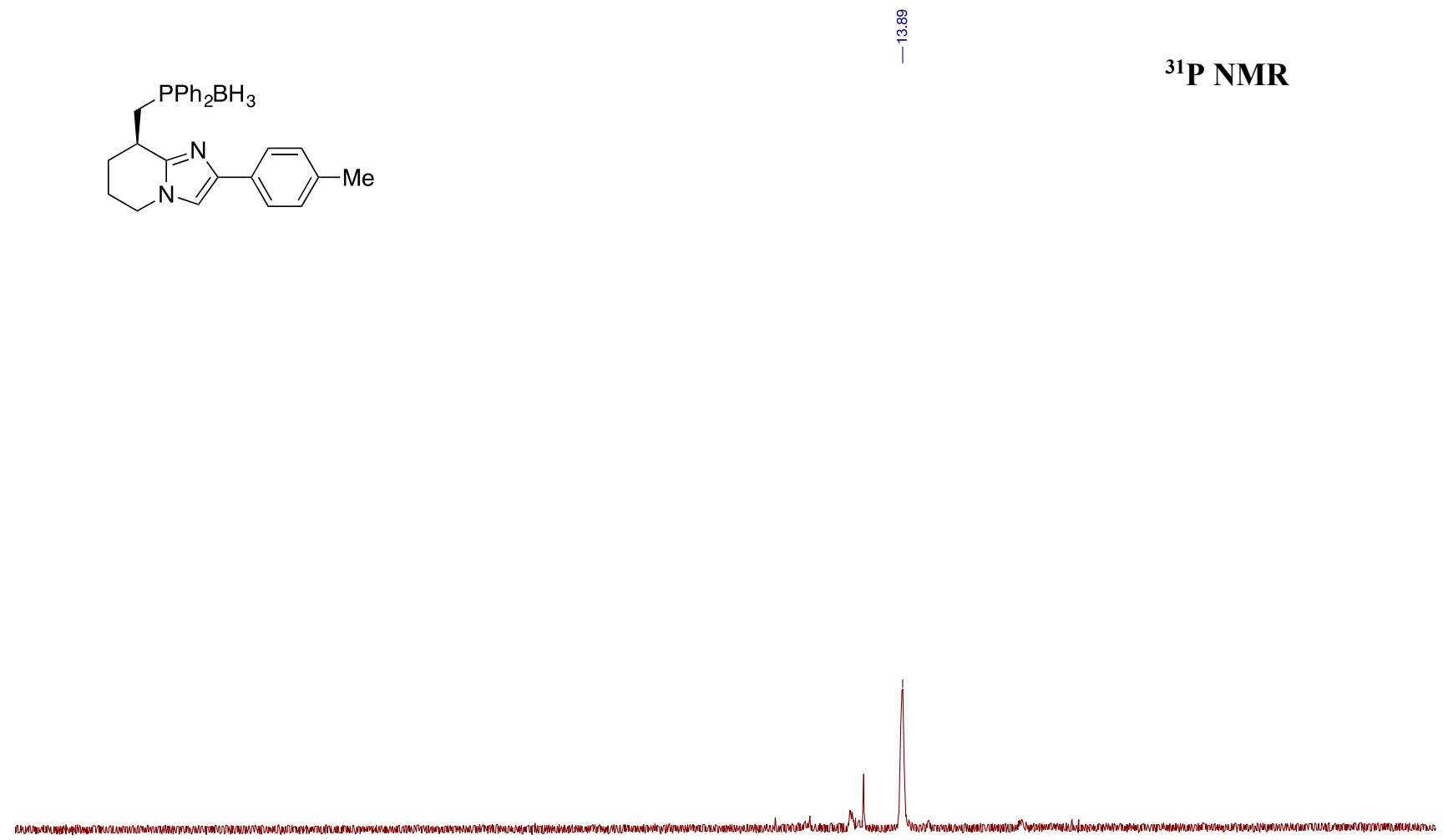

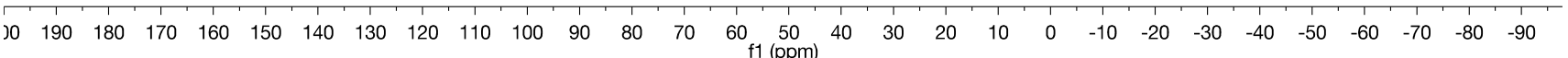


<smiles>Cc1ccc(-c2cn3c(n2)[C@@H](Cc2ccccc2)CCC3)cc1</smiles>
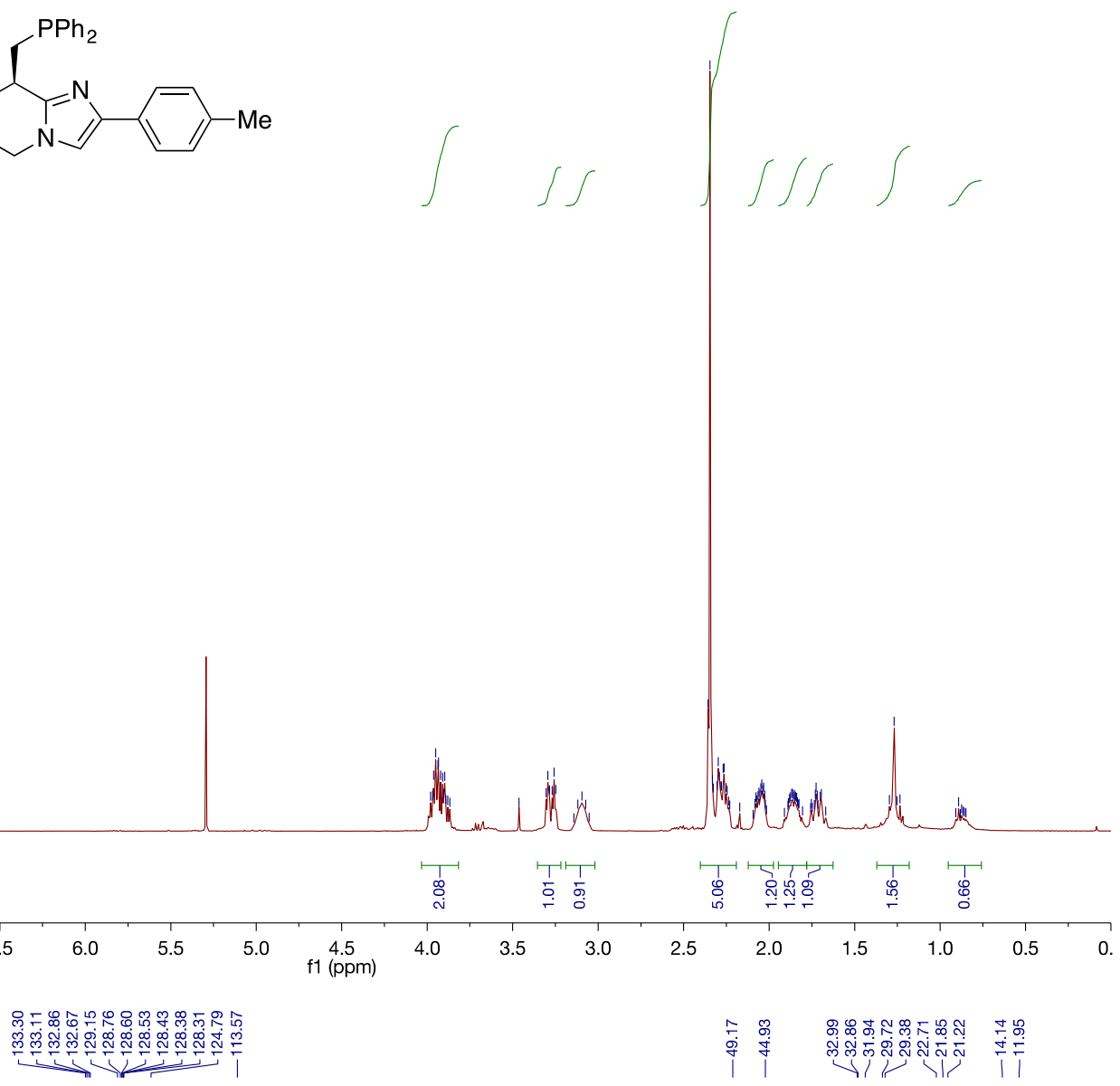

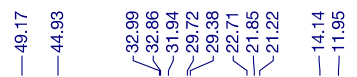

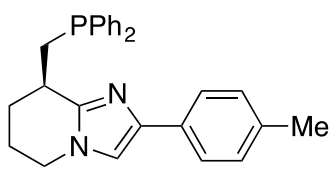

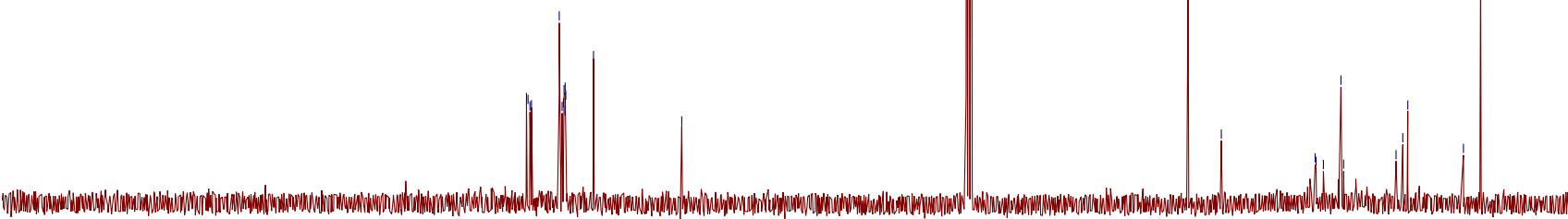

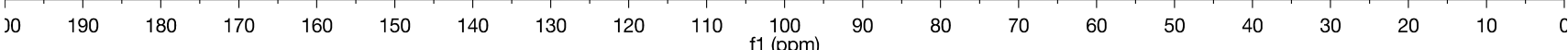


<smiles>Cc1ccc(-c2cn3c(n2)C(Cc2ccccc2)CCC3)cc1</smiles> 


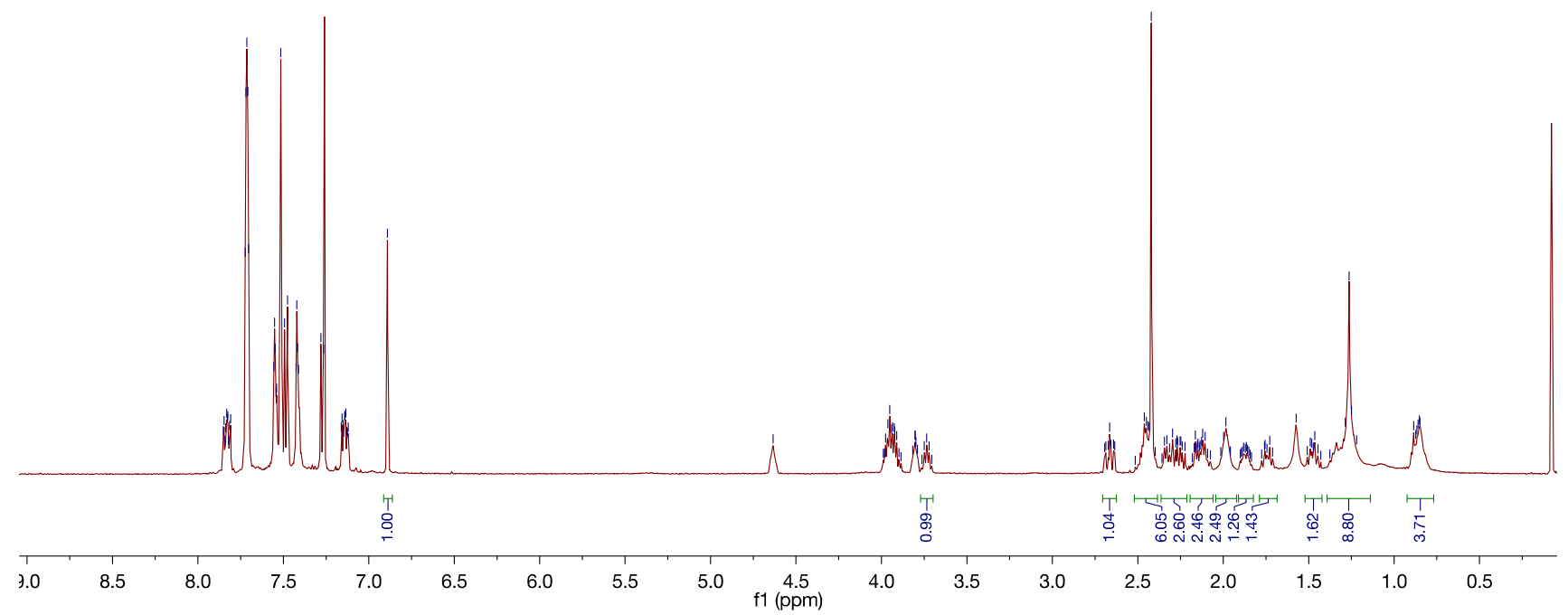

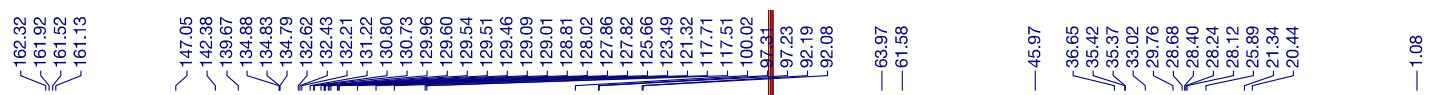

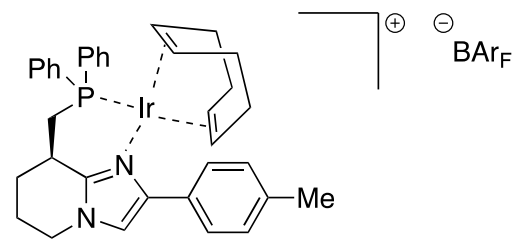

19.

\begin{tabular}{lllllllllllllllllllllllll}
\hline 0 & 190 & 180 & 170 & 160 & 150 & 140 & 130 & 120 & 110 & $\begin{array}{c}100 \\
\mathrm{f} 1(\mathrm{ppm})\end{array}$ & 90 & 80 & 70 & 60 & 50 & 40 & 30 & 20 & 10 & $\mathrm{C}$
\end{tabular} 


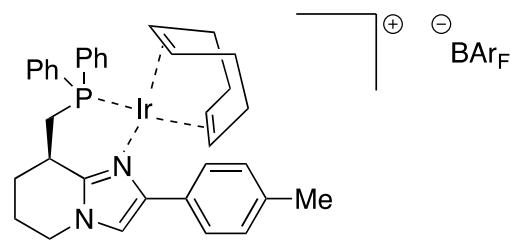

${ }^{31}$ P NMR

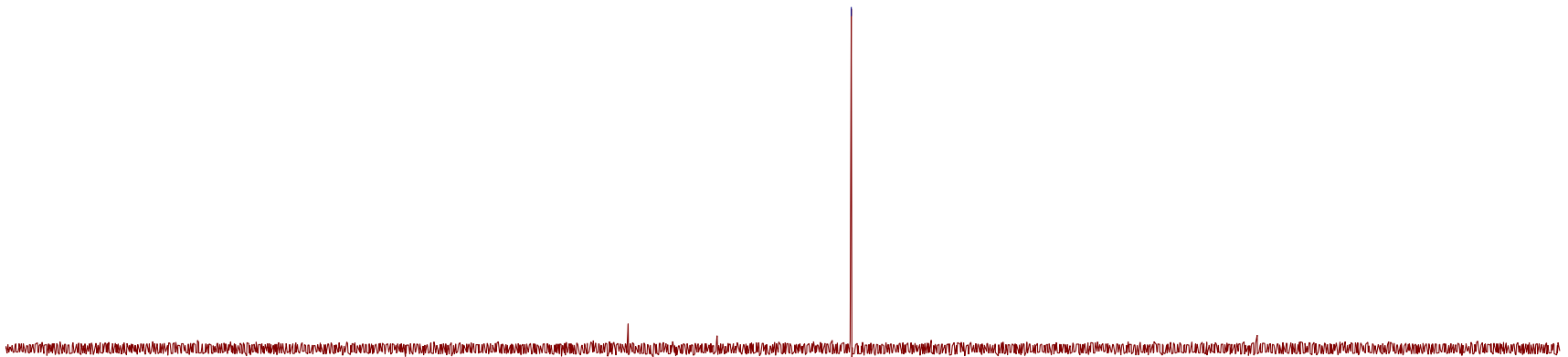

$\begin{array}{llllllllllllllllllllllllll}140 & 130 & 120 & 110 & 100 & 90 & 80 & 70 & 60 & 50 & 40 & 30 & 20 & 10 & 0 & -10 & -20 & -30 & -40 & -50 & -60 & -70 & -80 & -90\end{array}$ 


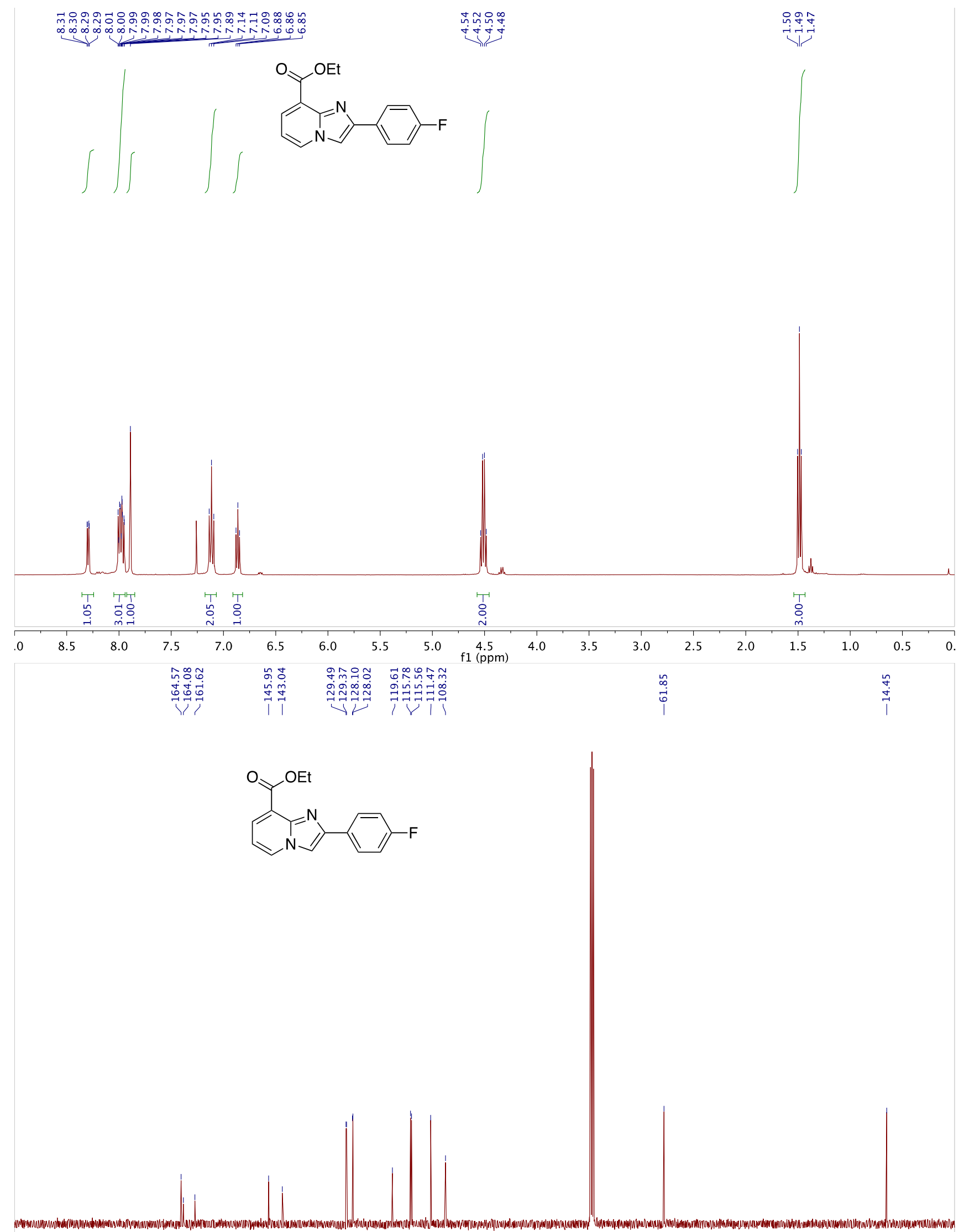

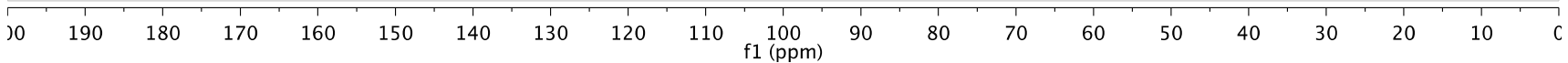




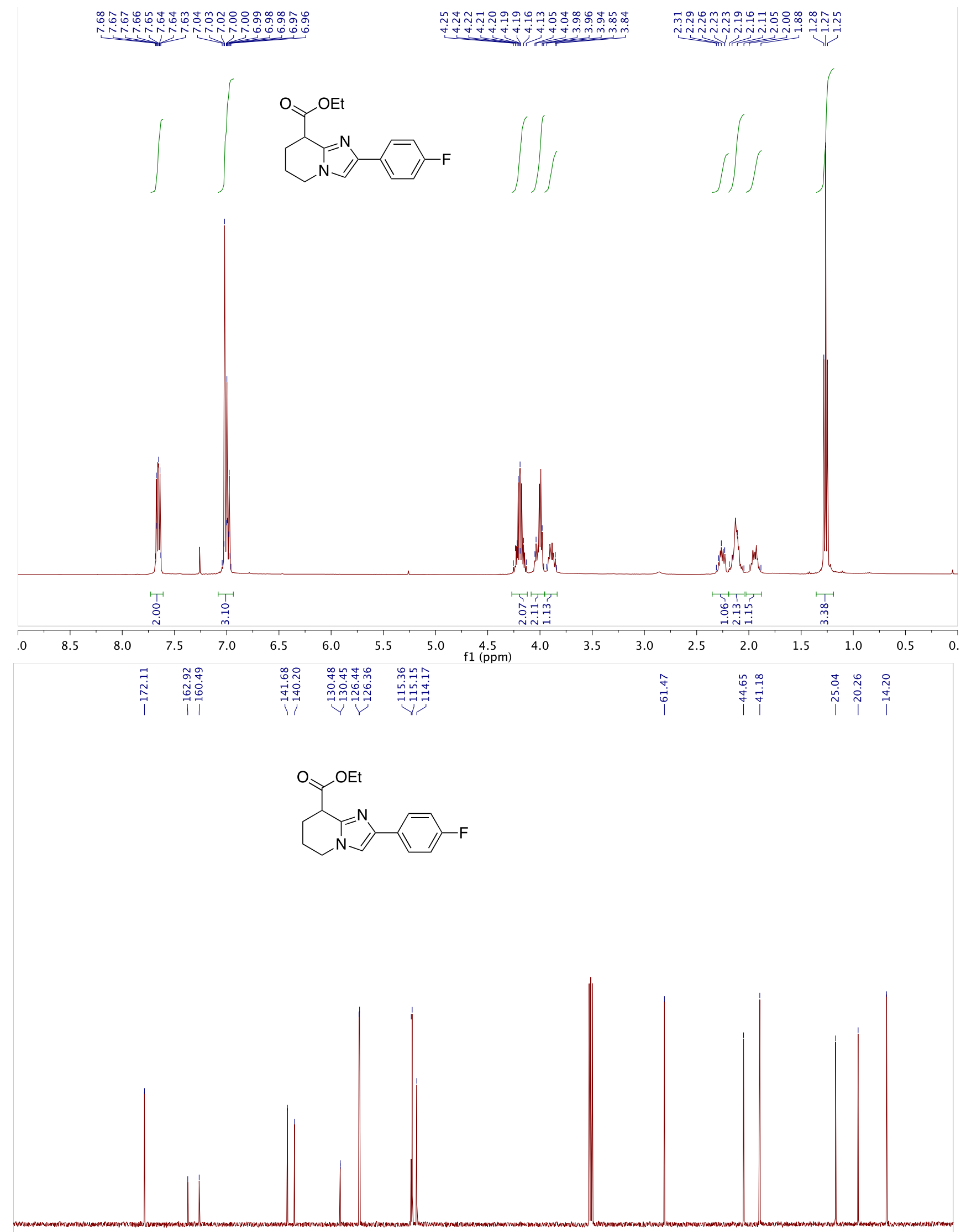

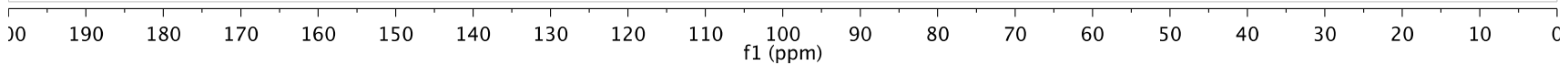




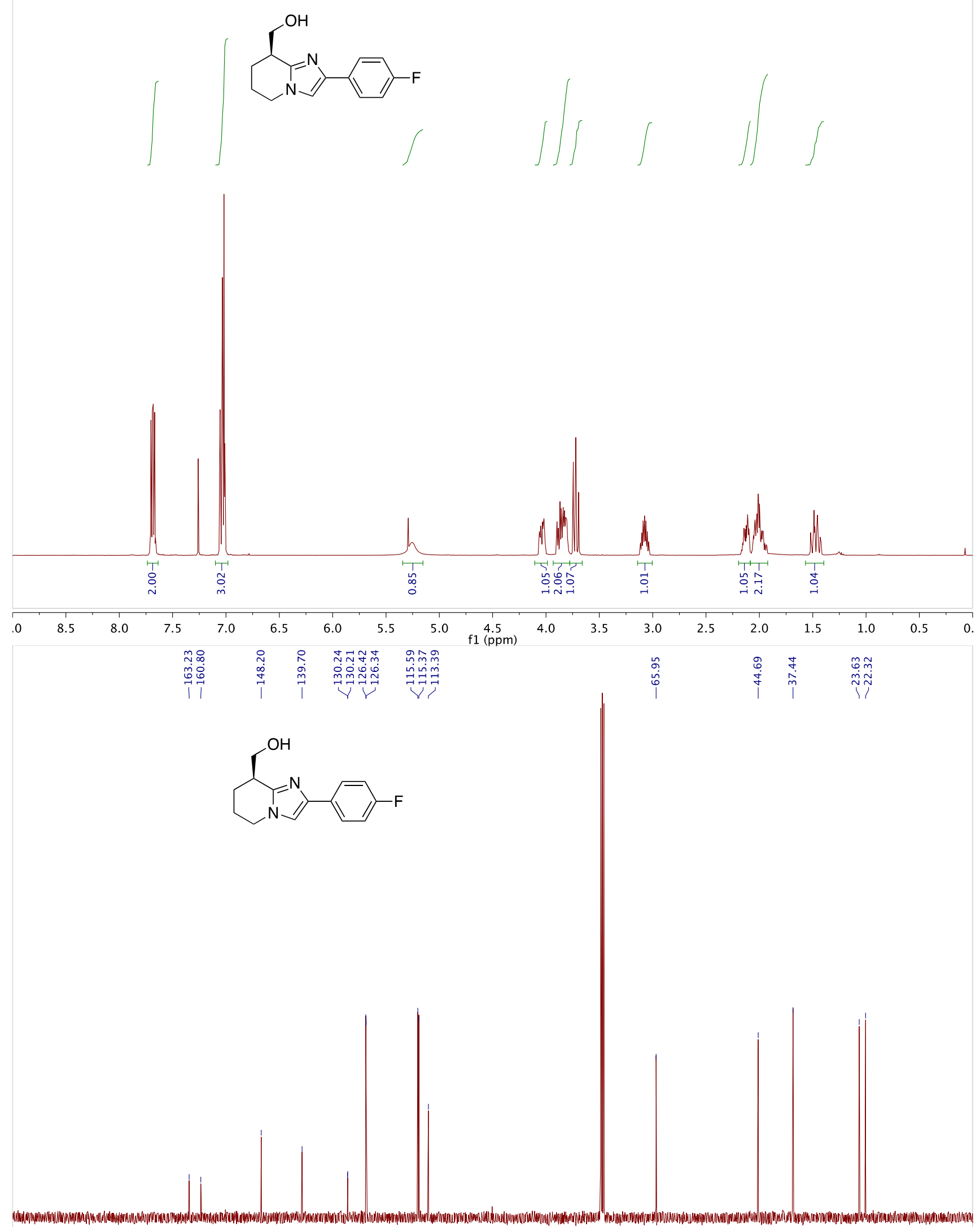

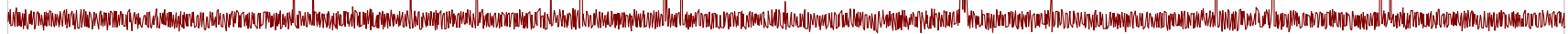

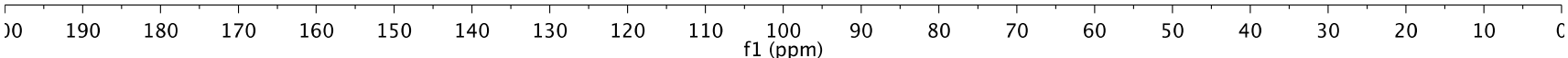




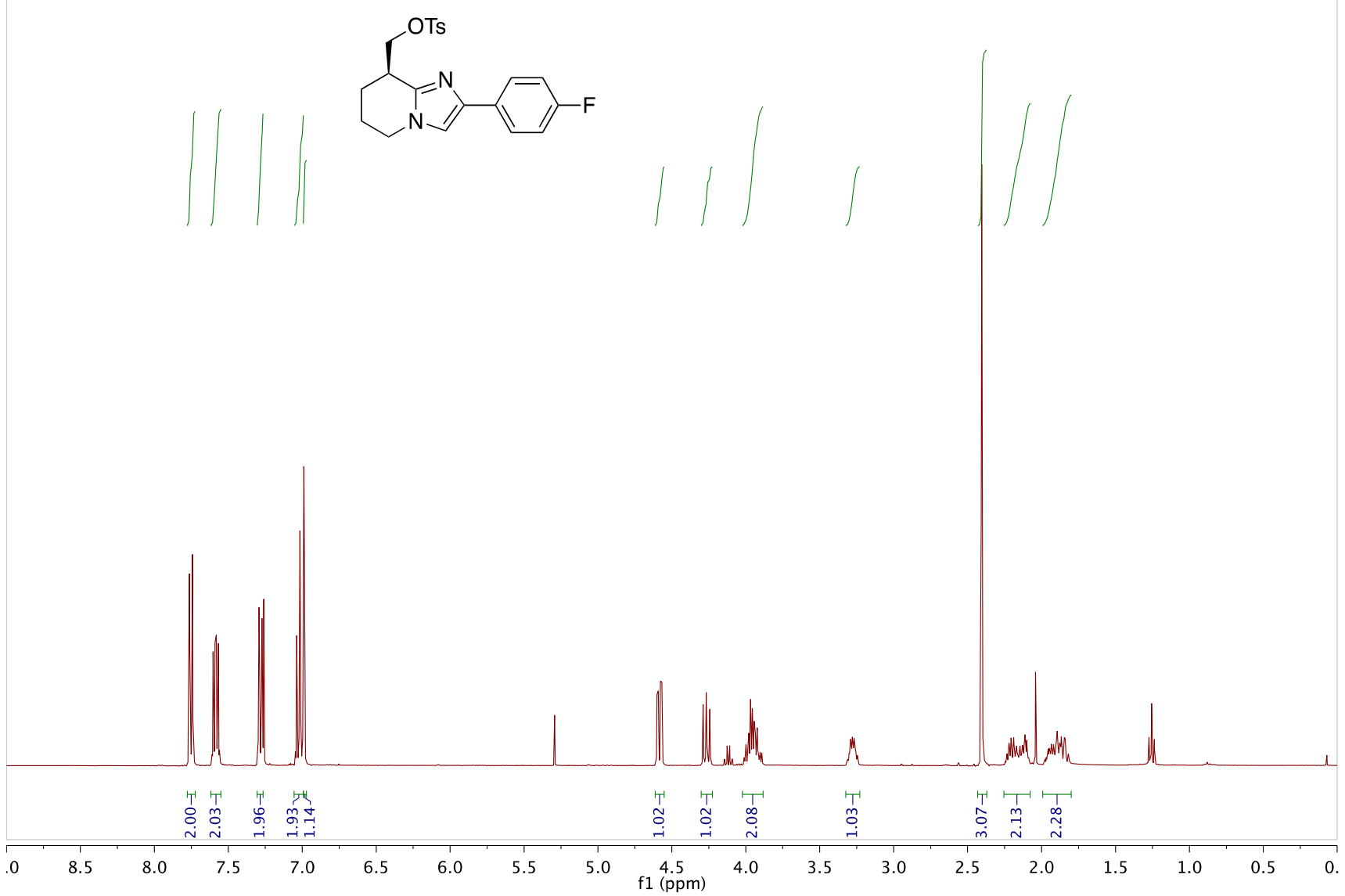

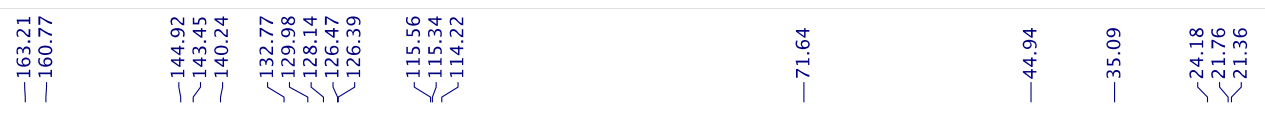
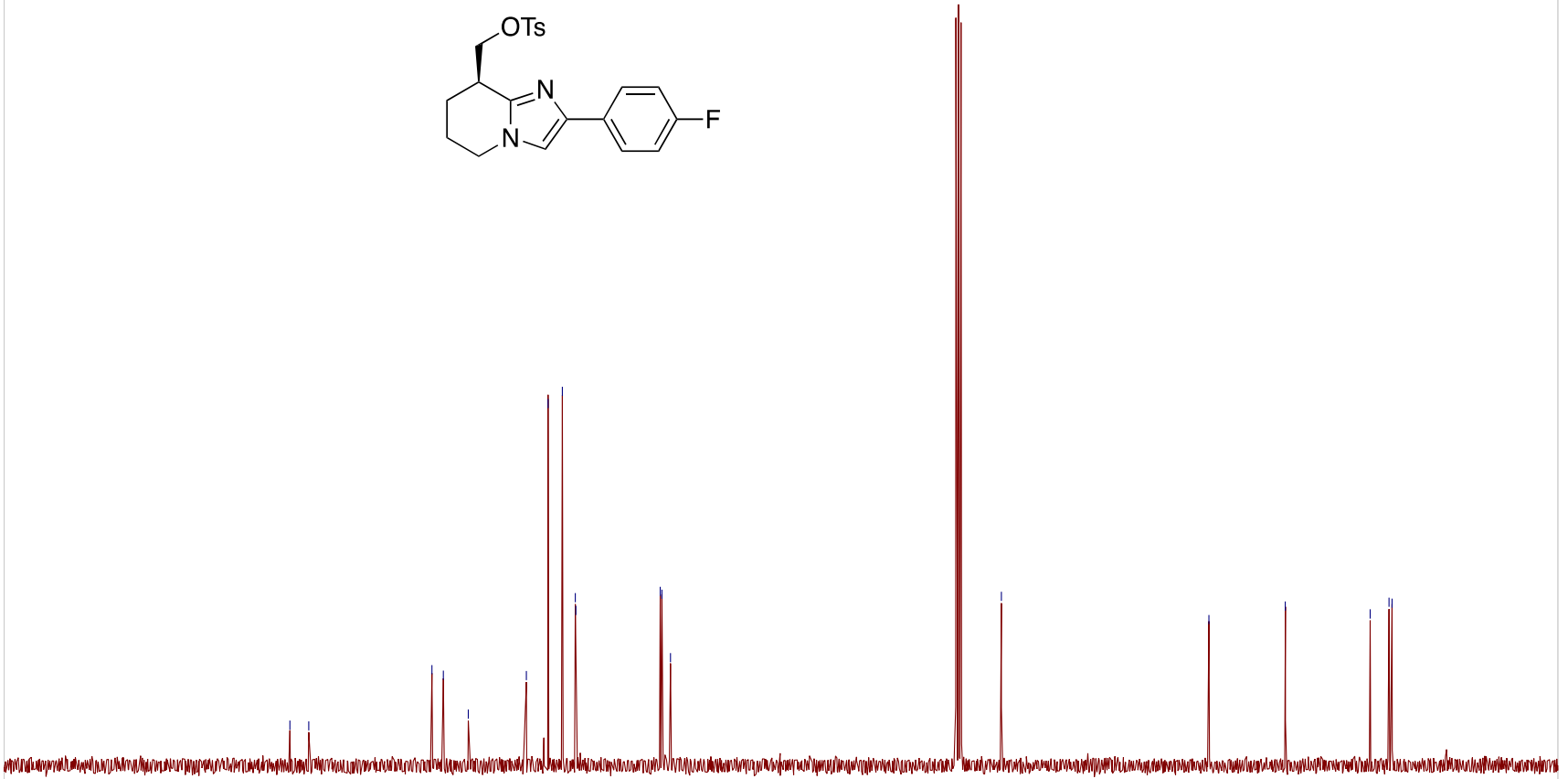

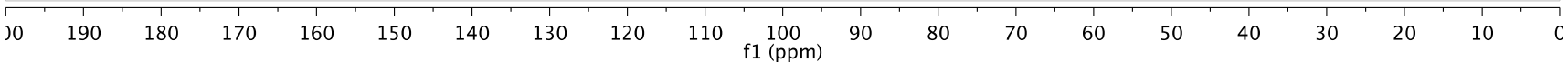



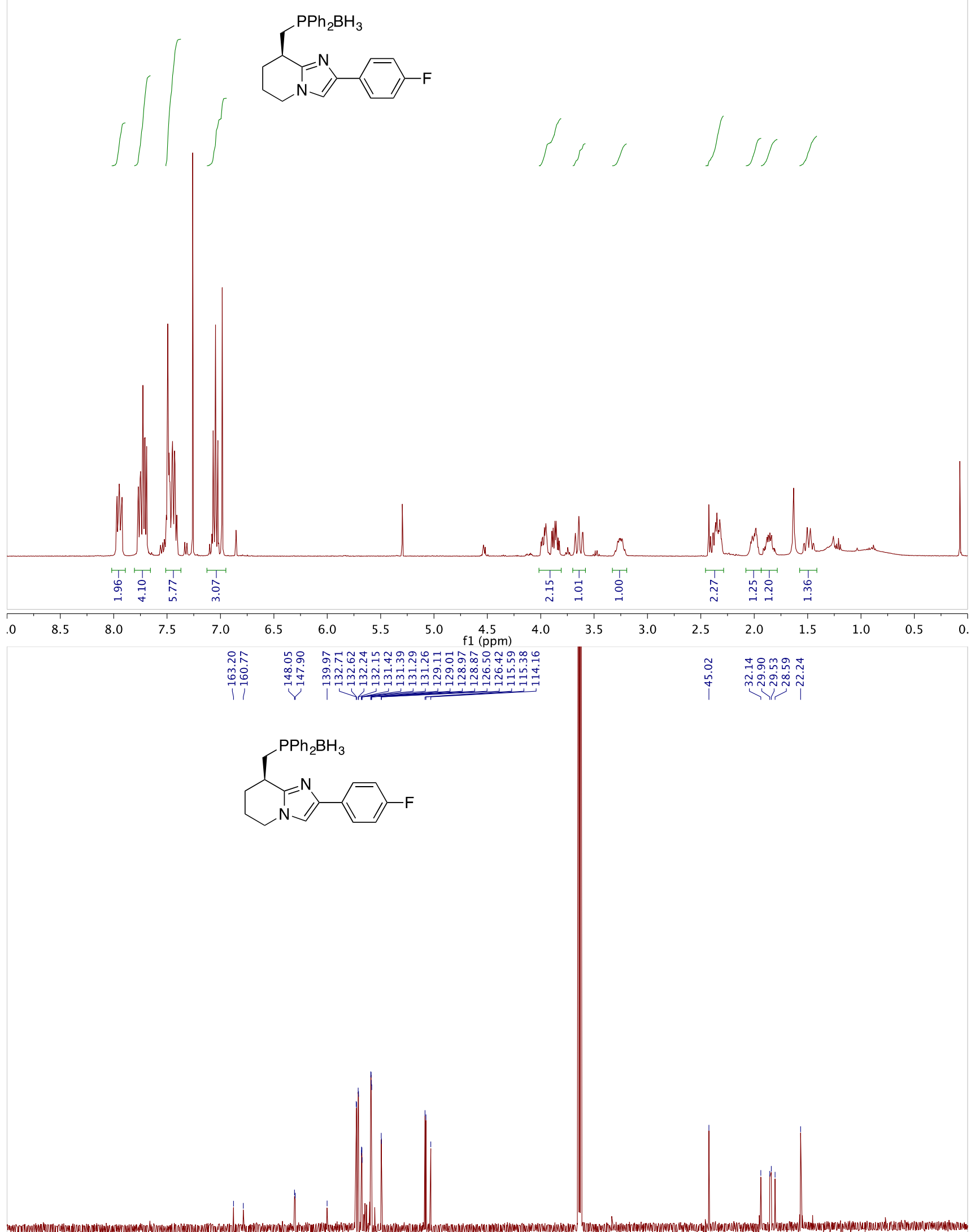

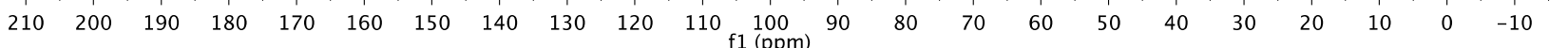



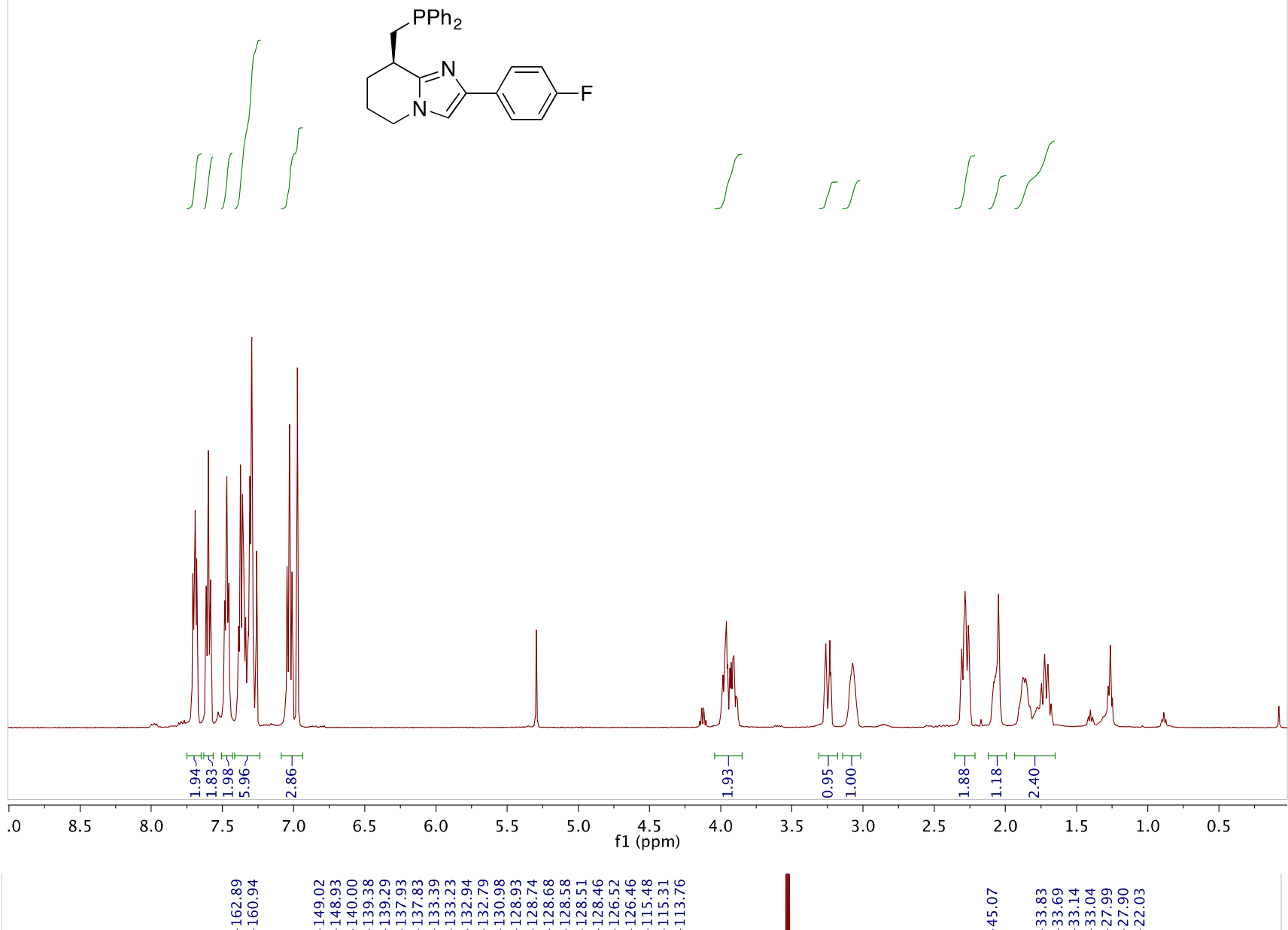

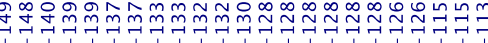

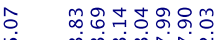

11

菂

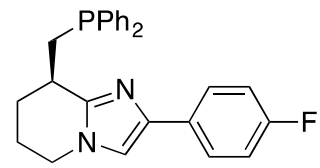

)0 190

$\begin{array}{lllll}90 & 180 & 170 & 160 & 150\end{array}$

140130

120

10100

90

$80 \quad 70 \quad 60$

50

40

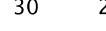


<smiles>Fc1ccc(-c2cn3c(n2)C(Cc2ccccc2)CCC3)cc1</smiles>

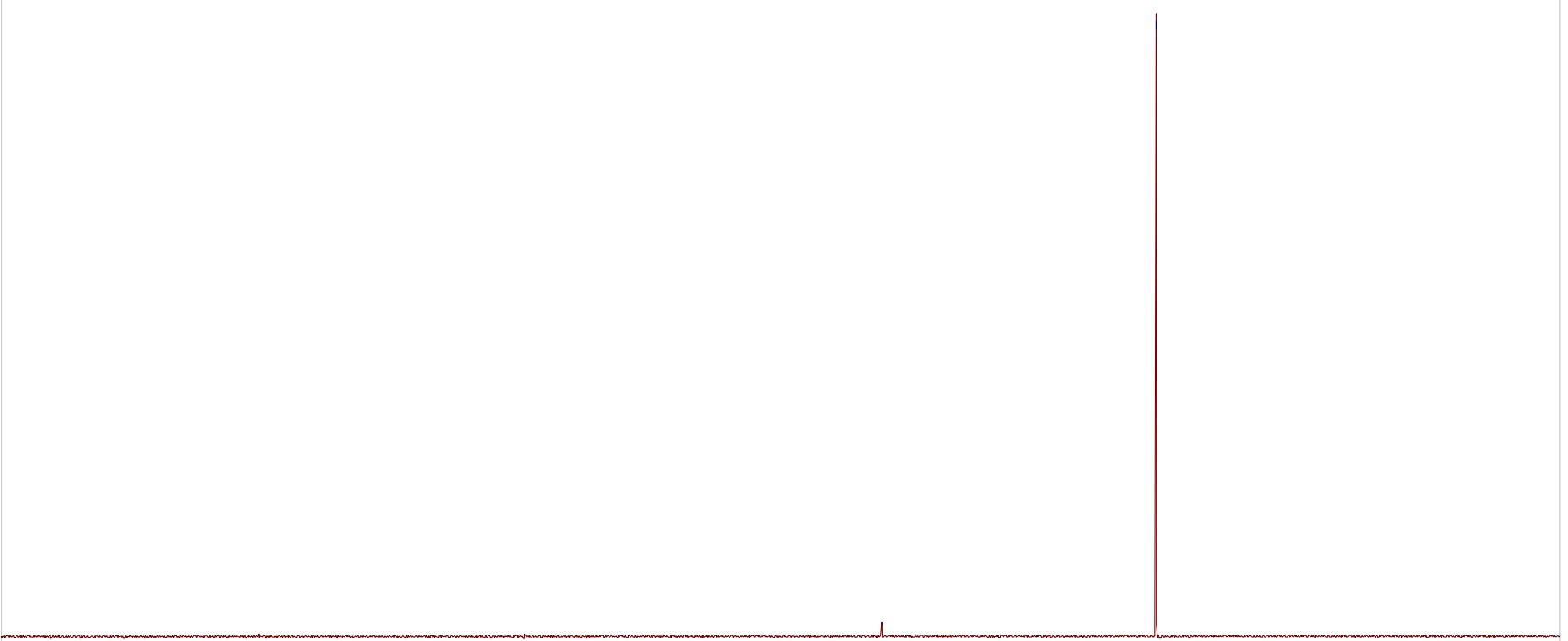

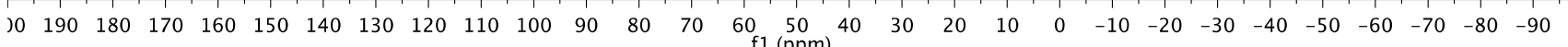




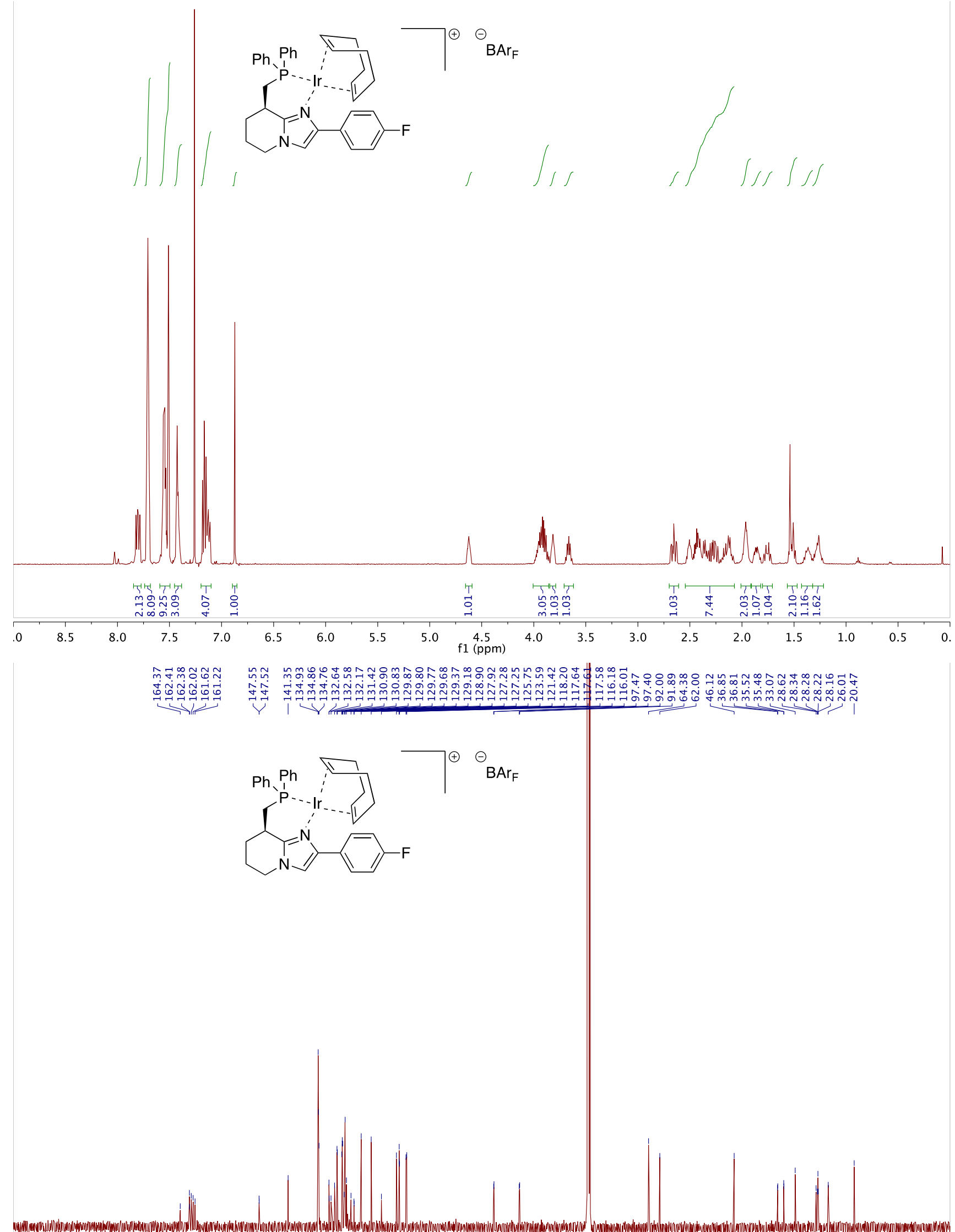

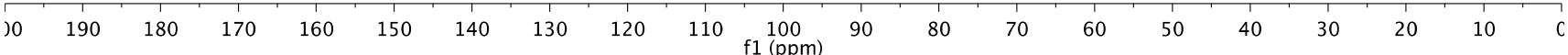




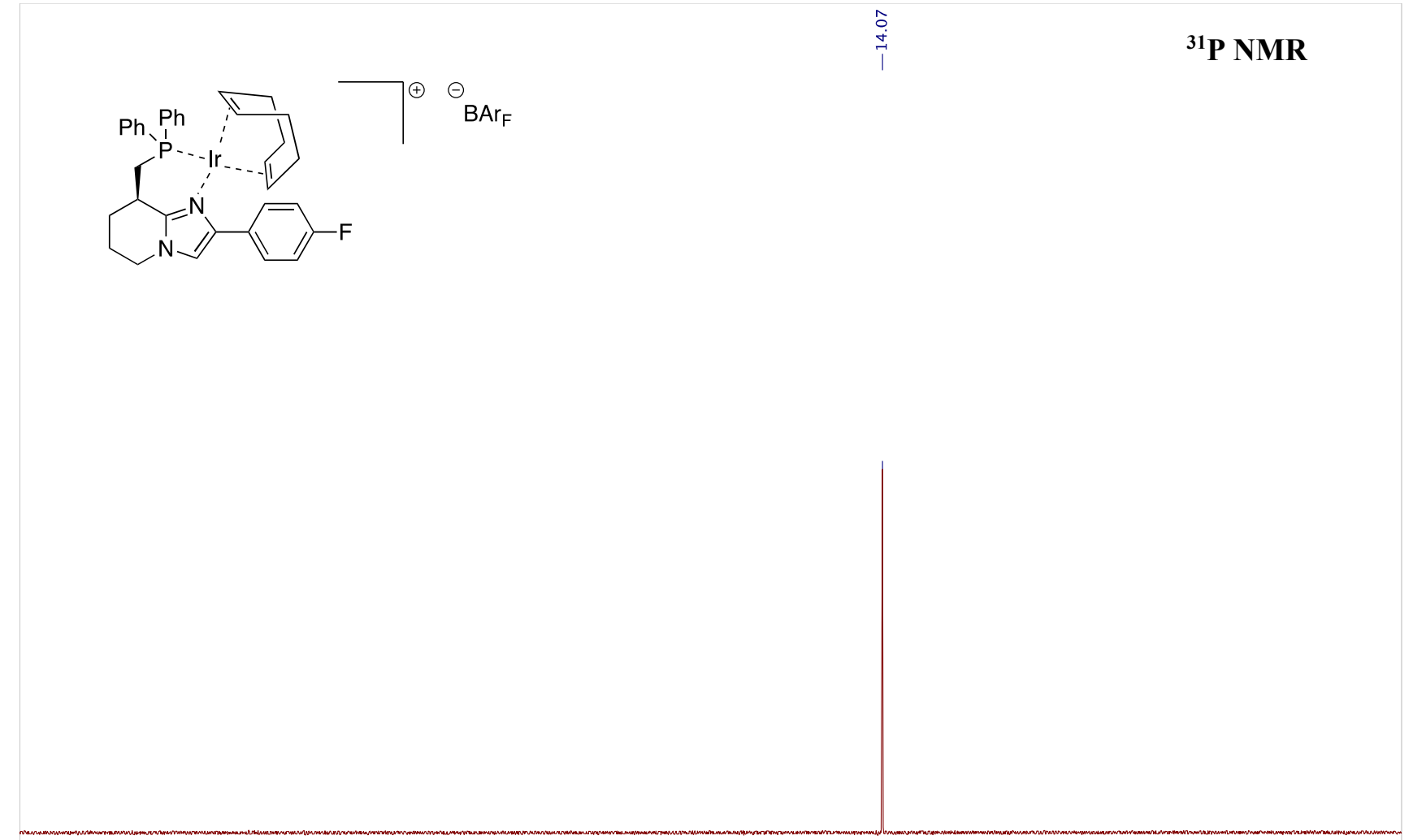

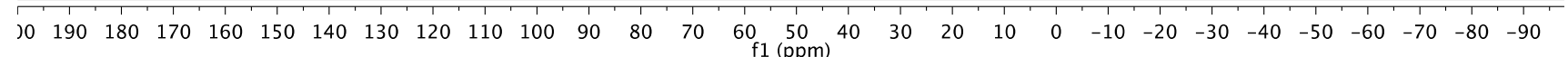




\section{Chromatograms}

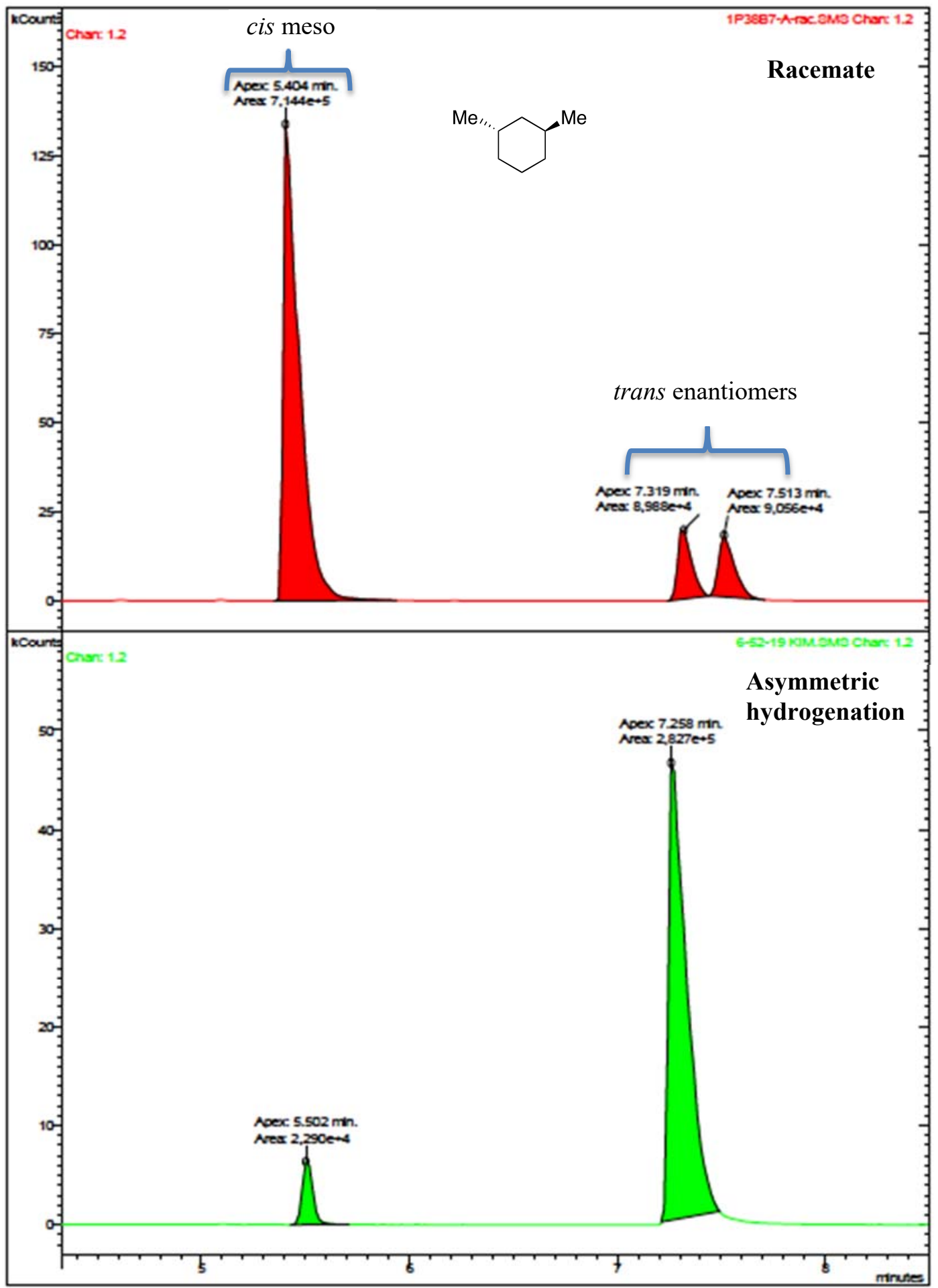




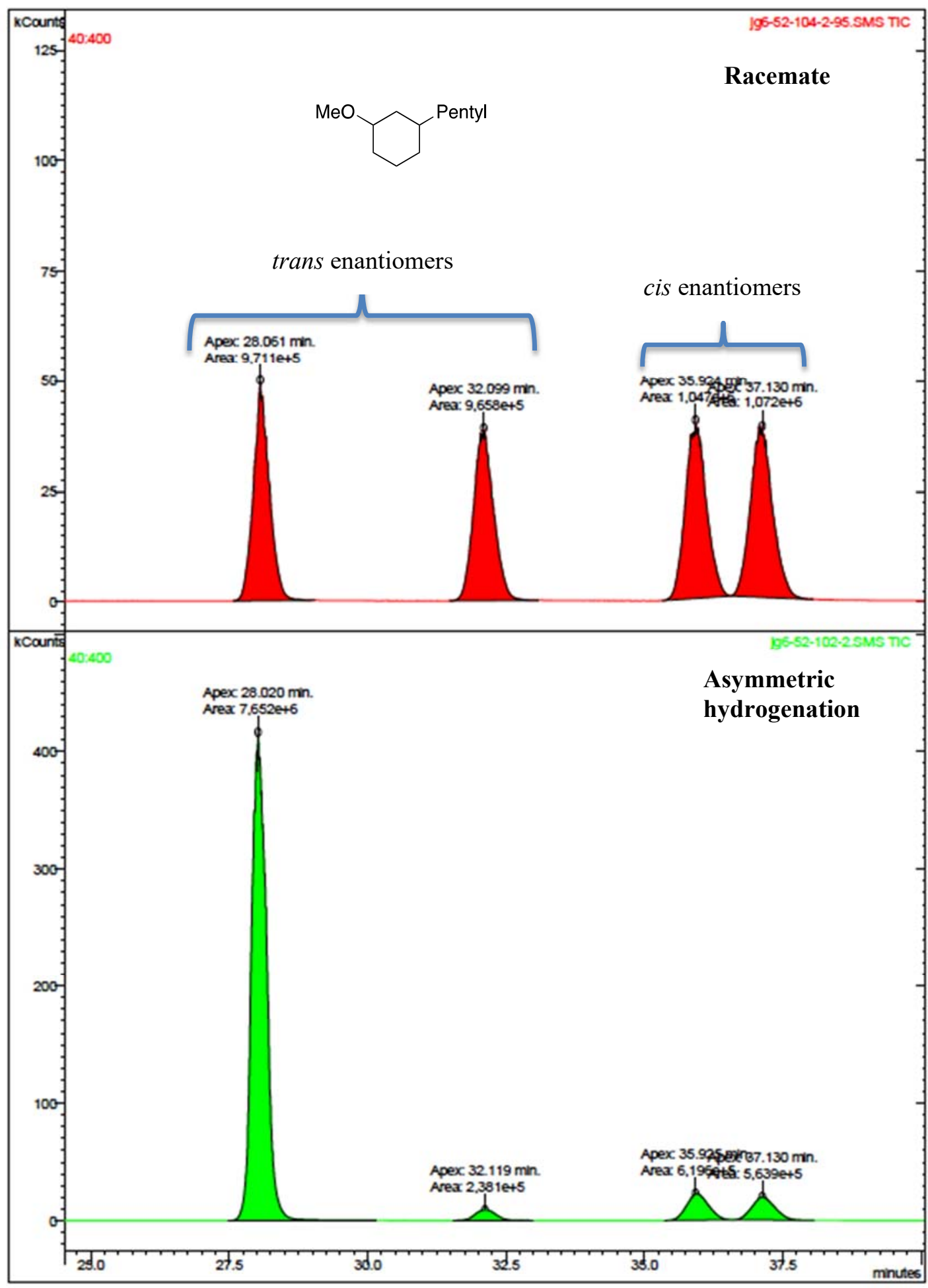




\section{Chromatogram Plots}

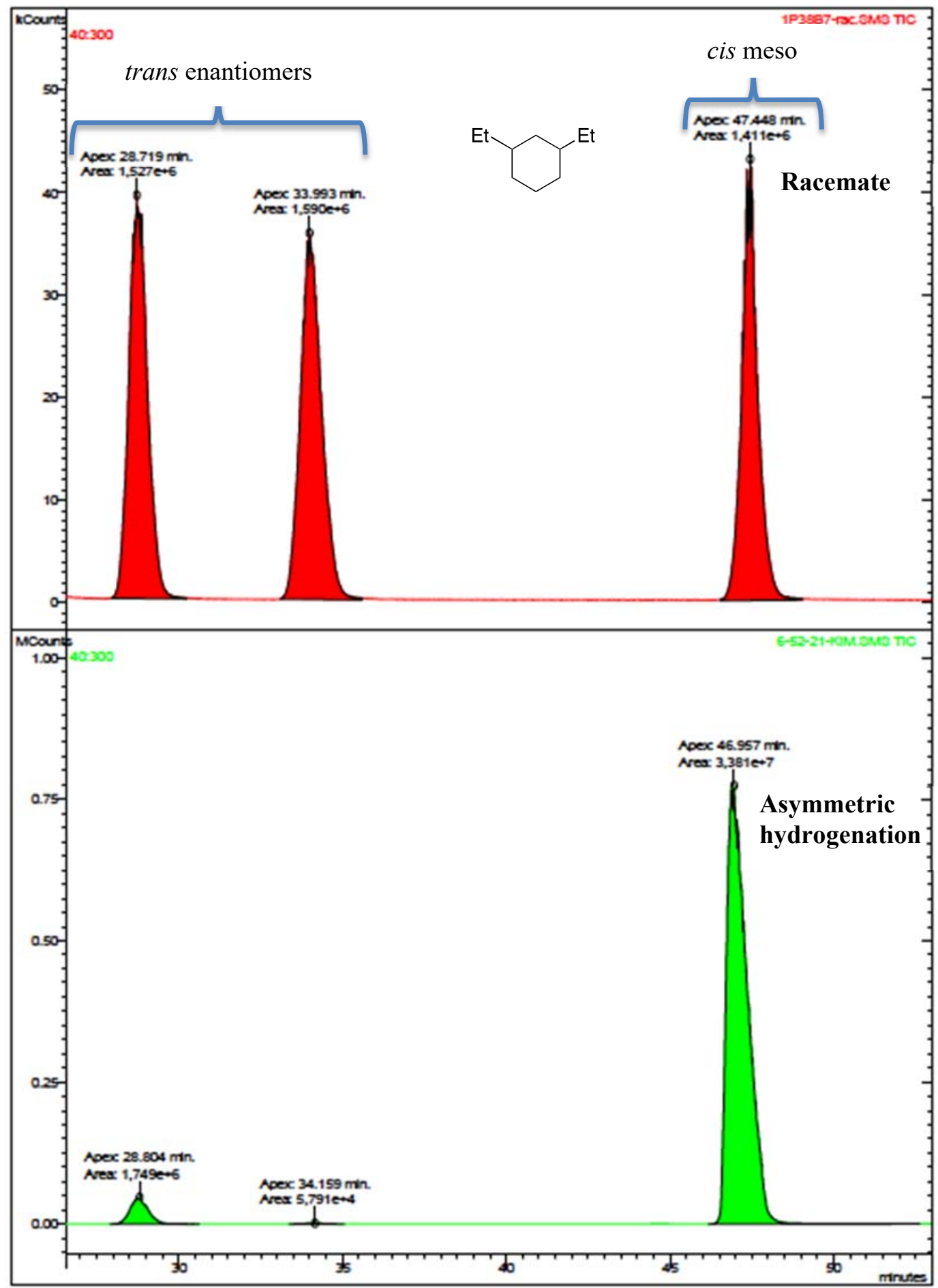




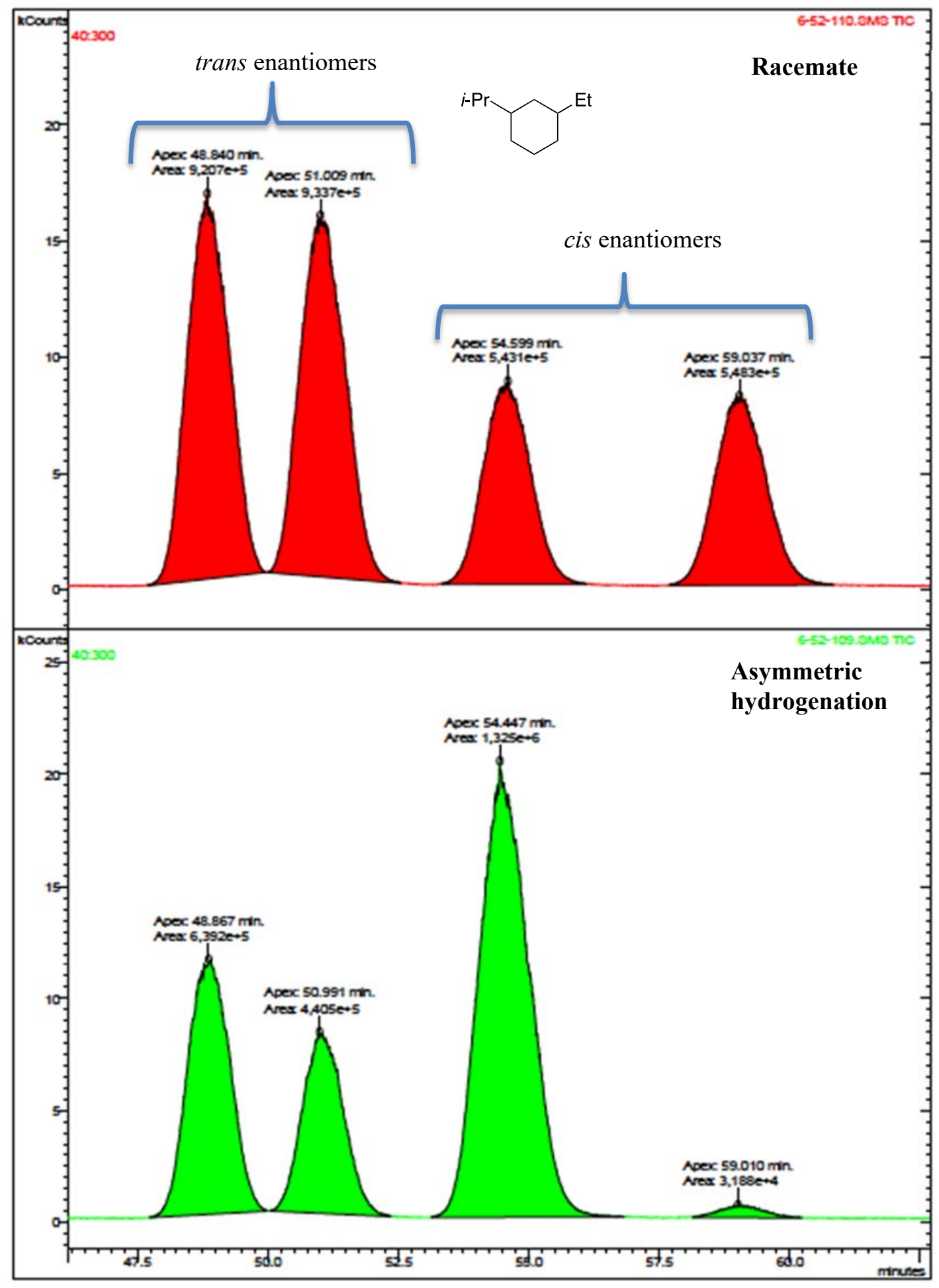




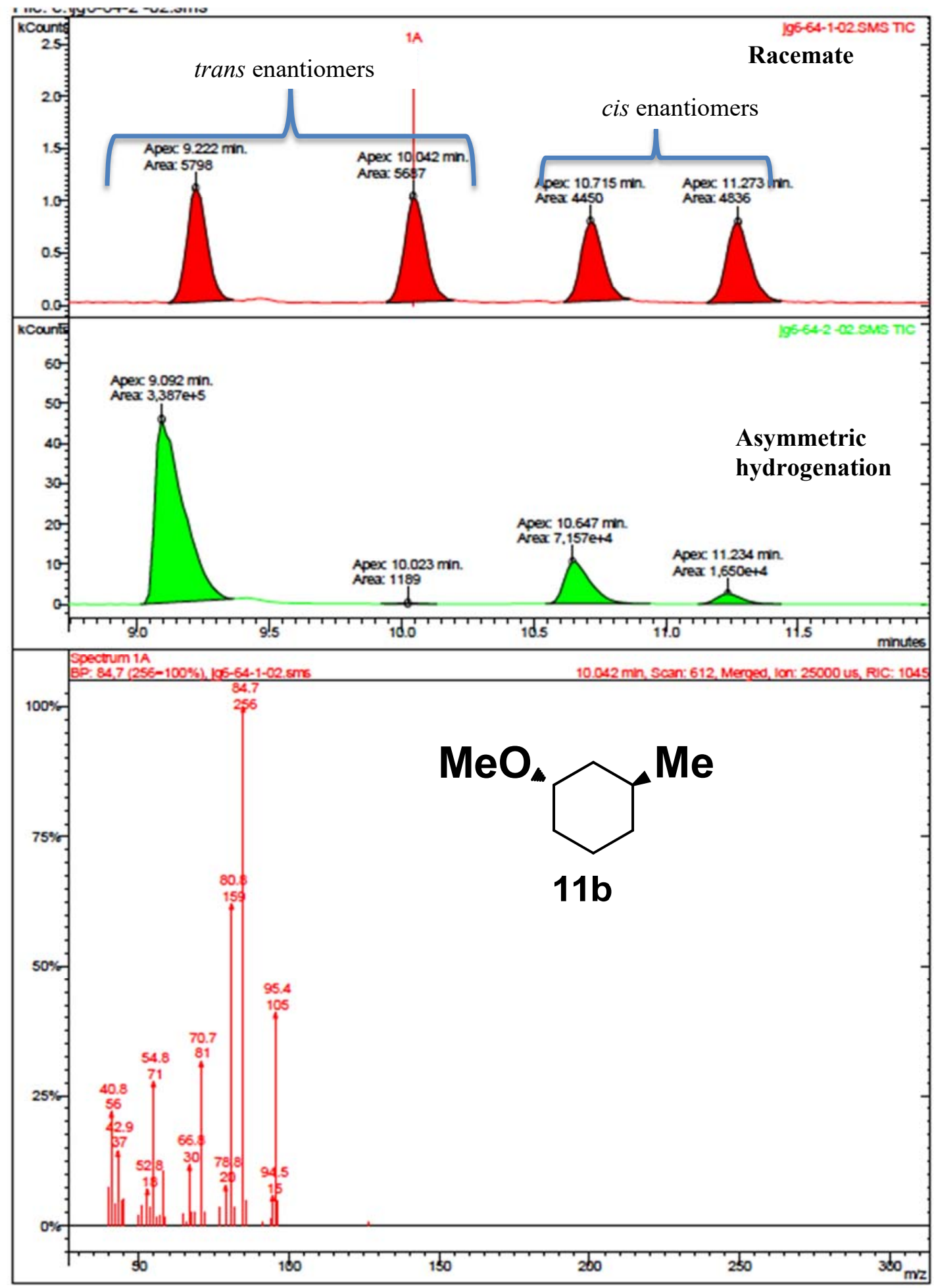




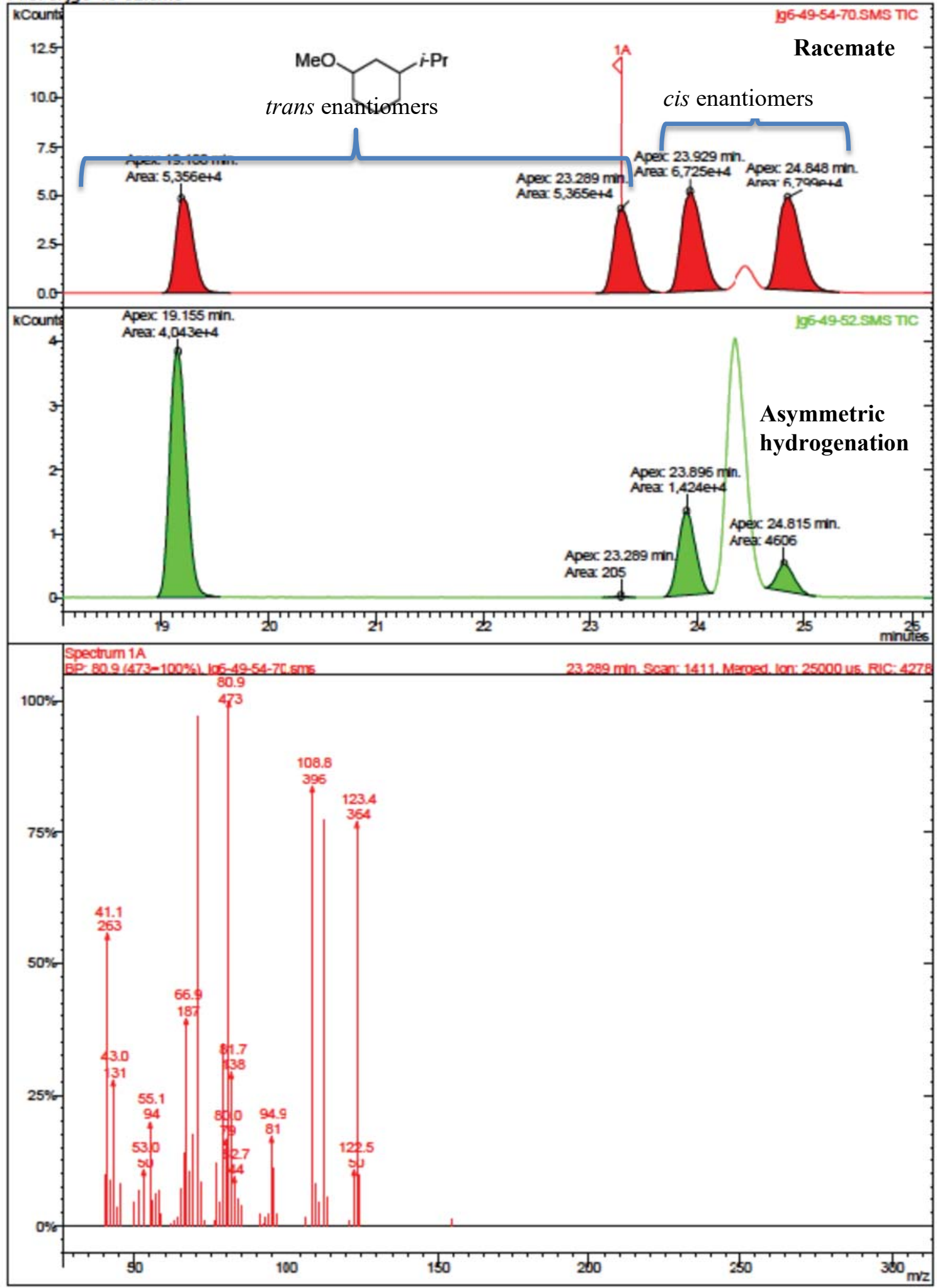




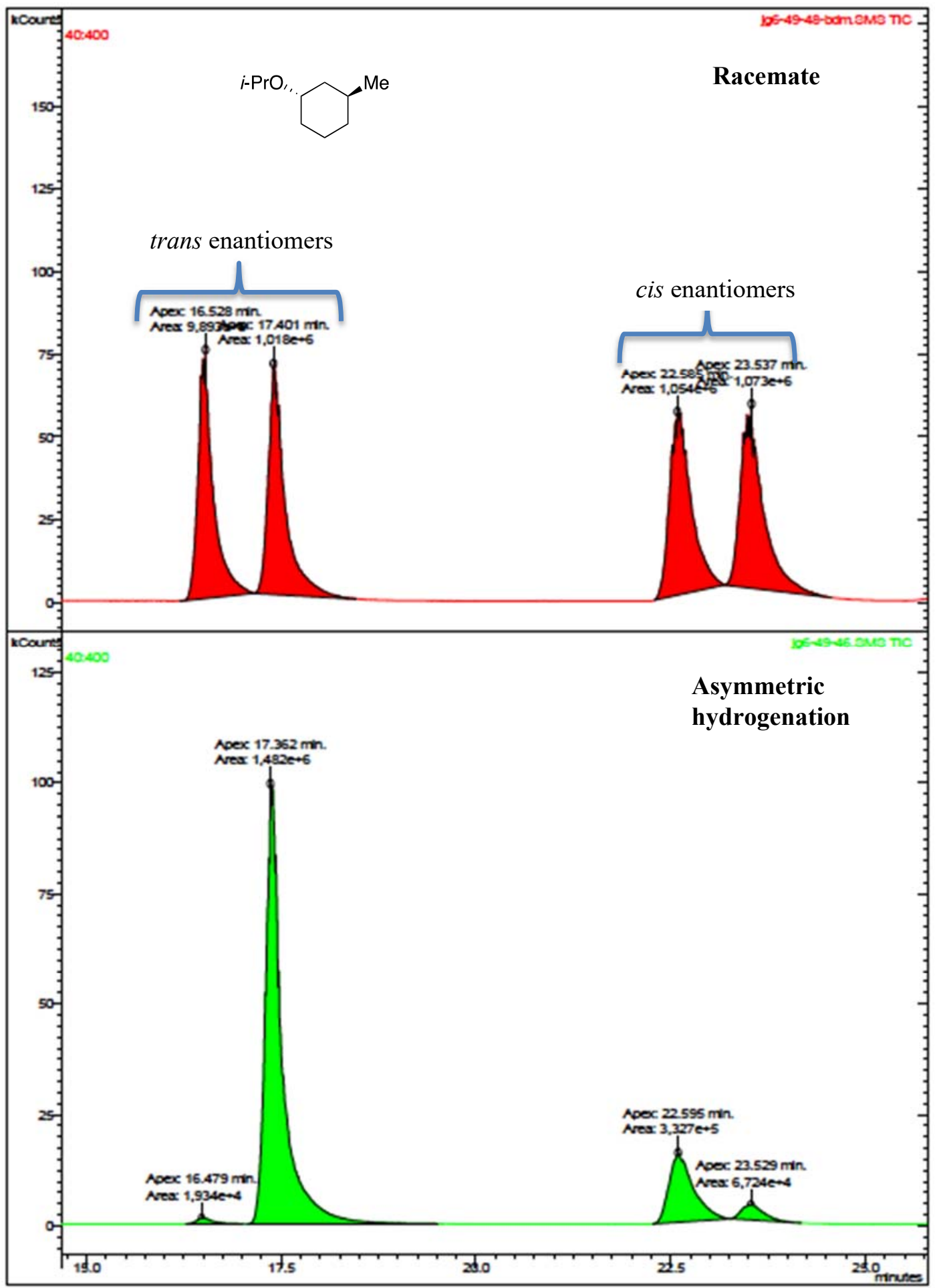




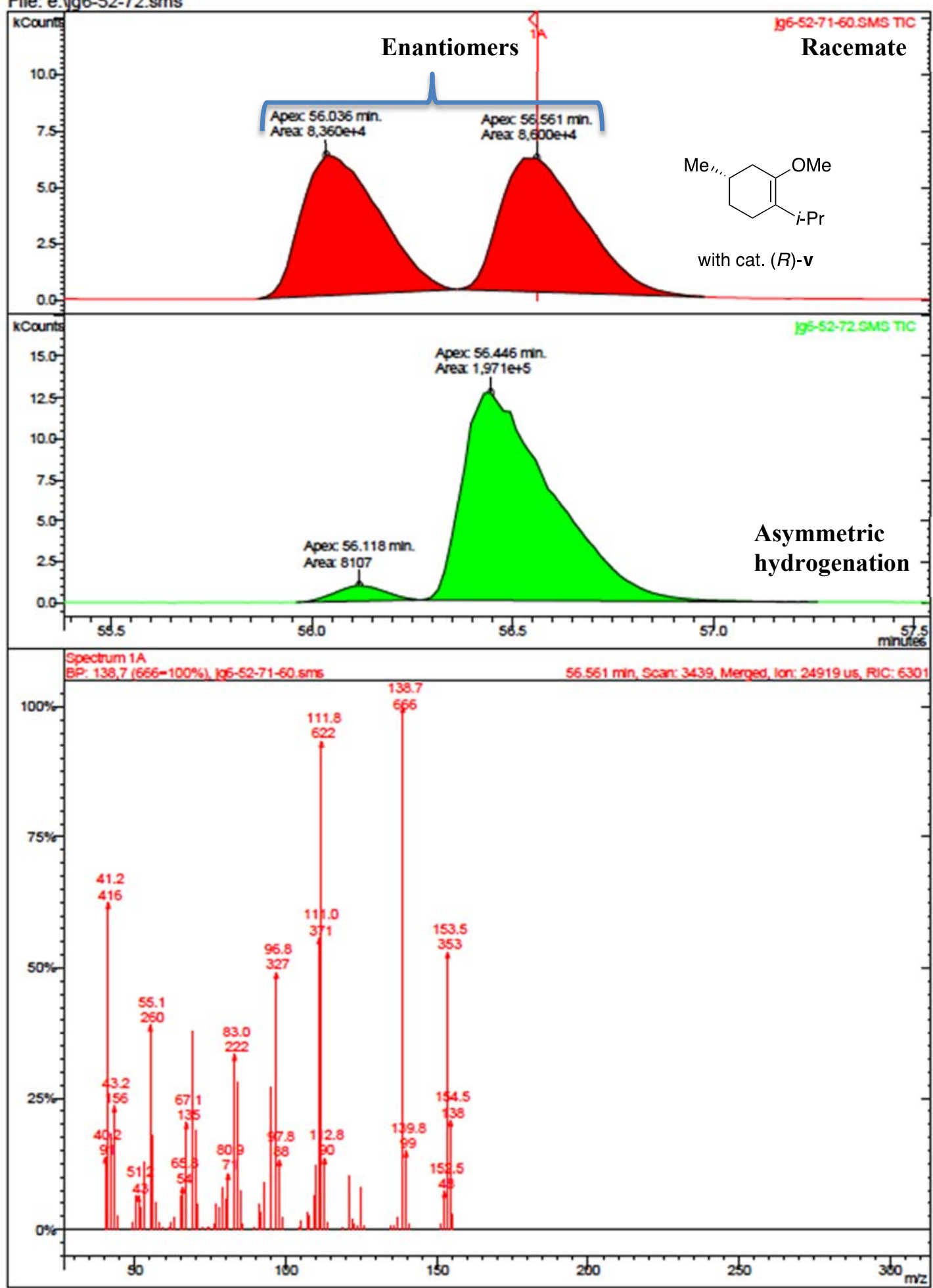




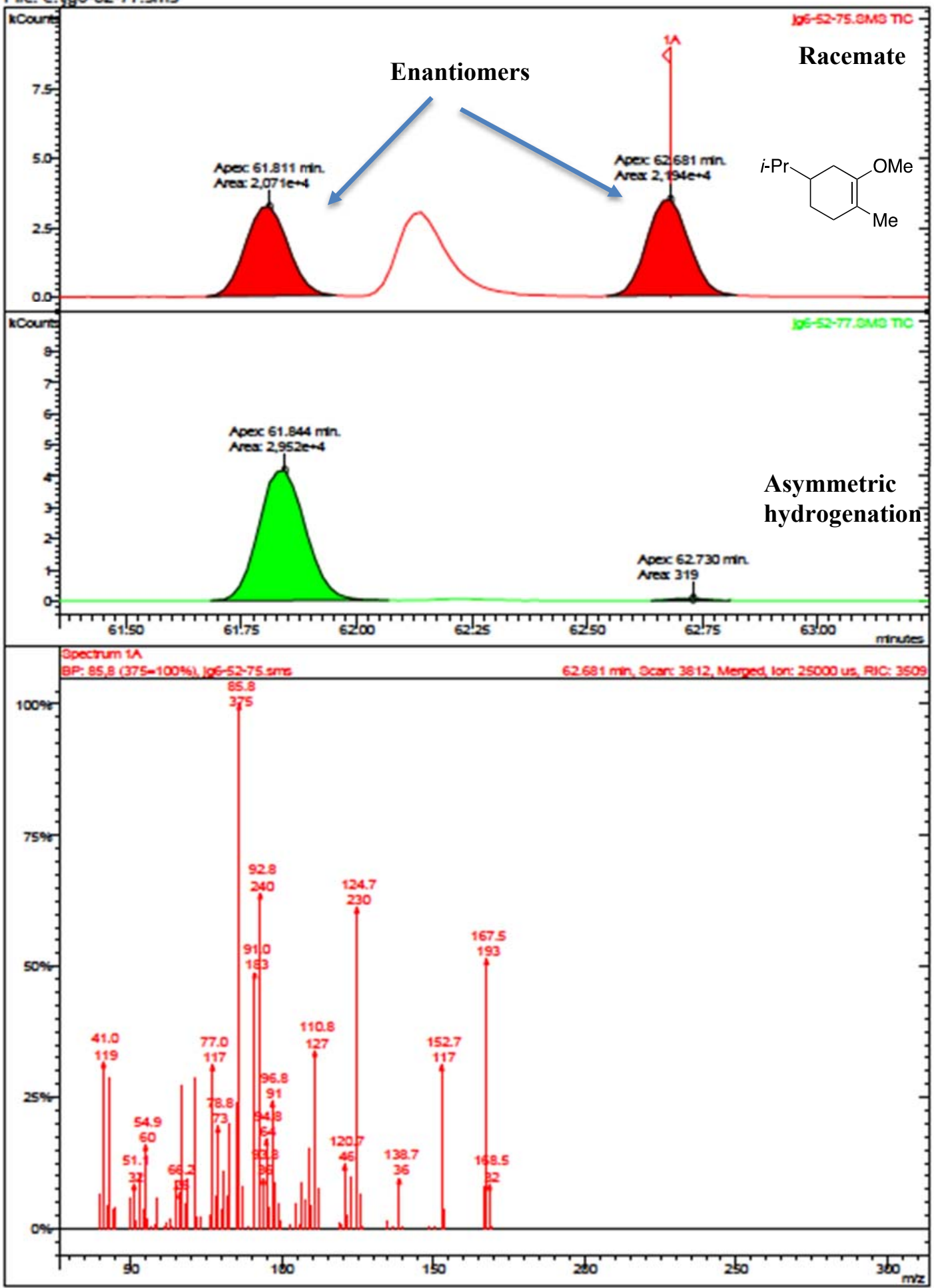




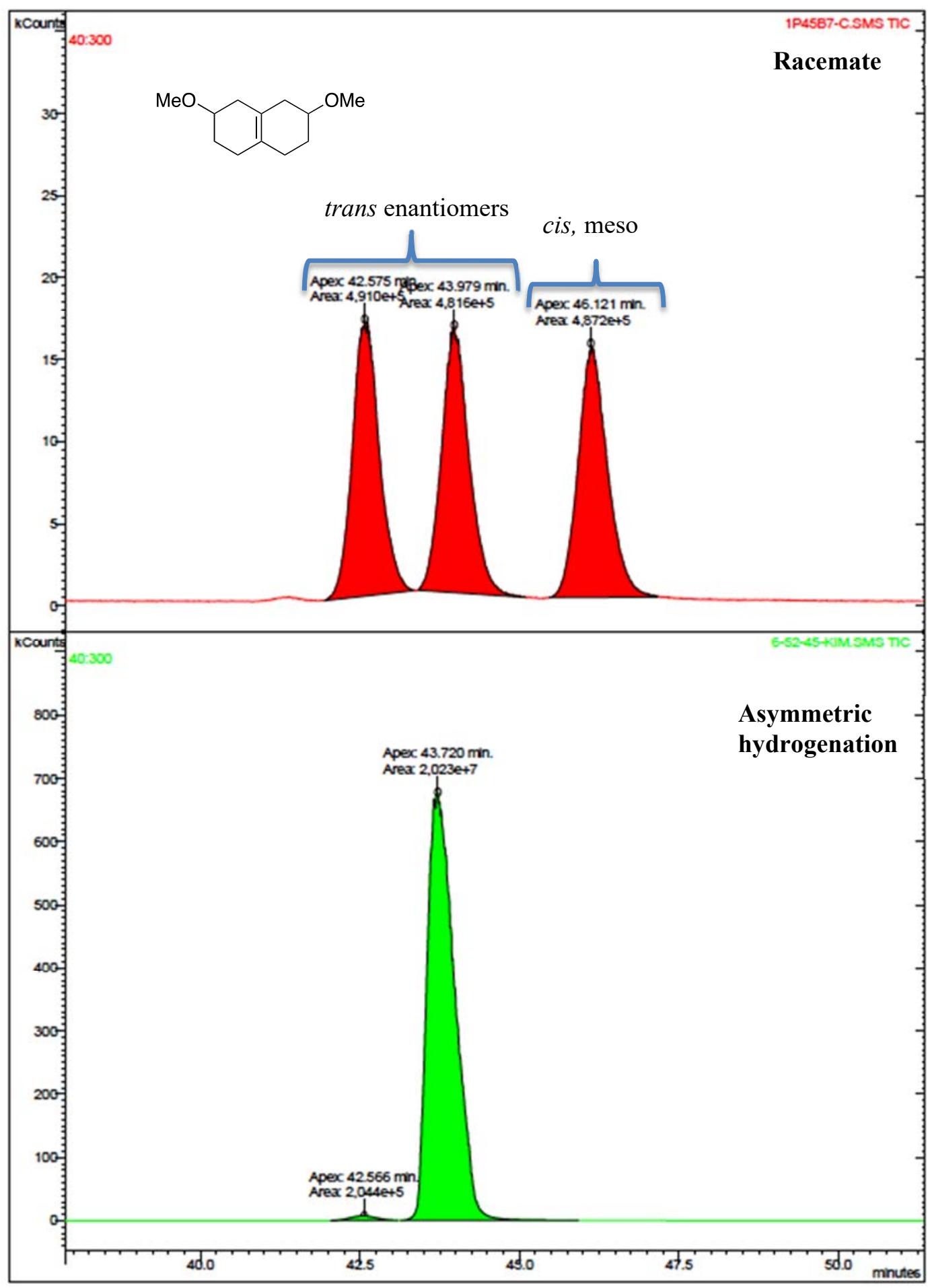




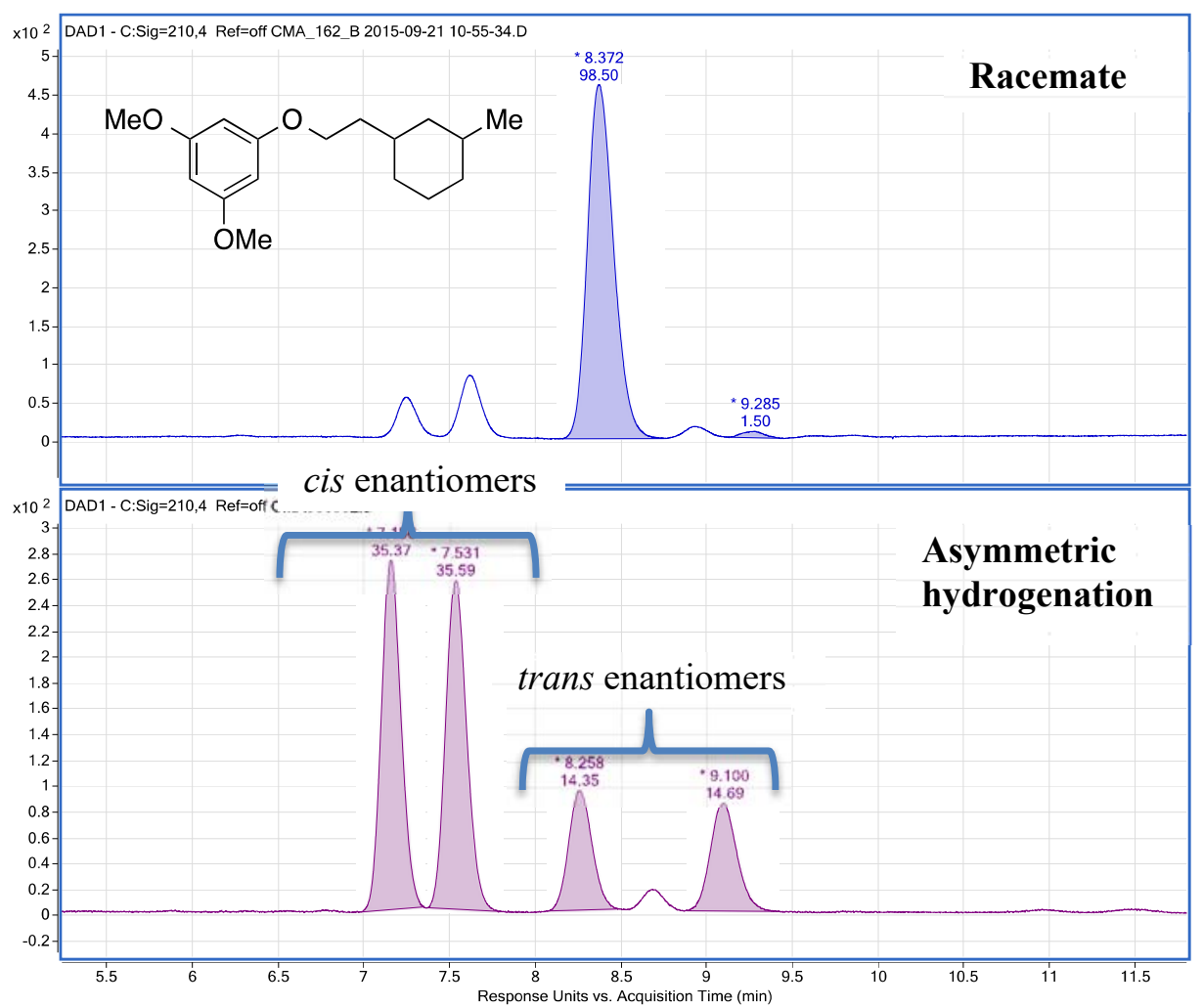


Print Date: 07 Jul 2016 17:30:14

\section{$\underline{\text { Chromatogram Plots }}$}

\section{Enantiomers}

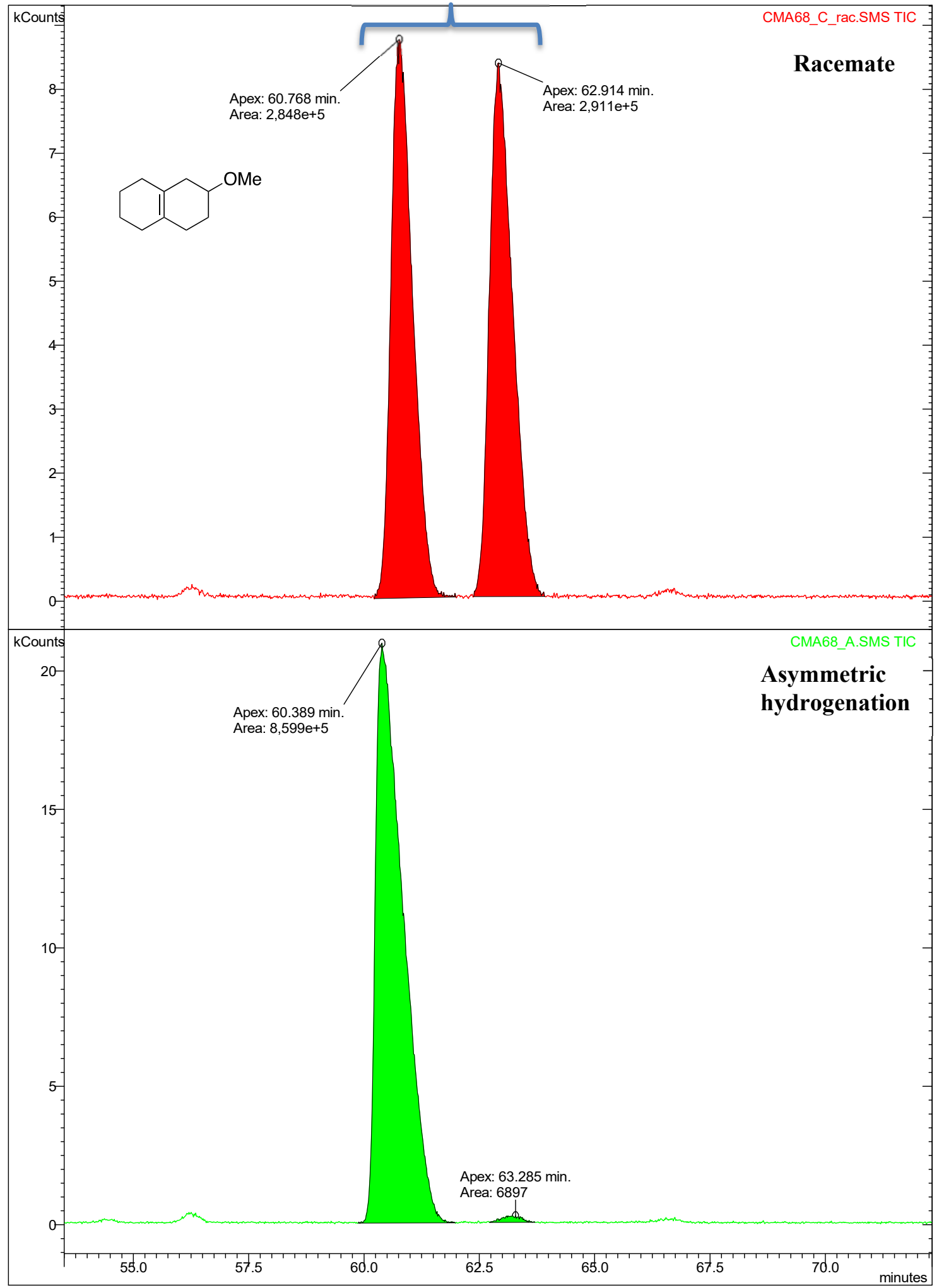


MS Data Review All Plots - 3/17/2016 10:56 AM

File: c:Ivarianwsldatalcris cis enantiomers ac.sms

File: f:lgc-msljg6-52-52.sı...

File: f:Igc-msljg6-52-54.sms

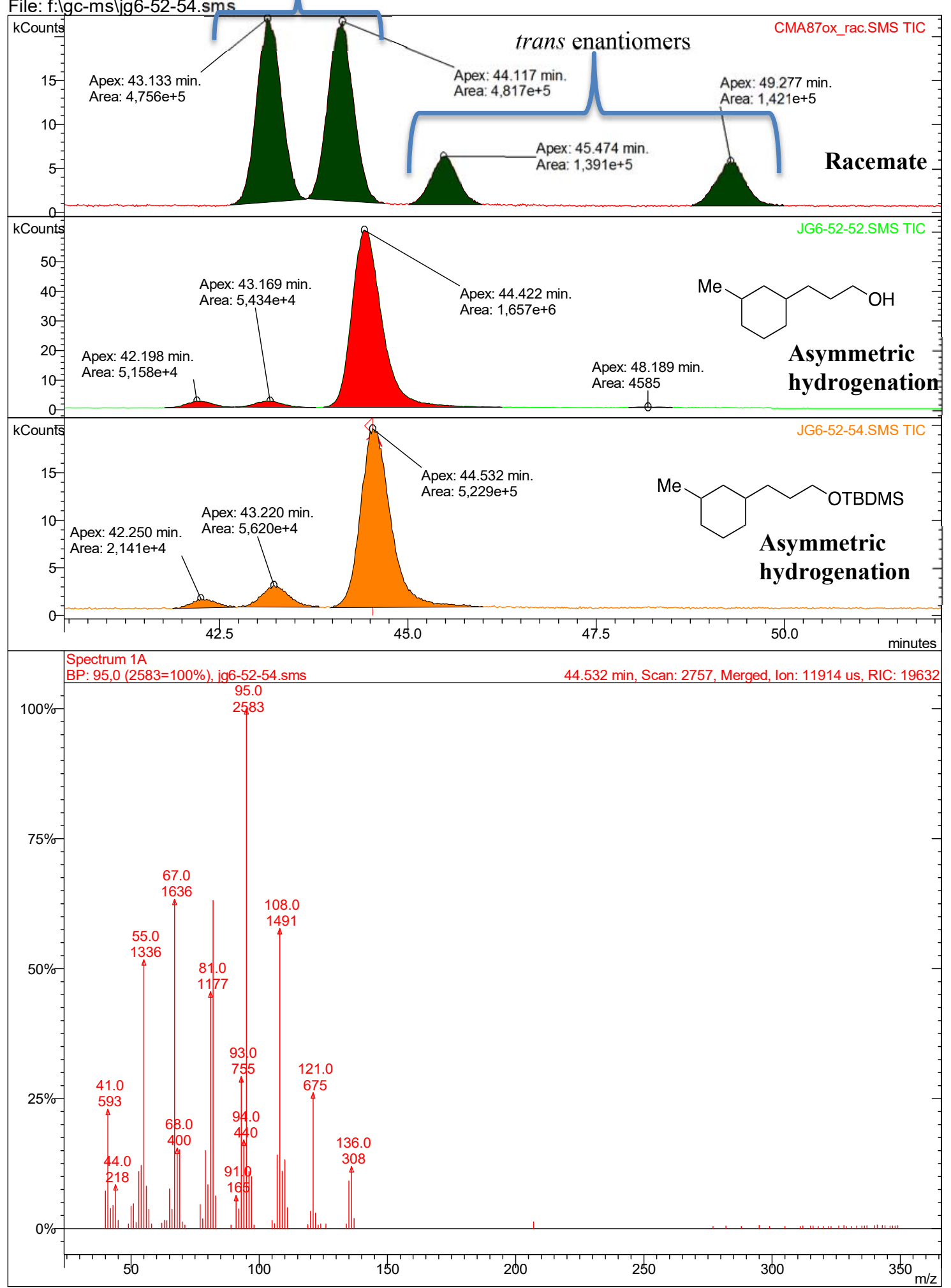


Chromatogram Plots

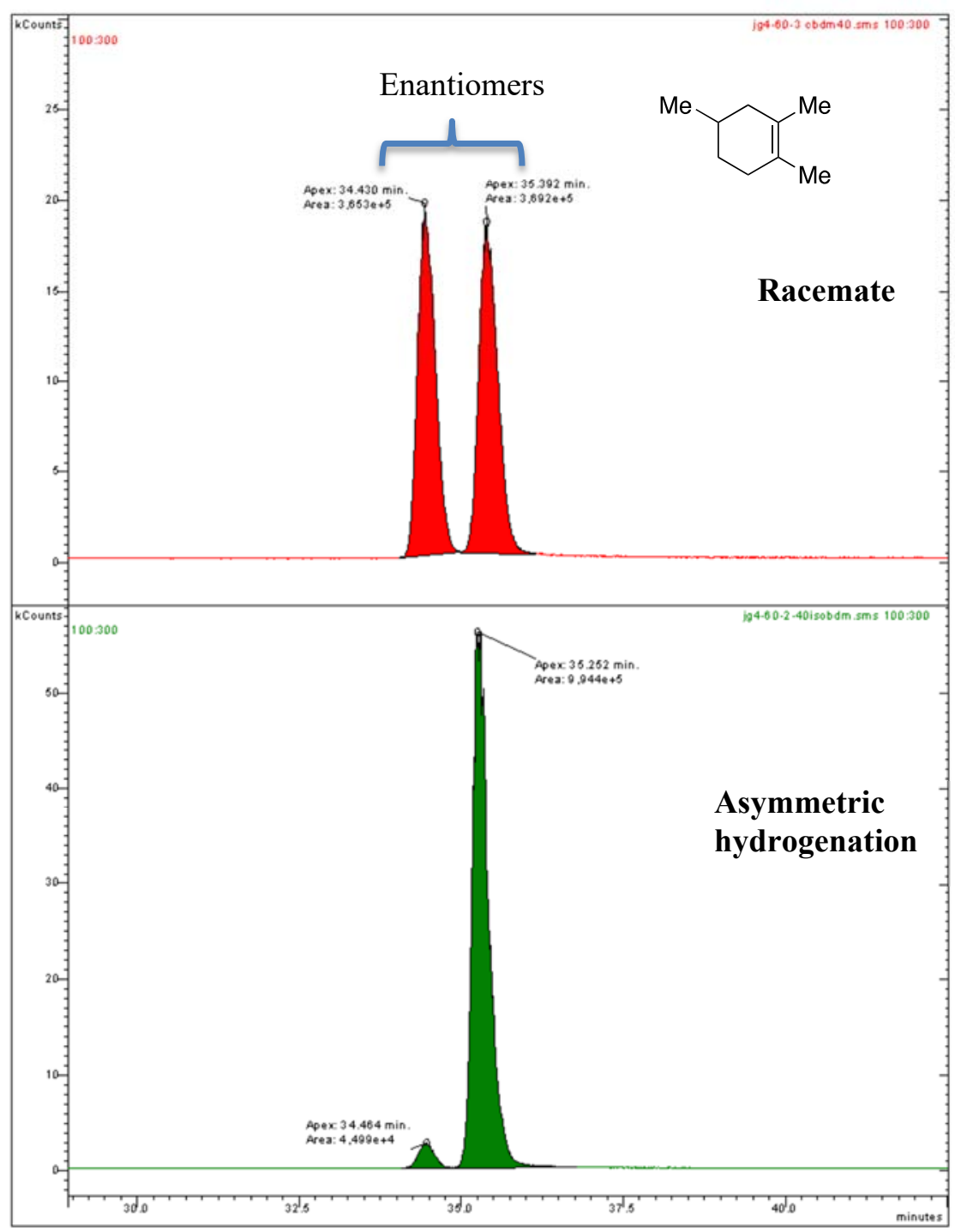




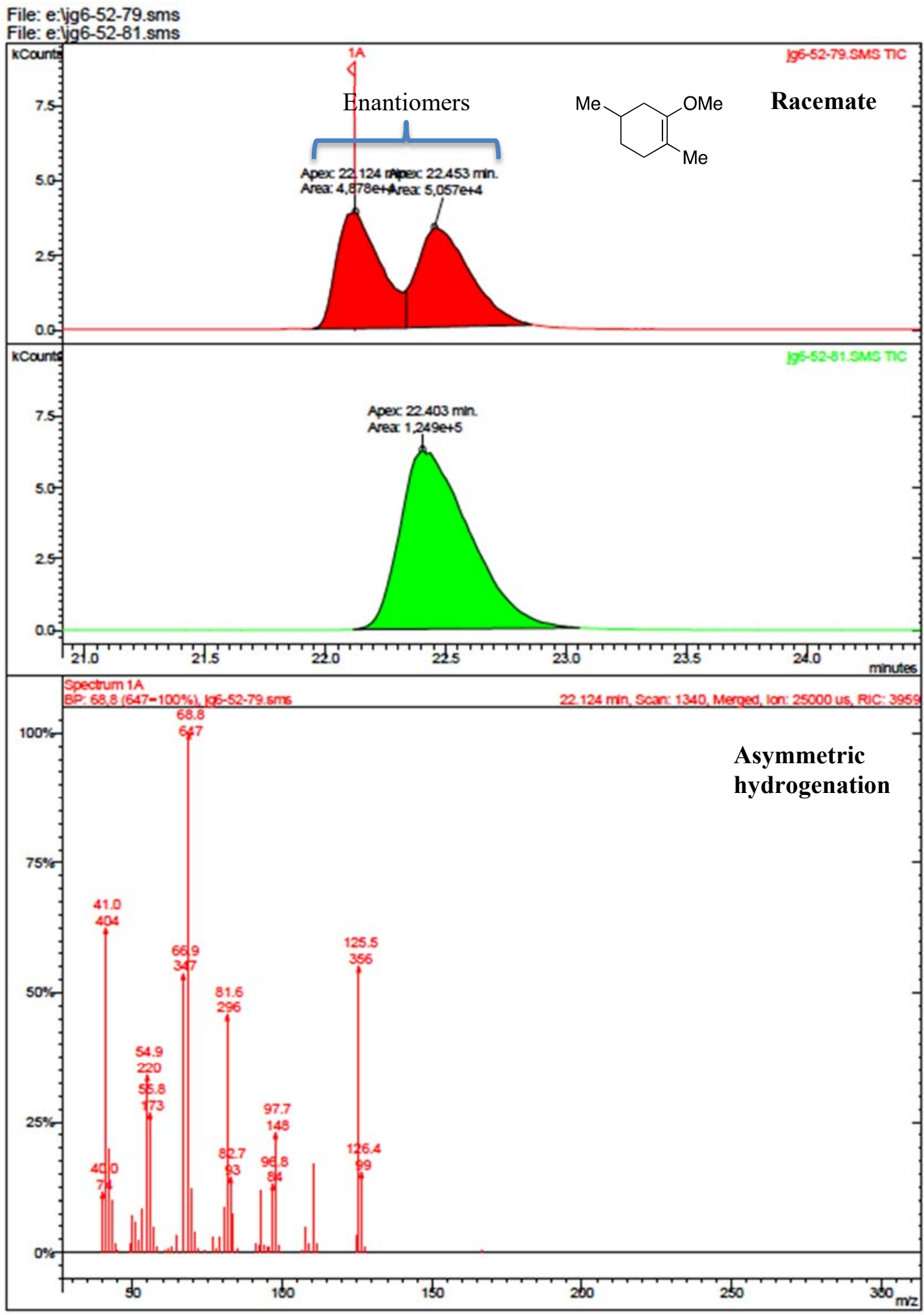




\section{Chromatogram Plots}

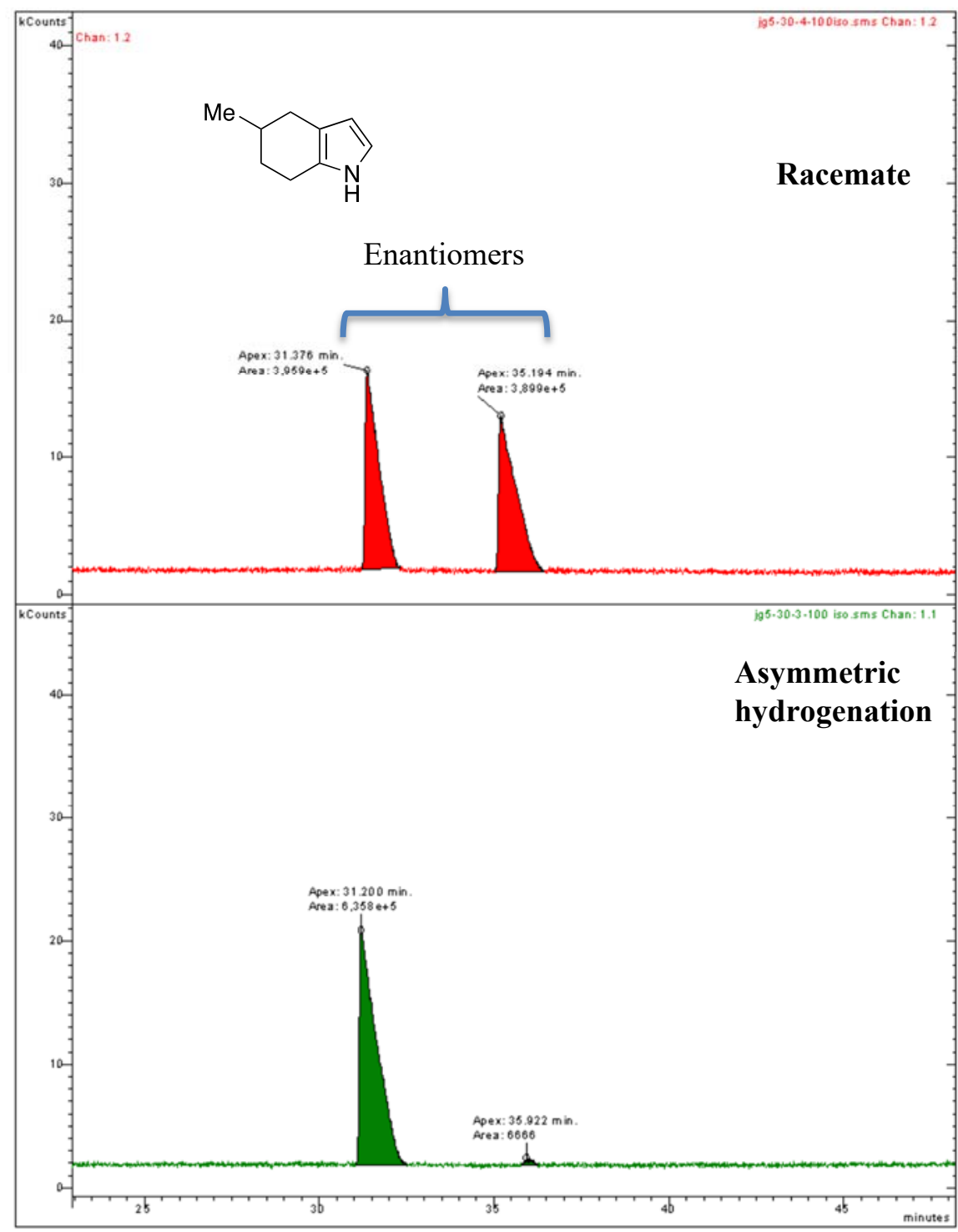




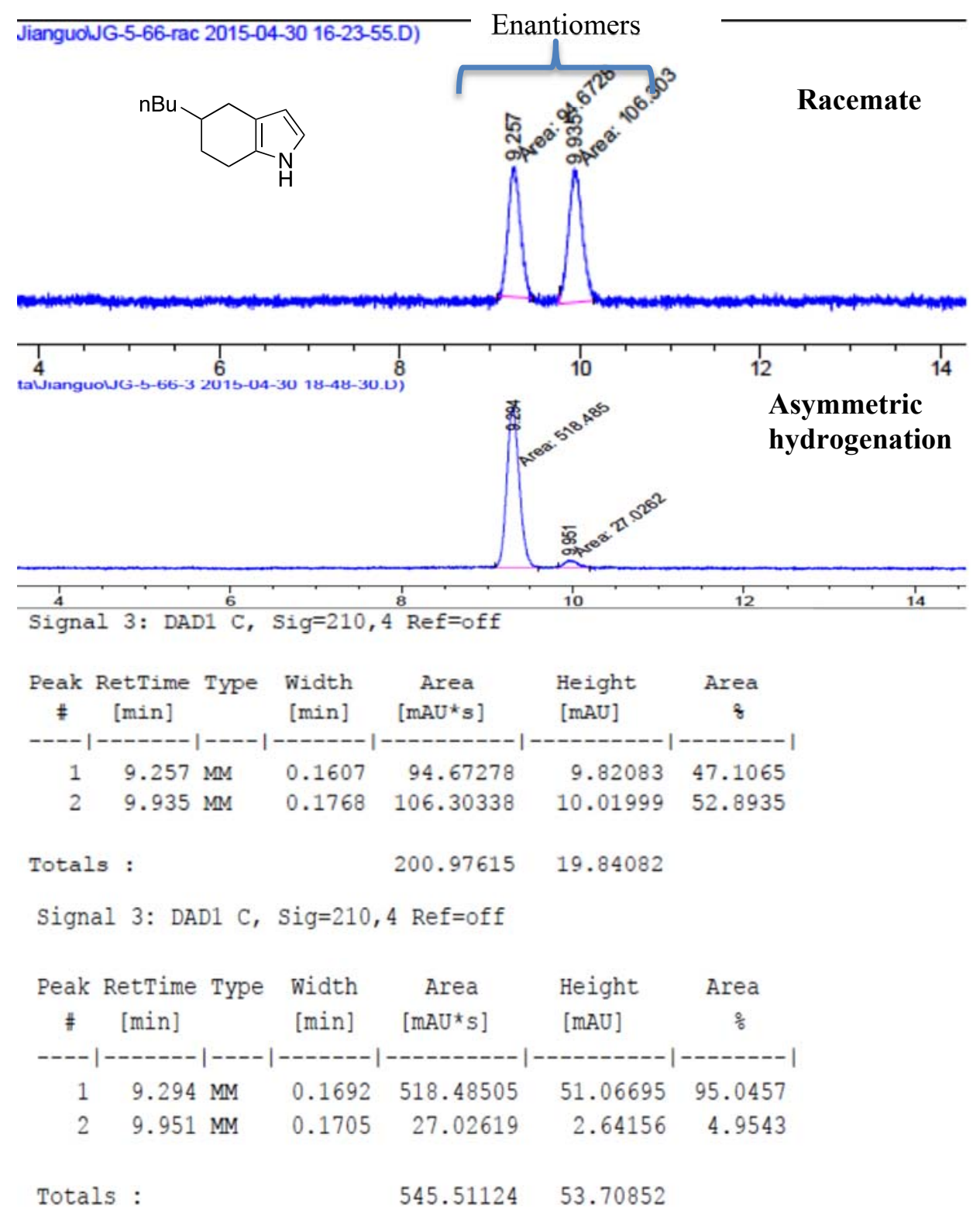




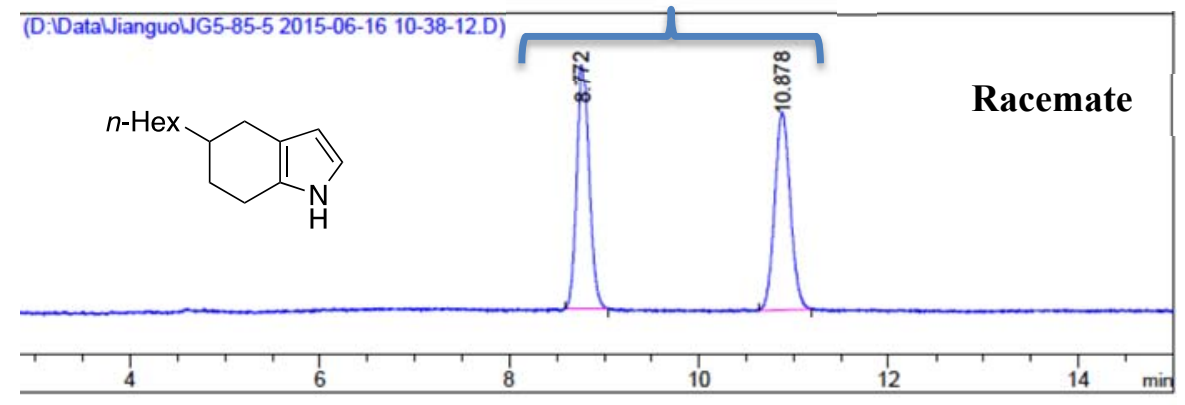

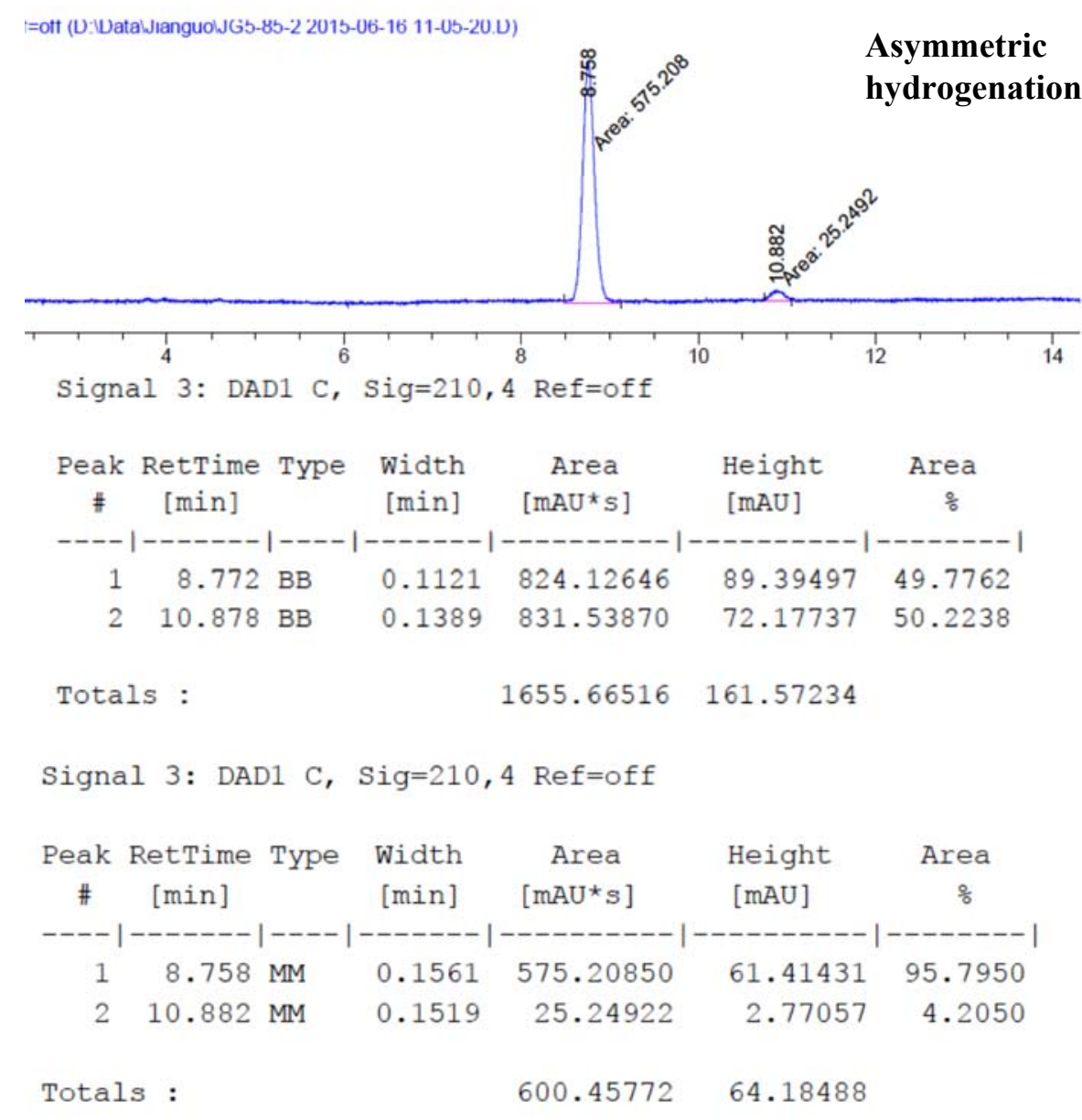




\section{Chromatogram Plots}

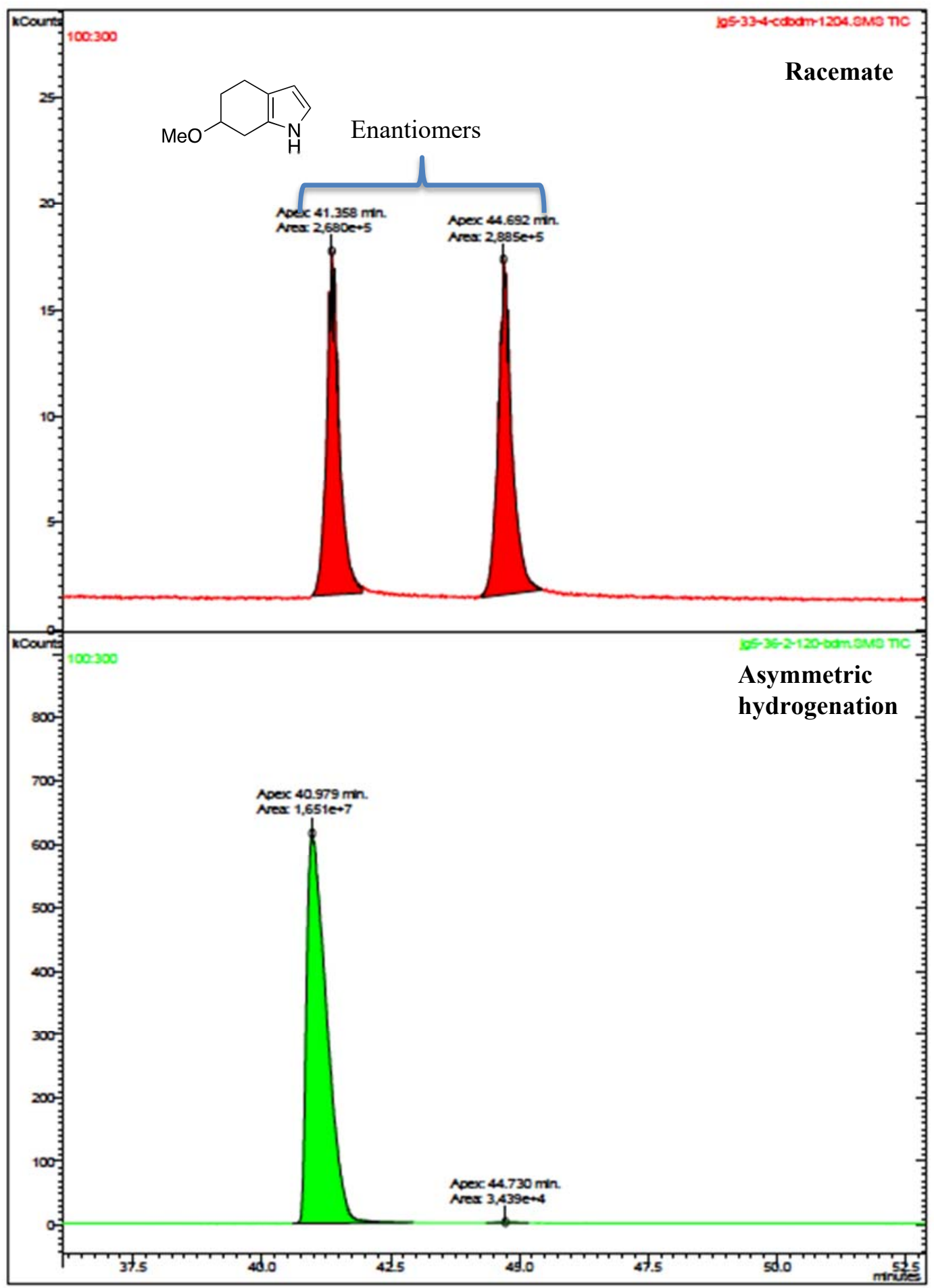




\section{MS Data Review All Plots - 10/29/2015 10:05 PM}

File: c:Ivarianwsidatalbyronlbp6lbp6-10-cd-bdm 7. sms

File: c:Ivarianwsidatalbyronlbp6lbp6-03_cd-bdm_2001.sms

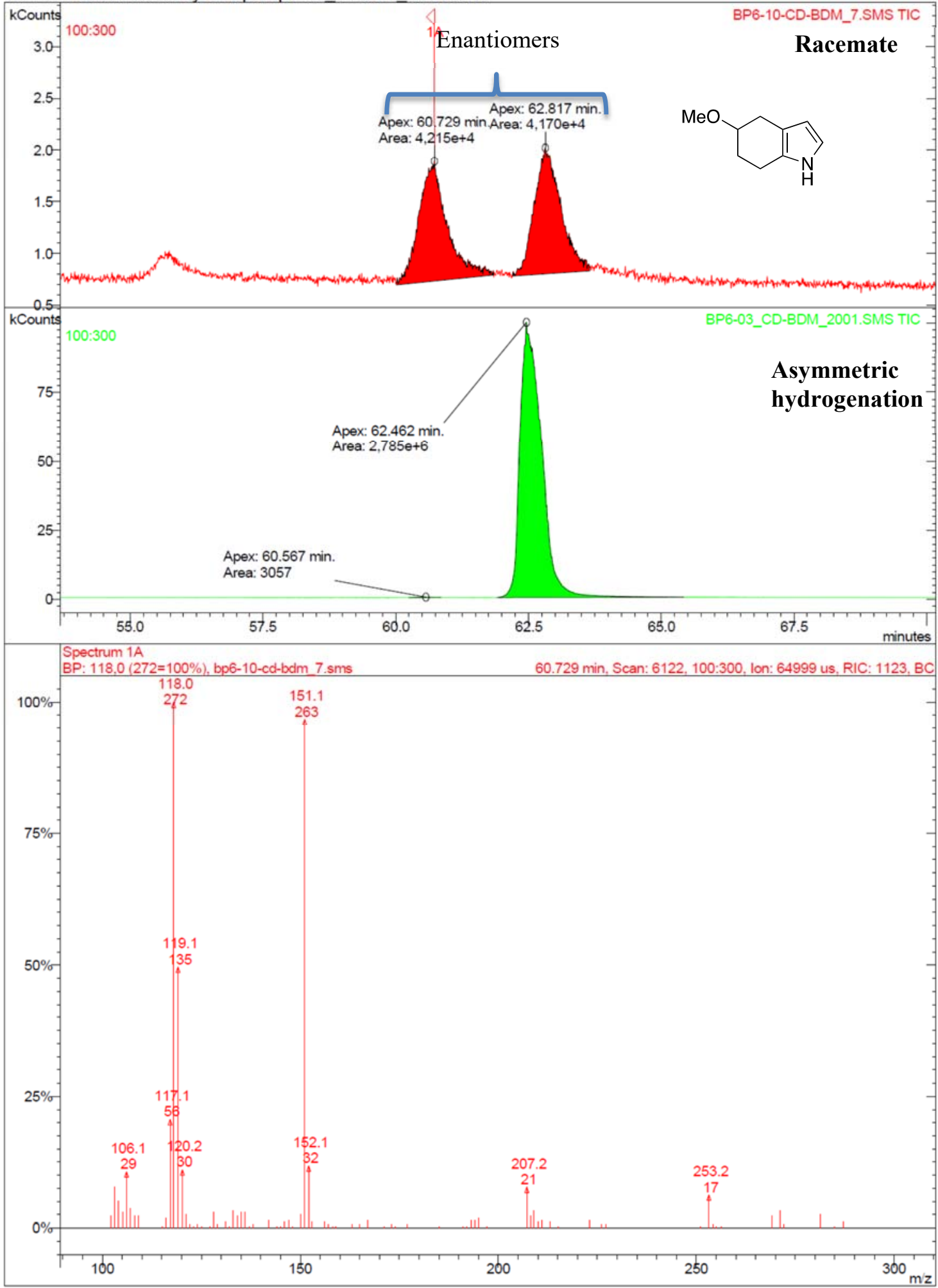


Print Date: 31 Oct 2015 16:44:50

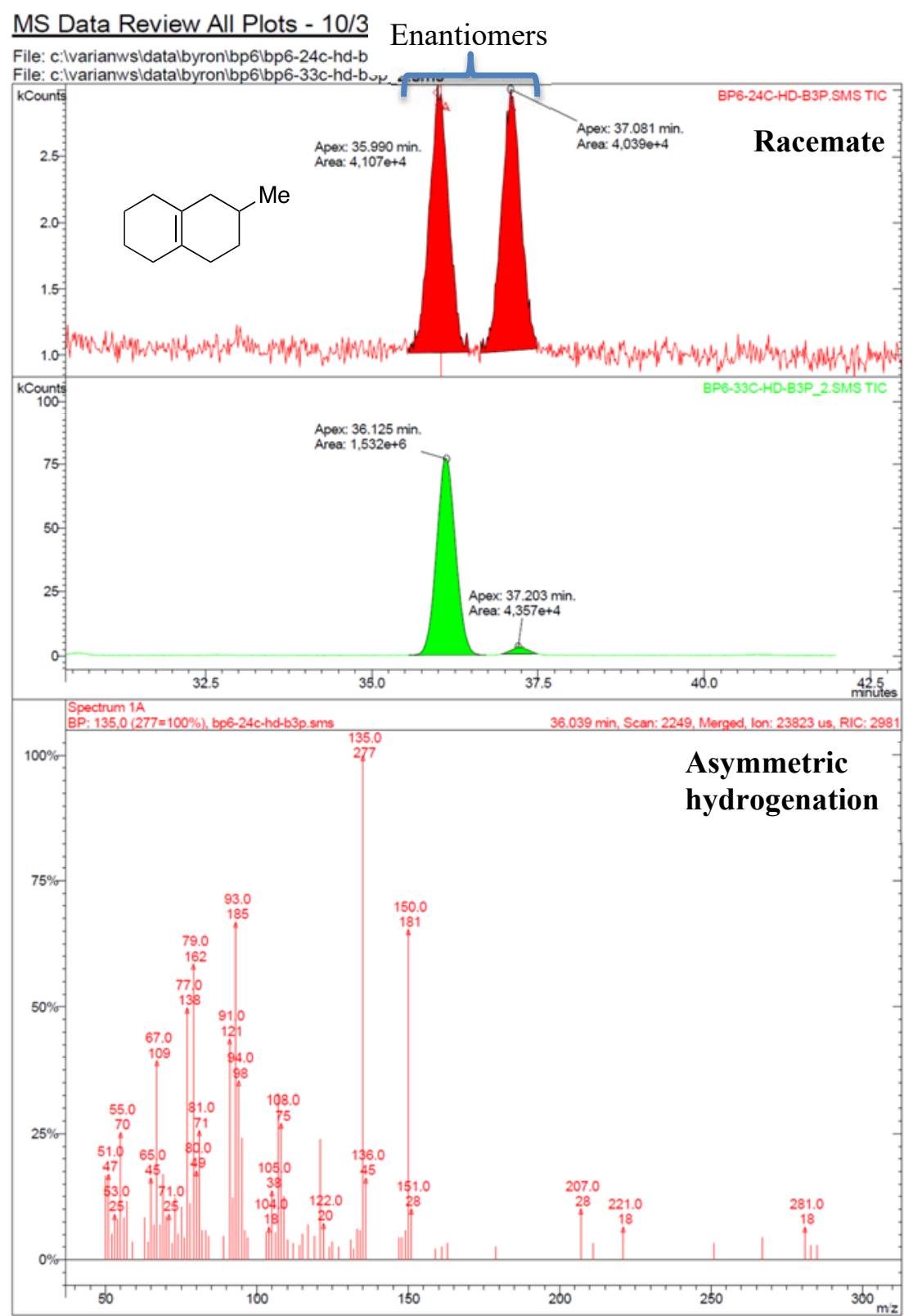


Print Date: 29 Oct 2015 22:24:46

\section{MS Data Review All Plots - 10/29/2015 10:23 PM}

File: c:lvarianwsidatalhuronlhn6lhn6-183c-cd-hdm_6.sms

File: c:lvarianws enantiomers $\mathrm{dm}^{-1 . \mathrm{sms}}$
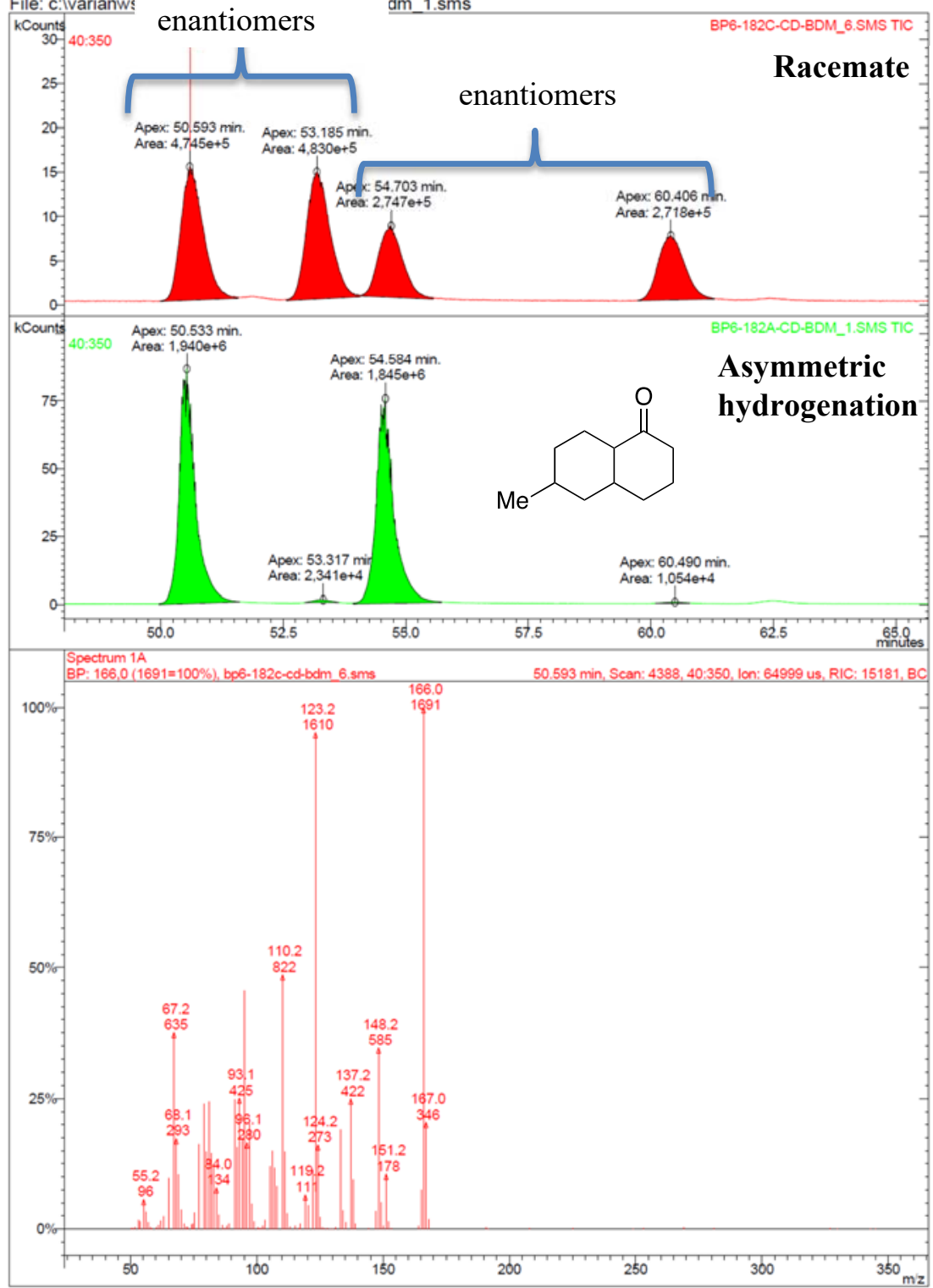


\section{MS Data Review All Plots - 10/29/2015 9:48 PM}

File: c:Ivarianwsidatalbyronlbp6lbp6-70d_b6tbdm_2.sms File: c:Ivarianwsidatalbyronlbp6lbp6-70c_b6tbdm_1.sms

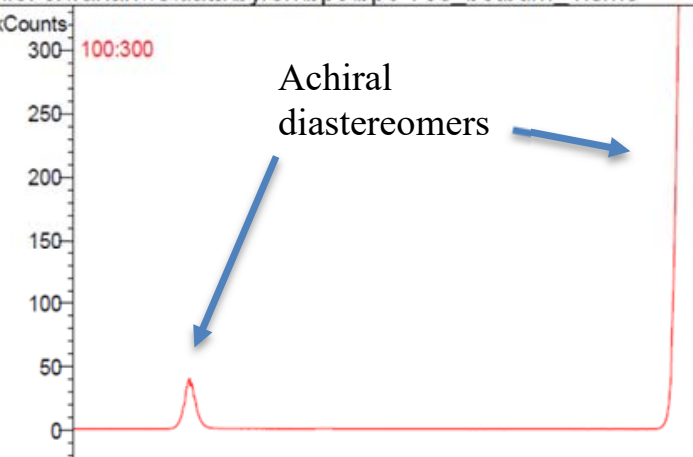

BP6-70D_B6TBDM_2.SMS 100:300

analysed as:

$\overbrace{M e}^{C O O M e}$
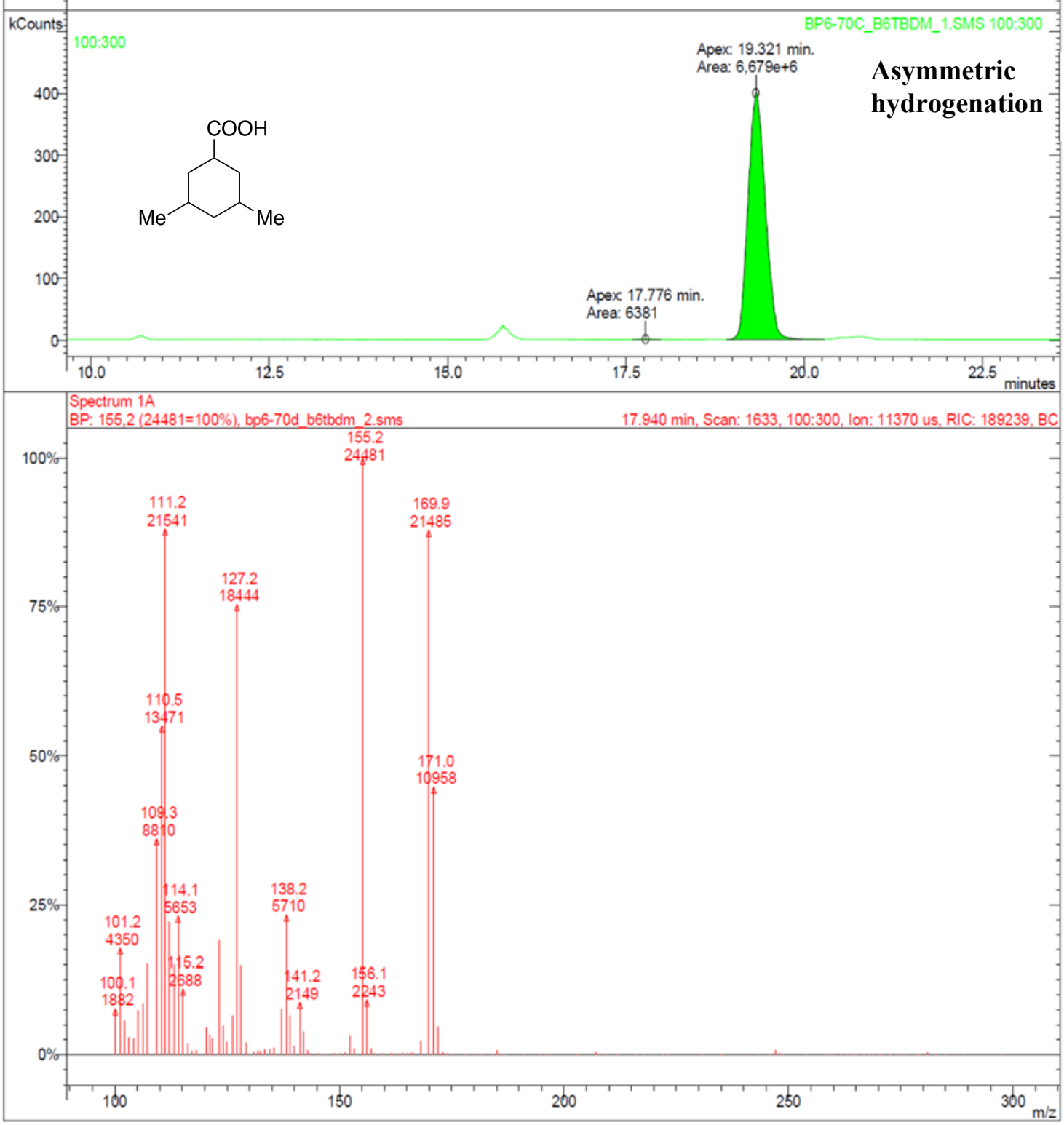
Print Date: 29 Apr 2016 11:44:15

MS Data Review All Plots - 4/29/2016 11:43 AM

File: e:lcma-gc-160322lcma2-46-b.sms

File: e:Icma-gc-160322ljg6-49-62.sms kCounts

kCounts.

(1)

(1)

(2)

(2)
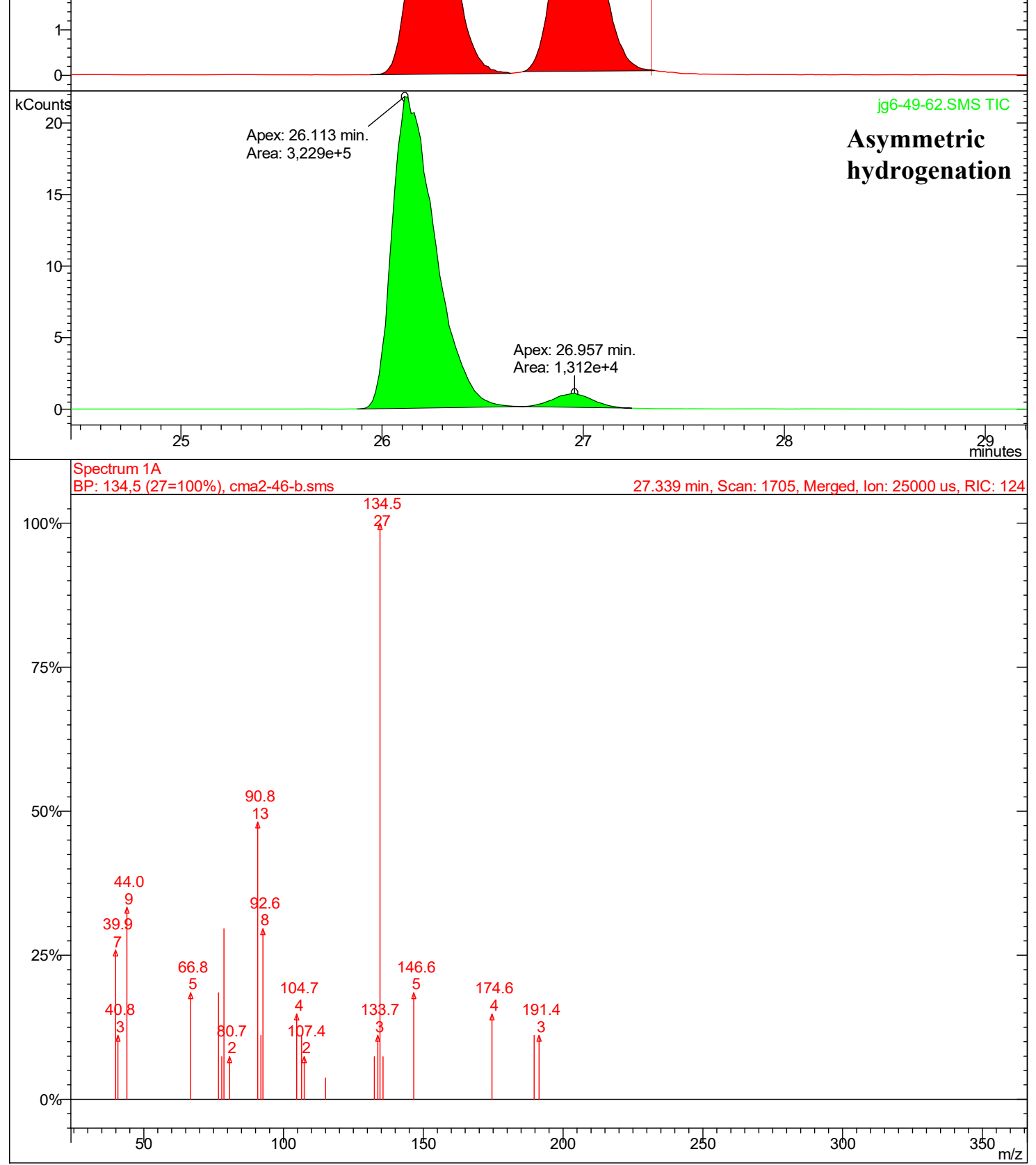
Print Date: 31 Jan 2016 01:00:49

MS Data Review All Plots - 1/31/2016 1:00 AM

File: e:Ibp6-159a-hd-b3p_3.sms

File: e:lbp6-159b hd-b3p_2.sms

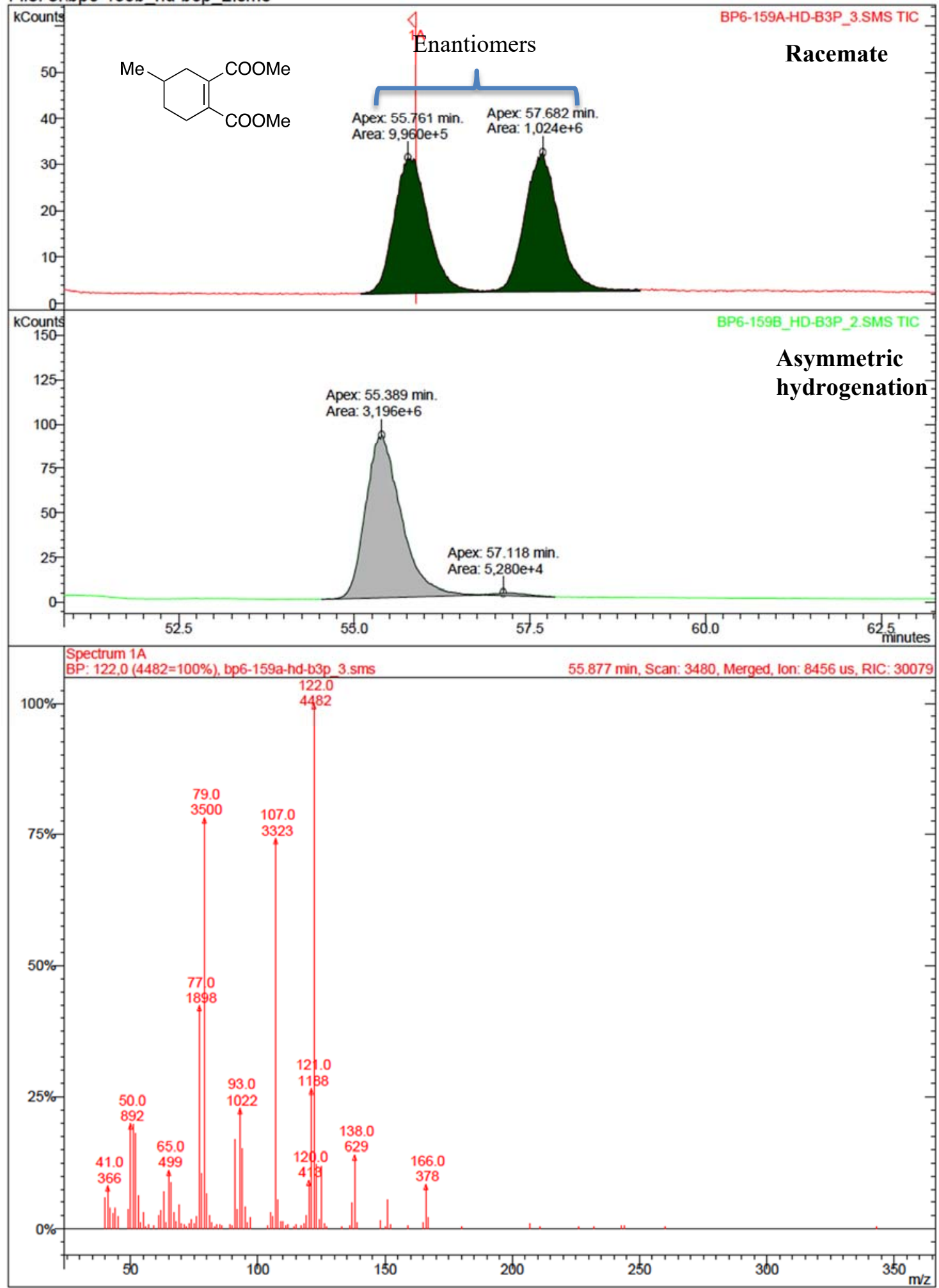


Print Date: 26 Apr 2016 16:21:32

\section{MS Data Review All Plots - 4/26/2 ( Enantiomers}

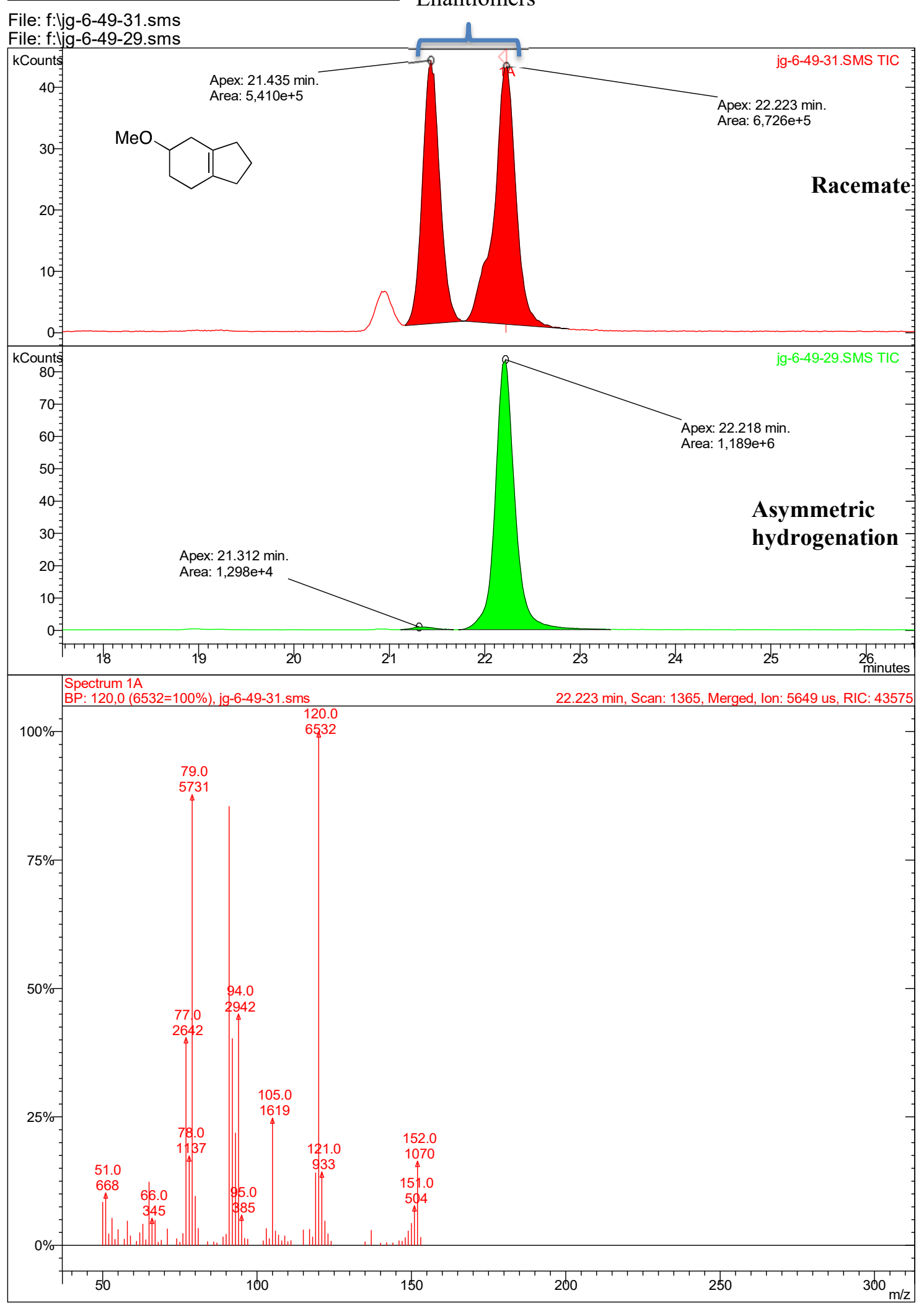


Print Date: 26 Apr 2016 15:38:44

\section{MS Data Review All Plots - 4/26/2016 3:38 PM}

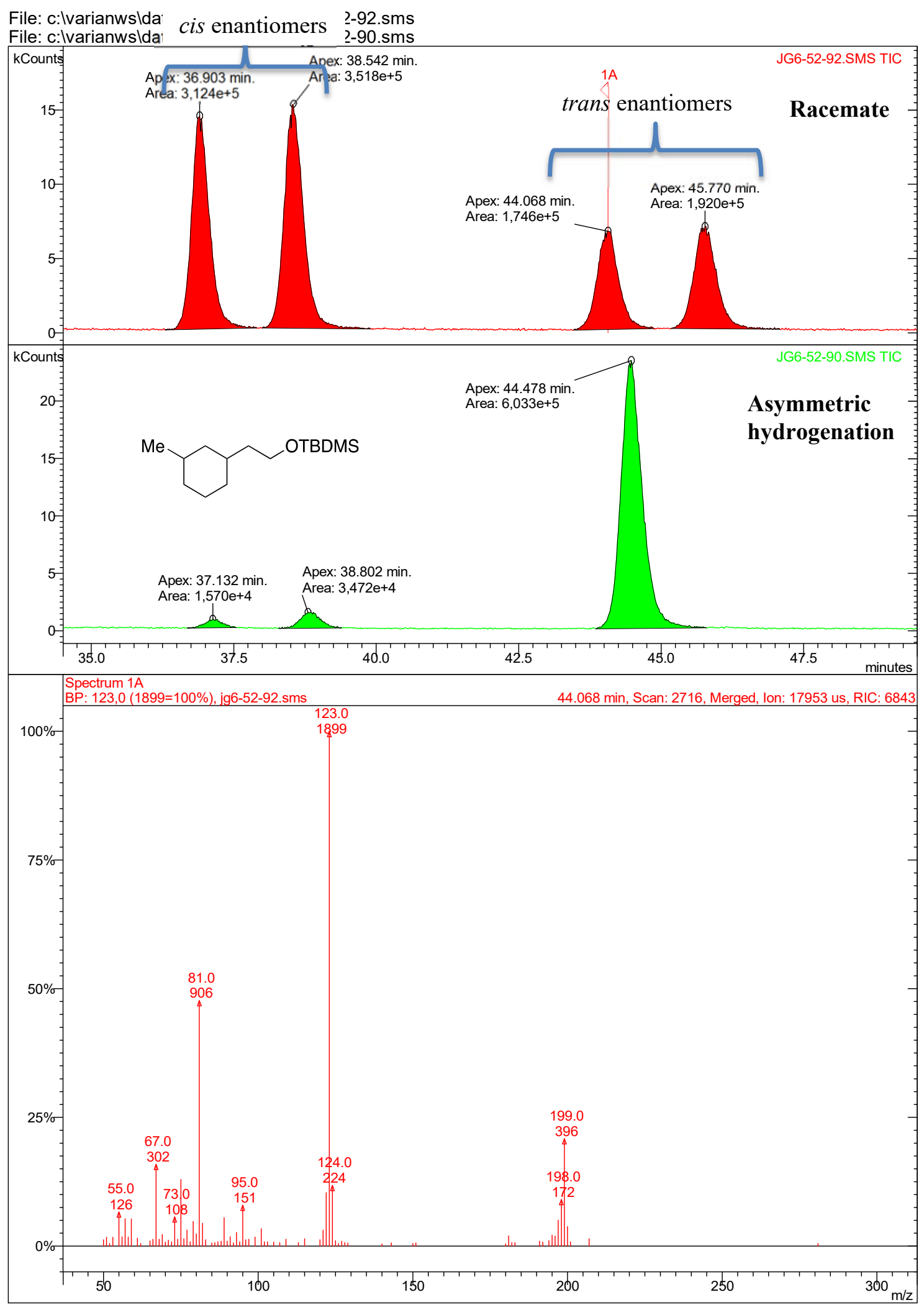


Print Date: 19 Feb 2016 11:22:59

MS Data Review All Plots - 2/19/2016 11:22 AM File: c:Ivarianwsldatalwangchuklbirchlpiv
File: c:Ivarianwsldatalwangchuklbirchlpiv trans enantiomers

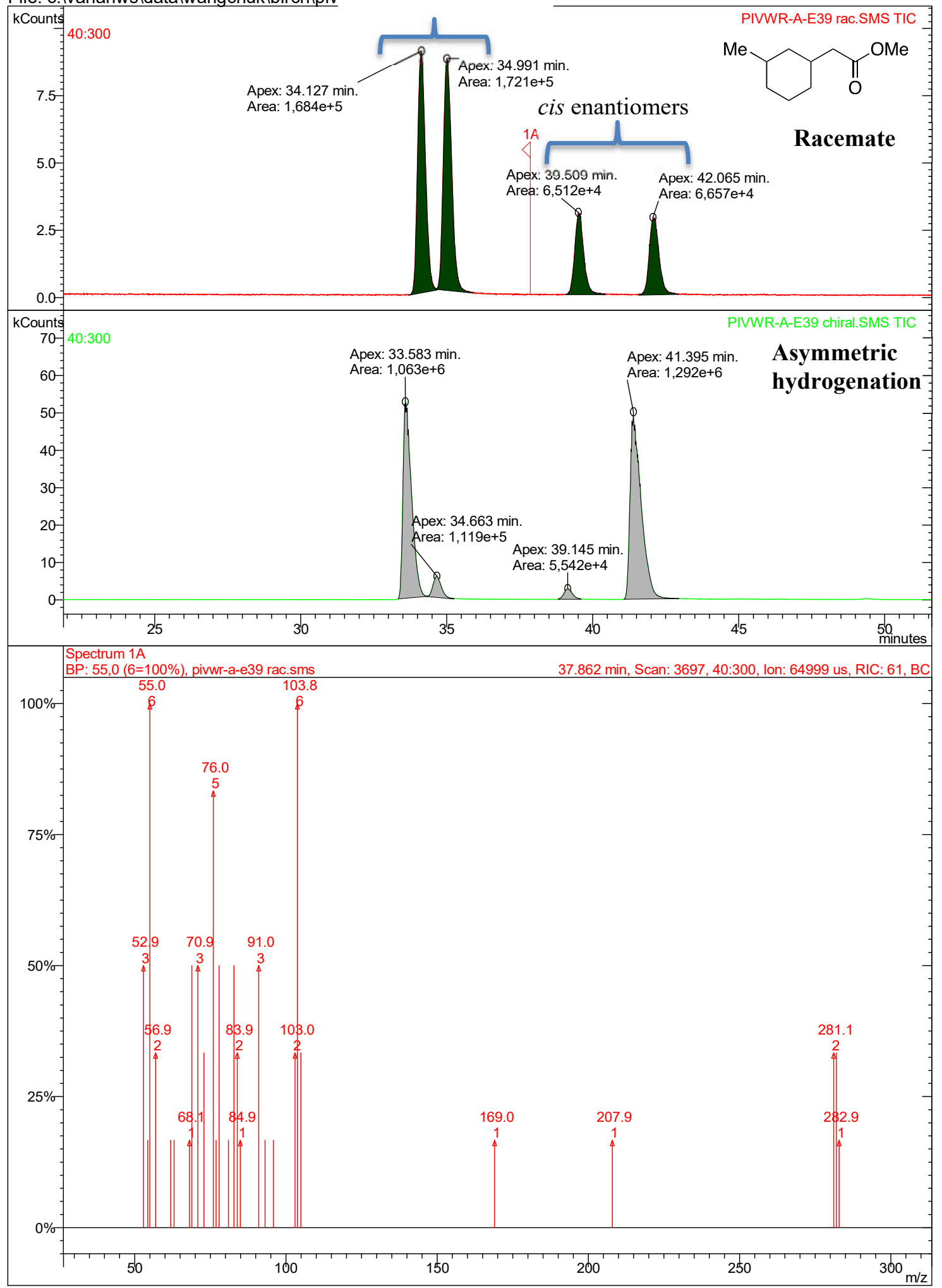




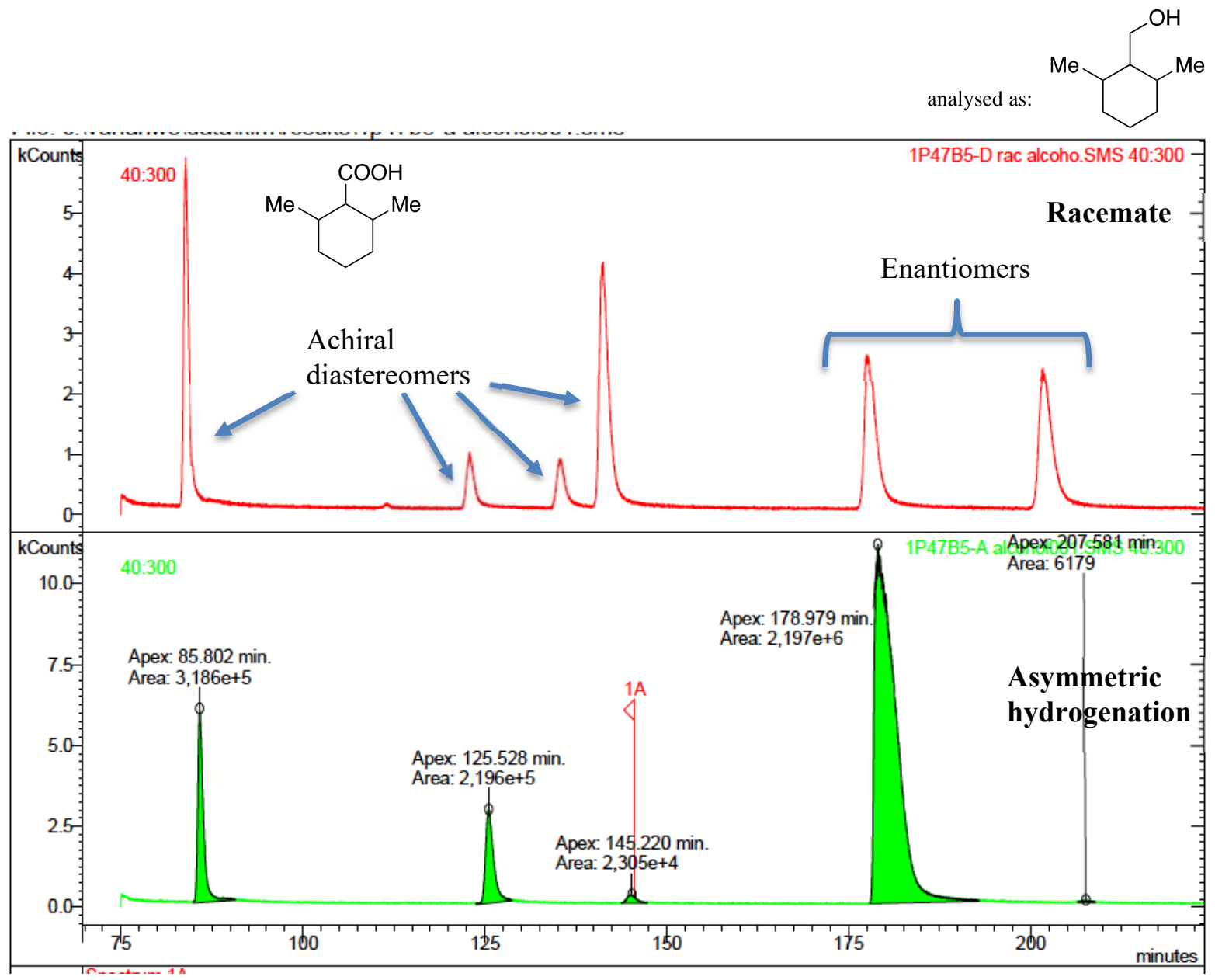


Print Date: 05 Feb 2016 12:25:31

\section{Chromatogram Plots}

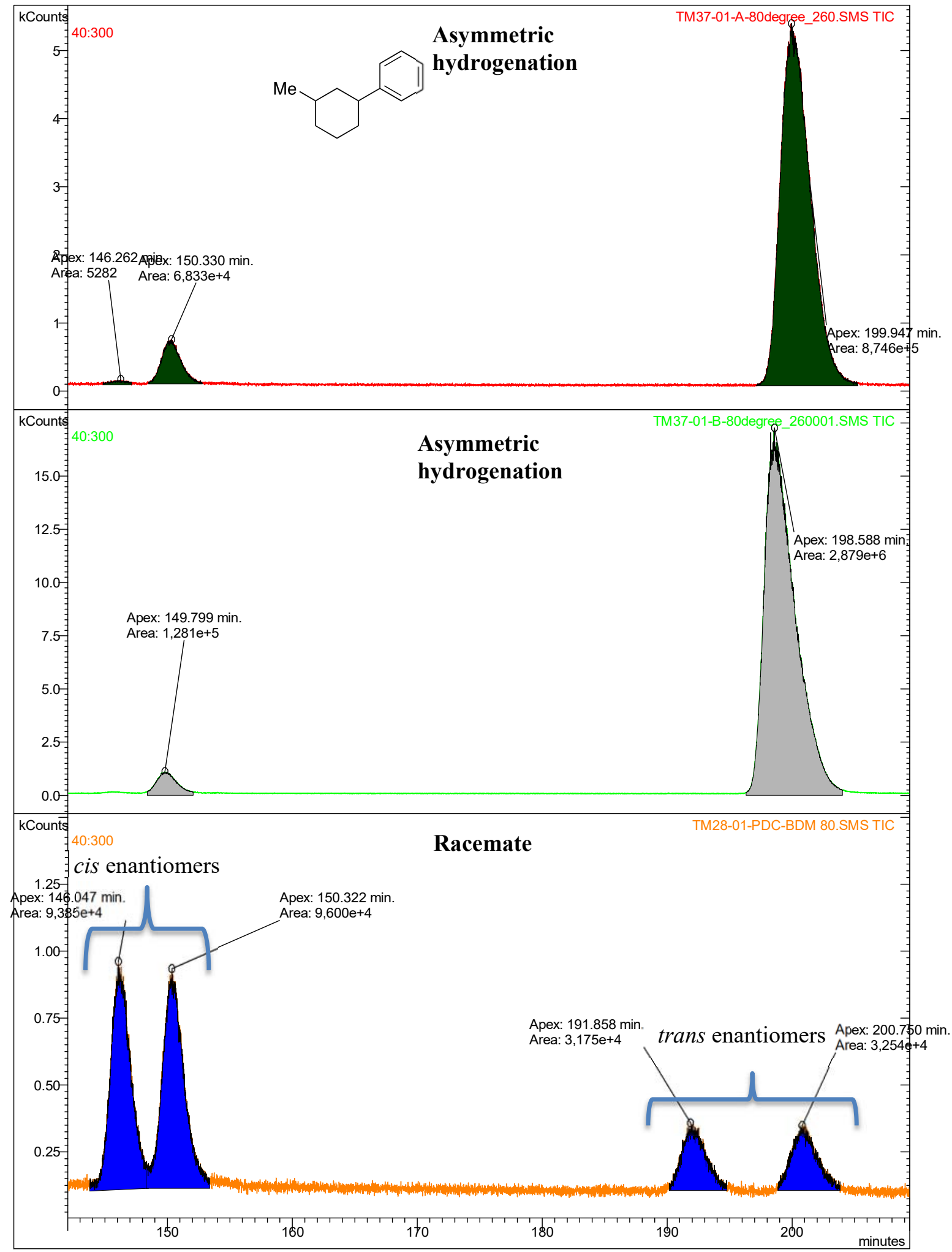


Print Date: 05 Feb 2016 16:00:00

\section{$\underline{\text { Chromatogram Plots }}$}

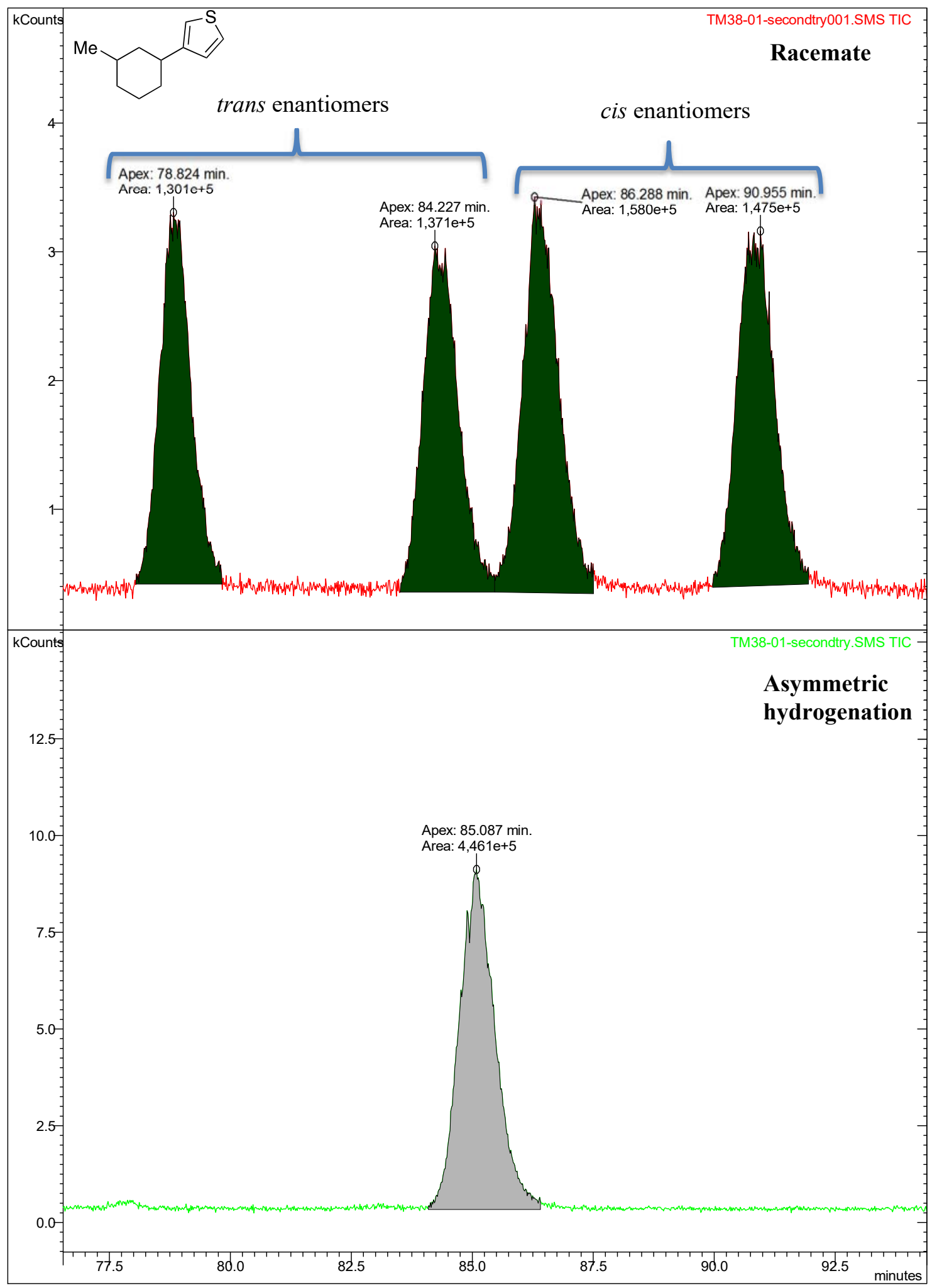




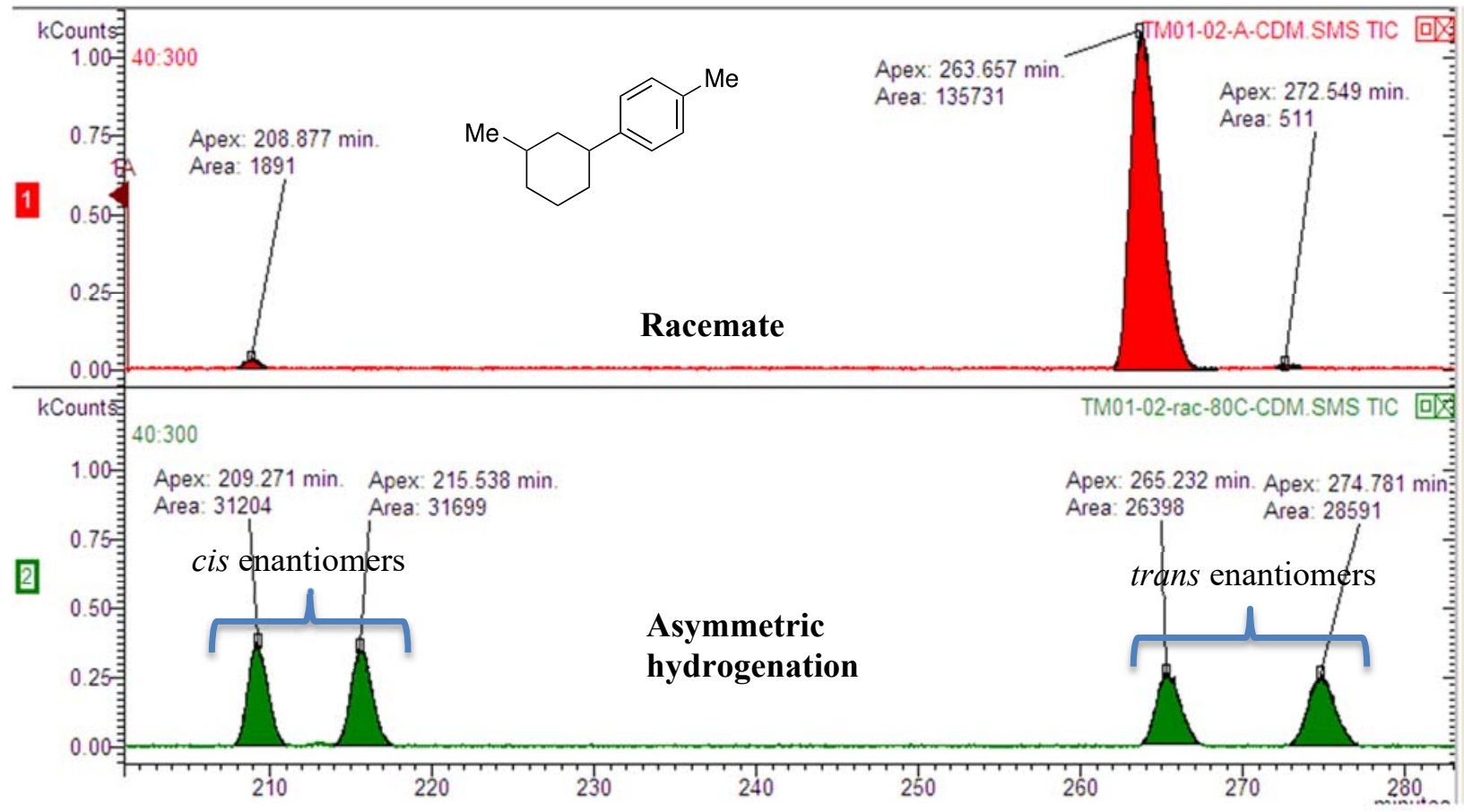




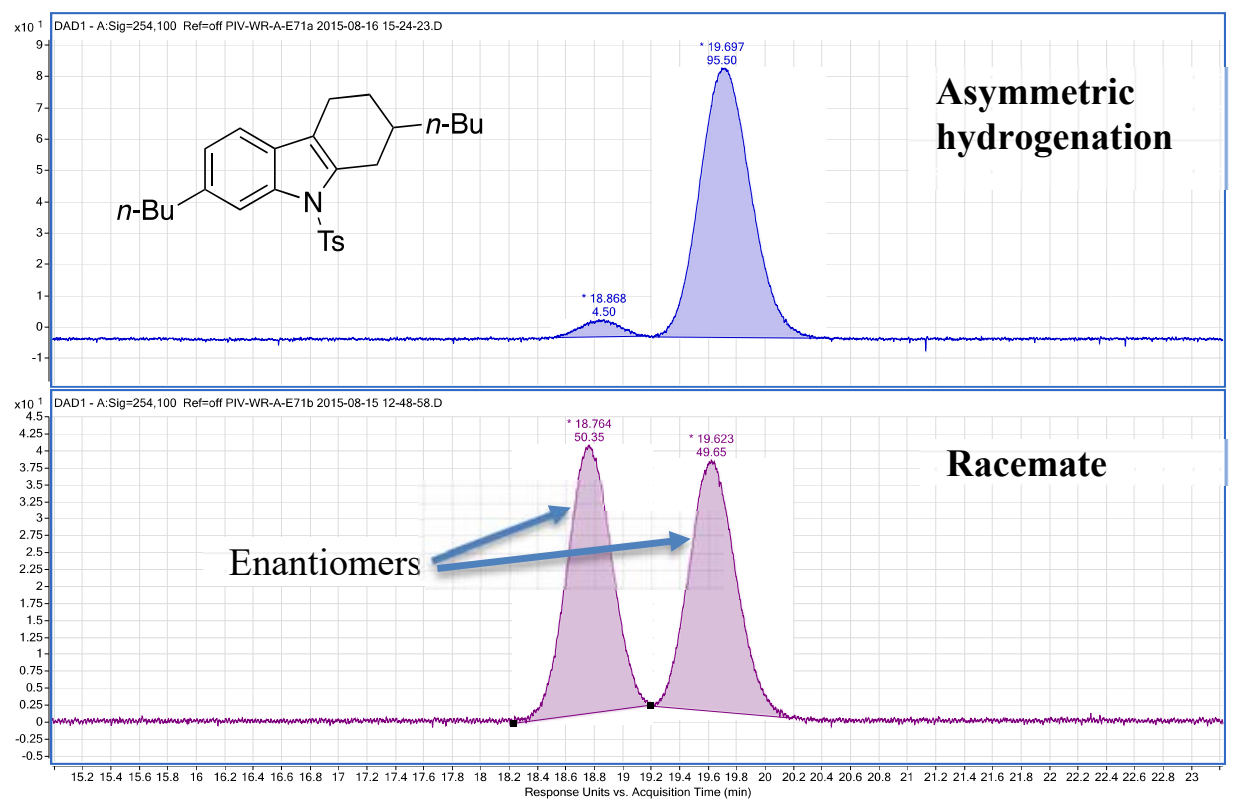


Print Date: 26 Apr 2016 17:15:21

\section{MS Data Review All Plots - 4/26/2016 5:14 PM}

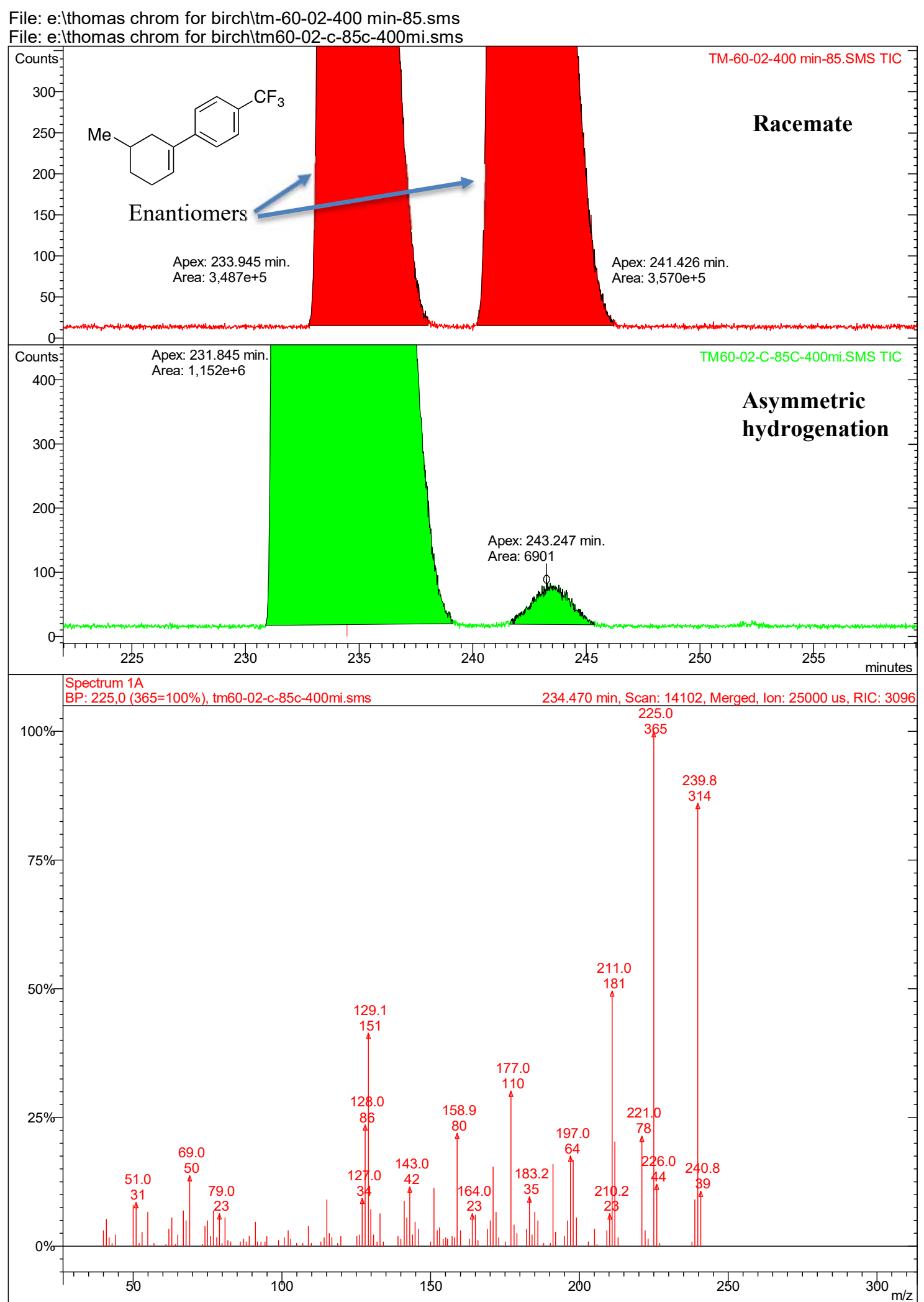


Print Date: 26 Apr 2016 17:23:45

\section{MS Data Review All Plots - 4/26/2016 5:23 PM}

File: e:Ithomas chrom for birchltm-jg-85-85c-200min.sms

File: e:Ithomas chrom for birchltm-jg-83-85c-200min.sms

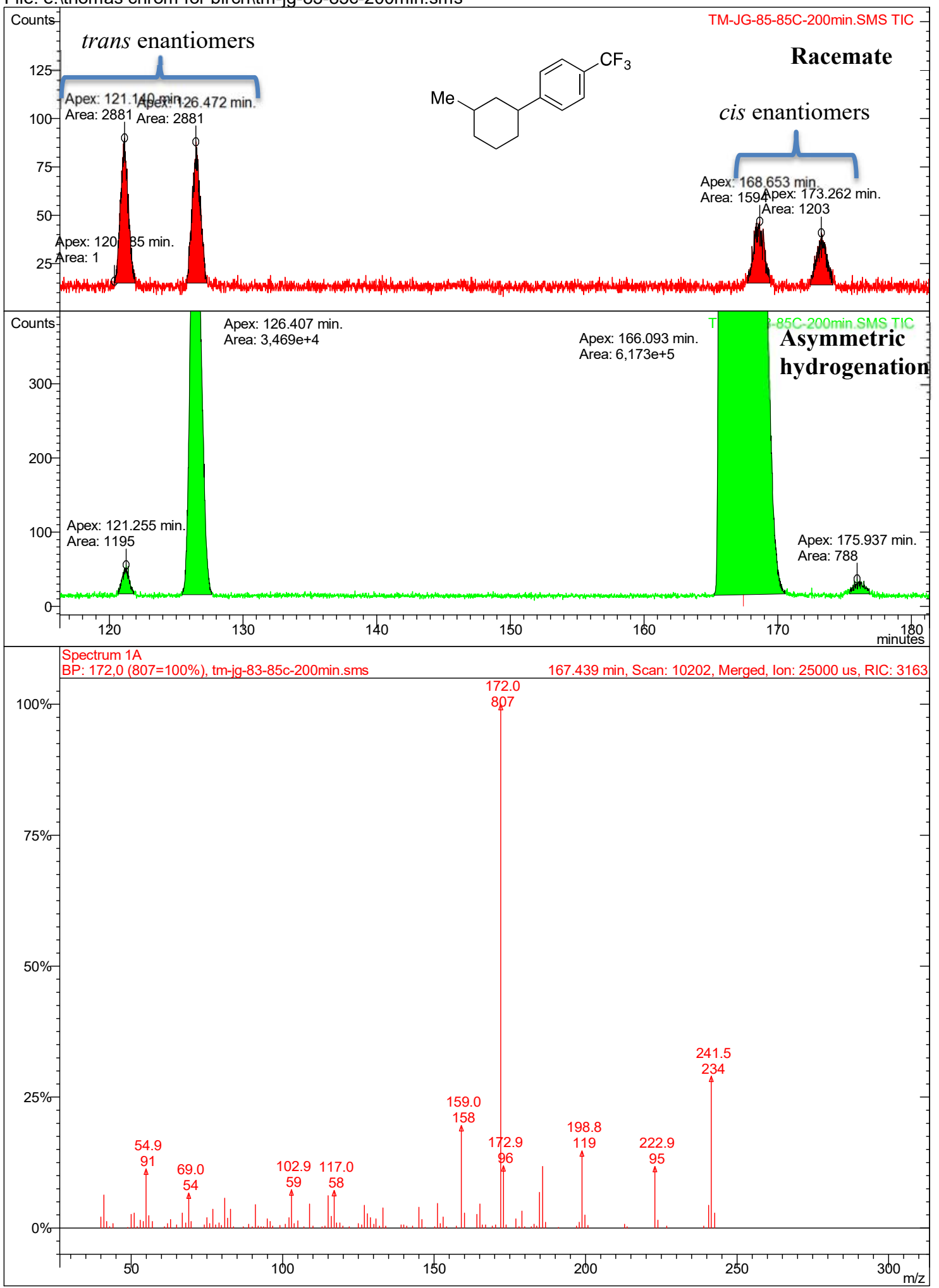


Print Date: 26 Apr 2016 17:31:22

\section{MS Data Review All Plots - 4/26/2016 5:31 PM}

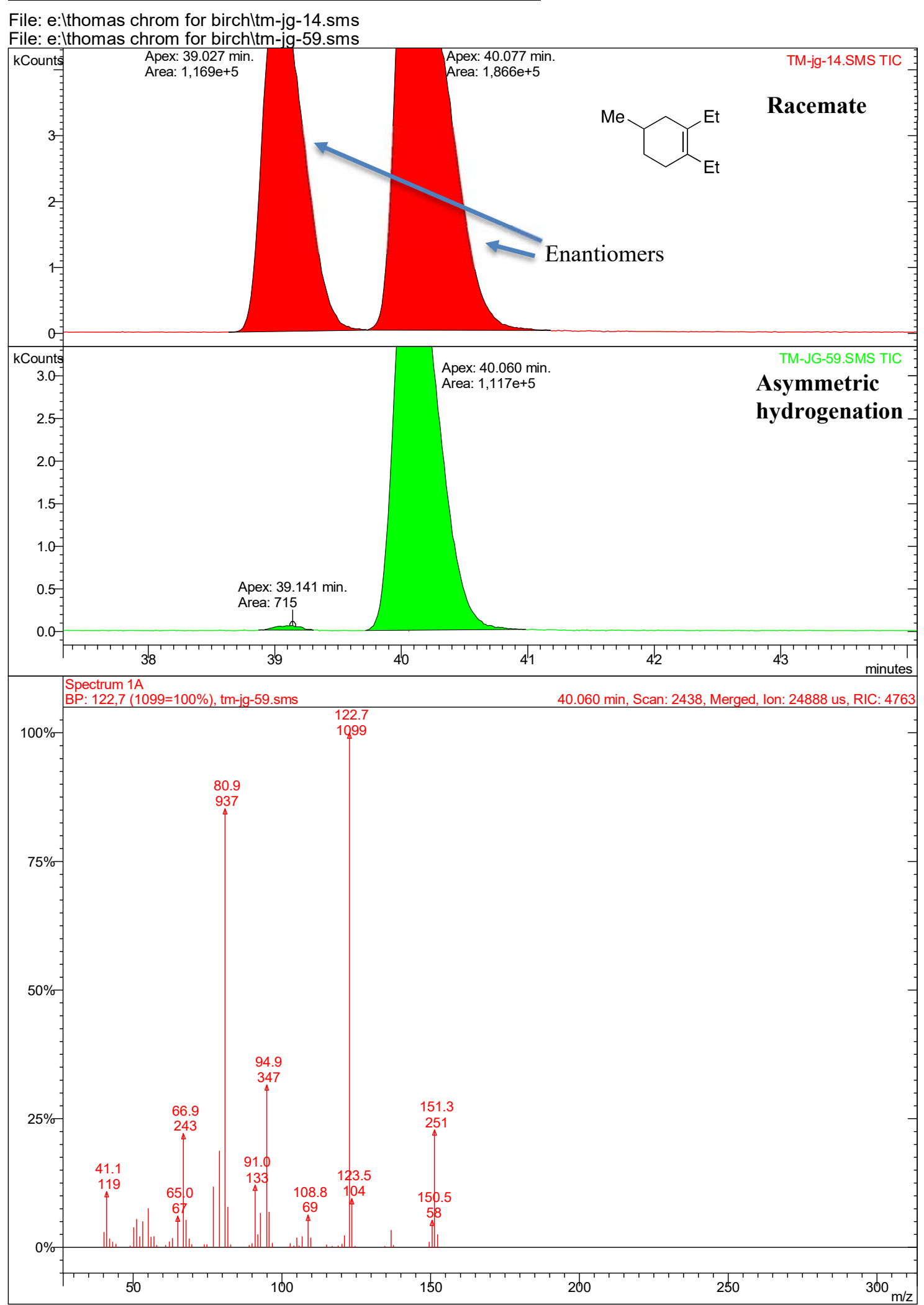


Print Date: 26 Apr 2016 17:35:04

\section{MS Data Review All Plots - 4/26/2016 5:34 PM}

File: e:Ithomas chrom for birchljg6-49-37.sms

File: e:Ithomas chrom for birchljg6-49-35-bdm.sms

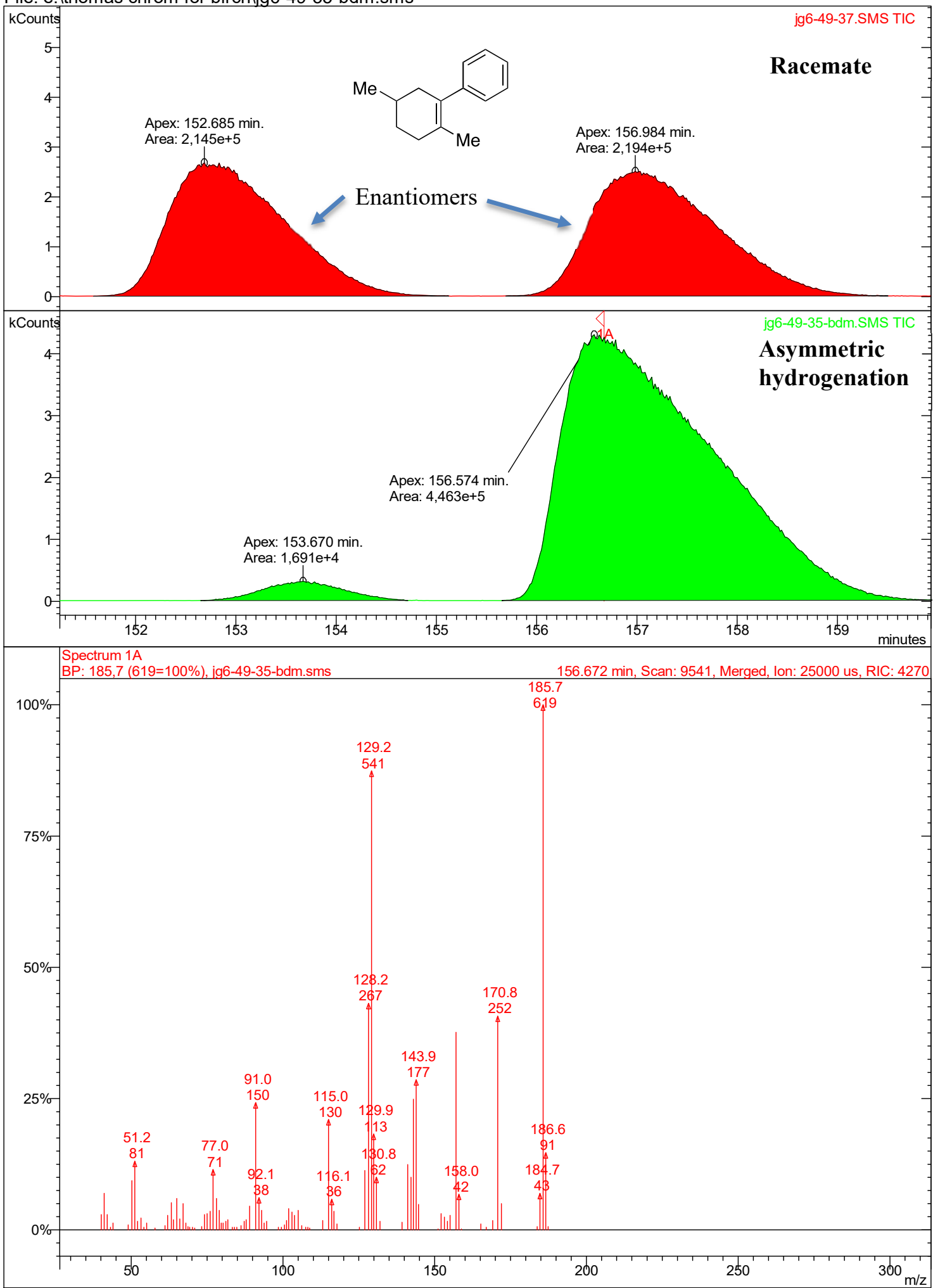



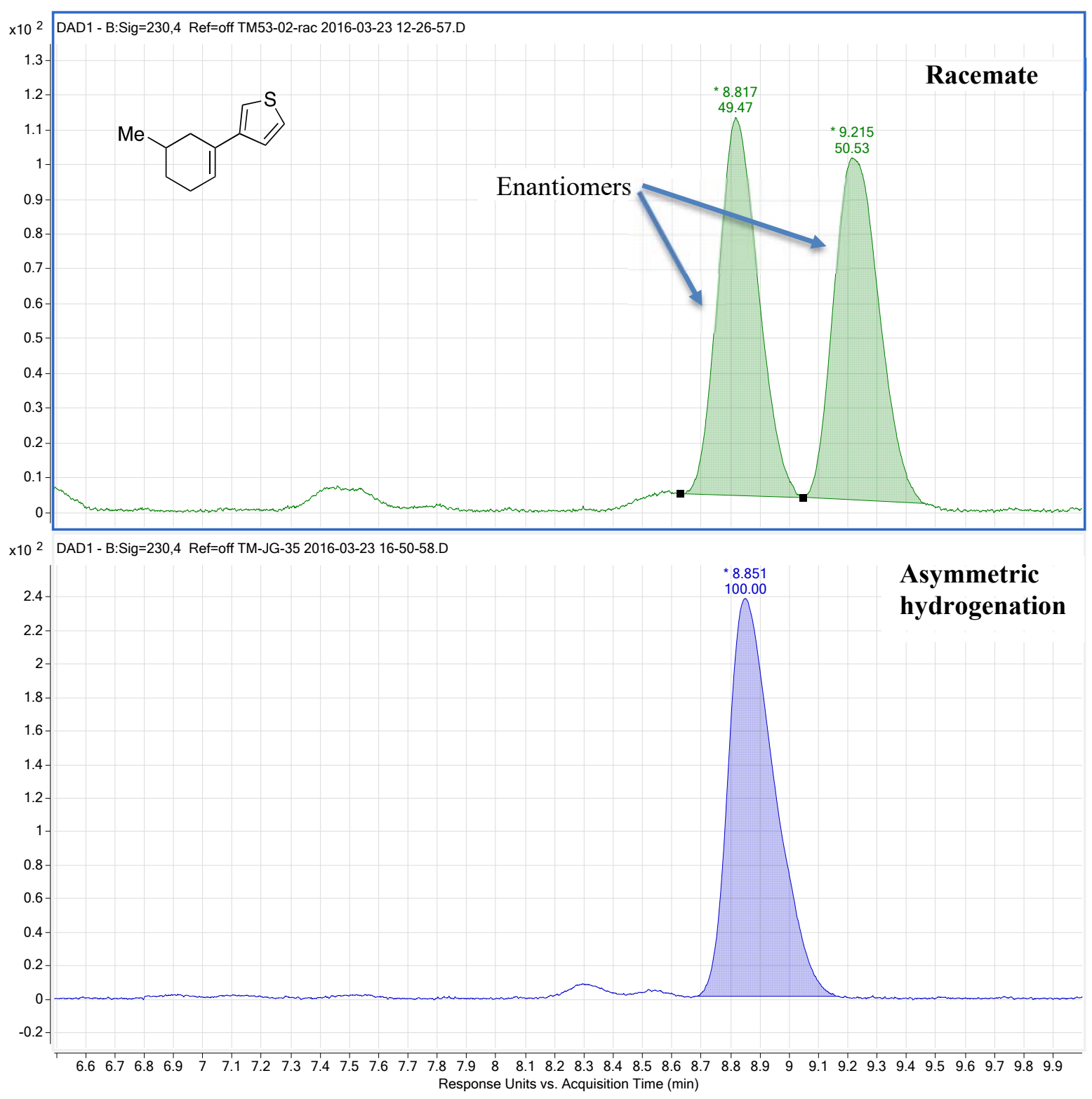

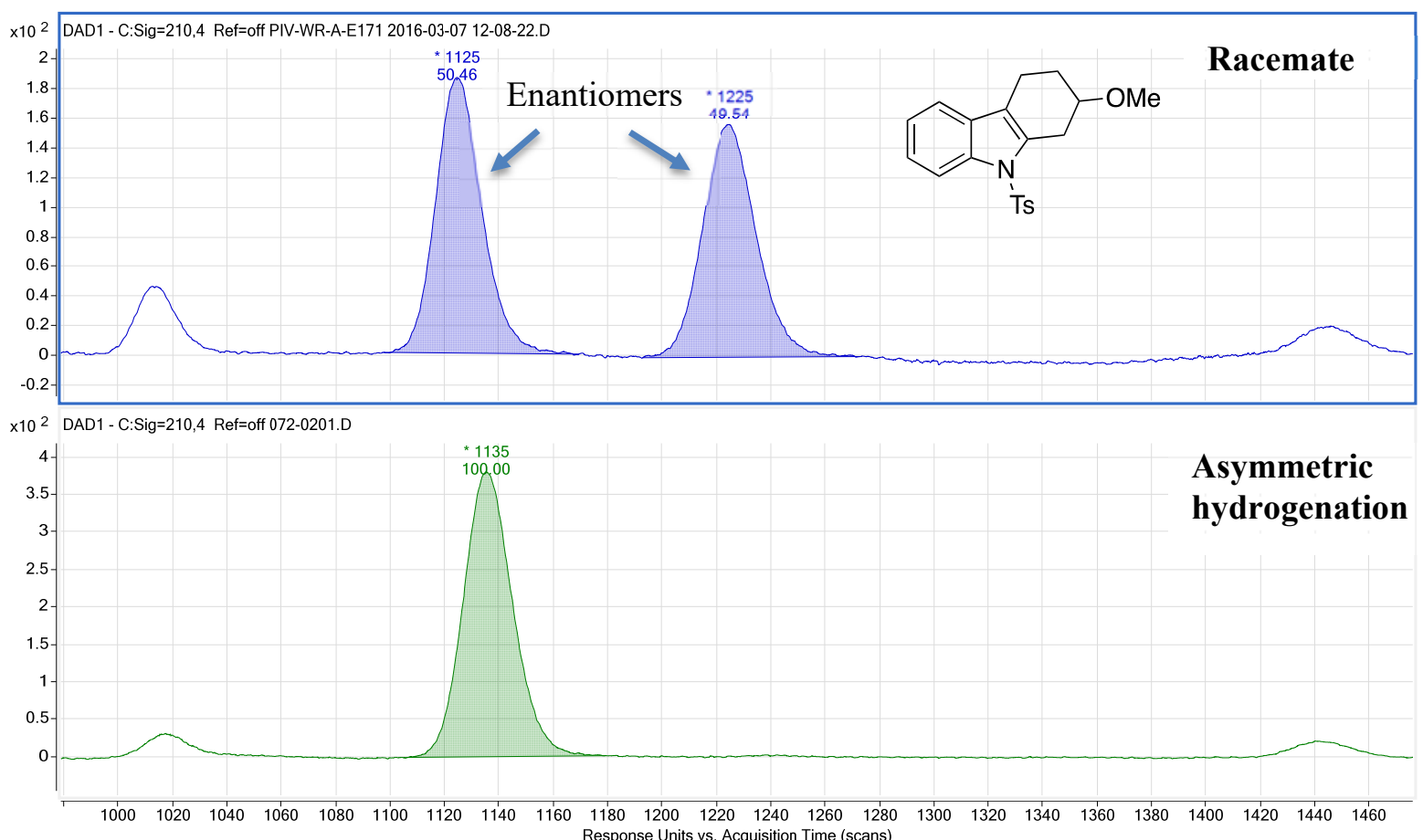